\title{
ENCYCLOPEDOIE SCIENTIFIQUE
}

PERY

BHYHORTEQU

DE $700 \mathrm{LOGH}$
DrREWTUR

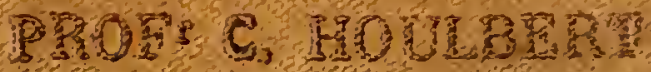

\section{Les Insectes}

Anatomio el Phyziologio genorales

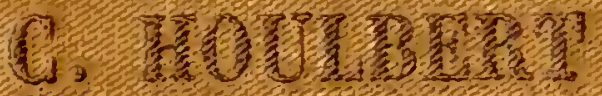


R.C. Sthannon 


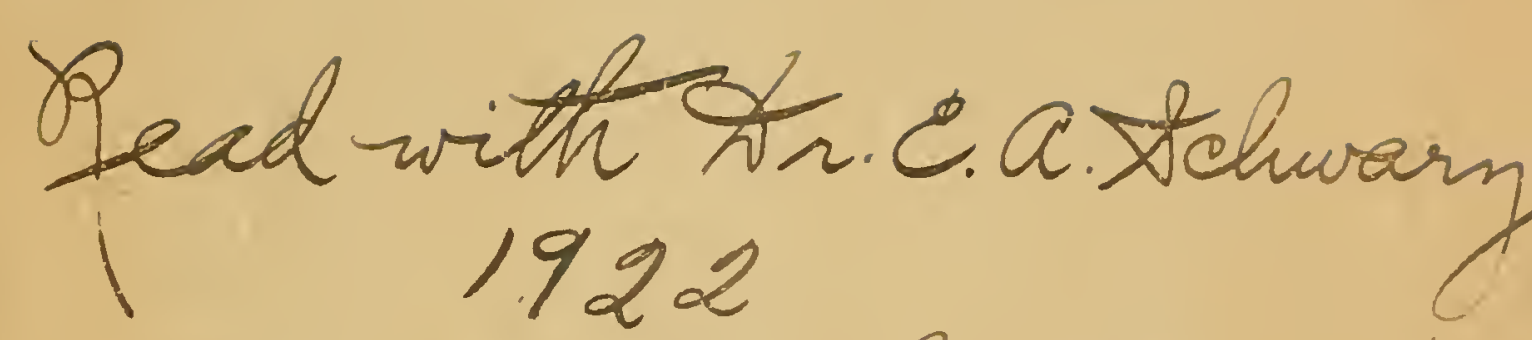
R.e.thannon.

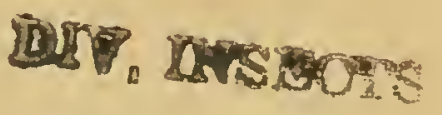



Librairie Octave DOIN, Gaston DOIN, éditeur, 8, place de l'Odéon, Paris.

\section{ENCYCLOPÉDIE SCIENTIFIQUE}

Publiée sous la direction du Dr Toulouse

\section{BIBLIOTHEOUL DE ZOOOLOGIE}

\section{Diregteur : DrGustave LOISEL}

Directeur de Laboratoire à l'École pratique des Hautes Études Professeur de Zoologie des Cours secondaires, à la Sorbonne.

La Bibliothèque de Zoologie, qui formera cinquante volumes environ, comprendra l'étude des grands groupes zoologiques considérés au point de vue général ainsi que la systématique des faunes française ou européenne.

Les volumes qui traiteront des grands groupes zoologiques feront connaître, sous forme d'introduction ou autre, l'histoire des animaux dansle passé, leurs rapports avec l'art, la religion, les légendes, la vie économique des peuples, etc. Ils traiteront ensuite de l'anatomie et de la physiologie comparées du groupe considéré, de son ontogénèse et de sa phylogénèse, de sa classification et enfin de sa distribution géographie qui mettra en évidence ses adaptations diverses aux différents milieux et climats.

Les volumes qui seront consacrés à l'étude particulière de telle partie de la faune seront conçus, non plus seulement au point de vue systématique, comme on l'a fait jusqu'ici pour des ouvrages semblables, mais dans un sens nettement biologique, visant à montrer les différentes modalités de la vie des animaux observés dans leur milieu naturel d'action. Ces ouvrages seront donc autre chose que des catalogues raisonnés. Ils comprendront, d'abord, une sorte d'introduction dans laquelle l'auteur, tout en expliquant les termes spéciaux dont il pourra avoir besoin, saura situer son sujet, en donnant, par exemple, les caractères généraux, anato- 
miques ou biologiques, propres au groupe considéré et en en montrant ses rapports avec les groupes voisins.

Dans le corps même de l'ouvrage, des diagnoses trìs complètes seront accompagnées de tableaux dichotomiques basés, s'il est possible, autant sur l'anatomie que sur la morphologie externe, et donneront toujours les différences propres à l'âge et au sexe. Tous les genres des faunes française et européenne seront déterminés mais, pour certains groupes, l'étude des espèces devra être limitée aux formes les plus typiques on à celles qui présentent un intérêt spécial. Par contre, les auteurs s'étendront sur la vie même des espèces envisagées par rapport aux différents milieux cosmiques et biologiques, ce qui leur donnera l'occasion de montrer le degré de variabilité de ces espèces. De même, il. parleront longuement des mœurs des individus : habitat, régime, moyens d'attaque et de défense, adaptation, parasites, instincts, sociabilité, phénomènes de reproduction (époques é circonstances de la ponte et du rut, accouplement, eufs et nids, incubation et grestation, éducation et développement des petits, métamorphoses, mues, etc.). Enfin ils indiqueront leurs rapports avec l'espèce humaine, soit en ce qui concerne leurs différentes sortes de nuisance, soit en parlant de leur utilité aux points de vue agricole, commercial, industriel ou médical.

Les rolumes de la bibliothèque ainsi conçus, s'adresseront non seulement aux étudiants et aux licenciés des Facultés des Sciences, mais encore aux amateurs éclairés qui sont si nombreux dans les diverses sociétés ou académies scientifiques des grandes villes. Ils ne formeront pas une œuvre de simple vulgarisation; leur but est plus éleré, et bien qu'ils n'aient pas la prétention de répondre à tous les desiderata des naturalistes qui se sont étroitement spécialisés, ils renfermeront une bibliographie assez complète pour qu'ils constituent le vademecum nécessaire de toute personne travaillant un sujet donné.

Tous les volumes, écrits par des auteurs choisis parmi les personnalités les plus autorisées en chaque matière, seront illustrés, sous la direction de l'auteur, par le moyen de photographies ou de dessins, autant que possible originaux.

La Bibliothrque de Zooiogie ne se contentera donc pas de venir donner le reflet de données actuellement acquises; ses ouvrages auront une autre prétention, celle d'être des 
incitateurs à des recherches nouvelles, surtout à l'étude et à l'observation de l'animal vivant, faites dans un sens nettement biologique et expérimental.

Trop longtemps, on n'a considéré chez nous l'animal, que comme objet de musée, de table à dissection ou d'étuve à inclusion pour coupes microspicoques; dans nos nombreuses stations zoologiques, presque toutes situées au bord de la mer, l'on ne peut guère faire que des travaux d'anatomie ou de morphologie comparées, alors que partout autre part, en Amérique aussi bien qu'en Europe, fonctionnent déjà depuis plusieurs années des stations de zoologie expérimentale terrestres aussi bien que marines. De semblables activités ne sauraient tarder à se manifester dans notre pays, croyons-nous, et c'est en partie pour aider à leur, éclosion, pour orienter les zoologistes français dans ces voies nouvelles essentiellement fécondes, que les auteurs de cette bibliothèque ont été chargés d'écrire leurs ouvrages.

Les volumes seront publiés dans le format in-18 jésus cartonné ; ils formeront chacuu 350 pages environ arec figures dans le texte. Chaque ouvrage se vendra séparément.

Voir, à la fin du volume, la notice sur l'ENCYCLOPÉDIE SCIENTIFIQUE, pour les conditions générales de publication. 


\section{TABLE DES VOLUMES \\ ET LISTE DES COLLABOHATEURS}

Les volumes parus sont marqués d'un *

1. Les Protozoaires, 1 rol. par M. FAuré-Fremiet, attaché au College de France.

2. Les Splougiailes, 1 vol par M. TOPSENT, maitre de conférences à l'Université de Caen.

3. Les l'olypes, I vol. par M. Roule, professeur à l'Université cle Toulouse.

4. Les Echimodermes, I vol. par M. Rémy Perrier, chargé de cours à la Faculté des sciences cie l'Université de Paris.

5. Les Insectes, par MM. W'ILAEM; Houlbert, Professeur a l'Université de Remnes; Gúkin, préparateur au Museum d'Histoire naturelle; PICARD, préparateur à l'Institut Pasteur de Paris, etc., etc.

* a. Les Insectes. Anatomie et physiologie générales. - Introduction à l'étude de l'entomologie biologique, $2^{e}$ édition, par C. Houlbert.

b. Les Coléoptères, 3 volumes par C. Houlbert.

6. Les Myriapodes et les Onychophores, par M. WLHEM, professeur à l'Université de Gaud.

7 . Les Arathnides.

8. Les Crustacés.

9. Les Vers.

10. Les Rotifìres, les Bryozoaires et les Brachiopodes.

11. Les Mollusques, 5 vol. pas MM: Varssières, professeur à ]'Université d'Aix-Marseille; QuiNTARET, préparateur à I Université d'Aix-Marseille et DISTASO, attaché au Laboratoire zoologique de Villefranche et à l'Institut Yasteur de Paris.

12. Les Tuniciers, 1 vol. par M. HERDMAN, professeur à l'Université de Liverpool.

13. Les Poissons, 3 vol. par M. Cligny, directeur de la Station aquicole ce Boulogne-sur-Mer.

14. Les Batraciens, 1 vol. par E. Boulenger, D. Se., D. Phil., membre de la Société Royale de Londres.

15. Les Reptiles, 1 vol. par M. Boulename.

16. Les Oiseaux.

17. Les Mammifères, 3 vol. har M. 'Trocessant, professeur de mammalosie au Museum d'Histoire naturelle. 


\title{
ENCYCLOPÉdIE SCIENTIFIQUE
}

\author{
PUBLIÉE SOUS LA DIRECTION
}

du Dr TOuLOUSE, Directeur de Laboratoire à l'École des Hautes-fudes

Secrétaire général : H. PIÉRON

\section{BIBLIOTHĖQUE DE ZOOLOGIE DIRECTEUR: Dr Gustave LOISEL}

Direcienr de Laboratoire à l'École pratique des Hautes-Études Professeur de Zoologie des Cours secncaires, à la Sorlonne.

\section{LES INSECTES}





\title{
LES INSECTES
}

ANATOMIE ET PHYSIOLOGIE GÉNÉRALES

\section{INTRODECTION A L'ÉTLIE: \\ DE L'ENTOHOLOGIL BIOLOGIOCE}

\author{
PAR \\ C. HOULBERT
}

PROFESSEUR A L'UXITERSITÉ DE REXXES,

École Ie I Edecise et de Phardacie

I.AUREAT DE L'IXSTITUT.

DEUXIÈME ÉDITION REVUE ET COPRIGÉE

Avec 207 figures dans le texte.

\section{PARIS}

LIBRAIRIE OCTAVE DOIN GASTON DOIN, EDITEUR

S. PIACE DE L'ODÉT, 8

1920

Tous droits réservés. 



\section{PRÉFACE}

Cet ouvrage s'adresse à ceux qui débutent dans la science difficile des Insectes, et à ceux qui, ne faisant pas de cette étude l'objet principal de leurs occupations, désirent cependant en posséder les notions les plus élémentaires et les plus générales.

Nous nous sommes appliqué, dans ce travail, à présenter l'Entomologie telle que nous la concevons aujourd'hui, c'est-à-dire sous son aspect nettement biologique ; toutefois, l'espace nous étant mesuré, nous nous sommes borné, le plus souvent, à résumer les faits sans les discuter.

Nous nous sommes appuyé de préférence sur les vues d'ensemble, qui permettent d'envisager, d'un seul coup d'œil, les problèmes si divers de la biologie. Pour cela, il va sans dire que nous avons largement mis à profit les œuvres de nos devanciers : Lacordaire, Jacquelin du Val, Packafi, Félix Henneguy, Berlese, etc.

Nous ne voulons pas terminer cette préface sans adresser l'expression de notre reconnaissance la plus vive à MM. Charles et René Oberthür, les savants entomologistes rennais, qui nous ont fourni tant de renseignements précieux, et dont les merveilleuses collections ont été mises à notre disposition avec une bienveillance qui ne s'est jamais démentie.

Rennes, le 12 Juillet 1919.

C. HOULBERT. 


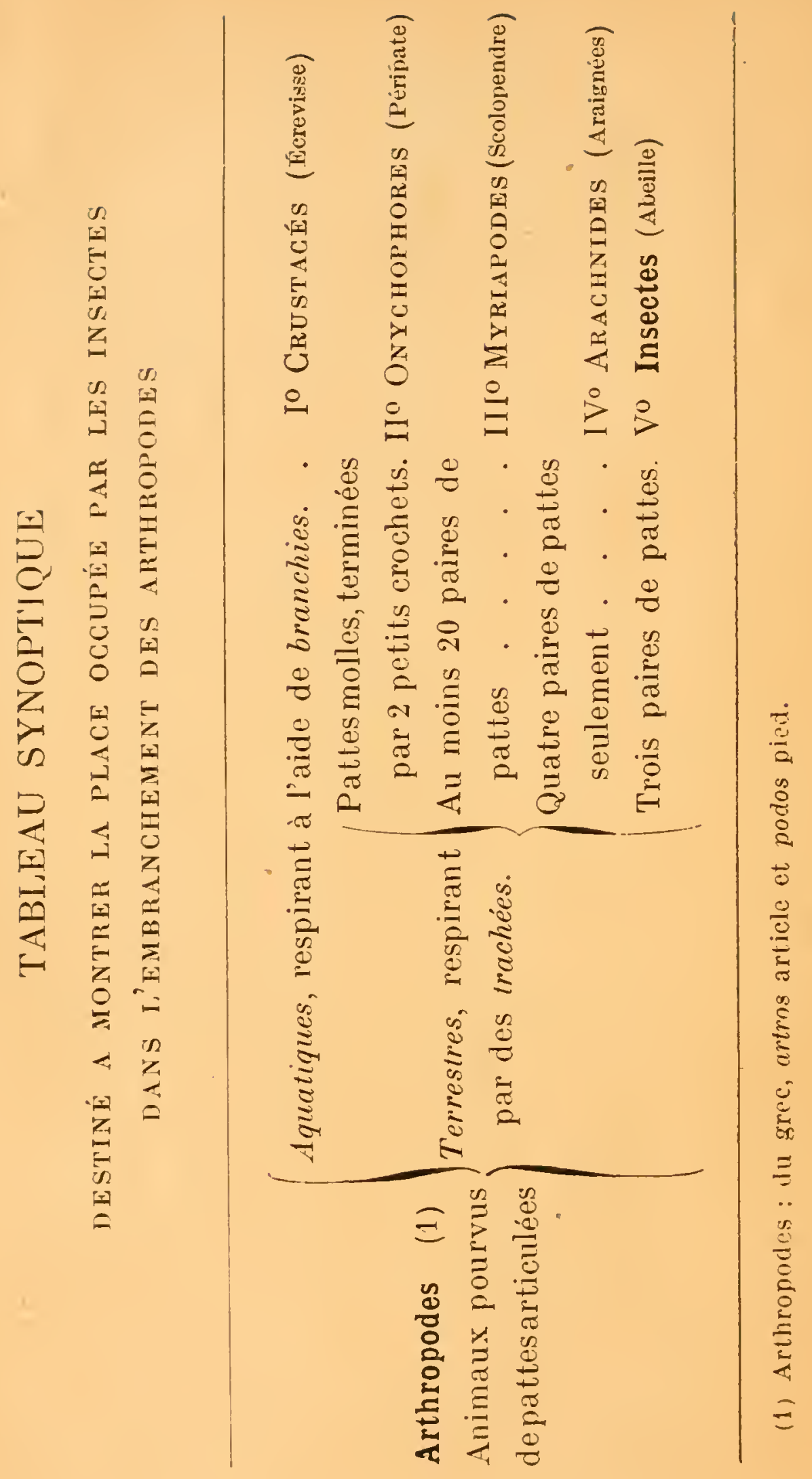




\section{LES INSECTES \\ ÉTUde ÉLÉMENTARE d'EnTOMOLOGIe BloLogloue}

\section{I. - GENERALITÉS SUR L'ERTOMOLOGIE}

Les Insectes, ainsi qu'on peut s'en rendre compte par le tableau qui précède, constituent la 5 e classe du raste embranchement des Arthropodes.

Cette classe est assurément, et de beaucoup, la plus norntreuse et la plus riche de tout le règne animal, puiscru'elle en représente les $4 / 5$ à elle seule.

Plus de 300.000 espèces sont aujourd'hui cataloguées dans les collections particulières et dans nos Musées; mais ce nombre est certainement bien au-dessous de la vérité, puisqu'il existe des régions entières du globe qui n'ont pas encore été explorées ou qui ne l'ont été qu'imparfaitement.

Bien que dérivant sûrement des formes terrestres aptères, les Injectes actuels sont presque tous pourvus d'ailes (1) ét admirablement adaptés à la vie aérienne. Possédant les habitudes les plus rariées "tantôt marchant, tantôt volant", on peut les rencontrel pariout: sous les pierras, sous los débris organiques, dans les

(1) Exception pour les espèces parasites et pour quelques formes secondairement réadaptées. Nous parlons, bien entenchu, des Insectes irinltes.

LES INSECTES. - 2e é.it. 
2 ÉTUdE ÉlÉnENAIRE D’EXTONOLOGIE BIOLOGIQUE eaux douces ou marines, sur les lleurj et mème dans les̉ cavernes les plus obscure?

La science des Insectes se nomme l'Entomologie, du

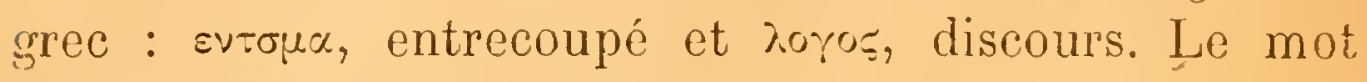
Insecte (insectum, contraction du mot latin intersectum) n'est lui-même que la traduction du terme grec correnpondant.

Par conséquent, si l'on devait s'en tenir strictement aux données de l'étymologie, l'entomologie aurait à s'occuper de tous les animaux articulés, et il en a été ainsi, en elfet, pendant longtemps ; mais aujourd'hu! on est convenu de réserver à cette science un domains plus restreint; elle s'applique exclusivement à l'étude des Arthropodes à trois paires de pattes, tels qu'ils ont été définis par Lamarck en 1801 (1).

\section{II. - DÉFINITION DU TYPE INSECTE}

Le nom d'Insecte n'avait pas, pour les Anciens, le sens précis qu'il a aujourd'hui ; il s'appliquait non seulement à tous les animaux articulés, mais encore, indistinctement, à tous les Invertébrés de petite taille, telles que les Limaces et les Étoiles de mer.

Aucun auteur, cependant, n'est allé aussi loin dans cette voie que notre illustre physicien Réaumur; il voulait faire rentrer dans la catégoric des Insectes, tous les animaux que leur forme ne permettait pas de placer parmi les "Quadrupèdes ordinaires " les Oiseaux et les

(1) Iamarci (A) - Systeme des Animaux sans vertibres nu 'Tabue gén'rub des classes, des ordres et des genres de ces animaux. Paris, 1801. V111-13:1\% in-80. 
Poissons. "La grandeur d'un animal, disait-il, ne doit pas suffire pour l'òter du nómbre des Insectes." A ce titre, " un Crocodile serait un furieux Insecte», et Réaumur arouait, en effet, qu'il n'aurait eu aucune peine à lui donner ce nom, ainsi qu'à tous les Reptiles.

Ces conclusions bizarres indiquent combien l'anatomie comparée était peu avancée au début du xrirr siècle, puisque des savants de distinction n'avaient encore aucune notion des trpes organiques et pouvaient placer, dans un même groupe, des êtres aussi différents que les Mollusques, les Insectes et les Vertébrés.

Aujourd'hui, la classe des Insectes est limitée d'une façon précise ; et, pour tous les naturalistes, les animaux qui la composent peurent être définis, à l'état adulte, par les caractères suirants :

10 Ils respirent presque tous l'air' en nature à l'aide de trachées (d'où le nom de Trachéates).

20 Leur corps est divisé en trois parties: tête, thorax et abdomen:

$3^{0}$ Le thorax porte toujours trois paires de pattes et, généralement, deux paires d'ailes.

40 L'abdomen est complètement dépourvu d'appendices locomoteurs.

Il a fallu arriver jusqu'à Lamarck (1801), c'est-à-dire jusqu'au commencement du siècle dernier, pour voir l'expression d'Insectes exclusivement réservée aux Arthropodes à trois paires de pattes; ce ne fut mème qu'en 1817, qu'un auteur anglais, Leach, en établissant le groupes des Myriapodes, fixa les limites définitives de la classe des Insectes. 
4 Étude Élémentaire d'entomologie biologique Ces vues entrèrent d'ailleurs presque aussitôt dans le domaine de la pratique sous l'influence de Latreille qui, en 1832, publia, dans son Cour's d'entomologie (1), une classification des Articulés, où les Insectes sont très nettement définis sous le nom d'Hexapodes ou Condylopes à six pattes.

(1) LAtreille (P. A.) - Cours d'entomologie ou de l'histoire naturelle des Crustacés, des Arachnides, des Myriapodes et des Insectes. Paris, Roret, 1831, avec atlas de $2 \pm$ planches. 


\section{INTRODUCTON}

\section{HISTOIRE DE L'ENTOMOLOGIE}

Il est bien difficile d'établir avec précision une histoire complète de l'Entomologie, dans le cadre d'un ouvrage aussi restreint que celui-ci. Les diverses branches de la science se sont d'ailleurs déreloppées simultanément et il conriendrait d'examiner chacune d'elles isolément, dans sa marche et dans ses progrès; mais, comme cette méthode nous exposerait à de nombreuses répétitions, nous sommes nbligé de suivre, purement et simplement, l'ordre chronologique.

Au début, la science est seulement descriptive ; les auteurs n'envisagent guère que l'aspect extérieur des organes et leur usagé immédiat; plus tard, lorsque les détails de la forme deviennent mienx connus, on épronve le besoin de les comparer entre eux, afin de grouper rationnellement les espèces ; on arrive ainsi, petit à petit, à la, classification.

Puis les naturalistes s'aperçoivent que les particularités de l'organisation interne peuvent très utilement servir à compléter les classifications ; ils étudient alors la structure anatomique, le développement et les métamorphoses.

Nous envisagerons, dans l'histoire de l'Entomologie, les trois époques suivantes, qii correspondent plus ou moins exactement aux tendances des périodes indiquées ci-dessus :

$1{ }^{\circ}$ L'entomologie dans l'antiquité et au moyen àge ;

$2^{\circ} \cdot$ L'entomologie dans les temps modernes;

: I J'entomologie dans les temps actuels.

I. L'ENTOMOLOGIE DANS L'ANTIQUITÉ ET AU MOYEN AGE

\section{$1^{0}$ Égypte et Grèce.}

On trouve fréquemment des Insectes peints ou sculptés sur les monuments de l'ancienne Égypte; Latreille, dans son opuscule sur

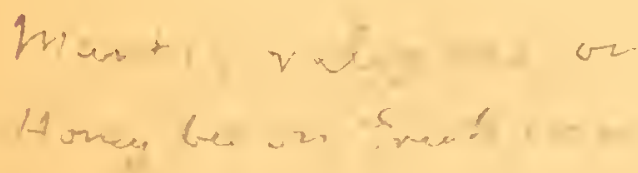


les insectes sacrés, indique le Sphex, l'Abeille et le Scarabée rouleur de boules (1) ; ce dernier surtout était hautement vénéré des Égyptiens, qui lui attribuaient une multitude de vertus réelles ou symboliques.

D'un autre côté, un certain nombre d'Orthoptères sont cités dans le livre de l'Exode, parce que les Hébreux furent souvent obligés de se nourrir de Criquets dans le cours de leurs pérégrinations à travers les déserts de l'Arabie Pétrée ; Moïse pouvait même déjà, semble-t-il, distinguer entre eux quelques genres voisins, tels que les Grillons, les Locustes, et les Truxales, car il les indique en parlant des animanx qu'il est permis ou défendu de manger (Voir p. 302).

Il est à peine besoin de faire remarquer ici que l'une des plaies d'Égypte mentionnées dans l'Écriture (la $\mathrm{X}^{\mathrm{e}}$ ), n'est autre chose que l'mne de ces terribles invasions de Sauterelles qui se reproduisent à peu près périodiquement dans ce pays (2).

Wais tout cela, dit Lacordaire, n'est pas encore de la science; pour voir l'Fntomologie prendre, en quelque sorte, une forme arrêtée, il faut se transporter en Crèce, all temps d'Aristote, dont les écrits peurent être considérés comme le résumé de tout ce que l'antiquité connut en ceite matière (3).

Aristote divisait tous les animaux en deux grandes catégories . les Enaïma, qui avaient du sang (4), et les Anë̈ma qui n'en avaient pas. C'est à ce dernier groupe qu'appartenaient les ENTOM A (5), c'està-dire les animaux poulvus de pieds et ayant le corps divisé par des incisions plus ou moins profondes.

En réalité, quarante-sept insectes seulement sont mentionnés dans les écrits d'Aristote; mais, comme ils ne sont désignés que par le nom du genre et qu'aucune description ne les accompagne,il est à peu près

(1) Latreiche (P). - Des Insectes peints ou sculptés sur les monuments antiqucs de l'Egypte. Paris, 1819, $21 \mathrm{pp} .1 \mathrm{pl}$.

(2) Voir : Les Orthopteres E S. : Acridium agyptium et Pachytilus miyratorius.

(3) Le résumé listorique que nous publions ici est, en grande partie, emprunté A I.Acordsinr, pour le $\mathrm{xv}^{\mathrm{e}}$ et le $\mathrm{XvI}^{\mathrm{e}}$ siècle : Introduction à l' Entomologie. $2^{9}$ rol., b. 619 .

(1) Il faut entendre du sang rouge, par conséquent bien visible; les Ancïma compreniaient tons les animanx dont le sang est ineolore, c'est-ì-dire ì pen près tous les invertébrés.

(5) D'oul le mot : entomolosie. 
inrossible de savoir avec lesquelles de nos espèces actuelles ils pourraient être identifiés (1).

\section{$2^{\circ}$ Rome et l'Europe aux premiers siècles.}

Les Romains, uniquement préoccupés de guerres ou de commerce, ne s'intéressèrent pas aux sciences naturelles; ILLIXf (l'Ancien) ne fait donc que reproduire les idées d'Aristote. Il les déforme même lo plus solivent en les encombrant des légendes et de toutes les croyances populaires gui avaient cours de son temps. De lui, on ne doit retenir qu'une chose, c'est que, contrairement à l'opinion du grand philosophe grec, il croyait pouvoir admettre - oh! bien timidement d'ailleurs, - qu'il existe quelqque chose d'analogue au sang chez les Insectes : sanguinem non esse in his fateor, sicut ne terrestribus quidem cunctis, rerum simile quiddan (Livre XI, p. 379. édit. Lemaire).

Puis, la nuit du moyen âge s’étendit sur toutes les connaissances humaines; dans ce long intervalle de temps. l'entomologie, elle aussi, fut presque totalement oubliée; rien ne fut ajouté au fonds de connaissances transmis par les Grecs et les Romains. AleERT i.E (irand lui-même, le savant évêque de Ratisbonne (1193-1280), qui consacra un livre entier de ses cuvres à l'histoire des insectes, ne fit que recopier, comme il.l'avoue loyalement, les écrits d'Aristote.

Et il en fut ainsi jusqu'an milieu du xriI ${ }^{\mathrm{e}}$ siècle.

\section{L'ENTOMOLOGIE DANS LES TEMPS MODERNES}

1. -- I.'invention de l'imprimerie et la découverte du NouveauMonde paraissent avoir été le signal d'un réveil général de l'esprit humain ; que!ques naturalistes eurent l'audace- de penser qu'Aristote n'avait peut-ritre pas tout vu; ils cherchèrent alors, dans l'observation directe de la nature. la solution de certains problimes que l'étude des écrits des anciens ne pouvait leur donner: mais l'influence du grand philosophe ionien se fit encore sentir bien longtemps, à tel point que Coxran Gesser (1516-1565), surnommé le Pline moderne, Aldrovaxn lui-même, "le plus infatigable compi-

(1) ARISTote. - Histoire des animaux, texte grec avec traduction française Paris, Camus, 1783,2 vol. in $-4^{\circ}$. 
lateur qui ait jamais existé ", ne s'en libérèrent jamais complètement.

L'ouvrage du premier sur les Insectes était inachevé quand il mourut; il ne fut publié que très longtemps après sa mort, en 1634, par Théodore de Mlayerne, l'un des médecins de la cour de Charles I ${ }^{\text {er }}$; il n'est d'ailleur's remarquable que par la prodigieuse érudition de !'auteur (1).

L'ouvrage d'Aldrovandi (2) renferme une première ébauche de classification qui contribua, dans une certaine mesurn, à accélérer les promrès de l'entomologie naissante.

Nous ne sommes pas encore sortis, comme on le roit, des tâtonnements de l'antiquité; mais nous touchons cependant à une époque où le règne des anciens va prendre fin; l'observation va l'emporter sur l'érudition, et, dans le courant du xvirre siècle, la science va, pour ainsi dire, se rénover de fond en comble. GeDART ouvre la voie, arec son Traité sur les métamorphoses des Insectes (3), fruit de quarante années d'observations; et Fraxcors ReDi, appuyé sur le guide le plus solide qui soit au monde, celui de la méthode expérimentale, porta le premier coup aux stériles spéculations de. l'antiquité, en démontrant l'inexactitude de la fameuse théorie de la génération spontanée (4).

2. -- Une ère nouvelle s'ouvre pour les sciences naturelles avoc la découverte des instruments grossissants ; on ne s'était guère attaché jusqu'alors qu'à l'étude des caractères extérieurs des Insectes, on peut maintenant s'appliquer aux détails de leur organisation interne.

Malpighi, célèbre médecin de Bologne, fit paraître, en 1669 , le premier traité sur l'anatomie des Insectes, à l'occasion de ses recherches sur la Chenille du ver à soie (5). Ce petit volume de 100 pages, orné de douze planches, n'est, selon l'expression enthousiaste de Réaumur, riu'un tissu de découvertes, où l'on peut prendre

(1) Fut publie sous ce titre: Insectorum sive minimorum animalium theatrum. Londres, 1634 .

(2) Ardrovandi (Ulysse). 1522-1607, professeur al l'Université de Bologne : De animalibus Insectis, libri VII, Bononioe, 1602.

(3) CigD.sRT (J.) - Metamorphosis et historia naturalis Inscctorum. Medioburgi. Jac. Fierensium 166:-1667, 3 vol. pet. in- $8^{\circ}$.

(4) "Dalle earni degli animali morti non s'iugenerio i vermi, se in quelle da altri animali viventi non se sienn portate le semenze. "

(5) Malpign (M.) - Dissertatio cristolica de Bombyce cum figuris. Londres, 1669 , in-4". 
"plus de connaissances sur l'admirable composition des Insectes, que dans tous les ouvrages ensemble qui l'ont précédé. "

Mais, si remarquables que fussent les travaux de Malpighi, ils furer $t$ cependant bientôt dépassés rar ceux du hollandais Swammerdam.

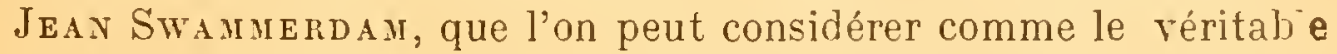
fondateur de l'anatomie entomologique, était fils d'un apothicaire d'Amsterdam (1637-1680). Repoussé par sa famille qui ne lui pardonnait pas d'avoir abandonné la médecine pour les sciences naturelles, malade et presque sans ressources, il ne put publier, de son vivant, que la plus faible partie de ses travaux. Ses manuscrits circulèrent de main en main pendant près de cinquante ans; enfin son compatriote, le célèbre Boerhaave, les racheta en 1729 , et, après les avoir mis en ordre, les publia à Leyde, huit ans après, sous le titre de Bible de la nature (1).

De son temps (1838), Lacordaire considélait encore cet " ouvrage admirable " comme indispensable à celui qui voulait connaître l’anatomie des Insectes. Jusqu'à cette ér oque on avait, en effet, toujours cru, qu'au cours deses métamorphoses, la chenille se changeait brusquement en chrysalide; et celle-ci, de même, brusquement en Papillon. Swammerdam démontra que le Papillon était déjà tout formé sous la peau de la chrysalide, ce qui est rrai dans une certaine mesure ; toutefois, quittant alors le domaine de l'observation directe pour celui de la spéculation, il crut pouroir avancer que les organes de la chrysalide sont à leur tour renfermés, à tout âge, dans la chenille; il alla même jusqu'à admettre que le Papillon est déjà tout formé dans l'œuf. "La Chenille, dit-il, est le Papillon même revêtu d'une membrane qui nous cachait tous ses membres. Les nymphes sont cachées dans le ver, ou plutôt sous la peau, de la même manière qu'une fleur tendre est renfermée dans un bouton."

Parti de faits parfaitement exacts, ilen arriva à formuler sa lameuse théorie de la préformation ou de l'emboîtement des germes, qui arrêta pendant cent ans tous les progrès de l'embryogénie.

Cependant, on doit être reconnaissant à Swammerdam d'avoir

(1) SwAMmerdu (J.). - Biblia natura, seu historia insectorum, belgice cum versione latina H. D. Gaubii, et rita auctoris, Leyde, 1737-1738, 2 vol. in-folio. Sur la fin de sa vie, Swגumerd.u s'engoua des idées mystiques de Mlle Bourignon, et, croyant offenser Dieu par ses étucies anatomiques, fit jeter au feu ses propres

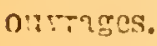


introduit les procédés de la technique expérimentale en micrographie; c'est lui qui, le premier, employa les réactifs fixateurs et durcissants pour étudier plus facilement les parties molles des organes ; c'est lui qui imagina la méthode des injections, qui a rendu et rend encore tant de services dans l'étude de la vasologie: il excellait dans l'art de préparer les Insectes et surtout les "Chenilles par insufflation ".

Parmi les auteurs qui s'illustrèrent encore à cette époque dans l'étude de l'entomologie, nous citerons seulement Martin Listra, qui publia, après les avoir améliorés, quelques-uns des travaux de Gredart et de John Ray ; puis Melle Sibylra de Mérian qui, dans un élan d'enthousiasme, s'exila pendant deux ans à la Guyane hollandaise, afin d'étudier les métamorphoses des insectes de ce pays. Le principal ouvrage de cette habile artiste. qui parut à Amsterdam en 1705 , n'est remarquable que par sa rareté et par la splendeur des planches qu'il renferme (1). Une édition française en a été donnée par Buchoz en 1771.

Enfin Leuvenifeck et Vallisieri firent connaître le développement de plusieurs espèces vulgaires; c'est au premier que nous devons presque toutes nos connaissance sur les métamorphoses de la Puce commune; il fabriquait lui-même les microscopes dont il se servait; et, c'est à l'aide de ces instruments primitifs, qui ne grossissaient guère au delà de soixante diamètres, qu'il fit, comme on le sait, ses plus remarquables découvertes.

3. - Presque seules jusqu'ici, les études anatomiques et descriptives avaient retenu l'attention des entomologistes; mais un auteur parait qui, de suite, s'acquiert une gloire immortelle pour la sagacité et la patience avec lesquelles il a scruté les mœurs des Insectes, c'est Réaumur.

René Antoine Ferchaud na Réaumur naquit à la Rochelle en 1683 ; à la fois physicien, naturaliste et mathématicien, il s'illustra dans presque toutes les branches des sciences; mais, sa célébrité universelle, il la doit surtout à son talent d'observateur et à la disposition ingénieuse des expériences qu'il entreprit (2). Aucun auteur,

(1) Meriax (Sibylle de). - Metamorphosis insectorum surinamensis. Walk, Amsterdam, 1705, gr. in-folio avec $60 \mathrm{pl}$.

(2) Riaubur (Ferch. de). - Mémoires nour servir à l'histoire nalurelle ct à l'anatomie des Insectes. Paris, 1734-42, 6 vol. in $4^{\circ}$. 
peut-̂̂tre, n'a contribué autant que lui à rendre l'entomologie sttrayante et populaire.

Ce grand savant mourut accidentellement d'une chute de cheval à l'âge de 7 '́t ans; ses restes reposent dans une pauvre église de la Mayenne (1).

Les travaux de Réaumur suscitèrent de nombreuses et fécondes vocations ; c'est ainsi que CharLes Boñet, de Genève, découvrit, à l’âge de vingt ans, la parthénogénèse des Pucerons. Ayant isolé un Puceron du Plantain, il put le suivre et le voir se reproduire, sans accouplement, jusqu'à la dixième génération.

4. - La gloire d'avoir fondé les méthodes et fixé les règles de la nomenclature appartient à Linné. CHAfLEs LINxé ne fut pas un entomologiste au sens restreint de ce mot, car son activité s'étendit ¿̀ l'universalité des êtres vivants; mais son génie clairvoyant sut établir la classification des Insectes sur une base solide, en utilisant des caractères de premier ordre, habilement hiérarchisés ; tels, par exemple, le nombre et la conformation des ailes (2).

Il y aurait bien quelques réserves à faire à propos de la classification de Linné; mais, en somme, à part l'absence du groupe des Orthoptères, qui ne fut délimité que cinq ans plus tard par le baron de Geer; à part la suppression du groupe des Aptères et quelques autres modifications de détail, les ordres d'Insectes que nous distinguons aujourd'hui, sont encore ceux qui furent établis par le grand naturaliste suédois.

Ciarles de GEer, dont nous parlions tout à l'heure, tient à la fois de Réaumur et de Linné ; comme le premier il fut un anatomiste $\mathbf{h}$ ibile et un excellent observateur; comme le second, mais avec moins de bonheur, il se distingua aussi dans les travaux de systématique. Ses ourrages forment sept volumes in $-4^{\circ}$ avec 238 planches ; chose remarquable, bien que composés par un suédois et imprimés à Stockholm, ils sont rédigés en français (3).

(1) A Saint-Julien-du-Terroux, canton de Lassay.

(2) Linné (Ch.). - Systema nature, sive regna tria naturce systematice proposita per classes, ordines, genera et species. Th. Haak, Lugduni-Batavorum (Leyde) 1735, gr. in-fol.

(3) Grer (Ch. de). - Mémoires pour servir à l'histoire des Insectes. Stockholm $1752-1778,7$ vol. $1 \mathrm{n}-4^{\circ}$.

A cette époque, le français était déjà considéré comme une langue savante, et remnlaçait fréquemment le latin. 
La période anatomique du Xrme siècle doit être close avec Pifri E LYoNeT, né à Maestricht en 1707. Le premier travail de cet auteur fut la traduction de la Théologic des Insectes de Lesser; mais son chef-d'œuvre, l'incomparable Traité anatomique de la Chenille du Saule (Cossus ligniperda), peut être considéré comme l'un des plus admirables travaux qui aient jamais été produits sur l'anatomie des Insectes (1). Cet ouvrage renferme dix-huit planches sur cuirre, gravées par l'auteur lu:-même, et chacune de ces planches est un chef-d'œu vre d'exécution.

C'est aussi dans les travaux de Lyonet que " nous trouvons indiqués, pour la première fois, les corps qu'on désigne aujourd'hui sóus le nom de disques imaginaux ou d'histoblastes " et dont le rôle est si important dans le développement des organes appendiculaires des Insectes.

5. - Les travaux d'entomologie deviennent si nombreux et si variés à cette époque, qu'il est impossible de les citer tous, et plus difficile encore de les relier les uns aux autres; ce sont toutefois les études de systématique qui vont dominer; et, parmi les auteurs qui nous intéressent le plus, nous Français, il faut citer le célèbre médecin parisien, Geoffroy, qui publia, en 1762, la première faune loçale sous ce titre : Ḧistoire abrégée des Insectes des environs de Paris, Paris, 1762. 2 vol. in $4^{\circ}, 22 \mathrm{pl}$. - Latreille estimait hautement cet 0 !rrage ; "si quelque main habile le rajeunissait, disait-il, il contribuerait plus que tout autre à populariser la science (2). "C'est aussi Geoffroy qui employa. le premier, le nombre des articles aux tarses pour classer les Coléoptères.

Un autre auteur, Jean-Cirristian Fabricius, professeur à líel, chercha, luı aussi, les bases d'un système de classification des Insectes, et il crut trouver ces bases dans la conformation des pièces de la bouche, dans les "instruments cibaires", comme il les appelle

Il ne faut pas oublier que Fabricius est l'un des plus fervents disciples de Linné ; comme son maître illustre, il pensait que les caractères les plus importants de la classification doivent être tirés d'un seul et même organe, et il voulut appliquer sa manière de voir à l'entomologie.

(1) Lyonet (P.). - Traité anatomique de la chenille qui ronge lé bois de saule. La Haye, 1760 , in-4 600 pages avec $18 \mathrm{pl}$.

(2) Discours prononcé le 19 février 1832. ì l'ouverture de la première séance de la société entomologique de lirance. 
Les ouvrages de Fabricius sont très nombreux et nous ne pouvons pas les analyser ici ; c'est dans un Supplément à la $2^{\mathrm{e}}$ édition de son Entomologie systématique (1), qu'il faut aller chercher l'exposé complet de son système de classification.

Nous pourrions encore noter, à cette époque, les travaux de CiairVILLE et d'ILLiger; nous nous bornerons seulement à rappeler le nom d'Antoine Olivier, auteur de l'Histoire naturelle des Insectes et de l'Encyclopédie méthodique, parce qu'il ajouta l'ordre des Orthoptères à la classification de Linné (2).

6.-Avecle xix siècle s'ouvre, en quelquesorte, une ère nouvelle pour l'entomologie. AxtorneLAURENT DE JUSSIEU vient d'appliquer la méthode naturelle (3) à la classification du règne végétal (1789); Latreille s'efforça d'appliquer les mêmes principes à la classification des Insectes (4).

Pierre-ANDré LATREILle, né à Brives en 1762, mort à Paris en 1833, peut être considéré comme. l'un des plus

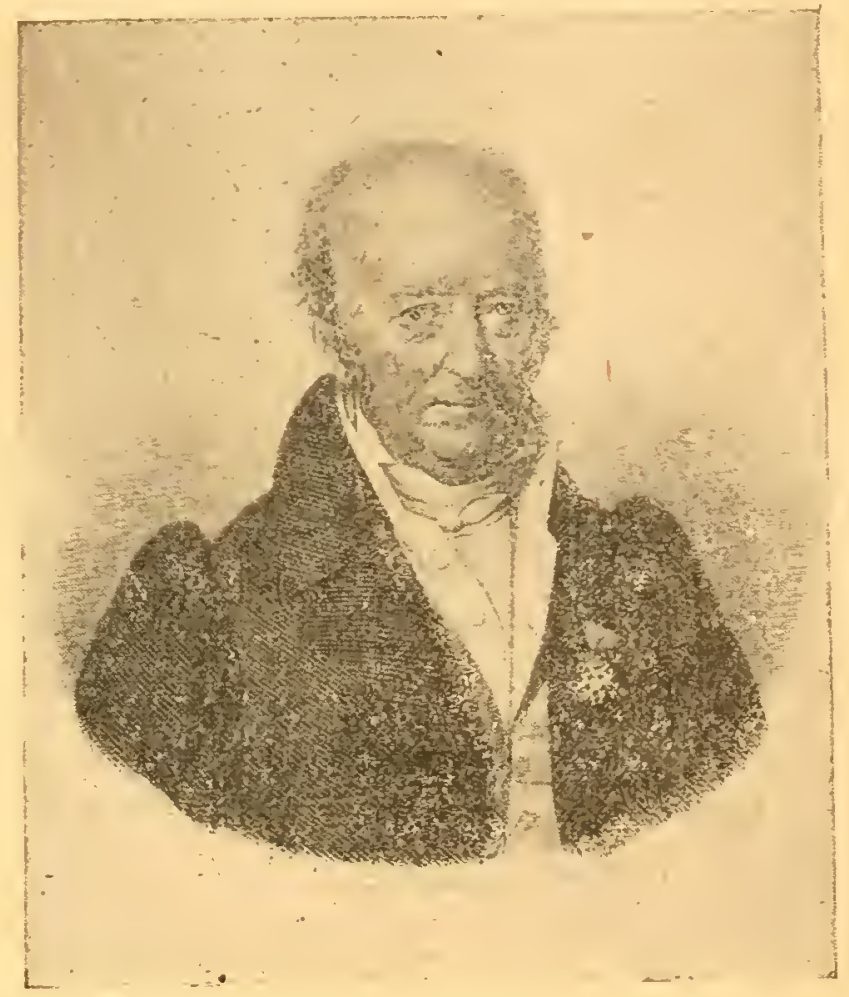

Fig. 1. - L.ATreille (Fierre-André), né à Brive-la-Caillarde, le 29 norembre 176?: mort à Paris le 6 férrier 1833. grands entomologistes qui aient jamais paru (Fig. 1); le premier,

(1) FABricius (J. C.). - Entomolagia systematica th aucta, 1792-1798, 5 rol. in- $8^{\circ}$. - Le $5^{\text {e }}$ volume constitue le Supplément en question.

(2) Il ne fant pas oublier cependant que cet ordre arait déjà été proposé par De Geer en 1774 sons le nom de Dermaptères.

(3) Par opposition aux néthodes artificielles empioyées jusqu'ici, on désigne sous le nom de mẻthode naturelle celle qui base la classification sur l'ensemble des caractères et non plus sur un seul.

(4) Latreille (P. A.) - Précis des caractères génériques des Insectes disposés dans un ordre naturel, Brives, an Y (1796), in $8^{\circ} .208 \mathrm{pp}$. 
il eut l'idée de ranger les Insectes par familles; puis il donna à ces premiers groupements des noms correspondant à leur origine: acrydiens, asiliques, bombyliers, bostrichines, etc.; etc., voulant s'assurer par là, dit-il, "la priorité exclusive de l'établissement des principales familles."

Voici, en ce qui concerne spécialement les Insectes, l'arrangement systématique de Latreille, celui qu'il proposa dans son Cours d'Fntomologie, un an à peine avant sa mort (1). (Voir tableau, page 15.)

A l'exception des Parasites, qui ne constituent pas un ordre homogène, on voit que la classification de Latreille a été conservée dans ses grandes lignes. Quant aux Dermaptères (Forficulides), on nréfère aujourd'hui les réunir aux Orthoptères, mais il n'y aurait aucun inconvénient à les maintenir en un ordre à part.

La vie de Latreille ne fut pas exempte de vicissitudes; en 1793, lorsqu'il n'était encore que curé de Brive-la-Gaillarde. il fut arrêté avec les autres prêtres du Limousin et devait être déporté à la Guyane; il ne dut son salut qu'à la découverte d'un insecte nouveau et grâce aux démarches pressantes de M. Bory de Saint-Vincent (2).

Bien qu'il ne se soit pas occupé exclusivement des Insectes, nous ne pouvons pas négliger de mentionner ici le nom du grand naturaliste Georges r'uvier, le créateur de l'anatomie comparée.

Dès sa jeuncsse, alors qu'il était encore élève à l'Académie de Stuttgart, il employait déjà ses loisirs à récolter des animaux et à illustrer les cuvres de Buffon. Vers la fin de sa vie, "il se propposait de traiter avec quelques détails, l'histoire si merveilleuse des métamorphoses des Insectes. "

Avec ces grands noms se termine, en quelque sorte, l'ère brillante de la Systématique générale; les auteurs qui suivent sont plutôt des monographes, comme PAYkul, qui décrit les Coléoptères de la Suède (3); Mergen, l'un des premiers historiens des Diptères (4) ; Kirny et les deux IüBer, qui étudièrent les Abeilles et les Four.

(1) Les trois elasses qui précèdent sont celles : des Crustacés, des Arachnides et des Myriapodes.

(2) Voir la relation de cet épisole émouvant de la vie de Latreille dans le volume des Coléoptères (E. S.), à propos du genre Necrobia.

(3) PAykuld (G.). - Faunasuecica: Insecta, 3 vol. Upsal 1800, in-8.

(4) Mer(ten (J. W.). - Kilassificat. u. Beschrcib. der curopaischen zuciflügeligen. Insekten, Braunschw. 1804, in-4º. 


$$
\text { INTROD UCTION }
$$

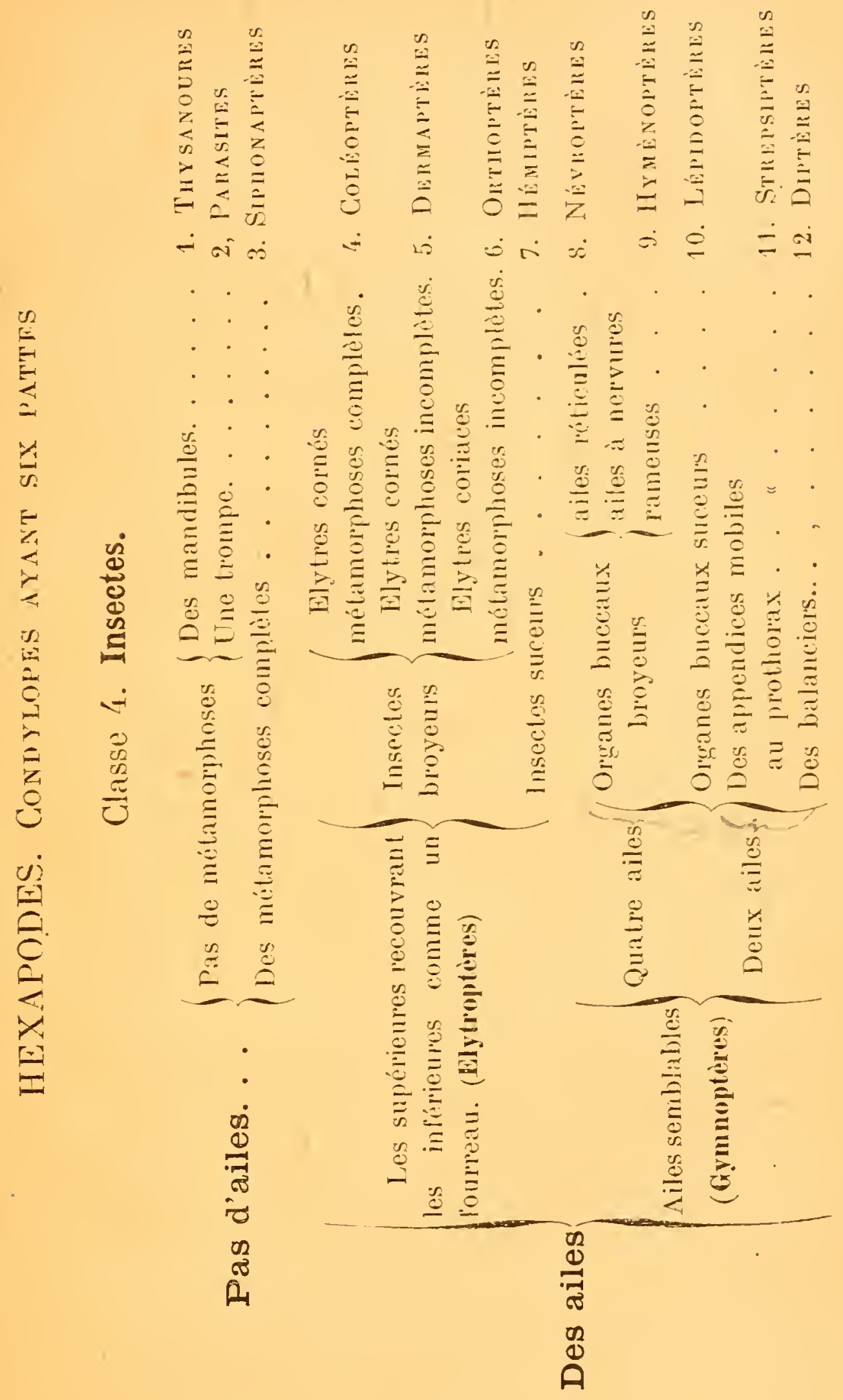


mis ; Schaxherr, l'infatigable ourrier de la synonymie des Coléoptères (1).

\section{L'ENTONOLOGIE DANS LES TEMPS ACTUELS}

1. Dans les périodes précédentes, comme on l'a vu, les entomnlogistes se sont surtout préoccupés de rassembler des faits et de "créer la classification".

L'époque actuelle fait un pas de plus ; elle cherche à généralisc:" ces faits; elle est, si l'on peut dire, nettement caractérisée par ses tendances philosophiques.

Il ne faudrait pas croire, cependant, que cette tendance se soit manifestée brusquement et comme à jour fixe ; elle est déjà en germe dans beaucoup d'écrits des anciens ; mais, r'est de nos jours seulement, qu'elle revêt son caractère de méthode précise et qu'ellc trouve sa voie.

L'un des ouvrages où perce déjà nettement l'esprit philosr phique est celui de LEAch, en 1817 (2) ; le premier, comme nqus l'arons déjà dit (p. 3), il sépara les Myliapodes des Insectes proprement dits, et distribua les animaux de cette dernière classe en denx grandes catégories, suivant qu'ils subissent ou non des mét:imolphoses, (Amétabola et Métabola).

Plus tard, Kin By et Spexce, ainsi que Burueister, adoptèren $t$ des classifications qui diffèrent si peu de celle de Leach, qu'il est inutile de les faire connaître ici en détai].

Nous laissons aussi complètement de côté le système trop compliqué d'Oren et les fantaisies cabalistiques de Mac-LEay sur les "analogirs circulaires"; ces vues, presque exclusivement spéculatives, relèvent de la métaphysique et nullement de la science expérimentale (3).

2. - Mair, si nous quittons les édificateurs de systèmes pou:

(1) Schonierr (C. J.). - Synonymia insectorum od. Synon. aller belannten Insekten (Coleopteren). 3 Thle Stocklı. u. Skara, 1806-17, in- $8^{\circ}$.

(2) LEACII (G. E.). - The zoologieal miseellany, being descriptions of new or inters'ing animats. Lon ion, 1814-17. 3 rol. in-8º.

(3) Je ne critique ici, bien entendu, que les vues systématiques de Mac-Leay ; la plupart de ses autres travaux sont des plus remarquables pour l'époque où ils ont èté écrits. 
revenir à l'cbservation directe des $\mathrm{f}$ ¡its, la lignée des anatomictes va nous fournir des données nouvelles et de la plus haute impcrtance. La célèbre théorie de l'unité de plan de composition, déjà en germe dans les écrits de Buffon, vient d'être formulée à nouveau par F. Geoffroy-Saint-Hilaire (1). Malgré la formidable opposition de Cuvier, certains naturalistes avaient cherché également à reconnaitre cette unité de plan dans les Invertébrés ; c est donc ici qu’il convient de mentionner les suggestifs travaux de Savigny sur la bouche, et de Victor Audouin sur le thorax des Insectes.

Jules Lelorgye ne Savigny, de même que Goffroy-Saint-Hilaire, fit partie de la Commission scientifique qui accompagna l'armée française en F́grpte, en 1799. Dans le premier fascicule de ses Mémoires sur les Animaux sans sertèbres, qui parut en 18i6, il établit que la bouche des Insectes est toujours constituée par les mêmes pièces et que les variations, parfois très profondes. qu'on y' remarque, suivant les classes, proviennent exclusivement d'adaptations diıes à des différences de régime.

Jusqu'ici aucun fait n'est venu infirmer cette manière de voir. Audouin arrive à des conclusions analogues en ce qui concerne la constitution du thorax; Latreille lui-même se croit obligé d'admettre l'identité d'origine des ailes et des pattes (2).

3. - C'est maintenant l'étude du développement des Insectes et des transformations qui se produisent au cours des métamorphoses, qui va prendre une importance de plus en plus grande. IIÉrold signale déjà, en 1S15, quelques modifications des organes génitaux et du système nerveux chez les Lépidoptères. Líox Dufour, dans de nombreux Mémoires, pose en quelque sorte les bases de l'anatomie comparée des Insectes; enfin, la précieuse monographie de Strauss-Durgkhein, sur l'anatomie du Hanneton, est un véritable chef-d'œuvre, qu'on a cherché bien des fois à imiter, mais qui n'a jamais été surpassé.

Si l'étude de l'anatomie et des métamorphoses continue à faire de très grands progrès, il est juste de reconnaître que la systématique, elle aussi, conserve de fervents adeptes ; elle acquiert même,

(1) Geoferoy-Santi-Hrdatre (Et.). - Sur les Makis.Philosophie anatomique, t.I. Des organes 1818-1823; Paris, 96.2 vol. in- $8^{\circ}$ et 2 atlas in- $4^{\circ}$.

(2) Latreille (P.).- De la formation des ailes des Insectes, Paris, sans date, in-8. - Nous verrons cependant qu’il y a lieu de faire des réserves sur ce sujet, ๖. 83. 
de jour en jour, un caractère plus précis et plus scientifique en se spécialisant, et par l'habitude que prennent les entomologistes de se constituer des collections privées; il arrive alors que des groupes, jusqu'ici très délaissés, trouvent tout à coup leur historien ; c'est ainsi qu'un pauvre organiste de Stolberg, GuILı,AUME MFigen, après avoir passé vingt ans de sa vie à étudier les Mouches, publia l'un des monıments les plus complets qui aient jamais été consacrés à cet ordre dédaigné ; sa Description systématique des Insertes Diptères d'Europe parut à Aix-la-Chapelle en 1818. Malgré son importance, cet ouvrage n'aurait jamais pu voir le jour, si le professen' Wiedemann n'avait lıonoré l'auteur de son appui et de son amitié.

La collection de Meigen fut acquise par M. Maccquart et rétrocédée par lui au Muséum de Paris.

Un autre ordre d'Insectes, celui des Lépidoptères, fut également, vers la même époque, étudié par Augustr Dupnxchel. Lié d'amitié avec Latreille et Duméril, mais ayant été obligé, pour des motifs d'ordre politique, de quitter l'armée à l'âge de 42 ans, Duponchel entreprit de continuer seul le grand ouvrage qu'il avait commencé avec son ami Godart (1). Ce travail immense coûta vingt années d'efforts assidus; on y trouve des observations de toute nature sur les habitudes de 4.134 espèces de Papillons.

De son côté, un modeste employé du Ministère de la guerre, Jean-Guillaune Audinet-Serville, avait porté sa curiosité veri les Opthoptères; mais, il faut lui rendre cette justice qu'il connaissait également bien tous les ordres d'Insectes. Initié à la science entomologique par la charmante $M^{m e}$ de Tigny, il avait étudié les Papillons sous la direction de Duponchel et les Hyménoptères avec Lepelletier de Saint-Fargeau.

Walgré ses vastes connaissances, Audinet-Serville était l'humilití et la modestie mêmes.

En dehors de l'Encyclopódie incthodique, qui fut terminée par lui, l'un des ouvrages les plus connus d'Audinet-Serville est son Histoire nnturelle des Insertes Orthopteres, Paris, Roret, 1839, in-80, qui fait partie des Suites à Buffon; cet ouvrage apporte une très grande clarté dans cet ordre qui jusque-là " n'arait été qu'un chaos".

4. - Au cours de cette époque, de 1825 à 1850, glorieuse entre

(1) Godart et Duponchin. (A.). - Histolre naturelle des Lépidopteres ou Papillons de France. Paris, 1821-1842, 18 vol. gr. in- $-8^{\circ}$ avec 546 planches coloriées au pinceau. 
toutes pour l'entomologie française, nous trourons encore un nom qui jouit pendant longtemps d'une immense notoriété : c'est celui du général comte Dejean.

Auguste Dejean naquit à Amiens en 1780 ; à 15 ans il était déià soldat. Passionné pour l'entomologie. il profite de ses nombreuses campagnes pour récolter des Insectes dans les différentes régions de l'Europe, et devient ainsi, de très bonne heure, possesseur d'une des plus riches collections qui aient jamais existé (1). Ayant aussi été obligé de quitter l'armée, il occupa ses loisirs à mettre en ordre les richesses qu'il avait accumulées. Ce travail dura trois années; il le couronna en 1821, en publiant le Catalogue des Coléoptères de sa collection, où se trouvent déjà énumérées 6.692 espèces.

On peut dire que ce Calalogue a été le point de départ de l'étude spéciale des Coléoptères; car, le seul Species, qui existât à cette époque, était le Systóma Eleutheratorum de Fabricius, ouvrage déjà vieilli et dont la partie générique avait été bouleversée par les travaux de Latreille.

Fn 1837, le général Dejean publia une $3^{\epsilon}$ édition de son Cata logue (2) ; sa collection renfermait alors 22.39 ? espèces. Cet ouvrage manque malheureusement de références bibliographiques ; c'est le seul reproche qu'on puisse lui faire, car son influence a été capitale pour la formation des collections de Coléoptères (3).

La collection du comte Dejean, trop nombrense pour être acquis: nar un seul acheteur, fut mise en vente par sa farnille; elle fut ainsi dispersée en de nombreuses mains.

5. - Les auteurs français n'étaient pas seuls à s'illustrer dans l'étude de l'entomologie; nous derons au moins, dans ce court résumé historique, citer le nom de 7.ETTERSTEDT, illustre naturaliste, suédois, né à Miölby en 1785. Son chef-d'curre: Diptera scandinavix disposita et descripta, Lund 18'12-1860, modèle du genre, représente un effort de plus de ringt années.

(1) Officier de dragons sous l'empire, on raconte qu'il avait fait doubler de liège l'intérieur de son casque; apercerait-il un insecte pendant une marche, il mettait pied à terre et piquait sa capture dans cette boîte d'un nouvean genre.

(2) Deifar (P. F.). - Catalogue des Colsoptères de la Collection de M. Le baron Deiean, $3^{e}$ édit. Paris, Méquignon-Marvis, 1837. in-8º.

(3) En 1826, le comte Dejean était cevenu acquéreur cie la Collection ce Latreille. 
L'ordre des Névroptères tronva à son tour un scrupuleux historien dans le Dr Ranbur (Fig.

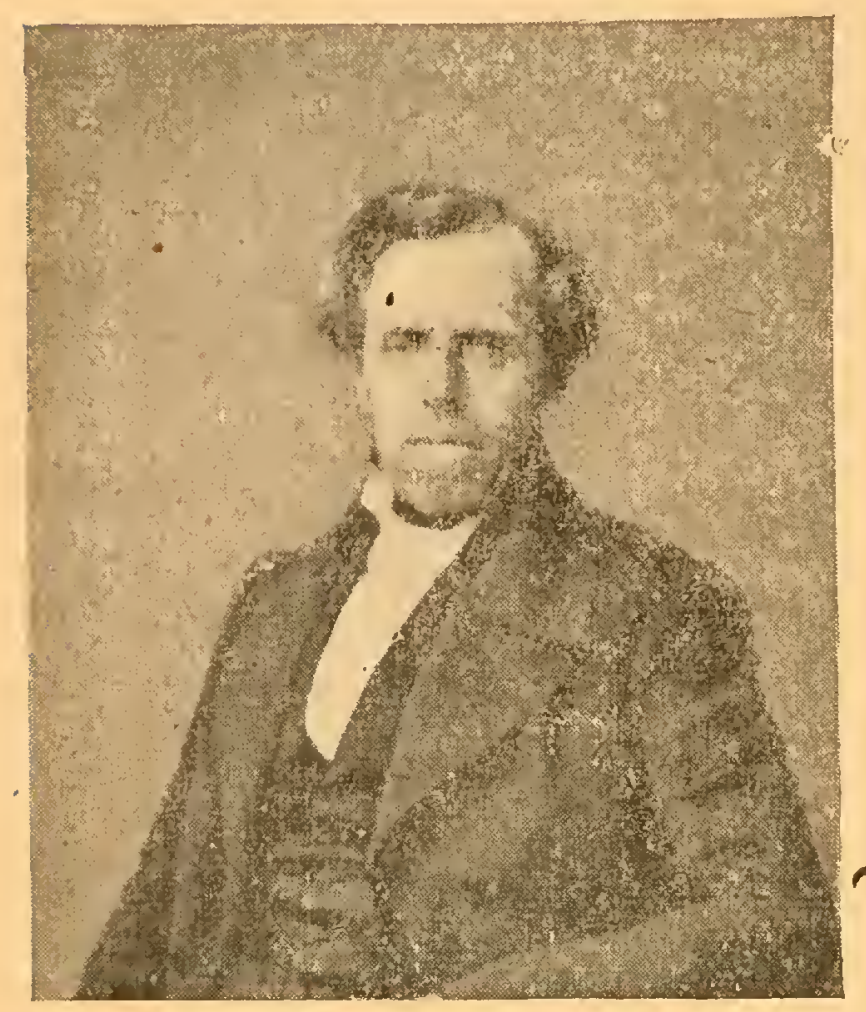

Fig. 2. - D Pierre RAMRTR, né à Ingrandes, le 21 juillet 1801 ; mort à Genève le 10 aồt 1870. 2 ) ; les nombreux travaux de ce sarant entomologiste sont, en général, dispersés dans les grandes publications périodiques de l'époque; quelques-uns sont malheureusement restés inachevés. Ainsi, sa Faune entomologique de l'Andalousie, qui, dans la pensée de l'anteur, devait comprendre tous les ordres d'Insectes, fut interrompue en 1840 ; seuls, deux rolumes accompagnés de cinquante planches ont partu. L'ourrage le plus connu du DrRambur est l'Histoire naturelle cies Insectes Nésroptères, publiée en 1842 dans les Suites à Buffon.

Nous devons encore citer ici le nom dn Dr Aubé, qui fut l'un íns membres fondateurs de la Société entomologique de France et le possesseur d'une des plus belles collections de Coléoptères qu'on ait jamais vues. Le Dr CH. Auré excellait à reproduire au trait la physionomie des petits Insectes ; aussi, sut-il utiliser avec profit les détails les plus minutieux des antennes, des pièces buccales et des tarses, dans la description des espèces. Le premier aussi, il eut l'idée de disséquer ces petits organes dans un milieu transparent, tel que l'essence de térébenthine ou le baume de Canada.

Sa famille, ne roulant pas que sa collection fît vendue, la confia au Dr Grenier ; elle est aujourd'hni, en ce qui concerne les Coléoptères européens, propriété de la Société entomologique de France.

6. - A part quelques rares exceptions, l'étude des Insectes adultes 
semble avoir, jusqu'ici, exclusirement préoccupé les entomologistes ; nous allons voir maintenant l'étude des larres prendre une importance de plus en plus grande. En tête de ce moure. ment nous trourons Édouard Perris, riceprésident du Conseil de Préfecture des Landes, l'un des entomologistes les plus exacts du $x_{\mathrm{J}} \mathrm{x}^{\mathrm{e}}$ siècle. (Fig. 3).

Observateur rigoureux et patient, voyant profondément, (occulatissimus, disait L. Dufour), tous les travaux de Perris sont empreints de la plus grande précision. L'un des plus admirables peut-être est son Traité des Insertes du

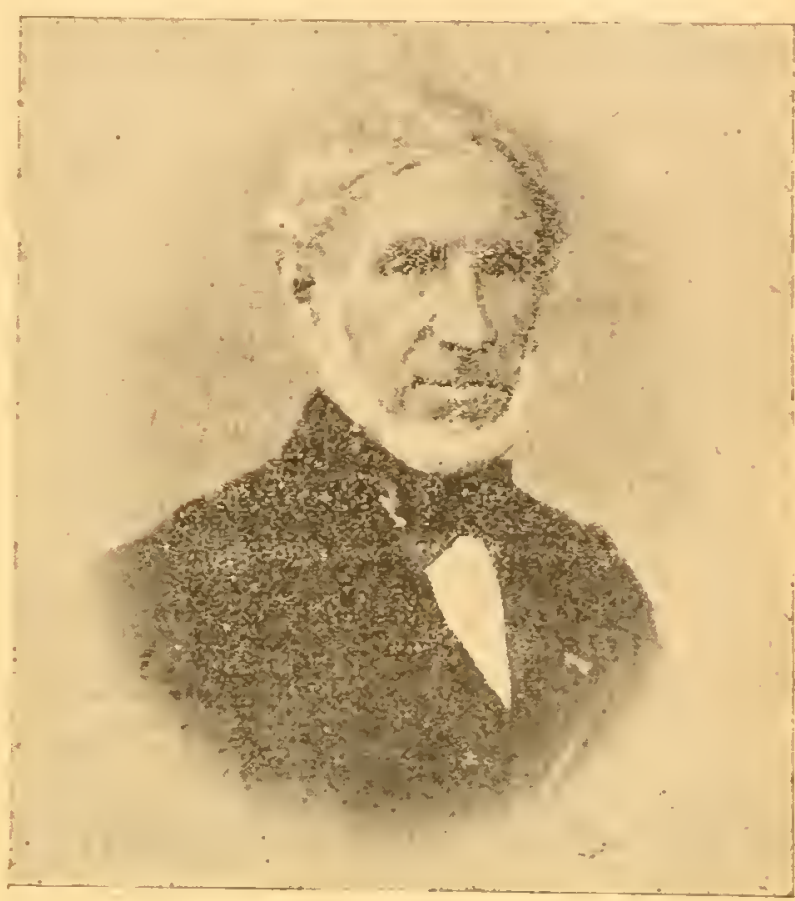

Fig. 3. - PERRI: (Edouard), né à Pau, le $1 \frac{1}{4}$ juin 1808 ; mort à Hont-de-1larsan le 10 férrier 1878 Pin Maritime, que, par suite d'un entêtement bizarre, il ne voulut jamais terminer. L'entomologie était pour lui un sacerdoce. "La science des Insectes, disait-il, ne consiste pas uniquement en une classification, elle est quelque chose de plus vaste et de plus élevé. On ne connait une espèce quelconque que lorsqu'on a précisé ses formes, sa structure, son habitation, ses conditions d'existence, ses instincts, ses mœurs, ses industries et au besoin ses migrations."

Inaccessible à. l'ambition, Perris refusa toujours les emplois élerés qu'on lui offrait pour l'attirer à Paris; il préféra rester près des siens, dans la région qu'il affectionnait. Il était accueillant à tous ; sa parole était captivante et son affabilité proverbiale (1).

Son ouvrage le plus connu : Larses de Coléoptères, Paris, Dey-

(1) La collection"très importante de larres, réunie par PerRis se troure actuellement à l'Ecole nationale d'Agriculture de Aontpellier. 
rolle, 187\%, est dédié à Mulsant; l'impression n’en était pas encore acherée quand il mourut.

Perris n'a pas écrit ses dernières volontés; il légua serbalement sa collection au Dr Gobert, son élève et son ami.

7.-. L'étude des Hyménoptères, jusque-là peu avancée, devient plus facile avee Gaspard Brullé, qui fut l'un des premiers secrétaires de la Société entomologique de France et, plus tard, doyen de la Faculté des sciences de Dijon. Outre son grand ouvrage, en que tie volurnes, qu'il publia de 1836 à 1846, en collaboration avec Lepelletier de Saint-Fargeau (1), on peut encore citer de lui l'Histoire nalurelle des Hyménoptères dans les Suites à Bufjon.

Je ne veux pas quitter "l'époque glorieuse "sans joindre au nom de Brullé, ceux de Robinean-Desvoidy et du colonel Goureau, car l'impulsion que ces sarants ont donnée à l'entomologie n'est pas près de s'éteindre au pays dle Bourgogne.

Malgré son isolement, à Saint-Sauveur-en-Puisaye, près d'Auxerre. le Dr RobineaU-Desvoiny consacra la plus grande partie de sa vie à l'étude des diptères Tachinaires (2), (qu’il désignait sous le noın de Myodaires). On "a pu dire de lui qu'il a été "le dernier des diptéristes français " et, de lait, depuis Meigen (p. 18), malğré sa richesse et son étendue, cet ordre important avait été presque entièrement négligé.

Les riches collections diptérologiques du Dr Robineau-Desvoidy, ainsi qu'une partie de sa bibliothèrque, ont été léguées par lui à la Société des sciences naturelles d'Auxerre.

Son compatriote, le colonel Charles Gouriau, s'acquit aussi, dans le monde savant, ver's la même époque, une très grande notoriété par ses travaux d'entomologie appliquée.

Goureau avait une très haute idée de sa science favorite; aussi arait-il coutume de dire que les savants "qui ont étudié au microscope l'anatomie délicate des Insectes, qui I'ont décrite et dessinée, ont fait preuve d'une dextérité qui surpasse de beaucoup les travaux des plus habiles anatomistes du corps humain."

(2) LEPELADTIR DE SAINT-FALGLAU et BRULA:- Histoire naturelle des Inscetse IIymennptères. Paris, 1836-1846, 4 vol. gr. in-80 avec 18 pl. coloriées.

(1) Roprimau-Drsvony (Dr). - Essai sur les Mvolaires. Iravax des savant; étrangers. Vol. II. Paris, in-10. 
Son Essai sur la Stridulation des Insectes (:1), 18:7, ful traduit dars plusieurs langues ; mais ses ourrages les plus importants sont ceux qu'il publia de 1857 à 1872 sur les Insectes nuisibles, dans le liulletin des Sciences historiques et naturelles de l' I'onne.

8. - Si les travaux de biologie et de systématirue éfaient en grand honneur en France, l'entomologie, à l'étranger, n'était pas non plus sans éclat ; nous citerons seulement iri les noms de Bunners. TER, ÉRICISON, KOLla li: WESTWOOD, et surtou! celui du professeur HERMAN SchaUn, digne émule et successeur d'Érichson à l'Unirersité de Berlin. L'un des premiers il dressa le Catalogue de toutes les espèces de Coléoptères connus de la faune européenne (2). Lacordaire a pu dire, en parlant de l'érudition et de l'esprit critique de cet auteur, que, sous plus d'un rapport, il n'a jamais été

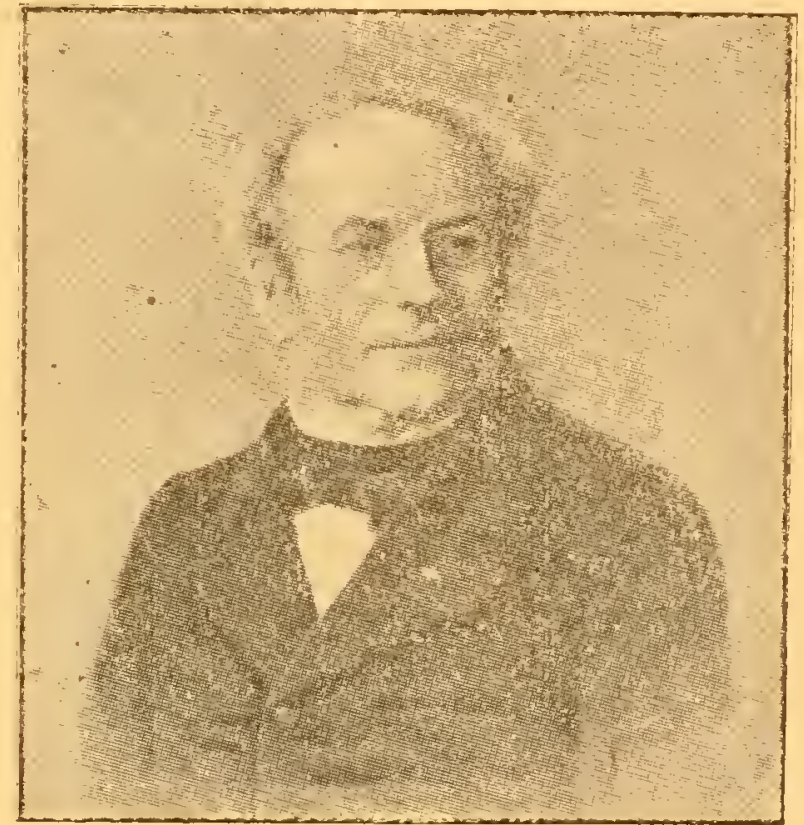

Fig. 4. - T.1Cordirne (Jeall-Théodore),

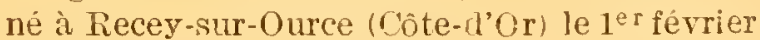
1801 ; mort ì Liège en 1870. surpassé ni même égalé.

LACORnAIRE, encore un fils de l'opulente Bourgogne : La Francr: dit quelque part Albert Fauvel, aura toujours à regretter de n'avoir pas su retenir ce savant, qui eût été une des lumières de son enseignement, comme il est l'une des gloires de l'entomolngie.

Frère de l'illustre prédicateur dominicain, Lacordaire (Jean-Théodore) naquit à Recey-sur-Ource (Côte-d'Or), en 1801 (Fig. 4). Après avoir fait d'excellentes études au collège de Dijon, son goût l'entraìna

(1) Govreau (Ch.). - Essai sur la Stridulation des Insectes (Ann. de la Soc. entom. de France. T. VI, 1837. Parì in $-8^{\circ}$ ).

(2) Schaum (H. R.). - Catalogus coleopterorum Europu, 2e édit. Berlin, Nicclai 1862 , in $-8^{\circ}$. 
vers les voyages ; c'est ainsi qu'il visita l'Amérique du Sud de 182.5 à 1832. A son retour en Europe, après avoir tout d'abord collaboré à la Rerue des Deux-Mondes, il accepta une chaire de Zoologie it l'T'niversité de Liège.

Son principal ouvrage, chef-d'œuvre d'érudition et de clarté, est son Genera des Coléoptères (1).

I.n nous rapprochant de l'époque actuelle, nous allons trouver des figures de plus en plus familières : qui ne connaît, en effet, le nom de Jacruelin du Val ? Son Genera des Coléoptères d'Europe (1857-1862), si artistement illustré par le peintre Migneaux, est un ouvrage que les Coléoptéristes consultent charque jour. La santé de l'auteur ne lui ayant pas permis d'y mettre la dernière main, cet important travail fut achevé par l'un des plus savants entomologistes de notre temps, M. LÉox Fairuairf, dont la science déplore la perte récente.

La riche collection de Jacquelin du Val appartient maintenant au Muséum de Paris.

9. - Ce n'est pas une tâche facile de résumer en quelques pages l'histoire de l'entomologie française. Tant d'hommes éminents se sont distingués dans cette science que mon embarras est grand pour rendre exactement à chacun l'hommage de reconnaissance qui lui est dû; j'ai un regret profond de ne pouvoir citer toutes nos gloires dans les limites restreintes de cet ouvrage ; pourtant qu'on veuille bien me permettre de consacrer encore quelques lignes aux plus illustres.

Parmi les monographes, c'est un lépidoptériste: le Dr DechaurFOUR-DE-BOISDUVAL (Fig. 5) qui ouvrit en quelque sorte la période brillante du siècle dernier : combien il me serait agréable de reproduire ici la Notice émue que notre savant collègue, M. Charles Oberthür, lui a consacrée en 188n, dans les Annales de la Société entomologrique dê France.

La précieuse collection de Lépidoptères du $\mathrm{D}^{r}$ Boisdural, linne des plus belles et des plus riches de l'époque, a été acquise, en 1376 , par M. Charles Oberthür.

lci doit encore être cité le nom de Berce (Jean-Étienne), l'un des

(1) Lacondan: ('Ih.). - Histoire naturelle des Insectes. Genera des Coléoptires ou exposé méthodirue et critique de tous les genres, etc. Puris, Roret, 185-1866. 7 vol. in- $8^{0}$ a vec planches. 
plus anciens membres de la Société entomologique de France et son président en 1867. L'œuvre capitale de Berce est sa Faune des Lipidoptère:ide France(1) en six volumes, qui parut de 1867 à 1878 et qui valut à l'auteur l'honneur d'être le premier lauréat du Prix Dollfus, en 1873.

Le hasard des dates nous ramène vers les Coléoptéristes; notre attention doit s'arrêter maintenant sur l'œuvre immense de Mulsant.

Né en 1797, à Marnand, canton de Thizy, près Villefranche( Rhône), Mulsant fut le condisciple et le rival de Lamartine au collège de Belley; aussi, son premier travail: I.ettres à Julie sur l'Entomologie, est-il aussi littéraire

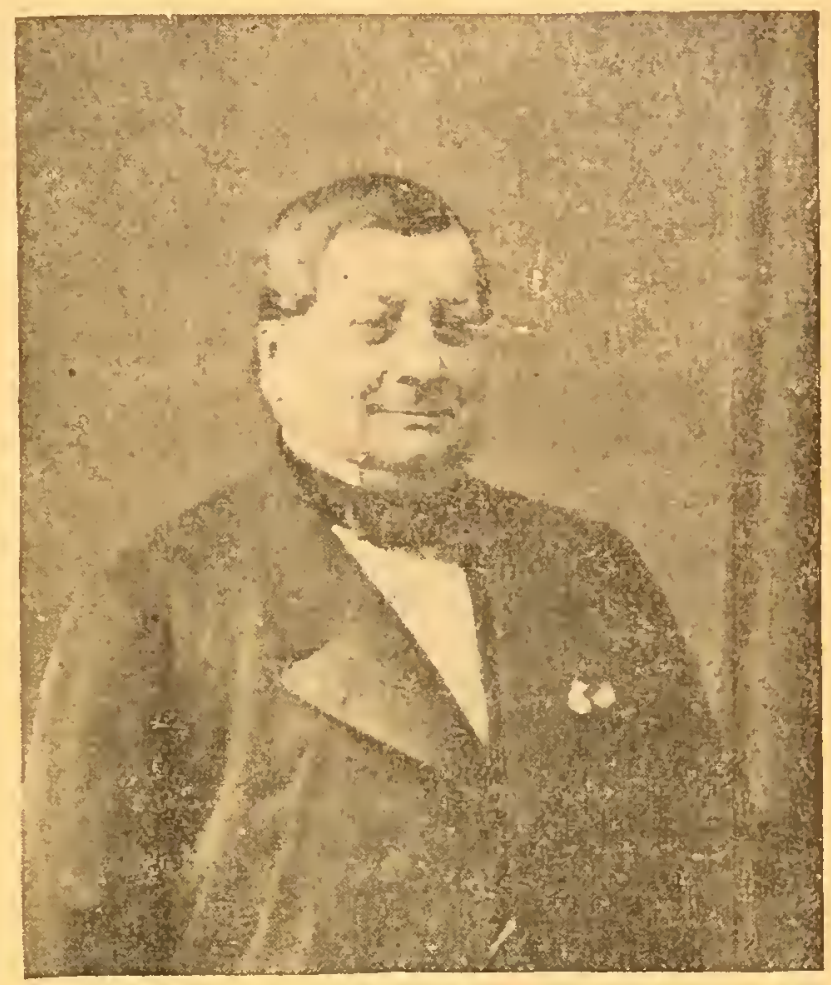

Fig. 5. - J.-B. Déchauffour de Boisdural. Docteur en médecine, né à Tícheville (Orne) : mort le 30 désembre 1879. que scientifique (2).

Tout d'abord sous-bibliothécaire de la ville de Lyon, il devint ensuite professeur au Lycée (1843); c'est là qu'il enseigna les sciences naturelles pendant plus de trente ans. A cette époque, il avait déjà entrepris la publication de son Histoire naturelle des Coléoptères de France, ouvrage qui lui valut de suite une réputation européenne. En Allemagne surtout; où ses travaux sont très estimés, on l'a surnommé le "pater entomolngirus".

(1) BERCE (J. E.). - Faune entomologique francaise, Lépidoptères: description de tous les papillons qui se trouvent en France, Paris, Deyrolle, 1867-1878; 6 rol. in $-8^{\circ}, 65$ planches coloriees.

(2) La muse de Mulsant existait réellement, c'était M1le Julie Ronchivolle, qu'il épousa en 1815 ; il avait 18 ans, elle en avait 15.

LES INSECTES. - $2^{\mathrm{e}}$ édit. 
Il décrivait les Insectes avec un soin minutieux, aussi lui repıche-t-on parfois ses descriptions d'une longueur excessive ; mais il

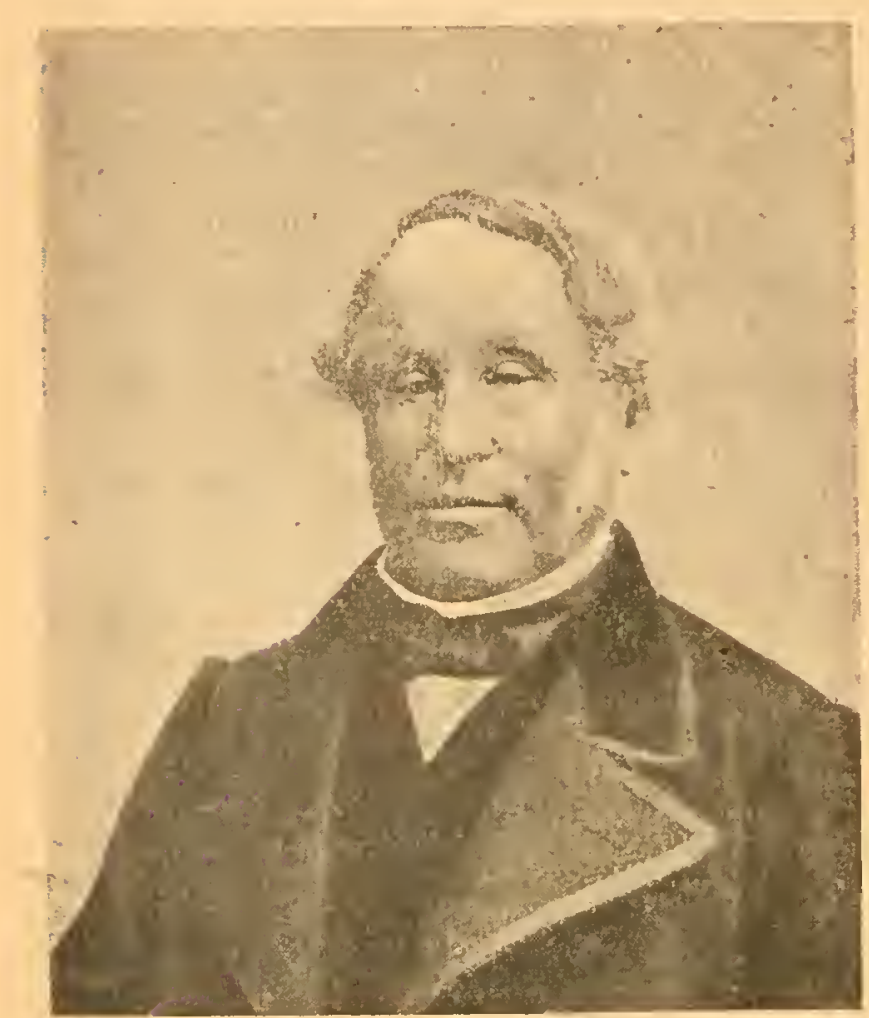

Fig. 6. - Guevér (Achille), lépidoptérologiste, né à Chartres le $1^{\mathrm{e}}$ janvier 1809 ; mort à Châteaudun le 30 décembre 1880 . faut convenir qu'elles sont toujours claires et parfaitement exactes.

Mulsant propagea aussi, le premier, l'usage des tableaux synoptiques, jusqu'alors peu usités ; après les avoir tout d'abord appliqués aux tribus, il les étendit plus tard aux genres et même aux espèces.

A sa mort, son importante collection resta entre les mains de son fils, M. l'abbé Victor Mulsant.

Chaque auteur apporte sa pierre à l'œuvre commune.AcHLLEGUENÉE fut exclusivement un lépidoptériste ( Fig. 6). Le premier, il s'occupa de l'étude des chenilles, et, comme il attachait une importance capitale aux détails biologiques, il les fit entrer dans la caractéristique des genres. Il eut souvent, à ce sujet, de vives discussions avec Duponchel, mais, finalement, l'avenir lui donna raison.

Sa collection, d'une très grande valeur, comme celle du Dr Boicduval, a été acquise par notre éminent collègue $\mathrm{M}$. Charles Oberthür, de Rennes.

10. - Quoique russe, le nom du baron Maximilien de Ciravoln indique cependant une origine frariçaise; il descendait, en effet, d'une famille d'émigrés.

Lorsqu'il vint en France pour la première fois, il acquit, du masquis de Laferté-Sénectère, la célèbre collection de Carabiques 
du comte Dejean, à laquolle avaient déjà été réunies celles d? Reiche et de Gory. Cette circonstance lui permit de publier un nombre immense de monographies sur cette famille si vaste. Lorsque la mort le surprit, en France, à Amélie-les-Bains (1881), il était encore occupé à une révision du groupe des Harpalides.

Sa merveilleuse collection de Carabiques est à Rennes; elle fut acquise, en 1880, par M. René Oberthür. Six ans auparavant, il arait cédé sa collection de Cicindélides (713 esp.) au Muséum d'histoire naturelle de Paris.

Il nous plaît encore de citer avec orgueil un autre savant étranger, Jonn Le Conte, de Philadelphie, que l'on regarde comme l'un des premiers entomologistes du monde. Ses ancêtres étaient également français et originaires de la Normandie.

Son œurre capitale est la Classificaiion of the Coleoptera of North America, dans laquelle il fit connaître une faune presque entièrement nouvelle. Le premier aussi, il démontra que le singulier Platyp. sillus, parasite des castors, n'était pas un Aphaniptère, ainsi qu'on l'avait cru jusqu'alors, mais bien un Coléoptère.

J. Le Conte a légué sa riche collection au Museum of comparatise Zoology, de Cambridge. Mlass.

Un autre savant coléoptériste de cette époque fut encore AUGUSTE Chevrolat, qui eut pour maîtres Latreille et Duméril. Son exigence pour l'exactitude des déterminations était telle, qu'il se rendit exprès en Angleterre pour vérifier les collections de Banks et de Linné, et de même à Kiel pour consulter celle de Fabricius.

Il fut l'un des membres fondateurs de la Société entomologique de France.

11. - Jusqu'ici, comme toujours, c'est surtout l'étude des Insectes adultes qui a retenu l'attention des collectionneurs et des entomclogistes; ; çà et là des observations sur les larves ont bien été faites, mais ces observations sont restées isolées, Schiödte entreprend de combler cette lacune pour l'un des groupes les plus importants.

Jorgen-Christian Schiödte, né à Copenhague en 1815, fut certes l'un des plus habiles observateurs de son temps; il me suffira de citer ici la belle série des Mémoires qu'il publia de 1861 à 1883 sur les larves des Coléoptères (1) ; c'est là une cuvre considérable et

(1) Schiôdte (J. C.). - De Metamorphosi Eleutheratorum obseriationes. Pars III. Naturhist. Tidsskr. t. IV. Kjobenharn, 12 Theile, 1862-1883, in-Sº 
pleine d'indicatirns les plus précieuses sur les premiers états de ces Insectes.

Schiỏdte mourut à Copenhague en 1884 ; on pent dire que sa vis n'a été qu'un long travail consacré aux progrès de l'entomologie.

12. - Avec Maurice Girard, nous enregistrons une nouvelie tendance; entomologiste de grande valeur, il entreprit de vulgariser la science. Son petit ouvrage : Métamorphoses des Insectes, Paris, Hachette, 1874, si intéressant et si vivant, fut couronné par l'Académie des sciences; mais son œuvre maîtresse fut son Traité élmmentaire d'entomologie, en 4 volumes, où se trouvent réunis une foule de renseignements bibliographies extrêmement précieux.

Avec le baron Edgard von Harold, la littérature entomologique s'enrichit d'un Catalogue où sont mentionnées toutes les espèces de Coléoptères aujourd'hui connues. Ce travail immense, commencé en collaboration avec le Dr GEMminger, en 1868, n'a été terminé qu'en 1876 (1) ; mais, comme il continue à être tenu au courant des découvertes nouvelles à l'aide de suppléments, il rend toujours aux Coléop. téristes les plus grands services. Il n'est personne qui n'ait eu l'occasion de consulter le fameux "Catalogue de Munich ".

13. - Nous ne voudrions pas.allonger outre mesure ce résumé historique de l'entomologie ; cependant nous ne pouvons pas passer sous silence le nom de Lichtensteiv, ni omettre de rappeler ses vues si originales sur le polymorphisme et les migrations des Pucerons, qui le conduisirent, en quelque sorte d'instinct, à découvrir le véritable état civil du Phylloxera.

Un lépidopiériste bourguignon, (Millière 1811-1887): acheva de f zirn connaître la faune des Papillons de France, en décrivant un nombre considérable de chenilles inconnues avant lui. La plus grande partie de la merveilleuse collection qu'il arait réunie passa malheureusement à l'étranger.

Mentionnons encore ici l'importante collection de Coléoptères de M. Reiche qui, malheureusement, fut aussi, à sa mort, dispersée en de nombreuses mains. Les Lamellicornes, dont Reiche arait fait ses études de prédilection, furent acquis par M. de Marseul : ils sont maintenan: au Muséum de Paris.

(1) Gemmingis et Harold. - Catalogus Colcopterorum hucusque descriptio. sunonym. et syst. 12 vol. Monachii, 1868-1878, in-8 . 
Je suis fier de rencontrer ici le nom d'un mayennais; M. l'abbé S.-A. DE Marseul est, en effet, né à Fougerolles-du-Plessis (Mayenne) Tous les entomologistes connaissent sa Mlonographie des Histérides (1) et son Calrilogue synonymiqi:e (2).

Sa collection et sa bibliothèque, toutes les deux très riches, furent léguées au Laboratoire d'entomologie du Muséum d'histcire naturelle de Paris. L'abbé de Marseul ne cessait de s'élever, avec raison, contre l'abus qui consiste à multiplier outre mesure les espèces basées sur des caractères trop fugaces.

Un souvenir à Charles Brisout De Barveville, qui connut si admirablement les habitudes des Insectes et qui, surtout, savait les découvrir avec une perspicacité qui n'a jamais été dépassée.

14. - Nous ne voudrions pas étendre davantage cet exposé historique, déjà trop long; qu'il nous soit cependant permis, avant de terminer, de jeter un rapide coup d'œil sur quelques-uns des plus récents progrès de l'anatomie et de la biologie entomologiques.

Il nous faudrait encore citer les noms d'une multitude d'auteurs qui ont contribué, à des degrés divers, aux progrès de la science entomologique; mais ces noms seraient si nombreux que l'énumération seule, avec l'indication de leurs travaux, en serait à peine possible

Il est un fait de biologie sur lequel nous devons cependant appeler l'attention. On savait, depuis l'antiquité, que les Insectes qui, comme les Lépidoptères (Papillons), subissent des métamorphoses complètes, passent successivement par les phases de larve (chenille) et de nymphe (chrysalide) avant d'arriver à l'état parfait.

Or, en 1867, un savant naturaliste d'Avignon, HexrIFABre (Fig. 7) découvrit, chez les Cantharides, un mode de développement encore plus compliqué, en ce sens que la larve, avant de passer à l'état de nymphe, revêt trois formes d'adaptation intermédiaires ; après avoir été tout d'abord carnassière, elle devient ensuite mellivore (3) ; c'est vers la fin de ce régime qu'elle passe à l'état de pseudonymphe. On a désigné le cycle de ces transformations sous le nom d'hypermétamorphose, et on a constaté qu'il n'était pas spécial aux larves des

(1) Marseul (S. A. de). - Essai monoyraphique sur la fam. Ies Histérides (Aun. de la Soc. entom, de France 1853-1859, Paris, in- $8^{\circ}, 38$ pl.).

(2) Marseul (S. A. de). - Cat. synonym. et géograph. des Coléopteres de l'Ancien Monde. Paris 1889, in-12, 562 pp.

(3) C'est-à-dire mangeuse de miel. 
Cantharides; on le retrouve, en effet, chez tous les Coléoptères de la famille des Vésicants

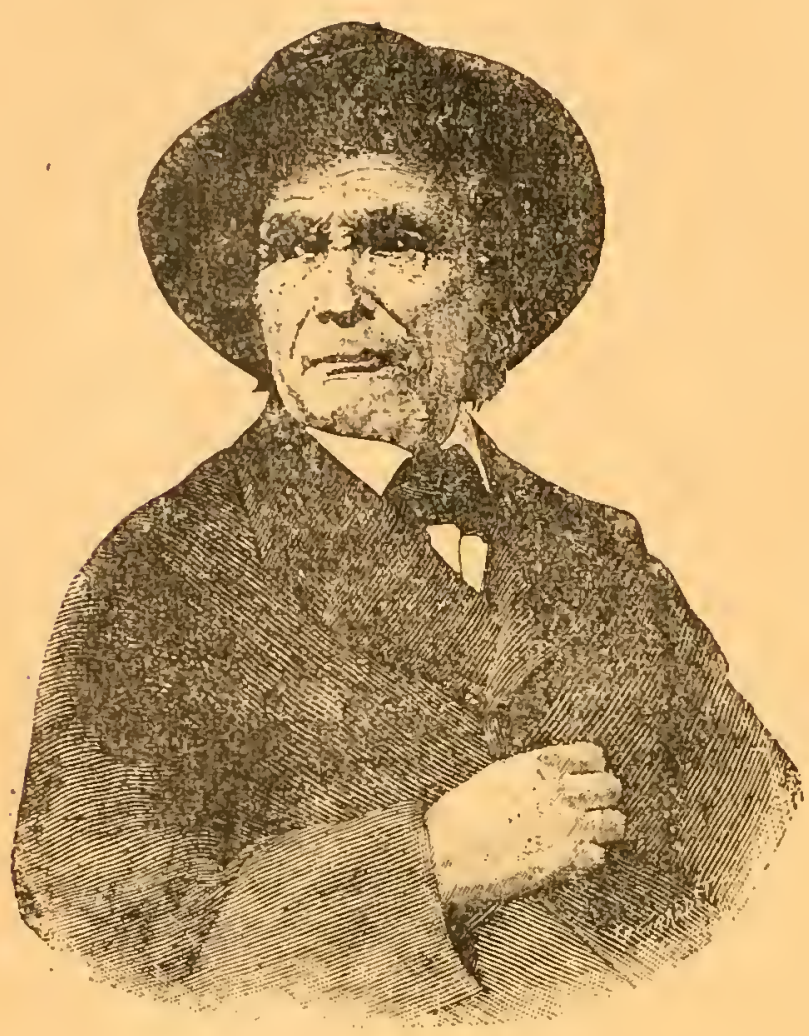

Fig. 7. - FABRE (J. Henri), L4 né à Saint-Léons (Aveyrô1) en 1823 mort à Sérignan (Vaucluse) le 11 octobre 1915.
(Mélö̈des) (1).

15. - Enfin, pour terminer, la vie et l'muvre de Riley vont nous montrer quelle importance peuvent avoir les études entomologiques pourl'avenir économique d'un grand pays.

C.harles Riley peut être considéré comme l'un des plus grands entomologistes des ÉtatsUnis, cependant il avait une origine européenne; il était né à Londres, en 1843. Il termina son éducation en France, (à Dieppe), et en Allemagne (Bonn), puis de là, de bonne heure, partit pour l'Amérique avec l'intention d'y installer
ultats n'ayant pas, tout de grandes exploitations agricoles. Les résultats n'ayant pas, tout d'abord, répondu à ses espérances, il accepta, en 1864, de collaborer à un journal, le "Prairie Farmer"; il devint bientôt "l'âme du journal n, et c'est alors qu'il commença à publier les remarquables études sur les métamorphoses des Insectes qui l'ont rendu célèbre dans le monde entier.

Je voudrais pouvoir présenter ici le tableau saisissant que Riley soumettait, en 1897, à l'Université de Washington, sur le rôle des Insectes niusibles; il démontrait, en effet, avec chiffres authentiques à l'appui, que la seule Chenille du Coton (Cotton (rorm), con̂tait plus

(1) КАВік: (Н.). - Mémoire sur l'Hypermétamorphose et les mours des Méloïles (Ann. des se. naturelles zool. 'T. V'II et IX, Paris in-8 ${ }^{\circ}$ 1857-58). 
de vingt millions de dollars, par semaine, aux États du Sud; que la Chrysomèle de la pomme de terre (Doruphora decemlineata), avait à peu près empêché la culture de cette solanée dans les États de l'Ouest, jusqu'à ce qu'on eut appris à la combattre; que la Mouche de Hesse (Cecidomyia destructor) ruinait des hectares entiers de blé et que 50 millions de dollars ne suffiraient pas à couvrir les pertes occasionnées par les Sauterelles de 1873 à 1875 .

Certes Riley, comme tout entomologiste expérimenté, ne croyait pas à la possibilité de détruire complètement tous les Insectes nuisibles, mais il démontra qu'il était possible d'atténuerleurs ravages et par conséquent de sauver une bonne partie des récoltes.

Après avoir occupé, pendant dix ans, les fonctions d'Entomologiste d'Ftat à Saint-Louis, Riley fut appelé à Washington, comme Directelir du Service de l'Entomologie au Ministère de l'Agriculture. Il mouruł d'un accident banal, une chute de bicyclette, en 1895, à l'âge de cinquante-deux ans. M. L. O. Howard, son collaborateur et son ami. lui a succédé et continue, avec une activité qui ne se dément pas, à perfectionner l'organisation d'un service d'entomologie qui s'impose à l'admiration du nıonde entier (1).

Les détails qui précèdent étaient nécessaires pour relier le présent au passé et pour donner une idée nette des progrès accomplis dans l'étude de l'entomologie. Nous avons surtout essayé de présenter la science en marche, hésitante et naïve au début, étendant plus tard son domaine par l'observation, et abordant enfin, aujourd'hui, aux Iumières de l'expérience, les problèmes les plus délicats de la biologie.

Nous ne voulons rien dire des entomologistes vivants, car le recul du temps est nécessaire pour juger les hommes avec impartialité, mais certes, les œuvres puissantes auxquelles beaucoup d'entre eux ont attaché leur nom, auront aussi un jour leur place marquée dars l'histoire des sciences.

(1) Les clichés de tous les portraits utilisés dans cette ze édition, nous ont été gracieusement offerts par M. Charles Oberthür, l'auteur bien connu des Etudes a'Entomologie et de Lépidoptérologie comparée. 



\title{
LIVRE PREMIER
}

\author{
PREMIERE PARTIE
}

Miorphologie externe.

\section{CHAPITRE I}

\section{CARACTIRES GÉRERAUX DES INSECTES}

TEchnique. - Suivre les développements de ce chapitre sur un Hanneton, une Abeille ou une Libellule.

I. _ Il a fallu arriver jusqu'à Lamarck (1801), pour voir la classe des Insectes renfermée dans les limites que tout le monde s'accorde à lui reconnaître aujourd'hui.

On désigne sous le nom d'Insectes, tous les animaux dépourvus de squelette interne qui, à l'état adulte, ne possèdent jamais plus de trois paires de pattes, et dont le corps est divisé en trois régions bien distinctes : une Abeille, une Mouche, une Sauterelle, un Papillon sont des Insectes.

La plupart d'entre eux subissent des métamorphoses plus ou moins compliquées.

Les trois régions du corps d'un Insecte sont :

10 LA TÊTE, qui porte les organes des sens (yeux et 
antennes), ainsi que les nombreuses pièces de l'appareil buccal.

$2^{\circ}$ LE thorax ou corselet, sur lequel sont fixés les organes locomoteurs (pattes et ailes).

$3^{\circ}$ L'ABDomes, formé d'anneaux emboîtés les uns dans les autres, et sur chacun desquels s'ouvrent les

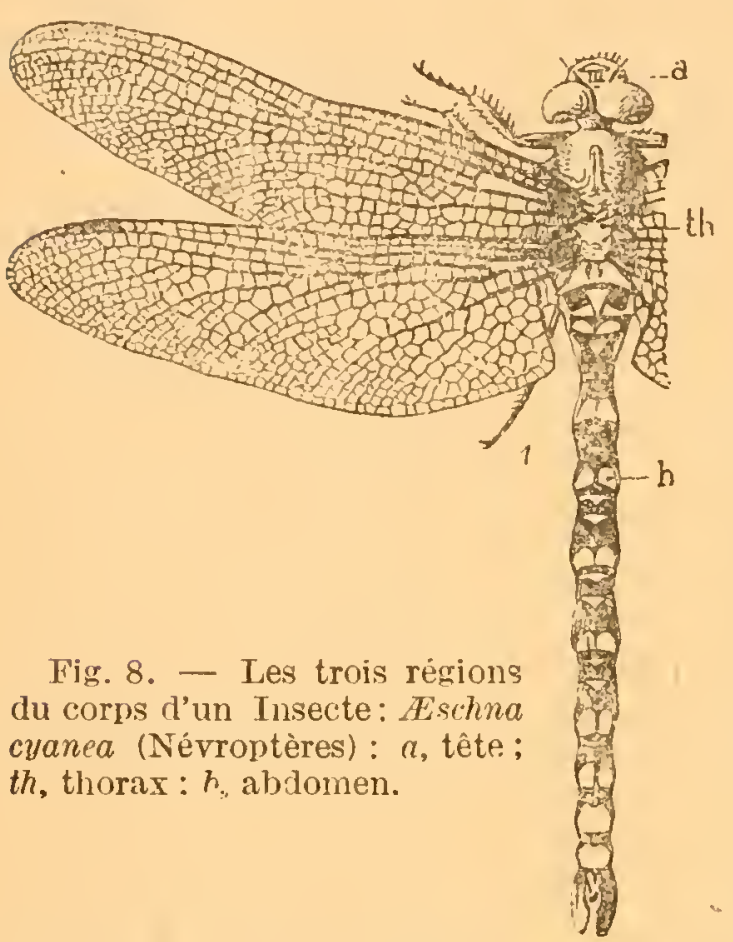
petits orifices (stigmates), quipermettent àl'air extérieur de pénétrer à l'intérieur du corps (Fig. 8).

Cette définition de l'Insecte n'est établie que sur les caractères extérieurs les plus apparents, sur ceux qui sont immédiatement visibles, mais personne ne sera surpris d'apprendre qu'on peut également trouver, dans l'ensemble de l'organisatiou interne, un certain nombre d'autres caractères importants qui, joints aux premiers, contribueront à fixer le type de la classe tout entière et à définir son caractère zoologique.

Ces caractères distinctifs spéciaux, ont été bien indiqués, en 1875, par Paul Mayer.

Par une intuition très heureuse, cet auteur est parvenu à établir un schéma très vraisemblable de ce que fut probablement, dans le passé, le type ancestral des Hexapodes; ce type synthétique, il le désigne sous le nom de Protentomon (1) et il lui attribue les caractères suivants,

(1) Du grec: protos premier et entomon insecte. 
que l'on retrouve, en effet, sauf modifications secondaires, chez les Insectes de tous les ordres.

Paul Mayer prévient toutefois que les considérations qui vont suivre ne peuvent pas s'appliquer aux Thysanoures; il explique pourquoi à la fin de son travail. Le Protentomon ne représente, cela va sans dire, que l'ancêtre schématique des Insectes adultes et nullement celui des larves.

II. - Théorie dy Protentomon (Fig. 9). - Voici résumés, d'après le travail de Paul Mayer, les caractères, de ce type ancestral de tous les Insectes.

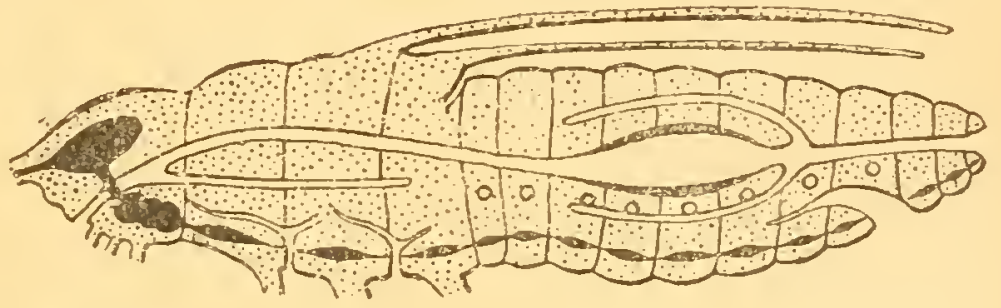

Fig. 9. - Le Protentomon: représentation idéale du type primitif des Tnsectes (d'ap. P. MAYER).

10 Un corps segmenté, dans lequel on peut distinguer trois régions : la tête, le thorax et l'abdomen. La tête porte une paire d'antennes filiformes, trois paires d'appendices buccaux (1), une paire d'yeux composés, et, probablement, trois ocelles. Le thorax, constitué par trois métamères très distincts (prothorax, mésothorax et métathorax), porte une paire d'appendices locomoteurs à la partie ventrale de chacun de ces métamères et une paire d'ailes à la partie dorsale de chacun des deux derniers (2). L'abdomen, formé de onze anneaux, ne porte ni ailes ni appendices locomoteurs.

$2^{\circ}$ Les appendices locomoteurs (pattes) sont des prolongements, en forme de tubes creux, des parois du corps; ils sont égaux entre eux et composés de cinq segments. Les ailes, égales entre elles, sont des vésicules transparentes, aplaties, dont la surface externe est une lamelle de chitine homogène.

(1) Les recherches récentes ont montré qu'il faut, en réalité, compter quatre paires d'appendices buccaux (voir p. 56) chez les insectes les plus voisins du type ancestral.

(2) Il faudrait encore ici modi fierla formule du Protentomon, car on sait que certains insectes des temps primaires possédaient trois paires d'ailes (voir p. 27j). 
$3^{\circ}$ Le tube digestif, qui s'étend depuis la bouche placée à la partie inférieure de la tête jusqu'à l'anus, situé sur le dernier anneau abdominal, comprend trois régions : l'intestin antérieur, l'intestin moyen ou estomac et l'intestin postérieur. La paroi de l'estomac est formée par une seule, assise de cellules digestives et dérive de l'entoderme (1), tandis que l'intestin antérieur, et l'intestin postérieur d'origine ectodermique, sont tapissés, comme l'hypoderme tout entier, par une membrane chitineuse. A la partie antérieure du tube digestif, une paire de cæcums constitue deux glandes salivaires, tandis que, de même, à l'origine de l'intestin terminal (rectum), deux paires de tubes constituent les organes excréteurs ou tubes de Malpighi (2).

$4^{\circ}$ Le système nerveux consiste en une masse ganglionnaire susœsophagienne ou masse cérébroïde, unie par un collier à une masse ganglionnaire sous-osophagienne; celle-ci est rattachée elle-même à une chaîne ventrale comprenant trois ganglions thoraciques et o.lze ganglions abdominaux, réunis entre eux par deux connectifs longitudinaux.

$5^{\circ}$ L'appareil circulatoire (raisseau dorsal) est un tube placé dans la région dorsale, au-dessus du tube digestif, il est divisé métamériquement en chambres dans la région abdominale, mais non segmenté dans sa partie antérieure où il constitue une sorte d'aorte.

$6^{\circ}$ Les trachées, placées immédiatement sous l'épiderme, résultent d'invaginations ectodermiques; elles restent en relation avec l'extérieur par des ouvertures ou stigmates, et se ramifient à l'intéricur da corps. La tête et le prothorax sont dépourvus de stigmates.

$7^{\circ} \mathrm{La}$ grande partie de la cavité viscérale est remplie par le colps adipe ux, dans les lacunes duquel circule le sang.

$8^{\circ}$ Les organes génitaux sont constitués pas-une paire de glandes ayant chacune leur conduit propre, et venant s'ouvrir à la partie ventrale de l'abdomen entre le huitième et le neuvième anneau.

Les caractères sexuels secondaires manquaient probablement.

D'après les remarques que nous venons de faire, il est facile de voir que le schéma de Paul Mayer au-

(1) Ce caractère, vraisemblahlement ancestral, ne se trouvera plus chez les insectes actuels, ou les trois parties de l'intestin dérivent de l'ectoderme (voir 1). 2225).

(2) Les tubes de Malpighi sont bien, en effet, caractéristiques des Insectes; mais les homologues des glandes salivaires, sur le proctodceum, sont plutôt des glandes anales. 
rait besoin de subir aujourd'hui quelques retouches.

Si nous avions eu, pour notre part, à établir les caractères du Protentomon primitif, nous ne lui aurions pas attribué d'ailes. Les Insectes dérivent, évidemment, d'ancêtre aptères voisins des Campodés actuels; ils devaient avoir, au début, un caractère larval très prononcé ; le Protentomon de Mayer est déjà plus perfectionné que n'importe quel Thysanoure; tel quel, il ne saurait donc être consicléré comme leur ancêtre réel ni même comme le prototype des Hexapodes.

Il est juste de reconnaître que cette difficulté n'a pas échappé à Paul Mayer, puisqu'il a pris soin, ainsi que nous l'avons vu, d'indiquer que son Protentomon répresente la souche primitive des adultes et non celle c'e: larves. 


\section{CHAPITRE II}

\section{TÉGUMENTS DES INSECTES}

TECHNIQue. - Prendre un Hanneton, une Sauterelle ou un Carale, pour étudier l'aspect des téguments et le mécanisme des mouvements. Les ramollir par une immersion de huit à dix heures dans une solution concentrée et chaıde de potasse caustique, afin ce pouvoir faire ensuite des coupes transversales de la paroi chitineuse.

Il n'est personne qui ne connaisse l'Abeille ou le Carabe doré; tout le monde a pu voir que le corps de ces animaux est recouvert, dans toute son étendue, par une sorte de carapace rigide, amincie seulement aux articulations, ce qui permet aux différents anneaux de se mouvoir les uns sur les autres.

Cette carapace existe chez tous les Insectes sans exception; elle est formée d'une substance complexe, que l'on désigne sous le nom de chitine (1) et dont la fonction chimique est encore inconnue.

Origine de la membrane cuticulaire. - Si nous faisons une coupe transversale de la peau d'un Insecte quelconque, à l'état adulte, nous y trouvons toujours deux couches bien distinctes : une couche externe, continue, peu perméable aux liquides, c'est la cuticule. Au-dessous se trouve une membrane formée d'une seule assise de

(1) Chitine : du grec kiton revêtement. 
cellules cubiques ou faiblement aplaties, c'est l'hypoderme (1) (Fig. 10).

La paroi des cellules hypodermiques reste mince dans ses parties internes et latérales; mais, dans sa partie Jibre, c'est-à-dire celle qui est en contact avec l'extérieur, elle s'épaissit considérablement. Les parois externes épaissies forment alors, en se réunissant les unes aux autres, une lame continue, flexible, plus ou moins stratifiée. C'est ainsi que se forme le revêtement si caractéristique, qui protège le corps de tous les Insectes, et même de tous les Arthropodes.

Guticule. - Les auteurs ne sont pas d'accord sur le mode de formation de la cuticule; pour les uns, elle résulte simplement d'une modification de la paroi des cellules hypodermiques; pour les autres, c'est un produit d'excrétion qui se dépose en couches successives stir la surface libre des cellules hypodermiques, épaississant peu à peu leurs parois, de telle sorte que les nouvelles couches repoussent continuellement les anciennes vers l'extérieur.

Quoi qu'il en soit, la membrane cuticulaire, issue de l'activité des cellules hypodermiques, ne s'est pas seulement modifiée dans son épaisseur pendant sa formation, elle s'est aussi profondément modifiée dans sa constitution chimique, et s'est incrustée de cette substance spéciale, à laquelle Odier, en 1821, a donné le nom de Chitine.

Il convient donc d'étudier, avec quelques détails, la structure intime et le développement de la Cuticule, ainsi que les propriétés chimiques de la chitine. C'est ce

(1) Le nom d'hypoderme ne convient pas : cette assise de cellules n'est pas sous la peau, c'est la peau elle-même. 
que nous allons faire, d'après les recherches récentes de M. J.-W. Tower (1)

Structure de la cuticule. - A l'origine, c'est-à-dire à la sortie de l'oruf et pendant les premières phases de la vie larvaire, le corps de l'Insecte est limité par une seule assise de cellules ectodermiques: c'est le futur hypoderme.

1. Cuticule primaire. - La cuticule apparaît de très bonne heure, à l'extérieur de cet hypoderme, sous l'aspect d'une membrane mince recouvrant toute la surface du corps, sans aucune solution de continuité. Cette membrane ne cesse de s'épaissir, jusqu'à ce qu'elle ait atteint la solidité qui caractérise l'espèce considérée : c'est la cuticule primaire; elle donnera naissance aux écailles, aux poils et à tous les autres prodüits de l'activité tégumentaire.

2. Cuticule secondairc. - Pendant la nymphose, au moment oì la cuticule primaire cominence à se durcir, par suite de sa transfor.mation en chitine, l'hypoderme entre dans une nouvelle phase d'activité ; il sécrète, rapidement, une deuxième membrane culiculaire (cuticule secondaire), plus épaisse que la première et donnant une consistance rigide à la paroi du corps (Fig. 10).

Cette cuticule secondaire diffère notablement de la première par

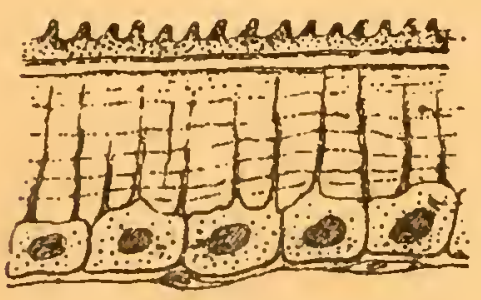

Fig. 10. - Structure de la cuticule au cours de la nymphose (d'ap. W. L. Tower). sa structure et par ses réactions: elle est formée de couches alternantes ayant des indices de réfraction différents, qui lui donnent un aspect stratifié; elle est, de plus, traversée par de très fins canaux, qui se terminent toujours à la cuticule primaire.

Cies petits canaux sont extrêmement nombreux; ils sont, en réalité, remplis par de fins prolongements des cellules hypodermiques, et jouent un rôle important au moment de la formation des pigments.

Chez les larves, qui mangent avec avidité, la cuticule secondaire s'accroît continuellement; mais, chez l'adulte, elle ne s'épaissit que si l'hypoderme continue à recevoir de la nourriture; lorsque l'insecte cesse de s'alimenter, cas fréquent chez les adultes, le développement s'arrête et l'hỵpoderme dégénère.

(1) Towrin (J.-W.) D. 22-20. (Voir : Index bibliograph zque, 1). 351). 
Propriétés de la Chitine. - I a Chitine, qui parait être aux téguınents des Arthropodes ce que l'osséine est aux os des Vertébrés, présente une très grande résistance à l'action des réactifs. Elle est à peine altérée, à l'ébullition, par des solutions failles d'acides ou d'alcalis, ainsi que par l'alcool, l'éther, les huiles essentielles, etc. : mais en revanche, elle se ramollit sous l'action des hypochlorites (1) et se dissout facilement dans les solutions concentrées d'acide chls:1 hydrique ou d'acide sulfurique.

Les auteurs qui ont essayé d'analyser la chitine en ont donné des Pormules différentes; cela tient à ce que la composition de cette substance est variable suivant les espèces, et, très probablement aussi, suvant les diverses parties du corps d'un même Insecte. Nous donnons ici l'une des formules les plus récentes, celle qui a été établie par Sundwick, en 1881 (2).

$$
\mathrm{C}^{60} \mathrm{H}^{100} \mathrm{~N}^{8} \mathrm{O}^{38}+\mathrm{n} \mathrm{H}^{2} \mathrm{O}
$$

Les deux couches cuticulaires chitinisées ne forment pas simplement un revêtement protecteur pour le corps des Insectes, elles jouent encore un rôle très important dans la réparation des tégcments. Verhoeff a, en effet, observé que, chez un Carabe, à la suite d'une blessure, la paroi du corps fut très rapidement reconstituée, non pas uniquement par un caillot de sang, mais par de nouvelles couches rhitinisées.

Métamérie. - L'un des caractères les plus remarquables des Insectes, caractère qu'ils possèdent d'ailleurs en commun avec les autres Arthropodes et même avec les Vers, c'ést la segmentation de leur corps en anneaux distincts, placés les uns à la suite des autres. Ces anneaux, que l'on désigne sous les noms de somites, de zoonites ou de métamères (3), visibles dans toute l'étendue du corps chez les larves, ne, le sont plus guère, chez l'adulte,

(1) On utilise cette propriété, dans la technique histologique. lorsqu'on veut pratiquer des coupes minces dans les téguments, pour les étudier au microscope.

(2) Dans cette formule $n$ varie de 1 is 4.

(3) Somite: du grec soma corps. - Zoonite, de zoon animal : les segments clu corys, chez les vers, étant quelquefois consi dérés comme des animaux soudés. - Métamères: du grec meta (un changement) et meros partie, d'où le nom de Hétamerie. 
que dans la région abdominale. Cette segmentation du corps est évidemment un caractère héréditaire que les Insectes tiennent des Vers, leurs ancêtres probables.

Sclérites. - La membrane chitineuse qui forme l'enveloppe du corps et des appendices ne présente aucune solution de continuité, mais elle ne possède pas la même épaisseur ni la même rigidité en tous ses points. Au niveau des articulations, cette membrane reste mince et flexible, ce qui permet les mouvements ; mais dans l'intervalle qui sépare deux articulations, la chitine forme des plaques solides, symétriquement distribuées et variables suivant la région que l'on considère. Ces plaques ont été désignées sous le nom de sclérites (1).

Constitution schématique d'un métamère (segment). - Victor Audouin (2), qui a cherché, en 1814, à découvrir, dans les diver's anneaux du, squelette tégumentaire des Insectes, un plan commun d'organisation, ainsi que Savigny l'avait déjà fait pour les pièces de la bouche (V. Introd. p. 17.), crut pouvoir admettre que, dans un métamère typique, comme par exemple l'anneau médian du thorax, on trouvait toujours un nombre invariable de sclérites; il créa, pour ces diverses pièces, une nomenclature très simple, mais en même temps très philnsophique, de sorte que, "connaître la constitution

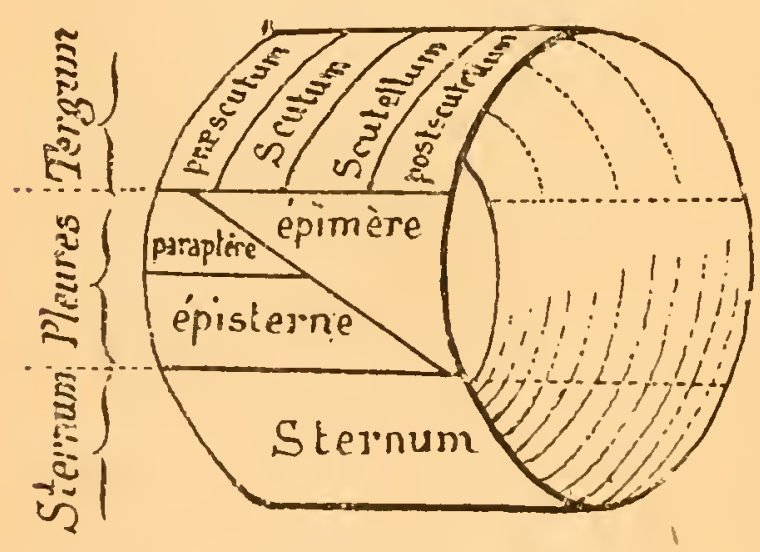

Fig. 11. - Schéma de la constitution d'un métamère thoracique (d'après Comstock).

de cet anneau typique, c'est connaître celle de tous."

$1^{\circ}$ D'après Audouin, un an' neau complet peut être consi déré comme formé de deux demi-roûtes, disposées en sens inversel'une de l'autre, et réunies de chaque côté pas des pièces latérales (Fig. 11).

La demi-voûte supérieure porte le nom de tergum (ou encore notum) et les pièces (sclérites) qui la composent sont les torgites; la demi-voûte inférieure est le sternum, de même

(1) Du grec skleros, dur.

(2) Audourn (J. V.). - T. I. p. 97-135:410-432. 
ronstituce paı les sternites. Quant aux pièces latérales, servant rúunir le tergum et le sternum, elles ont reçu le nom de pleures (1).

Tergum. - Le tergum se compose de quatre tergites, qui son1, d'avant en arrière : le proscutum, le scutum, le scutellum et le post. scutellum.

Sternum. - Le sternum reste indivis en apparence.

Pleures. - Les pleures sont au nombre de trois de chaque côté : ce sont: l'épisterne, l'épimère et le paraptère.

Audouin avait encore désigné, sous le nom d'entothorax, un certain nombre de lames internes, saillantes à l'intérieur du corps et qui ne sont autre chose que les replis accolés de deux sclérites contigus. On donne aujourd'hui à ces organes le nom d'apodèmes (Fig. 12. a) ; ils servent de point d'attache à de nombreux muscles et abritent même parfois la chaine nerreuse ou le tube digestif.

L'épımère est une dépendance de l'arceau supérieur, avec lequel il est, en général, très largement articulé; l'épisterne,

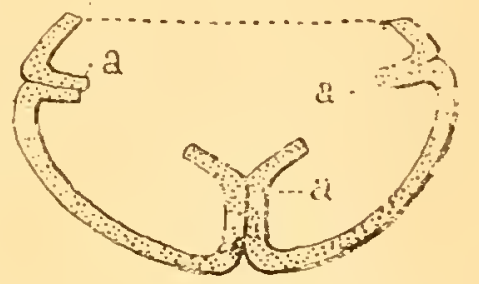

Fig. 12. - Replis des téguments constituant les apodèmes

(d'après T'. Audouin). au contraire, fait partie de l'arceau inférieur qu'il complète de chaque côté. Quant aux paraptères, on ne doit pas les considérer comme des sclérites du thorax : ce sont deux petites pièces sura. joutées, dépendant vraisemblablement de la base des ailes antérieures.

Si nous ajoutons que les pièces impaires de chaque anneau (tersum et sternum), doivent être considérées comme formées, en réalité, de deux sclérites symétriques soudés sur la ligne médiane du corps, nous verrons alors que chaque segment squelettique, à son maximum de complication, est constitué par quatorze sclérites : dix pour le demi-segment supérieur et quatre pour le segment inférieur (2).

$2^{\circ}$ Sur chaque anneau thoracique, les pattes s'insèrent toujours entre les épimères et les épisternes ; les ailes, lorsqu'elles existent, sont également fixées, de chaque côté, entre les épimères et les tergites.

Disons enfin, pour terminer que, à l'origine - bien longtemps

(1) Du latin pleuro, flancs.

(2) Lès parantères comme nous renons de l'indiquer, ne cevant pas entrer en ligne de compte. 
sans doute avant l'existence du problématique Protentomon - chaque segment du corps devait porter une paire d'appendices. Cette disprsition, conservée chez les rares représentants du groupe des Onıih pphores (Peripatus), est très visible encore sur certains embryor', pendant les premières phases du développement.

Telle est, théoriquement, la composition d'un anneau thoracique complet de l'exosquelette des Insectes; nous pouvons maintenan entrer dans le détail des trois principales régions du corps : tête, thorax et abdomen; ensuite viendront l'étude des appendices et celle, non moins importante, de l'ornementation de la paroi et de la protection des téguments. 


\section{CHAPITRE III}

\section{LA TETE ET SES APPENDIGES}

TeCHAique. - Pour suivre les développements de ce chapitre, prendre le Carabe doré, la Sauterelle serte ou une Libellule de grande taille.

10 Constitution de la tête. - La tête des Insectes résulte de la fusion d'un certain nombre de métamères; mais les nombreux sclérites qui la constituent sont si modifiés, si intimement fusionnés les uns avec les autres, qu'il est absolument impossible de déterminer la limite des divers anneaux. L'ensemble forme une capsule, une boite chitineuse continue et de forme variable suivant les groupes; seules, l'embryogénie, et une étude anatomique très minutieuse, analogue à celle qu'a faite Charles Janet pour les Fourmis (1), pourront nous donner quelques renseignements précis sur la constitution métamérique de cette partie du corps.

Les entomologistes descripteurs ont distingué, à la partie supérieure de la capsule céphalique, un certain nombre de régions assez faciles à reconnaître chez les Insectes broyeurs (Coléoptères, Orthoptères, Nésroptères), et même chez la plupart des Hyménoptères ; mais, fort difficiles ou même quelquefois impossibles à homologuer

(1) JANET (Ch.). - Essai, 1899, n. 31-65.

3. 
chez les Lépidoptères et les Diptères. Ces régions sont : l'épistome, le postépistome et l'épicrâne (Fig. 13); de

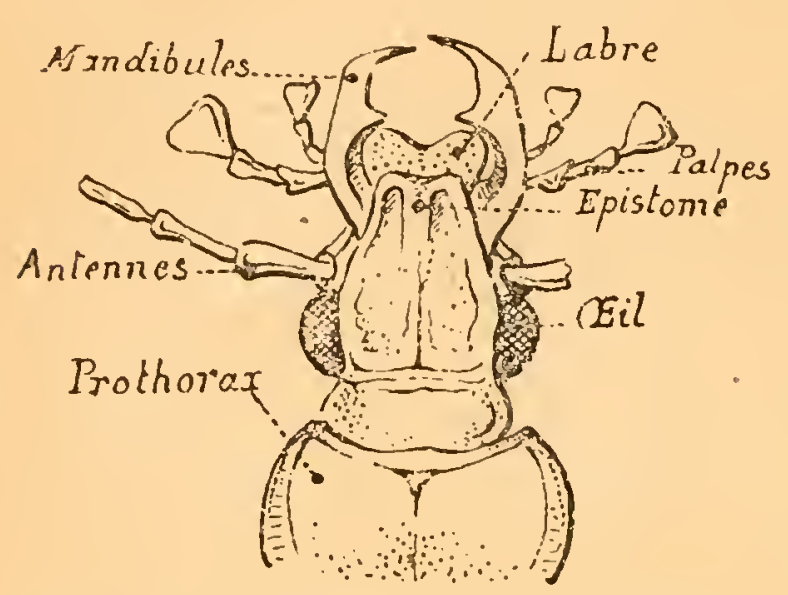

Fig. 13. - Tête d'un Carabe vue en dessus. même, à la face inférieure, nous devons distinguer la pièce basilaire et la pièce prébasilaire.

10 FACE SUPÉRIEURE DE LA TÊTE. - L'épicrâne, en particulier, c'est-à-dire lá partie la plus étendue et la plus apparente du dessus de la tête, est, le plus souvent, décrit avec un grand luxe de détails dans les ouvrages d'entomologie descriptive; il présente à considérer un certain nombre de subdivisions conventionnelles, d'étendue très variable, sans limites précises, que l'on désigne sous les noms de front, vertex, tempes, joues, occiput, etc.

Epistome. - L'épistome (Fig. 14, 2), encore désigné sous les nonis de chaperon et clypeus, est généralement bien développé chez les Coléoptères; cependant, quand il est intimement fusionné avec le postépistome ou l'épicrâne, ainsi que cela se produit chez les Cétonides, sa limite postérieure devient impossible à fixer.

Chez les Orthoptères et les Névroptères, l'épistome est presque toujours très risible entre le labre (1) et l'épicrâne (3); mais, nulle part, il n'est aussi bizarrement construit que chez les Hémiplères notamment chez les Cigales, on il forme une saillie antérieure striée transversalement, et divisée

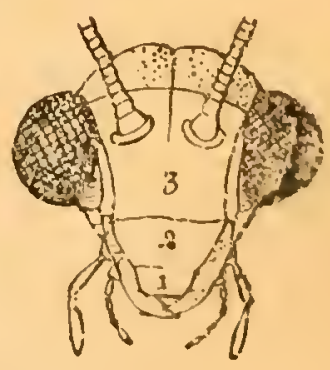

lig. 14. - Face antérieure de lia tête d'un Névroptère : Myrmeleon diversum (d'il). I'ACKARD). dans le sens de la longueur par un sillon (Fig. 19,1,)

Chez les Diptères, il se réunit généralement aux joues poul douner un bec conique. 
Postépistome. - Cette pièce est confondue avec l'épistome dans la grandë majorité des Insectes (F ig. 13) ; cependant, on la trouve très distincte chez certains Coléoptères de la famille des Chrysomé! ides (1) ; elle fournit ainsi de bons carartères à la classification.

On peut également l'observer chez les Névroptères (Libellulines) et, contrairement à l'opinion de Lacordaire, beaucoup plus diffir lement chez les Orthoptères.

Ailleurs, elle est presque toujours extrêmement étroite ou tout à fait distincte.

Fpicrâne. - L'épicrâne, ainsi que nous l'arons déjà dit, constitue toujours, en dessus, la partie la plus étendue et la plus apparente de la tête (Fig. 14,3). Dans son intérieur, se trouve un système varié de lames chitineuses, donnant insertion aux muscles moteurs des mâchoires et servant à supporter le cerveau. Cet ensemble a été désigné sous le nom de tentorium, il est facile à étudier chez l' Hydrophile, ou il est formé par trois paires d'invaginations des téguments.

2u Face inférieure de la tête. - Pièce basilaire. - Ainsi nommée par Strauss, elle occupe la partie inférieure de la tête et limite, en partie, le trou occipital. Très distincte chez la plupart des Coléoptères (2), des Orthoptères et des Hémiptères, elle est au contraire à peine risible dans les autres groupes.

Pièce prébasilaire. - La présence de cette pièce n'est pas constante; on ne peut même l'observer que chez un nombre très restreint d'espèces, surtout parmi les Coléoptères (Hydrophile, Carabe, etc.).

$2^{\circ}$ Appendices céphaliques. -- Les appendices de la tête sont, comme nous l'avons dit, les yeux, les antennes et l'appareil buccal.

Les yeux seront décrits plus tard avec les organes des sens (p. 166i).

10 Antennes. - Les antennes, vulgairement désignées sous le nom de cornes, sont des appendices articulés, de forme très variée, insérés latéralement sur l'épicrâne;

(1) Très visible dans les Sagra, le postépistome manque chez les Donacies; on a donc lì un moyen de distinguer facilement ces deux genres.

(2) Elle est remarquable par sa grandeur chez le Hanneton, où elle forme une large plaque luisante à la kase du cou. 
elles sont au nombre de deux chez tous les Insectes sans exception.

Le mode d'articulation des antennes avec la tête est celui bien connu sous le nom d'articulation à genoux. En effet, la base du premier article se renfle en une sorte de rotule parfaitement lisse, reçue dans une cavité cor-

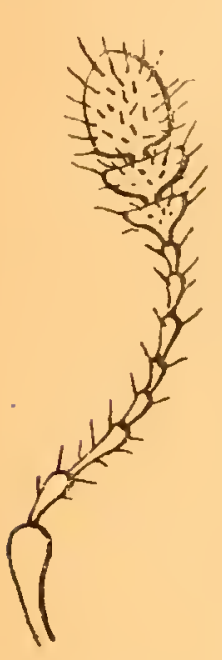

Fig. 15. Une antenne ie Coléoptère Heoticus serratus Gull. respondante de l'épicrâne, ce qui, grâce aux muscles logés à l'intérieur, permet à l'antenne de se mouvoir dans tous les sens.

Quelle que soit leur forme, on distingue théoriquement trois parties dans une antenne: le premier article a reçu le nom de scape (1); le deuxième est le pédicelle; puis, l'ensemble de tous les autres articles constitue le funicule ou flagellum (Fig. 15). La forme du flagellum est tellement variable qu'il est impcssible d'en donner une description générale; tout ce qu'on peut dire c'est que, dans cerlains cas, cette forme peut servir à caractériser plusieurs familles: les Lamellicornes et Longicornes parmi les Coléoptères; les Bombycides et les Sphingides parmi les Lépidoptères.

Le nombre des articles aux antennes subit également de grandes variations suivant les groupes. Bien que ce nombre soit typiquement de onze chez les Coléoptères, 11 descend à trois dans quelques Platypus; il peut arriver à deux chez les Paussus et les Andranes, et même à un, dans le curieux autant que rare Articerus armatus, appartenant à la famille des Clavigérides.

Chez les Orthoptères, le nombre des articles peut

(1) Le Scape est ordinairement très développé chøz. Jes Curculionides. 
varier entre onze (quelques Phasmides) et cent cinquante, par exemple chez les Blattidés.

Les Lépidoptères ont les antennes composées d'un nombre très grand d'articles, souvent si peu distincts qu'il est presque impossible de les compter; il en est de même, en ce qui concerne les Hyménoptères de la tribu des Ichneumonides.

Chez les Diptères, on peut distinguer deux types : les Némocères (ex. : Tipulides) qui ont, en général, des antennes de quatorze à seize articles, et les Brachycères (ex. : Mouches) où le nombre des articles n'est jamais supérieur à trois.

Bien entendu, toutes ces variations des antennes, relativement à la forme et au nombre des articles, sont des résultats d'adaptation; ce sont même quelquefois de simples variations sexuelles : ainsi, par exemple, dans le curieux Prionus imbricornis, les antennes des femelles ont neuf articles, tandis qu'elles en ont jusqu'à vingt chez les mâles, et ce cas n'est pas isolé.

\section{PIÈCES BUCCALES}

Théorie de Savigiy:- Lorsqu'on étudie la constitution de la bouche dans la série entomologique, on constate que les organes qui la composent sont formés dé façon très variable suivant les différents groupes et surtout suivant le mode de nutrition.

Certains Insectes, en effet, tels que les Carabes (Coléoptères), les Libellules (Névroptères) et les Grillons (Orthoptères), doivent brọer forrement les aliments solides dont leur nourriture se compose; leur bouche est, pour cela, armée défortes mandibules et de mâchoires triturantes; ils constitient la catégorie des INSEctes erovecra. D'autres, au contraire, te's que les Punaises (Hémiptères). les 
Papillons (Lépidoptères), ne'peuvent absorber qu'une nourriture liquide; les pièces de leur bouche se sont alors modifiées en conséquence et on a, suivant les cas, les INSEctes PIQUeUrs et les IXsectes suceurs.

Toutefois, il existe tout un groupe d'Insectes, dont la bouche est intermédiaire entre celle des broyeurs et celle des suceurs; c'est le groupe des Insectes lécheurs, dans lequel se range l'ordre nombreux et remarquable des Hyménoptères.

Enfin, il faut encore remarquer que, chez certains Insectes dont le mode de nutrition varie aux diverses phases de leur développement, la conformation de la bouche change avec l'âge, ce qui montre bien que cesvariations sont simplement adaptatives et qu'elles ne sont autre chose que des modification d'un thème fondamental unique, le même pour tous les Insectes. Tel est, par exemple, le cas des Lépidoptères ; sous leur état de Chenille, ils se nourrissent de substances régétales et leur bouche est conformée sur le même type que celle des Insectes broyeurs; mais, lorsqu'ils deviennent Papillons, ils ne peuvent plus absorber que le liquide sucré, formé par le nectar des fleurs, alors leur bouche se modifie; elle prend l'aspect d'une sorte de trompe, ils sont devenus suceurs. C'est SAv1. G.XY qui, le premier, en 1816, dans son travail sur les animaux sans vertèbres (1), établit l'homologie des différentes pièces de la bouche chez les Insectes. mais afin de bien comprendre ce qui se passe chez les divers types que nous venons d'examiner, voyons tout d'abord comment la bouche est constituée chez les Insectes broyeurs.

1. Insectes broyeurs. - Prenons, par exemple, le Carabe doré, si commun partout dans les jardins et dans les champs; c'est un cliasseur infatigable, un carnassier de premier òrdre.

Les organes dont sa bouche est armée, et qui servent à la fois à saisir et à broyer les aliments, sont au nombre de six; si nous les examinons, de dessus vers le dessous, l'insecte étant placé sur ses pattes, dans la position ordinaire du repos, nous trouverons, dans l'ordre suivant (Fig. 16):

$1{ }^{\circ}$ Une pièce médiane impaire appelée labre ou lèsre supérieure; cette pièce est formée par la soudure de deux sclérites contigus et s'articule en arrière, avec le bord antérieur de l'épistome.

(1) Lelorane de Savigny (J.). - Mémoircs sur les animaux sans rertèurcs. 1 Partie, Paris, 1816 , in $-8^{\circ}$. 
20 Sous le labre, on remarque une paire de grosses dents appelées mandibules $(M d)$. Ces organes s'articulent avec la tête au moyen de deux ou trois apophyses dont leur base est munie.

Dans quelques espèces, comme par exemple chez. les Lucanes (Cerfs-solants), ce sont les mandibules qui se développent d'une

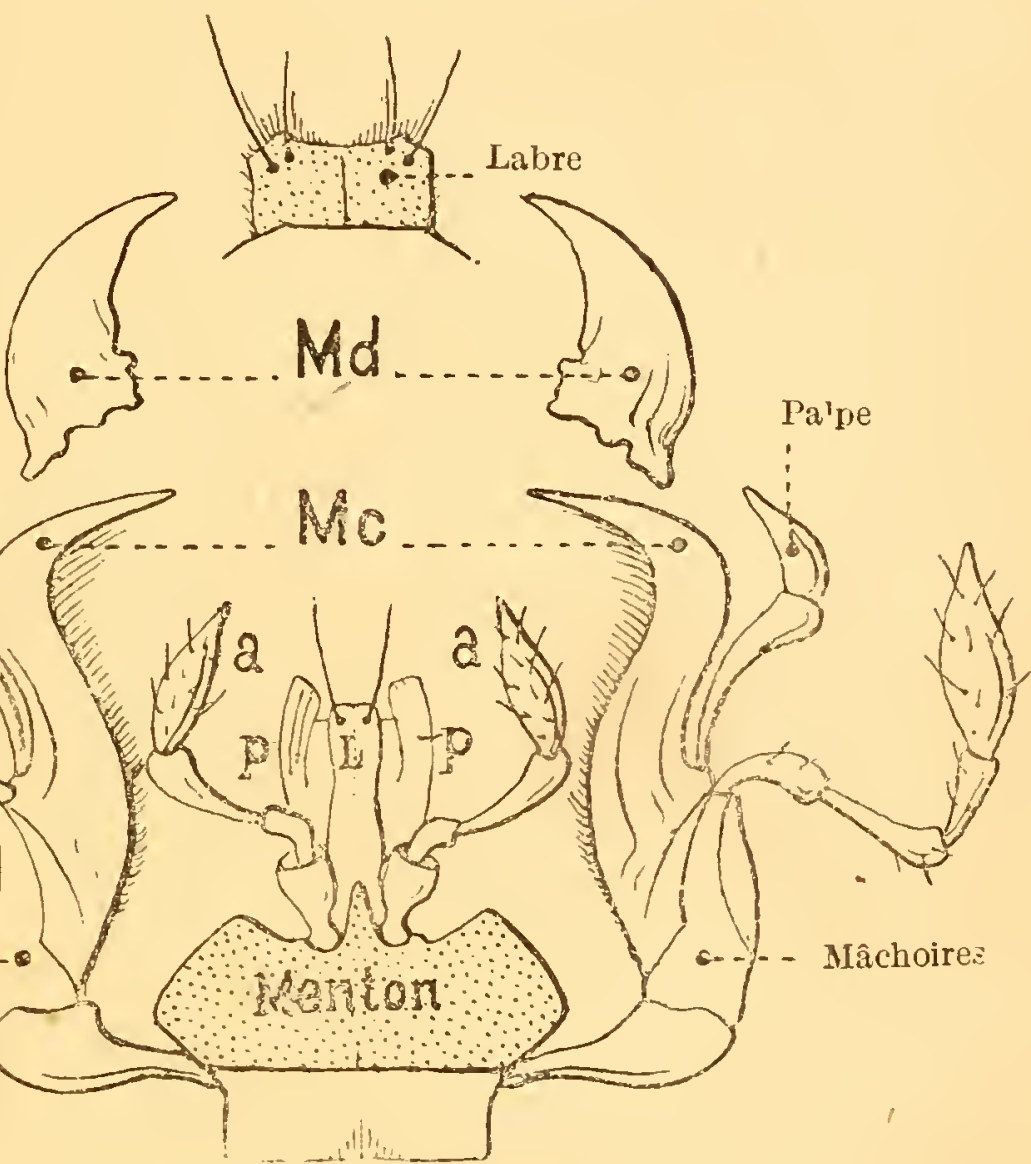

Fig. 16. - Schéma dez pièces de la bouche. vues en dessous, chez un Insecte broyeur: Carabus auratus (Coléopt).

façon démesurée et forment la pince puissante que tout le monde connaît (Fig. 119).

$3^{\circ}$ En dessous des mandibules, se trouvent les mâchoires ( $\boldsymbol{H}$ c), également au nombre de deux. Les mâchoires étant destinées à diriser les aliments, chacune d'elles porte, en dedans, une lame coupante garnie de dentelures. En dehors, les mâchoires présentent uin ou deux petits appendices appelés palpes maxillaires; les palpes servent à flairer les aliments, grâce aux nombreux poils dont ils sont revêtus.

$4^{\circ}$ Enfin, on appelle lèse inférieure ou simplement labium, une pièce impaire asseż compliquée, opposée au labre, et située à la 
partie inférieure de la bouche ; elle porte aussi une paire de palpes, ce sont les palpes labiaux (a.a.).

La lèvre inférieure se compose de plusieurs parties qu'il est nécessaire de connaître, parce qu'on les utilise quelquefois dans les tableaux de détermination.

La piẹce la plus importante est le menton qui s'articule, en arrière, avec la pièce prébasilaire (p. 47) et qui sert à protéger, en dessous, les organes buccaux. Le prolongement antérieur et souvent cartilagineux du menton, a reçu le $\mathrm{nnm}$ de languette $(L)$. Enfin, très souvent, entre les palpes labiaux. et la base de la languette, existent deux petits appendices menbraneux et ciliés qu'on a désignés sous le nom de paraglosses (Fig. 16, p.).

Examinons maintenant les modifications de la bouche dans les antres types.

20 Insectes lécheurs. - Les modifications les moins profondes se présentent dans l'ordre des Hyménoptères (Fig. 17), c'est-à-dire

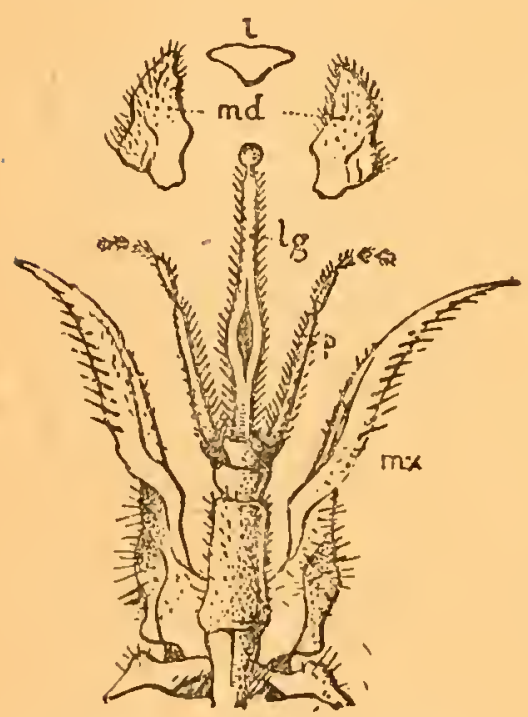

Fig. 17. - Les pièces buc. cales d'un Insecte lécheur : Abeille $n$, palpes labiaux; $m a$. mâchoires. dans le groupe d'Insectes qui renferme l'Abeille, la Guêpe, etc. Ici, la bouche présente une conformation intermédiaire entre le type broyeur et le type suceur; en effet, les pièces de la partie supérieure de la bouche, c'est-à-dire le labre et les mandibules $(m d)$, nc diffèrent en rien d'essentiel des mêmes pièces chez. les Insectes broyeurs; c'est seulement la partie inférieure de la bouche qui se modifie; les mâchoires et surtout la languette $(l g)$ s'allongent fortement; cette dernière prend la forme d'une gouttière garnie de poils à son extrémité (langue), et l'ensemble devient une sorte de trompe servant à aspirer les aliments liquides qui conviennent ì ces Insectes.

En résumé, les Ifyménoptères ont donc la moitié supéricure de la bouche conforméc pour déchirer les aliments, (type broyeur) et la moitié inférieure disposée pour aspirer (tıpe suceur). Au moyen de leurs mandibules, ils peuvent découper les substances dont ils se nourrissent et les matériaux dont ils composent leurs nids; la 
trompe sert ensuite à pomper les sucs des fruits dont ils ont préalablement déchiré l'enveloppe.

$3^{\circ}$ Insectes suçeurs.-Chez les Insectes suceurs, qui comprennent l'ordre entier des Lépidoptères, les divers appendices de la bcucho se modifient tellement que, pendantlongtemps, on ne les a pas crus construits sur le même plan que ceux des Insectes broṿeurs.

T'out le monde sait que la bouche des Papillons est prolongée par une trompe qui s'enroule en spirale à l'état de repos, et qui s'allonge en un tube flexueux quand ils veulent aller puiser leur.nourriture au fond de la corolle des fleurs (Fig. 18).

Cette trompe cylindrique est formée de deux demi-gaines (C) qui correspondent aux mâchoires des autres Insectes; on retrouve, en effet, à leur base, des palpes velus (A. p.) et de petits filaments, représentant les autres pièces de la

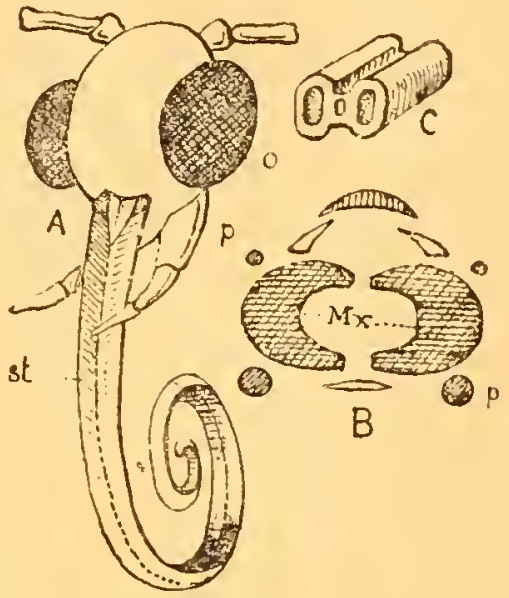

Fig. 18. - La bouche r'un Insecte suceur (Lépidontère). A. $o$, œil ; $p$, palpes ; st. trompe ; B. , $m x$, mâchoires ; $p$, palpes: C. coupe transversale de la trompe.

(d'ap. Mtali et Denny'). bouche profondément modifiées.

40 Insectes piqueurs. - Chez. ces Insectes, qui comprennent l'ordre entier des Hémiptères (Punaise, Cigale), et une partie de celui des

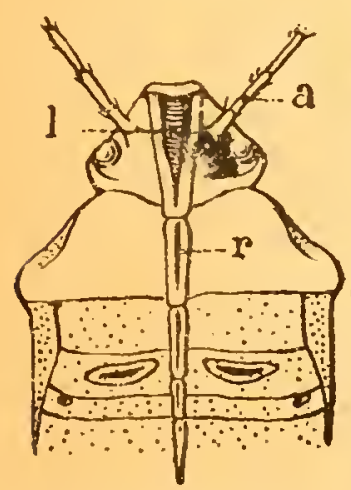

Fig. 19. La bouche d'un Insecte piqueur $l$, épistome ; $a$, antennes.

Diptères (Consins), la bouche est encore conformée d'une autre façon; les parties qui prennent ici le plus grand développement, ce sont les deux lèvres. La lèvre inférieure, surtout, s'allonge en formant un tube ayant l'aspect d'une aiguille creuse (Fig. 19). A l'intérieur de cette aiguille, que l'on désigne sous le nom de rostre $(r)$, on trouve quatre petits stylets garnis de dentelures et d'épines; ce sont les deux mandibules et les deux mâchoires modifiées.

Chez les Hémiptères, le rostre sert à percer la peau des animaux ou les tissus des végétaux dont ces Insectes se nourrissent; le plus souvent, le rostre est grêle et appliqué, au repos, sous la poitrine en passant 'ntre les pattes. 
Chez les Mouches (Diptères), la trompe représente aussi la lèvre inférieure modifiée, cette origine est attestée par les palpes qu'on observe encore à sa base, mais cette trompe est terminée par deux tampons charnus, qui fonctionnent comme des ventouses, et au centre desquels se trouve l'entrée du tube digestif.

Chez d'autres Diptères, les Cousins, par exemple, le suçoir n'est ras dilaté à son extrémité en un disque charnu; il contient plusieurs soies rigides qui servent d'arme vulnérante à l'Insecte et qui repré. sentent aussi les mandibules et les mâchoires modifiées.

\section{SIGNIFIGATION DES APPENDIGES CÉPHALIQUES.}

Quelle que soit la variété des organes que nous venons de décrire, les travaux d'Audouin, de Savigny, de Brullé, ainsi que des expériences toutes récentes, ont montré que les antennes, les yeux, de même que les autres pièces mobiles de la bouche, n'étaient que les appendices des anneaux céphảiques, profondément modifiés et adaptés à des fonctions spéciales. Chacun d'eux possède donc la valeur morphologique d'un membre; il est facile de le démontrer.

10 Antennes. - En ce qui concerne les antennes, il. est nécessaire, pour établir l'homologie, de recourir aux

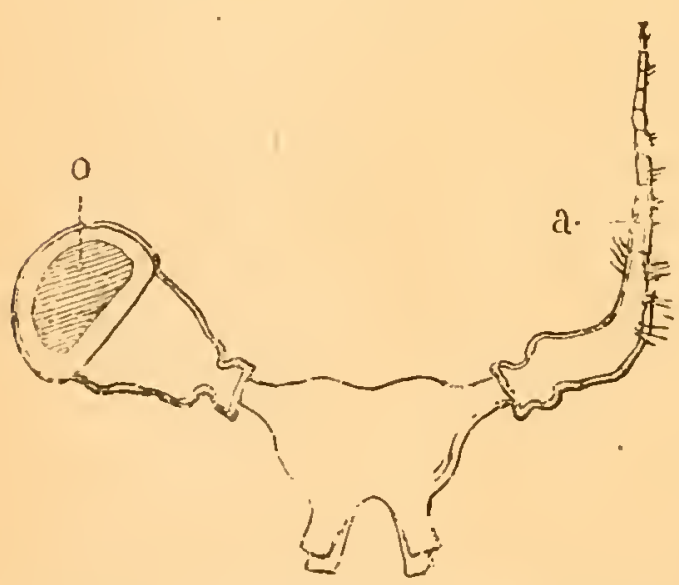

Fig. 20. - Exemple remarquable d'hẻtéromorphose ; un neil a été enlevé, il s'est forms une antenne à la place

(l'après IIKRIssT).

premières phases du développement larvaire; on voit alors que ces organes se développent en même temps que les pattes et par le même processus : elles représentent les membres profondément modifiés du deuxième anneau céphalique.

$2^{\circ}$ Yeux. - Les yeux peuvent être considérés comme des appendices du premier segment céphalique (Fig. 24). ou anneau postérieur de la tête; malgré l'opi- 
non contraire de la plupart des auteurs, ils sont, comme 'I s'antennes, des membres modifiés; une récente expérience de Herbst sur les Crustacés (Palaemon, Palinurus, ctc.), le démontre nettement; lorsqu'on enlève un ozil, dans certaines conditions déterminées, c'est une antenne (a) qui se reconstitue à la place de l'œil détruit (Fig. 20).

Il n'y a qu'une seule manière d'expliquer cette curieuse hétéromorphose, la plus remarquable, dit Loeb, "qu'on ait observée jusqu'ici (1) », c'est d'adméttre que l'ail et l'antenne sont deux organes de même valeur morphologique.

$3^{o}$ Pì̀ces buccales. - L'homologie des pièces buccales est beaucoup plus claire, cela se conçoit : on peut même y retrouver les principales subdivisions des membres des Crustacés. Considérons, par exemple, une mâchoire isolée et légèrement schématisée d'un Carabe doré (Fig. 21). Nous y trouverons, à la base, une pièce articulaire (c) correspondant au coxopodite; en dessus vient une tige plus ou moins aliongée (s) correspondant au basipodite; à son exirémité libre, cette tige est bifur-

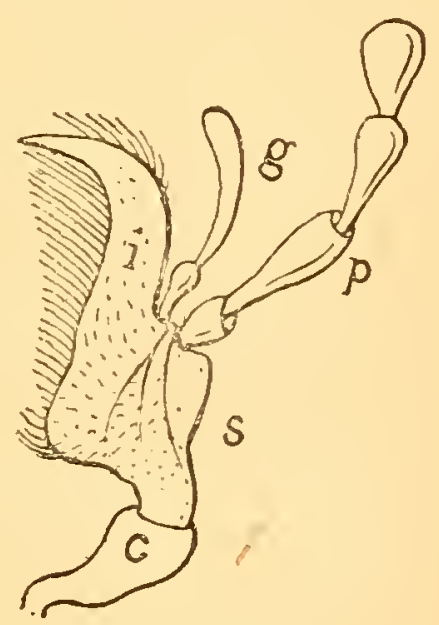

Fig. 21. - Homologie des pièces de la bouche chez les Insectes et les Crustacés (d'après BoAs) quée; sa lame interne (i), ciliée, devient masticatrice, elle représente le premier article de l'endopodite; sa lame externe (galea. g) en représente le deuxième article. Chez les Coléoptères carnassiers, Cicindélides, Carabides et Dytiscides, cette lame se segmente en s'adaptant aux

(1) L.ab (J.). - La dynamique des phénomènes de la rie, Paris, Alcan, 1908, in-8 '“rad. H. Daudin et G. Schœefer, p. 376). 
fonctions tactiles : elle devient un véritable palpe interne, ce qui fait que ces Insectes sont fréquemment désignés sous le nom d'Hexapalpes. Quant au palpe normal (p) il correspond à un exopodite (1).

La constitution des mandibules s'explique maintenant d'elle-même. D'après ce qui précède, il suffit, en effet, d'admettre que l'exopodite (palpe) a disparu et que l'endopodite est réduit à sa lame interne.

Lèsre inférieure. - La disposition de la lèvre inférieure

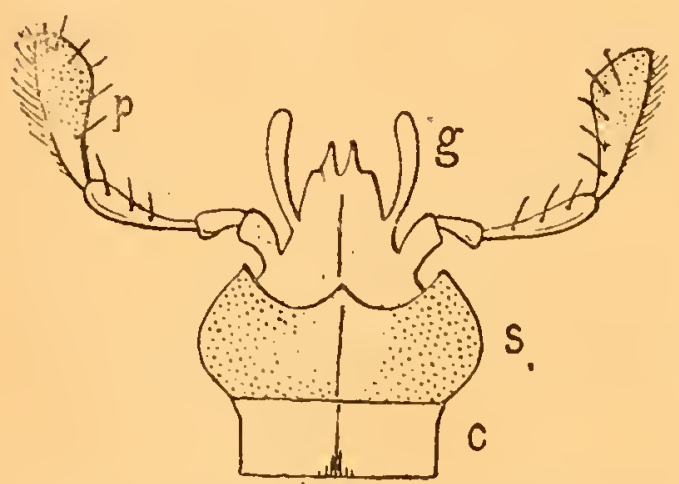

Fig. 22. - Lèvre inférieure d'un Insecte. (Schéma d'après BOAs ; mêmes lettres que dans la fig. précédente). n'est pas moins facile à comprendre; on peut la considérer comme formée par deux mâchoires accolées et soudées sur la ligne médiane; les deux coxopodites réunis forment la pièce basilaire; les deux basipodites forment le menton (Fig. 22).

Les endopodites peuvent se comporter de deux façons différentes ; tantôt leurs pointes ne sont pas bifurquées, clles se soudent en une pièce médiale qui constitue la ligule; d'autres fois, au contraire, les deux pointes restent séparées, les lames internes seules s'accolent et forment l'organe auquel on a donné le nom de langriette ; quant aux lames externes restées libres, elles constituent alors les paraglosses (g.).

\section{Hypopharynx et Epipharynx.}

1. Hypopharynx. - Il existe encore, à l'intérieur de la bouche, particulièrement chez les Insectes lécheurs, un organe sur lequel la

(1) Nous supnosons que le lecteur est familiarisé avec la nomenciature aclontée pour les articles des pattes che\% les Crustacés. 
sagacité des entomologistes s'est bien des fois exercée depuis Swammerdam ; l'entente n'est pas encore faite non plus au sujet de sa signification morphologique.

Chez les Insectes broyeurs, où il se présente avec ses caractères les moins différenciés, cet organe, que Savigny désignait sous le nom de langue ou d'hypopharynx, se présente sous l'aspect d'un bourgeon membraneux, $(l g)$ fixé sur le plancher de la lèrré inférieure. Ce bourgeon est couvert de papilles ou de soies rigides; il représente probablement un organe tactile et gustatif hautement différencié (Fig. 23).

Chez d'autres Insectes, notamment chez les Abeilles, l'hypopharynx, très spécialisé, forme un conduit rétractile couvert de soies nombreuses et servant à aspirer le nectar des fleurs.

Cher les liptères piqueurs, l'hypopharynx existe de même, sous forme d'un long tube chitineux ouvert à con extrémité.

2. Epipharyni..- A la voûte supérieure de la bouche, sous le labre et l'épistome, se trouve de niême un repli membraneux couvert de papilles auquel on a donné le nom d'épipharyzir (rig. 23).

3. Che q. quelques Insectes inférieur's, l'hypopharynx se présente sous forme d'une

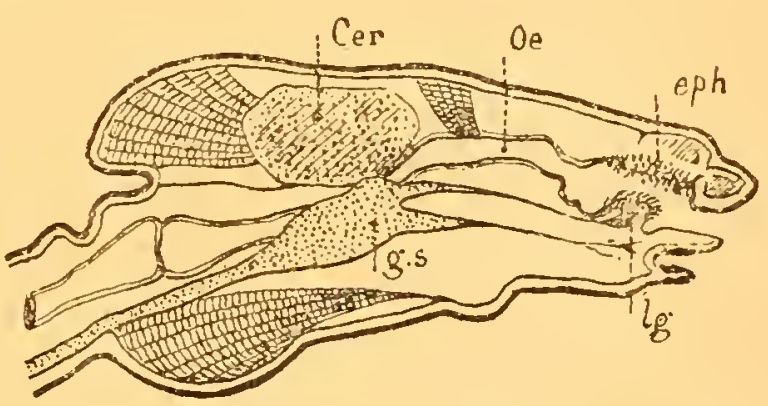

Fig. 23. - Section longitudinale de la tête d'un Insecte Anaphtalmus Telkampii colécpt. bour montrer l'épipharyux $(\epsilon p h$.) et l'hypopharynx (lg.). Cer. cerveau : $\alpha$, cesophage; gs ganglions sous-œesophagiens

(d'après PACKARD). lame portant, de chaque côté, de petits palpes articulés. Cette disposition a conduit certains auteur. à penser que l'hypopharynx possède la valeur morphologique d'un métamère; d'autres, pour des raisons analogues, ne sont pas éloignés d'accorder la même valeur à l'épipharynx.

n'après cette manière de voir, la tête des Insectes ne comprenArait done pas six zoonitea, mais huit, en réalité. M. Charles Jane: ar.ive, par une autre voie, à des conclusions du même ordre parl'étude apnrofondie quil a faite de la métamérivation de la tête de Myrmica rubra. Nous regrettons de ne pouvcir entrer dans la discussion de ce curieıx point de morphologi ', très intéressant, il est vraí rrais I rès diffieile à élucidar. 


\section{ORIGINE MÉTAMÉRIQUE DE LA TÊTE}

L.e nombre des anneaux qui entrent dans la constitution de la tête des Insectes: constitue l'un des problèmes les plus difficiles de l'anatomie entomologique.

Les entomologistes descripteurs considèrent la tête comme formée d'un seul segment; les embryologistes, au contraire, et les anatomistes la considèrent comme formée de plusieurs, mais ils ne sont pas d'accord sur le nombre. Ces divergences de vues sont impos. sibles à concilier lorsqu'on re considère que la tête de l'Insecte adulte; il faut ćemander des éclaircissements à l'embryologie. Toutefois une chose est nettement établie, c'est que; dans la région postorale de la tête, de même que dans les deux autres régions du corps (thorax et abdomen), on trouve toujours une paire de ganglions nerveux (neuromères) dans chaque'segment; il ne s'agit done, alors, pour résoudre le problème de la métamérie de la tête, que de déterminer le nombre de neuromères, constituant ce qu'on est convenu d'appeler les ganglions céréboïdes ou sus-œsophagiens.

D'après les recherches de Patten, de Wheeler et plus spéciale-
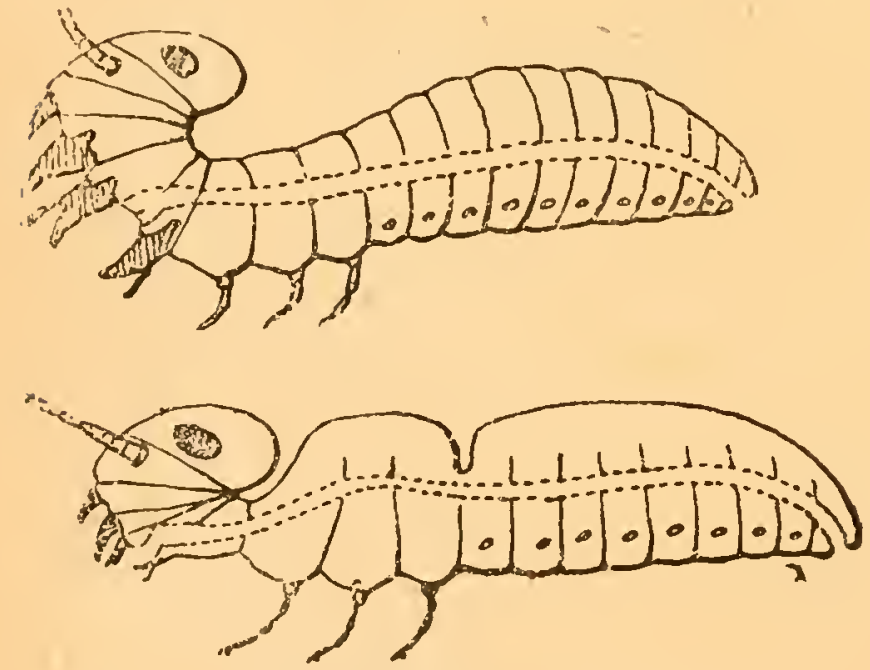

Fir. 24. - Schéma destiné à nıntrér le mode de spécialisation des métamères. ment d'après celles de Viallanes, il semble bien que le cerreau des Insectes est divisé en trois segments primitifs (1).

Les antennes sont innervées par la division moyenne ou deutocerebron. Parailleurs, le segment oculaire, qui porte les yeuxsimples ou les yeux composés, est supposé représenter le premier neuromère céphalique; ce fait n'a rien qui doive nous surprendre puisque les yeux, comme nous l'avons vu, sont les équivalents morphologiques des antennes (Fig. 25.).

(1) Il serait bou que le lecteur revint sur ce paragra!he ajres avoir lu tout ce gui concerue l'évolution du systène nerveux, 1). 246. 
Le troisième segment de la tête est très obscurément indiqué ; les faits qui militent en faveur de son existence supposent une connaissance approfondie de l'embryogénie des Insectes, et ne peuvent guère être exposés ici.

Disons seulement qu'Heymons, après Viallanes et Wheeler, a également découvert ce segment dans l'embryon du Lepisma, et qu'il l'a désigné sous le nom de segrment prémandibulaire.

La détermination des autres se@ments céphaliques se fait ensuite sans difficulté, grâce aux appendices dont ils sont pourvus; le segment mandibulaire parait former une partie notable de la région postanteanaire de l’épierâne, ainsi que l'indique l'insertion des larges muscles moteur's des mandibules sur cette partie de la

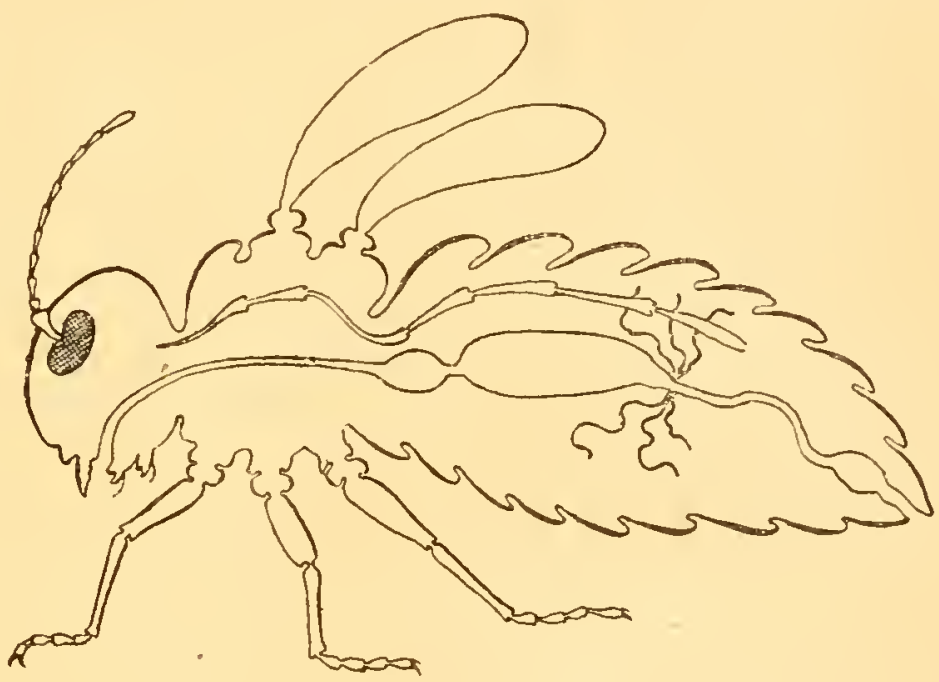

Fig. 24 bis. - Un Insecte théorique.; la spécialisation de métamères est complète. tête.

Le premier segment maxillaire est caractérisé par l'avortement de sa région tergale.

Quant au deuxième segment maxillaire (labial), il paraît être représenté, dans sa partie tergale, par la région occipitale de la tête.

Ainsi donc, d'après le nombre des appendices et d'après les données embryogéniques leś plus probables, la tête des Insectes peut être considérée comme formée de six segments intimement fusionnés, qui sont (Fig. 24):

$1^{\circ}$ Le segment oculaire, sur lequel sont fixés les yeux.

$2^{\circ}$ Le segment antennaire, ayant pour appendices les antennes.

$3^{\circ}$ Le segment prébuccal, correspondant à la lèvre supérieure (labre).

$4^{\circ}$ Le segment mésobuccal ou mandibulaire (mandibules).

$5^{\circ}$ Le segment métabuccal ou maxillaire (mâchoires avec leur's pa]pes).

$6^{\circ}$ Le segment labial ayant pour appendices les deux moitiés de la lèvre inférieure (labium) avec leurs palpes. 


\section{CHAPITRE IV}

\section{ZE THORAX ET SES APPENDICES}

Techrique. - Suivre les développements de ce chapitre sur un Orthoptère (Lncustæ riridissima) ou sur une Libellule.

I. Les trois parties du Thorax. - Le thorax constitue la division moyenne du corps; il est formé, dans la grande majorité des cas (1), de trois segments dont les limites sont surtout bien distinctes en dessous, chez les Orthoptères (Fig. 25).

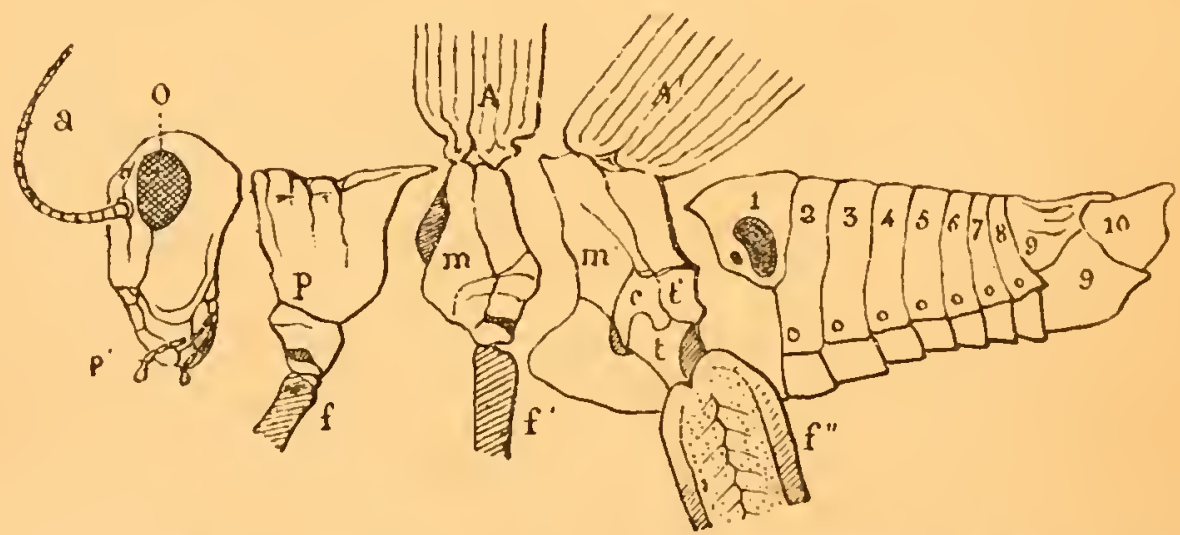

Fig. 25. - Les trois yarties du thorax cu Mélanoplus spretus (Orth.). a, antenue. $p^{\prime}$ yalpes; $f f^{\prime} f$ " pattes; $A, A^{\prime}$ ailes; $1-10$, anneaux de l'abdomen (d'après $\left.1^{\prime} A c k i l i v\right)$

En allant de la tête vers l'abdomen, nous rencontrons trois anneaur, qui ont reçu les noms suivants: prothorax (p. en avant), mésothorax (m. au milieu) et métathorax ( $m$ '. en arrière).

(1) Voir 11. 64, la curiense anonalie présentée var certains Hyménoptères. 
Ces trois anneaux portent, en dessous, chacun une paire de pattes; de plus (exception pour les Diptères), le deuxième (mésothorax) et le troisième (métathorax), sont munis, en dessus, d'une paire d'ailes bien développées. Par conséquent, à part l'exception ci-dessus indiquée, le thorax porte done toujours deux paires d'ailes et trois paires de pattes.

Ainsi que nous l'avons expliqué p. 42, chaque anneau du thorax se compose d'une pièce médiane supérieure le notum (ou tergum), d'une pièce inférieure le sternum et de pièces latérales les pleures. Nous avons donc, en dessus pour les trois parties du thorax précédemment définies, le pronotum, le mésonotum, et le métanotum, auxquels correspondent, en dessous, le prosternum, le mésosternum et le métasternum.

Très fréquemment (Coléoptères), le pronotum est la seule partie du thorax qui soit bien visible en dessus (prothorax), car les autres arceaux de la région dorsale sont presque toujours cachés par les ailes et les élytres (1).

La forme et les dimensions du thorax sont extrêmement variables suivant les différents ordres d'Insectes, et ceci tient au développement plus ou moins grand que prennent les diverses pièces qui entrent dans la constitution d'un somite thoracique. Ainsi, par exemple, chez les Insectes qui marchent plutôt qu'ils ne volent: Coléoptères, Orthoptères, un grand nombre d'Hémiptères, le prothorax acquiert un très grand développement et se sépare, dans une certaine mesure, du mésothorax et du métathorax. C'est le contraire qui arrive chez les Insectes

(1) Pour une étude plus complète des pièces du thoras ct des modi flcations dont elles sont susceptibles, roir le volume traitant des Colécptères (E.S.).

LES INSECTES. - $2^{\mathrm{e}}$ édit. 
bons voiliers, tels que les Hyménoptères, les Lépido tères, les Diptères, etc., ici, le mésothorax et le métathorax, étroitement unis, prennent un développement énorme, tandis que le prothorax se trouve réduit à des dimensions très exiguës. La raison d'être de ces variations se comprend d'elle-même.

Examinons maintenant la composition de chacun des trois anneaux thoraciques.

1 Prothorax. - Dans aucun ordre d'Insectes, on ne peut distinguer, dans le prothorax, les quatorze sclérites fondamentaux qui entrent, d'après Audouin, dans la composition d'un anneau typique; en général, un ou deux des sclérites du tergum manquent toujours complétement (1). Chez les Coléoptères, ceux qui restent se soudent intimement et constituent la pièce unique que l'on désigne sous le nom de pronotum ou prothorax; les deux pièces disparues sont le scutellum et le post-

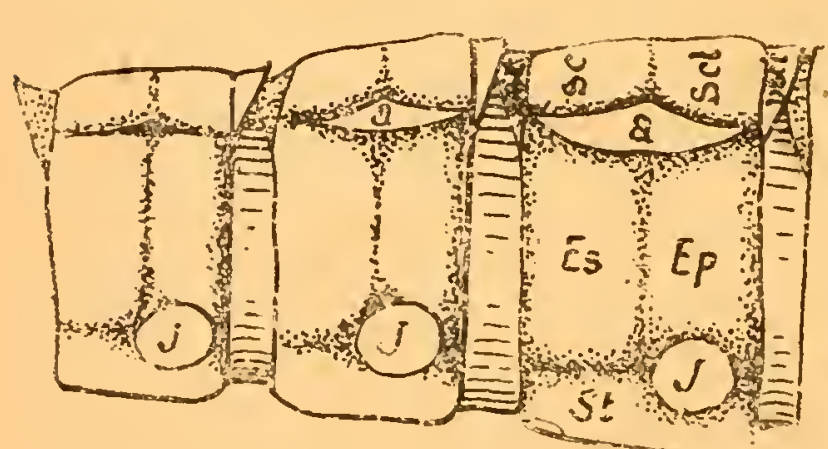

Fig. 26. - Constitution schématique des anneaux du thorax d'un Névroptère. '.I. insertion cies jam. bes ; $a$, insection des ailes ; st. stcrmum (inité ảe WoonwonTH). scutellum.

Par contre, chez un certain nombre d'Orthoptères (Phasgonuridés), on distingue assez nettement les quatre divisions; il en est de même chez les grands Névroptères de la famille des Odonates (Anax) (Fig." 26), bien que là, le prc." thorax soit très petit.

Quant aux pièces pleurales épisternes (Es) et épimères

(1) Nous rappelons les noms de ces pièces : proscutum ( $p s c$. ), scutum (sc.), scutellum (1.) et postscutellum (psct.) (Fig. 26). 
(Ep), elles sont quelquefois séparées et bien visibles, notamment chez les Névroptères.

L'épisterne est une pièce située à la partie antérieure et latérale du prothorax; elle s'appuie, par sa base, sur le prosternum, mais sa forme et ses dimensions sont trop variables pour qu'on puisse les décrire ici.

En arrière de l'épisterne et toujours soudée avec lui, se trouve l'épimère. Après avoir un instant longé le protergum, cette pièce se recourbe, en général, pour former la cavité qui reçoit la hanche antérieure.

L'épimère n'abandonne jamais la hanche avec laquelle elle s'articule; c'est à ce caractère qu'on le reconnaîtra toujours.

2 Mésothorax. - Le mésothorax, ainsi que son nom l'indique, constitue la subdivision moyenne du thorax ; il porte, en dessous, chez tous les Insectes, la deuxièms paire de pattes et, en dessus, la première paire d'ailes. Les sclérites mésothoraciques sont généralement mieux développés et beaucoup plus distincts que ceux du prothcrax, surtout chez les Insectes ailés. Ici, les quatre mésotergites (1) sont toujours bien individualisés; cependant, dans la plupart des ordres, le proscutum est très petit, et ne forme souvent qu'un étroit liséré infléchi vers l'intérieur ; c'est chez les Névroptères (Odonates) qu'il est le mieux dévelop-

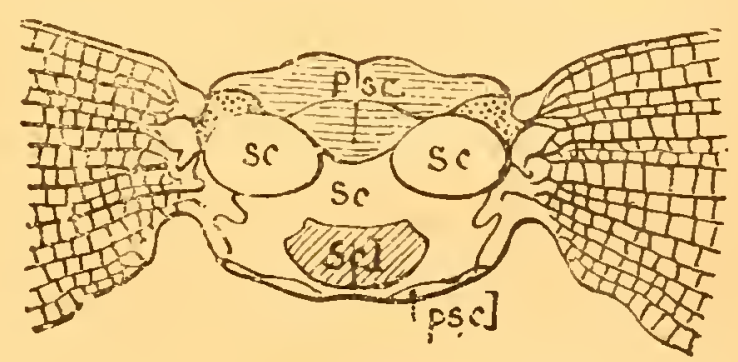

Fig. 27. - Constitution du mésothorax chez le Pteronarcys californica (Névropt.) (imité de PACKARD). pé, et qu'on peut le plus facilement l'observer (Fig. 27).

(1) Mésotergites: proscutum (psc.), scutum (sc.), scutellum (sct.), postscutellum (psct.) (Fig. 27). 
Le second mésotergite, scutum, est toujours le plus important du mésothorax ; c'est sur lui que la première paires d'ailes est articulée; il varie, d'ailleurs, considérablement dans ses dimensions ; chez les Hyménoptères, les Lépidoptères, les Diptères, etc., il constitue la partie la plus visible et la plus étendue du mésonotum. Au contraire chez les Orthoptères, les Coléoptères et les Hémiptères hétéroptères, il est presque toujours caché

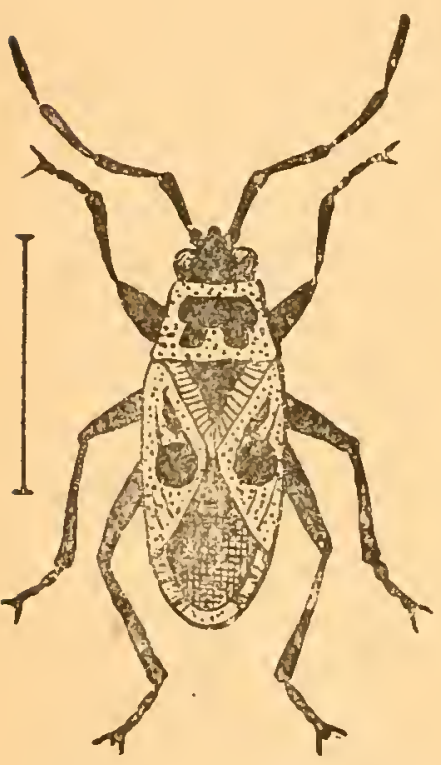

Fig. 28. - Un Hémiptère, Pyrrhocoris apterus pour montrer le grand développement de l'écusson. (D'après Guérix et Pf́NeAU). sous le bord postérieur du pronotum. Chez les Odonates, le scutum paraît divisé par une carène médiane, ce qui indique qu'il est formé par la soudure de deux moitiés symétriques (p. 65).

La partie visible du scutellum (mésoscutellum) est la pièce triangulaire située à la base de la suture des élytres et qu'on désigne, en entomologie descriptive, sous le nom d'écusson (Fig. 28).

Très développé chez les Hémiptères (Scutelléridís), où il recouvre l'abdomen presque tout entier ; encore très visibles chez un grand nombre de Coléoptères (Cétonides); il disparaît, par contre, chez beaucoup d'autres (Copridiens).

Enfin le postscutellum est, en général, la pièce la moins développée du mésonotum; il est le plus souvent caché par la partie antérieure du métanotum. C'est chez les Libellules qu'on peut l'observer avec le plus de facilité; là, il est très distinct et ressemble au scutellum, sauf qu'il est plus déprimé et que ses dimensions sont un peu plus faibles. 
Le mésosternum existe toujours, mais sa largeur dépend de l'écartement des hanches intermédiaires ; il est bien développé chez les Orthoptères, où il offre de bons caractères pour la classification.

Les mésopleures (épimérite et épisternite), de même que dans l'anneau précédent, offrent de grandes variations de forme et sont fréquemment utilisés en taxinomie.

Dans son ensemble, le mésonotum est découvert et bien distinct chez les Névroptères, les Hyménoptères, les Lépidoptères et les Diptères, tandis qu'il est cạché par les élytres (sauf le scutellum = écusson), chez les Coléoptères, lorsque ceux-ci sont fermés.

3 Métathorax. - Le métathorax constitue la dernière subdivision thoracique; il fait suite au mésothorax et porte, en dessous, la troisième paire de pattes ; en dessus, les ailes de la deuxième paire lorsqu'elles existent. Ses dimensions sont variables, et correspondent au développement plus ou moins grand des ailes inférieures; cependant il est preque toujours moins développé que le métathorả. A son maximum de complication, il offre à étudier les mêmes pièces que les anneaux précédents; mais toutefois, dans sa partie tergale, ce sont presque toujours le scutum et le scutellum qui sont les plus visibles.

Chez les Libellulides, la partie postérieure du métathorax présente toujours une particularité bizarre; elle est échancrée en forme d'X, par suite de la soudure de deux pièces à peu près symétriques: le scutellum et le postscutellum ; la même particularité s'observe d'ailleurs au mésothorax (Fig. 29).

Quant au postscutellum, il est généralement indistinct 
et soudé au scutellum; cependant on peut l'observer facilement chez la plupart des Névroptères (I ibellulides) et chez les Diptères.

Les pleures métathoraciques sont souvent utilisées

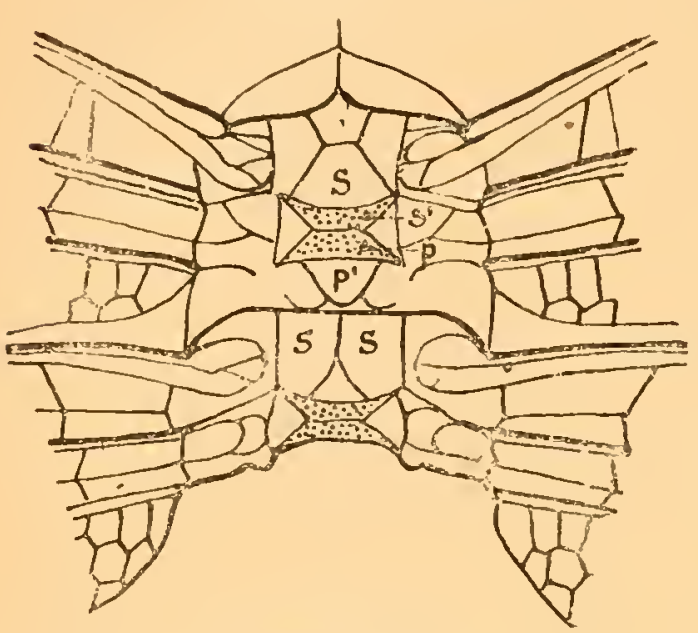

Fig. 29. - Structure du métathorax chez Afischna cyacea (Névropt.) (imité de WoopwortH). dans la classification, surtout chez les Coléoptères et les Orthoptères.

Les dimensions du métathorax sont toujours, comme nous l'avons dit, en rapport avec le développement des ailes; elles diminuent à mesure que la seconde paire prend de moins en moins part au rol.

Constitution Spéciale du thorax ciez Certains Hymínoptíres. - La composition normale du thorax, telle que nous venons de la décrire, présente une variation très remarquable chez certains Hyménoptères, principalement chez ceux qui sont pourvus d'une tarière ou d'un aiguillon : ici le thorax présente quatre anneaux au lieu de trois, comme c'est la règle. Cette disposition curieuse est due à ce fait que, au moment de la transformation de la larve en nymphe, le segment basal de l'abdomen perd

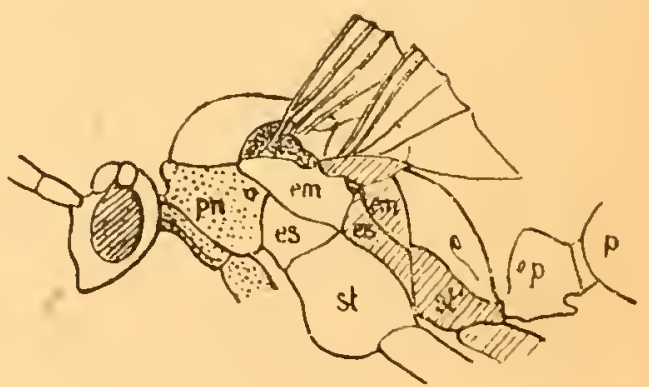

Fig. 30. - Le métathorax des Hymé. noptères $n$, segment médiaire (d'après G. EMERY). sa forme ordinaire et vient s'unir étroitement avec le métathorax (Fig. 30 p.).

Latreille, à qui cette particularité n'avait point échappé, avait donné à ce quatrième anneau thoracique le nom de segment médiaire.

Chez les Hyménoptères phytophages, le premier segment abdominal ne prend aucune part à la constitution du thorax; la disposition typique se trouve donc conservée. 


\section{APPENDICES DU THORAX}

\section{(PATTES ET AILES)}

Techique. - Pour l'étude des pattes prendre le Carabe doré et le Dytique. - Pour les ailes, prendre le Hanncton, le Papillon blanc du chou ou un Orthoptère de grande taille (Locusta siridissima).

C'est sur le thorax que sont fixés les organes de la locomotion : en dessous, l s trois paires de pattes, organes adaptés à la marche ou à la natation; en dessus, les deux paires d'ailes (une seule paire chez les Diptères), organes de la locomotion aérienne.

10 Pattes - Les pattes sont au nombre de six chez tous les Insectes, d'où le nom d'Hexapodes, qui leur a été donné par de Blainville.

Les pattes sont disposées par paires; chacune d'elles est reçue dans une cavité (cavité coxale) formée par les pièces pleurales et le sternite correspondant. Chaque patte est formée de cinc segments mobiles, à l'intérieur desquels se trouvent des muscles, des trachées et des nerfs. Ces cinq segments, dont la grandeur et

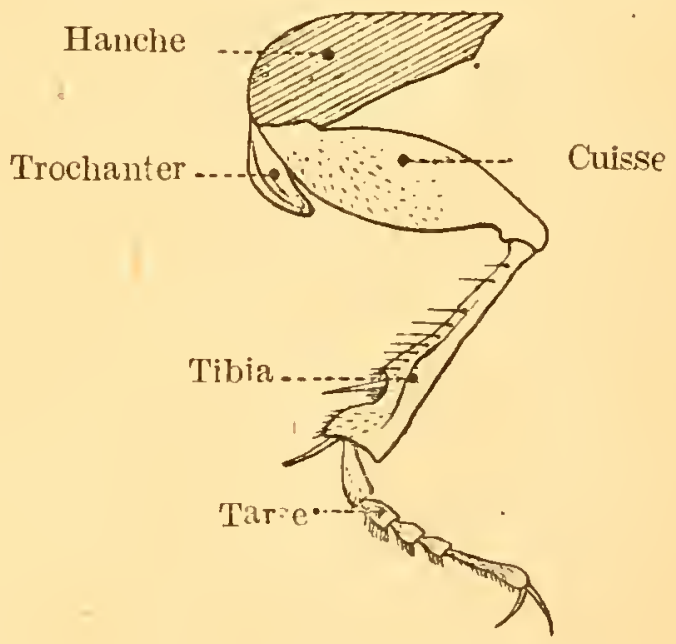

Fig. 31. - Patte d'un Coléontère carabique, Chlonius velutinus. la forme varient suivant les groupes que l'on considère, sont, de la base vers l'extrémité libre : la hanche, le trochanter, la cuisse, la jambe et le tarse. Ce dernier se compose lui-même d'articles en nombre variabie te se termine généralement par deux griffes recourbées (Fig. 31). 
Hanche. - La hanche ou coxa est une pièce courte, enchàssée dans le prothorax pour la première paire de pattes, dans le mésothorax pour la paire moyenne et dans le métathorax pour la paire postérieure. La forme de la hanche est variable; il en est de mème pour son mode d'articulation qui est identique pour les deux dernières paires de pattes, mais qui est presque toujours différent pour la paire antérieure.

Il est, en général, facile d'établir l'homologie entre les différentes régions de la patte des Insectes et celle des Crustacés; on voit alors que la hanche correspond au coxopodite.

Trochanter. - Le trochanter, quelquefois peu développé, correspond au basipodite des Crustacés ; c'est un petit article presque toujour's sphérique ou ovoïde, très

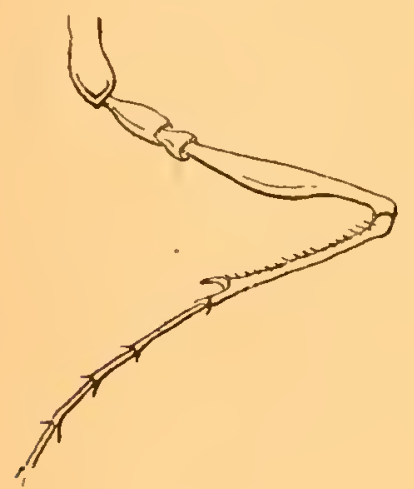

Fig. 32. - Une patte d'Ichneumonide Ephialtcs manifestator

(imité de KOLBE). court, sur lequel la patte peut tourner dans tous les sens.

Par une exception remarquable le trochanter est double chez un grand nombre d'Hyménoptères parasites (Ichneumonides) (Fig. 32). Chez les Insectes marcheurs, comme les Coléoptères, le trochanter des pattes postérieures quitte, petit à petit, la position qu'il a normalement dans l'axe de la patte, et vient se placer latéralement à la base de la cuisse. Dans ees conditions, cet organe paraît avoir perdu sa fonction; c'est un article devenu inutile (Fig. 31).

Cuisse. - La cuisse ou fémur est le troisième segment de la patte; cette pièce varie beaucoup dans sa forme et dans ses dimensions; morphologiquement, elle cor- 
respond au premier article de l'endopodite des Crustacés.

La cuisse est remarquable, tantôt par sa longueur, cornme chez les Sauterelles et les Criquets (Orthoptères), tantôt par sa grosseur, comme chez les Altises et les Orchestès (Coléoptères), qui sautent avec facilité. Elle est souvent, de plus, ornée de pointes, d'épines, de membranes, de raînures ou d'arêtes plus ou moins saillantes, que l'on utilise dans la classification.

Jambe. - La jambe ou tibia est la quatrième partie de la patte, celle qui correspond au deuxième article de l'endopodite. Elle est placée entre le tarse et la cuisse et présente presque autant de variations que le lémur, dont elle a généralement la longueur et la solidité.

La conformation de la jambe varie à l'infini suivant ses usages ; elle est courte, aplatie avec son bord antérieur dentelé chez les Insectes fouisseurs (Courtilière, Scarabée, etc.) ; elle est plus ou moins richement garnie de cils dans les Insectes nageurs (Dytiques, Notonectes); son bord externe est garni de brosses ou poils rigides chez un grand nombre d'Hyménoptères (Abeilles) ; on y remarque même des épines mobiles chez les Hydrophilides.

Tarse. - Le tarse, qui correspond au reste de l'endopodite, est la partie la plus importante de la patte au point de vue de la classification ; toutefois, il n'est guère utilisé que dans l'ordre des Coléoptères (1).

Le tarse est formé de plusieurs segments ou phalanges, dont le nombre varie de un (Poduridés) à cinq (Blattides), (Hanneton, Libellule); il n'y a aucune règle fixe à ce sujet lorsqu'on considère l'ensemble des Insectes; le

(1) Houlbert (C.) 2. Miscéll. entomol. T. III., p. 8-27. 
dernier segment tarsal porte, en uutre; deux griffes recourbées.

De même que pour les autres parties de la patte, la conformation des articles des tarses est toujours en rapport avec les mœurs de l'espèce. Ainsi, les Insectes dont les pattes postérieures présentent une grande longueur, sautent plutôt qu'ils ne marchent (Locustaires, Acridiens) ; chez les Dytiques et les Gyrins, excellents nageurs, les tarses sont aplatis et ciliés, ils fonctionnent comme des rames. Chez ceux qui peuvent marcher sur la surface lisse des vitres, comme les mouches, on observe, sur l'avant-dernier article, deux ventouses qui favorisent l'adhérence.

Enfin, on connaît aussi des Insectes chez lesquels les pattes antérieures, très réduites et repliées contre le thorax, ne servent plus à la marche; elles échappent alors très facilement à l'observation, de sorte qu'au premier abord on se croirait en présence d'Insectes pourvus de quatre pattes seulement. Plusieurs papillons diurnes, qui ne marchent presque jamais, sont dans ce cas ( $V a$ nesse, Nymphalides, etc.).

Le dernier segment tarsal, ainsi que nous l'avons dit, est très fréquemment terminé par une paire de crochets mobiles (ongles) ; on observe, en outre, entre les ongles, un petit appendice ayant souvent l'aspect d'une palette ou d'un petit coussinet; les auteurs on' donné un grand nombre de noms à ce petit appendice ; nous le désignerons simplement sous le nom de plantule; certains pensent que la plantule n'est autre chose qu'un sixième segment tarsal atrophié (Fig. 33).

En général, les Insectes utilisent les épines ou les poils de leurs pattes pour le nettoyage de leur tête, de leurs 
antennes, de leurs palpes ou de leurs ailes. Tout le monde a pu voir les brosses délicates avec lesquelles les Abeilles se nettoyent les yeux, lorsque ceux-ci se trouvent recouverts par la poussière ou par le pollen des fleurs.

$2^{\circ}$ Ailes. - Les ailes des Insectes sont constituées par deux replis membraneux de la peau, soutenus, à l'intérieur, par des nervures chitineuses plus ou moins solides. Elles sont portées, comme les pattes, par les pièces du thorax, mais ne sont nullement, comme on pourrait le croire, les homologues des membres articulés.

Lorsqu'il n'y a ciu'une paire d'ailes, comme chez les Diptères, elles prennent naissance sur le mésothorax, ou plus exactement, sur le scutum mésothoracique (Voir p. 63); s'il y en a deux paires, comme chez tous les autres Insectes ailés, les premières nais-

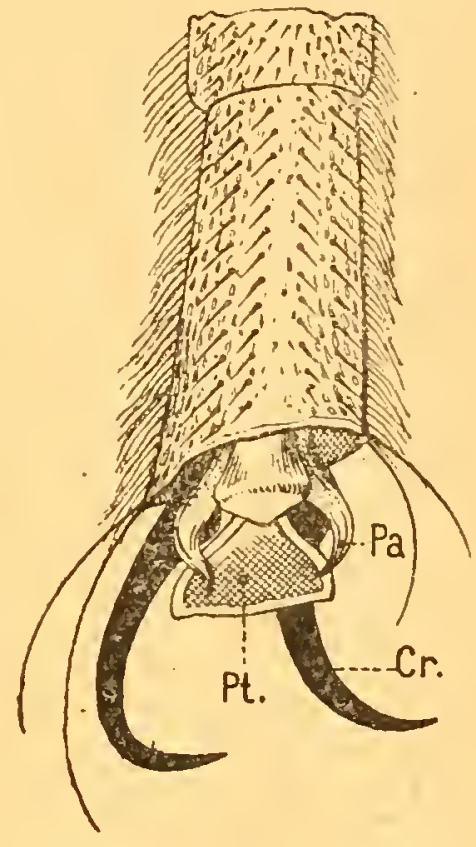

Fig. 33. - Patte de Castnie (lépidopt.) vue de côtė pour montrer la plantule. Pt.(Orig. $\times 45$ ). sent sur le mésothorax et les autres sur le métathorax. Jamais, chez les Insectes actuels, le prothorax ne porte d'appendices en dessus (1).

La présence ou l'absence des ailes, leur nombre, leur forme, leurs dimensions relatives, les différentes manières dont elles se replient, ont fourni des caractères de première importance à la classification.

(1) Beaucoup d'Insectes primitifs, parmi ceux qui vivaient dans les tenips primaires, étaient, au contraire, pourvus de trois paires d'ailes (voir p. 277 ) ; la paire antérieure était alors fixée sur le pronotum. 
On a donné le nom d'Aptères aux Insectes qui n'ont pas d'ailes du tout, comme les Poux; mais il est bien probable que ce groupe rentre dans un ordre plus étendu, dont il n'est, sans doute, qu'un rameau dégradé par le parasitisme.

Tous les Insectes qui ont deux ailes, comme les Mouches, les Cousins, les Tipules, forment l'ordre des Diptères. Ceux qui ont quatre ailes, comme les Hannetons, les Abeilles, les Libellules, les Papillons, pourraient être rangés sous la dénomination générale de Tétraptères.

Parmi les Tétraptères, les uns ont des ailes semblables, sensiblement égales, et parcourues par des nervures innombrables, comme les Libellules : on leur donne le nom de Névroptères. Mais, si les ailes sont couvertes d'écailles, se détachant comme une fine poussière, au moindre contact, on a l'ordre des Lépidoptères (Papillons).

Chez d'autres, tels que les Cynips, les Guêpes, les Abeilles, les quatre ailes ont encore le même aspect et

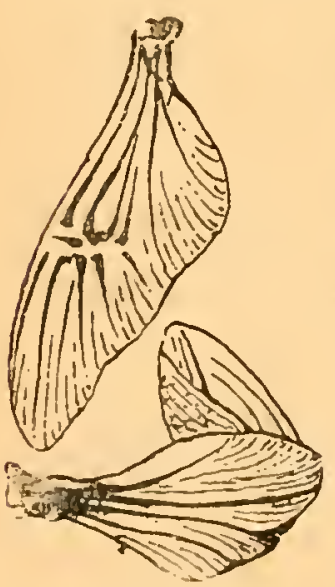

Fig. 34.- Ailes membraneuses du Hanneton. la même structure, seulement les nervures sont moins apparentes et beaucoup moins nombreuses que chez les Névroptères ; de plus, les ailes supérieures sont toujours plus grandes que les ailes inférieures : ce sont les Hyménoptères.

Lorsque les ailes sont complètement dissemblables, les supérieures s'incrustent généralement d'une substance chitineuse plus ou moins abondante et portent le nom d'élytres ; elles recouvrent alors et protègent les ailes inférieures qui restent membrancuses. 
Trois cas peuvent se présenter :

10 Si les ailes inférieures se replient transversalement sous les élytres, comme chez les Hannetons (Fig. 34), les Lucanes, etc., on a l'ordre des Coléoptères, le mieux connu et peut-être le plus riche de tous.

$2^{\circ}$ Si les ailes inférieures se replient en long sous les élytres, à la manière d'un éventail, on a l'ordre des Orthoptères (Fig. 35).

30 Enfin, il peut exister une disposition intermédiaire entre ces deux dernières; les élytres peuvent n'ètre durs que darıs leur région basilaire et rester membraneux à leur extrémité libre; cette conformation est fréquemment réajisée duns l'ordre des Hémiptères qui

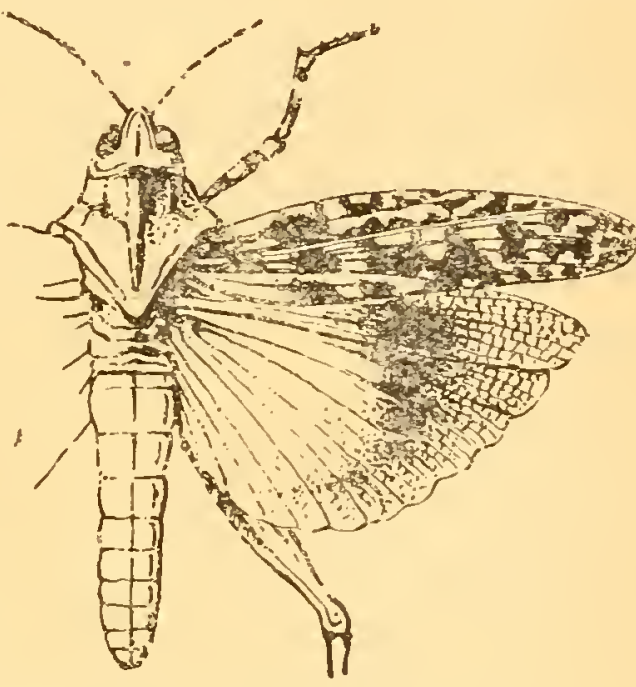
Fig. 35. - Ailes développées d'un Orthoptère. Acridium peregrinum. possèdent alors, comme on dit, des hémiélytres.

Il conviont encore de dire que l'on connait des Insectes dont las ailes, au lieu d'être formées

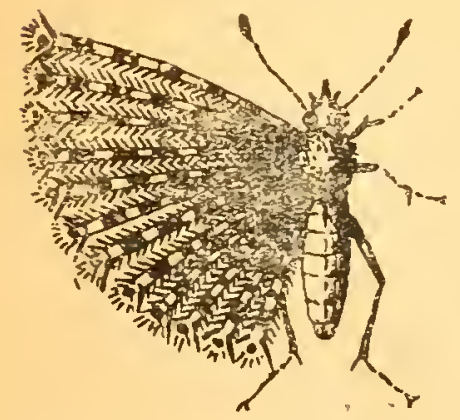

Fig. 36. - Ailes dégradées des Lépidoptères ( $O r^{\circ}$ néodes). d'une membrane réticulée, sont lacérées en un grand nombre de rayons semblablés à des plumes minuscules (Fig. 36).

Cette structure s'observe chez certains Papillons nocturnes, très communs à l'automne dans les prairies humides, et qu'on désigne sous les noms de Ptérophores et d'Ornéodes. Cette division des ailes est regardée comme une dégradation. 
Enfin, quand les ailes postérieures manquent, comme chez les Diptères, on trouve à leur place de petits filets mobiles terminés en massues : tels sont les balanciers des Mouches et des Tipules.

\section{Classification des Insectes \\ d'après les caractères fournis par les ailes.}

Io Pas d'ailes (Thysanoures). . . . . . Aptères.

I Io Deux ailes membraneuses seulement . . Diptéres.

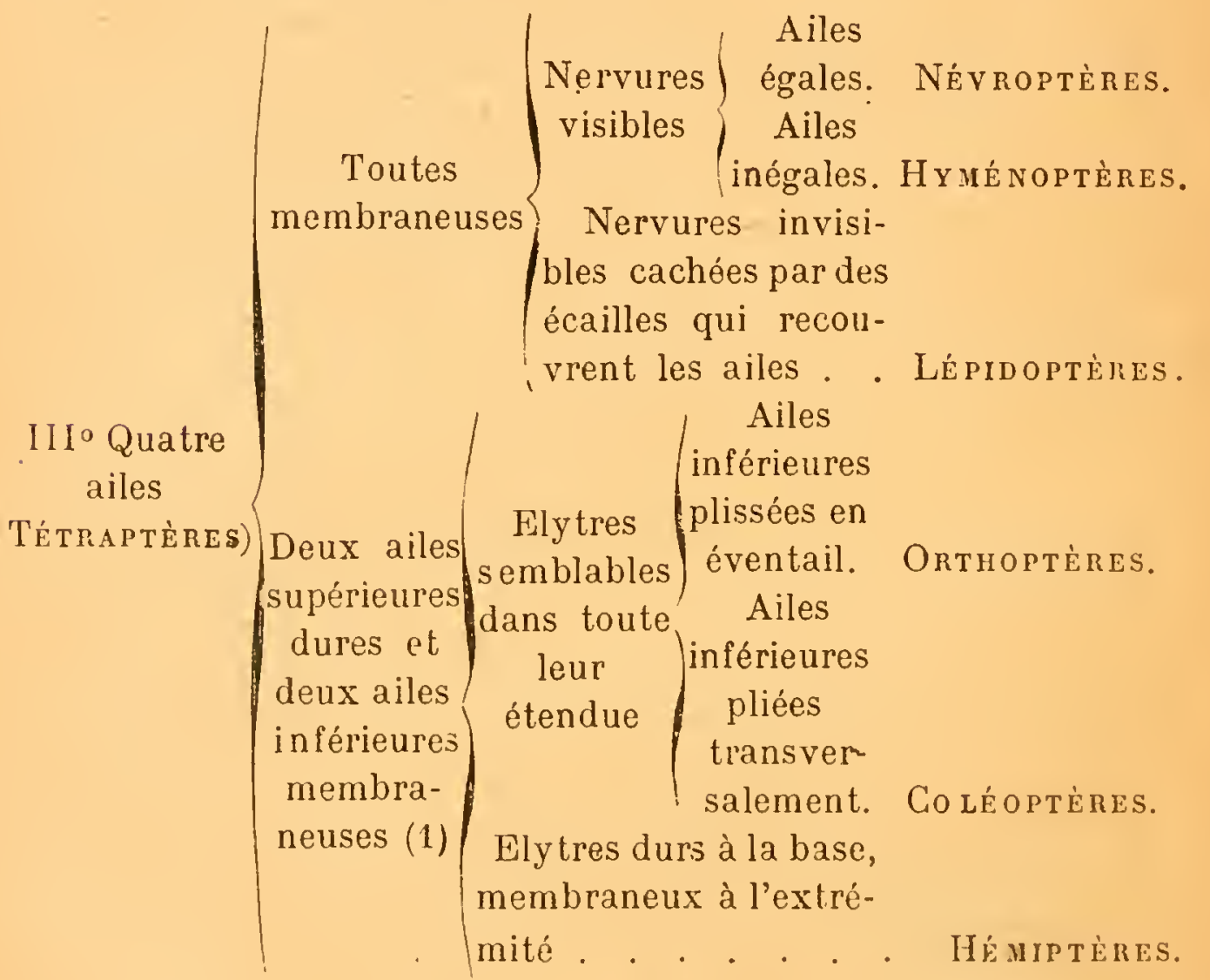

C'est ici le lieu de mentionner certains petits organes très curieux observés dans différents ordras d'Insectes et dont l'origine et les fonctions sont encore trùs mal connues, nous voulons parler des paraptìres et des patagia.

Paraptères. - On donne ce nom, ou encore ceux de tegulx, pté.

(1) Beaucoul) de Coléoptères sont privés d'ailes membraneuses sous les élytres: ceux-ci suffisent í les earactériser. Il existe aussi certains Névropteres. dont les ailes inférieures sont très petites ou complètement atrophiées (Ephémères). 
rygodes, hypoptères, épaulettes ou squamules, à deux petites nièces chitineuses, de forme variable, qu'on peut observer sur le mésothorax, à la base des ailes antérieures, dans différents groupes d'Insectes.

Chez les Diptères, ces pièces sont allongées, très étroites et placées le long de la bordure inférieure de l'aile.

Chez les Hyménoptères (Fig. 37), elles prennent générakement. l'aspect de deux petites écailles et sont placées au-dessus de l'aile (p.). Ces pièces paraissent manquer chez les Coléop. tères, les Orthoptères et les Hémiptères.

Patagia. - Enfin, chez les Lépidoptères on trouve, sur les côtés du prothorax, deux appendices généralement mobiles, de forme ovale et

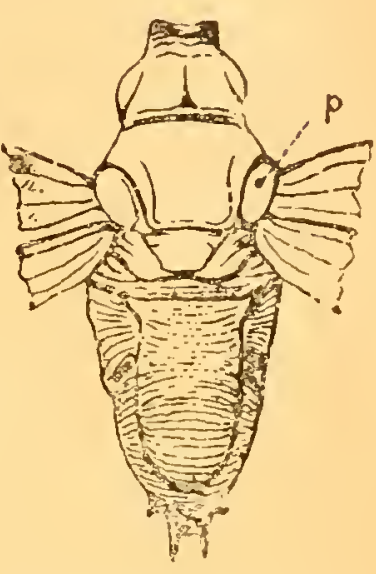

Fig. 37. - Tue ciorsale d'un IJyménoptère stphex chrysis

(d'ap. SHARP). érectiles, ce sont l Is patagia (pt.); dans certains cas, ces appendices deviennent très grands comme chez Geometra papilionaria (Fig. 38).

Les auteur's ne sont pas d'accord sur la signification morpholngique des patagia. Cholodkowsky a montré que

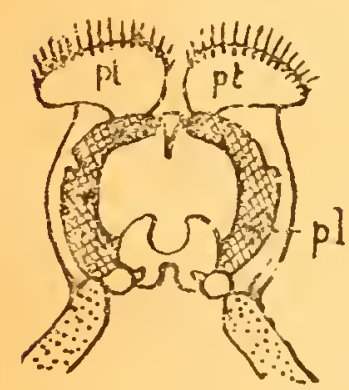
ces écailles sont creuses et remplies par du sang, dans lequel baignent de nombreux rameaux tra chéens; il a cru alors pouvoirles considérer comme une paire d'ailes prothoraciques atrophiées. D'autres auteurs ne voient dans les patagia que des dépendances de la base de l'aile antérieure; et, de ce fait qu'on en trouve de semblables, mais plus Fig. 33. - Pro- petits, sur le mésothorax de certains autres Lépi-

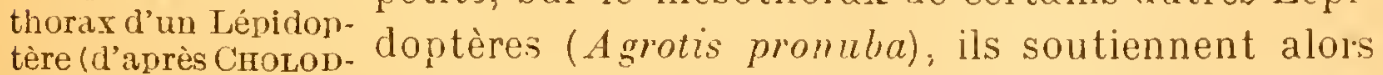
коwsкY). $p l$. pleures. qu'ils sont seulement les homologues des paraptères. Il nous semble que ces deux opinions ne sont point fondamentalement contradictoires; si chaque segment peut théoriquement porter des appendices, et cela n'est pas douteux, on peut, en effet, penser que les patagia (du prothorax) sont des ailes atrophiées; les paraptères (du mésothorax) sont les appendices du prossutum, tandis que les ailes véritables seraier. $t$ ceux du scutum.

Je sais bien que PACKard n'admet point une origine segmenteir? 
pour les mésotergites; selon lui, ces pièces ne sont que le résultat de la différenciation des ailes, puisqu'elles sont très réduites ou même effacées chez les Insectes aptères. Nous ne méconnaissons pas la valeur de cette objection; toutefois, si le scutum porte des appendices, nous ne voyons pourquoi on refuserait d'admettre qu'il puisse en naître également sur le proscıtum.

Normalement, les ailes s'articulent toujours avec le deuxième tergite méso- ou métathoracique (scutum); mais, dans un but de consolidation, le scutellum peut aussi quelquefois prendre part à l'articulation ; Audo ain a donné le nom d'épidèmes d'articulation (1) aux petitcs pièces solides qui servent à réunir la base de l'aile aux parois du thorax et qui donnent ainsi à l'articulation toute la flexibilité qu'exigent les mouvements des ailes.

Balanciors des Diptères. - La signification morphologique des balanciers des Diptères sera discutée dans le volume de l'E.S. qui

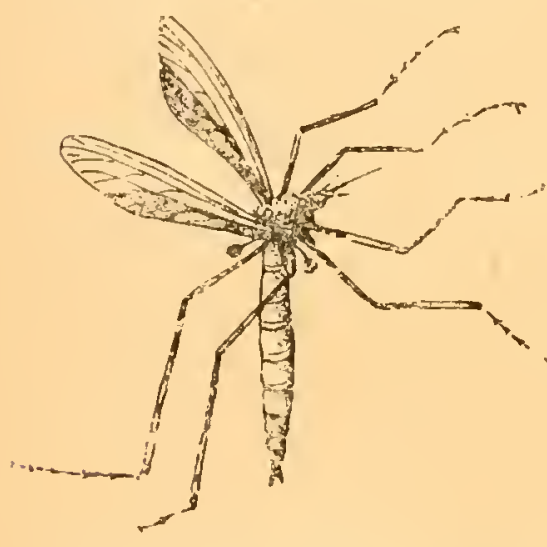

Fig. 39. - Un diptère (Timule) vu de côté, your montrer les ba. Ianciess. traitera de ces Insectes. Tous les auteur's paraissent maintenant d'accord pour les considérer comme des ailes modifiées; rnais malgré l'opinion de Jacquelin du Val (2), leur position véritable, sur le troisième segment thoracique ou sur le premier segment abdominal, n’a jamais été clairement élucidée (Fig. 39).

Outre l'importante articulation des ailes avec le thorax, il convient encore de faire raemarquer que, dans certains groupes d'Insectes, surtout chez les Insectes bons voiliers, les deux paires d'ailes présentent encore, entre elles, des modes d'union particuliers. Ainsi, par excmple, chez un grand nombre d'Hyménoptères, les ailes postérieures, sont en quelque sorte accrochées aux ailes antérieuses à l'aide d'un nombre variable de petits harpons,

(1) D'inutres anteurs (Chabrier,jurine) préfèrent le nom d'osselets.

(:) J.eQtrux de V.M. (C). - Genera 1) XC, vol.1. 
nommés hamuli. Dans les Lépidoptères hétérocères (1), on trouve : Ine disposition réalisant le même résultat, mais par un autre mécanisme; ici il existe, en effet, à la base des ailes postérieures, une sorte de soie rigide (frein) qui s'engage dans une coulisse de l'aile supérieure (2).

Ces dispositions curieuses, reliant ainsi les ailes postérieures aux ailes antérieures, tendent incontestablement à rendre leurs mouvements synchrones, à donner ainsi, à la rame aérienne, une plus rrande surface et, par suite, à rendre le vol plus soutenu et plus puissant.

(1) Nocturnes et Crépusculaires. Cette disposition, ne se rencontrant jamais chez les Rhopalocères (diurnes), permet de distinguer immédiàtement les deux groupes.

(2) Morphologiquement parlant, ce crin n'est autre chose que la nervure costale qux s'est dégagée de la partie antérieure des ailes secondes. Nous verrons la conséqucnce de cette adaptation, Iorsque nous étudierons la nervation des ailes chez les Lépidoptères. 


\section{CHAPITRE V}

\section{LA INERVATION DES AILES}

TFCINIQUe. - Pour lire ce chapitre avec fruit, il sera bon de se procurer un Phyrgane, une Libellule, une Blatte et une Mouche; les divers groupes de nervures sont faciles à distinguer sur les ailes de ces Insectes.

Pour étudier la structure des nervures sur une coupe transversale, il faut s'adresser aux élytres des Orthoptères.

\section{LA NAISSANGE DES PREMIERES NERVGIES}

Les nervures sont des tubes ou des baguettes de chitine, placées entre les deux membranes alaires et destinées à donner une plus grande rigidité aux rames aériennes que sont les ailes. Suivant leur grosseur et leur direction, mais surtout suivant leur mode de développement, on doit, d'après les récents travaux de

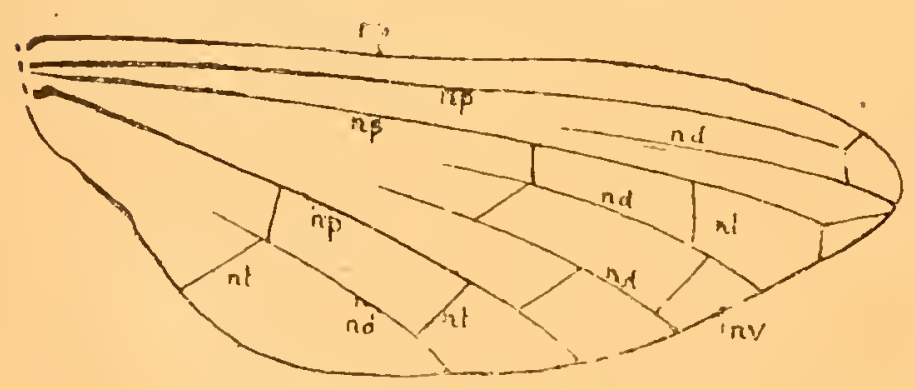

Fig. 40. - Une aile théorique (schéma d'après les idées de Woodwortu).
C. W. Woodworth, distinguertroisespèces de nervures. I $^{\circ}$ Les nervures principales (np.) (primaires) : ce sont celles qui partent de la base de

l'aile, et s'avancent plus ou moins loin vers le sommet (Fig. 40).

$2^{\circ}$ Les nervures indépendantes naissent entre les prin- 
cipales sur le bord de l'aile (nd.), surtout vers son extrémité libre, et s'avancent plus ou moins loin vers la base (Fig. 40).

$3^{\circ}$ Enfin, les nervures transverses partent à angle droit des précédentes (nt.) et les relient dans toute l'étendue de l'aile (Fig. 40).

Bien entendu, par suite de l'accroissement de la surface alaire, un certain nombre de ces dernières peuvent perdre leur position primitive et devenir plus ou moins obliques. Dans certains cas, leur nombre peut augmenter (Névroptères), dans d'autres, il peut diminuer (Hyménopteres), de sorte qu'une multitude de combinaisons peuvent être réalisées, suivant les différents groupes d'Insectes et suivant les exigences de la locomotion aérienne.

Il ne saurait plus être question aujourd'hui, comme l'ont fait jusqu'ici presque tous les entomologistes, d'admettre un plan commun de la nervation alaire chez tous les Insectes; les interprétations si diverses des auteurs, en ce qui concerne l'homologation des nervures, montrent suffisamment que ce plan n'existe pas.

Il n'y a qu'une chose, dont l'existence soit certaine, c'est le besoin, pour chaque Insecte, de réaliser un appareil de locomotion aussi exactement adapté que possible aux conditions de son existence.

$\mathrm{Si}$, pour les Insectes d'un même groupe, vivant dans le même milieu, ce besoin a créé des ressemblances, il n'y a là rien qui puisse surprendre un naturaliste; que ces caractères, une fois acquis, puissent se transmettre héréditairement, cela est encore très naturel ; mais rien de plus ne doit ètre cherché dans les aspects si variés de la nervation des ailes. Ne voit-on pas à chaque ins- 
tant, dans un même ordre d'Insectes, des dispositions s'éloignant notablement du type le plus fréquemment réalisé.

Nous ne pouvons pas nous étendre davantage sur ce sujet; mais, dans les volumes spéciaux de cette colle?tion, on étudiera les curieuses variations dont la nervation alaire est susceptible, en essayant toujours de donner à ces variations l'explication rationnelle qui leur convient.

Nous nous bornerons à dire ici que, si l'on veut cependant étudier comparativement les différents types de nervation - en tenant compte des réserves que nous venons de faire - on peut diviser les Insectes en trois groupes : les Neuroptères; les Elytroptères et les Néoptères.

10 Neuroptères. - L'étendue du groupe des Neuroptères doit s'entendre ici dans le sens linnéen ; on doit y faire rentrer toutes les anciennes formes fossiles désignées sous le nom de Palæodictyoptères

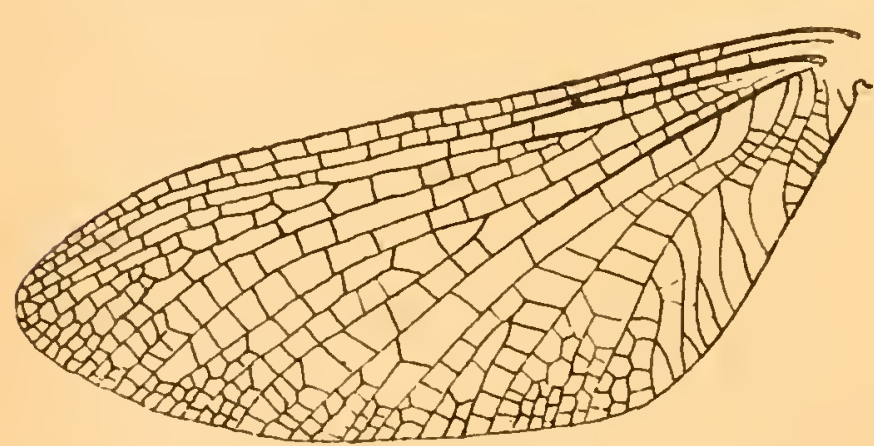

Fig. 41. - Une aile d'Ephéméride (Hexagenia bilineata), pour montrer la richesse du réseau transversal (d'après WOODWORTH).

par Scudder. Il com prend ainsi tous les Insectes primitifs où il y a prédominance des ner vures primaires, avec riche développement du réseau transversal (Fig. 41).

$2^{\circ}$ Flytroptères. Incontestablement, le groupe des Élytroptères n'est pas naturel au point de vue systématique, en ce sens qu'il n'y a aucun motif de croire à des relations de parenté entre tous les groupes rassemblés sous cette dénomination.

Le nom choisi exprime seulement que les ailes antérieures, toujours plus ou moins incrustées de chitine, présentent une tendance à la 
'i jparition des nervures. Ce groupe comprend les Crthoptères (inclus Islatidés et Forficulidés), les Hémiptères (malgré l'importante exceptiơn des homoptères), et les Coléoptères.

$3^{\circ}$ Néoptères. - Les Néoptères, comprenant les Hyménoptères, le; Lépidoptères et les Diptères actuels, forment un groupement assez naturel, mais dont les relations sont difficiles à saisir, si l'on ne ræmonte pas jusqu'à leurs ancêtres neuroptériques (Fig. 42).

Vraisemblablement, aucun représentant de ce phylum n'a vécu pendant l'ère paléozoïque ; la nervation des ailes, dans les trois ordres, est caractérisée par le nombre relativement petit des nervures. Toutes les veines transversales sont spé. cialisées, c'est pourquoi elles sont devenues presque aussi

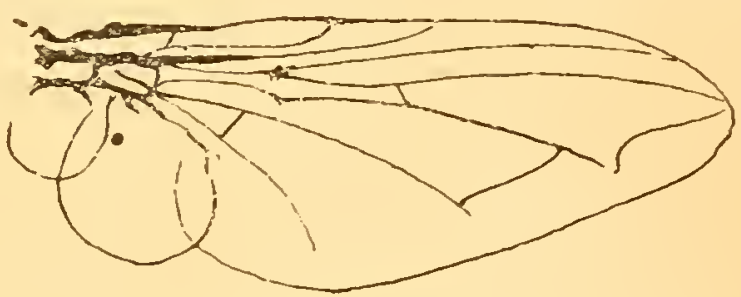

Fig. 42. - Aile de la Mouche commune (d'après Woodworth). constantes et aussi importantes que les veines longitudinales.

Quelles sont maintenant les causes qui ont amené ces différences de conformation ? Étudions d'abord, avec M. Woodworth, l'évolution de la réticulation alaire ; ensuite, il nous suffira de considérer quelques adaptations secondaires, pour voir toutes les variations s'expliquer d'elles-mêmes.

\section{Evolution et Classification Des Nervures}

Nervures principales. - L'observation des ailes aux premiers stades de leur développement, ainsi que l'étude des types ailés les plus inférieurs, montrent que la première nervure qui apparait est la nersure marginale (Fig. 43): qui semble résulter d'une simple modification des cellules de la bordure de l'aile.

Premier stade. - Cette nervure marginale (n.m.) n'est pas également développée sur tout le pourtour de l'aile; presque toujours aussi, elle est plus épaisse sur la bordure antérieure que sur la bordure postérieure; souvent même elle est entièrement absent: dans la région distale de l'aile.

Deuxième stade. - Une deuxième nervure, ontogenétiquement beaucoup plus importante que la marginale, est celle que Woodworth désigne sous le nom de nervure primaire (n.p.); en général, cette 
nervure, tonjours la plus forte des veines présentes, naît dans le tier's antérieur de l'aile (Fig. 43). Chez certains Phasmides cependant, la nervure primaire paraît rejetée tout à fait en arrière, à cause du
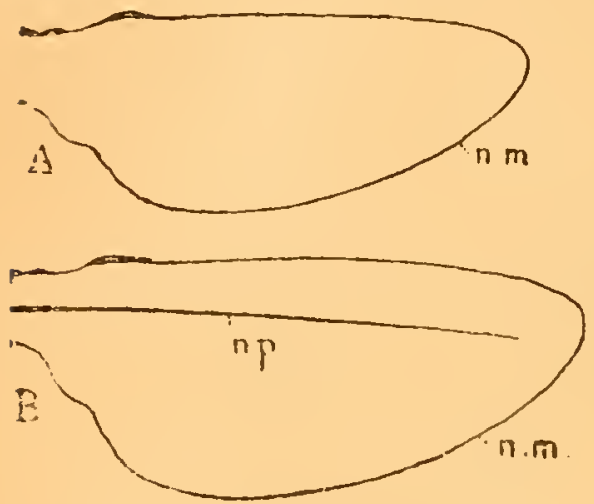

Fig. 43. - Marche du développement c'es nervures d'après la théorie de Woodworth. A, premier sta te ; B. deuxième stade. développement exagéré de la partie antérieure de l'aile.

La position typique de la nervire primaire est déterminée, cela se conçoit, par les conditions mécaniques du vol, surtout p.ar ce fait que le plus grand axe de l'aile représente une ligne de moindre résistance, oì la membrane se trouve par conséquent facile à plier: le premier pli détermine donc. ainsi la position de la première veine, et nous verrons cette règle se poursuivre tout le temps, dans le cours de l'évolution du réseau alaire.

Quelle que soit la complication ultérieure de la nervation, la prééminence de la nervure primaire reste toujours très marquée. C'est done cette veine qui doit servir de point de repère principal dans l'étude de la nervation et dans la recherche des homologies, parce quelle est la plus ancienne, la plus importante, la moins variable . et la plus facile à reconnaître de toutes les nervures longitudinales.

La nervure primaire peut être simple ou richement ramifiée (Blattidés). Pour les ailes de faible étendue, la rigidité de la membrane est suffisamment assurée par la nervure primaire et ses ramifications ; mais, lorsque la surface de l'aile s'accroît, les premières nervures ne suffisent plus à consolider la membrane; aussi voit-on de suite se produire un accroissement corrélatif du réseau veineux. On peut évidemment citer quelques exceptions à cette règle, mais elles sont rares.

Troisième stade. - Denx autres séries de nervures partent également de la base de l'aile et se développent aussi de très bonne heure en avant et en arrière de la nervure prinaire. D'après leur position, ces groupes de nervures sont désignés sous les noms de nersures untérieures (na) et de nervures postéricures (ns) Fïg. 44).

(Chez la plupart des Insectes, on ne trouve qu'une seule veine antérieure; elle cesse de honne heure d'être en relation avec la paroi du corps et s'accole simplement à la base de la nervure primaire. 
Sauf chez les Orthoptères de la subdivision des Blattidés, la nervure antérieure est, en général, peu ramifiée.

En arrière de la nervure primaire, toutes les reines postérieures (à l'exception de la marginale), ne prennent aucune part à l'articulation de l'aile.

Si la nervure primaire et la nersure antérieure sont presque toujour. très faciles à distinguer, il n'en est pas toujours de même, dans la pratique, pour les veines postérieures, à cause des liaisons qu'elles contractent avec les nervures indépendantes ou avec les transversales.

Nervures indépendantes. - Ces nervures sont ainsi nommées, parce qu'elles n'ont, au début, aucune relation avec les nervures principales; elles se développent le long de la boràure de l'aile, de sorte qu'on pourrait, dans la majorité des cas, les considérer comme des ramifications internes de la veine marginale.

Chacune de ces veines

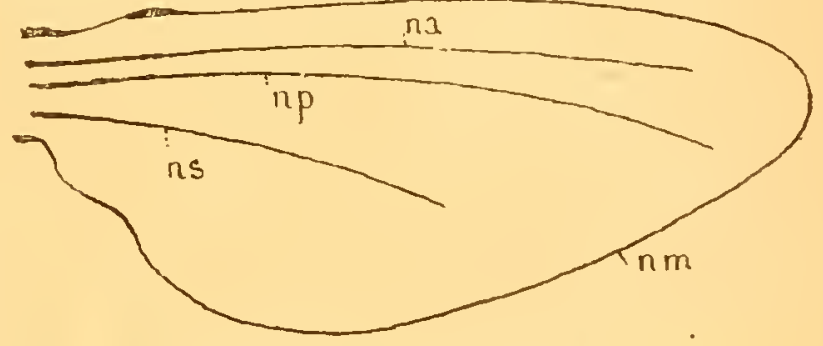

Fig. 44. - Troisième stade du développement des nervures (imité, de Woodworth). indépendantes s'étend vers l'intérieur de l'aile de manière à occu per l'espace vide laissé entre les extrémités des veines principales; ici encore, c'est la ligne de moindre résistance de la membrane alaire qui fixe la position de ces nervures. Leur nombre n'est pas fixe ; très faible ou nul dans certains Hyménoptères, il est au contraire très grand chez le Éphémérides (Fig. 41).

Nervures transversales. - Les veines transversales ressemblent aux veines indépendantes, tant dans leur constitution que dans leur structure, mais elles en diffèrent complètement par ce fait que l'ordre de leur développement n'est pas fixe; elles peuvent naitre simultanément, ou successivement, dans les divers points de l'aile, suivant que la membrane a besoin d'être consolidée.

En résumé, on voit qu'il est possible d'ébaucher une véritable théorie mécanique de la nervation, au lieu et place des anciens systèmes dontleschéma était arbitraire, et surtout à la place de celui qui considérait les nervures comme les vestiges d'un ancien réseau trachéen. Dans cette théorie moderne, l'utilité des nervures pour le vol est le facteur primordial; ce sont exclusivement des motifs 
d'adaptation qui règlentleur développement, leur nombre et leurcaractère; alors, les diverses classes de nervures deviennent déterminées, no. plus par leur structure, mais par leur direction et surtout par leur origine.

D'ailleurs, toutes les nervures, quelle que soit leur importance, se développent de la même façon, suivant les plis de l'aile, et par une modification sur place des cellules de la membrane.

Principales adaptations. - Maintenant que nous avons indiqué l'origine du plan primitif de la nervation alaire, plan qui donnera à l'aile son caractère en assurant sa stabilité, il nous faudrait suivre les variations de ce plan dans les différents groupes d'Insectes. Cette étude sera faite en détail dans les volumes suivants de l'Encyclopédie scientifique; nous nous bornerons à indiquer ici quels sont les principaux facteurs de variations qui ont prédominé dans les différents ordres.

En ce qui concerne les causes de la modification du plan primitif que nous venons d'établir, il faut considérer, en premier lieu, deux choses :

10 L'adaptation des ailes antérieures aux fonctions de protection (élytres) qui s'observe d'une faŗon plus ou moins complète chez les Elytroptères (Coléoptères, Orthoptères, etc., etc.) ;

$2^{\circ}$ Les liaisons qui peuvent s'établir entre les ailes antérieures et les ailes postérieures et qui obligent, dans une certaine mesure, les deux ailes à se mouvoir en même temps (Voir p. 76).

Sans aucun doute, la disposition la plus ancienne est celle où les deux ailes sont semblables dans leur forme, d'égales dimensions, et complètement libres l'une par rapport à l'autre ; c'est la disposition qui est encore réalisée aujourd'hui par la plupart des Névroptères (Fig. 335).

Au contraire, lorsqu'il existe une liaison quelconque (Hyménoptères, Lépidoptères), les deux ailes sont toujours inégales et les postérieures généralement beaucoup plus petites que les antérieures; il n'est pas douteux que ces dernières jouent le rôle principal dans l'acte du vol.

\section{ORIGINE DES AIIES}

Dans tous les Insectes dont le développement se fait sans métamorphose, les ailes apparaissent, de toute éridence, comme des 
expansions hypodermiques des bords latéraux du corps, dans la région du thorax (ए`ig. 45) (mésonotum et métanotum).

Comme on peut le voir, les rudiments des ailes sont, pendant longtemps, absolument continus avec le notum. Plus tard, pendant la période nymphale, une suture se produit à la base de l'aile, des trachées se développent dans son intérieur, et, finalement, quelques sclérites (osselets) se différencient à sa base, servant de point d'attache aux muscles qui doivent la faire mouroir. Il en résulte, qu'aussitôt après la mue nymphale, les ailes sont en état de fonctionner et de soutenir l'insecte dans l'air.

Munis de ces observations - qui ont été faites dans presque tous les ordres d'Insectes - nous pouvons maintenant chercher à déterminer l'origine morpho. Ingique des ailes.

Deux théories principales sont on présence; la première a été proposée par Gegenbaur. Partant de ce fait, qu'on observe. sur les côtés du corps, dans certaines larves aquatiques de.

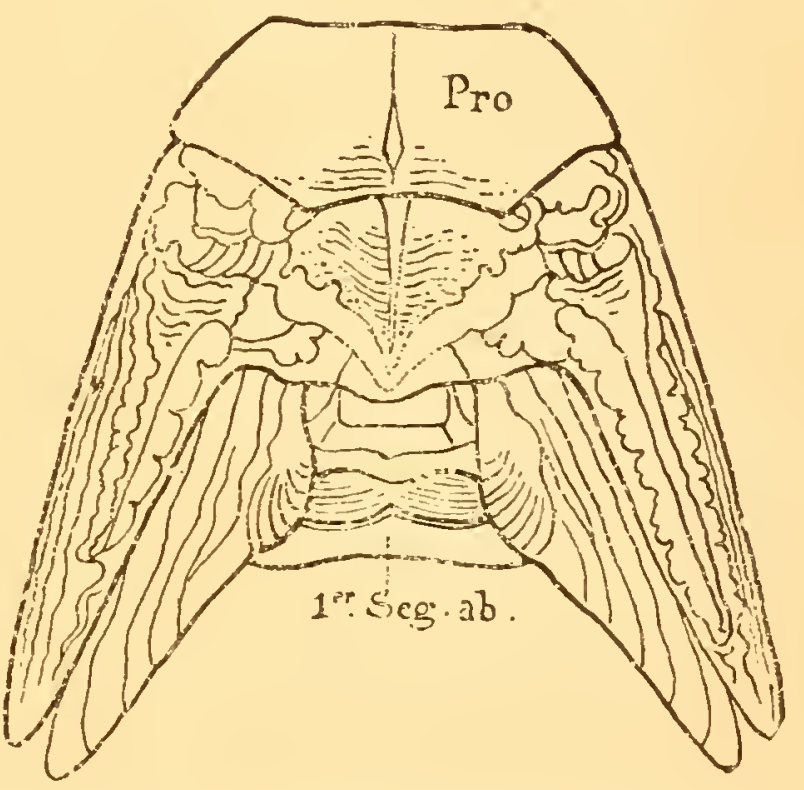

Fig. 45. - Origine des ailes (Aphrophora permutata Hémint.). Pro, prothorax (d'après PACKARD).

Pseudo-Névroptères (Ephémérides, Perlides), des branchies lamelleuses disposées par paires, sur les six ou sept premiers segments abdominaux, Gegenbaur admet que les ailes sont les homologues de ces branchies; en d'autres termes, par suite d'une adaption aux besoins de la locomotion aérienne, les branchies foliacées de la région thoracique se seraient, petit à petit, transformées en ailes.

De ce fait, dans certaines larves aquatiques, (notamment chez Cleon dimidiatum), on observe, en quelque sorte, le passage entre les branchies lamelleuses et les ailes.

La seconde théorie, dont le point de départ a été donné par Fritz-Müller en 1875, admet que, au début, les ailes ne furent autre chose que des duplicatures de la peau, destinées à protéger les parties latérales du thorax, et de fait, ces expansions, à peine indi- 
quées chez les Campodès, faiblement développées chez Japyx, sont, au contraire, déjà nettes et très riches en trachées, sur les trois anneaux thoraciques des Lépismes. Ce sont ces lames, simplement protectrices à l'origine, qui se seraient différenciées plus tard, de manière à fonctionner comme des organes de la locomotion aérienne.

La théorie de Gegenbaur est très séduisante à première vue, et beaucoup d'excellents naturalistes hésitent encore à l'abandonner. En ce qui me concerne cependant, il ne me con̂te nullement de dire qu'elle me parait inacceptable, parce que les trachées sont essentiellement des organes de respiration aérienne; tout Insecte pourvu de trachées ne peut descendre que d'ancêtres aériens; les larves des Ephémérides ne sont donc devenues aquatiques que par suite d'une adaptation secondaire. Les ailes et les branchies lamelleuses sont bien des organes homologues, mais les premières ne dérivent pas des secondes; ce serait plutôt l'inverse qui serait vrai; les expansions membraneuses du tergum, déjà riches en trachées, se seraient transformées on branchies lamelleuses chez les larves aquatiques, en ailes chez les Insectes aériens; cette origine commune explique aussi pourquoi ces deux sortes d'organes coexistent chez certaines larves d'Ephémérides (Cleon). 


\section{CHAPIT'RE VI}

\section{LABDOMEN ET SES APPENDICES}

TeGInique. - La structure de l'abdomen et de ses appendices devra être étudiée sur des Orthoptères de grande taille (Locusta riridissima ou liecticus rerrucisorus). Les cerques sont bien développés chez les Perce-Oreilles.

I. - Abdomen des Insectes. - L'abdomen comprend toute la partie du corps située en arrière du thorax; il est, en général, plus volumineux que les autres régions, parce qu'il renferme les organes de la nutrition et de la génération.

L'abdomen est toujours nettement métamérisé ; les anneaux qui le composent s'emboîtent les uns dans les autres, parce que leur diamètre diminue à mesure qu'on s'avance vers l'extrémité postérieure.

Sauf quelques rares exceptions (voir p. 90), l'abdomen des adultes ne porte ni pattes, ni ailes; aussi, la constitution de ses anneaux est-elle toujours beaucoup plus simple que celle des parties correspondantes du thorax; chaque anneau de l'abdomen n'est formé que de deux sclérites (1), l'un correspondant au demi-anneau

(1) Pour abréger le langage, Packard a désigné sous le nom d'urosome, l'abdomen de tous les animaux articulés. Dans ces conditions, les sclérites geront désignés, suivant leur position, sous les noms d'urotergites et d'urosternites. Chaque segment, dans son ensemble. est un uromère. Nous emploierons quelquefois ces expressions. 
supérieur (urotergite), l'autre constituant le demi-anneau inférieur (urosternite) (Fig. 46).

Quoi qu'il en soit, c'est généralement sur la membrane

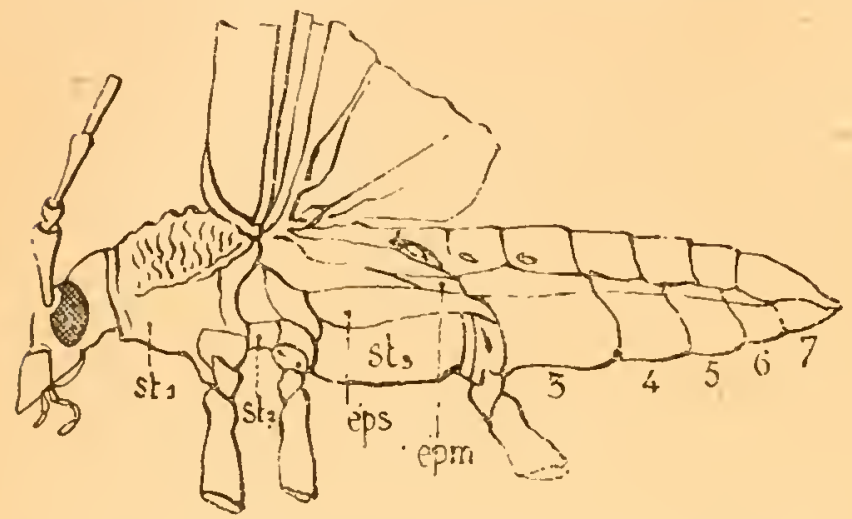

Fig. 46. - Un Coléoptère vu de côté (Cerambyx rerdo) $s t_{1}, s t_{2}, s t_{3}$. les trois régions sternales du thorax; éps, épisterne : épm, épimère: $3,4,5,6,7$, les cinq anneaux r'e l'abdomen risibles en ciessons. (D'après KíOLBE). molle qui réunit latéralement les sternites et les tergites que viennent s'ouvrir les stigmates, c'est-à-dire les petites ouvertures qui laissent pénétrer, à l'intérieur du corps, l'air nécessaire à la respiration.

Typiquement, chez les Insectes adultes, le nombre des segments abdominaux paraît être de dix; mais ce nombre est sujet à variation, par suite des réductions qui peuvent se produire, soit à la partie antérieure, soit le plus souvent, à la partie postérieure du corps.

Chez un rertain nombre d'Insectes, la partie inférieure des premiers anneaux abdominaux est tellement refoulée par le métasternum, qu'elle disparaît complètement; les premiers uromères ne se trouvent done plus constitués que par le demi-anneau supérieur (urotergite) ; c'est là la raison pour laquelle on ne compte souvent (ex. : Coléoptères) que six à sepí segments visibles à la partie inférieure de l'abdomen, tandis que la face supérieure en laisse voir sept à huit (ex. : Hanneton) et mème davantage (Fig. 47).

La base de l'abdomen est, en général, largement articulée avoc la face postérieure du thorax; cependant, cher. un grand no ubre 
d'Hyménoptères, l'abdomen est rattaché au thorax par un filet très grêle désigné sous le nom de péroncule.

C'est dans la partie postérieure de l'abdomen que se produisent les plus grandes modifications des uromères; ces modifications affectent surtout le dernier segment qui, en s'adaptant aux fonctions de reproduction, arrive à former un ensemble de pièces qu'on a désigné sous le nom d'armure génitale.

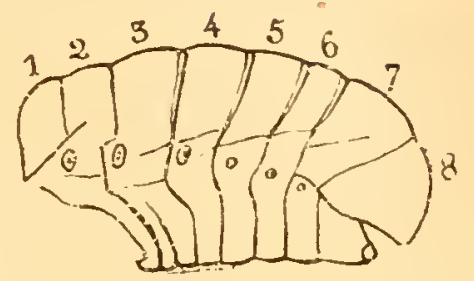

Fig. 47. - Abdomen vu de côté, pour montrer la réduction des urosternites (Xylotrupes Gideon.Coléopt. (d'après KoLBE).

Quelle que soit la forme qu'il affecte, le dernier segment visible de l'abdomen est fréquemment désigné sous le nom de pygidium.

II. - Appendices de l'abdomen. - L'abdomen ne présente, dans la plupart des cas, aucun appendice; il n'en est cependant pas toujours ainsi ; et, chez un certain nombre d'Insectes appartenant à la famille des Thysanoures, on trouve, sur les côtés des arceaux inférieurs, des appendices que l'on peut considérer comme des membres abdominaux atrophiés. Dans les autres groupes d'Insectes, on trouve encore des appendices analogues chez, un certain nombre de larves, mais ils disparaissent génér:jement, chez l'adulte.

La présence des membres abdominaux est considérée comme la persistance d'un caractère ancestral ; et, du fait qu'on l'observe surtout chez les embryons et chez les Insectes les plus inférieurs (Campodea, Machilis), cela indique, à n'en pas douter, que les ancêtres des Hexapodes devaient être pourvus de membres abdominaux (Fig. 48-49).

Enfin, tout à fait à la partie postérieure du corps, on trouve des filets ou des lames étroites, paraissant 
sortir de l'ouverture anale. Suivant leur structure et surtout suivant leur origine, on désigne ces appendices sous les noms de stylets ou de cerques.

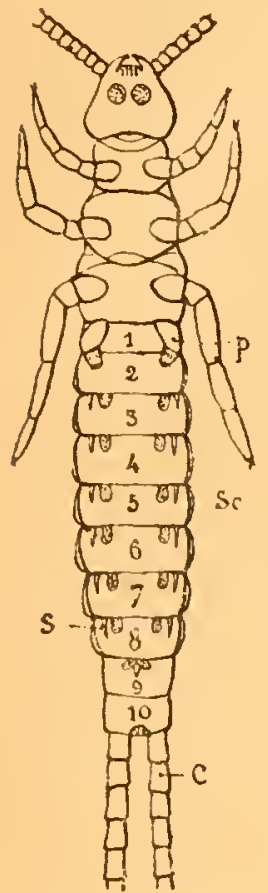

Fig. 48. Appendices abdominaux d'un Campodé (d'ap. VAYSSIÈRE)

Nous allons exáminer succinctement ces différents appendices.

\section{0 - Membres abnominaux des Thysanoures. -} Si nous examinons la face inférieure de l'abdomen chez Campodea staphylinus. curieuse espèce de Thysanoure habitant la vase humide, on trouvera, sur le premier segment, une paire de pattes très courtes et vaguement articulées ( $p$.$) . Sur les$ sept anneaux qui suivent, les appendices sont toujours présents, mais ce ne sont plus que des styles mobiles, non segmentés; en dedans, à leur base, se trouve une poche membraneuse dévaginable $(s c$.$) , pouvant$ faire saillie au dehors sous l'aspect d'un petit mamelon (Fig. 48).

Sur le $9^{\mathrm{e}}$ anneau, les appendices manquent, mais les membranes dévaginables persistent. Enfin, sur le dixième anneau, on ftrouve deux longs filamenis articulés, re sont les cerques (Fig. 48).

Une disposition analogue s'observe chez Japyx gigas, avec cette différence que tous les segments

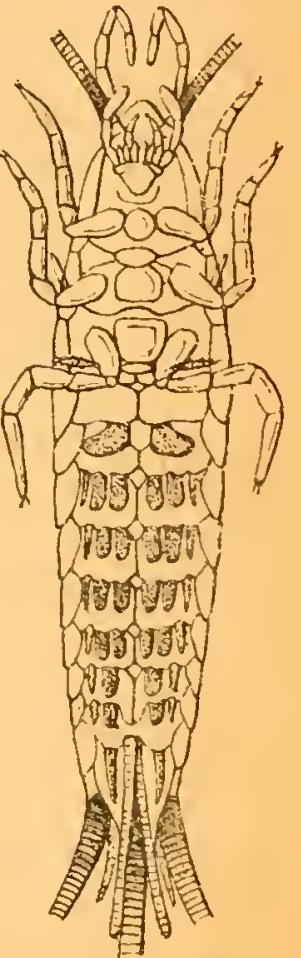

Fig. 49. $\rightarrow$ Ap. pendices abdominaux du Machile (d’ap. VAYSSIITRI). de l'abdomen sont dépourvus de sacs ventraux à
l'exception du deuxième; mais les stylets existent, comme chez Campodea, sur tous les anneaux jusqu'an huitième inclus. Le dixième se termine par deux forts appendices analogues à la pince des Forficule; ce sont là évidemment des cerques modifiés.

Enfin, on pourrait peut-être encore rapprocher des sacs membraneux des Campodès, les curieux tubes saltatoires des Collemboles. D'ailleurs, la comparaison de tous ces organes (sacs membraneux et stylets) avec la disposition analogue qui existe chez certains Myria- 
podes, Scolopendrella immaculata, par exemple, permet de se rendre compte que ce sont là, sans aucun doute, des restes d'organes appartenant aux articles coxaux des membres disparus.

$2^{\circ}$ - Styles et Cerquas chez les alitres Insectes. - On désigne sous le nom de styles, des appendices que l'on rencontre chez un certain nombre d'Insectes. Ce sont des filaments mobiles, presque toujours recouverts de poils mais non segmentés. De ce fait qu'ils s'insèrent toujours au bord postérieur du neuvième urosternite (Orthoptères et Hyménoptères), ou, par exception, sur le bord du septième (certains Coléoptères : Hydrophilus), on admet qu'ils équiralent, morphologiquement, aux stylets abdominaux des Thy cirorus, st, styles; $c$, cersanoures (Fig. 50).

Quant aux cerques, ce sont des appendices plus ou moins lonors, portés par le dixième ou par le onzième sternite; les auteurs ne sont pas d'accord à ce sujet.

Il est probable que les cerques sont des styles modifiés, et adaptés à des fonctions spéciales; c'est l'opinion d'Heymons. Quoi qu'il en soit. dans la plupart des cas, on ne possède aucun rensergnement sur le rôle de ces organes. Ce qui est certain, c'est qu'ils sont tout à fait distincts des pièces de l'armure génitale, avec lesquelles on serait parfois tenté de les confondre. 


\section{CHAPITRE VII}

\section{ORNEMENTATION Ét ARMATURE DES TÉGUMENTS}

Teginique. - Prendre le Hanneton et sa larve (Ver blanc); le Papillon blanc du Chou et sa Chenille; la Chenille velue d'un Bombycidé quelconque.

La cuticule solide qui revêt le corps des Insectes, constitue un appareil de protection très efficace, lorsqu'elle est suffisamment chitinisée, c'est ce qui a lieu, le plus souvent, chez les adultes. Dans ce cas, les téguments peuvent être lisses, brillants (Colépotères, Nésroptères, Orthoptères, simplement ponctués ou granulés. Lorsqu'on y observe des tubercules, des épines ou des cornes, ce sont, ou des organes de défense (Lucanides), ou des caractères sexuels secondaires (Dynastides).

Le corps reste nu chez les larves qui vivent en parasites à l'intérieur des végétaux. Inaccessibles à leurs ennemis, et protégées contre le froid ou la dessiccation par le milieu même où elle ont trouvé un abri (1), ces larves n'ont pas besoin d'un appareil protecteur spécial ; aussi leur corps reste-t-il généralement très mou, et c'est à peine si l'on observe quelques soies ou quelques tubercules servant à favoriser les déplacements. Ces larves meurent très vite lorsqu'elles sont extraites de

(1) A n'importe quelle période de leur existence, les Insectes craignent plus la dessiccation que le froid. 
leur milieu naturel (ex.: Anthonome; larves lignicoles des Cérambycides; mineuses des feuilles, etc.)

Il en est de même des larves qui vivent en terre, à une profondeur assez grande (Hanneton, Ver-gris) ; mais on observe que celles qui vivent dans le sol, à une faible profondeur (5 à 10 centimètres, ex. : Ver-fil-de-fer, larves de Carabiques), possèdent une carapace déjà passablement résistante, et, de plus, garnie de poils ou de tubercules.

Il y a toujours, comme on le voit, une corrélation très nette entre la vestiture de l'Insecte et le milieu où il vit.

Certaines régions du corps, particulièrement les pattes, sont garnies de poils rigides et de soies formant de véritables brosses, pour les soins de propreté : tels sont, par exemple chez les Abeilles, les cils rigides qui garnissent les jambes et qui leur servent à enlever le pollen tombé sur leurs yeūx.

Pour le moment, nous allons nous occuper uniquement de l'armature protectrice ou ornementale du corps, c'est-à-dire des poils, des écailles et des glandes cutanées.

1. Poils et leurs principales adaptations.- Quelle que soit leur forme, les poils ne sont autre chose que des prolongements creux de la cuticule primaire; ils doivent leur origine à une cellule hypodermique (Fig. 52 e.) généralement pyriforme et plus grande que les autres; cette cellule, désignée sous lo nom de trichogène par Graber, donne un prolongoment protoplasmique qui passe dans un canal de la cuticule secondaire ét arrive ainsi dans l'axe du poil de façon à le nourrir.

Les poils naissent, soit au sommet d'un petit tubercule, soit dans une dépression des téguments en forme de fossette annulaire ; généralement les poils sont souples et flexibles, alors ils se conservent bien à la surface du corps ; mais, s'ils sont très incrustés de chitine, ils deviennent cassants et tombent au premier frottement. 
Modifications des Poils. - Les poils sont le plus souvent simples; on en rencontre cependant quelquefois qui sont ramifiés ou plumeux, comme par exemple chez les larves de certains Dermestidés (Anthrenus, Dermestes) et des Coccinellides (E.pilachna, Cassida). Lorsque les

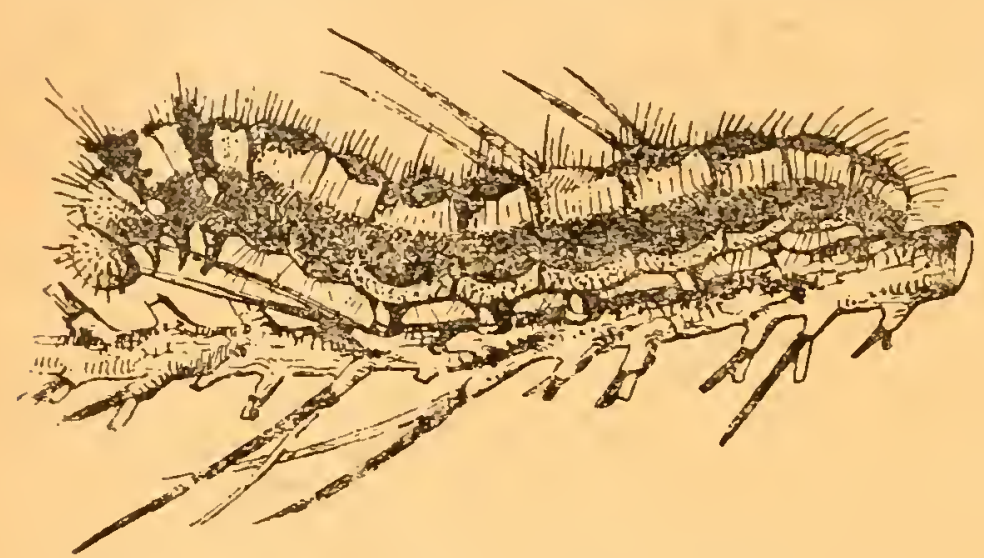

Fig. 51. - Chenille cu Lasiorampa pini, couverte de poils urticants. poils sont allongés et rigides, ils prennent le nom de soies; s'ils sont. massifs et très solides, ils constituent les épines.

Poils slanduleux. - On rencontre, chez certains Insectes, de fines soies tubulaires qui laissent suin-

ter, par leur extrémité, faiblement lenflée et probablement percíe de pores très fins, un liquide clair, légèrement visquenx. C'est à cette catégorie qu'appartiennent les poils adhésifs qui garnissent les pattes des Mouches, et qui leur permettent de se maintenir sur les surfaces lisses des vitres, ou de marcher, dans une pocition renversée, au plafond de nos habitations.

Chez un grand nombre de Chenilles apparienant aux familles des Saturnides et des Lasiocampides (Fig. 51), le corps est revêtu de poils glandulaires sécrétant un liquide urticant (1). Ces poils sont creux; le poison dont ils sont remplis est produit par une grosse cellule hypodermique située à leur base (Fig. 52). Si l'on vient à toucher une de ces Chenilles sans précaution, les poils se brisent en pénétrant dans la peau où ils produisent une douleur cuisante.

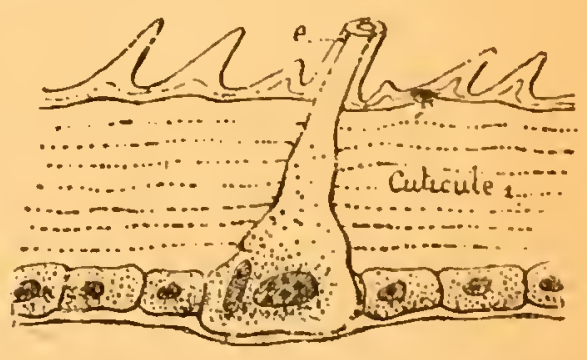

Fig. 52. - Origine des poil; urticans (d'après Towlı).

Poils tactiles. - Nous étudierons l'organisation de ces poils avec les organes des sens (p. 163).

(1) Urticant se dit des substances qui prolujsent une irritation rappelant Iit siçûre rles Orties. 
$2^{\circ}$ Écailles. - Les écailles, que l'on observe sur les ailes des Papillons, sur le corps des Lépismes et sur les élytres de certains Coléoptères (Hoplies), ne sont autre chose que des poils aplatis et modifiés; et, de fait, on a pu, dans un certain nombre de cas, observer tous les passages (Fig. 53) entre les poils ordinaires, cylindriques et les véritables écailles. Semper a, prouvé d'ailleurs (1857), que le déreloppement de ces deux espèces d'organes était absolument identique.

Il y a certains groupes d'Insectes où la présence des écailles est générale et absolument caractéristique, tels sont, par exemple, les Lépidoptères (1) et les Thysanoures; d'autres fois, elles ne se rencontrent que dans certains genres, (ex. : Lixus, Ptinus, pour les Coléoptères, Culex pour les Diptères).

Les écailles présentent souvent de très brillantes colorations; ces couleurs sont dues, évidemment, à des pigments développés dans leur intérieur (coloration chimique), mais, laile du Sphinx pinastri (KoLBE). très souvent aussi, à des phénomènes de diffraction, comparables à ceux qui donnent naissance aux réseaux en optique.

Nous citerons seulement à ce sujet les magnifiques écailles colorézs de l'aile des Morpho, qui présentent de si brillants reflets métall:ques; on paut compter, sur certaines de ces écailles, jusquà 1.400 stries longitudinales par millimètre.

Développenent des écallees. - L'origine et le mode de déveoppement des écailles ont été suivis par Semper en 1857, sur l'aile dos Sphinx et des Saturnidés (2). Il a montré que ces écailles pro. veraient de certaines grandes cellules hypodermique, de forme ovale (e), qui produisaient, an dehors, un prolongement destiné à sécréter la jeune écaille (Fig. 54).

Pour plus de détails sur ce sujet, voir le volume de l'E.S. trai!ant. des Papillons.

(1) Du grec lepidos ècaille et ptéron aile.

(2) Semper (C.). - Reobachtungen über die Bildung der Flügel-Schuppen und Haare bei cen Lepicopteren (Zeitschr. fur Wissensch. Zoologie). T. VIIT. pp. 326-339 
$3^{\circ}$ Glandes cutanées. - En plus des éléments hỵpodermiques qui donnent naissance au revêtement pileux et aux écailles, on trouve encore quelquefois, dans la peau de certains Insectes (Coléoptères) de très grandes cellules adaptées aux fonctions sécrétrices :

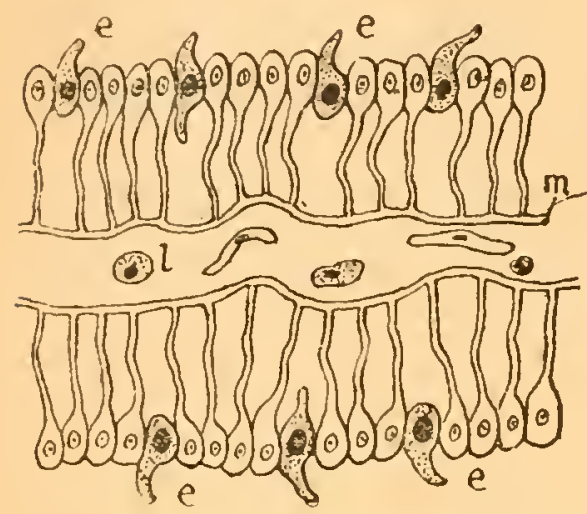

Fig. 54: - Développement des écailles; $e$, cellules s'allongeant en écailles: $m$, membrane fondamentale ; $l$, leucocytes.

(D'après PACKARD). ce sont les glandes cutanées, qui produisent un liquide destiné à la dé. fense de l'Insecte. Leydig a signalé depuis longtemps celles qui existent chez les Téléphores et qui répandent l'odeur âcre que l'on perçoit lorsqu'on vient à toucher ces Insectes. C'est à la même cause, très probablement, qu'il faut rapporter l'odeur des Galéruques et de certains Coccinellides; enfin, Plateau a encore fait connaître, sur le prothorax des Dytiscides, des cellules globulaires sécrétant un liquide blanchâtre très fétide.

Couleur des Insectes. - Cette importante question sera traitée, avec tous les détails qu'elle comporte, dans le volume de l'E.S. consacré aux Coléoptères. 


\section{DEUXIÈME PARTIE}

\section{Fonctions de Nutrition.}

\section{CHAPITRE VIII}

\section{L'APPAREIL DIGESTIF ET SES ANNEXES}

Technigue. - Prendre le Carabe doré, un Dytique ou la grande Sauterelle verie ; après avoir enlevé les ailes et les élytres, inciser les tíguments avec des ciseaux à pointe fine, le long de la ligne médiane du dos, jusqu'à la tête. Etaler ensıite le corps de l'Insecte et le fixc: avec des épingles sur le fond liégé d'une cuvette à dissection. Recou!virir d'eau afin que les organes flottent et s'étalent bien; puis, dilacérer avec un scalpel et une aiguille emmanchée, les brides de tissu c nnjonctif qui maintiennent le tube digestif en place, jusqu'à ce que celui-ci soit bien isolé des organes voisins.

L'appareil digestif des Insectes, comme celui des animaux supérieurs, comprend deux parties principales:

1 o Le tube digestif, destiné à recevoir les aliments après qu'ils ont été divisés par les mâchoires.

$2^{\circ}$ Les or ganes annexes, servant à produire les sucs qui doivent effectuer la transformation des aliments.

\section{Io TUBE HIGESTIF.}

Le tube digestif est un canal irrégulier, s'étendant de la bouche à l'extrémité de l'abdomen; l'ouverturt

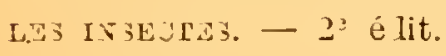


postérieure du tube digestif (anus) est toujours située sur le dernier segment abdominal, au-dessous d'une pièce chitineuse de forme varjable, qu'on désigne sous le nom de plaque suranale. Tantôt court et presque droit (ex.: Nésroptères) ; tantôt, au contraire, beaucoup plus long que le corps et décrivant de nombreuses circonvolutions, le tube digestif est toujours sensiblement situé dans l'axe de la cavité générale, entre la chaîne nerveuse et le vaisseau dorsal (Fig 55). Il est maintenu en place par des brides fibreuses qui le rattachent, en

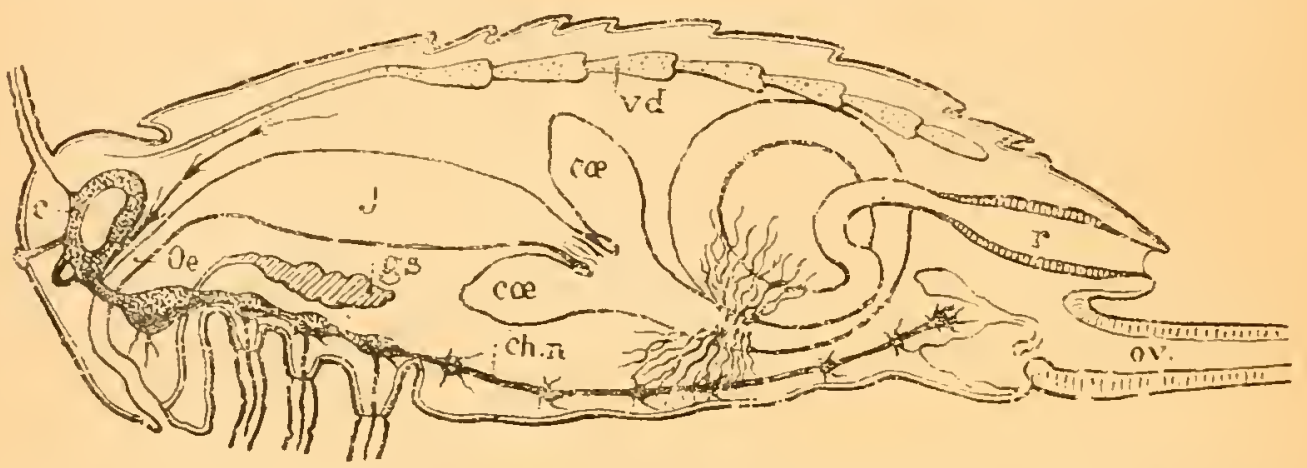

Fig. 55. - Section Iongitudinale d'un Orthoptère (Anabrus), pour montrer la

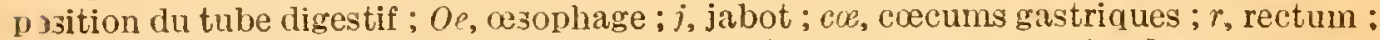
on, oviscapte; $v d$, vaisseau dorsal; chn, chaine nerveuse; gs, glandes salivaires ; c, cerveau. (D'après P.Ackarn).

dessus, à la paroi du corps ; mais, en dessous, il est largement supporté par les nombreuses ramifications qui partent des plus gros troncs trachéens.

La longueur du tube digestif varie, non pas tant, comme on pourrait le croire, avec le régime de l'Insecte, mais, suivant les observations de Newport, d'après la qualité et surtout d'après le degré de digestibilité des aliments consommés.

Considéré dans son ensemble, et principalement chez les Insectes broyeurs, où il parait atteindre son maximum de complication, le tube digestif peut se diviser 
cn trois régions bien distinctes, qui sont déjà indiquées dans l'embryon; ce sont :

10 L'intestin antérieur ou stomodeum (1)

20 L'intestin moyen ou mésenteron.

$3^{\circ}$ L'intestin postérieur ou proctodeum.

Examinons ces différentes régions sur un Carabe doré, insecte facile à se procurer dans tous les pays.

10 L'Intestin antérieur, ou stomodeum, résulte d'une invagination ectodermique se produisant à la partie antérieure du corps; il comprend les parties suivantes, qui font suite à la bouche : le pharynx, l'œsophage, le jabot et le gésier. Le développement de ces deux derniers organes varie beaucoup, suivant le régime alimentaire des Insectes (Fig. 61).

Pharynx. - Le pharynx,qui est renfermé dans la tête, n'est autre chose que la partie antérieure et légèrement dilatée du tube digestif. Il a, en général, la forme d'un entonnoir et sa partie postérieure, quelquefois difficile à délimiter, est garnie de plis longitudinaux.

Chez les Insectes suceurs, Hémiptères, Lépidoptères ct Diptères, la région pharyngienne est développée en un large sac membraneux, rattaché aux parois de la tête par des brides musculaires. Ce sac pharyngien est disposé de telle façon que, lorsqu'il se dilate, il se produit un vide à son intérieur et les liquides alimentaires sont aspirés par la trompe de l'Insecte; au contraire, lorsqu'il se contracte, les aliments sont chassés dans l'œsophage.

C'est dans la région du pharynx que viennent déboucher les glandes salivaires lorsquelles existent (ex.: Orthoptères.)

(1) Stomodeum, du grec strma, bouche. 
Cssophage. - L'osophage est un tube rectiligne qui lait suite au pharynx et se termine au jabot; sa longueur varie suivant les espèces.

Jabot. - Le jabot n'est autre chose qu'un renflement de la base de l'œesophage, servant à emmagasiner les aliments ; il doit son nom à ce fait qu'il occupe la même position et remplit probablement les mêmes fonctions que l'organe du même nom chez les Oiseaux (Fig. 61, b).

La forme et les dimensions de ce renflement sont très variables; le plus souvent il est ovoïde et lisse intérieurement ; mais, lorsqu'il est distendu par les aliments, il peut se trouver déjeté sur le côté ; c'est ce qu'on observe chez les Névroptères du groupe des Hémérobides.

Cette disposition s'accentue chez les Insectes suceurs - Hémiptères, Lépidoptères) ; le jabot constitue alors une sorte de poche reliée à l'osophage par un pédoncule rétréci, on l'a alors désigné sous le nom très impropre d'estomac suceur (Fig. 56, J.). Chez un certain nombre de

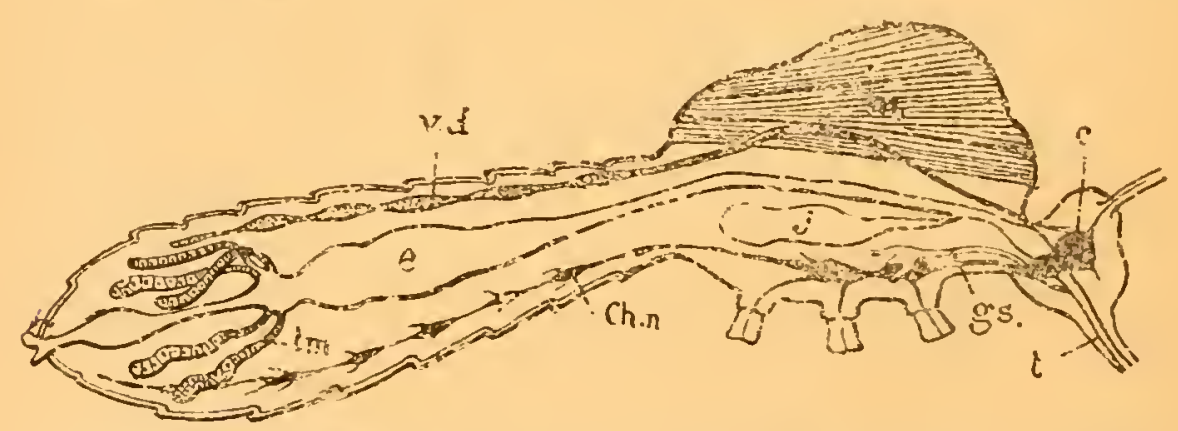

Fig. 56.-Coupe longitudinale du corps d'un Moustique; $t$, estomac $t m$, tubes de Malpiglif; $j$, jabot; $g s$, glandes salivaires; $f$, trompe: chn, chaine nerveuse; $c$, cerreâu, (D’aurès H. Polatlron).

Coléoptères carnassiers, le jabot est très développé sur le côté ; il porte, en outre, des sillons qui lui donnent un aspect côtelé (ex. : Carabes).

Chez les Abeilles, Bourdons, etc., c'est dans le jabot que s'opère la translormation du nectar en miel. 
Gésier. -- Le gésier, lorsqu'il existe (ex. : Orthoptères, Coléoptères), vient immédiatement à la suite du jabot; dans ce cas, il précède toujours l'intestin moyen (Fig. 61, c). ce qui fait qu'on le désigne quelquefois sous le nom de proventricule (1).

Comme il est généralement garni, à l'intérieur, de pièces chitineuses dentelées, on l'a longtemps considéré comme un appareil masticateur ; mais Plateau a démontré, en 1873, par une série d'expériences ingénieuses (2), que les aliments qui l'ont traversé se retrouvent dans l'estomac avec la même forme et le même volume. Cet organe n'est donc probablement qu'un appareil valvulaire, destiné à arrêter les aliments jusqu'à ce qu'ils soient bien ramollis par les sucs digestifs.

Le gésier est bien développé chez les Insectes broyeurs, qui se nourrissent de substances relativement dures (Orthoptères, Nérroptères, Coléoptères) ; par contre, il l'est beaucoup moins chez ceux qui absorbent des aliments liquides (Lépidoptères, Hémiptères, etc.).

$2^{c}$ L'Intestin moyen, ou mésenteron, comprend tout d'abord une région renflée, le plus souvent recouverte de villosités à l'extérieur (Fig. 61, d.) : c'est le ventricule chylifique. A la suite vient une deuxième région, s'étendant depuis le ventricule chylifique jusqu'au point d'insertion des tubes de Malpighi. Cette deuxième région, plus ou moins développée selon les Insectes, n'a pas reçu de nom particulier; on pourrait lui donner le nom de duodenum, car son aspect est déjà celui de l'intestin qui lui fait suite.

Au point où commence l'intestin moyen et où finit.

1) Parce qu'il précède le rentricule chylifique, c'est-à-dire l'intestin moyen.

2) Plateau (F.). - Acad. roy. Belg. T. XLI, p. 124. 
par conséquent, l'intestin antérieur, se trouve un épaississement.plissé de la muqueuse formant ce qu'on appelle la valoule ossopharienne (Fig. 57), souvent garnie de replis chitineux à son intérieur.

Contrairement à ce que l'on croyait autrefois, le mésenteron est aussi d'origine ectodermique, au moins chez. la plupart des Insectes (I); son développement est seulement plus tardif que celui des deux autres parties dn tube

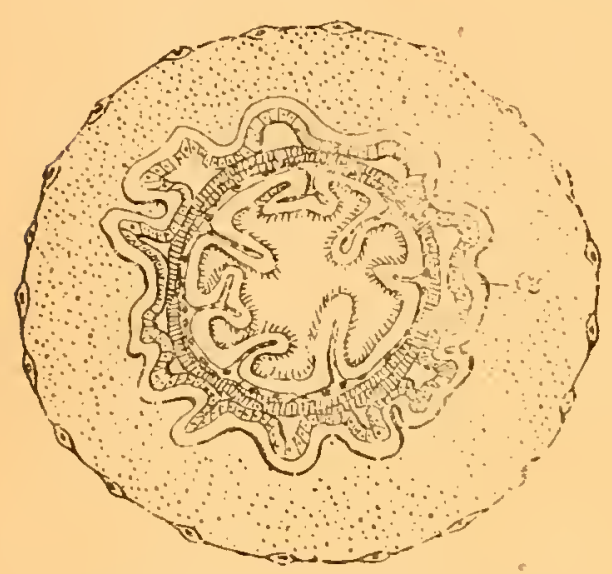

Fig. 57. - Valvule oesophagienne de la Cantharide, coupe trans. versale; $\propto$, membranebasale de l'intestin moyen. (D'ap. BEauregard). digestif; il se forme par un processus très intéressant que nous étudierons plus loin (p. 226).

L'intestin moyen joue le rôle principal dans l'acte de la digestion, aussi le compare-t-on volontiers à l'estomac des animaux supérieurs; de fait, c'est dans son intérieur que les aliments subissent les transiormations qui les amènent à l'état de chyle.

$3^{\circ}$ L'Intestin postérieur, ou proctodeum, est un tube étroit, plus ou moins contourné; il commence au point d'insertion des tubes de Malpighi et se termine à l'anus. Vers sa base, il porte un renflement ovoïde qui est le rectum, tandis yue sa partie antérieure rétrécie reçoit le nom d'iléon.

L'intestia postérielir doit aussi son origine à une invagination de l'ectoderme se produisant à la partie postérieure du corps. Cett région du tube digestif présente généralement moins de variations que les précédentes : si l'on y renoontre parfois dos dispositions spéciales, ces

(1) Toir les travaux de Lécaillon sur le développement des Chrysoméli 'es. 
dispositions sont purement spécifiques, et ne peuvent, sinon en des cas très rares, servir à la classification (Fig. 61, 1.).

Rectum. -.. Le rectum s'ouvre constamment dans un véritable cloaque, au niveau du dernier segment abdominal ; c'est dans son intérieur que viennent déboucher les glandes anales, lorsqu'elles existent (1).

\section{Anatomie du tube digestif.}

A part les adaptations spéciales exigées par le régime, la structure et les fonctions du tube digestif paraissent être identiques dans tcus les ordres d'Insectes.

Nous nous bornerons donc à ranpeler ici les faits anatomiques le-plus généraux et à indiquer quelques-unes des particularités des. plus intéressantes signalées chez les Insectes.

La paroi du tube intestinal est toujours constituée par trois tuniques, comme chez les animaux supérieurs, une tunique externe ou péritonéale de nature conjonctive, extrêmement mince et quelquefois difficile à distinguer : une tunique moyenne, de nature musculcire et enfin, une tunique interne (muqueuse), de nature épithéliale.

D'après Henneguy, la tunique musculaire possède une structure différente suivant qu'on la considère dans l'intestin antérieur, dans l'intestin moyen ou dans l'intestin postérieur.

Chose remarquable, chez l'homme et les animaux supérieurs, ce sont toujours, 'comme nous le savons, des fibres musculaires lisses, se contractant lentement et sans l'intervention de la volonté, qui forment, en majeure partie, la tunique moyenne du tube digestif. che? les Insectes, au contraire, cette paroi est entièrement formée de fibres musculaires striées (2).

(1) Certains auteurs réservent le nom de rectum au tube très court qui fait suite au renflement anal; c'est une opinion soutenable; mais ici, avec la majorité des anatomistes, nous clonnons ce nom à la partie terminale élargie et fortenent musculeuse $(r)$ du tube digestif (Fig. 61).

(̊) Quelques auteurs y ont, il est vrai, signalé la présence de fibres lisses; ma:s ces fibres, lorsqu'elles existent, sont toujours en très petit nombre chez l'adulte, et localisées dans l'intestin moyen; tandis que, d'après Mingazzini, elles se rencontrent constamment chez les larves. 
10 ÉPITUÉLIUM DE L'INTESTIN ANTÉRIEUR. - Bien qu'elle soit généralement constituée par une seule assise de cellules, l'épaisseur će la tunique interne diffère notablement suivant les diverses régions du tube digestif. Cette tunique est revêtue d'une cuticule dans toute son étendue, et ce.fait s'explique facilement, puisque le tube digestif résulte de deux invaginations des téguments externes.

$\Lambda$ la limite de l'intestin antérieur et de l'intestin moyen la muqueuse se plisse et s'épaissit fortement; elle-forme ainsi ce qu'on a appelé la salsule osophagienne (1).

Dans certains cas, la valvule œesophagienne s'invagine plus cu moins loin dans l'estomac; elle forme ainsi une sorte de bec flottart ¿iu'Anton Schneider a désigné sous le nom de trompe. Cette disposition a aussi été signalée par Beauregard chez la Cantharide et par Mingazzini chez les larves des Lamellicornes phytophages. Nous donnons ici, d'après Kowalevsky (Fig. 58), un schéma de cette

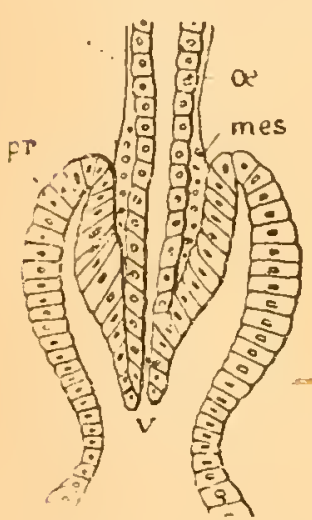

Fig. 58. - Ouverture de la valvule cesophagienne dans le proventricule. (D'aurès KowALEVSKI)。 valvule (๑) pour montrer la part importante qu'elle prend à la constitution du proventricule (gésier).

Entonnoir. - Il ne faut pas confondre la trompe saillante qui termine l'intestin antérieur avec un autre organe très curieux désigné sous le nom d'entonnoir.

L'entonnoir n'est autre chose qu'un repli chitineux, prenant naissance à l'orifice de la valvule œsophagienne (ou de la trompe quand elle existe), et s'étendant dans tout le reste du tube digestif jusqu'à l'anus; ce tube, qui flotte en quelque sorte librement à l'intérieur de la cavité digestive, n'est pas formé par toute la paroi prolongée de l'intestin antérieur, mais uniquement par la couche cuticulaire qui le tapisse; il est donc très mince, et son rôle paraît être de conduire jusqu'à l'anus les particules dures et non digérées des alimenls, afin qu'elles ne puissent, en passant, blesser les cellules épithéliales qui tapissent l'estomac à l'intérieur.

Ce tube chiniteux (entonnoir), s'allonge continuellement; mais comme il se détruit sans cesse à son extrémité postérieure, sa longueur reste invariable; les parties détruites sont expulsées avec les aliments.

(1) Cette expression doit être préférée à celle de valvule cardiaque. 
20 Épithéliun De l'intestix moyen. - La tunique épithéliale de l'intestin moyen présente une particularité intéressante; elle comprend deux espèces de cellules, les unes très grandes, les autres beaucoup plus petites. Les auteurs ont beaucoup discuté sur la signification de ces deux espèces de cellules ; l'opinion la plus généralement admise arjourd'hui, et qui semble, en effet, la plus probable, est que les petites celìules sont des éléments jeunes destinés à remplacer les grandes cellules au fur et à mesure de leur disparition.

Bizzobero a, en effet, constaté, chez l'Hýdrophile, que la membrane épithéliale tombe tous les deux ou trois jours et disparait ensuite par résorption dans l'intestin; c'est alors que les petites cellules se multiplient activement de manière à régénérer l'épithélium caduc (1).

Il convient aussi de noter une particularité très importante de la structure du plateau des cellules épithéliales. Cette particuliarité, signalée tout d'abord par Frenzel en 1885, a été confirmée plis tard par Mingazzini chez les larves de Lamellicornes : ce sont des cils vibratiles rigides, à mouvements très lents, et qui paraissent jouer un rôle important dans la progression des aliments. La présence de ces cils n'a rien qui puisse nous surprendre; c'est là un phénomène d'adaptation comme on en rencontre à chaque instant chez les animaux, et nous ne nous y arrêterions même pas si l'on n'avait toujour's jusqu'ici considéré les Arthropodes comme des orgánismes complètement dépourvus de cils vibratiles. Cette règle, comme on le voit, n'est pas absolue.

3o Épitílium de l'intestix postérifur. - Dans l'intestin postérieñr, l'épithélium présente les mêmes caractères que dans l'intestin antérieur; il n'est formé que d'une seule épaisseur de cellules et porte six replis caractéristiques dont l'existence parait générale chez les Insertes.

\section{II ${ }^{0}$ Organes annexes du tube Digestif}

Aux diverses régions du tube digestif sont annexés un certain nombre d'organes qui jouent un rôle plus ou moins important dans la transformation des aliments ou l'épuration de l'organisme, ces organes sont: les glandes salivaires pour l'intestin antérieur;

(1) Ces petites cellules sont ce véritables histoblastes pour l'épithélium c.e l'intestin moyen (roir 1, 267). 
les cocums gastriques pour l'intestin moyen; les tubes de Malpigh et les glandes anales pour l'intestin postérieur.

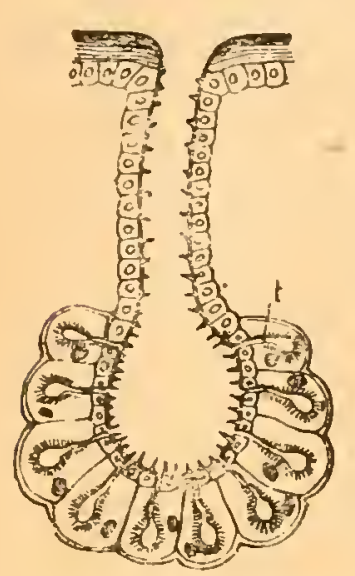

Fig. 59. - Glande acineuse simple; $t$, tube efférest des cellules glandulaires (d'après BERLESE).

10 Glandes salivaires. - Les glandes salivaires n'existent pas chez tous les Insectes; et, sous ce rapport, ce sont les Coléoptères qui paraissent les plus mal partagés; par contre, elles sont très développées chez les Orthoptères ainsi que dans certains autres ordres. La structure de ces organes est égaiement fort variable; on peut observer les dispositions suivantes:

a) De simples cellules isolées, généralement de grande taille, situées, soit dans la paroi de l'œsophage, soit sur les parties membraneuses de la bouche (lères); chacune de ces cellules possède un prolongement servant de canal excréteur (ex. : Coléoptères).

b) Des tubes simples, tapissés intérieurement par des cellules sécrétantes, et pourvus d'un canal excréteur très court (ex.: Psoques).

c) Des glandes acineuses, comprenant un petit nombre de renflements tapissés par des cellules sécrétantes; chacun de cès renflements possède un canal excréteur propre; mais, le plus souvent, tous res canaux se réunissent en un conduit commun (ex. : Coccidés) (Fig. 59).

d) Enfin des glandes en grappes, plus ou moins ramifiées, et de tout point analogues à celles des animaux supé rieurs (ex. : Orthoptères, Hémiptères) (Fig. 60).

Chez certains Insectes, les glandes salivaires présentent des adaptations remarquables; ainsi, par exemple, la soie, dont s'entourent les Chenilles des Lépidoptères nocturnes au moment où elles vont se

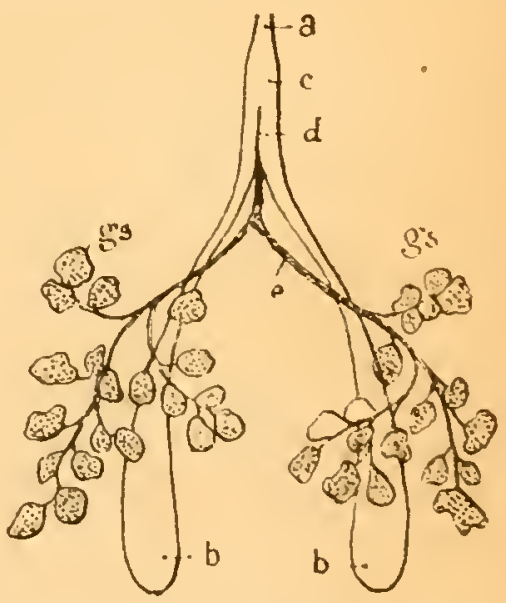

Fig. 60.- Glandes salivaires de la Blatte: $a$, ouverture de la glande; $b b$, réservoirs salivaires; $c$, canal de la glande: 1. conduit excréteirr commun ce la partie acineuse; e. ca. naux excréteurs: $g s$, glandes salivaires acineuses (d'atprès KOII31:). transformer en chrysalides (cocon), est un produit des glandes salivaires modifiées : le venin, qu'un certain nombre d'Ilémiptères (Punaises) et de Diptères (Moustiqucs) dé- 
versent dans la blessure quand ils piquent avec leur trompe, est encore un produit ayant même origine.

2 . Cœcums gastriques. - Les cœcums gastriques (Fig. 61, d.), comme leur nom l'indique, sont de petits tubes areugles ayant l'aspect de papilles ou de villosités ; ils recouvrent toute lasurface extérieure du ventricule chylifiquo chez les Coléoptères carnassiers et c'est à la présence de ces petits tubes que cet organe doit son aspect velouté. La structure de ces tubes est très simple; ils ne sont formés que par des éraginations de la muqueuse gastrique; ils traversent done purement et simplement la tunique musculaire, qui ne prend aucune part à leur constitution. D'après Léon Dufour, l'illustre anatomiste landais, les cœecums gastriques serviraient à faire passer les aliments digérés et lirquides, le chyle en un mot, de l'intérieur du tube digestif dans la cavitégénérale du corps où se ferait ensuite directement le mélange avec le sang. Ainsi compris, les cœcums gastriques seraient de véritables organes d'absorption analogues aux villosités intestinales (Fig. 61).

Il est d'ailleurs probable que

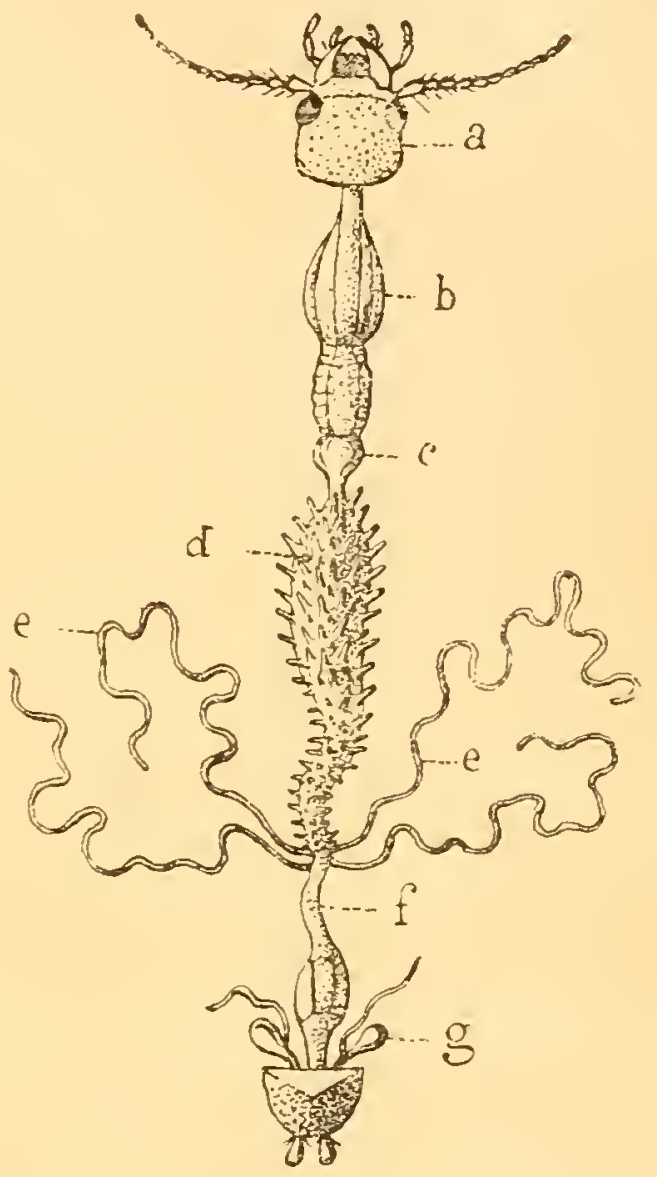

Fig. 61. - Appareil digestif c’un Coléoptère (Zabrus gibbus), pour mon. trer les coecums gastriques; $a$, tête; $b$, jabot; $c$, gésier: $d$, ventricule chylifique arec les cœecums gastriques; $e$, tubes de Malpighé : $f$, intestin $g$, glandes anales (d'après L. Dufour). leur rôle est double, car on retrouve, dans leurs parois, les grandes cellules glandulaires dont nous arons parlé précédemment (p. 106).

Il serait désirable que des recherches lussent entreprises en vile d'établir le rôle exact des villosités gastriques. Burmeister, Hope Seyler et Plateau admettent que le liquide sécrété possède des propriétés analogues au suc pancréatique des Vertébrés, ce qui explique pourquoi il est capable d'émulsionner les graisses et de saccharifier l'amidon. 
Chose remarquable, ces organes n'ont été rencontrés jusqu'ici que chez les Coléoptères, car on ne peut vraiment pas leur comparer les gros tubes aveugles que l'on observe à l'entrée de l'intestin moyen chez les Orthoptères, et autour desquels la couche musculaire est continue (Fig. 55 . cœ.).

$3^{\text {n }}$ Tubes de Malpighi. - On désigne sous ce nom, en mémoire du célèbre anatomiste italien qui les a le premier découverts, des canaıx filiformes, très allongés et très flexuenx, naissant généralement au point de jonction de l'intestin moyen et le l'intestin postérieur. Ces tubes flottent dans la cavité générale ou rampent à la suriace du ventricule chylifique; leur diamètre est généralement le même dans toute leur étendue ; ils s'ouvrent, à leur base, dans l'intestin, mais ils sont fermés à leur extrémité libre (Fig. 61. e.).

Le nombre des tubes de Malpighi est très variable (1) ; ils manquent complètement chez un certain nombre de Collemboles; mais, à part cette exception, on les rencontre chez tous les autres Insectes.

On en trouve deux chez les Hémiptères et chez un bon nombre de Lépidoptères; quatre chez les Diptères et les Siphonaptères; de quatre à six, jamais plus, chez les Coléoptères; de six à huit chez la plupart des Névroptères; de cinquante à soixante chez les Odonat :

les Perlides et les Éphémères; plus de cent chez les Orthoptères ef lo; Hyménoptères.

Si le nombre des tubes de Malpighi est, comme on le voit, parfois tr: grand chez les Insectes adultes, il n'est, par contre, janais supérieur à six chez. les larves.

Structure des tubes de Malpighi. - La structure des tubes de Malpighi diffère notablement de celle des crecums gastriqucs. Résultרnt d'invaginations latérales du proctodeum, ils sont, en général, Cormés d'une couche épithéliale très nince revêtue d'une membiane caitineuse à son intérieur (Fig. 62) ; à l'extérieur, on n'observe pas denveloppe musculaire complète, mais senlement un réseau très compliqué de fibres contractiles, ce qui explique les mouvements varniculaires qu'on a signalés dans certaines espèces.

Fonctions des tubes de Malpighi. - Les tubes de Malpighi sont prob iblement les seuls organes d'excrétion des Insectes, mais lear' rôle a lé le sujet de nombreuses discussions.

Los anciens auteurs, considérant qu'ils débouchaient fréquemment

(1) Sauf quel(ques exceptions très rares (Culicides et P'sychola), ce nombro est t)ujours deux ou un multiple de deux. 
dans l'intestin, un peu au-dessous du ventricule chylifique, comme le canal cholédoque des Vertébrés supérieurs, leur avaient, vraisemblablement sans autre motif, attribué des fonctions analoguns à celles du foie. Cependant, dès 1810, Robiquet avait signalé la présence de l'acide urique dans ces canaux, chez les Cantharides; mais ce fut Rengger (1817) qui, à la suite des travaux de Brongnatelli, suggéra le premier l'idée que ces organes remplissaient plutôt une fonction d'excrétion analogue à la fonction urinaire ; cette manière de voir fut adoptée en 1836 par Audouin, qui découvrit de véritables calculs dans les canaux du Lucanus capreolus.

Il est démontré aujourd'hui que la fonction hépatique des tubes de Malpighi était une pure hypothèse; ces canaux sont des organes urinaires et rien de plus.

En concordance avec la manière de voir des auteurs qui considèrent les tubes de Malpighi comme des organes segmentaires modifiés, Brauer a divisé les Insectes en oligonéphridés et polynéphridés (1). Les oligonéphridés, où le nombre des tubes varie de deuxà vingt, comprennənt les Névroptères, les Lépidoptères, les Diptères et les Colénptères; aux polynéhpridés (nombre des tubes supérieur à 20) se rattachent les Hyménoptères et les

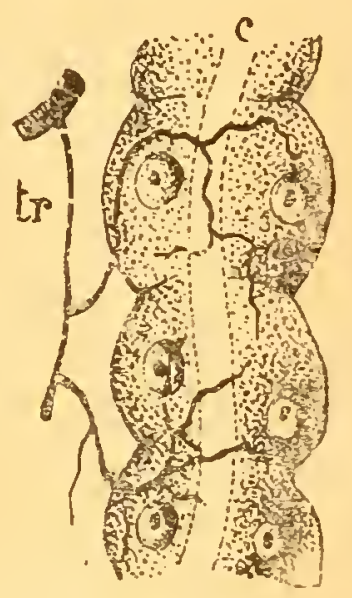

Fig. 62. - Structure d'un tube r'e Malpighi ; $c$, canal intérieur; $t r$, trachées (d'ap. GeGENBATr). Orthoptères.

Développement des tubes de Malpighi. - D'après Grassi, l'origine des tubes de Malpighi serait des plus curieuses. Leur apparition est très précoce et ils se forment par des invaginations de l'ectoderme en des points où se trouvent de petites fossettes, placées symétriquement, sur les deux ou trois derniers anneaux de l'abdomen.

C'est précisément aussi, à cette même époque de la vie de la larve que le proctodeum prend naissance; en s'avançant vers ''intérieur du corps, ce canal entraîne les derniers segments abdominaux, rui rentrent, en quelque sort? par tólescopage, dans les précédents; les tubes partent donc dı proctodeum au lieu de vesir s'y terminer, comme on le croyait autreínis. Cria explique poureugi les tubes de Malpighi se trouvent toujours fixés à la limite du proctorleum et de

(1) Brauer (F). - Systematisch. zsol. Siudien. s. B. Akad. Wien. Bd: XCI. 1885. 
l'intestin moyen, bien qu'ils aient pris naissance à la partie postérieure du corps.

Les tubes de Malpighi se forment par le même- mécanisme que les trachées; et sil'on remarque, que chez les oligonéphridés tout au moins, leur nombre est toujours égal à celui des derniers anneaux abdominaux privés de stigmates, on arrive facilement à admettre qu'ils ne représentent autre chose que des trachées détournées de leurs fonctions et adaptés à l'épuration de l'organisme ; cela explique aussi pourquoi les tubes de Malpighi sont toujours tapissés à leur intérieur par une cuticule chitineuse.

4. Glandes anales. - On désigne sous ce nom des organes de défense de forme variable qu'on rencontre surtout clız les Coléop. tères, et qui viennent déboucher près de l'anus, tout à fait à la partie postérieure de l'intestin. C.es glandes ressemblent tout il fait aux glandes salivaires et présentent les mêmes variations.

Le liquide sécrété par ces glandes est projeté au dehors, à volonté par l'Insecte; comme il a généralement une odeur repoussante et qu'il peut être très corrosif, on comprend sans peine l'efficacité de son rôle défensif. (Pour plus de détails, voir le volume de l'L.S. consacré aux Coléoptères.)

\section{III ${ }^{\circ}$ Physiologie de la digestion}

Bien que, dans leur ensemble, les phénomènes physiologiques soient les mêmes que chez les animaux supérieurs, il ne faudrait pas croire cependant que les différentes parties du tube digestif des Insectes se comportent comme celles qui portent les mêmes noms ches les animaux vertébrés; il est à peine nécessaire de faire remalquer que la similitude de nom n'implique nullement la similitude de fonctions. On a donné aux diverses parties du tube digestif des Insectes les mêmes noms que chez les Oiseaux, pour ne pas créer des noms nouveaux, mais la structure et la fonction peuvent différer — et diffèrent généralement beaucoup.

Malgré les nombreuses recherches des auteurs, la physiologie de la digestion est encore peu connue chez les Insectes. Tous les liquides sécrétés par le tube digestif sont neutres ou à réaction faiblement alcaline, excepté, paraît-il, dans la partie postérieure de l'intestin moyen. Bien qu'on n'ait pu encore en isoler aucun, ces liquides doivent agir comme de coutume, grâce aux diastases qu'ils renferment.

10 Digestion salivaire. - Les aliments subissent l'action de la 
salive dans la bouche, dans l'œsophage et probablement aussi dans le jabot, où ils ne séjournent que peu de temps; ils arrivent ainsi rapidement dans l'estomac en franchissant le gésier. C.et organe (gésier) ne broye pas les aliments, mais, grâce aux valvules chitineuses qu'il renferme, il les tamise en quelque sorte de façon à n'admettre, dans l'estomac, qu'une pulpe tellement fine, qu'il est déjà presque impossible de distinguer la nature des substances alimentaires consommées.

D'après Rengger, la salive est un fluide aqueux, incolore ou légèrement opalescent, à réaction alcaline.

Les organes qui la sécrètent (glandes salivaires) n'existent, parmi les Insectes broyeurs, que chez ceux qui sont plus ou moins phytophages; ils manquent complètement dans les espèces carnivores. Chez ces dernières, la salive parait remplacée par le suc des glandes esophagiennes et celles du jabot.

Comme chez les animaux supérieurs, la salive agit sur les substances amylacées pour les iransformer en dextrine, puis en glucose assimilable.

20 Digestion stomacale. - Las expériences de Hope Seyler, de Krukenberg et de Plateau, ont établi que la transformation a surtout i'eu dans le ventricule chylifique sous l'action d'un liquide sécrété par l s cocums gastriques. Ce liquide, par son action, se rapprocherait du suc pancréatique, c'est-à-dire qu'il serait à la fois, hydrolisant pour' les substances amylacées et peptonisant pour les substances albuminoïdes.

Absorption. - Il n'existe pas d'appareil absorbant spécialisé, à moins que l'estomac, aver ses cocums gastriques, ne remplisse un double rôle ; c'était là l'opinion de Strauss et de Ramdhor ; il est, én effet, très probable que les substances alimentaires, au fur et à inesure qu'elles sont digérées, passent directement, au travers de la paroi de l'estomac, dans la cavité générale, où elles se mélangent directement au sang.

3o Digestion intestinale. - On ne sait que fort peu de chose sur la nature des phénomènes physiologiques dont l'intestin est le sièg'e. Dans certains groupes, au moins, il semble bien que l'absorption se continue et qu'elle ne se fait pas exclusivement au nireau du ventricule chylifique. 


\section{CHAPITRE IX}

\section{APPAREIL GIRCULATOIRE ET CIRCULATION}

Teснарue. - L'Insecte qui permit à Carus de découvrir la circulation du sang était la larve transparente de l'Agrion puella; mais toutes les larves des Agrionides et des Libellulides peuvent servir. On trouve ces larves, dans les fossés d'eaux claires, au printemps.

On peut, d'une manière générale, utiliser toutes les larves ; mais, les fausses chenilles des Tenthrèdes, à peau nue et transparente, sont très avantageuses.

Le vaisseau dorsal se distingue facilement par les fluctuations auxquelles il est continuellement soumis; le liquide, contenu dans son intérieur, semble poussé par des mouvements onduleux de la partie postérieure du corps vers la tête.

Historique de la circulation. - On a cru, jusqu'au siècle dernier, que le sang des Insectes était immobile et qu'il baignait simplement les organes à l'intérieur du corps. Bien que Malpighi eût décrit depuis longtemps le vaisseau dorsal de la chenille du Ver à soie, et qu'il ait regardé cet’organe comme un véritable cœur, ce fut en 1826 seulement, au congrès de Dresde, que Carus démontra l'existence d'une véritable circulation du sang chez les Insectes. Deux ans plus tard, Strauss-Durkheim fit connaître la structure valvulaire du cœur et ses propriétés contractiles.

I'ar suite d'un entêtement bizarre, de grands anatomistes, comme Cuvier et Léon Dufour, se refusèrent toujours à admettre la possibilité d'une circulation chez les Insectes; Marcel de Serres ne voyait autre chose, dans le vaisseau dorsal, que l'organe sécréteur du corps adipeux.

\section{I. - APPAREIL CIRCULATOIRE}

L'appareil cuntral de la circulation chez les Insectes est une sorte de muscle creux, en formo de tube allongé, s'étendant, comme un cordon, dans la région supé.. 
rieure du corps, d'où son nom de vaissean dorsal (Fig. 63, vd.). Le plus souvent, ce tube est légèrement renflé dans sa partie médiane (cœur, str. s) et rétréci à ses deux extrémités; la partie antérieure se prolonge assez loin du côté de la tête, sous forme d'un tube frrèle, que l'on désigne sous le nom d'aorte; l'aorte est presque toujours simple, mais elle peut cependant se bifurquer en deux courtes branches rappelant ainsi, quoique de très loin, la disposition que l'on rencontre chez les Crustacés.

10 Vaisseau dorsal. Le vaisseau dor'sal est placé presque immédiatement au-dessus du tube digestif, dont il n'est séparé que par une très mince couche de tissu

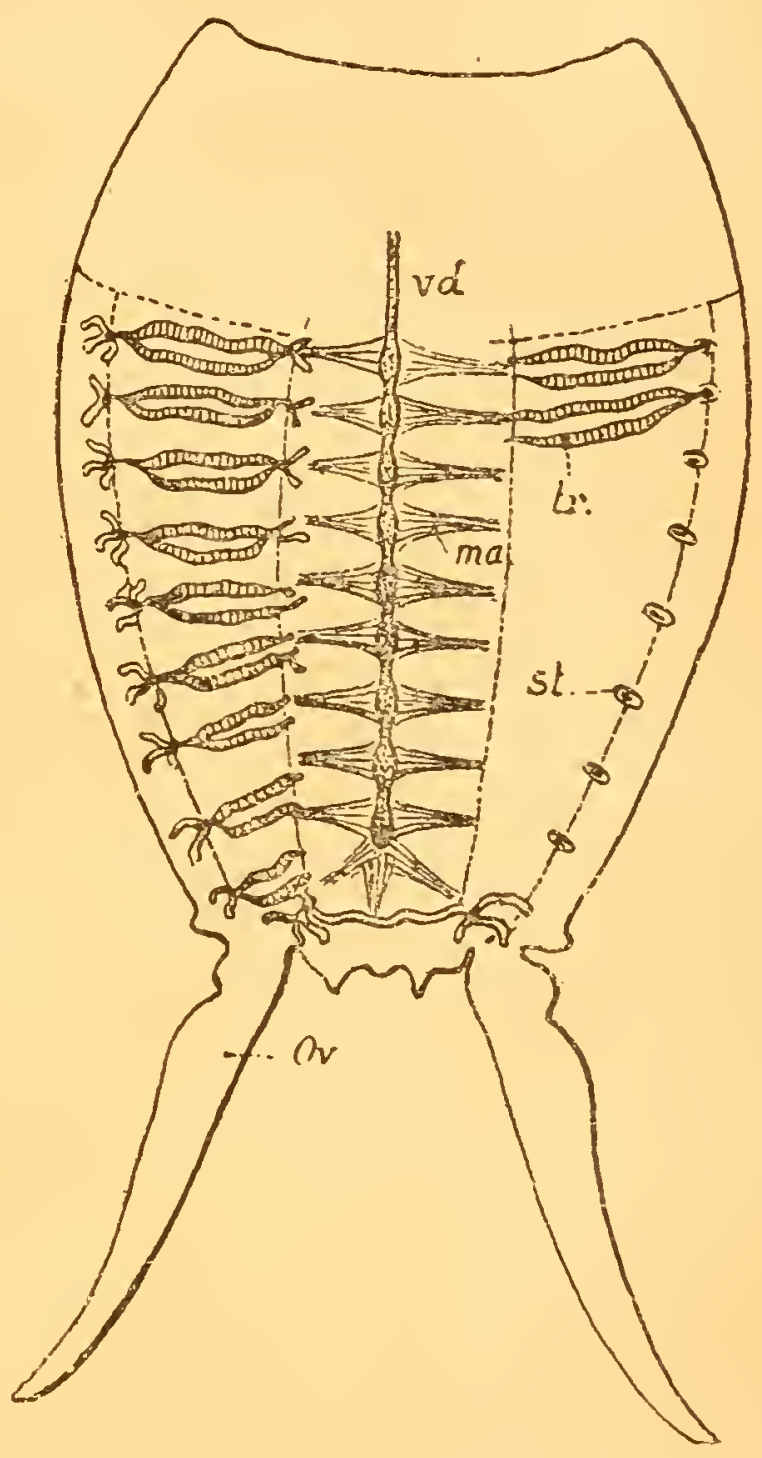

Fig. 63. - Toyographie générale des appareils circulatoire et respiratoire chez la Sautertle verte; $r d$, vaisseau dorsal; $m a$, muscles aliformes; $t r$, trachées fasciculeuses ; st, stigmates; ov, oviscapte. conjonctif (Fig. 64).

Le corps des Insectes étant, comme on le sait, divisé en trois régions par de très forts étranglements, le vaisseau dorsal suit forcément la courbure des téguments. 
Arrivé à la partie antérieure de l'abdomen, on le voit s'infléchir subitement vers le bas pour franchir l'articulation thoraco-abdominale; il s'abaisse une seconde fois pour passer du thorax dans la tête. En un mot, le

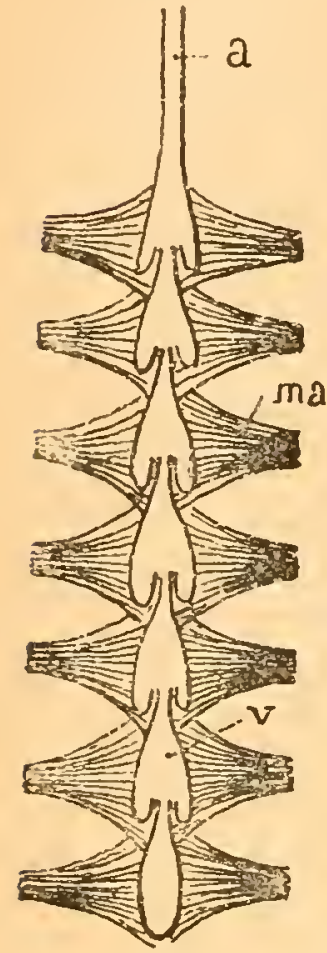

Fig. 64. - Vais seau dorsal ilu Hanneton reposant sur son plancher musculaire : $r$. ventriculites; ma, muscles aliformes ; $a$. aorte. trajet du vaisseau dorsal est toujours en rapport avec la forme générale du corps.

CEuR. - I I Il convient de réserver ce nom à la partie du vaisseau dorsal située dans l'abdomen; cette partie est, en effet, seule contractile; elle est divisée, par des étranglements, en chambre successives (ventriculites), chacune correspondant sensiblement à un anneau. Le tube cardiaque est fermé en arrière, et suspendu en quelque sorte au milieu d'une grande lacune sanguine, qu'on désigne sous le nom de péricarde; sa division en chambres séparées estréalisée par des replis internes de la paroi musculaire tout entière (Fig.64.); ces replis forment des valvules et sont disposés de telle manière que le liquide sanguin peut facilement passer d'une chambre à l'autre mais ne peut refluer en arrière; le sang est donc continuellement chassé vers l'avant; finalement, il vient sortir par l'orifice antérieur de l'aorte, pour se répandre librement dans toutes les lacunes de l'organisme. Chaque ventriculite possède de plus, à sa partie postérieure, deux petits orifices valvulaires (ostioles) qui le mettent en communication arec la lacune péricardique et qui permettent au sang de repénétrer dans son intérieur (Fig. 65). Le nombre des chambres cardiaques (ventriculites) est variable 
$(7,8,9$ et mème quelquefois 11$)$; mais les renseignements que nous possédons sur cette question sont trop peu nombreux pour décider si les différents groupes d'Insectes sont caractérisés sous ce rapport ou s'il existe des variations irrégulières. En ce qui nous concerne, nous avons constamment trouvé neuf ventriculites chez les Locustides (Orthoptères), que nous avons étudiés.

$2^{\circ}$ Le cour n'est qu'une
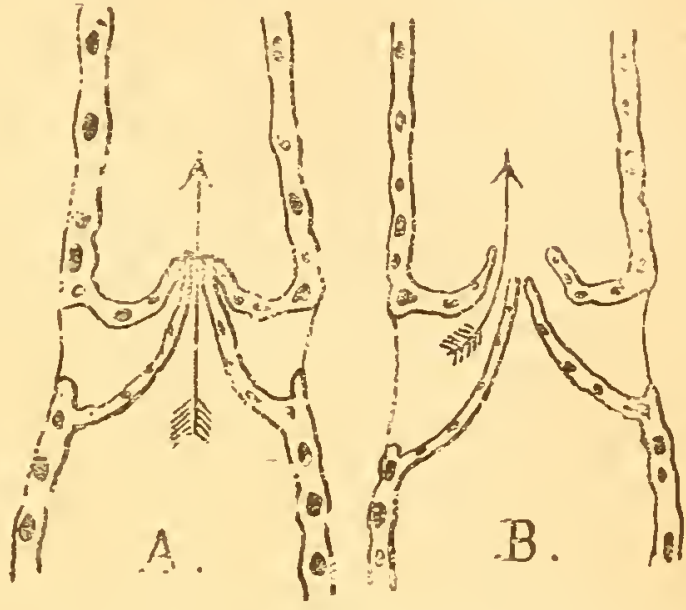

Fig. 65. - Mode de communication des ventriculites. $A$, pendant la systole ; $B$, pendant la diastole

(d'après CH. JANET). partie de l'appareil pro-

pulseur du sang; il faut encore tenir compte de la disposition suivante, dont le fonctionnement a été expliqué par Graber.

A la face inférieure du vaisseau dorsal, il existe un plancher de muscles triangulaires (ailes du coeur) à raison d'une paire pour chaque ventriculite; l'extrémité libre de ces faisceaux musculaires est très effilée et va s'attacher, d'autre part, à la paroi solide du corps. L'ensemble de ces muscles aliformes constitue une sorte de diaphragme qui limite incomplètement, en dessous, la grande lacune (péricarde) dont nous avons parlé ; lorsque ces muscles se contractent le diaphragme s'abaisse (Fig. 66), la cavité péricardique s'agrandit et le sang, provenant des lacunes voisines, passe dans son intérieur ; lorsqu'ils reviennent, au contraire, à la position de repos, le sang, comprimé dans un espace étroit, pénètre dans les compartiments du cœur et se trouve lancé dans l'aorte. 
Aorte. - L'appareil circulatoire des Insectes est certainement plus compliqué qu'on ne le décrit habi-

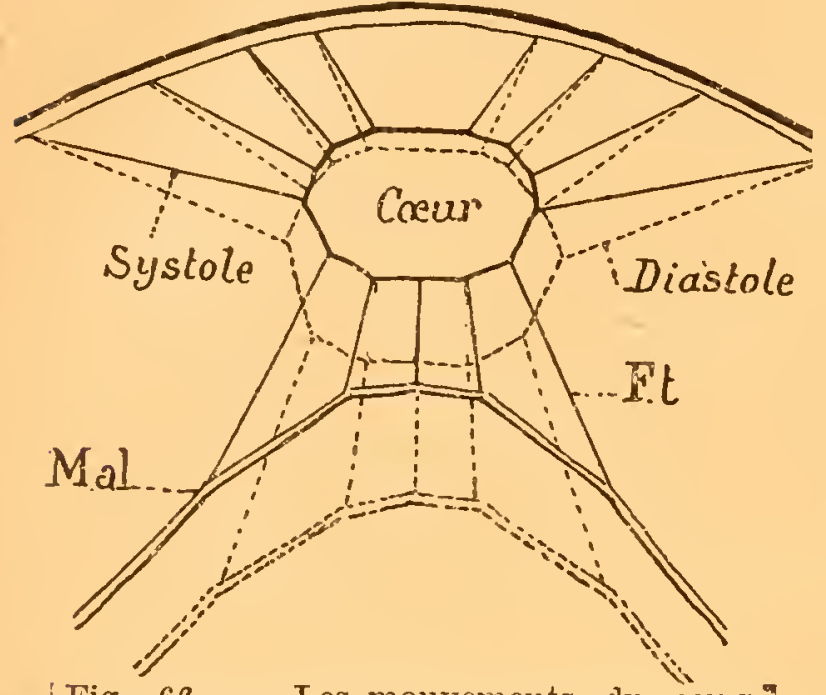

Fig. 66. - Les mouvements du cour.? 11. al muscles aliformes; $F t$. filaments tenseurs (d'après CH. JANET). tuellement; et, à notre avis, il y aurait lieu de reprendre complètement cette étude en bien des points.

Ainsi, par exemple, d'après Newport, chez Vanessa urticœ, et probablement chez tous les Lepidoptères, l'aorte, que l'on décrit généralement comme simple, se ramifie tout d'abord en deux grosses branches qui se répandent dans les deux côtés de la tête; chacune de ces branches se divise à son tour en trois rameaux qui se recourbent en arrière, mais qu'il est très difficile de suivre plus loin, en raison de leur extrême ténuité. Même avant le départ de ces deux gros trones, on en aperçoit deux plus petits qui paraissent se rendre aux parties de la bouche et aux antennes. Lorsqu'clle se ramifie, l'aorte ne se divise jamais qu'à son extrémité; aucune branche latérale ne naît sur ses côtés.

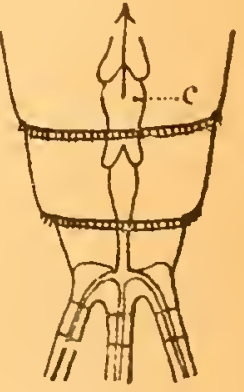

Fig. 67. $V$ a is s e a $x$ sanguins péné trant dans les appendices $\mathbf{a b}$ dominaux.

De véritables vaisseaux sanguins paraissent encore exister dans l'appendice caudal des Ephémérides (Cloëon dipterum) (Fig. 67).

Les Insectes ne sont donc pas entièrement dépourvus de vaisseaux sanguins, comme on l'a cru jusqu'ici. 
$2^{\circ}$ Mécanisme de la circulation. -.. Les contractions du vaisseau dorsal ne me paraissent pas suffisantes pour expliquer le mouvement du sang à l'intérieur du corps des Insectes; mais il existe encore, chez un certain nombre d'espèces, peut-être chez toutes, une grande lacune ventrale agissant sur le sang à la façon d'un aspirateur

Sinus ventral. - Cette lacune, que l'on peut considérer comme un sinus ventral, est placée au-dessus de la chaîne nerveuse et fermée, en dessus, par un diaphragme, semblable à celui qui forme le plancher du péricarde.

Cette disposition curieuse, découverte tout d'abord par Réaumur chez la Mouche commune, fut ensuite retrouvée par Graber chez les Libellules et les Locustides. Au moment de la contraction, la membrane se soulève et le sang afflue dans la lacune ventrale. Les deux sinus (péricarde et sinus ventral), par suite de leurs mouvements concordants, impriment donc au sang un véritable mouvement circulaire ; cependant nous ne sommes pas en présence d'un. appareil circulatoire fermé, car il n'existe pas de vaisseaux complets (veines) pour ramener le sang vers le cœur; ce sont les mouvements du corps et les contractions des organes, qui repoussent ce liquide vers la cavité péricardique.

Organes pulsatiles des jambes. - Les Hémiptères du groupe des Népides présentent une particularité qui jusqu'ici, à ma connaissance du moins, n'a encor zété signalée chez auc ın autra Insecte.

On observe, en effet, dans les tibias des trois paires de pattes, immédiatement au-dessous de leur articulation avec le fémur, un système d'organes pulsatiles très curieux. Ces organes, qui ont été observés pour la première fois par Behn en 1835, ont la forme de fuspaux allongés (whip-like), ils sont rattachés aux téguments de la patte par de longues fibres prolongeant leurs extrémités; leur 
rôle paraît être d'aider la circulation, qui doit être, en effet, très gêr.ée aux articulations : ce soni donc des sortes de cœurs en relais, spécialement affectés à la nutrition des pattes.

Exceptionnellement, ches les Ranatres, l'organe pulsatile des jambes antérieures est situé à l'origine de l'insertion du tarse, tout près de son articulation avec le tibia.

\section{II, - LE SANG DES INSECTES}

Le sang des Insectes est un liquide incolore ou très légèrement teinté, qui remplit, dans la cavité générale, tous les espaces non occupés par les organes; ainsi, par exemple, le tube digestif est complètement entouré par le sang.

On peut suivre facilement la marche du sang chez les

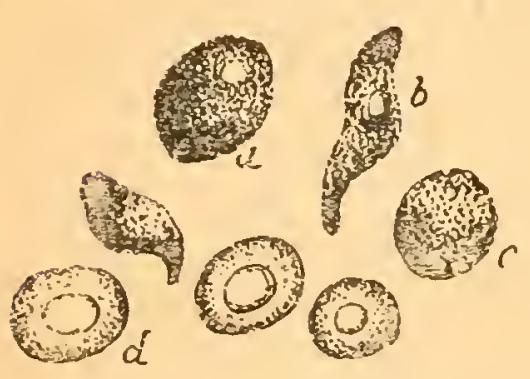

Fig. 68. - Eléments figurés du sang Ces Insectes. $a$. $b . c$, corpuscules sanguins; $d$. leucocyte: (l'après GraBer). Insectes dont le corps est transparent (larves d'Ephémérides). Ce qui est remarquable, c'est que le sang hématosé baigne immédiatement le cerveau à sa sortie de l'aorte; cet organe reçoit donc du sang très oxygéné.

Dans ce liquide incolore, qui est en réalité un mélange de sang et de chyle, on trouve des corpuscules amiboïdes qui correspondent aux globules blancs (leucocytes) des Vertébrés (Fig. 68). En somme, le sang des Insectes, comme celui de tous les animaux, est formé de deux parties :

10 Une partie liquide, le plasma.

$2^{\circ}$ Une partie solide, les globules, en suspension.

Flasma. - Lorsque le sang des Insectes est coloré, il doit sa coloration à une substance spéciale en dissolution dans le plasma. On ne connaît pas la composition exacte du plasma; en l'absence de tout renseignement, on peut. 
admettre qu'il se comporte comme la lymphe des Vertébrés.

D'après Cuénot, le sang des Méloë renferme, en plus des córpuscules amiboïdes, un abondant fibrinogène pouvant former un caillot; on y trouve, de plus, un pigment (uranidine), qui s'oxyde à l'air et se précipite ; une substance albuminoïde (hæmoxanthine), qui remplit à la fois, comme l'hémoglobine, les fonctions nutritives et les fonctions respiratoires; et, finalement, de la cant'zaridine dissoute.

Le plasma du sang peut quelquefois servir de liquide défensif, s'il contient, en dissolution, des substances âcres ou toxiques; c'est ainsi, par exemple que, d'après Leydig, le liquide jaunâtre qui suinte par les articulations des pattes de certains Insectes (Coccinella, Timarcha, les Méloïdes), n'est autre chose que du plasma sanguin (Fig. 69).

Leucocytes. -.. Les leucocytes sont généralement allongés, ovales ou apla- femoro-tibiale chez Zonatis, et nucléés; ils présentent souvent (d’après Hollante). des propriétés amiboïdes (Fig. 68).

Le nombre des globules varie avec le stade du développement, mais, c'est au début de la nymphose qu'ils paraissent être le plus nombreux; vers la fin de la période nymphale, le sang diminue en quantité ; il est cacore moins abondant à l'état adulte. Commo on le voit, la quantité de sang est en rapport avec l'activité ds la nutrition. 


\section{CHAPITRE $\mathrm{X}$}

\section{LA RESPIRATION}

Techique. - Observer les stigmates sur les côtés du corps d'une Chenille nue (Piéride du Chou) ou sur la larve du Hanneton(Verblanc).

Pour les adultes, choisir le Hanneton, l'Hydrophile, la grande Sauterelle r'erte, etc.

L'étude des branchies trachéennes se fera facilement avec les larves d'Ephémérides, qu'on trouve, rampant sur la vase, au fond des rivières et des ruisseaux où l'eau est limpide et bien aérée.

Historique de la respiration. - Bien qu'il eut le premier proclamé cette vérité fondamentale que "l'air est indispensable à la vie comme à la flamme ", Aristote enseignait que les Insectes ne respiraient pas, et son opinion prévalut pendant dix-huit siècles.

Ce fut Malpighi qui, en 1669, découvrit les organes respiratoires chez le Bombyx du mûrier; il démontra aussi que l'air estabsoliment nécessaire à la vie; et que, sous ce rapport, les Insectes se comportent comme tous les êtres vivants.

\section{E I'APPAREIL RESPIRATOIRE}

L'appareil respiratoire des Insectes se compose de canaux très fins, situés à l'intérieur du corps et ramifiés à l'infini. Ces canaux, désignés sous le nom de trachées, se. distribuent dans toute l'étendue de l'organisme : à l'intérieur des membres, autour des viscères, dans les nervures des ailes; ils pénètrent mème dans la profondeur des organes, pour apporter aux cellules l'oxygène dont elles ont besoin. 
L'arbre trachéen communique avec l'extérieur de différentes manières, suivant que les Insectes se sont adaptés à la vie aérienne ou à la vie aquatique.

Dans le premier cas - le plus fréquent - l'air pénètre à l'intérieur des trachées par de petits orifices appelés stigmates (1), disposés métamériquement, de chaque côté du corps, l'un à droite, l'autre à gauche, sur chacun des anneaux de l'abdomen et même sur ceux du thorax (Fig. 70).

Dans le deuxième cas, on observe plusieurs dispositions intéressantes à signaler, suivant que l'adaptation au milieu aquatique est plus ou moins complète.

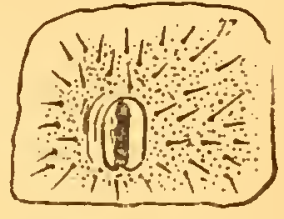

Fig. 70. - Un stigmate isolé du Dectique verrucivore (fig. orig.)

Ainsi par exemple, chez les Dytiques, Coléoptères plutôt amphibies que véritablement aquatiques, les stigmates s'ouvrent normalement snus les ailes, dans la position habituelle; lorsque ces Insectes veulent respirer, ils montent à la surface de l'eau, et là, on les voit, soulevant légèrement l'extrémité postérieure de leurs élytres, faire une nouvelle provision d'air pur. De cette façon l'air arrive dans les trachées sans que l'eau ellemême puisse y pénétrer.

Chez les Hydrophiles, autres gros Coléoptères aquatiques, les stigmates thoraciques et abdominaux se ferment; seuls, les deux derniers stigmates, situés à l'extrémité de l'abdomen, restent ouverts et continuent à fonctionner. C'est pourquoi on peut voir, périodiquement, les Hydrophiles venir puiser l'air à la surface de l'eau, en se plaçant la tête en bas, de manière à faire émerger l'extrémité de leur abdomen.

(1) Ce sont les spiracles des auteurs anzlais. 
Chez certains Hémiptères aquatiques (Nèpes, Ranâtres), l'adaptation au milieu aquatique est plus prononcée; ici encore tous les stigmates abdominaux sont oblitérés, à l'exception des deux derniers. Ces deux stigmates s'ouvrent au fond d'un tube allongé, formé luimême de deux moitiés creusées en gouttières. Lorsque les Nèpes veulent respirer, elles grimpent le long des plantes aquatiques, et émergent, pendant un instant, leur tube respiratoire hors de l'eau ; ayant alors renouvelé leur provision d'air, elles regagnent promptement la vase où elles vivent.

Enfin, lorsque l'adaptation à la vie aquatique est

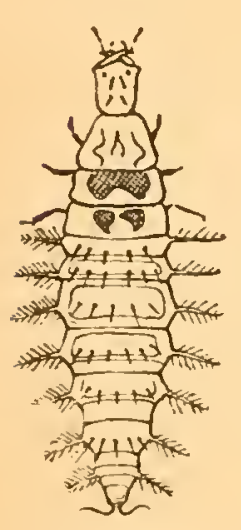

Fig. 71.

Larve de l'Hy drous caraboï. des (Col.) avec ses branchies abdominales (d'après ScHIODDTE). complète, ce qui est le cas pour un certain nombre de larves de Névroptères, les stigmates ont complètement disparu ; mais alors on trouve, sur les côtés du corps, des expansions variées : lamelles membraneuses ou filaments ciliés, au travers desquels s'effectuent les échanges gazeux; on a donné à ces organes, quelle que soit leur forme d'ailleurs, le nom de branchies trachéennes.

Ainsi done, partout, dans les Insectes, à l'inverse de ce qui se passe chez les animaux supérieurs, c'est l'air qui va chercher le sang pour lui céder l'oxygène dont il a besoin.

L'ordre des Coléoptères offre peu d'exemples de branchies trachéennes; on ne peut guère citer que les larves des Gyrinides et celles de l'Hydrophilus caraboides (Fig. 71) ; ces organes sont, au contraire, très répandus parmi les larves des Névroptères (Ephémérides, Phryganides, Sembiides, etc. ).

Les larves d'un grand nombre de Diptères sont égale- 
ment pourvues de branchies trachéennes, ex. : Chironomus ; cet ordre est aussi le seul où ces organes s'observent chez les nymphes. Enfin, dans l'ordre des Lépidoptères, on ne connaît qu'un seul exemple de branchies, il est fourni par la larve de l'Hydrocampa stratiotalis, qui vit sur les feuilles des plantes aquatiques dans les eaux stagnantes.

\section{IO INSECTES AÉRIENS}

Techninue. - Étaler dans une goutte d'eau, sur une lame de verre (recouvrir d'une lamelle), quelques fragments des viscères pris à l'intérieur du corps d'un Insecte. Examiner au microscope, bien entendu.

On aperçoit généralement de nombreuses trachées, reconnaissables à leur aspect brillant et au filament spiralé qui soutient leurs parois.

1. Trachées. - Les trachées sont, comme on le sait, des tubes ramifiés à l'infini et destinés à conduire l'air dans toutes les parties du corps; les orifices qui font communiquer les trachées avec l'extérieur ont reçu le nom de stigmates.

D'après le mode de ramification des trachées, on peut distingue trois types principaux dans l'appareil respiratoire des Insectes, ce sont:

$1^{\circ}$ Les trachées fasciculeuses.

$2^{\circ}$ Les trachées tubuleuses.

$3^{\circ}$ Les trachées vésiculenses.

A. Trachées fasciculeuses. - Dans ce type, en quelque sorte primitif, que l'on peut observer chez les Mléloïdes, on voit partir, de chaque stigmate, un petit tronc trachéen qui se divise en un grand nombre de rameaux; ces rameaux se distribuent dans les organes. On a donc ainsi une sorte d'arbuscule aérifère, complètement indépendant des arbuscules voisins. Ici, la distribution de l'air est notoirement métamérique comme chez les Myriapodes.

B. Trachées turuieuses. - Les trachées tubuleuses, comme leur nom l'indique, sont cylindriques; elles prennent aussi leur origine à l'orifice de chaque stigmate par un gros tronc qui se bifurque presque aussitôt. Les deux branches ainsi formées, branche anłé- 
rieure et branche postérieure, vont se réunir à des branches semblables issues des troncs voisins; il en résulte que tous les stigmates. des deux côtés du corps, sont reliés les uns aux autres, par deux canaux longitudinaux s'étendant d'une extrémité à l'autre de l'abdomen. On peut même observer, dans un certain nombre de cas, notamment chez Mélolon.ha vulgaris, et probablement aussi chez. un grand nombre de Lamellicornes, que certaines branches trachéennes, d'assez fort calibre, appartenant aux six derniers anneaux de l'abdomen, partent de ces troncs longitudinaux, et, se dirigeant transversalement, vont se réunir, sur la ligne médiane du corps, à leurs correspondantes du côté opposé. Il résulte de cette disposition - indépendamment bien entendu des autres complications qui peuvent se présenter - que les trachées du côté droit du corps sont en communication avec celles du côté opposé. C'est là un perfectionnement qui a certainement pour but une répartition plus parfaite de l'air dans toute l'étendue de l'organisme.

C. Trachéf.s vésiculeuses. - Les trachées vésiculeuses ne sont, à vrai dire, qu'une modification du type précédent, particulières aux Insectes bons voiliers. Elles sont caractérisées par l'existence de renflements, véritables sacs aériens, de volume variable, disposés sur le trajet des trạchées tubuleuses.

On peut observer ces sacs aériens chez le Hanneton, les Cétoines, les Géotrupes; l'examen de la Fig. 72, mieux qu'un long texte, pourra donner une idée de la disposition de ces vésicules.

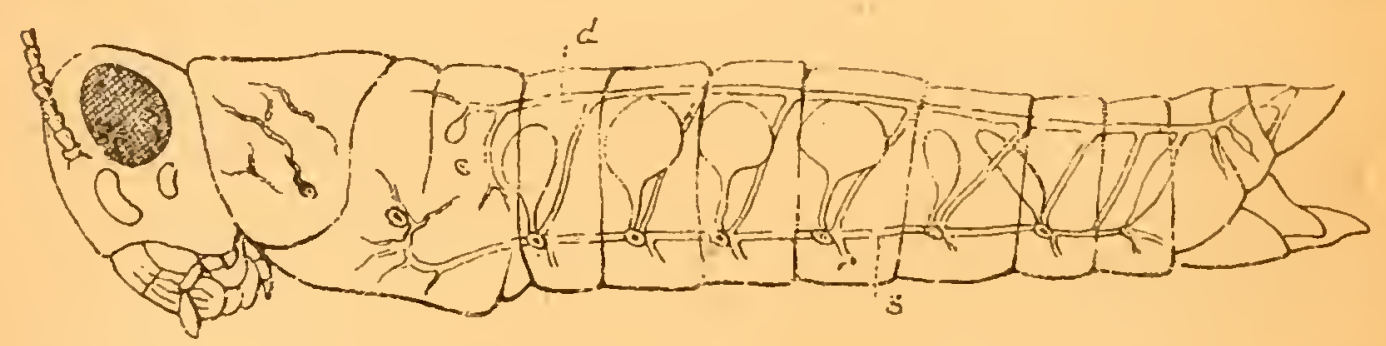

Fig. 72. - Distribution des trones trachéens et des sacs à air dans le corps du Melanoplus femur-rubrum. Orthopt. (d'après PACKARD).

20 Structure des trachées. - Lastructure anatomique des trachées paraît identique chez tous les Insectes : ainsi que Bütschli l'a démontré en 1870; leurs parois sont constituées, comme celles des téguments, dont elles dépendent : on y distingue, en effet, deux couches essentielles; l'une, externe ou membrane ectotrachéale, n'est 
autre chose qu'une invagination de l'hypoderme tégumentaire; l'autre, l'interne, désignée sous le nom d'intima (membrane endotrachéale), correspond à la cuticule sécrétée par les cellules hypodermiques, ce qui explique pourquoi elle tombe, à chaque mue (Fig. 73'.

Le fil élastique spiralé si caractéristique, qui soutient la paroi pour maintenir les trachées toujours ouvertes, et que Macloskie considère comme formé par àes replis de la cuticule, est, en réalité, constitué par des épaississements de l'intima; d'après Packard, ce sont des cercles très rapprochés et non de véritables spires.

Dans tous les cas, la vieille théorie des trois membranes, développée par Meyer et MilneFdwards, doit être complètement abandonnée.

Les membranes indiquées ci-dessus ne sont bien déreloppées que dans les plus gros troncs trachéens; dans les branchies les plus fines, l'intima et le fil spiralé disparaissent petit à petit: la paroi, réduite alors à la membrane ectotrachéale amincie, permet l'échange facile des gaz dans l'acte respiratoire.

Inutile de parler ici du mode de terminaison des trachées à l'intérieur des organes ; c'est un sujet très peu connu et sur lequel les auteurs ne sont pas d'accord.

Origine des trachées. - Les trachées se

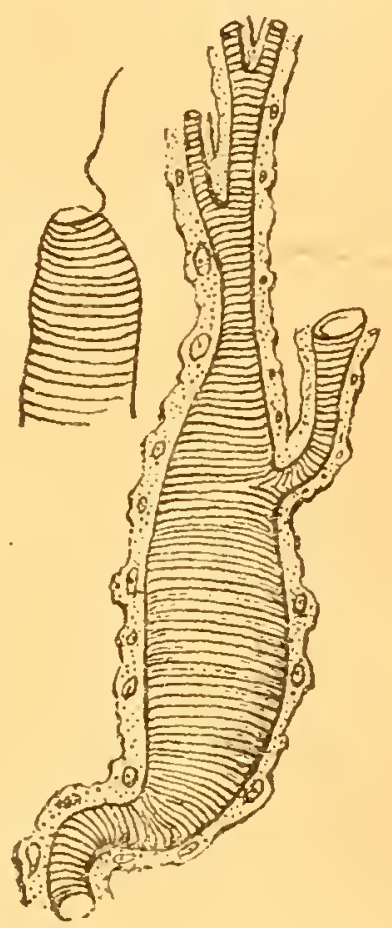

Fig. 73. - Vésicule trachéenne du Dectique verrucivore (fig. orig. gross. 120 diam.). développent de très bonne heure dans le cours du développement embryonnaire. Elles prennent naissance, sur les côtés du corps, par des invaginations de l'ectoderme, qui, au début, apparaissent sur tous les métamères, à l'exception des deux derniers segments abdominaux.

Au début, toutes ces invaginations sont distinctes ; plus tard seulement, elles s'unissent à l'intérieur du corps, ainsi que nous l'avons indiqué p. 124, pour former les grands troncs longitudinaux, d'oli partent ensuite touies les ramifications qui se rendent anx organes.

Comme les trachées ne comniuniquent, à aucune période de leur développement, avec la cavité générale, il est difficile d'admettre qu'elles soient les homologues des organes segmentaires (néphridies) d ss Vers; il est plus probable, all contraire, selon l'opinion de 
Moseley, qu'elles doivent leur origine à des glandes cutanées, ada :tées à des fonctions spéciales ; et de fait, d'après Henneguy, "toutes les glandes cutanées que l'on peut observer, soit chez les larves, soit chez les adultes, ont pour origine des invaginations de l'hypoderme, semblables à celles qui donnent naissance aux trachées, aux glandes salivaires et aux tubes de Malpighi. "

3o Stigmates. - Les stigmates sont toujours placés sur les côtés du rorps ; ce sont des ouvertures en forme de boutonnières, bordées par un cadre chitineux rigide désigné sous le nom de péritrème (Fig. 74 ).

Les stigmates s'ouvrent généralement sur la membrane molle

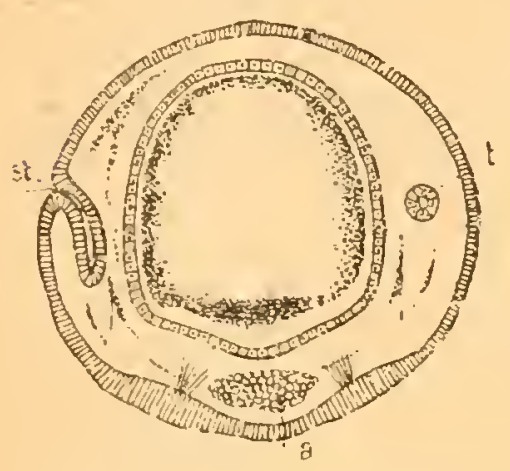

Fig. 74. - Coupe transversale d'un embryon, montrant l'invagination ectodermique st, qui donne naissance aux trachées. (d'après KowALEVSKI) qui relie le sclérite dorsal (tergum) au sclirite ventral (sternum) mais cependantleur situation peut changer; ils peuvent passer, tantôt sur l'arceau supérieur, tantôt sur' l'inférieur, suivant le mode de vie des Insectes, et suivant le développement des pièces squelettiques. La pénétration des corps étrangers (poussières, gaz délétères), à l'intérieur des trachées est empêchée soit par des membranes convenablement disposées, soit par des garnitures de poils, qui constituent, pour l'air, un appareil flltrant.

Le nombre des stigmates est variable; la tête n'en porte jamais. A l'état adulte, sauf exception, le thorax n'en montre jamais plus de deux paires, l'une sur le mésothorax, l'autre sur le métathorax; comme, par ailleurs, le dernier anneau abdominal en est constamment dépourvu, il en résulte que le nombre de ces orifices ne peut jamais être supérieur à vingt (dixipaires). C'est ce nombre que l'on trouve chez la plupart des Coléoptères, des Orthoptères, des Lépidoptères, mais on observe aussi, dans certains grroupes, (Hyménoptères 16 ; Hémiptères, 10 à 18; Diptères 12-14) de notables réductions; c'est ainsi, notamment, que chez les Libellulides (N'éroptères), il n'existe jamais, semble-t-il, plus de quatre orifices stigmatiques distincts; enfin, le maximum de ces réductions s'observe chez les Insectes aquatiques, qui n'ont plus que deux stignates placés à l'extrémité de l'abdomen (p. 121).

Structure des stigates. - D'après leur forme, d'après la présence on l'absence d'un péritrème, d'après lia disposition des mem- 
branes ou des poils qui en garnissent l'entrée, Packard a distingué six types de stigmates que l'on peut réunir en deux groupes principaux :

10 Stigmates sans péritrème. - Il représentent, évidemment, la forme primitive, la plus ancienne de toutes; ils consistent, généralement, en une simple fente linéaire ouverte dans les téguments et garnie le cils sur ses bords.

On n'a trouvé jusqu'ici cette disposition, très simple, que dans les stigmates thoraciques des Hémiptères, de certains Coléoptères (Dytiscus marginalis) et des Libellules. Très sourent, les bords de la fente stigmatique se rapprochent jusqu'à se toucher; mais, plus fréquemment encore, ils présentent des dentelures qui s'engrènent les unes dans les autres et rendent la fermeture plus hermétique.

$2^{\circ}$ Stigmates munis d'un péritrème. - Il existe ici un certain nombre de rariations (Fig. 75).

Dans un premier cas, que l'on rencontre chez 1e: Coléoptères et chezla plupart des Hémiptères (stigmates abdominaux), l'ouverture stigmatique est pourvue d'un anneau corné ou cartilagineux, dont les bords sont nus ou simplement

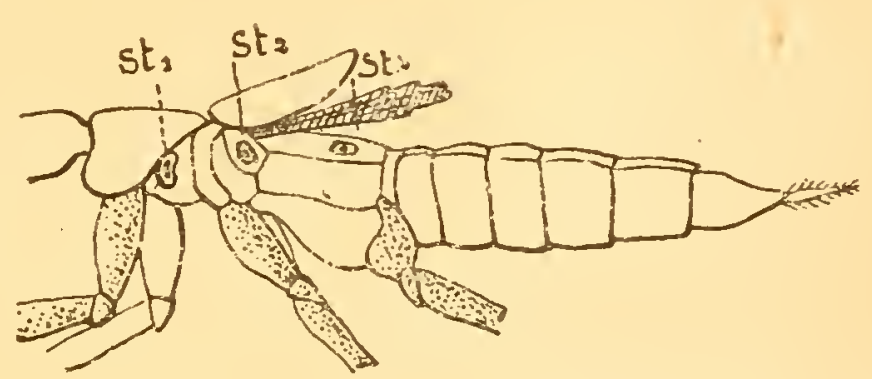

Fig. 75. - Tue latérale d’un Coléoptère IStaphylinus nebulosus) pour montrer les trois premières paires de stigmates, $\mathrm{st}_{1}, \mathrm{st}_{2}, \mathrm{st}_{3}$,

(d'après KOLBE). ciliés (Fig. 74).

Dans un deuxième cas, la fermeture se complique par l'existence. à l'in térieur du péritrème, d'un prolongement valvulaire membranfux c s qui fait que l'ouverture stigmatique est, en quelque sorte, ferr te par un diaphragme. L'une de ces valves ou paupières, selon l'expression de Réaumur, est ordinairement plus grande que l'autre, et toutes deux sont souvent ornées de cils simples, ou ramifiés en feuille ie fougère.

On trouve des exemples de cette disposition dans le premier stigmate abdominal de Lucanus cercus, dans tous les stigmates abdominaux de l'Hydrophilus piceus et chez un très grand nombre d'autres Insectes.

Enfin, une troisième disposition, qui se rapproche encore des précédentes, est celle où au lieu des cils garnissant les valves du 
péritrème, on trouve une membrane criblée de petits trous à travers lesquels l'air pénètre dans les trachées.

Appareil d'ogclusion des stigantes. - Lorsque le pourtour do

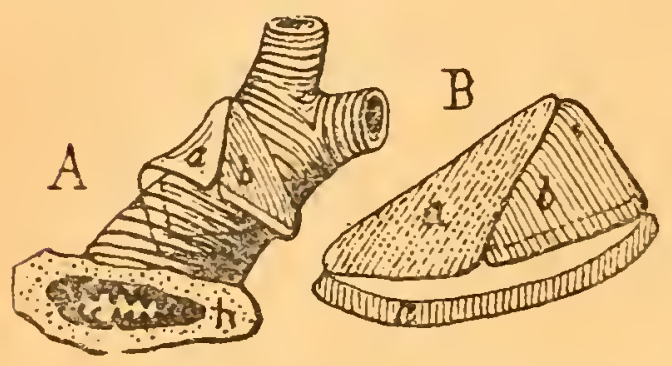

Fig. 76. - Appareil de fermeture des trachées: $A$, trachée avec son appareil d'occlusion : $B$, appareil d'occlusion isolé; $a$ et $b$, pièces chitineuses triangu. laires ; c. arc chitineux (d'ap. JUDEICH et Nitsche).

l'orifice stigmatique est pourvu d'un péritrème, l'ouverture de cet organe est toujours béante; mais, dans uncertain nombre d'Insectes, notamment chez les Coléoptères, il existe un appareil de fermeture assez compliqué, qui leur permet de fermer à volonté leurs orifices respiratoires lorsqu'ils se trouvent menacés d'asphyxie, par exemple, lorsqu'ils sont plongés dans un gaz non respirable ou toxique.

L'appareil d'occlusion est fixé à l'intérieur du corps, un peu alldessus de l'ouverture stigmatique, et avant le point où se fait la première ramification du tronc trachéen. Cet appareil est formé de trois pièces chitineuses élastiques et d'un muscle destiné à les faire mouvoir. "Quand le muscle se contracte (Fig. 76), les deux pièces $a$ et $b$ qu'il réunit, basculent et se rapprochent de la concavité $c$; elles compriment ainsi le tronc trachéen, dont la lumière se trouve plus ou moins oblitérée."

La description donnée par M. Ch. Janet, de l'appareil d'occlusion des stignıates chez. Myrmica rubra, diffère notablement de celle qui précède ; elle a cependant le mérite d'être beaucoup plus claire; mieux qu'une longue description, le dessin schématique que nous reproduisons ici, avec la bien-

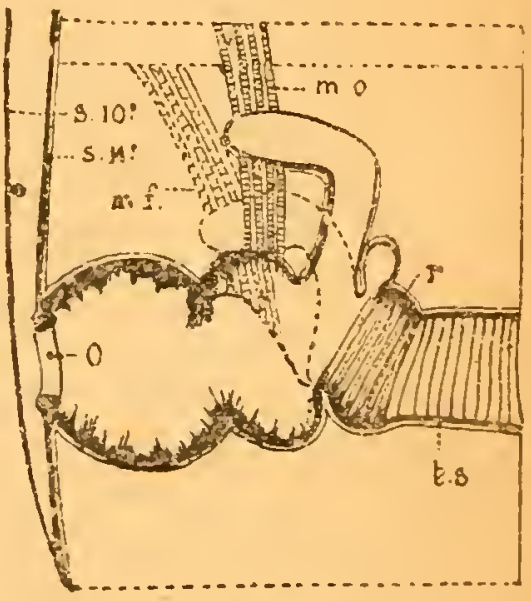

Fig. 77. - Appareil d'occlusion de la Fourmi rouge ; en traits pleins mo. pendant l'ouverture. en traits pointillés $m f$. pentant le fermeture; $s 10^{\mathrm{e}}$, sclérite dorsal du dirième anneau; s. 11". sclérite dorsal du $11^{\mathrm{e}}$; mo. et $m$ f. muscles d'ouverture et ie fermeture : $o$, orifice stigmatique $: r$, raidisseur.

(l'après CH. J.ANET). veillante autorisation de l'auteur, fera comprendre le mécanisme de cet appareil (Figr. ;7).

Pour le développement des stigmates, voir la fig. Tí. 


\section{INSECTES AQUATIQUES}

$1^{\circ}$ Adultes. - A l'exception des larves que nous avons précédemment citées (Nésroptères, quelques Cyrinides, etc.), aucun Insecte adulte n'est franchement adapté à la vie aquatique; l'appareil respiratoire est, en effet, toujours un appareil aérien, avec des stigmates et des trachées, et tous sont obligés de venir, de temps en temps, renouveler leur provision d'air à la surface de l'eau.

Les Insectes aquicoles adultes ne se rencontrent guère que parmi les Coléoptères (Dytiscides, Hydrophilides, etc.) et les Hémiptères (Nipes, Notoriectes, etc.) ; mais en réalité, la plupart d'entre eux ne sont guère plus aquatiques que les Palmipèdes; et. bien que passant leur vie dans l'eau, comme ces derniers, ils sont aussi, comme eux, obligés de venir respirer l'air en nature à la surface.

$2^{\circ}$ Larves des Insectes aquatiques. - Il n'en est pas de même parmi les larves; un certain nombre de celles-ci, principalement dans l'ordre des Névroptères, sont organisées pour mener une existence franchement aquatique, en ce sens qu'elles sont capables, pour se procurer l'oxygène dont elles ont besoin, d'utiliser l'air en dissolution dans l'eau.

Pour cela, ces larves possèdent, comme nous l'avons vu, des organes particuliers en forme de filaments ou de lamelles foliacées qu'on désigne sous le nom de branchies. Il ne faut pas oublier que ces branchies sont des organes acquis, par suite d'une adaptation secondaire au milieu aquatique; le fait qu'un réseau trachéen se distribue dans leur intérieur, indique que les anrêtres de ces Insectes ont, à ce moment donné de leur existence, mené une vie aérienne.

30 Braychies PERMANExtes des adultes. - Un très petit nombre d'Insectes conservent, à l'état adulte, les branchies trachéennes que possèdent leurs larres. L'exemple le plus connu et le plus remarquable est celui d'un Perlide de l'Amérique du Nord, Pteronarcys regalis, signalé pour la première fois par Newport, en 18'千́. Cet Inserte porte, en effet, trcize pairas de branchies filamenteises, placées symétriquement à la surface inférieure du thorax et sur los deux premiers segments abdominaux. Dans chaque houppe branchiale, le nombre des filaments varie de vingt à cinquante. En plus des houppes branchiales, le Pteronarcys possède aussi des stigmates, ce qui lui permet d'utiliser, avec une égale facilité, l'air en nature ou l'air en dissolution dans l'eau (Fig. 78). 
Le Pteronarcys regalis est un Insecte nocturne, qui ne vole que le soir ou à la fraîcheur de la nuit; fréquemment, en volant, il vient tremper dans l'eau la face inférieure de son corps, de manière à maintenir ses branchies toujours humectées. Pendant le jour, il s'abrite sous les pierres ou dans les crevasses humides des rochers.

L'ensemble de ces conditions permet, d'après Barnston, d'expliquer la persistance des branchies trachéennes chez les Pteronarcys qui vivent continuellement dans une atmosphère saturée de vapeur d'eau (Fig. 78).

40 Mécanisme De la respiration. - Les mouvements du corps, qui déterminent l'entrée de l'air dans les trachées, ou son expulsion,

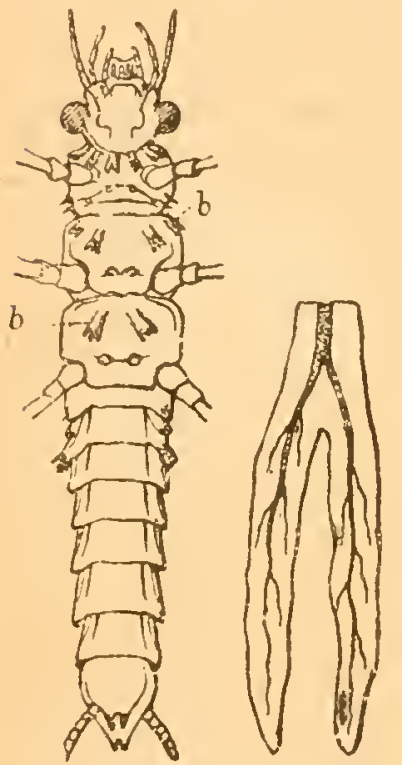

Fig. 78. - Le Pteronarcus regalis Névropt. ru en dessous : it droite une branchie limelleuse isolée

(d'anrès Neñport). se font vraisemblablement par le même mécanisme chez tous les Insectes, et paraissent sous la dépendance des muscles abdominaux.

L'air pénètre à l'intérieur du corps par suite de l'extension des parois, d'où résulte un agrandissement de la cavité générale; il en sort par un mouvement inverse; mais on possède très peu de renseignements sur ce sujet.

Il n'existe pas de muscles inspirateurs pour produire l'extension des parois du corps; lorsque les muscles abdominaux se contractent, la cavité générale se trouve diminuée et l'air esl chassé au dehors ; mais lorsque - l'action d s muscles cessant -- les parois du corps reviennent à leur état primitif d'équilibre, par suile de leur élasticité, l'air rentre dans les trachées. En somme ici, contrairement à ce qui se passe chez les Vertébrés aériens, l'expiration seule est active ; l'inspiration, purement passive, ne se fait que grâce à l'élasticité des parois de. l'exosquelette. En général, les mouvements respiratoires sont localisés dans l'abdomen; le thorax ne prend qu'une part négligeable i) l'accomplissement de cette importante fonction.

Plateau a établi que le mécanisme des mouvements respiratoires pouvait être rapporté à trois types principaux (1).

Dans un premier type, que l'on peut observer chez les Caléoptères.

(1) Platenu (F.). -Móm. Acad. Belg. 1884. T. XLV. p. 219. 
les Hémiptères hétérop tères et les Blattina (Fig. 79, 4.), la partie supérieure de l'abdomen seule est mobile et s'abaisse sous l'influence de muscles spéciaux pendant l'expiration ; la partie ventrale, très convexe et fortement chitinisée, reste immobile.

Dans le deuxième type, qui comprend les Orthoptères, les Diptères, les Hyménoptères et la plupart des Névroptères, le tergum et le sternum, tous deux mobiles, se rapprochent pendant l'expiration (Fig. 79, C.).

Tans le troisième type, que l'on peut observer surtout chez les Lépidoptères, la contraction des parois de la cavité abdo minale se fait dans toutes les directions (Fig. 79, B.).

Enfin, les observalions faites sur les grands Coléoptères

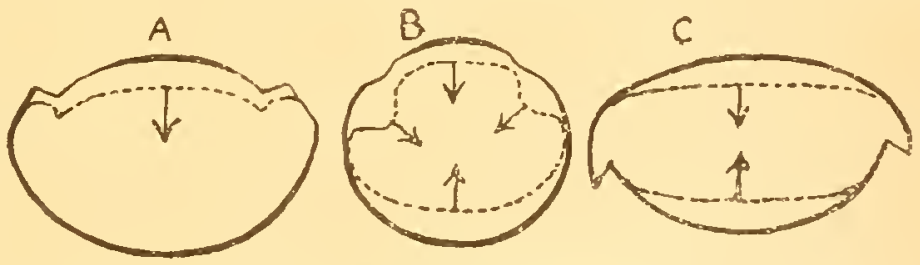

Fig. 79. - Schéma des mourements du corps pendant la respiration: 4 , chez un Coléoptère; $B$, chez un Lépidoptère ; $C$, chez un Hyménoptère (d'après Piateau).

ont montré que le mouvement d'inspiration (extension des parois) était toujours beaucoup plus lent que celui de l'expiration (contraction) : ce dernier pouvant même être quelquefois très brusque.

Quant à la pénétration de l'air à l'intérieur des rameaux les plus fins des trachées - qui doivent ofirir une grande résistance au mouvement des gaz - e'est encore un sujet de discussion parmi les physiologistes.

Phénomènes chimiques de la respiration. - Les phénomènes chimiques de la respiration sont les mêmes chez tous les êtres vivants : ils consistent toujours en une absorption d'oxygène par les cellules et une émission correspondante de gaz carbonique. 


\section{CHAPITRE XI}

\section{APPAREILS DE SECRÉTION ET D'ÉPURATION}

Technique. - Pour étudier les glandes séricigènes du Ver à soie, il faut anesthésier la Chenille en la plaçant pendant un quart d'heure dans une solution étendue d'hydrate de chloral. - Ensuite, l'ouvrir en dessus avec des ciseaux fins, et chercher les glandes, qui se présentent sous l'aspect de deux longs filaments blancs placés en dessous du tube digestif.

Épuration de l'organisme - Les fonctions d'excrétion sont très peu connues chez les Insectes. Cependant, comme le nôtre, leur organisme a besoin d'être épuré et les déchets de l'activité cellulaire doivent être rejetés au dehors.

Parmi les organes chargés de ces fonctions, nous ne connáissons bien que les tubes de Malpighi, dont il a été question à propos de l'appareil digestif (p: 109.), et dont les fonctions paraissent analogues à celles des reins chez les animaux supérieurs.

Nous nous bornerons donc à indiquer ici quelques-uns des produits spéciaux élaborés par l'organisme des Insectes.

$1^{0}$ Soie et glandes séricigènes. - Les glandes séricigènes, ainsi que nous l'avons vu p. 106, sont des glandès salivaires modifiées et adpatées à la production de la soie.

Sauf quelques rares exceptions, la soie, chez les Insectes, est toujours sécrétéz r ar des larves. Parmi les adultes on ne connoît guère que la femelle de "'Hydrophile. qui soit pourvue d'un appareil séricigène.

Par contre, les larves productrices de soie sont très 
nombreuses ; elles se rencontrent chez tous les Lépidoptères, surtout chez les chenilles des Bombycidés, qui, à l'aide de cette soie, fabriquent un cocon pour abriter leur chrysalide.

La soie, étant surtout utilisée pour la protection des nymphes, se rencontrera par conséquent chez les Insectes à métamorphoses complètes ; et, de fait, on observe des glandes séricigènes chez les Lépidoptères, les Névroptères, les Coléoptères (Donacies) et même chez quelques Diptères. (Pour les détails, voir les columes spéciaux de l'E. S.).

LÉPID OPTÈ RES. - Les glandes séricigènes des Lépidoptères sont très simples; ce sont deux tubes très longs, d'un blanc brillant, placés en dessous du tube digestif et aboutissant à une filière située à l'extrémité de la lèvre inférieure. La longueur de ces tubes

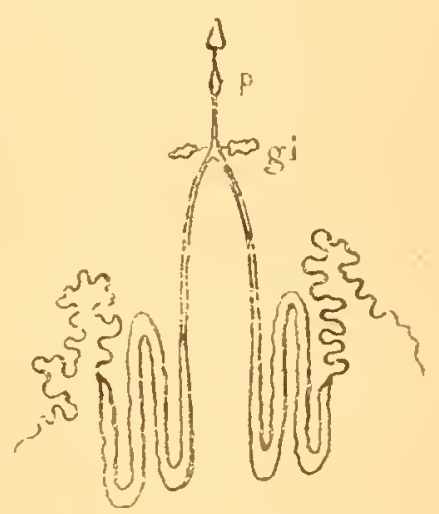

Fig. 80.- Glandes sé1i. cigènes du Ver à soie. $p$. presse; $g l$. glandes de Filippi (d'après Gillson). est variable, suivant la quantité de soie dont la larve a besoin pour la construction de son cocon (Fig. SO).

La filière se présente sous l'aspect d'un tube fin, faisant une légère saillie entre les deux palpes labiaux; on peut la considérer comme formée par une invagination de la langue; les deux canaux vecteurs des glandes séricigènes viennent se réunir en un conduit unique à la base de cet organe. Contrairement à l'opinion de Réaumur, la filière ne possède qu'un seul orifice à son extré. mité libre; cet orifice est taillé cn bec de plume, ce qui permet à la larve de l'appliquer très étroitement sur les corps où elle veut fixer son fil.

LES INSECTes. $-2^{\mathrm{e}}$ édit. 
Névroptères. - Chez les Phryganes, les glandes séricigènes sont construites comme chez les Chenilles des Lépidoptères;-mais, chose remarquable, dans les larves carnassières des Fourmilions, e'est le rectum qui constitue l'organe sécréteur pendant la vie nymphale; il perd sa fonction sécrétante lorsque l'Insecte passe à l'état parfait. Ici, la filière n'est, par conséquent, autro chose que la partie postérieure du tube digestif qui s? prolonge en un tube corné rétractile, que l'Insecte fait saillir à volonté quand il veut agglutiner les grains de sable dont se compose son cocon.

HyMénoptères. - Un grand nombre d'Insectes parasites (Ichneumonides), des Apiens et mème des Formicides, se construisent également des cocons de soie pour s'abriter pendant la nymphose; Seurat a démontré (1893) que l'appareil séricigène des larves de Braconides consistait en deux gros tubes sinueux, se réunissant dans le thorax, et débouchant sur la lèvre inférieure : il est donc construit sur le même plan que celui des Lépidoptères.

Dans tous les cas, lorsque l'Insecte passe de l'état de larve à celui de nymphe, les glandes séricigènes disparaissent; ce sont done des organes essentiellement transitoires, dont le développement a été provoqué par le besoin d'assurer la protection de la nymphe, à l'aide d'un cocon.

Constitution de la soie. - La soie n'acquiert les propriétés qui la caractérisent que lorsqu'elle est sortie des organes qui la produisent. $\Lambda$ l'intérieur des glandes, elle se présente sous l'aspect d'un fluide visqueux et transparent. L'analyse chimique a montré qu'elle se compose de trois substances: sécrétées dans des régions diflérentes de la glande : la ribroüne, la sćricine ou grès et la mincoüdine. 
La fibroïne prend naissance dans toutes les cellules dı tube sécréteur; elle constitue la partie centrale et homogène du fil de soie.

La séricine possède une structure finement granuleuse d'où, probablement, son nom de grès ; elle se produit surtout dans la partic postérieure du réservoir et se dénose à la surface du cylindre de fibroïne, lorsqu'il se présente pour traverser cette région.

On n'est pas fixé sur l'origine véritable du mot "grès "; la subslance désignée sous ce nom présente un aspect gommeux et granulé. M. Howard estime que ce terme est l'équivalent de grège (grecque) : et, de fait, on donne le nom de soie grège à la soie brute, non débarrussée de son enduit gommeux. C'est sous cette forme que les soies d'Asie arrivaient à Cos, avant d'être manufacturées, au commence. ment du rve siècle.

C'est de Grèce également que les dames romaines reçurent les premiers tissus de "gaze légère ", qui se vendaient au poids de l'or, et que les poètes vantaient pour' leur souplesse et leur transparence.

La mucoïdine est produite par la partie antérieure du réservoir ; le rôle de cette substance, qui n'est qu'une sorte de mucns, est probablement de favoriser le glissement du fil de soie dans la filière.

Lorsque la soie est teintée naturellement, c'est en passant dans le canal du réservoir que le cylindre de fibroïne se charge de matière colorante ; on ne connaît pas le mécanisme de cette coloration : les expériences de Blanc, entreprises en 1890 sur ce sujet, n'ont donné, semble-t-il, aucun résultat positif.

La soie résiste à l'action de la plupart des réactifs : le grès seul est dissous par les solutions alcalines; on utilise cette propriété iorsqu'on veut dévider les cocons : la fibröne résisle, car elle n'est soluble que dans les acides très concentrés.

La soie est tellement visqueuse à l'orifice de l'appareil séricigène, qu'elle ne saurait être simplement déversée au dehors, comme le serait un produit liquide; e:le s'étire à la manière des substances ductiles; la Chenille, en effet, après avoir fixé l'extrémité dı fil à ur. corps voisin, relève la tête dans diverses directions, dans le but d'allonger ce fil ; elle donne ainsi, à son cocon, la forme et les dimensions qui lui sont utiles; la Chenille est donc une tapissière; son travail progresse de dehors en dedans. 
Enfin, il nous paraît encore utile de faire remarquer que le fil de soie, pendant son étirement, est obligé de traverser un petit organe, fixé sur le parcours du canal excréteur commun, et auquel on a donné le nom de presse : c'est cet organe qui est la véritable filière. Grâce aux dispositions de la presse, le diamètre du fil de. soie se trouve régularisé; il prend une forme aplatie et son épaisseur est réglée, suivant la volonté de la larve; si la Chenille veut se suspendre, la presse, fonctionnant comme des tenailles, peut arrêter complètement l'étirement du fil ; la presse est donc, en somme, ㄴ.. filière à lumière variable.

Le cylindre de fibroine se solidifie à l'air au moment où le fl sort de la filière, mais la couche de grès qui l'entoure reste molle, ce qui permet à la larve de coller entre elles les différentes couches du fil qui constituent la paroi du cocon.

$2^{\circ}$ Glandes cirières. - Depuis les beaux travaux d'Huber, on sait que la cire n'est pas fabriquée directement par les Abeilles, avec le pollen des fleurs, ainsi que le pensait Réaumur; la cire est le résultat d'une sécrétion.

Les glandes qui sont chargées de l'élaboration de la cire, rentrent dans la catégorie de celles que nous avons décrites sous le nom de glandes cutanées (p. 96); elles sont constituées par des amas de cellules isolées, et le canal excréteur de chaque cellule vient déboucher, au sommet d'une petite éminence, dans une région membraneuse des téguments. On peut facilement mettre les membranes sécrétantes à découvert en exerçant une légère traction sur l'abdomen, de manière à dégager les segments les uns des autres; on aperçoit alors, à droite et à gauche de la ligne médiane, de petites plagges pentagonales de couleur jaunâtre (Fig. 81). C'est sur ces plages que la cire est sécrétée; elle s'y accumule sous forme de minces lamelles que l'Abeille détache à l'aide de ses pattes postéricures. 
Chez les Hémiptères homoptères du groupe des Aphidiens, les glandes cirières sont distribuées sur presque toute la surface du corps; de plus, les deux iubes dorsaux (cornicules), que beaucoup de Pucerons. portent à la partie postérieure de l'abdomen et qui sont en rapport avec une cellule hypodermique sécrétante, produisent, de même, une substance cireuse fluide qui leur sert, très probablement, à se défendre contre leurs ennemis (larses de Coccinelles).

Enfin, le bouclier solide qui protège le corps des Coccidés (Cochenilles), est encore un produit d'origine analogue; il est formé, selon Witlaczil,

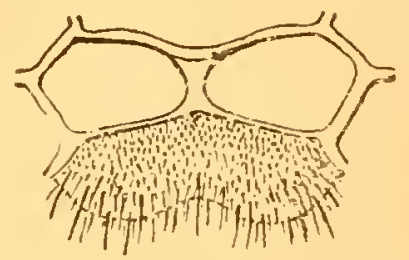

Fig. 81. - Glandes cirières abdominales de l'A beille. par un feutrage serré de filaments cireux entrelacés.

$3^{\circ}$ Glandes venimeuses. - Les glandes venimeuses des Insectes se présentent sous deux aspects différents. Tantôt on les rencontre dans la tête, où elles représentent des glandes salivaires spécialisées (Hémiptères, Diptères et parmi les Hyménoptères, les Fourmis) : ce sont alors des armes offensives; ailleurs, au contraire, ces glandes sont des dépendances de la partie postérieure du corps (Abeille, Hyménoptères porte-aiguillon): ce sont des armes défensives.

Mais, comme la présence de ces glandes n'est pas générale dans les Insectes, nous décrirons les appareils à venin des Hyménoptères, des Diptères, etc., dans les volumes de l'E. S. qui traiteront de ces ordres.

Quant aux produits élaborés par les glandes venimeuses, on n'a que des renseignements incomplets sur leur nature; ils sont nombreux et leur constitution parait assez variable. Le plus connu est celui qui est sécrété 
avec abondance par les Fourmis; Scheele qui l'a l'un des premiers préparé vers 1770 a reconnu sa nature acide et l'a désigné sous le nom d'acide formique. Le venin des Abeilles renferme également beaucoup d'acide formique. Enfin, la glande venimeuse des Moustiques (Cousins,

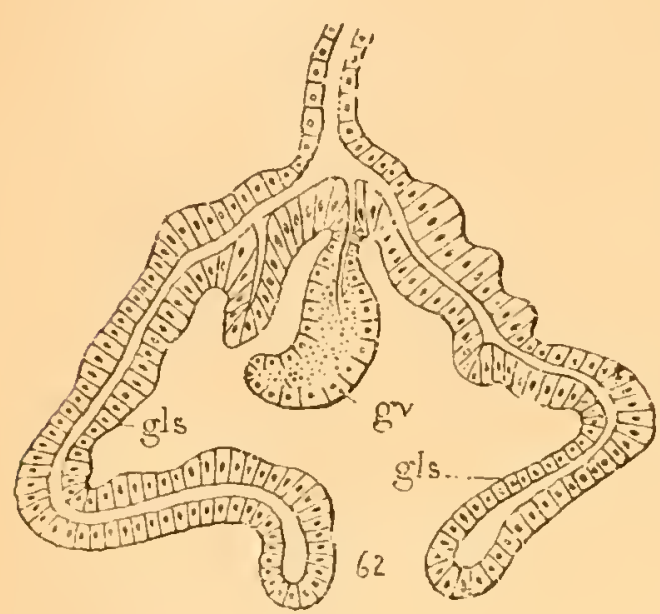

Fig. 82. - Glandes salivaires de I'Anopheles claviger Dipt. les deux lobes latéraus gls, secrètent la salive; le lobe méciian $g v$. est la glande à venin (d'après (Grasst). Anophèles) (Fig. 82) est bien connue, mais la nature exacte du poison qu'elle sécrète n'a pas encore été déterminée.

4. Glandes défensives. Les glandes que l'on désigne sous ce nom, se rencontrent chez un grand nombre d'Insectes; elles produisent un liquide tantôt corrosif, tantôt simplement odorant, vraisemblablement destiné à la prot:ction de l'espèce en éloignant ses ennemis. Tant par leur forme que par leur position, ces organes doivent être considérés comme des glandes coxales adaptées à des fonctions particulières.

Un certain nombre d'Hémiptères émettent, surtout lorsqu'ils sont irrités, un fluide d'odeur nauséabonde, produit par des glandes situées dans la région médiane interne du segment mésothoracique ; le canal excréteur de ces glandes vient déboucher au dehors entre les cuisses de la troisième paire de pattes.

On ne sait rien sur la nature du fluide sécrété par les Hémiptères; on a pa seulement constater que son odeur rappelle de très mrès celle de la Corriandre. Tout 
le monde a remarqué la mauvaise odeur et le goût désagréable que prennent les fruits sur lesquels les Punaises (!) ont marché.

Au groupe des glandes défensives et odorantcs appartiennent encore les glandes anales des Coléoptères, que nous décrirons dans un autre volume (Fig. 83).

Enfin, les Chenilles d'un grand nombre de Lépidoptères se protègent également par l'émission de fluides spéciaux. Ainsi, par exemple, on sait, depuis les travaux de De Geer (1750), que la larve " à queue fourchue" de Dicranura, produit une sécrétion que Bonnet a considérée, en 1755, comme étant un véritable acide piquant et corrosif. L'appareil excréteur de Dicranura sinula a été bien décrit par Rengger et Klemensiewicz.

Klemensiewicz a également décrit les deux tubes orangés (osmeterium) que les Chenilles des Papilionidés émettent à la partie supérieure du segment prothoracique lorsqu'elles sont irritées; ces organes répandent une odeur désagréable de melon. On ne connaît pas la nature exacte du liquide excrété; on sait seulement qu'il a les propriétés

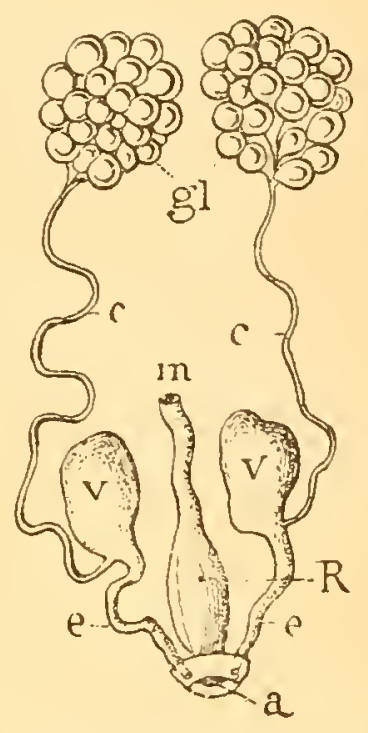

Fig. 83. - Glandes anales in Carabus a?:ronitens Col,; gl. grappe glandulaire ; v. vésicule séceptrices du venin ; $c, c$, canaux eftérents; $e, e$ canaux excréteurs terminaux; in, intestin; $R$. rectum : a, anus, (d'ap. I. BORDAS). d'un acide et qu'il peut rougir la teinture de tournesol.

50 Glandes à cément (Glandes sébifiques). - Ces glandes, très répandues chez les Insectes, seront décrites en même temps que l'appareil sécréteur particulier à

(1) Il s'agit ici, bien entendu, de la Punaise des bois, grand insecte de couleur g-:se qu'il ne faut pas confondre arec la punaise des lits. 
chaque groupe; il nous suffira de dire ici qu'un grand nombre d'entre elles sont en connexion plus ou moins intime avec l'appareil génital. Le produit de ces glandes sert, en général, à agglomérer les œufs au moment de la ponte ou à fabriquer, pour ces mêmes cufs, des enveloppes protectrices (oothèques, coques osigères, etc.). Le liquide sécrété par ces glandes renferme de nombreux petits cristaux et se coagule rapidement à l'air.

$3^{\circ}$ Glandes dermiques. - On donne ce nom aux glandes unicellulaires hypodermiques répandues sur toute la surface du corps; le seul fait intéressant à noter est que la distribution de ces glandes est toujours plus ou moins métamérique. 


\section{CHAPITRE XII}

\section{RESERVES NUTRITIVES}

TECHNIQUe. - Pour étudier le corps adipeux, il sunfit d'ouvrir une Chenille vers l'époque où elle va se transformer en nymphe. Ce tissu se présente alors sous l'aspect d'une pulpe blanchâtre, placée immédiatement sous la peall et entre les organes.

10 Corps adipeux et ses dérivés. - On désigne sous ce nom des amas de grosses cellules arrondies ou polyédriques, de couleur jaunàtre, et formant, à l'intérieur du corps, principalement sous la peau et autour des organes, des réseaux lacuneux très irréguliers, ou des cordons allongés, disposés métamériquement. Ce tissu est plus abondant chez les larves que chez los Insectes parfaits, et il ne saurait en ètre autrement; en effet, pendant toute la période de temps qui s'écoule depuis l'éclosion jusqu'à la transformation en nymphe, la larve se nourrit abondamment; sa principale préoccupation est donc d'accumuler d'abondantes réserves nutritives en prévision de l'abstinence forcée qu'elle subira pendant la durée de la nymphose. Ce sont ces réserves, constituant le corps adipeux, qui, au cours de l'histogénèse, seront utilisées par les histoblastes pour l'édification du nouvel organisme en voie de devenir l'Insecte parfait (Fig. S4).

Les auteurs qui ont étudié le corps adireux dans ces 
vingt dernières années, sont arrivés à conclure que ses fonctions étaient multiples et qu'on devait, en réalité,

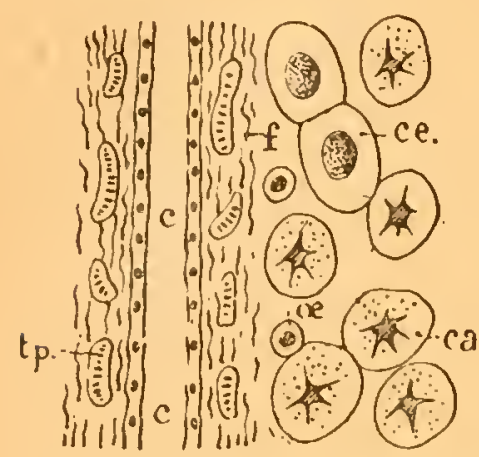

Fig. 84. - Le cocur, $c$, entouré du corps adipeux. $C a$, cellules adipeuses; $c \theta$, cellules excrétrices ; $\propto$, œnocytes (d'ap. Ch. JAxLT). distinguer, dans ces amas de tissus. semblables en apparence, plusieurs groupes de cellules, savoir :

Les cellules adireuses proprement dites, qui sont, comme toujours, des éléments de réserve nutritive.

Les cellules péricardiques, signalées par Graber, et qui, comme leur nom l'indique, se rencontrent particulièrement sur les côtés de l'appareil circulatoire.

Les cellules spléniques, étudiées par Kowalersly chez les Orthoptères sous le nom de rate.

Les onocyles, signalés par Wielowiejski chez les larves des Diptères.

En ce qui concerne les origines de ce tissu, nous en dirons quelques mots lorsque. nous exposerons l'anatomie et le développement des larves.

\section{Cellules adipeuses.}

Examinées au microscope, les cellules adipeuses se montrent formées d'un protoplasma granuleux renfermant un gros noyau; disséminées à l'intérieur

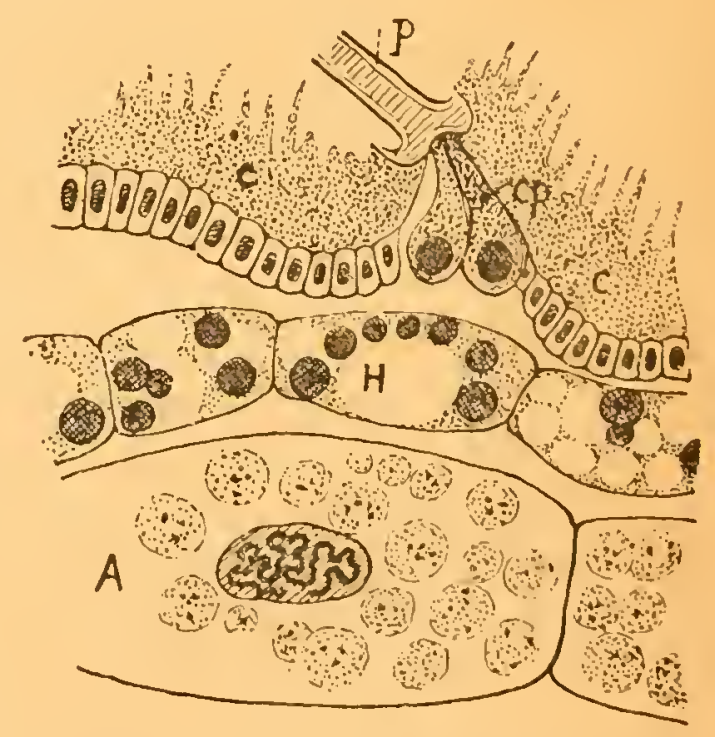

Tig. 85. - Te corys alipeux cliez un Insecte récemment né. 1 , tissul larvaire; $H$. tissu imaginal; $c$. cuticule: $P$. poil: $c p$. cellules hypoderniques it la base du poil ( (laap. Bralese). du protoplasma, on trouve également des gouttelettes giaisseusses en grand nombre et des cristaux d'acide urique (rig. 85).

De nnmbreuses trachées se distribuent dans la masse des cellules 
adipeuses, ce qui porte à penser que ce tissu joue un rôle important dans les phénomènes de nutrition; la présence des gouttelettes graisseuses permet de le considérer comme un tissu de réselve; et, de fait, dans les Chenilles des Lépidoptères nocturnes et crépusculaires - où il est particulièrement abcindant -- on le voit se résorlie: lentement pendant la nymphose, au point qu'il a presque totalement disparu lorsque le Papillon sort de son enveloppe.

C'est aussi à ses dépens que vivent les parasites entomophages. Ainsi, par exemple, lorsque les petites larves du Mirrosaster se développent à l'intérieur de la Chenille de la Piéride du Chou, elles dévorent, tout d'abord, le tissu graisseux en ayant soin d'épargner les organes essentiels de la digestion et de la respiration.

2 Cellules péricardiques. - On donne ce nom à des bandelettes de grosses cellules, de couleur rougeâtre ou jaune verdâtre, situées de chaque côté du vaisseau dorsal. Malgré leur aspect, ces cellules se distinguent des cellules adipeuses par leur constitution et par leurs propriótés.

En effet, chaque cellule possède, en général, deux ou plusieurs noyaux; de plus, elles donnent naissance à de nombreux filaments protoplasmiques qui les mettent en relation, d'une part avec les parois du vaisseau dorsal, d'autre part avec le diaphragme souscardiaque. Comme précédemment, de nombreuses trachées viennent se terminer entre ces cellules, en formant des anses qui les contournent.

Au point de rue de leurs fonctions, l'expérience à démontré que les cellules péricardiques doivent être considérées commes des éléments excréteurs, capables d'éliminer les produits de désassimila. tion à réaction acide, tandis que les tubes de Malpighi sont, eux. adaptés à l'élimination des déchets alcalins.

3'. Enocytes. - Les cellules que l'on désigne sous ce nom, par allusion à leur couleur qui est d'un jaune vineux (1), sont les plus volumineuses de toutes celles qu'on rencontre dans l'organisme des Insectes. Wielowiejski, qui les a le premier signalés chez les larves de Chironomue et de Conrthra, a attiré l'attention sur leur disposition en grappes, sur leurs relations avec les trachées et sur leur distribution métamérique (Fig. 81).

Jien qu'ils soient toujours plus ou moins rattachés aux autres cel-

(1) Du grec : oinos vin et kutos cellule. 
lules du corps adipeux par de fins prolongements, les onocytes doivent avoir une signification absolument différente, ils tirent, $\in n$ effet, leur origine de l'ectoderme, tandis que le corps adireux aurait, comme on l'a vu (n. 140). une origine entoderry ique.

Je sais qu'il ne faut pas attarher une importance trop grande à ces questions d'origine; mais, il n'en est pas moins vrai qu'ici, la disposition métamérique de ces csllules, leur apparition précoce et leur existence chez tous les Insectes, indiquent que ce sont des organes importants. Nalheureusement, jusqu'ici, les fonctiors des senocytes sont totalement inconnues.

40 Organes lumineux. - I es organes lumineux que j'on peut observer chez un certain

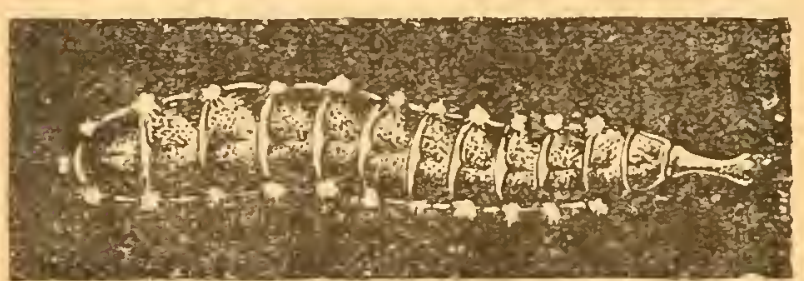

Fig. 86. - La nymphe lumineuse du Phengotes latirollis. Col. (d'après F. HeNxEGUY). nombre d'Insectes, sont constitués par diverses régions spécialisées du corps adipeux. Ils consistent, généralement, en amas de ceilules polygonales, situées immédiate. ment au-dessous de l'hypoderme et au milieu desquelles se distribuent des nerf s et re nombre':ses trachées (Fig. S6).

On peut olserver les organes lumineus principalement chez les (oléoptères, (Lampyre, Pyropho"e, etc.) ; les autres groupes d'Insectes où l'on a égalemient signalé des espèces phosphorescentes ne sont ras très nombreux; voici ceux qui sont connus juscqu'à re iour :

Parani les Névroptères, deux gerıres d'Fiphémérides. Conis et Teloganodes.

Farmi les Lépidoptères, deux chenilles de Noctuélides : 1 grotis occulata et Mamestra oleracea.

Enfin, plusieurs larves de Diptères: Culex, Chironomus, Thyyreophora, etc.

Il est très probable que, parmi les cas de phosphorescence observés chez les Insectes, un certain nombre sont dus à la présence accidentelle de bactéries photogènes, soit à la surface du corps, soit dans l'intérieur des tissus : quelques-uns n'ont, en eflet, été ol)servés qu'une fois. C'est ainsi que la phosphorescence de Lipurr noctiluca, signalée par R. Dubois, et même celle du classique Fulorore porte-lanterne, décrite par Mile Sybille de Merian, n'ont .jamais 
être constatées à nouveau nar les entomologistes qui ont examiné ces Insectes à l'état virant.

Quoi qu'il en soit, comme les propriétés photogéniques sont surtout répandues chez les Coléoptères, nous ferons l'étude complète des organes producteurs de lumière dans le volıme de l'E.S. qui traitera de l'anatomie de ces Insectes.

50 Organes spléniques. -- Enfin, nous signalons ici, simplement à titre documentaire, les amas cellulaires que Kowalewsky a désignés sous le nom de rate, et qui n'ont jusqu'ici été étudiés que chez les Orthoptères. Cuénot, qui les a retrouvés chez les Gryllus, pense qu'il ne s'agit, en réalité, que de simples amas de phagocytes; ce sont, pour lui, de jeunes globules sanguins adaptés à la défense de l'nrganisme. 


\section{TROISIÈME PARTIE}

\section{Fonctions de Relations.}

Chez les Insectes, de même que chez. les animaux supérievrs, les fonctions de relations sont accomplies par:

$1^{\circ}$ L'appareil locomriteur, constitué, comme toujours, pardeux sort 's d'organes; des organes passifs, pièces de l'exosquelette, déjà décrites, Chap. II ; et des organes actits, ensemble des muscles.

$2^{\circ} \mathrm{L}$ 'appareil nerveux avec ses annexes les organes des sens.

\section{CHAPITRE XIII}

\section{APPAREIL MUSCULAIRE.}

Techxique. -- Enlever, avec une pince fine, la carapace chitineuse d'un Insecte ailé quelconque, au niveau de l'insertion des ailes; on découvre ainsi une masse musculaire volumineuse: ce sont les muscles jaunes des ailes. On découpe, avec des ciseaux fins, une tranche de cette masse, et on la dissocie dans une goutte d'eau sur une lame de verre avec une aiguilie. La tranche musculaire se. décompose d'elle-même en fibrilles. Pour bien distinguer la striaticn transversale des fibres, il faut employer un grossissement de 350 diamètres environ.

Io Disposition générale des muscles. - Il est impossible de dire quoi que ce soit de général sur l'arrangement des muscles, car aucun travail n'a été entrepris, en vue de 
comparer, sous ce rapport, les différents ordres de la classe des Insectes. La plupart des auteurs qui ont abordé l'étude de l'appareil locomoteur actif́, n'ont eu en vue que le fonctionnement ou la structure intime de la fibre musculaire. Pour les études d'ensemble, on ne peut ģuère citer que la célèbre monographie du Hanneton, par Straus-Durkheim, écrite il y a déjà bien longtemps et où l'appareil musculaire est décrit avec un grand luxe de détails; puis les travaux, si minutieux et si précis, de Charles Janet sur l'anatomie des Fourmis. Toutefois, c'est au travail de Straus-Durkheim (p. 17) que nous cmprunterons presque tous les détails qui vont suivre, relativement à la forme et à la distribution des muscles dans le corps des Insectes (1).

En général, la disposition des muscles, comme celle ¿lu corps lui-même, est métamérique; chaque anneau possède un système particulier de muscles, qui servent cn même temps à le rattacher aux anneaux voisins. Toutefois, dans la région thoracique, les muscles perdent leur disposition primitive, et il s'en développe d'autres, uniquement destinés à produire les mouvements des ailes et des pattes; en général, ces muscles, d'origine adaptative, s'insèrent, non plus sur la bordure des sclérites, mais sur des prolongements internes de la carapace chitineuse (apodèmes).

Les muscles sont formés de fibres faciles à dissocier par l'action de l'álcool; ces fibres sont toujours striées transversalement; les fibres lisses étant, comme on le rait, rares chez les arthropodes.

(1) Le travail de cet auteur sur le Melolontha ruloaris (1828) et l'anatomie de ta Chenille du Cossus ligniperda par I,yonet (1762) sont jusqu'ici les seuls ourrages dù la myologie des Insectes soit traitée c'une façon à peu près complète. 
Enfin, les diverses parties du squelette des Insectes présentant les mêmes modes d'articulation que chez les Vertébrés, il en résulte que les muscles peuvent être rationnellement classés d'après leurs fonctions : extenseurs, fléchisseurs, abductcurs, etc., plutôt que d'après leur forme. Le nombre des muscles étant très grand (1), nous nous contenterons de citer les principaux parmi ceux qui, dans les trois régions principales du corps, remplissent les plus importantes lonctions.

A. Muscies de ta tête. - I Les muscles de la tête peuvent se partager en deux groupes, ceux qui déterminent les mouvements généraux de la tête (muscles céphaliques) et ceux qui appartiennent aux appendices ( $m$. masticateurs et $m$. antennaires).

10 Muscles céphaliques. - Ils sont au nombre de quatre paires; les trois premières servent aux mouvements de flexion et d'élévation de la tête ; suivant l'action qu'ils doivent produire ils s'attachent, par l'une de leurs extrémités, au bord supérieur ou inférieur de l'ouverture prothoracique, et, par l'autre, au bord correspondant de l'ouverture occipitale.

La $4^{\circ}$ paire consiste en deux larges muscles fixés sur les côtés des ouvertures prothoracique et occipitale. Si l'un d'eux se contrarte seul, il fait, selon le cas, tourner la tête à droite ou à gauche; s’ils se contractent tous les deux en même temps, le cou rentre dans l'intérieur du prothorax.

20 Muscles masticateurs. - Les mandibules, n'étant composées que d'une seule pièce, n'ont que deux muscles, un extenscur pour les écarter et un fléchisseur pour les rapprocher.

Les mâchoires, au contraire, étant formées de plusieurs pièces et portant des appendices mobiles (palpes maxillaires), sont mises en mouvement par neuf paires de muscles symétriques. Chacun des palpes possède également deux muscles antagonistes, l'un extenseur', l'autre fléchisseur'.

(1) Lyonet en a compte 4061 dans lin chenille du Cossus ligniperta, mais il rearardait comme tels de simples fibres isolées, qui n'anraient yas dû entrer en ligne ce compte. 
$3^{\circ}$. Iuscles antennaires. - Les antennes possèdent chacune trois muscles principaux : un extenseur pour les écarter à droite et à gauche; un élévateur pour les mouvements verticaux de bas en haut; puis, enfin, un flérhisseur antagoniste des deux premiers.

En résumé, dans la tête des Coléoptères - il en est probablement de même cher tous les Insectes - il n'y a pas moins de quarante muscles, sans compter ceux qui servent à faire mouroir isolément les articles des palnes et ceux des antennes.

B. MUscles du THorax. - Le thorax possède, comme on pouvait s'y attendre, deux espères de muscles : les uns servent à relier entre eux les trois segments thoraciques; les autres sont destinés aux mouvements des pattes et des ailes (Fig. 87).

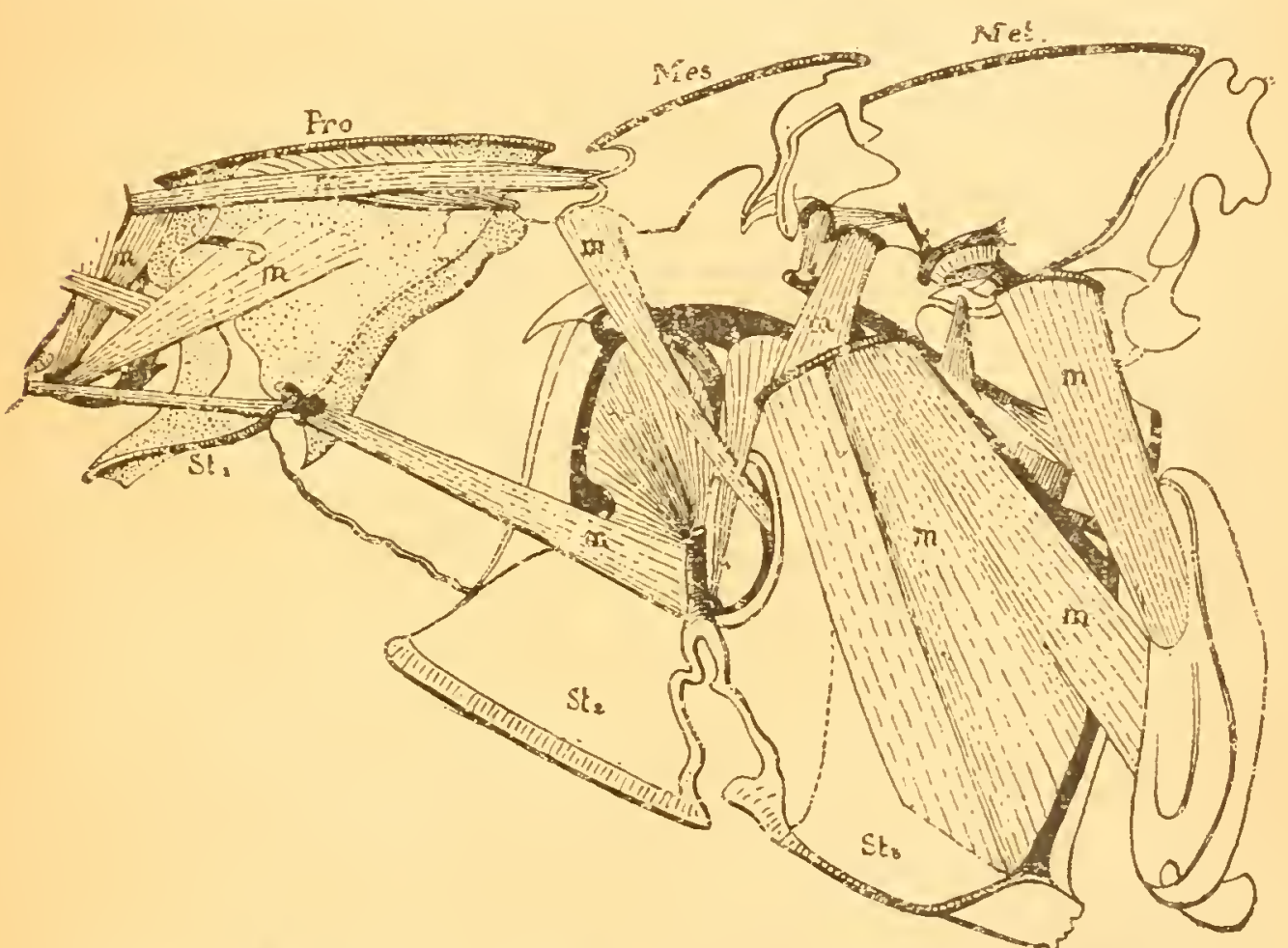

Fig. 87. - Musculature du thorax chez un Insecte : Hydrophilus piceus, Pro, pronotum ; Hes, mésonotum ; .Iet, métanotum ; St, prosternum ; Str. mésosternum : Stz, métasternum (d'ap. BERLESE).

10 Muscles propres du thorax. - Cies muscles sont au nombre de vingt: quatre paires dans le prothorax et trois paires dans chacune des deux subdivisions suivantes; chose remarquable, presque tous ces muscles ont l'une au moins de leurs insertions sur les bords du mésothorax. 
2. Muscles des ailes. - Les trois paires de muscles propres du mésothorax et du métathorax jouent un rôle actif dans les mouvements des ailes; toutefois, leur rôle n'est que secondaire et indirect. Les véritables muscles des ailes sont au nombre de trois, deux extenseurs et un fléchisseur. Le principal extenseur naît sur le bord la téral du sternum; il se porte ensuite transversalement (obliquement) à la base de l'aîle du côté opposé, où il s'insère par un tendon aplati. L.e second extenseur, plus grîle que le précédent, naît un peu en arrière, également sur la paroi latérale du sternum : il suit la direction du premier jusqu'à la base de l'aile, mais là, il s'en sépare poul' s'insérer sur la nervure costale.

Il convient de noter que, chez lẹs Coléoptères, les extenseurs des élytres sont très grêles puisque ces organes n'exécutent aucun mouvement pendant le vol. ("est l'inverse chez. les Hémỵnoptères, oì "les ailes inférieures sont dépourvues de musculature et resteraient immobiles si elles n'étaient accrochées aux ailes supérieures et entraìnées par elles."

Le fléchisseur, beaucoup plus faible que les extenseurs, nait " de la partie supérieure des branches des entothorax (apodèmes) et se porte aux ailes du côté opposé, où il se divise en plusieurs branches qui s'attachent sur artant de ces petites pièces cornées qui existent à la base de l'aile et que Jurine a décrites sous le nom d'csselets."

Il y a de sérieuses réserves à faire ici, relativement au rôle et a la disposition des muscles moteurs de l'aile indiqués par Lacordaire (lic. cit. p. 259). Les figures schématiques données par Graber (loc. cit. p. 207) et par Henneguy (loc. cit. p. 110), ne concordent pas avec la manière de voir de Straus. Ces derniers auteurs, notamment, ne parlent pas du croissement des muscles indiqué par StrausDurckheim ; il serait cependant bien extraordinaire que cette disposition fût particulière aux Coléoptères.

Théorie du vol. - Un grand nombre d'auteurs ont essayé, avec peu de succès d'ailleurs, d'expliquer le mouvement des ailes chez les Insectes; le seul point sur lequel ces auteurs soient d'accord, c’est que le mécanisme du vol, chez les Insectes, n'est, en aucune façon, comparable à celui des Oiseaux.

Les expériences de Marey l'ont amené à conclure que, dans l'acte du vol, le mourement imprimé aux ailes par les muscles du thorax est peu compliqué ; c'est un simple mouvement de ra-et-vient dans 
u plan perpendiculaire à l'axe du corps ; la résistance de l'air o llige ensuite l'aile à s'incliner dans toutes les directions; il résulte is ce double mouvement qu'un point fixe de l'extrémité de l'aile décrit une trajectoire en forme de 8 (Fig. 88.) Mais comment, dit Ch. Janet, les muscles vibrateurs du $v$ l, qui ne s'insèrent pas directement si:r l'aile, mais sur squelette tégumentaire du mésothorax, peuvent-ils prodire ce mouvement de va-et-rient? ("est là, en effet, le point délicat de la rizestion. Les auteurs n'étant pas d'ac. ford sur ce sujet, nous allons envisager la. deux opinions qui nous paraissent rendre le mieux compte des cas ob- d'une Guêpe pendant le vol servés.

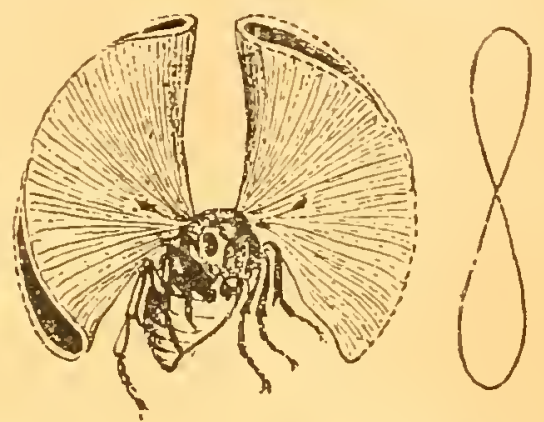

D'après Graber, la nervure principale de l'aile se prolongerait à l'intérieur du thorax sous forme d'un bras de levier rigide, très court, qu'il désigne sous le nom de pédicelle; le point d'appui de ce levier sərait au contact du sclérite dorsal (Fig. 89); deux muscles $h$

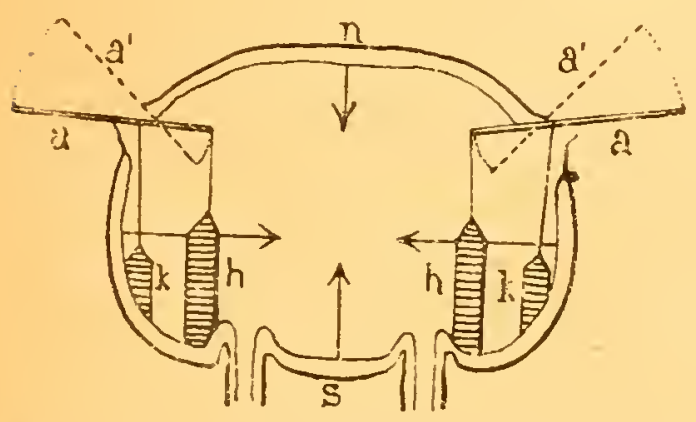

Fig. 89. - Schéma destiné à expliquer le mouvement des ailes, $a, h$, muscles élévateurs ; $k$ muscles abaisseurs ; $n$, notum : $s$, sternum (d'après GRABER). et $k$, sont fixés de chaque côté du point d'appui; lorsque le muscle h (élérateur) se contracte, l'aile est soulevée; elle est au contraire abaissée; lorsque c'est le muscle $k$ qui se raccourcit. Cette explication, trop simpliste assurément, ne tient malheuleusement aucun compte des mouvements vibratoires qui animent les parois du thorax pendant le vol. Ces mouvements czpendant sont très nets et on peut les percevoir facilement lor'squ'on place le doigt sur le dos d'un Insecte (Hyménoptères, Diptère) alors que les ailes fonctionnent.

L'explication proposée par M. Charles Janet me parait beaucoup p'us acceptable.

Les ailes, couchées longitudinalement le long du corps, sont tout d'abord amenées dans la position voulue pour le vol par de petits muscles de mise en place. Ensuite, la contraction des muscles vibra- 
tours transversaux abaisse la voûte supérieure du thorax, ce qui im. prime un mouvement de bascule â la plaque chitineuse supportant la base de l'aile : celle-ci se trouve donc soulevée ; elle s'abaisse au

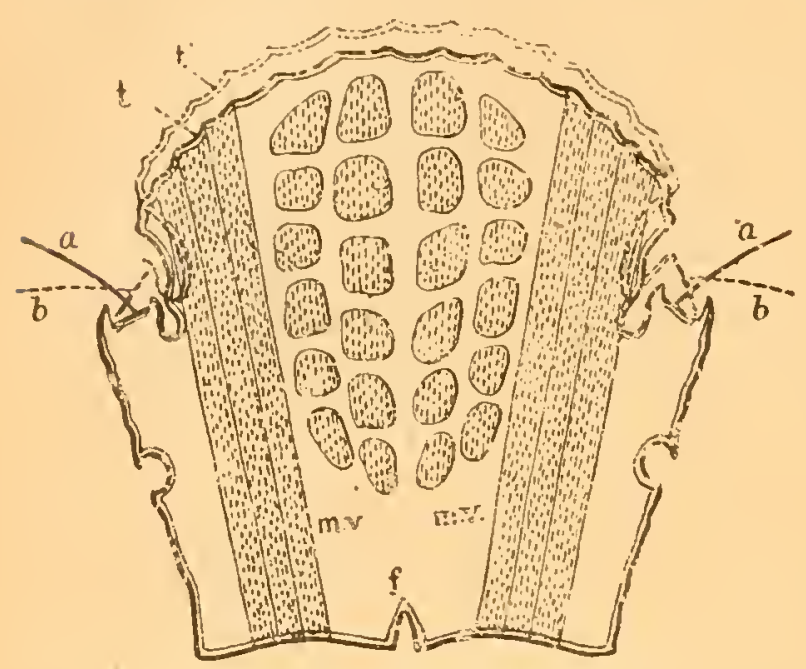

Fig. 90. - Positions successive; des ailes $a$ et $b$. pendant les mouvements de vibration dn thorax: $t$, paroi supérieure du thorax pendant la contraction des muscles; $t$ ', la 'même pendant le relâchement: mv, muscles vibrateurs ; $t$ furca (d'après CH. JANET).

chez les Agrionides, étudiés par Lowne, les ailes se meuvent presque parallèlement à l'axe du corps; Landis que chez certains

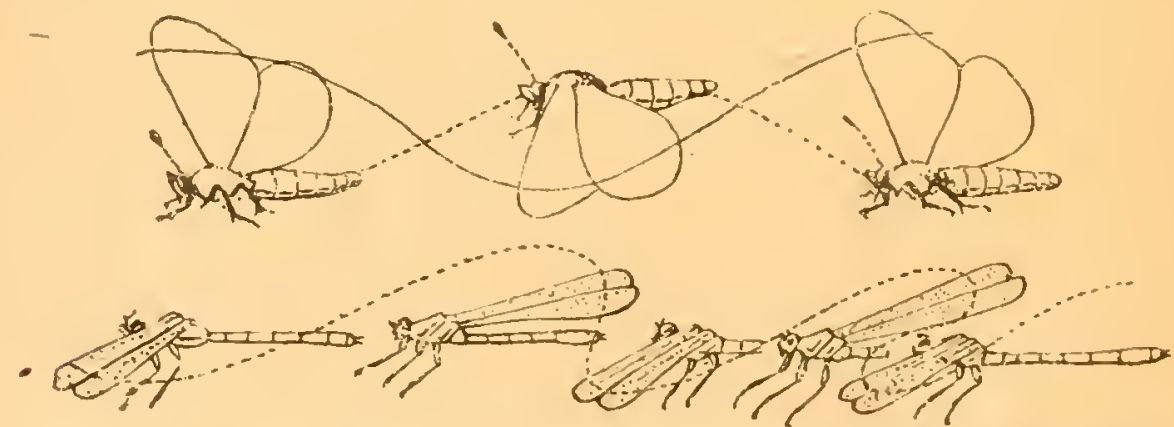

Fig. 91. - Diagramme du vol chez les lnsectes : en dessus chez les Lênirioptères : en dessons chez les Nérroptères (d'alurès WOODWOITH).

Lépidoptères (Piérides), elles battent dans une direction sensiblement verticale (Figr: '91).

$3^{n}$ Muscles des pattes. - F.tant données la grande mobilité de ces organes cl leur division en articles, la musculature des palles est beauroup jlus riche que celle des ailes. Ainsi, il existe, dans les 
pattes du Hanneton commun, d'après Straus, quatre extenseurs et un fléchisseur poul les hanches antérieures et postérieures; denx extenseurs seulement et trois fléchisseurs pour les hanches intermédiaires. Tous ces muscles prennent naissance à la partie interne du tergum et s'insèrent ensuite, à l'intérieur des hanches, suivant les mouvements qu'ils doivent produire.

La cuisse est actionnée par un seul muscle, dont le corps est contenu dans le trochanter.

Les muscles, produisant les monvements des tibias, sont logés dans les cuisses; il existe un extenseur et un féchisseur. Chacun des articles des tarses possède aussi deux muscles; ces muscles sont logés dans les tibias correspondants et se prolongent jusqu'aux divers articles à l'aide de tendons allongés. Enfin, le dernier article des tarses renferme deux muscles destinés aux mouvements des crochets qui le terminent.

C. M LiSCLES DE I'APDOMEN. - L'absence de membres entraine une simplification notable de la musculature dans cette région; on n'y trouve guère, en eflet, que les muscles destinés à faire mouroir les anneaux les uns sur les autres, ou bien l'abdomen dans son ensemble. Presque tous les muscles abdominaux se ressemblent: ce sont de minces bandelettes aplaties et dépourrues de tendons, qui s'insèrent, d'une part au bord antérieur' d'un segment, de l'autre all bord opposé de l'anneau suivant. Il en résulte que, lorsque les faisceaux musculaires dorsaux se cortractent, l'abdomen se relève : il s'abaisse au contraire, si ce snnt les muscles ventraux qui agissent.

IIo Structure des muscles. - La structure intime et les propriétés des muscles sont à peu près identiques dans toute la série animale; les Insectes présentent cependant, sous ce rapport, certaines particularités qui les distinguent des Vertébrés et qui sont l'indice d'une très lointaine adaptation. Nous résumerons ici les faits les plus importants, mis en lumière par les belles recherches de Leydig (1855) et de Weismann (1862) (1).

(1) Il y a de si grandes variations clans la terminolorie des auteurs que nous ne pouvoñ songer à rétablir ici les concorrances. Nous adoptons la terminologie ie Prenant, Cytologie, Paris, Schleicher, 1904, 1. 429. 
Weismann a reconnu trois types principaux de faisceaux primitifs (fibres musculaires) chez les Insectes.

10 Chez les larves, chaque fibre possède un sarcolemme (enveloppe) renfermant une substance contractile ; entre le sarcolemme et la masse contractile se trouvent généralement des noyaux; nous avons donc là l'aspect ordinaire de la fibre musculaire jẹne chez les Vertébrés (Fig. 92).

$2^{\circ}$ Dans les muscles moteurs des pattes, chez les

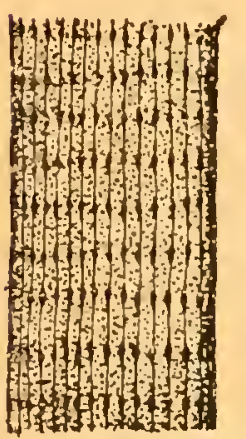

Fig. 92. - Fibres musculaires et une fibrille isolée (d'après IJANG). Insectes adultes, chaque fibre mus: culaire est encore revêtue d'un sarcolemme, mais la substance contractile interne, ainsi que Leydig l'a indiqué le premier, est parcourue par un canal, à l'intërieur duquel on trouve de nombreux'noyaux alignés (Fig. 93).

$3^{0}$ Dans les muscles moteurs des ailes (muscles jaunes de Leydig), les fibres sont dépourvues de sarcolemme; elles sont composées chacune d'un paquet de fibrilles séparées les unes des autres par une substance demi fluide, ce qui explique pourquoi la dissociation de ces fibres est si facile.

Nous ne discuterons pas le fait de savoir si ces fibrilles, des faisceaux de l'aile, sont réellement les unités physiologiques du muscle; ou bien si, comme le veulent Grumbach et Ranvier, elles sont les homologues des colonnettes musculaires des Vertébrés.

Striation transsersale des fibres. -- Nous avons déjà dit que par une remarquable exception - presque tous les muscles du corps des Insectes étaient striés, même ceux du tube digestif; li ctriation est même parfois tellement compliquée (ex. : I.ucanc) qu'on 
n’en a jantais observé de pareille chez les Vertébrés. Nous citerons sa slement ici l'exemple classique de l'Hydrophile. Une fibrille is lée des muscles jaunes du thorax, nous mintre la succession ordinaire des disques clairs et des disques sombres, arec, au milie!: de ces derniers, la strie de Hensen (Fig. 92). The fibrille analogue, mis prise dans les muscles de la patte, présente deux stries de Hensen. D’autres Insectes, tels que le L : cane, les Orthoptères montrent fréquemment des fibrilles d'une striation encore plus riche (Fig. 92).

Notons enfin, pour terminer ce sirjet difficile, l'intéressante obser$v$ :tion faite par notre collègue, M. le $D$ - Charles Lefeuvre, sur le pattes $\Lambda^{\prime}$

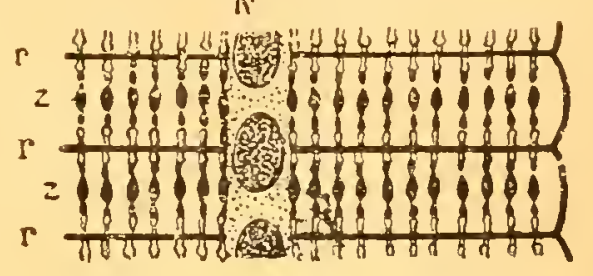

Fig. 93. - Schéma de la striation d'une filorille; $N$. noyaux; $r$, réseau transversal produisant la ligne de Dobie ; $c$, épaissisement des trabécules longitudinaux produisant la strie de Hensen (d'après Chr. JANET). sauteuses des Orthoptères (Dectiri-

dés); M. Lefeuvre a constaté, sur des fibrilles isolées, que le systè ne de striation du muscle extenseur était beaucoup plus compliqué que celui du fléchisseur. C'est la première fois, il nous semble, q l'une variation de structure aussi nette est signalée sur deux m iscles de même fonction, mais accomplissant un travail différent.

On peut en conclure que la striation transversale du myoplasma est réellement fonction du travail que la fibre musculaire exécute.

III Insertion des muscles sur les téguments. -- On a cru prendant longtemps que les fibres musculaires des Insectes venaient sattacher sur la paroi chiniteuse des téguments en traversant simplament l'hypoderme avec lequel elles n'avaient aucune relation. Papuis les beaux travaux de M. Ch. Janet, on sait que cette manière dz voir n'est point exacte. En rêalité, au moment où la fibre musculaire va s'attacher au tégument, elle commence tout d'abord par s'accoler à une cellule hy podermique (Fig. 9'́, 1); on roit alors naître, à l'intérieur de cette cellule, des filaments chitineux destinés à ratticher la fibre à la paroi solide du corps; il est rare que les choses ea restent là ; ordinairement, l'insertion a besoin d'une plus grande solidité, alors la cellule hypodermique tout entière se soulève autour da l'extrémité de la fibre; celle-ci se trouve donc logée dans une sorte de cupule fibreuse très résistante, ayant ses racines tendineuses insque clans l'épaisseur de la cuticule (Fig. 94, B). 
Si la filme musculaire paraît traverser l'hypoderme, ce n'est qu'une apparence; en réalité, c'est une cellule hypodermique modifiée et

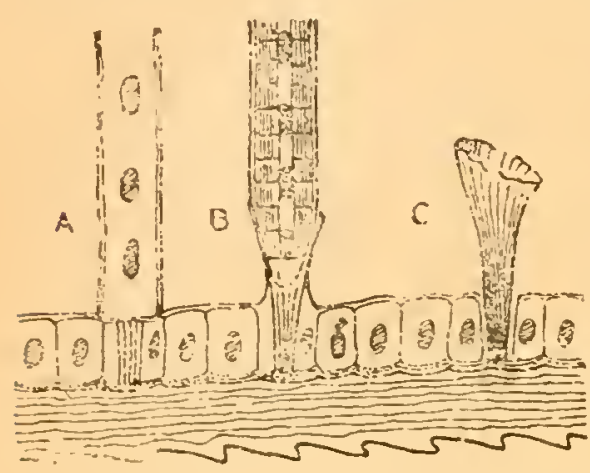

Fig. 94. - T anports des fibres musculaires avec les té:rument: Schéma (l'après CH. JANET).

allongée en forme de tendon qui lui a fourni l'insertion dont elle avait besoin (Fig. 9', C).

IV Force uUscutalre nes IXsecTrS.- Les Insecto: font preure d'une force musculaire remarquable, lnrsqu'ils marchent sur une surface horizontale; sous ce rapports ils sont très supérieur's aux Vertébrés. Ainsi, par exemple, un Lucane peut tenir, entre ses mandibules, en élerant et en abaissant alternativement la tîte et 1. thoras, une règl: d'acier pesant 400 grammes, tindis que son propre poids a lui-même, ne dépas:e pas denx grammes ( $M$. de Lury).

Pour rendre ces faits plus saisissahlec, F. Plateau (1) ? réalisé un série d'expériencez ingénieuses ef très démorstratives ; il a fait trałner des petits chøriots, véritables balances dynamométriques, par de lourds Hannetons transformés en bœufs, et il a constaté ce fait important : c'est qu'en général, la puissance musculaire est en raison inverse de la taille, les plus petits Insectes étant capables de déployer les piris grands efforts.

Finfin, Plateau a encore établi que, toutes proportions gardées, un Hanneton est vingt fois plus fort qu'un cheval : en effet. un cheval ne peut exercer un effort supérieur à $1 / 67$ de son poids, alors qu'un Hanneton peut facilement trainer 14 de ses pareils.

(1) Puatiau (F.). - 1. T. AX. 1. 732-757 ; T. XXIl. 1) 1) 283-308. 


\section{CHAPITRE XIV}

\section{APPAREIL NERVEUX ET ORGANES DES SENS.}

Techxicur. - Les grands Orthoptères (Locuste ou Dectique) coniennent très bien pour cette recherche.

Après avoir ouvert l'Insecte en dessus, on enlève tous les organes internes. La chaîne nerveuse se distingue alor's facilement : elle est placée sur la ligne médiane du corps, en contact aver le tégument inférieur.

Les recherches modernes ont montré qu'il existe, chez les Insectes, de même que chez les animaux supérieurs, un double système nerveux, l'un (système siscéral ou stomoga-uıue) commande Lous les organes de la vie végétative; l"autro (système central) préside à la coordination de tous les phénomènes sensitifs et locomoteurs.

10 Système nerveux central. - Si nous appliquons les idées de Paul Nayer à l'étude des organes de la sensibilité, nous pouvons poser en principe que le Protentomon, c'est-à-dire le tỵpe ancestral des Insectes, possédait un système nerveux ainsi constitué : 10 dans la tête, une masse nerveuse mamelonnée (ganglions cérébrö̈des) reposant immédiatement sur l'œsophage et paraissant correspondre au cerveau des Vertébrés (Fig. 45 ); cette masse nerveuse sus-œsophagienne est réunie, par des cordons passant à droite et à gauche du tube digestif (collier xesophagien), à une autre masse placée, celle-là, au-dessous de l'œesophage, et nommée, à cause de ce fait, masse sous-oesophagienne (ganglions sous-oesophagiens). 
Les ganglions cérébroïdes envoient des ramifications nerveuses aux yeux, aux antennes et à la lèvre supérieure; la masse sous-osophagienne innerve toutes les autres pièces de la bouche et les glandes salivaires, lorsqu'elles existent. Les ganglions sous-:esophagiens

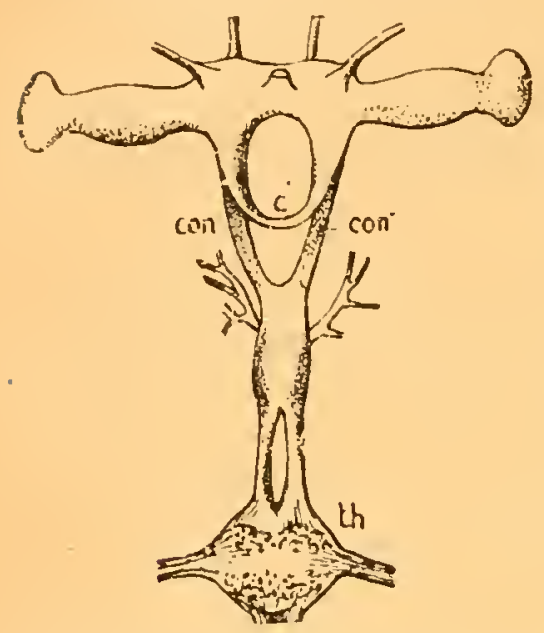

Fig. 95. - Cerveau d'un insecte Névroptère, con, connectifs formant le collier cesophagien (d'après VAsssitire). sont eux-mêrnes réunis à une séric de ganglions, disposés par paires dans toute l'étendue du thorax et de l'abdomen, et qui constituent la chaîne nerveuse ventrale (Fig. 96). Théoriquement, on devrait toujours trouver trois paires de ganglions thoraciques et onze paires de ganglions abdominaux; mais cette disposition primitive ne so rencontre guère que chez les larves; chez les adultes, il se produit. fréquemment des contractions de la chaîne nerveuse qui amènent la fusion des masses ganglionnaires; quelquefois même, cette fusion est poussée si loin, que toute la chaîne ventrale est contractée en une grosse masse nerveuse située dans le thorax (Sarcophaga carnaria).

Cierveau (ganglions sus-osophagiens). - Il est impossible de comprendre la morphologie du cerveau si l'on ne possède quelques notions préalables sur son développement.

Tout à fait au début de la vie embryonnaire, ainsi que l'a établi Wheeler, le système nerveux d'un Insecte cumprend dix-neuf paires de ganglions primitifs, appelés neuromères (1); disposées les unes à la suite des autres,

(1) Le nombre théorique devrait fitre vingt. 
dans toute l'étendue du corps (Fig. 97) ; les six paires antérieures de ces ganglions rentreront dans l'intérieur de la tête; quant aux treize autres paires qui suivent, elles selviront à former la suite de la chaîne ventrale, dans le thorax et dans l'abdomen. Ce sont surtout les neuromères céphaliques dont le sort nous intéresse; elles correspondent, cela se conçoit facilement, aux six métamères dont nous avons déjà parlé (p. 58), et qui se sont fusionnés pour former la tête de l'insecte.

Ce sont les trois premières paires de neuromères céphaliques, qui vont se fusionner pour donner le cerveau proprement dit; et les masses nerveuses, qui résulteront de cette fusion, correspondent précisément aux trois régions que Viallanes avait déjà distinguées chez les adultes et qu'il avait désignées sous les noms de protocerebron, deutocerebron et tritocerebron.

Les trois paires suivantes de neuromères céphaliques (nos $4,5,6)$, se rapprochent également les unes des autres, et, de leur fusion, résulte la masse ganglionnaire sous-cesophagienne; toute cette masse nerveuse, placée comme on le sait, en arrière de la bouche, et au-dessous de l'œsophage, est, en réalité,

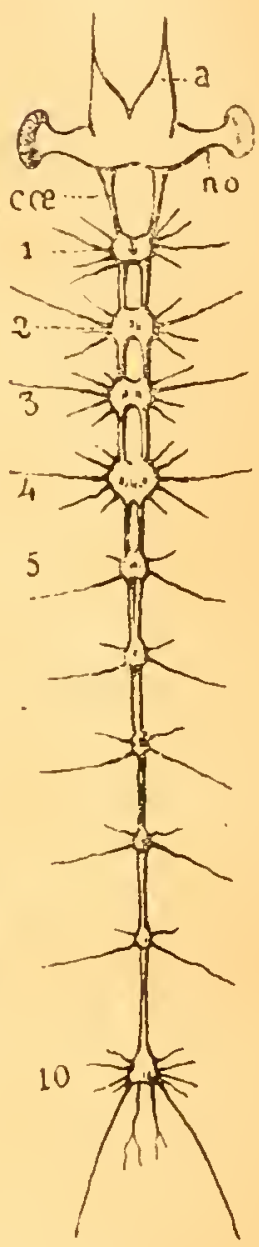

Fig. 96. Le cerveau et la chaine ventrale de la $L o$ custe verte. - 1 . ganglions bousœesophagiens : 2.3.4.ganglions thoraciques : 5-10 gangl. abdominaux. le début de la chaîne ventrale.

Le cerveau est déjà hautement spécialisé (Fig. 98); et, il y a déjà bien longtemps, qu'un des plus savants maîtres de notre Université, le professeur Dujardin, avait proclamé qu'il existe une relation entre sa compli- 
cation et le degré d'intelligence des Insectes; c'est, en effet, chez les Hyménoptères sociaux (Abeilles, Guêpes,

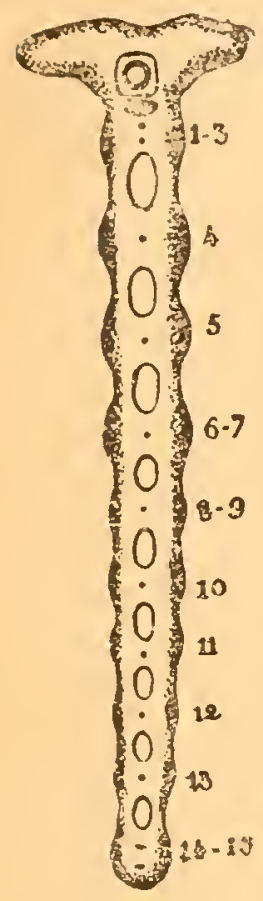

F!g. 97. Déréloppement r?u cerveau et de la chaîne ventrale du $\mathrm{X} i$ phillium ensiferum. Orth. (d'al)rès IV HEsLER). Fourmis), qu'il présente son maximum de différenciation.

Il en est de même, d'ailleurs, en ce qui concerne les organes des sens; chez les Libellules, par excmple où les yeux sont très larges, les ganglions optiques correspondants sont très développés. Par contre, chez les Insectes privés d'yeux, tels que les Coléoptères cavernicoles (Anophthalmus), les ganglions optiques sont complètement atrophiés; ils disparaissent même, ainsi que les nerfs, chez les Adelops, lesquels conservent cependant, comme on le sait, des vestiges de facettes oculaires.

Chaine ventrale. - La chaîne nerveuse ventrale, qui commence aux ganglions sous-oesophagiens, s'étend normalement — lor'squ'il ne s'est produit aucune concentration, -... jusqu'à l'extrémité postérieure du corps; elle est généralement protégée, dans la région du thorax, par des replis internes des téguments. La

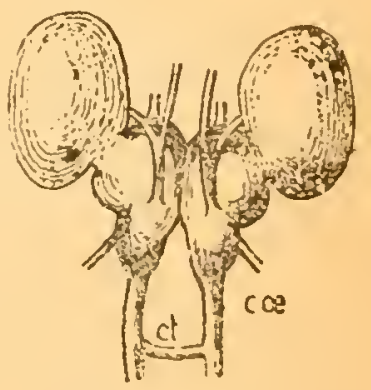

Fiæ. 98. - Cerveau du Criquet bleuâtre : ca, connectifs asophagiens; tt. commissure transverse ce lannesu cesophagien (d'ar. Vtaluanfs). dernière masse nerveuse de la chaîne ventrale résulte, presque toujours, de la fusion de deux ou trois paires de ganglions, qui envoient des ramifications nerveuses au rectum et à l'appareil génital (Fig. 96).

les nerfs, qui naissent de la chaine ventrale, renfer- 
ment deux sortes de fibres : des fibres sensitives, dont les racines paraisscnt se rattacher à la face inférieure des ganglions, et des fibres motrices se rattachant à la partie dorsale. Il parait même résulter, des recherches de Fairre et de Viallanes, que les fibres nerreuses qui proviennent des ganglions cérébroïdes subissaient un véritable entre-croisement.

20 Système nerveux viscéral. -- Le sýstème nerveux viscéral présente, chez les Insectes, une très grande uniformité; d'après Blanchard, on peut y distinguer trois parties principales (Fig. 99):

10 Les ganglions et les nerfs de l'appareil digestif.

20 Les ganglions et les nerfs du raisseau dorsal avec ceux des trachées.

$3^{\circ}$ Les ganglions et les nerfs des stigmates.

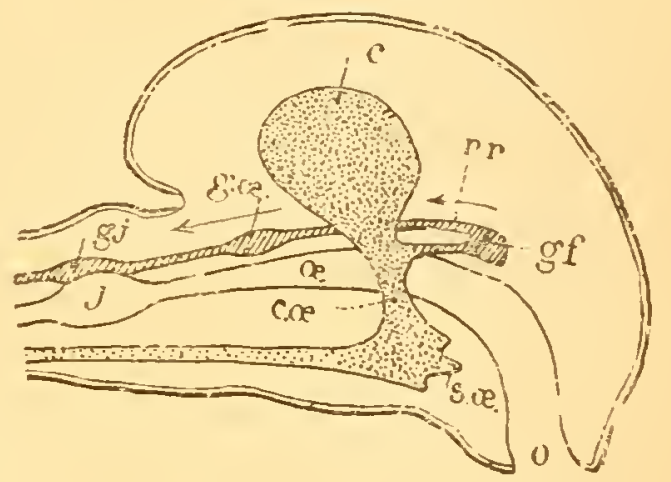

Fig. 99. - Coupe longitudinale de ia tête d'mn Insecte. Blatte orientale; $n$, bouche; $c$. œsophage; i, jabot; $c$, cerveau ; cce, collier œsophagien; sce, gallglions sous-cesophagiens ; af, ganglion frontal; $m r$, nerf récurrent; gce et $g j$, ganglions du tube digestif (inuité de L.IYG).

$1^{\circ}$ Innersation du tubr digestif.- 11 existe trois renflements nerveux (ganglions intestinaux) situés en dessus, sur la ligne médiane du tube uigestif.

Depuis Lyonet, tous les auteurs ont donné, an premier de ces amas nerveux, lo nom de ganglion frontal, parce qu'il est toujours placó en avant du cerveau,dans la partie antérieure de la tête (Fig. 99, gf).

Ce ganglion, en forme de triangle renversé, est relié de chaque côté au cerveau par deux cordons nerveux qui viennent s'insérer à la base des neris antennaires. De són sommet part, en arrière, un nerf unique $(n r$.) qui suit la Iigne médiane de l'œsophage et va passer sous les ganglions cérébroïdes : c'est le nerf récurrent. Ce nerf continue à descendre le long de la face supérieure du tube digestif et 
se renfle, en deux petits ganglions ovoïdes, allongés, qui émettent des branches nerveuses très fines s'étendant sur le gésier et jusque sur le ventricule chylifique.

20 Innervation du vaisscau dorsal et rles trachées. - L Les ganglions destinés à l'appareil circulatoire (ganglions angéiens) sont au nombre de deux paires ; ils sont placés à la partie supérieure de l'œsophage (Fig. $100 a$ et $a^{\prime}$ ) et appliqués, en dessous, contre le vaisseau dorsal ;

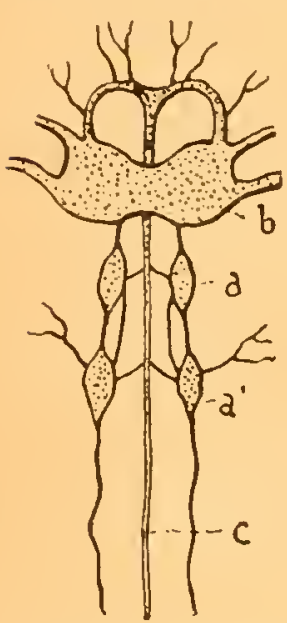

Fig. $100 .-$

Relations entre le système nerveux central et le système viscéral (d'après J. MuLLER). ces deux ganglions sont réunis par une commis sure très courte; ils sont, de plus, l'un et l'autre, ratlachés au cerveau (b) par de minces filets nerveux, et les ramifications qui en partent se distribuent au coum et à l'arte.

A la suite des ganglions angéiens, se trouvent les ganglions trachéens, reliés aux plemiers par un connectif très court; ils fournissent des branches nerveuses aux trachées de la tête, principalement.

30 Innerration res stigmates. - Enfin, il existe un troisième appareil, homologue du premier, impair comme lui, et dépendant, celui-là, non plus du cerveau, mais de la chaine ventrale. Il est constitué par un filet nerveux médian, naissant en arrière de chaque ganglion et descendant entre les deux connectifs. Arrivé près de la masse nerreuse qui suit, ce filet se bifurque et chacune de ses branches, après s'être cllemême renflée en un petit ganglion très allongé, donne un filet grổle qui se rend à l'appareil obturateur de stigmates.

Sauf quelques lragètes modifirations de détail, les dispositions que nous venons de décrire se retrouvent chez tols les Insectes.

$4^{\circ}$ Physiologie de l'appareil neliveux. - Ce sujet a été jusqu'ici peu exploré, on peut admettre que l'appareil nerveux des Insectes fonctionne suirant les mêmes lois que celui des auimaux supérieurs. 


\section{CHAPIT RE XV}

\section{ORGA'NES DES SENS}

Techriqua. - Pourétudier la cornée de l'œil composé d'un Insecte. il faut prélever une parcelle du tégument rhitineux arec la pointe d'un scalpel. Examiner au microscope, dans une goutte de liquide éclaircissant (baume de Canada ou slucérime).

Bien que la littérature, sur ce sujet, soit très riche, nos connaissances sur les organes des sens sont peu nombreuses et peu précises; les organes tactiles et ceux de la vision sont les mieux connus; on sait fort peu de choses sur les autres : ouïe, goùt et odorat.

$1^{0}$ Sens du tollcher. - Il est certain que les Insectes, malgré la rigidité de leur carapace chitineuse, peuvent percevoir les impressions du toucher par toute la surface de leur corps : certaines régions cependant, ou mieux certains organes, tels que les antennes, les palpes et même les extrémités des pattes, paraissent plus spécialement adaptés à la réception de ces sensations.

Quelle que soit leur nature, les sensations tactiles sont perçues par des poils spéciaux (poils sensitifs), qui trarersent les téguments chitineux, et dans l'intérieur desquels pénètre un filament sensitif. Des ganglions cérébroïdes et des ganglions sous-oesophagiens partent des branches nerveuses qui se rendent dans les articles terminaux des antennes et des palpes. Là, de nombreuses ramifications latérales, renflées en forme de ganglions, fournissent des prolongements très fins qui pénètrent à 
l'intérieur des poils sensitifs. La Figure 101 rend très bien compte de cette lisposition.

L'hypothèse qui considère les palpes et les antennes

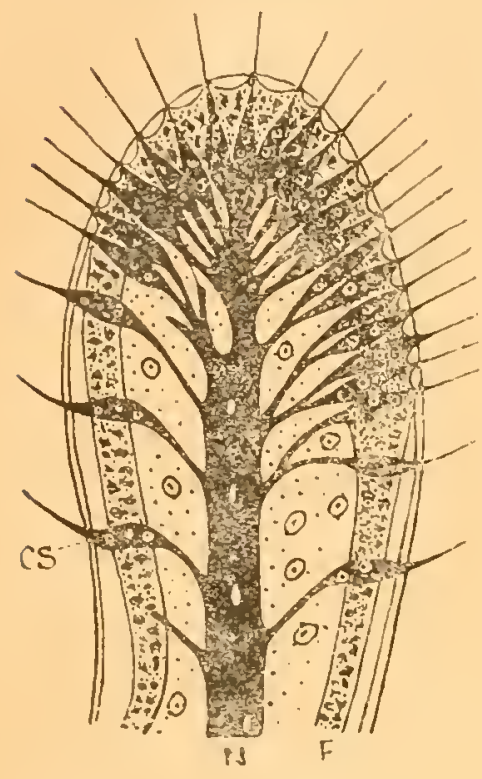

Fig. 101. - Section longitudinale du dernier article d'un palpe de la Sauterelle verte ; $\boldsymbol{x}$. nerf ; cs. poils sensitifs

(d'après ron RATH). comme les organes du toucher, n'a rien d'invraisemblable; lorsqu'on observe les Insectes à l'état vivant, ne les roit-on pas, en effet, agiter continuellement leurs antennes et leurs palpes, comme s'ils voulaient s'orienter et reconnaitre les objets environnants. Tout le monde sait également que, lorsque deux Fourmis ou deux Abeilles se rencontrent, elles se touchent réciproquement avec leurs antennes, afin de se reconnaitre et aussi peutêtre pour se communiquer leurs impressions.

La délicatesse du toucher est certainement très grande chez les Insectes; il est probable qu'ils peuvent acquérir, par cette roie, un grand - nombre de sensations dont nous n’avons aucune idée.

2 Sens du goût. - Tous les auteurs sont d'accord sur le siège des sensations gustatives; celles-cine peurant être perçues que par une surface spongieuse et humide. L'organe adapté à cette fonction devant forcément être en rapport immédiat avec les aliments, au moment de leur introduction dans le tube digestif, on en conclut qu'il doit se trourer dans la cavité buccale. Les membranes molles, qui tapissent la languette et l'épipharynx, réalisent les conditions nécessaires à cette fonction, et 
c'est là, évidemment, qu'on doit placer le siège c'e la gustation chez les Insectes.

Les organes chargés de recueillir les impressions gustatives sont des poils très délicats, situés au fond de petites fossettes dans lesquelles peuvent s'amasser des liquides. La distri- $p$ bution des poils gustatils rarie suirant les différentes classes d'Insectes.

\section{$3^{\circ}$ Sens de l'odorat. - Les Insectes sont} capables de percevoir les odeurs, ce fait est hors de doute; tout le monde sait, en effet, que les Nécrophores, les Bousiers, les Mouches, sont attirés de fort loin par les substances dont ils se nourrissent; les Abeilles savent très bien découvrir les aliments sucrés, les Guêpes perçoivent sùrement l'odeur des fruits. Mais, si l'olfaction est indiscutable, on est loin d'être exactement fixé sur le siège de cette importante fonction.

Nous n'entrerons pas dans le détail des (1'ardap).

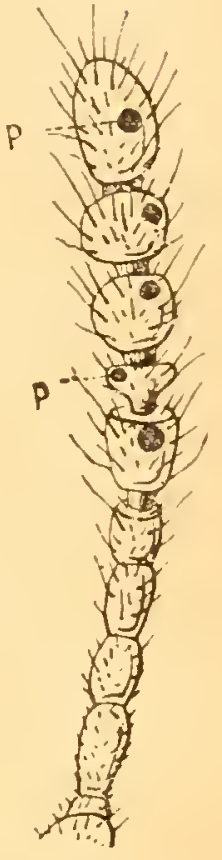

Fig. 102. Une antenne, Adelops hirtus laissant roir les fossettes olfactives

(d'après PAClongues discussions auxquelles ce snjet a donné lieu; nous nous bornerons à dire qu'aujourd'hui, en dépit des expériences contradictoires de Lehmann, de Hensen et de Landois, on peut conidérer les antennes comme étant " principalement " le siège de la fonction olfactive chez les Insectes (Fig. 102).

Il est d'ailleurs très difficile de distinguer les organes olfactifs des organes gustatifs, attendu qu'ils possèdent probablement, à très peu de chose près, la même structure anatomique. Des expériences seraient nécessaires pour séparer, d'une façon précise, ces deux catégories d'organes. 
4. Sens de la vision. - Comme nous l'avons déjà expliqué précédemment (p. 47), on trouve deux sortes d'yeux chez les Insectes : des yeux simples, stemmates ou ocelles, caractérisés par leurs dimensions, toujours très petites, et par leur cornée lisse ; puis des yeux composés ou à facettes, généralement beaucoup plus grands que les yeux simples et dont la cornée forme un réseau régulier, très élégant, de mailles circulaires (quelques Coléoptères) ou hexagonales.

A l'état adulte, un grand nombre d'Insectes possèdent, à la fois, des ocelles et des yeux composés ; les ocelles sont, en général, au nombre de trois, placés au sommet ou à la partie antérieure de la tête, entre les yeux à facettes.

A l'exception de quelques Névroptères et des Libellulidés, on ne trouve jamais, chez les larves, que des ocelles placés sur les côtés de la tête et non

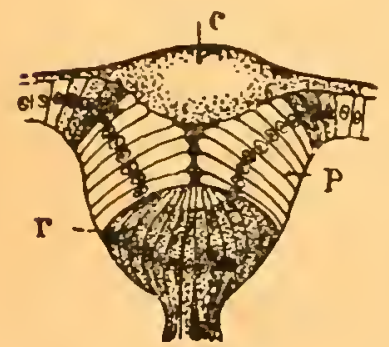

Fig. 103. - Section d'un ocelle chez une jeune larve de Dytique : $c$. lentille cornéenne; $p$. hypoderme modifié: r.cellules réti . niennes

(d'ap.GRENACHER). sur la ligne médiane du corps comme chez les adultes; leur nombre varie, de une à six paires; exceptionnellement, il s'élève jusqu'à vingt chez les larves des Panorpes.

Ocelles. - Réduits à leur plus simple expression, les ocelles consistent essentiellement en une réunion de bâtonnets cristallins, placés sous des cellules hypodermiques modifiées, formant une sorte de cornée transparente; le tout est recouvert par une portion de la cuticule épaissie en forme de lentille (Fig. 103). Les cellules hypodermiques, placées au fond de l'invagination oculaire (rétine), se continuent par des fibres dont l'ensemble 
contitue le nerf optique. Si rudimentaires qu'ils soient, ces ocelles sont déjà plus compliqués que les yeux simples des Crustacés inférieurs; on ne peut alors les comparer qu'aux yeux lentifères des Arachnides et des Myriapode.

Yeux couposés. - La structure des yeux composés, chez les Insectes, est absolument identique avec celle que l'on rencontre chez les Crustacés (1). Chaque œil est formé comme on le sait, par une réunion d'ommatidies, et chaque ommatidie se compose, d'après Grenacher, de deux parties bien distinctes: un appareil sensitif, la rétinule, surmontée d'un appareil dioptrique transparent. La rétinule comprend le rhabdome et les cellules rétiniennes ou rhabdomères, dont le pied s'effile en une fibre nerveuse (axone rétinulaire) qui s'échappe de l'œil à travers la membrane fenètrée pour gagner le periopticum; dans l'appa-

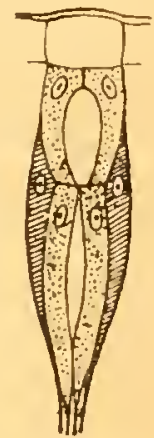

Fig. 104. - Une ommati die isolée (imité re GRE Y 1. CHER). reil dioptrique on trouve : la cornéule, les cellules cristalliniennes et le cône cristallinien. La rétinule et les cellules cristalliniennes sont entourées par des cellules pigmentaires (Fig. 104).

A chaque ommatidie correspond donc une cornéule ou épaississement de la couche cuticullaire; il en résulte que la cornée commune est constituée par autant de petites facettes distinctes qu'il y a d'ommatidies.

Grenacher distingue, chez les Insectes, trois espèces d'yeux composés, suivant que les cellules cristảlliniennes renferment ou non des cônes cristallins.

$\mathrm{I}^{\mathrm{O}}$ Les yeux acones (sans cristallin) se rencontrent

(1) Pour les détails, of : E. K.It.T. Anatomie et Physiologie comparées de l'apparcil oculaire (Encyclop. franc. d'opthalmologie, t. h. IT, 1905, p. 685-933. Voir pour les In ectes, 1. 736- 777 avec fig. 78579$)$. 
chez les Tipulidés, les Forficulidés, les Hémiptères hétéroptères et les Coléoptères (pentamères exceptés).

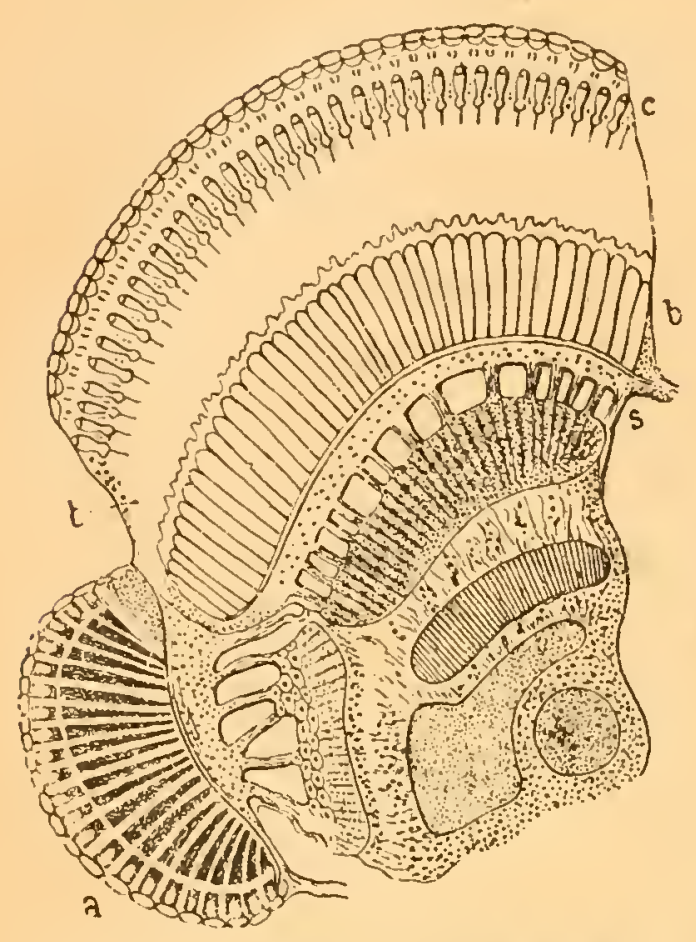

Fig. 105. - Section longitudinale de l'œil composé du Leptophlebia cinctx Névropt. $C$, zone des cônes cristallins: $s$, périphérie du nerf optique: $t$, zone de teminaison des trachées ; $a$, œil latéral ; $b$, zone inferieure des bâtonnets

(d'après C. Zinmer).

20 Les yeux pseudacones (cristallin fluide), s'observent chez un grand nombre de Diptères (Muscidés). $3^{\circ}$ Les yeux cucones (cristallin solide), caractérisont les Coléoptères pentamères, les Hyménoptères, les Orthoptères, les Lépidoptères, etc.

En réalité, la différence qui existe entre les. vaux simples et les yeux composés, n'est pas si profonde qu'cilc le paraît au premier abord; elle réside principalement en ceci, que l'œil simple possède une cornće commune, qui recouvre à

la fois toutes les ommatidies; tandis que, dans l'œil composé, chaque ommatidie possède sa cornée spéciale. Il en résulte que, si, comme on le croit, l'ommatidie constitue la partie élémentaire et fondamentale de l'oeil des Arthropodes, l'oeil à facette peut être considéré comme une agglomération d'yeux simples (Fig. 105).

Le nombre des facettes est très variabie suivant les Insectes; on en compte douze chez les Lépismes, ciuquante dans les Fourmis et plus de 4.000 chez la Mourh domestigue. Les Insectes les plus riches, sous ce rapport, sont les grands Libellulidés (Aschne) où l'on compte plus 
de 20.000 facettes (Fig. 106) ; un Coléoptère (Mordella) en a 25.000 au moins ; enfin, chez le Sphinx du Liseron, le nombre des facettes est, parait-il, voisin de 27.000.

ThÉorif DE I.A vision. - Maintenant que nous connaissons la structure des yeux, il resterait à expliquer le mécanisme de la vision; plusieurs hypothèses ont été proposées, mais jusqqu'ici aucune d'elles ne paraît absolument convaincante. Il est un fait certain, c'est que, derrière chaque cornéule, se produit une petite image totale et renversée des objets. Comment cette image impressionne-t-elle ensuite les éléments nerveux? C'est là ce qu'on ne sait pas; les cônes cristalliniens, qui viennent à la suite des cornéules, déforment notablement les images; ils ne transmettent aux rétinules que le faisceau très étroit des rayons lumineux parallèles à leur axe. L’impression reçue par chaque ommatidie se réduit donc, en quelque sorte, à une plage lumineuse uniforme sans aucun défail. Cependant les récentes études de M. Paul Vigrer, relatives aux

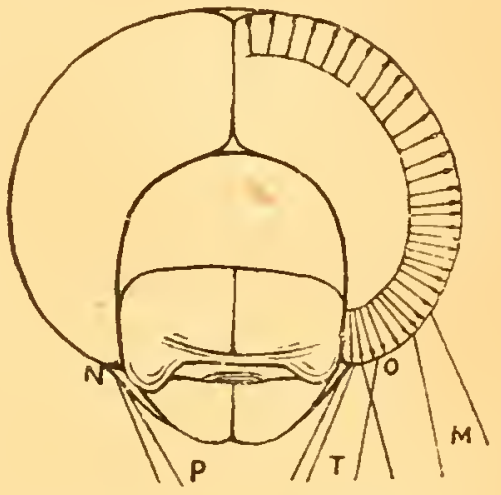

Fiæ. 106. - Schéma de l'appareil optique chez unc Libellule; $I, P, T$, rayons lumineux arrivant à la cor. née sous diverses inciclences; $o$, direction des rayons cans l'apparcil clioptrique (d'après TïMPEL). voies de transmission qui unissent les éléments photo-récepteurs aux centres visnels, semblent éclairer d'un jour noureau cette importante question. Filles expliquent notamment de quelle facon les petites images renversées, fournies p r les cornéules, peuvent se fusionner en une impression unique, 11.) véritable neuro photogramme, dans le périopticum (1).

\section{$5^{\circ}$ Sens de l'ouïe. - Bien que nos connaissances soient} cncore très incomplètes en ce qui concerne les organes auditifs, les recherches de Leydig et de Graber permettent cependant d'entrevoir par quel mécanisme les vibrations sonores peuvent être recueillies par les Insectes.

(1) Viaier (P). - Mecanisme de la sunthese les impressions lumineuses recueillies par les yeux composés dles Diptères (C. R. Acad. des Sciences, Paris, 1909, v. 1221).

LES IN3ECTEZ. - 2e édit. 
On trouve, en effet, fréquemment, chez ces anmaux, des organes ayant la disposition suivante.

Une cellule nerveuse, placée sous les téguments, présente deux sortes de prolongements : du côté de la peau, cette cellule se continue par un filament grêle terminé par un léger renflement (clou scolopal); ce filament, ainsi que son renflement terminal, sont logés à l'intérieur d'un tube chitineux (scolophophore) provenant de la modification.d'une cellule hypodermique (Fig. 107, sc); il en résulte que, par leur base, les scolophophores sont en contact

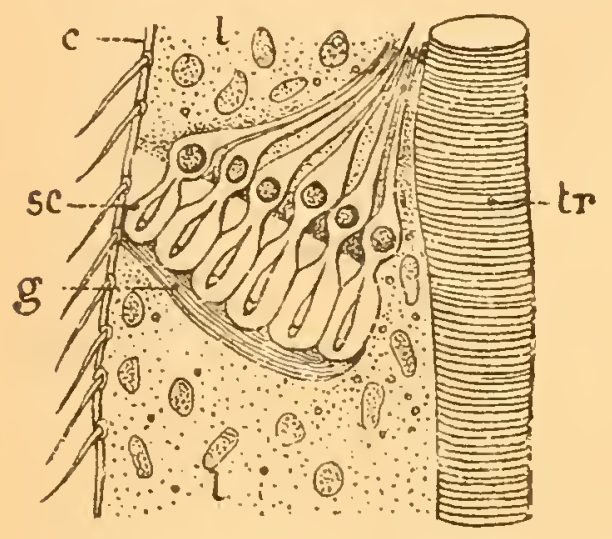

Fig. 107. - Siclıèna G'un aplareil chordotonal: sc. scolophophores a vec leur clou scololval à l'intérieur ; $t$, trachée ; $c$, cuticule ; $\theta$, ligament conjonctif reliant l'appareil chordotonal à la peau ; $l$. leucocytes (d'après GrABER). immédiat avec les téguments; ceux-ci, d'ailleurs dlans les régions auditives, sont toujours réduits à des membranes flexibles, fortement tendues.

Le prolongement qui part de l'autre extrémité de la cellule nerveuse se dirige vers l'intérieur du corps; ce n'est autre chose qu'une fibre acoustique, chargée de mettre l'organe récepteur en communication avec les centres; il va sans dire que la réunion de plusieurs fibres formera les nerl's.

Il est rare que les cellules nerveuses avec leur appareil scolopal restent isolées; généralement, elles se réunissent en groupes plus ou moins nombreux, de manière à constituer ces appareils auxquels on a donné le nom d'or'ganes chordotonaux (Fig. 107).

Orraves chondotonatix. - Jes organes chordotonanx onl ilé obserrés dans tous les ordres d'Insectes; ils sont ordinairement métamériques, surtout chez les larves. Ils peuvent se développer sur tous 
les points de la surface du corps; et, de fait, on les troure par groupes, de deux à deux cents, un peu partout; sur les antennes, les palpes, les pattes, les ailes et môme à la surface supérieure de l'abdomen.

Chez. quelques Insectes, l'appareil auditif, hautement différencié, est toujours localisé sur certains organes. C'est ainsi, par exemple, que chez les Locustidés et les Gryllidés (Orthoptères), il se présente extérieurement sous l'aspect de deux membranes ovales, tendues sur un cadre chitineux, et placées, de chaque côté; sur les tibias des jambes antérieures (Fï. 109). La membrane chargée de recueillir les vibrations sonores a été comparée à un tympan, ce qui fait que. l'on désigne, le plus souvent, l'appareil auditif des Orthop tères sous le nom d'organe tympanal, mais il ne faut pas oublier que ces appareils,

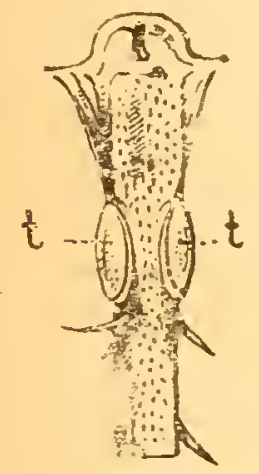

Hiy. 109. Jambe antérieure ile la $S a u$ terelle verte pour montrer la position les organes aujitifs, $t$. (d'ap. LANG). malgré leur apparente complication, ne sont autre chose que des organes chordotonaux localisés et constitués par un grand nombre de cellules nerveuses.

Chez les Acridiens, l'organe tympanal est situé sur les côtés du premier anneau abdominal.

En général, l'une des trachées qui passe au voisinage d'un organe tympanique, se renfle en une vésicule, constituant ainsi, probablement, un appareil de résonance. Très souvent aussi, des ligaments tendus rattachent l'ensemble de l'appareil auditif aux téguments externes; il en résulte que, si la paroi du corps reçoil des vibrations sonores, ces vibrations sont transmises, par le ligament, aux cellules nerveuses avec lesquelles il est en relation.

6 Bruits produits par les Insectes. - Les Insectes, tont le monde a pu le remarquer, sont capables de produire certains bruits. On admet généralement que ces bruits sont en rapport avec la fonction génératrice, et on les considère comme des appels sexuels; mais cependant. dans bien des ras, les sons émis paraiscent être éga- 
lement des moyens de défense, ayant pour but d'efirayer les adversaires ou les ennemis.

Nous nous bornerons à signaler ici le cas des Anobium (A. pertinax) où le bruit résulte uniquement d'un choc produit par l'Insecte en frappant sa tête contre les parois solides des galeries dans lesquelles il passe sa vie. Partout ailleurs, il existe des organes spéciaux pour la production des sons, et ces organes peuvent se rapporter à troịs catégories différentes.

$1{ }^{\circ}$ L'appareil bourdonnant (Hyménoptères et Diptères) paraît être, pour la plus grande partie, sous la dépendance des organes respiratoires et musculaires.

$2 \circ L^{\prime}$ appareil stridulant (Orthoptères et Coléoptères) comprenant toujours deux organes voisins, frappant l'un contre l'autre.

$3^{\circ}$ L'appareil tympanique des Cigales (Hémiptères), formé d'une Inembrane tendue et mise en vibration par les contractions rapides r'un muscle.

A. Bourdonnenent. - On croit volontiers, mais à tort, que le bourdonnement est produit par le mouvement rapide des ailes; il n'en est rien. Ce bruit particulier si caractéristiques de Hyménoptères (Bourdons, Aheilles, etc.) et des Diptères (Mouches, Cousins,etc.) continue ì se produire lorsque les ailes sont maintenues immobiles et mîme lorsqu'elles sont coupées. On sait aujourd'hui que le bourdon. nement est produit par les vibrations de la paroi du thorax, sous l'action des muscles tégumentaires et par le passage rapide de l'air ì l'orifice des stigmates.

On perçoit nettement ces vibrations de la paroi thoracique, en plaçant le doigtsur le dos d'un Bourdon.

B. Stridulation. - La stridulation est toujours produite par le frottement des organes les uns contre les autres: ainsi, par exemple, dans l'ordre des Coléoptères (Nórop'ures, Clythra, Criocires), ce sont les bords postérieurs des élytres qui viennent frotter contre deux sortes de petites rîpes situées à la partie supérieure des segments abdominaux ; chez les Géotrupes, la rîpe se trouve sur la cuisse de chaque patte postérieure, et elle frotte sur un appendice du troisième segment abdominal (1).

Chez les Acridiens, la stridulation, souvent très intense, est produite par le frottement des fémurs postérieurs contre le bord antérieur des

(1) Nous cevons prévenir que ces aplareils, dont le relief est très faible, ne se roient hien que sur des Insectes frais. 
élytres. Enfin, chez les Locustaires et les Crylloniens (Fig. 119), rui sont les mieux organisés sous ce rapport, l'appareil stridulant est toujours placé à la base des élytres ; l'élytre gauche (I ocustes) porto une grosse nervure dentelée en dessous, c'est l'archet ; dans la région correspondante de l'élytre droite, se trouve une membrane transparente (champ tympanal), dont les bords sont rugueux et constituent la chanterelle; ici, la stridulation est produite par'le frot tement de l'archet contre la chanterelle : le son se trouve considérablement renforcé nal la vibration de la membrane tympanalf ; lorsque les élytres sont trè réduits, comme cela a lier chez les Ephippigers, lc. champs vibraioires sont néannoins ronserrés. Chez les Grillons, les deux élytres sont orgranisés de la même manière, l'archet de l'un met en mouvement l'appareil vibratoire de l'autre.

Partout où il existe, dans l'ordre des Orthoptères (Acridiens, I.ocustiydes, Crillonniens), cesont toujours les mâles, seuls, qui utilisent leur appareil stridulant; cette particu-

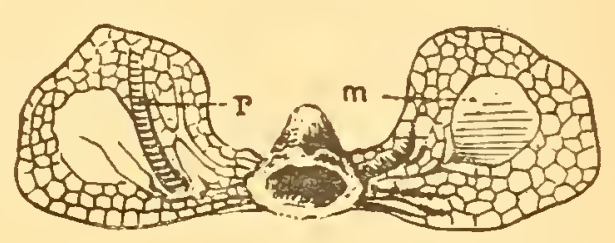

Fig. 110. - Appareil stridulant de l'Ephippiger vitium Orth. $m$. champ tympanal ; $r$, archet (d'après nature). larịté était bien counue des anciens, et le poète grec Xénarque félicite mélancoliquement les Grillons d'aroir" "des femmes silencieuses».

C. Apparerl ty upanigue des Gigales. -- L'appareil tympanique de la Cigale (Hémiptères) est le plus compliqué de tous les organes stridulants. Ainsi qu'Aristote l'avait déjà observé, il est placé "sous la ceinture des mâles ", c'est-à-dire à la base et sur lês côtés du premier segment abdominal.

Cet appareil est essentiellement constitué par une membrane parcheminée et convexe. la timbale, tendue sur un cadre chitineux; un petit muscle est fixé au milieu de la face interne de la timbale; par ses contractions rapides, ce muscle provoque un mouvement de va-et-vient de la membrane, qui rend un son très perçant; c'est, comme on le voit, le même principe que celui du jouet populaire connu sous le nom de cri-cri. Tout cet ensemble est placé à l'intérieur d'une carité faisant l'office d'une caisse de résonance et protégé par ın large opercule (colot).

Les Grecs aimaient beaucoup la voix des Gigales ; comme les Chinois de nos jours, ils les enfermaient dans de petites cages en osier pour se donner le plaisir de les entendre chanter. 


\section{QUATRIEME PARTIE}

\section{Fonctions de Reproduction}

\section{CHAPITRE XVI}

\section{APPAREIL REPRODUCTEUR}

TғснNiQ!e. - Ouvrir un Hanneton, comme nous l'avons indiqué à propos de l'appareil digestif (p. 97).

Guivant les cas, les gaînes ovariques ou les testicules, se voien! facilement, dans la partie postérieure de l'abdomen.

10 Généralités sur l'appareil reproducteur. - A part quelques cas tout à fait exceptionnels d'hermaphroditisme, les sexes sont toujours séparés chez les Insectes.

Les organes génitaux sont situés dans l'abdomen et les canaux, chargés de conduire au dehors les éléments reproducteurs, s'ouvrent toujours près de l'extrémité postérieure du corps, dans les intervalles qui séparent les derniers segments abdominaux, généralement entre le huitième et le neuvième.

Fondamentalement, aussi bien chez les màles que chez les femelles, les organes génitaux sont ćtablis sur le même plan; examinés dans les formes primitives (Thysanoures), ils comprennent, toujours, deux tuhes plus 
ou moins ramifiés et indépendants. Ces tubes peuvent rester libres et séparés pendant toute la vie, (Ephémérides); mais, le plus souvent, ils se réunissent à leur base de manière à constituer un canal évacuateur unique.

A ce canal s'ajoutent, le plus souvent, des organes accessoires:réservoirs séminaux, poches copulatrices, etc., ainsi que des appendices variés (armures génitales) destinées à favoriser l'accouplement.

Pour faciliter l'étude de l'appareil reproducteur, nous allons-donc distinguer deux groupes d'organes dans chacun des sexes.

10 Les organes essentiels ou primaires, qui comprennent les glandes sexuelles (ovaires et testicules) avec leurs canaux vecteurs et leurs accessoires, (canaux déférents, poche copulatrice, vésicule séminale).

20 Les organes secondaires ou armures génitales comprenant :

(a) L'appareil copulateur des mâles.

(b) L'appareil ovipositeur des femelles.

2 - Évolution de l'appareil reproducteur. - Tout à fait au début, c'est-à-dire pendant les première phases du développement Irvaire, les organes génitaux ont le même aspect et la même structnre dans les deux sexes; ils ne se différencient, dans leurs formes et dans leurs fonctions, qu'au moment de la maturité sexuelle, c'està-dire au cours de la période nymphale.

Les glandes génitales, ainsi que leurs canaux, tirent leur origine d'une paire de noyaux mésodermiques; mais les conduits vecteurs se relient, généralement, à des invaginations correspondantes de la cuticule, par conséquent d'origine ectodermique, desquelles dérivent, à leur tour, tous les organes secondaires, c'est-à-dire l'armure génitiile.

On peut considérer les glandes génitales comme des néphridies 
transformées, ce qui explique pourquoi le nombre des canaux excréteurs est toujours deux $(c d)$ : c'est, en effet, ce nombre qu'on rencontre dans les formes primitives, telles que les 'Thysanoures et les Éphémérides; on retrouve de plus, chez quelques-uns de ces Insectes, une disposition métamérique très netıe des testicules et des follicules ovariens (Fig. 111,li).

Dans tous les autres groupes d'Insectes, les deux can aux s'unissent

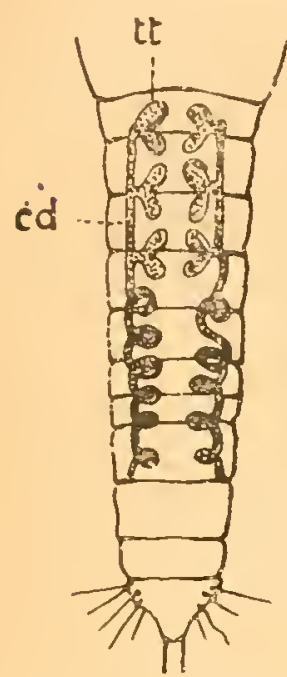

Fig. 111. -Appareil génital mâle du Lepisme, pour montrer la disposition métamérique ; $t t$, tes ticules ; cl, canaux déférents (d'ap. Gr.1ssi). à leur base de manière à constituer un canal unique. Déjà, chez certains Thysanoures, Japıx, Mcchilis, on voit se réaliser la soudure des canaux déférents; mais chez les Lépismes, pendant toute la durée de la période embryonnaire l'arıangement métamérique persiste, et les deux conduits séminaux s'ouvrent séparément à la base de l'abdomen (Fig. 111).

En ce qui concerne les autres Insectes terrestres ailés, la disposition la plus simple de l'appareil reproducteur s'observe chez les Orthoptères (Forfirulides) ; cependant, la concentration des organes, pait toujours disparaître la disposition métamérique.

Chez les Hyménoptères, l'appareil reproducteur est encore très simple, mais les variations sont déjà tellement nombreuses qu'il est impossible de donner aucune indication générale.

Enfin, chez les Lépidoptères, les recherches récenles de Jackson (1) ont montré qu'on pouvait reconnaître trois stades dans l'évolution des conduits génitaux : un stade éphéméridien, qui se termine à la fin de la vie larraire : un stade ortpoptéridien, indiqué pendant la période de repos qui précède la nymphose; enfin, un stade définitif ou lépidopuéridicn qui commence au début de la vie chrysalidienne.

\section{$3^{\circ}$ Appareil reproducteur des mâles. - L'appareil} génital des mâles comprend les parties suivantes :

10 I es deux testicules, destinés à produire les spermatozoïdes.

20 Les conduits séminaux (canaux déférents), sur le

(1) JACkson (W. H.). - Zool. Anzeig. 18 9. Jakr. XI1, 1. 6:22-8:6. 
trajet desquels s'obserre un renflement qui est la vésicule séminale.

$3^{0}$ Le canal éjaculateur commun terminé par le pénis.

$4^{\circ}$ Les glandes accessoires (glandes à mucus), dont le produit se mélange au sperme ou sert à l'agglomération des paquets séminaux désignés sous le nom de spermatophores.

Escherich a démontré que l'appareil le moins cornpliqué se rencontrait chez les Coléoptères (Carabiques), où les testicules se présentent sous la forme d'un simple tube, faiblement contourné à son extrémité (Fig. 112).

A.TeSticlif S.- Chez un grand nombre d'Insectes, les testicules sont constitués par un assemblage de petits tubes aveugles, pelotonné sur eux-mêmes et grénéralement enfermés dans une enveloppe commune ; chaque tube testicu-

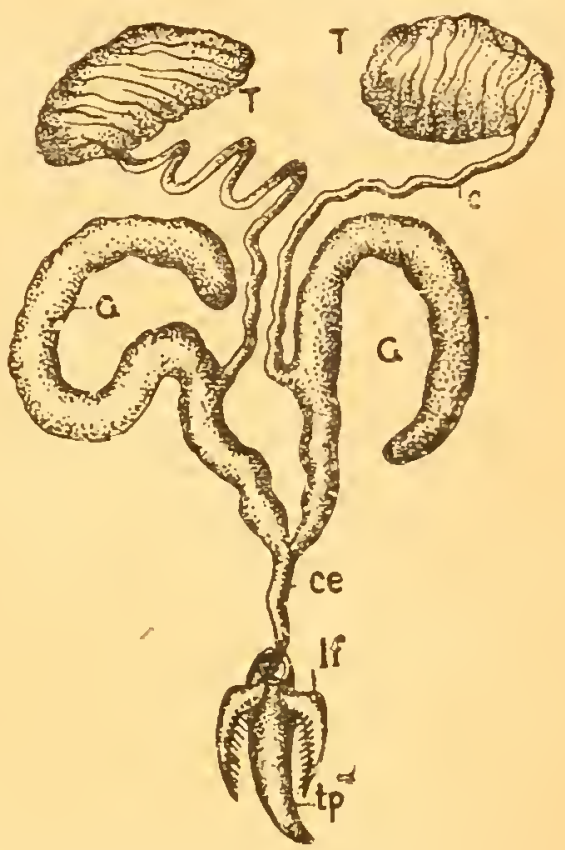

Fig.112. - Appareil génital mâle du Berosus cepha!otes (CoL).T, testicules: $c$, canaux dérérents : $G$. glandes accessoires ; ce, canal éjaculateur; $i f$, valves de l'armure génitale; $t p$, tube pénial (d'après L. BORDAS). laire est muni d'un petit canal excréteur ; tous ces conduits se réunissent ensuite pour former, de chaque côté, un canal déférent unique (c.)

Les deux testicules sont généralement séparés l'un de l'autre; cependant, dans un certain nombre d'Insectes, et notamment chez les Lépidoptères, ils sont si étroitement unis qu'on a, pendant longtemps, considéré leur masse impaire comme un testicule simple. On observe d'ailleurs, dans cet ordre, lorsqu'on suit le développement des larves, tous les degrés d'évolution, depuis les testicules séparés des Hépialides, jusqu'aux testicules soudés les plus répandus. C'est pour résumer ces faits si intéressants, que Cholodkowsky (1) a proposé de distinguer les quatre types suirants dans l'évo-

(1) Cholonkovisk (N. A.). - 1. T. ITT. n. $214-215$; T. VII n. 564-5c, 
Intion phylogénétique des glandes génitales chez les Lépidoptères: $1^{\circ}$ Le type primitif ou embryonal (hépialidien), où les deux testi. cules, ainsi que les follicules séminaux, sont entièrement libres (ex. : Hepialus humilis).

$2^{\circ}$ Le type larval (saturnidien), avec deux testicules séparés, mais dont les follicules sont enveloppés chacun dans une membrane scrotale (ex. : Saturnia pyri).

$3^{\circ}$ Le type pupal (lycrnidien), où les deux testicules sont réunis en une seule masse par une membrane frangée (ex. : Lycana).

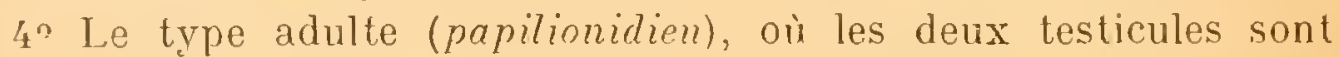
entièrement soudés et recouverts d'une membranc scrotale sous laquelle les follicules sont enroulés autour de l'axe de la glande (ex.: Pieris, Vanessa, etc.).

B. Ganaux déférents. - Ce sont des tubes très fins, dont la longueur varie beaucoup; au point où ils se réunissent commence le canal éjaculateur, qui résulte, lui, comme on le sait, d'une invagina tion hypodermique. Soit à leur base, soit en un point quelconque de leur parcours, les canaux déférents ce renflent en une espèce de sac. la "ésicule sćminale, servant à emmagasiner le sperme, au fur et à mesure qu'il est sécrété par les testicules (Fig. 112, G).

La forme et la position de la vésicule séminale varient à l'infini.

C. Canal éjaculateur. - Le rôle principal du canal éjaculateur est de porter le sperme dans la poche copulatrice de la femelle ; il hui faut, pour cela, une certaine rigidité. Dans ce but, sa partie terminale, fortement chitinisée et évacinable, est pourvue de puissints muscles qui permettent à l'animal de la laire saillir au dehors ou de la rentrer à volonté à l'intérieur du corps. Cette portion terminale chitinisée du canal éjaculateur a reçu le nom de pénis (tp.) ; son aspect el ses dimensions sont extrêmement variables suivant les Insectes. Le pénis est généralement protégé par des valvules ou des plaques de formes diverses: il est également garni de crocliets destinés à favoriser l'accouplement (Fig. 112, lf).

D. Grandes accessorres. -- La position des glandes accessoires est variable; elles viennent, le plus souvent, déboucher au point de jonction des conduits séminaux et du canal éjaculateur; suivant sa consistance, le liquide gu'elles sécrètent sert à diluer le sperme ou bien à former, antour des faisceaux de spermatozoïdes, des capsules qu'on a désignées sons le nom de spermatophores.

Spermatozö̈res. - Comme chez les animaux supérieurs, ce sont 
des rorpuscules excessivement petits, qui abondent dans le liquide séminal; ils ont la forme de filaments renflés en une tête à leur extrémité antérieure : leur corps est terminé par un long flagelle doué de mourements rapides. Chaque spermatozoüde représente une cellule modifiée, homologue de celles qui, dans les ovaires, donnent naisance aux ovules (Fig. 113).

Spermatogenese. - Nous ne nous arrêterons pas aux détails généranx de la spermatogénèse, parce que les phénomènes qui la caractérisent ne diffèrent pas, dans leur ensemble, de ce qui s'observe chez les autres animaux; nous nous bornerons seulement à signaler ici quelques particularités intéressantes, qui paraissent très réprandues chez les Insectes.

Ainsi, par exemple, Henking a trouvé que le noyau des cellules spermatiques primordiales, celles qui, morphologiquement, correspondent aux ovules primordianx, renferme toujours 24 chromosomes; retenons ce nombre, nous le retrouverons dans l'ovogénèse à propos du curieux phénomène d synapsis.

De plus, au lieu de nager individuellement dans. le liquide spermatique, les spermatozoïdes des Insectes restent ordinairement associés en faisceaux; dans les espèces où l'appareil copulateur est très réduit, ces faisceaux sont enreloppés par la sécrétion des glandes à mucus et ils constituent les spermatophores dont nous avons déjà parlé. C'est dans cetétat qu'ils sont introduits dans le vagin de la femelle ou simplement déposés à l'entrée des orifices génitaux.

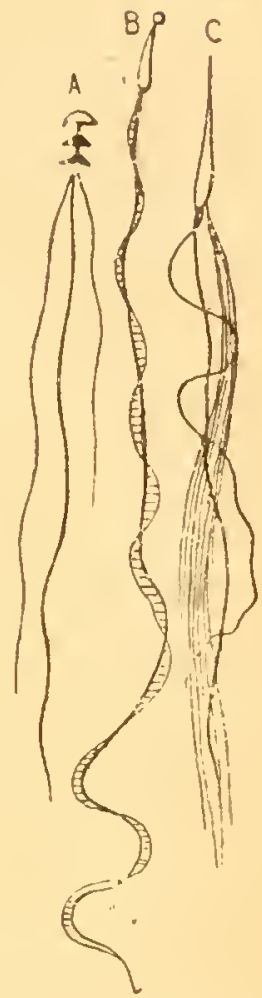

Fig. 113. Formes direrses des spermatozoïdes chezles Coléoptères.

1, chez un Carabique; $B$ et $C$. chez un Lamellicorne (d'après.i.. BALLOWITZ).

Spermatosyzigie. --- Le phénomène désigné par Ballowitz sous le nom de spermatosyzygie, et qui consiste en l'accollement transitoire de deux spermatozoïdes ou plus, par la région de la tête, est trop peu connu pour qu'il soit utile d'entrer dans quelques détails à ce sujet. On ne roit pas non plus, d'ailleur's, quelle peut en être la signification phrsiologique.

\section{Appareil reprodeuteur des femelles. - Construit} sur le mème plan que celui des màles, l'appareil génital des femelles comprend: 
10 Les deux ovaires avec leurs oviductes.

$2^{\circ}$ Le canal ovarien, résultant de la soudure des deux oviductes, et dont la partie terminale forme le vagin.

$3^{\circ}$ Le réceptacle séminal.

$4^{\circ}$ La poche copulatrice.

$5^{0}$ Les glandes accessoires.

On observe également un certain nombre de pièces squelettiques modifiées, servant à la ponte des œufs (oviscapte, tarière) ou d'appareil de défense (aiguillon), et dont l'ensemble constitue l'armure génitale.

A. Ovaires et gaines ovariques. - Il existe deux ovaires, symćtriquement placés, dans l'abdomen, de chaque côté du tube digestif ; chacun d'eux est formé d'un certain nombre de tubes coniques (aaines orariques), le plus souvent réunis en faisceau, et venant s'ouvrir, à leur partie inférieure, dans l'oviducte (Fig. 114).

Dans chaque gaine ovarique on peut distinguer trois régions : $1^{\circ} \mathrm{le}$

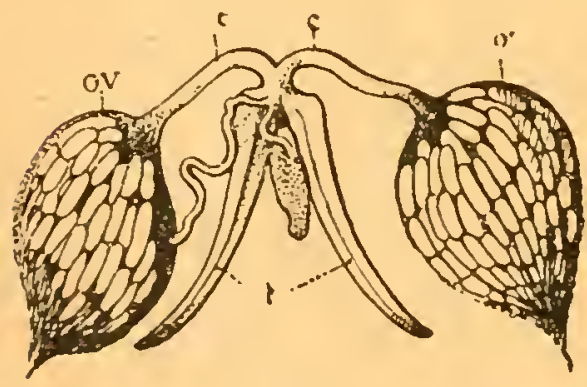

Fig. 114. - Appareil génital femelle du Dectique verrucivore ; $o r$, ovaires ; $;,$, oviductes ; $t$, oviscapte (d'après nature). filament terminal $; 2^{\circ}$ la chambre terminale $; 3^{\circ}$ le tube ovarien proprement dit, dans lequel se trouvent les ovules à divers degrés de développement.

Tous les filaments qui terminentles gaines ovariques se réunissent, de chaque côté du corps, en un seul faisceau; et ce faisceau, destiné à maintenir l'ovaire en place, va se fixer luimême au diaphragme qui sert de support au vaisseau dorsal.

La chambre terminale renferme des éléments cellulaires non diftérenciés (rellules ritelloñines); ce sont ces éléments qui, dans le cours du développement, produiront les œufs ainsi que toutes les parties constituantes internes des gaines ovariques.

I.a troisième région est divisée en chambres successives, et c'est dans ces chambres que l'on trouve les ovules à divers degrés de maturation; les plus âgés se trouvent à la base des tubes, prêts à passír dans les oviductes. 
Dans certains cas, les oviules se succèdent sans interruption dan: toute la longueur des gaines ovariques, et on ne trouve plus aucune trace des cellules nutritives dans la chambre terminale: cette disposition, qui parait la plus primitive de toutes, s'observe chez. les Thysanoures (Campodea excepté) et chez les Orthoptères.

D'autres fois, il existe, dans la chambre terminale, une réserve nutritive plus ou moins abondante, et chaque ovule reste en communication avec cette réserve par l'intermédiaire d'un petit pédoncule qui lui transmet la nourriture dont il a besoin. ( ${ }^{\top} n g r a n d$ nombre d'Hémiptères, Fig. 115).

Enfin, dans une dernière disposition, qui s'obserre chez les Hyménoptères, les Lépidoptères, de nombreux Coléoptères, des chambres"à cellules nutritives alternent régulièrement avec les chambres ovariennes; chaque œuf est donc, lans ce cas, accompagné de la réserve nutritive qui lui est nécessaire.

B. Oviductes et canal orarifax.Ces conduits, qui font suite aux gaines ovariques et qui servent à l'évacuation des œufs, ne présentent rien de particulier; après un parcours plus on moins long, suivant les espèces,

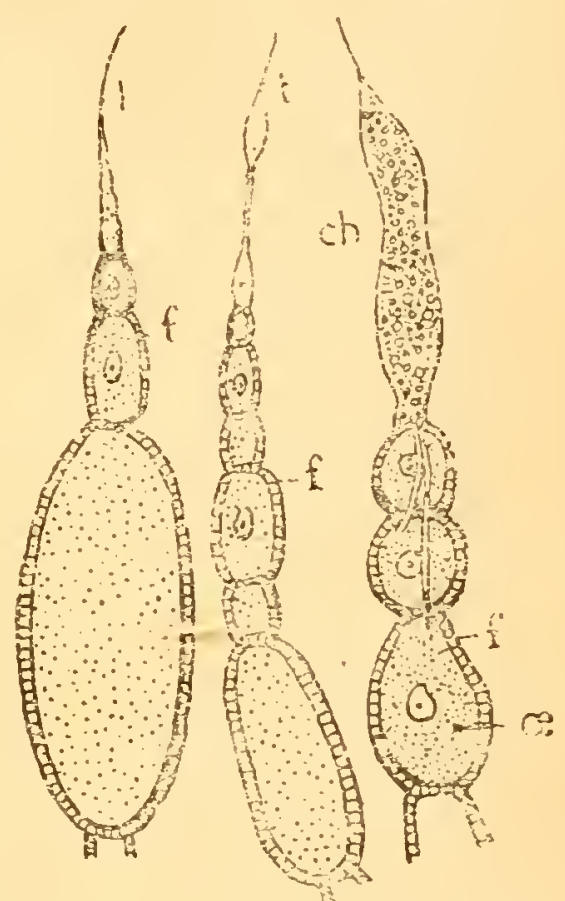

Fig. 115. - Gaines orariques isolées arec ces orules à divers degrés de maturité: t, filaments terminaux; ch, chambre terminale; $f$, chambres ovariennes ; $m$, ovule (d'arrès LANG).

ils se réunissent en un canal commun qui correspond, morpholo. giquement, au canal éjaculateur dẹs mâles (Fig. 116).

De même quéla partie terminale du canal éjaculateur forme un pénis, ici, également, la partie correspondante et chitinisée du canal ovarien devient le vagin ; comme le pénis, cette région tire son origine d'une invagination ectodermique, dont le résultat est de repousser à l'intérieur du corps les orifices des oviductes.

C. Pocile copulatrice et réservoir séminal. - Dans la plupart des Insectes, la poche copulatrice est une sorte de sac ou de tube allongé destiné à recevoir l'organe copulateur du mâle pendant l'union sex relle. Le liquide séminal (sperme) est généralement déposé dans la pache copulatrice pendant l'accouplement; il passe ensuite dans

LEs insectes. - $2^{e}$ édit. 
le réceptacle séminal, on il conserve ses propriétés fécondantes prndant fort longtemps.

La poche copula trice manque fréquemment (Hyménoptères, Diptires

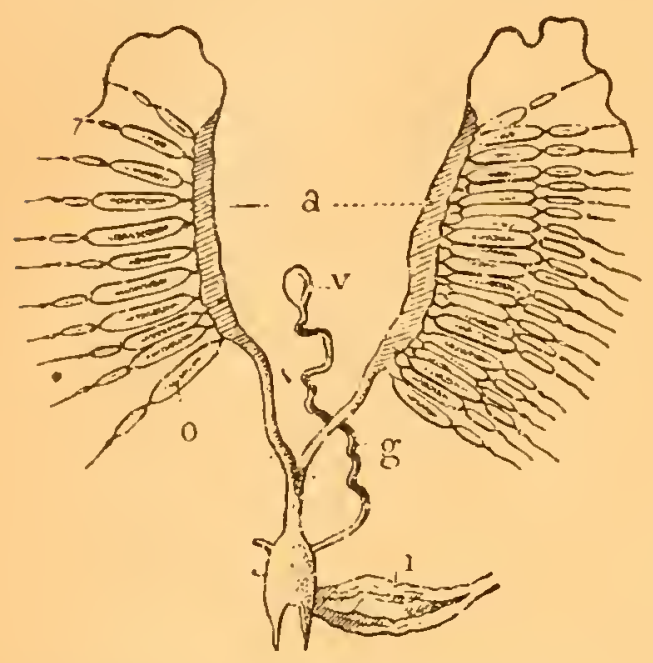

Fig. 116.- Aplrareil génital femelle du Mecostethus grossus (ORTHOPT.) où Is dispositlon segmentaire est conservड̃ : $a$, oviductes; $o$, follicules ovariens : $i$, intestin; $r$, glande à mucus ; $g$, canal e zcréteur. (Orig.)

s'emmagasine dans le réservoir séminal de la femelle; à l'époque d z la ponte, à l'instant précis où l'ovule passe de l'oviducte dans le vagin, une gouttelette de sperme est dévercé sur lui au passage. Un spermatozoïde pénètre alors dans l'intérieur par le micropyle et la fécondation s'effectue; l'ovule est alors devenu un auf.

Glandes accessoires. --. Il n'y a rien de particulier à dire des glandes accessoires (sébifiquos ou collétériqucs) qui débouchent, grénéralement, dans la partie telminale du vagin. La subctance sécrétée par ces glandes, insoluble dans l'eau, dans l'éther et dans l'alcool, est utilisée. chez les Orthoptères, pour la fabrication de leurs oothèques (p. 140); chez les autres Insectes, elle recouvre les ceufs d'une sorte de vernis, rili durcil très vite à l'air, et qui sert, par consépuent, à les firer it a surface des objets sur lesquels ils sont pondus.

Les grlandes à venin des Hyménoptères ne sont aulre chose que des sis landes sébifiques adaptées à des fonctions spéciales. 


\section{CHAPITRE XVII}

\section{ARMURES GENITALES}

'I'EGHIQUE. -- C'est sur les Orthoptères de grande taille : Locustidés, Acrididés et Gryllidés, que se fait, le plus facilement, l'étude des armures génitales.

Suivre les développements de ce chapitre, avec l'un de ces Insectes sous les yeux.

Depuis le grand travail de Lacaze-Duthiers, on donne le nom d'armure génitale à l'ensemble des pièces solides qui entourent les orifices externes de la génération. Ces pièces paraissent se développer toujours aux dépens d'un seul segment squeJettique (urite), et ce segment est celui qui vient immédiatement à la suite des ouvertures sexuelles (Fig. 117).
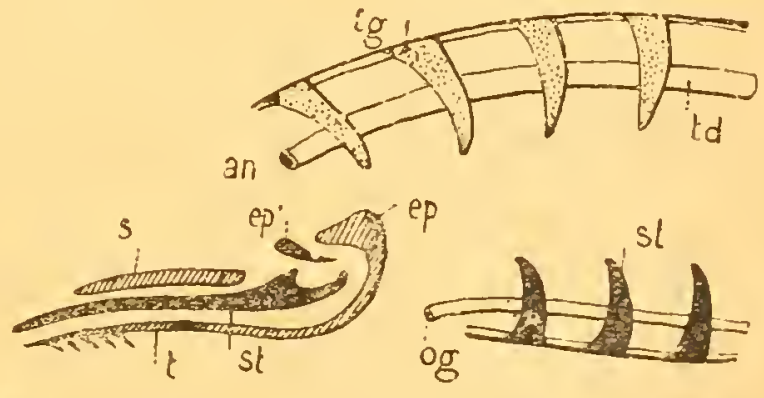

Fyig. 117. - Schéma destiné à montrer l'origine de l'armure génitale (Hymónol.tères): $t g$, tergites ; st, steruites; $t d$, tube digestif ; $a n$. anus: og. orifice des organes génitaux; $c p$, épimérite: $e p^{\prime}$, épisternite; $t$, tergorhabdite (stylet); $s$, sternorhabdite (d'après L.AC 1ZE-DUTHIERs).

Sauf quelques exceptions, connues de tout le monde (tarière des Icheumonides, oviscapte des femelles chez les Locustidés), il est rare que les pièces de l'armure génitale soient visibles au dehors en temps ordinaire; elles sont, le plus souvent, cachées dans l'abdomen par suite de l'invagination des derniers anneaux à l'intérieur de ceux 
qui les précèdent; pour les étudier, il est nécessaire de les faire saillir au dehor's par la pression, ou bien de les observer au moment de l'accouplement.

Nous n'entrerons pas dans le détail des longues discussions qui se sont élevées parmi les entomologistes sur la question de savoir quelle est l'origine et la signification morphologique des pièces de l'armure génitale; rappelons seulement leur rôle.

Chez les mâles, ces pièces sont surtout destinées à favoriser l'accouplement; elles servent à saisir la femelle

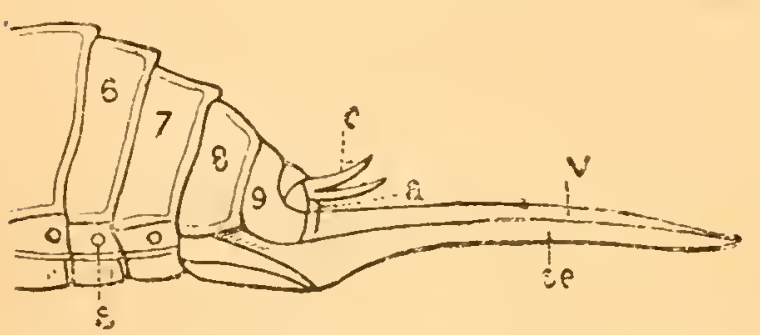

Fig. 118. - Extrémité de l'abdomen chez In Sauterelle verte; $c$, cerques; $s e, r$, valve $;$ de l'oviscape $; a$, anus; $s\rfloor$ stigmates : $6, \tau, 8,9$, les quatre dernier: segments abdominaux (d'après IKOLBE). et à faciliter l'introduction du pénis dans la poche copulatrice. Chez les femelles, les pièces principales de l'armure servent à la ponte des œufs; suivant sa structure et son déreloppement, la partie rigide et évaginable du canal ovarien porte les noms de tarière (Hyménoptères) ou d'oviscapte (Orthoptères) (Fig. 11S); elle peut même quelquefois devenir un forgane de défense, exemple : l'aiguillon des Abeilles, des Guêpes, etc.

1o Armure génitale des femelles. - I.'onverture de la vulve, cher les femelles, se trouvant le plus souvent sur la membrane molle qui réunit le se et le $9^{\circ}$ urite, c'est done le $9^{\circ}$ segment abdominal qui, en se modifiant, donnera naissance à l'armure génitale (Fig̣. 117).

A son maximum de complication, notamment chez les Orthoptères (Lorustidés) et chez les Hyménoptères, l'armure génitale des femelies est ainsi constituée :

1) Une pièce dorsale impaire représentant le tergite modifié.

2n I'eur épimérites et deux épisternites.

30 Une pice ventrale provenant du sternite. 
Chacun des épimérites porte un petit stylet inarticulé : tcrgorhakdite; les épisternites portent, de même, un sternôrhabdite (1). Ces appendices se déreloppent absolument comme les membres, aux dépens de petits bourgeons imaginaur prenant naissance dans l'hỵoderme.

La seule question qui divise actuellement les entomologistes est de savoir si ces appendices représentent réellement des membres abdominaux translormés (opinion de Packard, Dewitz, Verhoefi), ou si ce sont seulement des pièces supplémentaires, développées secondairement sur le squelette dermique (opinion de Peytoureau, Heymons, etc.). Cette distinction me parait un peu spécieuse, car enfin, que seraient donc ces organes, qui se déreloppent comme des membres et qui ne seraient pas des nembres?

Dans certains groupes d'Insectes, l'armure génitale femelle peut se simplifier considéralilement ; c'ost ainsi, par exemple. que chez les Courtilières, elle ne comprend que les deux pièces impaires, tergite et sternite ; chez les Forficules, elle est même réduite, d'après LacazeDuthiers, au tergite senl, toutes les autres pièces ayant arorté.

Notons enfin, nour terminer, que, rhez. les Coléoptères, l'armure génitale des femelles est peu développée ou même toıt à fait. rudimentaire.

2) Armure génitale des mâles. - I'armure génitale des mâles présente des variations plus nombreuses que celles des femelles, malheureusement elle est beaucoup moins connue. En plus du pénis tubuleur, qui n'est, comme on le sait, que la partie terminale et chitinisée du canal éjaculateur, on tronve des pièces accessoires qui constituent, soit des appendices protecteurs, soit des organes serrant à retenir la femelle nendant l'c couplement. Tout cet ensemble. est généralement caché à l'intérieur de l'abdomen ( $\mathrm{F}$ igg. 117).

Les rariations de structure de l'armure génitale sont tellement grandes chez les Insectes qu'il nous parait impossible d'en établir un schéma général applicable ì tous les cas. Le lecteur qui voudra se faire une opinion sur ce point si discuté de la mornhologie, devra consulter les volumes spériaux de l'E. S.

Il pourra lire aussi arec fruit l'exrellent ouvrage de ['eytourea!! ainsi que les intéressants mémoires de notre collègue M. L. Bordas.

(1) Du grec: terros dos, sternon sternum et rabdos baguette. 


\section{CHAPITRE XVIII}

\section{CARACTERES SEXUELS SECONDAIRES}

TECH viqu:- Il suffit ici de se procurer quelques-uns des exemples indiqués dans le texte.

On observe fréquemment, et cela dans tous les ordres d'Insectes, des différences profondes de forme, de taille ct de couleur entre les deux sexes d'une même espèce ; le mâle et la femelle sont parfois tellement différents cu'on serait tenté de les rapporter à deux espèces distinctes. Ce sont ces modifications, non en rapport direct avec les phénomènes essentiels de la reproduction, mais réanmoins liées au développement des glandes génitales, que l'on désigne parfois sous les noms de dimorFhisme sexuel et de polymorphismé saisonnier.

On ne connaît pas la raison d'être de ces modifications, il est probable que ce sont des adaptations qui favorisent, dans une certaine mesure, la conservation de l'espèce.

Il est bien évident que nous ne pouvons pas songer à passer en revue toutes les variations sexuelles secondaires qu'on peut trouver chez les Insectes; nous nous bornerons à indiquer les principales, convaincu que nos lecteur's remarqueront ensuite facilement d'eux-mêmes toutes celles qu'ils auront l'occasion de rencontrer.

10 Taille. - Les femrlles, dont l'abdomen est le plus souvent dilaté par la présence des aufs, sont, en général, plus grandes que 
les mâles cher. les Insectes; il existe cependant des exceptions à cetí règle; ainsi, dans certains Hyménoptères (Atieilles. Anthophores), chez la plupart des Libellulides et chez quelques Coléoptères (Lucane, Dynastes), les miles sont plus gros que les femelles.

Les différences de taille sont parfnis tellement accentuées qu'on a pu prendre les deux sexes d'un même lnsecte pour deux espèces distinctes. Mulsant ne décrivit-il pas, vers 1860 , sons le nom d'Athous 'Titanus, un petit Flatéride qu'on a reconnu, plus tard, n'être que li:

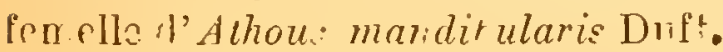

$2^{n}$ Yeux. - Les différences dans la crandeur des yeux ne sont pas très sensibles: cependant, on peut constatrr que, chez les Diptères (Syrphides, Muscides), chez bon nombre d'Hyménoptères (FauxBourdons) et che\% quelques Coléoptères (Anobiides), les ỵeux des mâles sont beaucoup plus gros et plus saillants que ceux des femelles.

$3^{\circ}$ Antennes. - Les antennes sont toujours plus longues, et leur massue, quand elle existe, est toujours plus développée chez $l_{S}$ mâles que chez les femelles. Cette particularité est, Iréquemment, très accusée chez. les Cérambycides (Coléopteres), par exemple dans le genre Acanthocinus.

Dans certains lialacodermes (Nrilus), Cetrio, les antennes sont courtes chez les femelles, tandis qu'elles sont longuement pectinées ou flabellées chez les mâles. Énfin, chez un grand nombre de Diptères némocères (Cul:x, Chi:onomus), les antennes des máles sont richement plumeuses, alors qu'elles ne sont garnies que de soies écartées chez les femelles.

4. Pièces buccales. - Les dittérences dans les pièces buccales, suivant les sexes, ne sont nulle part plus accentuées que chez les coléoptères: ce sont surtout les mandibules qui prennent parfois un énorme développement, ainsi unu'on peut l'observer'

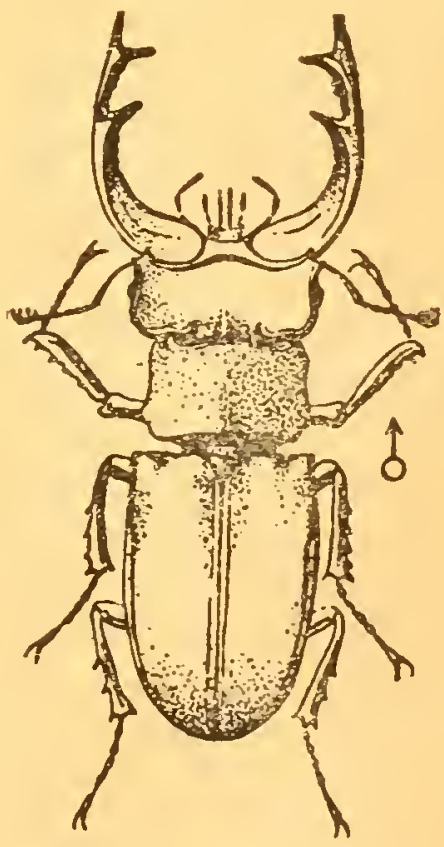

Fig. 119.-Lucane Cerfvolant, ठ (mále) pour montrer le grand développement des mandibules. dans les genres I.ucanus (Fig. 119) et Chiaso:na!hus.

Inversement, che\% les Diptères, qui se nourrissent du sang des 
animaux (Cousins, Taons, Anophiles), les femelles seules piquent les mâles n'ont qque des mandibules rudimentaires.

5. Cornes céphaliques et prothoraciques. -- Dans un certain nombre de Coléoptères (I amellicornes). les mâles sont souvent re. marquables par les appendices rigirles, cornes, pointes ou tubercules qu'ils portent, soit sur la tête (appeiddices céphaliques), soit sur le :orselet (appendices prothoraciques), snit sur tous les demy en même temps (Oryctes nasicornis).

Ainsi, par exemple, chez les Dynastides, le mâle de Dynastes Hercules, magnifique Coléoptère des Antilles et de Colombie, porte deux énormes pointes cornées, l'une recourbée en dessus et placée sur la tête, l'autre sur le prothorax et recourbée en dessous (Fig. 120). La femelle, par contre, très différente de forme et même de colo-

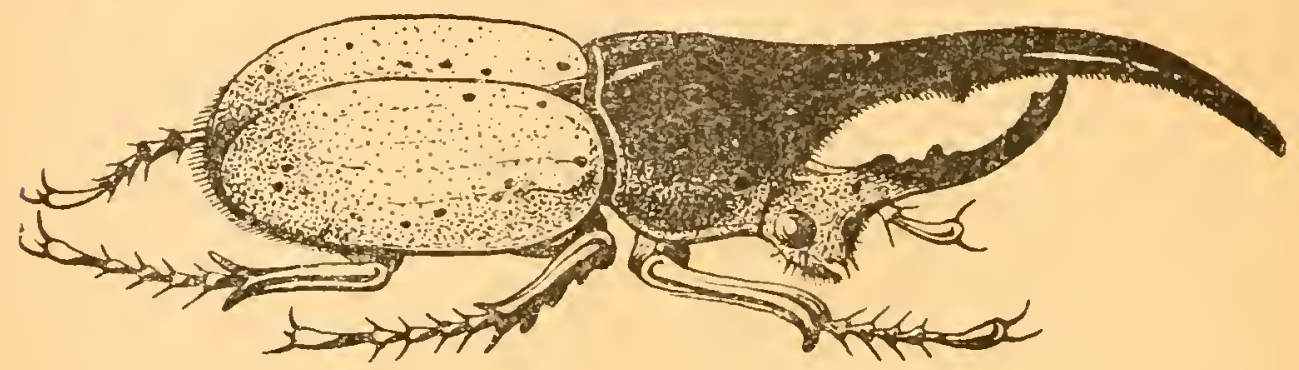

Fig. 120. - Dynastes Hercules mâle (d'après F. Unxxegur).

ration, ne présente rien de semblable. sauf un petit tubercule conique sur le milieu du vertex. Il en est de même dans les genres Colofa, A usasoma et Xylotrupes.

Le développement des cornes est lui même sujp̣t ̀̀ de nombreuses variations, car il existe toutes les transitions possibles entre les mâles à cornes développées et ceux chez lesquels ces ornements sont tellement réduits qu'on peut à peine les distinguer des femelles.

6'Pattes. - Les variations les plus intéressantes, cencernant cos organes, sont celles qu'on observe chez les Dytiscides (Colónutires); les trois premiers articles des tarses antérieurs des môles sont dilatés en une sorte de palette adhésive, destinée à s'appliquer fortement sur les élvtres des femelles pendant l'accouplement.

- 70 Ailes - La conleur et l'ornementation des ailes rarien! fréquemment d'un sexe à l'autre; les modifications les plus remar- 
quables se rencontrent surtout chez. les Lépirloptères : nous regret. tons de ne pouvoir représenter ici les curieux cas de dimorphismo sexuel q̧ue nous avons été souvent à même d'ohserver dans les mer. veilleuses collections de MM. Charles et René Oberthür.

Chez les Coléoptères, les diriérences de coloration et d'ornementation sont aussi parfois très accentuées.

Les élytres sont généralement lisses chez les mâles des Dytis us: (Coléoptères), alors qu'elles sont presque ioujours striées chez les femelles.

Fréquemment aussi, les femelles sont prirées d'ailes: Jampris, Drilus, parmi les Coléoptères, rieimatobia chez les Lépidoptères; mais, l'un des plus bearx exemples de dimorphisme sexuel, est celui que présente Paromia melanin. beau Papillon de la Nouvelle-Grenade décrit et figuré par M. Ch. Coerthür, F.tudes de Lépidoptérologie comparée, rasc. II, p. 22, r.. VII, fig. 1 et?.

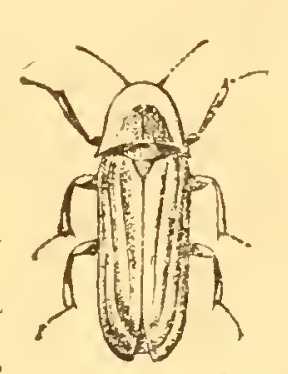

Fig. 121. - Lampyris noctiluca, mâle et femelle. (gr. nat.)

$8^{n}$ Organes lumineux. - Bien que la production de la lumière soit, généralement, l'apanage des deux sexes (Lucioles) chez les Insertes qui possèdent le pouvoir photogénique, dans le rulgaire $V$ cr-luisanc (Lampyris soctiluca (Fig. 121), l'appareil Lumireux de la lemelle est beaucoup plua développé que celui des mâles (voir p. 1/t2).

9. Aiguillon. - ret appareil de défense, dont soni pourrus un grand nombre d'Hyménoptères, n'existe toutefois que chez les fenielles et chez les our rières.

Q @uel que soit l'intérêt qui s'attache à l'étude de ces variations, il nous est absolument impossihle de les signaler toutes, car il n'est pas une partie du corps qui ne puisse varier chez les Insectes.

L'entomologiste utilise peu res rariations sexuelles dans la pra. tirque, soit pour la classification, soit pour la détermination des espèces; mais le biologiste ne peut pas s'en désıntéressel ; elle: montrent, en effet, le rôle important riue jouent les conditions dt milieu sur !e form:e extérieure des êtren. 


\title{
MODES DE REPRODUCTION
}

\author{
CHAPITRE XIX
}

\section{REPRO'DUCTION ASEXUEE}

La Parthénogénèse.

Techinge. - Fixer une forte loupe au dessus d'un rameau de Rocier envahi par les Pucerons ; on essayera d'observer en même temps, la parthénogénèse et la viriparité.

Bien que la reproduction sexuelle soit la règle chez les Insectes, on rencontre cependant, quelquefois, des femelles dont les œufs peuvent se développer sans fécondation préalable ; c'est ce phénomène, auquel les anciens paraissent avoir attaché peu d'importance, qu'on désigne aujourd'hui sous le nom de parthénogénèse (1).

10 Généralités sur la Parthénogénèse. - La parthénogénèse est normale dans un certain nombre d'espèces (Pucerons, Cynips, etc.) ; d'autres fois elle est irrégulière et purement accidentelle (quelques Papillons); mais, chose remarquable, dans tous les cas où on l'observe, les individus nés de la sorte, sans fécondation préalable de l'ovule, ont toujours un sexe absolument déterminé. Ainsi, par exemple, chez les Abeilles et chez les Guêpes, les générations parthénogénétiques sont toujours constituées par des mâles; celles des Pucerons, au moins au

(1) Du grec : parthenos vierge. 
début du printemps, ne renferment que des femelles. Il $\mathrm{y} a$, pour chaque espèce, une règle fixe, mais cette règle varie d'une espèce à l'autre.

Si l'on a pu soutenir, avec quelque vraisemblance, que les phénomènes de parthénogénèse chez les Abeilles avaient été découverts par Aristote, il ne faudrait pas croire, cependant, que les anciens se soient jamais représenté ces phénomènes sous le jour où ils nous apparaissent aujourd'hui.

Etant données les idées qui avaient_alors cours sur l'origine des êtres, tout cas de reproduction, sans l'union préalable des sexes, sans "baptême séminal " n'était pour eux qu'un cas particulier de la " génération spontanée ".

Pendant plus de vingt siècles, aucun fait expérimental ne parvint à ébranler cette théorie ; et, en 1667, l'observation du naturaliste hollandais Goedart qui, sans accouplement préalable, obtient des œufs féconds d'une femelle de Liparidé (Orgya gonostigma), passa elle-même complètement inaperçue.

Cependant, à partir de cette époque, les observations précises se multiplient.

En 1696, Hanneman indique que, pendant quatre ans, les descendants femelles d'une Araignée captive se reproduisirent sans le concours des mâles.

En 1706, Albrecht explique aussi, très formellement, qu'il a vu des œufs de Papillons se développer "sine prævia maris cum femella conjunctione ".

Enfin, en 1745, Charles Bonnet, de Genève, publia, sur la fécondation des Pucerons, les belles découvertes qui ont immortalisé son nom. Ayant isolé, sur les conseils de notre illustre compatriote Réaumur, une femelle 
d'un de ces Pucerons noirs qui vivent en si grande abondance sur les Fusains, il la vit, quatre fois dans l'espace de vingt et un jours et sans accouplement préalable, engendrer quatre-vingt-quinze petits Pucerons, de chacun desquels il constata "l'acte de naissance heure par heure ». En Suède, lo Sialon de Geer obtenait des résultats identiques.

Malgré toutes ces expériences, vingt fois répétées par Lyonet et par Réaumur lui-même, les naturalistes du siècle dernier n'admettaient pas la possibilité de la reproduction virginale; les uns ne voyaient, dans ces faits, qu'une confirmation éclatante de la théorie de l'emboîtement des germes, les plus sceptiques faisaient intervenir l'hermaphroditisme avec une sorte d'autofécondation.

Ce fut en 1845, qu'un apiculteur prussien, Dzierzon, curé de Karlsmarkt, en Silésie, établit définitivement l'existence de la reproduction virginale chez les Abeilles ; dix ans plus tard, Siebold (1856), observa le même phénomène pour les Lépidoptères du genre Psyché ; ce fut lui aussi qui appliqua, au fait de la reproduction ovipare, nun précédée de fécondation, le nom de "parthénogénèse " (1).

Cependant, les naturalistes n'étaient pas au bout de leurs surprises; ils reconnurent bientôt que la parthénogénèse se présentait avec de nombreuses variantes chez les Insectes : tantôt elle était régulière et, en quelque sorte normale, ainsi qu'on l'observe chez les Aphidés (Pucerons); d'autres fois, elle apparaissait comme facultative ou purement accidentelle, comme cela a

(1) Jugque-là, ce terme, créé par Oven, n'avait été employé que pour désigner la phase aseruée des générations alteruantes. 
lieu, par exemple, chez les Bombycidés (Lépidoptères) ; enfin, quelquefois, les larves elles-mêmes (Cécidomyes), présentant des phénomènes de maturation très précoce, peuvent se reproduire avant d'être arrivées à l'àge adulte; ce dernier phénomène, découvert par Nicolas Wagner en 1862, a été désigné sous le nom de Pædogénèse: bien que ce ne soit là, en réalité, qu'un cas de parturition virginale anticipée, nous distinguerons donc, avec les auteurs les plus récents, quatre modes principaux de reproduction parthénogénétique chez les Insectes.

10 La parthénogénèse normale, isoparthénogénèse (Abeilles).

20 La parthénogénèse cyclique, hétéroparthénogénèse, caractérisée par la succession régulière de nombreuses générations asexuées auxquelles succède une génération sexuée (Pucerons).

30 La parthénogénèse accidentelle, tychoparthénogénèse. (Bombycidés).

$4^{\circ}$ La parthénogénèse lavale, pædogénèse. (Cécidomyes).

Nous ajouterons quelques mots sur la parthénogénèse expérimentalc.

\section{$1^{\circ}$ Parthénogénèse normale (Isoparthénogéxése).}

\section{(Abeilles et Guêpes socinles).}

Ce mode particulier de parthénogénèsı, qui n'a été óbservé ¡usqu'ici que che? les Hyménoptères porte-aiguillon, est caractérisé par ce fail que les individus, issus d'un ouf non fécondé, sont toujours des mâles (1).

Voici, par exemple, ce qui se passe chez les Aheilles, où les phénomènes ont-été analysés avec le plus grand soin. Il y a, comme on

(1) C'est l'arrhénotokie de Siebold. 
le sait, trois sortes d'individus dans une ruche: 10 des Ouvrières en nombre très grand, femelles imparfaites et stériles; $\Sigma^{0}$ des FauxBourdons, mâles; 30 une seule Reine. - I a Peine est une femelle. normale, poulrue d'organes génitaux bien constitués, et dont le r.île exclusif est de pondre, pour assurer la conservation de lí colonie ; la Reine est, en somme, la mère de tous ses sujets. Or, cetíe femelle unique ne s'accouple qu'une seule fois dans sa vie, tout à fait au début de son existence, au moment du vol nuptial (1).

Cependant, si les circonstance l'empêchent de rencontrer un FauxBourdon, autrenient dit si, pour une cause ou pour une autre, la fícondation ne peut avoir lieu, la femelle-reine n'en accomplira pas moins sa destinée de pondeuse; comme de coutume, elle déposera ses œufs dans les alvénles, et ces œufs posséderont la faculté de se révelopper, mais la génération de jeunes qui en sortira sera exclusivement constituée par des Faux-Pourdons, c'est-à-dire par des mîics.

N’ayant à indiquer ici que les caractères généraux de la parthénogénèse arriénotoque, nous pourrions nous dispenser d'insister sur re sujet, mais nous tenons à relater les principaux faits qui ont mis hors de doute la manière de roir de Dzierzon; car cotte théorie, il faut bien le dire, rencontra, au début, de nombreux contradicteurs et suscita de violents débats.

Voici les faits sur lesquels elle s'appuic :

L.orsque la Reine fécondée pond, dans les conditions normales, elle dépose ses œufs, successivement. dans des alvéoles de dimensions variables, suivant qu'ils doivent donner naissance à une Ouvrière, à un Faux-Bourdon, ou à une nouvelle Reine (cellieles royales). Or Leuckart, et après lui, Siehold, en examinant, à un fort grossissement: les cufs fraîchement pondus dans les grandes cellules hexagonales oì doivent se développer les Faux Pouldons, ne purent jamais ! découvrir des spermalozoïdes, tandis qu'ils en ont trouré, au contraire, dans les oufs des femelles et des ouvrières.

On a aussi remarqué que, vers la fin de sa vie, l'Abeille reine. toujours féconde, produit une proportion de mâles quui va sans cesse en s'accrissant; il peut même arriver qu'elle ne produise plus aucun ouf de lemelle ni d'ruvrière ; cela provient de ce que la provision de substance fertilisante (sperme) renfermée dans soll réceptacle sémi-

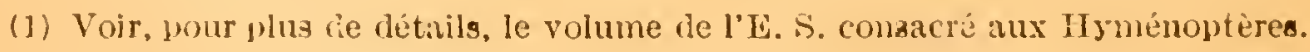


nal, est épuisée ; les cufs pondus n'étant plus fécondés ne peuvent donner naissance qu’à des mâles.

Finfin, lorsqu'on parvient à réaliser le croisement de delıx races d'Abeilles, différentes par la coloration, un mâle brun français, avec une lemelle jaune ligurienne, les reines et les ourrières qui en résultent sont des rnétis ; ils présentent, à la fois, les caraclères de la race française et de la race italienne, tandis que les mâles sont tous de race italienne pure.

Ces faits ne peuvent s'expliquer à moins d'admettre, avec Dzierzon, que l'Abeille mère fécondée peut pondre, à volonté, des œufs de mâles ou des nufs de femelles; il suffirait, en effet, pour cela qu'elle pût, par suite d'un réflexe dont le mécanisme nous échappe, déverser it volonté, sur l'œuf, au moment oì il passe devant l'ouverture du réceptacle séminal, le liquide fécondateur qu'elle tient en réserve.

C'est là, évidemment, le point faible de la théorie de Dzierzon; très justement seion nous, J. Pérez a contesté la possibilité de cet acte volontaire et en quelque sorte raisonné.; néanmoins, si la véritable cause de ces phénomènes est encore inexplicable, le fait lui-même de la reproduction parthénogénétique chez. les Abeilles ne saurait itre, aujourd'hui mis en doute.

\section{Parthénogénèse cyclique (HÉtÉroparthénogéxĖse).}

(Aphidés, (iynipidés, etc.)

C'est chez les Ifémiptères de la famille des Aphidés (Pucroris, Phr.lloxera, etc.) que ce mode de reproduction virginale se présente avec le plus de régularité.

Il est caractérisé, au moins dans les cas les plus simples, par ce fait que, à suite plus une ou moins longue de générations parthénogi... nétiques, assurant une large dissémination de l'espèce pendant la belle saison, succède une génération sexuée, destinée à assurer la conservation de l'espèce pendant l'hiver.

10. 1phidés. - Tout le monde a pu observer les Pucerons qui virent en nombre quelquefois prodigieux, sur la plupart des végétaux. Ces petits Insectes se fixent, à l'aide de leur rostre conique, sur lẹs parties molles des branches dont ils sucent la sève. La plante, ainsi parasitée, s'épuise lentement et finit par mourir. Le cycle évolutif est le suivant:

A. Examinons, par exernple. les innombrables Pucerons verts qui 
depuis le mois de mai jusqu'en septembre, envahissent les jeunes pousses et les feuilles du Rosier. Au début du printemps, on pourra constater que tous les individus sont dépourus d'ailes, ce sont des femelles qui, sans accnuplement préalable, donneront naissance chacune à 40 ou 50 petits vivants. Tous les petits Pucerons, nés ainsi par viviparité, sont également des femelles aptères ; ils se comporteront conıme leur mère, et alors, dans le cours de la belle saison, or pourra voir huit ou dix générations pathénogénétiques se succéder

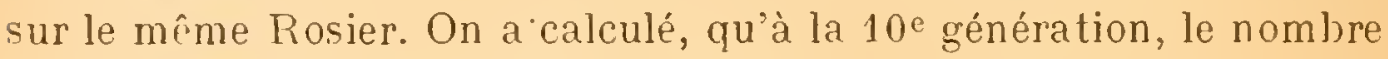
des descendants, provenant ainsi d'une seule de ces femelles vierges, était de 125.000 environ; on conçoit l'énormité des dégâts qui peurent en résulter. Nais ce n'est pas tout.

B. Dans le courant.de l'été, à peu près ver's l'époque où se produit la $3^{e}$ génération de femelles aptères, on voit apparaître quelques individus munis d'ailes transparentes. Ces pucerons ailés sont des migrateurs; ils quittent, en général, la plante nourricière et vont fonder une nouvelle colonie sur des Rosiers voisins; mais, ce sont toujours des femelles parthénogénétiques et vivipares; aussitôt fixés, ils seront le point de départ de générations nouvelles, qui se comporteront comme les prérédentes et ainsi de suite (Fịg. 122).

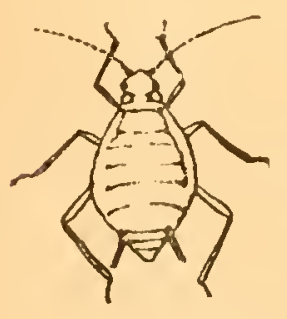

Fig. 122. - Différentes formes de Pucerons (d'aprèn nature)

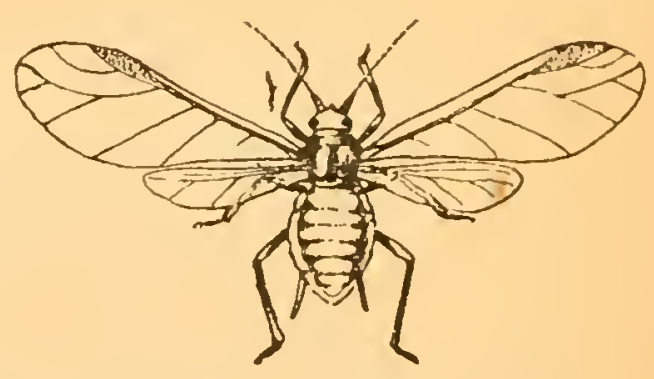

C'est donc par l'intermédiaire de ces Pucerons ailés, que se trouve assurée la dissémination de l'espèce dans l'espare.

C. Mais, vers la fin de l'été, lorsque la température s'abaisse et que la nourriture menace de devenir de plus en plus rare, les phénomènes changent; les Pucerons qui naissent, toujour's des lemelles parthénogénétiques, sont alors généralement ailés; nıais, rette fois, leurs organes génitanx sont bien développés; il y a des mâles et des femelles qui ront s'arcoupler entre eux.

Les femelles ainsi fécondées ne sont plus vivipares; elles ront 
pondre des œufs qui passeront l'hiver sous un abri déterminé et n'écloront qu'au printemps suivant. On a désigné ces cufs sous le nom d'cufs d'hiver : ils possèdent une coque épaisse, qui leur permet de résister aux intempéries : ils servent, par conséquent, comme on le voit, non plus à la dissémination de l'espèce dans l'espace, mais à sa dissémination dans le temps : ce sont des œufs de conservation.

Lorsque les oufs d'hiver éclosent, au printemps suivant, il en sort des fermelles aptères qui seront, comme l'année précédente, le point de départ de nombreuses séries de générations agames.

On donne, à l'ensemble de ces phénomènes, le nom de parthérngénèse cyclique, pour exprimer que la vie évolutive des Pucerons est constituée par une série plus ou moins nombreuse de générations virginales, toujours interrompues, à l'entrée de l'hiver, par une génération sexuée.

Il est bien certain que la température joue un rôle important dans les variations du cycle reproducteur des Pucerons, car on peut prolonger à volon!é, et en quelque sorte indéfiniment, la série des générations agames; il suffira de maintenir ces Insectes dans des conditions de température et d'alimentation convenables. I.es expériences les plus concluantes à ce sujet sont celles de Ky yer (1815). qui réussit à obtenir, pendant quatre années, 50 générations vivipariques du Puceron du Rosier, en prenant seulement la peine de conserver les rosiers dans une chambre chauflée pendant l'hiver. "L'effet d'une alimentation surabondante, dit Balbiani, s'étend à de nombreuses générations; avec la disette, surviennent l'épuisement et la stérilité de la lignée, mais survient le mâle, qui y rappelle la vitalité près de s'éteindre. "

Et de fait, l’idée rient à l’esprit de comparer ces phénomènes à ceux que Maupas a fait connaitre chez les Inlusoires ciliés, et qu'il a désignés sous le nom de rajeunissement car!ngamique.

Il existe bien, cela va sans dire, quelques petites variantes de reproduction chéz. les Pucerons; mais, ces légères modifications, qui ne sont d'ailleurs que des adaptations spécifiques, ne troublent nullement la régularité du cycle évolutif, tel que nous venons de la décrire. Ainsi, par exemple, on sait que, chez le Puceron lanicire, un certain nombre de femelles aptères persistent à l'automne sur le pommier; elles descendent alors le long du tronc et vont s'abriter dans la terre pour passe l'hiver. Au retour de la belle saison, ces femelles remontent vers les jeunes branches et vont se joindre at 
celles qui sont nées des œufs fécondés. Par la survie de ces femelles hibernantes, il n'y a donc qu'un arrît saisonnier dans la série des générations parthénogénétiques. Nous aurons encore l'occasion de iter d'autres curieux phénomènes d'adaptation, dans le volume de I'E.S. qui sera consacré aux Hémiptères.

On peut donc résumer, à l'aide dı schéma suivant, l'histoire évolutive des Aphidés (1).

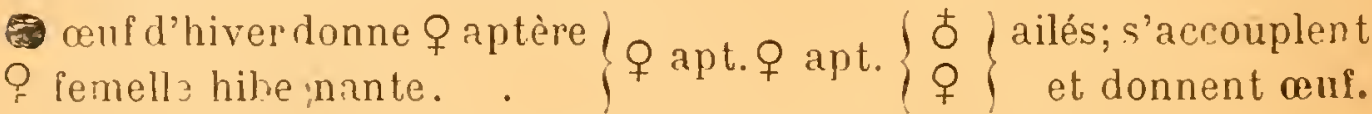

20 Cunividés. - L'évolution du Phylloxera, des Chermes et des Cynipides s'eflectue, dans ses grandes lignes, sur le même plan que rolle des Aphidés (Voir les volumes spéciaux). Enfin, il est bien probahle, que les cas de parthénngénèse cyclique irrégulière: signalés chez quelques Lépidontères du genre Psyché, rentreront dans les rèğles ordinaires, lorsqu'on connaîtra mieus le mode de vie des mâles.

$3^{\circ}$ Parthénogénèse accidentelle (TrchopakthéNogénèse).

Bombycidés).

On observe, de temps en temps, surtout chez les Lépidoptères, des phénomènes de parthénogénèse accidentelle, en ce sens qu'ils se produisent, sans régnlarité apparente, et sous l'infiuence de conditions qui nous sont tout à fait inconnues. Certaines femelles peuvent, en effet, sans accouplement préalable, pondre des nufs, chez lesquels il se produit un commencement de développement.

Il y a plus d'un siẹcle (1795) que Constant de Castellet signala, pour la première fois, le fait à Réaumur, en ce qui concerne le Pombyx du Mn̂rier ; mais Réaumur refusa d'y croire : "Ex nihilo nihil fit ", répondit-il.

L'expérience a d'ajlleurs démontré que ces œufs parthégénéliques se développaient beaucoup plus lentement que les autres; le plus souvent même, l'évolution s'arrête à une phase très précoce du développement; lorsqu'une petite chenille parvient à se former, il est rare qu'elle ait la force d'éclore, généralement elle meurt à l'intérieur de l'œuf.

(1) Le sikne $\delta$ indique les mîles: $q$ désigne les femelles. 
Jourdan, à la suite d'expériences nombreuses, publiées dans les Comptes-rendus de l'Académie des Sciences en 1861: avait cru pouvoir avancer que la parthénogénèse accidentelle était plus fréquente chez les races polyvoltines que dans les races univoltines (1); jusqu'ici ces rues n'ont pas reçu l'assentiment des naturalistes.

La proportion d'œufs, capahles d'éclore sans fécondation, est tolljours extrêmement réduite; à part quelques circonstances exceptionnelles, il est rare qu'elle atteigne 2 p. $0 / 0$; le plus souvent même, ainsi que nous l'arons déjà dit, les œufs présentent un commence ment de segmentation, mais ils ne peuvent pas arriver jusqu'à l'éclosion. Par conséquent, chez les Lépidoptères, la parthénogénèse no constitue donc jamais un mode de développement $\mathrm{n}$ zturel $\epsilon$ rigulier comme chez les Abeilles et chęz. les Pucerons.

Cependant Carlier, opérant sur I iparis disrar, en 1838, réussit à obtenir trois générations parthénogénétiques successires; malhevreusement ses expériences ne purent être continuées, parce que les wứs de la troisième génération ne donnèrent naissance à aucune femelle. Weijenbergh reprit avec succès, en 1870, les expériences cie Carlier; lui non plus ne put aller au delà de la troisième génération; bien que nombreux, tous les œufs qu'il obtint furent stériles

Là encore, nous voyons la parthénogénèse produire un épuisement rapide des éléments sexuels; seulı, très probablement, une conjugaison normale serait capable de ranimer la "vitalité près de s'éteindre ".

Des cas de parthénogénèse accidentelle ont été également observés chez les Hyménoptères (T'enthrédines), chez les Orthoptères(Phasmides), chez les Diptères (''écidom;es) et même chez quelques Coléoptères. Nous examinerons ces diflérents cas dans les volumes spéciaux consacrés à chacun de ces groupes.

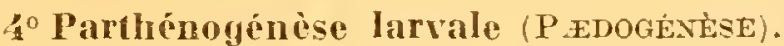

\section{(Cécidomyes).}

La plupart des auteurs ont constaté que, chez les Insectes parthénogénétiques, les cellules sexuelles apparaissent de très bonne heure

(1) Les races de Ver à soie, qui ne se reproduisent qu'une seule fois dans le cours b'une année, sont dites uniroltines; celles qui donnent deux générations et plus sont dites bivoltines et polyrolíines. 
au pôle de l'œuf. Chez les C'écidomyes et rhez. les Aphidés, on jeu.t les observer dès les premiers moments de la segmentation méme. avant la formation du blastoderme.

Imaginons que ces processus de développement précoce continuent à se produire, avec la même activité, pendant la période embryonnaire, on pourra alors rencontrer des larves, ou des nymphes, dont les éléments génitaux seront arrivés à maturation, avant que l'Insecte r'ait lui-même revêtu la forme de l'adulte. Si les œufs, produits par les larves femelles, peuvent se développer à cette époque, on a le phénomène que le professeur N. Wagner observa pour la première fois à Kasan, en 1862, sur des larves de Cécidomyes, et que von Baer désigna, l'année suivante, sous le nom de pædogénèse.

En ce qui concerne la Cécidomye étudiée par Wagner, les œufs ne sont jamais pondus; les petites larves se forment à l'intérieur du corps de leur mère, et celle-ci se détruit au moment de la mise en iberté des jeunes. Mais, dans une autre espèce de Diptère (Chironumus Grimmi), la reproduction se fait par de véritables œufs, pondus, non plus par la larve, mais par la nymphe.

Un simple retard dans le développement des produits sexuels suffit pour expliquer cette différence; pour un retard plus grand, on conçoit que la ponte ne pourra plus aroir lieu que chez. l'adulte et, de fait, A. Schneider a prouvé que la femelle de ces mêmés Diptères conservait, même dans l'âge adulte, la propriété de se reproduire parthénogénétiquement.

La pædogénèse et la parthénogénèse normale sont donc, comme on le voit, deux phénomènes-biologiquement identiques; il est probable qu'on peut passer de l'une à l'autre par toutes les transitions possibles, et la pædogénèse, en réalité, n'est autre chose qu'une parthénogénèse précoce.

La pædogénèse u'est d'ailleurs pas spéciale aux Insectes, on peut aussi la rencontrer dans d'autres groupes du règne animal ; ainsi, par exemple, la formation des Cercaires dans le sporocyste des Distomiens est un cas de pædogénèse parfaitement caractérisé.

Progénise. - Dans la règle, les Insectes, de même que les autres animaux, ne sont aptes à se reproduire sexuellement que lorsqu'ils sont arrivés à l'état adulte; on connaît cependant quelques larves qui font exception à cette loi générale, et qui, en outre, de la faculté qu'elles possèdent de donner des générations parthénogénétiques 
peuvent s'accoupler avant d'être arrivées àl'état parfait et produire des oufs féconds.

A. Giard, qui a le premier appelé l'attention sur ces crurieux phénomènes, en 1887, a désigné sous le nom de progénèse cette reproduction sexuelle des larves.

Comme exemples d'Insectes chez lesquels la progénèse peut être observée, on peut citer les femelles parasites des Stylops, qui conservent, pendant toute leur vie, la forme larvaire, ainsi que certaines femelles aptères et parthénogénétiques des Pucerons.

Il est possible que la progénèse ne puisse se rencontrerª que chez les espèces ayant déjà des tendances parthénogénésiques; mais elle se distingue néanmoins très nettement de la parthénogénèse normale en ce qu'elle implique toujours un acte préalable d'accouplement et qu'elle introduit, par conséquent, une légère variante dans les phénomènes de reproduction sexuée.

\section{Parthénog̣enèse expérimentale.}

Actuellement, l'une des questions les plus passionnantes de l'embryogénie, est celle de savoir si l'action fécondante du spermatozoïde sur l'œuf ne pourrait être reproduite, dans ses traits essentiels, par des moyens physiques ou purement mécaniques.

En ce qui concerne les Oursins, le problème vient d'être résolu affirmativement par M. Yves Delage, et il n'y a probablement aucune paison pour que ce merveilleux résultat ne puisse être généralisé.

La première expérience de parthénogénèse expérimentale parait présisément aroir été réxlisée sur les Insectes. En 1886, Tichomiroff montra, en effet, qu'on peut obtenir des érlosions d'cufs non fécondés de Ver à Soie, en les frottant légèrement avec un pinceau ou en les plongeant pendant deux minutes dans l'acide sulfurique concentré.

Malheuremenent, cette expérience n'est pas concluante; nous avons vu, en effet (p. 198), que les outs de cette espèce peuvent quelquefois se développer sans fécondation préalable. D'autre part, les expériences entreprises en vue de déterminer si le sexe d'un animal n'est pas déjà fixé dans l’ovule (1) ont montré que, dans le

(1) LrEP. (J). - Dynamique de la rị. p. 332-339. 
cas des Pucerons, par exemple, si l'on abaisse la température du milieu, si on dessèche l'air, ou si on modifie notablement l'état clinique de la plante nourricière, le cycle des générations parthénogénétiques est presque immédiatement arrêté; il se produit des mâles et des femelles qui s'accouplent bientôt. Inversement, si l'on maintient les plantes dans des conditions convenables de température et d'humidité ,le cours des générations agames peut ître prolongé pendant très longtemps.

Il n'y a donc pas de doute; le développement de l'œuf est fonction du milieu où il vit; reste à savoir si nous trouverons le ou les moyens scientifiques d'agil sur ce milieu de manière à obtenir, dans tous les cas, un résultat prévu et, à l'avance, déterminé. 


\section{CHAPITRE XX}

\section{DIVERS ACTES DE LA REPRODUCTION SEXUEE}

TECHNique. - Le Hanneton se prête très bien à l'étude des phénomènes d'accouplement.

La fécondation, chez les Insectes, s'accomplit comme chez les animaux supérieurs, il n'y a done pas lieu de s'arrêter à la description de ce phénomène; le seul point qui nous intéressera ici sera de rechercher comment, et à la suite de quels actes préparatoires, le spermatozoïde, élément màle, se trouve mis en relation avec l'ovule, élément femelle.

Nous avons déjà décrit, avec assez de détails, les appa reils génitaux dans les deux sexes, ainsi que les armures (organes accessoires) qui rendent possible le rapprochement des mâles et des femelles; il nous suffira donc de décrire maintenant les différents modes d'accouplement.

\section{$1^{\circ}$ Accouplement.}

L'acte essentiel de l'accouplement, c'est l'introduction du pénis du mâle dans le vagin de la femelle, de manière à déposer le fluide fécondant le plus près possible de l'ovule mưr. Cet acte est réalisé de façons très différentes, suivant les groupes d'Insectes; mais, dans l'immense majorité des cas, le mâle se place sur le dos 
de la fomelle et la retient embrassée avec ses pattes (Fig. 123); souvent aussi, suivant l'expression de

Lacordaire, si

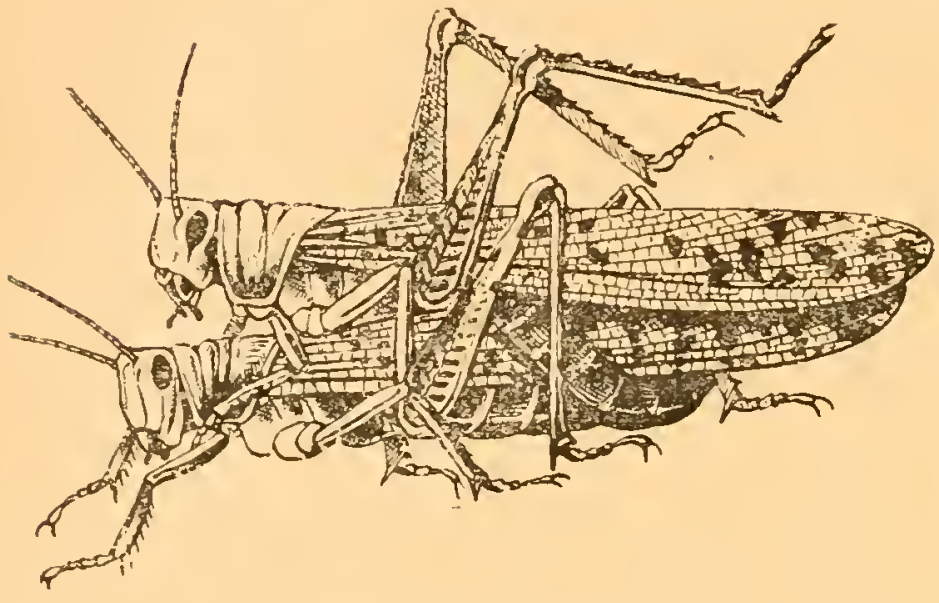

Fig. 123. - Accouplement du C'riquet pelerin (OrThopt.) (i'ajès Kuxckel d'Herctuas, in Henneguy) heureusement traduite par Henneguy, l'accouplement se pratique more canum, les deux sexes sont récnis bout à bout, et se tiennent la tête dirigée en sens inverse (Pentatomes).

Mais d'autres fois, le mâle et la femelle, bien que réunis bout à bout, se plaçent néanmoins l'un à côté d? l'autre, la tête dirigée dans le même sens ; cette position, également très commune, se rencontre chez un certain nombre de Lépidoptères nocturnes et chez quelques Hémiptères. Certains Névroptères (Bittacus Fig. 124), plusieurs Zygènes, les Cousins, les Notonectes et mème quelques Coléoptères (Atomaria), s'accouplent ventre à ventre. Chez les Tipulides

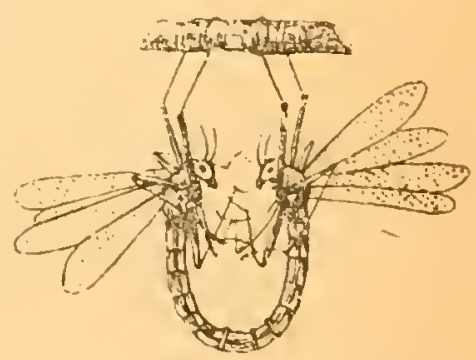

Fig. 121. - Acconplement du Bittacus lipularius Nevropt. (c'après BraUkr, in Henneguy). et un certain nombre d'autres Diptères, c'est la femelle qui se place sur le dos du mâle.

Conformément aux idées émises pour la première fois par Mouffet, e'est bien, parait-il, en dehors de la ruche qu'a lieu la fécondation de la Reine par un Faux-Bour- 
DIVERS ACTES DE LA REPPODUCTION SEXUEE 205 don 'liez les Ahei!les; l'accouplement s'opère dans l'air, pendant le vol nuptial, mais il semble n'aroir jamais été observé qu'une seule fois, par François Huber, le 29 juin $1788(1)$.

L'accouplement des Libellules présente des particularités qui ont intrigué les untomologistes pendant fort longtemps; aujourd'hui encore, je ne suis pas sùr qu'on en connaisse le mécanisme exact. Quoi qüil en soit, soici comment on l'explique.

L'appareil copulateur du mâle est situé, non pas à l'extrémité, comme on le croyait autrefois, mais près de la base de l'abdomen, dans une dépression ventrale, dépendant du deuxième et du troisième segment; la partie essentielle de cet appareil, fort compliqué, est con stituée par une sorte de tambour à l’intérieur duquel se trouve la résicule séminale. Le canal excréteur de cetté vésicule s'ouvre, à la base d'un crochrt mobile et triarLiculé, qui n'est autre chose que le pénis. Enfin, de chaque còté du réservoir séminal, se voient deux sacs ćlastiques, dont le rôle est de comprimer le réservoir afin d'en faire sortir, suivant la volonté de l'animal, lo liquide fécondateur.

Au moment de l'accouplement, le màle saisit la femelie par le cou, à l'aide de la pince située à l'extrémité de son abdomen, et l'entraine arec lui dans son rol ; après quelques instants, la femelle relève, à son tour, l'extrímité de son abdomen contre l'appareil copulateur du mâle (Fig. 125).

Un autre mode singulier d'accouplement est celui Les Lépidoptères du genre Psyché. Les femelles sont

i1) Fran:ois Hubert était areugle depuis l'âge de 15 ans; il faut entendre al e te fut par les yeux de son tiłèle domestiıue, Burnens, qu'il fit l'inportante observation indiquee ici-dessus.

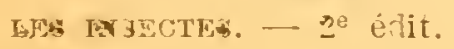


aptères; devenues adultes, elles ne quittent pas le fourreau qu'elles se sont construit étant chenilles; elles si tiennent, dans ce fourreau, la tète en bas, présentant

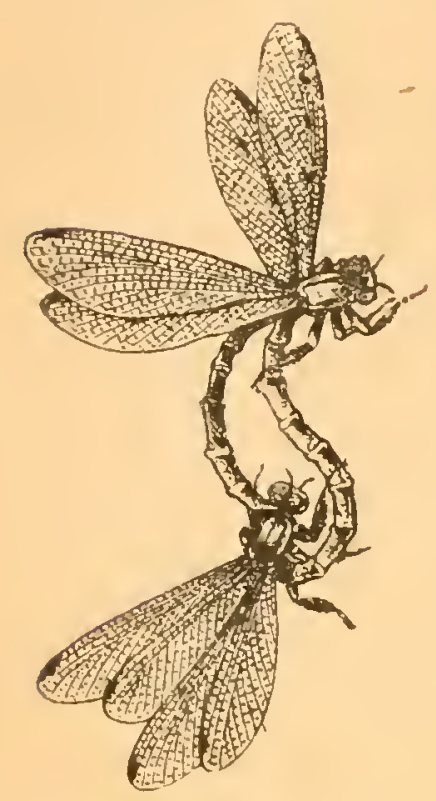

Fig. 125. - Accoublement des libellules (d'après F. ITwxpets). ainsi, du côté de l'ouverture, l'extrémité postérieure de leur corps. C'est dans cette position qu'clles reroivent le contact du mâle, dont l'abitomen est susceptible de s'allonger considérablement, afin de pénétrer à l'intŕrieur du fourreau.

Certains Coléoptères xylophagès (Anobiides, Scolytides), qui vivent à l'intérieur du bois ou sous les écorces, se comportent à peu près de la mème manière; les mâles sortent toujours de leur's galeries-; les femelles beaucoup plus rarement; clles se contentent de présenter l'extrémité postérieure de leur abdomen à l'orifice de leurs retraites.

Ces exemples suffisent à montrer qu'on ne peut pas encore formuler de règle générale en ce qui concerne l'accouplement des Insectes. On possède un certain nombre d'observations éparses, mais les exemples connus sont, en somme, relativement peu nombreux. Les aspects si variés de cet acte dépendent d'ailleurs, il était facilu de le prévoir, de la position des ouvertures génitales et de la conformation des armures.

Durée de laccouplement. - La dureve de l'acoumplement est ex. csssivement variable; lia encore on ne peul ren dire de grimer.y;

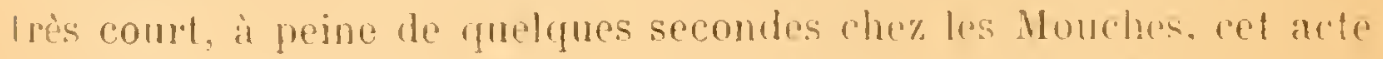

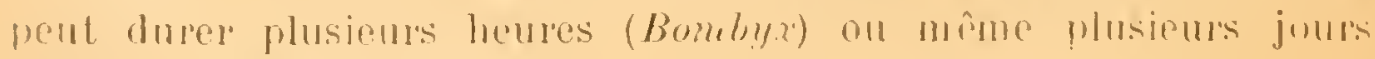
(1 i ? jours) rhez. le Hanneton. 


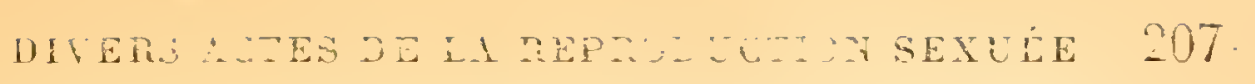

Le mâle meurt presque toujours à la suite de ces accouplemacsiss prolongés ; dans tous les cas, sa vie e-t beaucoup plus courte qle colle de la femelle, qui, elle, ne disparaît qu'après la ponte, lossqu'elle a épuisé toute sa fécondité.

Le plus souvent, l'accouplement commence à terre ou sur les plantes (Coléoptères) ; mais, pour un grand nombre d'Insectes bons voiliers, il se continue pendant le vol (Lépidoptères). et c'est alors la femelle, toujours plus vigoureuse, qui transporte le mâle. I'n certain nombre d'Insectes ne s'accouplent même que pendant le vol : Termites, Ephimères.

Paimi les Insectes aquatiques, les uns s'accouplent au repos, sur les plantes submergées (Ditiscides); d'autres, comme par ex^mple les Notonectes, restent réunis en nageant.

On ne saurait très exactement dire comment, chez les Insectes, les mâles sont avertis de la présence des femelles; par la vue sans doute, peut-être aussi par des sens spéciaux que nous ne connaissons pas; mais il semble aussi bien certain, d'après les expériences de Balbiani, que l'odorat et les antennes jouent le rôlc capital dans le rapprochement des sexes (1).

En général, la femelle des Insectes est monocarpique, aussi ne s'accouple-t-elle qu'une seule fois dans sa vie; cette règle paraît absolue, même pour les Insectes qui vivent plusieurs années, comme l'Abeille reine. Cependant, on connaît quelques espèces de Panorpes, et, parmi les Coléoptères, les Cantharides, dont les femelles s’accouplent, dans un espace de temps très court, avec plusieurs mâles différents ; mais la ponte est toujours unique.

L'accouplement des mâles, successivement avec plusieurs femelles est plus fréquent; de Geer a ru un Puceron s'accoupler avec cinq femelles différentes, et tout le monde connait l'exemple banal des Mouches.

\section{Ponte des aeuis.}

lo Nombre d'cufs. - Le nombre d'œufs pondus par les femelles est excessivement variable suivant les diflérents groupes d'Insectes. Dans une famille naturelle, et mème dans une espèce déterminée, il y a des variations, suivant

(1) B.LBI.Ixi (E.). - Note sur les antennes serunt aux Insectes pour la recherche des scxes. (Aljo. suc. entoni. re France, 1866, T. 11. Bu!l. 1. :38). 
le régime et stivant que la nourriture est abondante nu lare.

Ce sont les Colćoptères et quelques Diptères, qui paraissent, en movenne, produire le plus faible nombre d'ouls, 6 à 30 ; certains Lépidoptères, notamment Chelonia Caja, peuvent donner jusqu'à 1600 œufs; mais le record est certainement tenu par les femelles des Insectes sociaux, dont la ponte peut se prolonger, presque sans interruption pendant 4 ou 5 ans; on estime ainsi qu'une reine d'Abeille ne pond pas moins de 60.000 œufs dans le cours de son existence. Une espèce de Termite donnerait plus de 2 millions d'oeulis par an.

Toutefois, on le comprendra sans peine, le régime, la temperature, jouent un rôle important dans.la régularisation de cette fonction ; l'àge aussi amène, en général, une diminution notable de la fécondité. Sous ce rapport? le cas des Purerons est éminemment suggestif. Nous avons vu (p. 196) qu'au printemps, la fécondité des femelles parthénogénétiques est assez grande; mais, dans le courant de l'été, cette fécondation devint progressivement décroissante, à tel point que la femelle sexuée de la dernière génération agame, ne pond plus qu'un seul œeuf qui est l'œuf d'hiver.

$2^{n}$ I.ieu de ponte. - In certain nombre d'Insectes peunent soin de protégrer leurs nufs après la ponte, en les abrilant dans mne sorte de nid; les savantes constructions des Aheilles sociales, dus Vespirles. des Termites n'ont pas d'aulre signification. Ln antre rxemple classique, tonjoms cité, est celui des II ydrophiles. qui fi:briquent, a l'aide d'une soie grossicre, un coron ovoüde, minni d'rus appendice recombrá, dans tequrl elles déposent environ 50 ceufs.

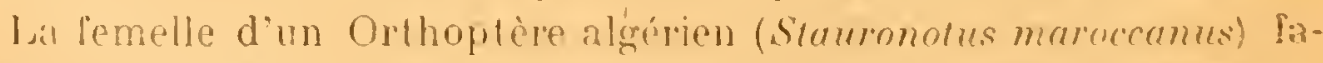
brique, en mêne lemps quelle pond, me solve d’étui terreux à l’airle doun murus qui agglomère les grains de sable, el ses a uls, an 
nombre de 60 à 70 , sont abrités dans cotte oothèque d'un nouveau genre (Fig. 126.) Le Criquet pèlerin, qui possède les même habitudes, peut, de plus, d'après Beauregard, placer son nid dans le sol à sept ou huit centimètres de profondeur.

Quelques Lépidoptères, notamment les Bombycidés (Liparis), déposent leur's œufs le long des branches des arbres; pour les protéger, ils les recouvrent d'une sorte de feutrage fabriqué arec les

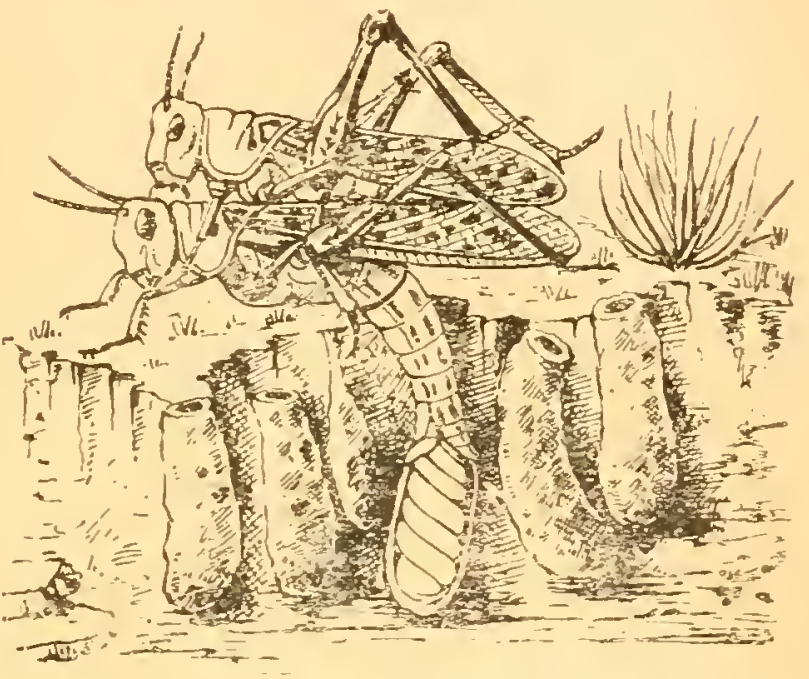

Fig. 126. - Femelle du staurunotus maroccanus Orthout. londant dans ses oothèques aussitôt après l'accouplement (d'après KracheL d'HERCtLus, in Henneguy). poils de l'extrémité de leur abdomen.

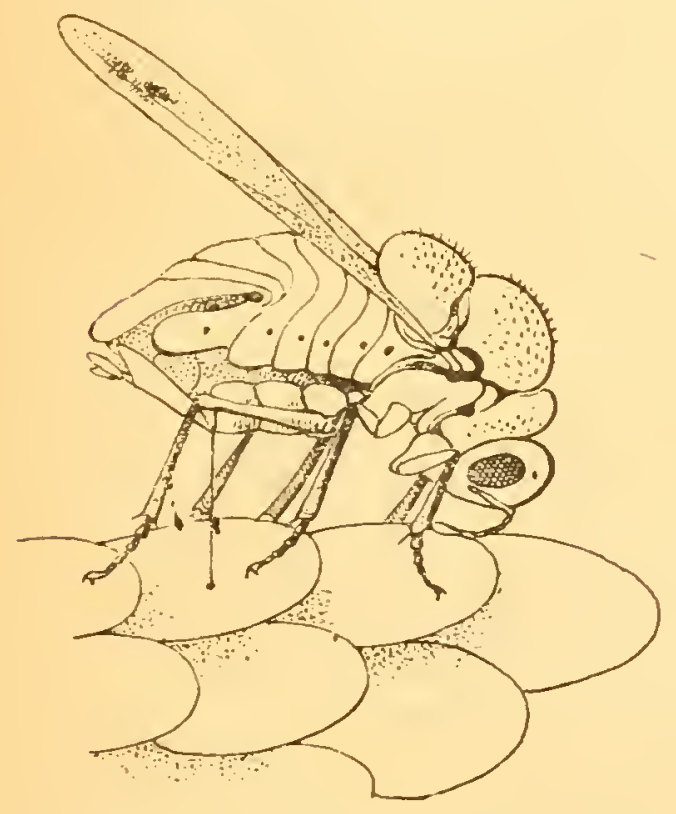

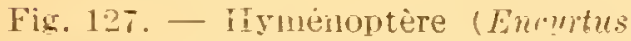
juscicollis ponrint dans les wufs de 'Hyponomente du ponmier (d'aprés M.ırchis).
Enfin, chez certains Hémip tères, les Coccidés notamment (I. ccanium), c'est le corps même de la femelle qui, vide et desséché, forme une sorte de bouclier chitineux, sous lequel les culs sont à l'abri de tout danger.

Les cas de prévoyance qui précèdent sont, dans une certaine mesure, exceptionnels ; le plus souvent, les femelles des Insectes se contentent de pondre leurs aufs dans le milieu où les larves doivent vivre ou à proximité de 1a nourriture qui leur convient. L'exemple des Mouches est caractéristique; il s'agit là d'un tropisme (1) dont l'exemple n'est

(1) Nous savüns très bien que ?a piupart rles faits attribués à l'intelligence ou à l'instinct sont des tronımes, mais nou₹ ne pourens pas nous étendre sur ces phénomènes, dans un ouvrage élémentaire comme celui-ci. 
pas isolé; tous les Insectes agissent de même; on pourrait citer des centaines d'exemples; mentionnons seulement les Charançons,

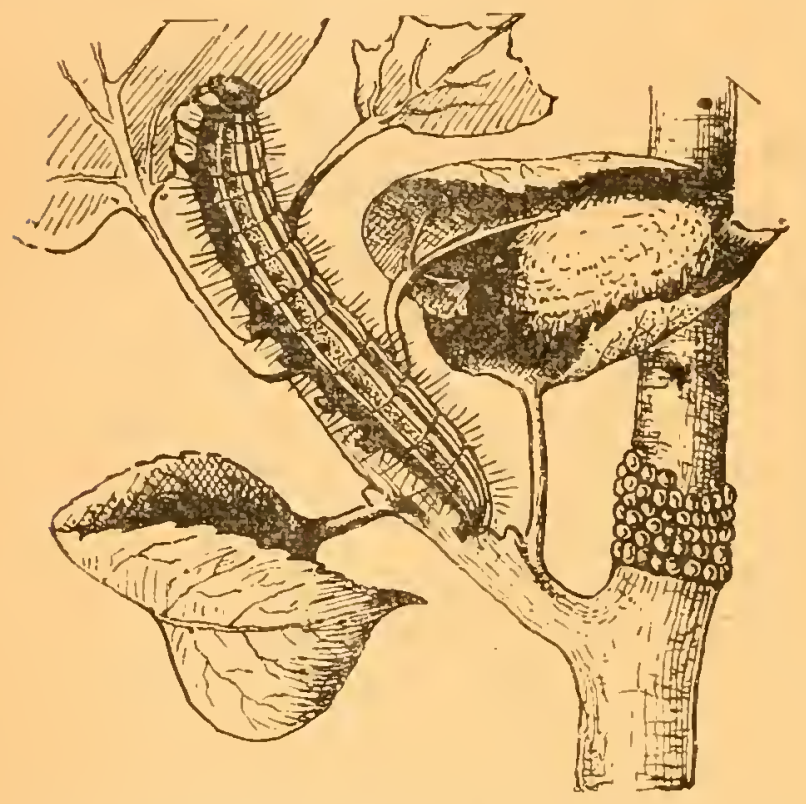

Fig. 128. - Bombya nenstria, $x$ uis, chenille et cocon renfermant !a Chrysalide. les Bruches, dont lies larves vivent dans les graines, et dont les eufs sont pondus sur l'ovaire lui-même au début de sa croissance; les Ichneumons sarent choisir les chenilles dans le corps desquelles leurs larves doivent se développer(Fig.127); les Cynips, en piquant les plantes, provoquent la formation d'un Cécidie \{salle? où leur progéniture tronvera à la fois la nourriture et l'abri. Tous les Insecte: Xylophages : Buprestide. Longicornes, etc., déposeni généralement leurs cuifs à la surface des arbres, dans les fisstres des écorres. Les Cousins pondent leurs ceufs à la surface de l'eau; il en est de même des Phyrganes, mais dans ce cas les cufs au lieu de flotter tombent an fond.

30 Mode de ponte. - La manière dont la femelle dépose ses $a$ uls dans le milieu qui convient le mieux à leur développement, est érralement très variable; ici encore, il n'y a aucune règle apparente mais on peut distinguer quatre cas principaux :

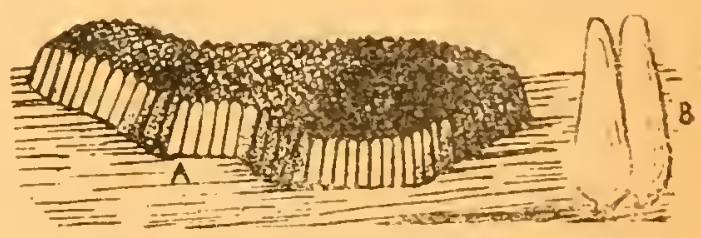

Fig. 1:2. - Oufs de Cousin, A, 11a:ell:? formée d’oufr placés côte à côte; $B$, deux

a. Dans le premiel cas, le ceufs isolé, groseis.

plus simple, les cufs sont pondus isolement. Ainsi, par exemple, la femelle du Dytique, étudiée par Regimbart, pratique une fente itroite, avec son oviscapte, dans une tige de Jonc on de Sagittaire, et, dans celte fente, introduit un seul ouf. Les Cynips percent, ia l'aide d'une longue larière, les tissus des plantes pour' y introduire

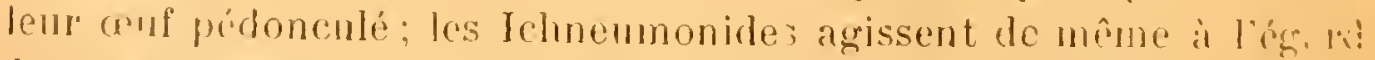
des chenilles on des neufs dans l'intérieur desquels leur larve re développera. 
b. Dans un cerlain nombre de cas, la femelle ne disperse pas ses ceufs ; ces derniers, tous déposés au même endroit, se trourent
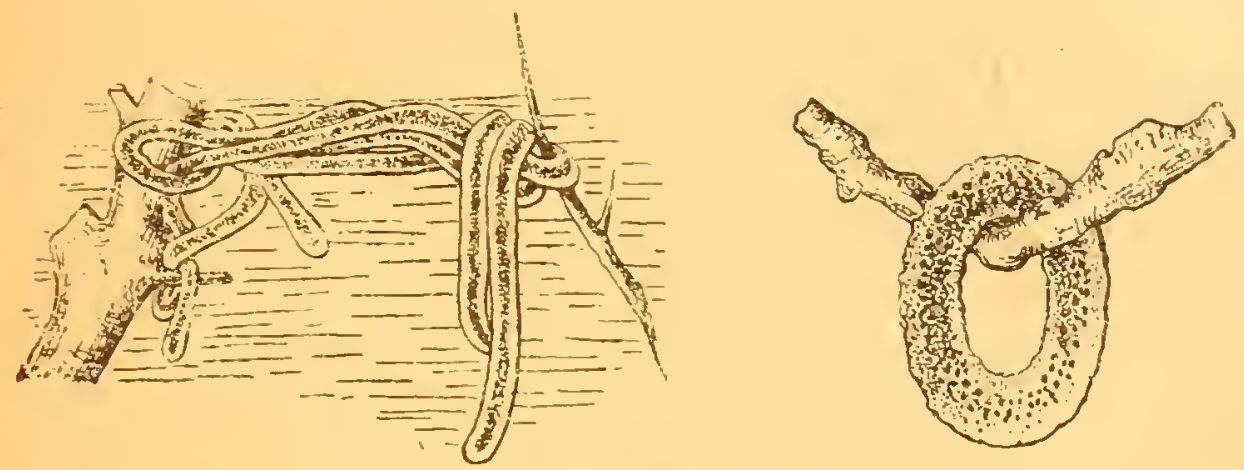

Fig. 130. - CEufs de Nérroutères aruatiques sous forme de rubans (Tetrosoneuria) ou d'anneaux gélatineux (T'richontères)

(d'alpès Nemdham et Better).

réunis, tantôt en amas irréguliers, comme par exemple ceux du Hanneton. tantôt en séries parallèles comme ceux des Ciyrins, on quelquefois mème en une élégante spirale à tours continus comme ceux du Bombyx neustria, autour des branches du poirier (Fig. 128).

Il en est encore de même des ceufs des Cousins qui, pondus côte à côte, forment de petites nacelles brunes fiottanl à la surface de l'eau (Fig. 129).

c. Dans un troisième mode de ponte, la femelle expulse ses coufs en une seule fois, sous forme de masses allongées ou lenticulaires, de consistance gélatineuse. Ce cas s'observe surtout chez les Insectes aquatiques (Diptères et Vérroptères (Fig. 136); mais on l’a également observé chez les Éphémères, chez les Phrỵanes et même chez un Lépidoptère c̀ larves aquatiques, le Botis potamogalis.

d. Enfin, dans quelques cas, les ceufs pondus sont renfermés dans une espèce de capsule qui leur assure une protection efficace; ces capsules, dont la forme rarie suivant les espèces, ont reçu le nom d'oothè-

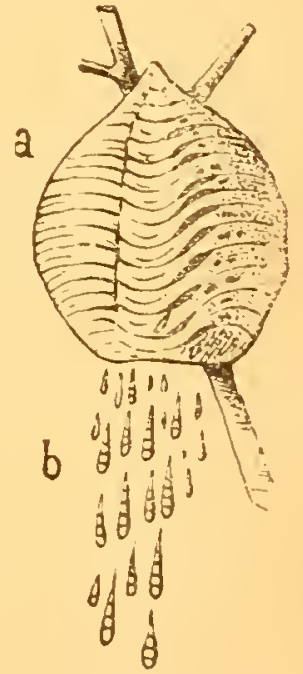

Fig. 131. Oothèque ce la Mante religicus; $a$, oothèque : $l$. embryous sortant cie l'oothèqueld'i . près BRONGNIART, ques (Fig. 131); on n'a rencontré jusqu'ici des oothèques que chcz les Orthoptères (surtout Blattidés et Mantidés).

Plusieurs auteurs ont essayé de suirre le mode de formation ćrs cothèques, et ils ont constaté qu'elles prennent naissance par i n produit de sécrétion des glandes sébifiques se durcissant à l'air. 


\section{CHAPITRE XXI}

\section{L'CUE ET SES ENVELOPPES}

Teснгiquf. - Prendre directement des œufs, à divers états de développement, dans les gaines ovariques des Insectes femelles : Hanneton, Bombyx du mûrier, Sauterelle s'erte, etc., etc. - Henneguy indique en outre un procédé très pratique, qui est le suivant:

"Pour étudier le développement des Pucerons, il suffit de prendre un de ces Insectes, de couper l'extrémité postérieure de l'abdomen et de faire sortir le contenu du corps dans une goutte d'eau salée à $0,6 \%$, en exerçant une légère pression sur l'abdomen. Les gaines ovariques sortent en entier ; on peut examiner leur contenu par transparence et faire agir sur elles différents réactifs qui font apparaître les détails de structure avec plus de netteté. " (HExNEGUY, Les Insectes, p. 40i.)

Il ra sans dire que ce procédé peut s'appliquer à tous les cas, lorsque la taille de l'Insecte ne permet pas la dissection.

$1^{\circ}$ Euf. - Aussitôt qu'il a subi l'action fécondante du spernatozoïde, l'ovule est devenu un œuf ; c'est alors qu'il est pondu, dans les conditions que nous avons indiquées précédemment (p. 207), et qu'il commence à se diviser, pour devenir un embryon, plus tard une larve, et, finalement, un Insecte notiveau.

Forme de l'œuf. - La forme et l'aspect extérieur de l'ceuf sont extrêmement variables; cependant, ses caractères paraissent asse\% constants dans certaines fanilles; étudiés avec attention, ils fourniratient même peut-être d'excellents caractères génériques ou spécifiques. 
Les œufs sont sensiblement sphériques ou orbiculaires chez un grand nombre de Papillons ; ils ont la forme d'un ovoïde allong; chez les Coléoptères et les Diptères, mais on en rencontre aussi ce plats et de coniques; quelquefois mème leur aspect est si singulicr qu'on hésiterait à les reconnaitre pour des œufs.

Ainsi, par exemple, les œufs de la Nèpe cendrée, sont oblongs $\epsilon i$ portent à leur extrémité supérieure une comronne de filaments grêles qui les font ressembler aux semences du Chardon-bénit.

Les oufs de certains Névtoptères (ex. : genre Chrysopa), pondis sur les écorces ou sur les fenilles, par groupes de dix à douze, sol t portés à l'extrémité d'un long pédoncule (Fig. 132. A.); c'est li, d'après Comstock, un acte de prévoyance de la femelle qui "sait "que si elle n'agrissait pas ainsi. le premier éclos de ses enfants dérorerait induljitablement les œufs où sont encore enfermés « ses frères et sœurs *

Beaucoup d Insectes, appartenant aux groupes les plus dirers, produisent ainsi des œufs pédonculés : le rôle de ce pédoncule paraìt varia ble; Adler, dans le cas des Chalcidites, le considère comme un appendice resp:ratoire; d'autres fois. comme parexemple chez les espèces entomophages (Ichneumonides), il sert à fixer l'ceuf sur le corps de la Chenille qui servira de pâture à la larve; chez un certain nombre d'Estrides, notamment chez Hypodema lineata, re pédicelle est admirablement adapté pour.

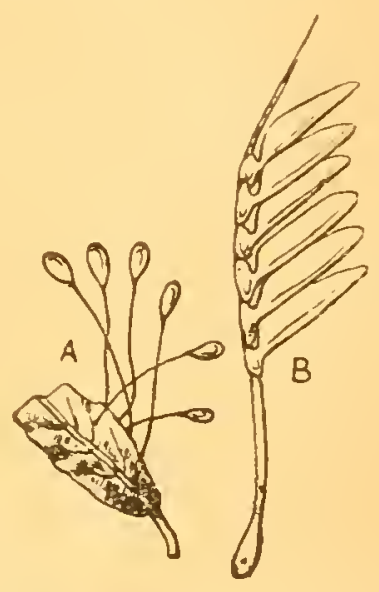

Fig. 132. - QEufs pédonculés et fixés sur un support, is. chez un Ephéméririe: $B$, chez les F.strides (d'ap. PACKARD). se fixer sur le poil (Fig. 132. B.). Enfin, certaines Mouches (Scatophaga, Drosophila), pour éviter la dessiccation de leur's aufs, dont l'enveloppe est très mince, les déposent à Y'intérieur de la substance même qui doit nourrir la larve; mais alors, pour que la petite larre ne soit pas exposée à être étouffée air moment de son éclosion, l'œuf porte, près de l'un de ses pôles. deux appendices divergents qui l'empêchent de s'enfoncer au delà des trois quarts de sa Iongueur. Latreille pense que les soies qui ornent l'cuf de la Nèpe cendrée ont un usage analogue, car le femelles les introduisent dans le parenchyme des plantes aquatiques, précisément jusqu'au nivean de ces appendices.

Si nous laissons de côté ces variations de forme pour examinc" 
l'aspect de la surface extérieure des aufs, nous trourons parf' is

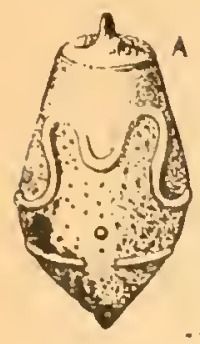

Fig. 133. - Aspe it exté rieur de quelques ceufs d'Insectes. A. chez un Phasmide: $B$. chez un Teppidoptère (d'aurès PACKARD). une ornementation des plus variée. S'il est vrai que certains œufs, comme par exer:ple ceux des Coléoptères et des Orthoptères, ont une enreloppe parfaitement lisse, or simplement granuleuse, il en esl d'autres dont la surface est très curieuscment ornée. Chez plusieurs Lépidoptères, l'enveloppe extérieure de l'œuf montre des réseaux très élégants (Fig. 133); mais les cufs les plus remarquables sous ce rapportsont certamement ceux des Phasmides.

Comme une longue description de ces particularités serait sans intérêt, nous nous bornons à donner ici les figures de quelques-uns de ces œuls, choisis parmi les plus caractéristiques (Fig. 133).

2。 Structure de l'œuf. - Les anteurs ont souvent compliqué outre mesure la terminologie des différentes parties de l'œuf; mais, si nous nous souvenons que l'élément reproducteur femelle a toujours la valeur d'une cellule, et qu'ici, en particulier, cette cellule est presque toujours pourvue d'abondantes réserves nutritives et protégée par une membrane annexe, il nous sera plus facile de comprendre les phénomènes qui se produiront dans le cours du développement, principalement au débul de la segmentation (Fig. 134).

Dans l'immense majorité des cas, l'eut est protégée par deux membranes: la membrane externe, plus ou moins épaisse, a été désignée sous le nom de Chorion. Cette membrane a été surajoutée pendant la maturation de l'ouf ; elle tire sou

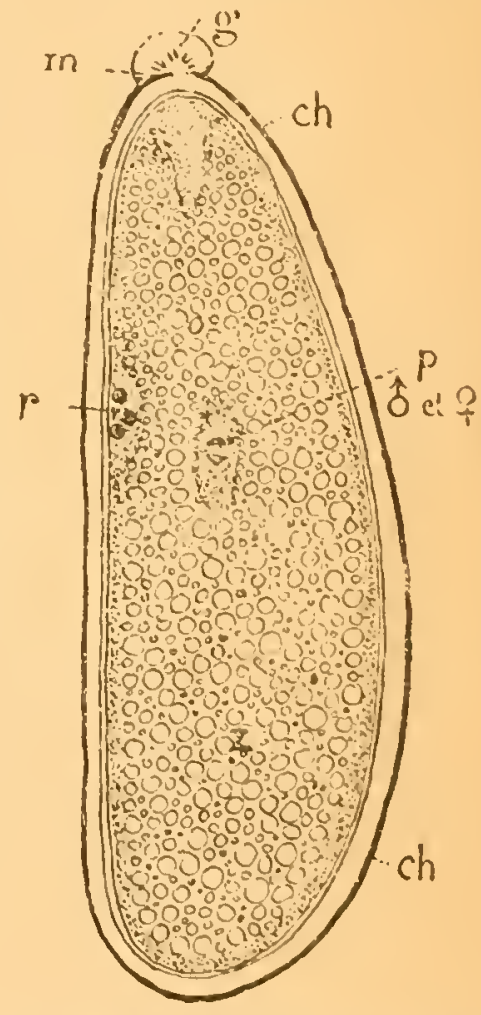

l'ig. 134. - Section honaitucinate d'un cenf de Monclie all moment de la fécondation; $m$, microllyle; ch chorion; $p$. promuclens mile et femeile: $r$ sphieres nibectries (rlapres KORACHELT et IIEHLR). origine de l'épithélium interne des graines ovariques, el c'est elle qui portel'ornementation remarquable que nous a vons signalée plus haul. 
E I dessous du Chorion se trouve une deuxième enveloppe très mince : c'est la membrane propre de la cellule-cuf (= membrane vilolline) ; sous cette deuxième membrane iient le protoplasma de l'aui (= vitellus formatif), au sein duquel la substance de réserve (= ritellus nutritif) est inégalement distribuéc. Le noyau de la cellile-œuf est souvent désigné sous le nom de résicule germinative.

O. observe, cela ra sans dire, daus la structure des cufs, un grand nombre de modifications dues, très probablement, au mode de vie des Insectes.

Vitellus. - On donne ce nom, comme nous venons de le voir, à la rúserve nutritive qui se forme à l’intérieur de l'œul pendant sa mituration.

Dans un grand nombre de cas, cette substance mutritive est irrégrulièrement distribuée à l’intirieur du protoplasma; l'cuf d.) bartient alors au type hélérolecithe (= mixolécithe (Hus$c(l)$; quelquelois cependant le vitollus nutritif paraît massé ver'z le centre de l'ouf; dans ce; casl'œul est dit centrolécithe (liparis); chez un grand nombre de Thysanoures, les cents prraissent même complète- du Chorion dans quelques Insectes; $a$, zone ment dépourvus de réserve externe, gross. 50 D (d'après F. Hexiecicy). nutritive (orufs alécithes).

On ne connait pas encore exactement la nature du ritellus nutritif ; l'analyse microhimique a seulement permis d'y raconnaitre des globules de graisse et des granulations prolíques.

Choriox. - Le chorion. ou enveloppe extérieure de l'cuf, dont la surface est fréquemment ornée de bandes en relief, d’alréoles ou de plis (Fig. 135), est une membrane d'épaisseul'variable suivant les Insectes. La structure de cette membrane, également très rariable. n'a étó étudiée juscu'ici que dans quelques cas particuliers; elle se compose, d'après Wheeler, de deux lamelles chitineuses réunies pxr da nombreux petits piliers de la même substance; ce sont les pointes saillantes de ces petits piliers qui donnení à certains cufs, leur aspect granulé. Henneguy a montré, de son côté, que la lamelle 
chitineuse externe de l'œuf d'un Phasmide (Phyllium curifolium)(1), rappelle, i s'y méprendre, la coupe d'un tissu végétal; or, par ailleurs, cet œuf ressemble extérieurement à un akène d'Ombellifère, de sorte que, chez cet Insecte remarquable, le mimétisme n'affecte: pas seulement les formes bizarres de l'adulte, mais il se poursuit jusque dans la structure de ses œufs (Fig. 131).

Micropyis.s. - Si l'enveloppe extérieure de l'œuf était continue, la fécondation serait impossible, car les spermatozoïdes ne pourraient pénétrer à l'intérieur du vitellus pour se fusionner avec le promu(leus femeile ; aussi, pour assurer cette pénétration, il existe toujours, dans l'épaisseur du chorion, une ou plusieurs petites ouvertures qu'on a désignées sous le nom de micropules. Lorsqu'il n'y a qu'une ouverture, sa position est très variable; elle est généralement placcée au pôle antérieur de l'cuf ; cependant (Acridiens), elle peut se trouver aussi au pôle postérieur. Dans certains cas même, les niicropyles ('slatte, Puce, etc.), très nombreux, sont répartis irrégulièrement on par groupes - sur toute la surface de l'œuf.

Fréquemment, l'orifice du micropyle est orné de papilles (Xèpe cendrée) ou d'appendices spéciaux; chez les Orthoptères, less Mouches, quelques grands Névroptères, il est même protégé par une sorte de capsult gélatineuse de grandeur variable.

3० Maturation de l'œuf. --. Pour que la conjugaison du spermatozoïde avec son équivalent morphologique, le pronucleus femelle, puisse produire un effet utile, autrement dit pors que la fécondation puisse avoir lieu, il faut que le volumineux noyau de la çilule-œuf (vé:icule germinative) subisse un certain nombre de mod!fications. Ces modifications consistert en une série de réductions chromatiques dont les plus importantes zont, sans contredit, l'ólimiin tion successive de deus globules polaires.

A la suite de ces éliminations, la substance chromatique hu noyau se trouve notablement diminuce; la vésicule germinative n'est plus qu'un très petit globule, auquel on a donné le nom de pionucleus femclle.

l'est ce pronucleus qui est, en réalité, le noyau actif de l'cuf ; "est lui seul qui, au moment de la fécondation, s'unira avec le spermatozoïde (pronucícus mâla).

En résumé, l’émission des globules polaires a lieu chez les Insectıs comme chez tous les antres anmaux; seulement ici, l'úpais chorion

(1) Hexsegicy (L. - F. ). Les Insectes 1\%204. 
qui protège l'œuf empêche leur sortie au dehors ; ils restent toujours inclus dans le protoplasma.

Certains auteurs ont cru pouvoir admettre que les œufs parthénogénétiques ne donnaient naissance qu'à un seul globule polaire, mais les avis sont très partagés sur cette question; les recherches récentes de Henkig (1892) tendent, au contraire, à prouver que tout se passe comme à l'état normal.

\section{Segmentation de l'œui。}

A. Segmentation superficielle ou endovitelline. - Aussitôt après la fécondation, le noyau de l'œuf commence à se diviser ; on voit donc apparaître, alı sein du vitellus, un grand nombre de noyaux plus petits qui s'entourent d'une légère couche de protoplasma: ce sont les noyaux vitellins de Bobretsky. Ces noyaux, avec le corps protoplasmique qui les entoure, sont de véritables cellules, ₹qui continuent à se développer par division. Mais comme ces cellules, plongées à l'intérieur du'vitellus, se trouvent dans de très mauvaises conditions pour respirer, elles émigrent bientôt vers la surface libre de l'œuf, et là, se disposant côte à côte, elles arrivent à former une membrane continue qui est le blastoderme (Fig. 136. bl).

Lorsque cette membrane est complète, les noyaux vitellins, qui continuent à se former à l'intérieur

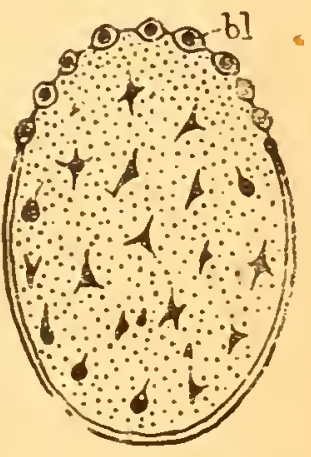

Fig. 136. Le début de la segmentation chez Pieris cratoegi Lél. (d'après

BOBRETZKY). du protoplasma, ne peuvent plus venir se placer à la surface, ils restent à l'intérieur du vitellus et forment ce qu'on a appelé les balles vitellines $(=$ cellules vitellophages).

Telle est, dans ses grands traits, la marche des premiers phéncmènes de la segmentation chez les Insectes ; ce mode de segmentation, superficielle en apparence, est évidemment. dî à l'abondance des réserves nutritives accumulées au sein du protoplasma de l'œuf. Les modifications légères qu'on a signalées dans certains groupes, par exemple, chez les Diptères, les Coléoptères et les Lépidoptères, aboutissent quand même, par une voie moins directe, ì la formelion d'un biastoderme continu (Fig 137) : c'est là lc fait capital çu'il faut retninir.

LES INSECTES. - $2^{e}$ édit. 
A ce moment, l'œuf esi donc constitué par deux groupes de cel. lilles bien distinctes : les cellules externes, formant une membrane continue à la surface, le blastoderme, et les balles ritellines internes. C'est là l'indication d'une première division du travail physiologique : les premières sont adaptées aux fonctions respiratoires ; les secondes à la nutrition.

B. Segmentation totale. - Il va sans dire que chez les Insectes où l'œuf est très pauvre en substances de réserves (œufs alécithes ou hololécithes), la segmentation se fait avec une plus grande régularité : elle est totale ou presque : tel est, par exemple, le cas d'un Polygaster, parasite de la Cécidomye du Saule, observé par Ganin en 1869.

En résumé, suivant la quantité de réserve nutritive accumulée dans le protoplasma de l'œuf, la segmentation peut être totale, inégale ou plus ou moins superficielle. Le premier mode, le plus simple, et certainement le plus ancien; n'a été observé, juśqu'ici, que chez quelques Thysanoures et chez les Hyménoptères parasites; le deuxième est celui qu'on observera

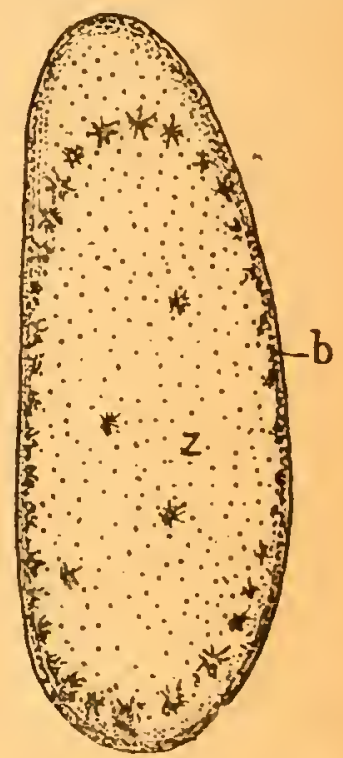

Fig. 137. - Formation du blastoderme, $b$, chez l'Hydrophile Col. probablement chez tous les autres Insectes; comme toujours, la segmentation aboutit à la formation d'une blastula, mais ici, c'est une blastula pleine dont la cavité est entièrement remplie par le protoplasma vitellin; la phase morula n'existe donc réellement, chez les Insectes, que là où les œufs subissent une segmentation totale (Thysanoures et Hyménoptères parasites). 


\title{
LIVRE DEUXIÈME
}

\author{
PREMIERE PARTIE
}

Embryogénie.

\section{CHAPITRE I}

\section{DÉVELOPPEIIENT DE L'EMBRYON}

Les deux points les plus importants de l'embryogénie sont ceux qui ont trait:

$1^{\circ}$ A la formation des membranes embryonnaires.

$2^{\circ}$ A la formation du corps de l'embryon lui-même.

$1^{\circ}$ For MATION DES MEMBRANES EMBRYNuARES.-Nous venons de voir que la segmentation aboutit à la formation d'un embryon tout d'abord indifférencié, dans lequel on ne peut distinguer que deux parties, une couche externe, blastoderme, puis une masse interne de granulations ritellines; la première modification importante qui se produit est celle-ci.

A un moment donné, le blistoderme forme, à la surface de l'œuf, un épaissisement longitudinal : ce premier épaississement constitue la zone germinative ou bandelette primitive. Bientôt après, cette bandelette se déprime suivant son axe en une gouttière plus ou moins marquée; on peut considérer la gouttière en question comme un blastopore allongé, et l'invagination n'est probablement elle- 
même autre chose qu'un rappel de la phase Gastrula. A peu près au moment où le blastopore va se fermer, on voit apparaitre le long des bords extérieurs de la bandelette primitive, deux replis allongés : ce sont les rudiments des enveloppes embryonnaires, si répandues et si importantes chez les Insectes.

En s'accroissant, les deux replis, dont nous venons de parler,

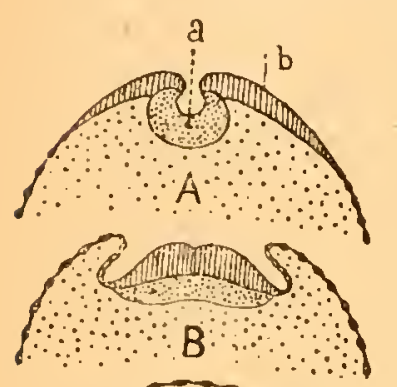
s'avancent l'un vers l'autre; ils empiètent de plus en plus sur la zone germinative et finissen $\imath$ bientôt par la recouvrir en se soudant par leur bord. La bandelette primitive se troure alors enfermée au fond d'une cavité qui a reẹı le nom de casité amniotique.

La paroi qui limite cette cavité comprens? deux couches de cellules: l'une interne qui se continue par son bord extérieur avec le blastoderme (Pig. 138) de la zone embryonnaire et constitue l'amnios proprement dit; l'autre externe, se confondant sur ses bords avec le reste du blastoderme de l'œuf, et formant ainsi une membrane protectrice entourant l'œuf tout entier: c'est l'enveloppe séreuse.

L'amnios et l'enveloppe séreuse n'ont aucun rôle à jouer dans la formation ultérieure de l'embryon; ce sont de simples membranes protectrices, qui disparaissent, en général, vers l'époque de l'éclosion.

Premiers linéaments de l'embryon. - En même

Fig. 138. - Origine des membraues enbryonnaires : $A$, formation de la plaque ventrale $a$; $B$, prennière apparition des replis anmiotiques; $C$, ammios complet $(\mathrm{am}$ )

d'après KioRscheLT et HEIDER ).

temps que la bandelette germinative se développe, elle s'étend à la surface du vitellus; puis, on y voit bientôt apparaître des sillons transversaux; c'est là le début de la métamérisation ; les segments qui vont alors se former correspondront à ceux de la larve et de !'adulte. Dès l'origine, l'aspect du premier segment antérieur est tout à foit caractéristique; il est divisé en deux larges lobes : ce sont les lobes procéphaliques, d'où dériveront, plus tard, les parties les plus importantes de la tête. L'invagination qui donnera naissance à la bouclıe et à l'intestin antérieur (stomodeum), celle qui donnera, de même, naisssance à l'anus et à l'intestin postérieur (proctodeum), se forment également, l'une à la partie antérieure, l'autre à la partie postérieure de l'épaississement embryonnaire. 
20 Modifications DANS LA FORMATION DES MEMBRANES EMBRYONNAIRs. - Maintenant que nous savons comment se forment les membranes embryonnaires, examinons les rariations qu'elles présentent chez les différents Insectes. Nous allons prendre pour guide l'important travail que Graber a publié sur ce sujet en 1888, mais en le débarrassant de la plupart des termes barbares, dont le savant professeur de l'Université de Czernovitz arait cru devoir l'émail$\operatorname{ler}(1)$.

10 Insectes dépourous de membrane embryonnaire. - Tout d'abord, si nous observons la marche du développement chez les Insectes inférieurs, nous constaterons que, chez la très grande majorité des Thysanoures, il ne se forme aucune membrane embryonnaire; l'eimbryon de ces Insectes est purement et simplement placé à la surface ventrale du vitellus, sans aucune enveloppe pour le protéger.

Toutefois, Uzel, en 1898, observa, chez Lépisma saccharina, une succession de phénomènes qu'il considère comme aboutissant à la formation d'un amnios rudimentaire.

$2^{\circ}$ Insertes à une seule membrane embryonnaire. - D'après Metchnikoff, l'embryon des Hyménoptères parasites (Pléromalides, Cunipides, etc.), n'est protégé que par une seule mémbrane embryonnaire; d'après ses recherches personnelles sur Smicra clavipes, Henneguy pense même que cette membrane ne se forme pas par le procédé habituel ; elle prendrait naissance "par simple délamination de la couche cellulaire superficielle de l'œuf segmenté ".

Il ne faut pas attacher une trop grande importance au mode irrégulier de formation de ce pseudo-amnios; le résultat définitif, c'est-à-dire la protection de l'embryon se trouve réalisée par un procédé différent, voilà tout.

$3^{\circ}$ Insectes entoptychiques. - Dans les Insectes de ce groupe, auquel appartiennent les Libellulides, la plupart des Hémiptères et quelques Orthoptères, la bandelette primilive, formée comme de coutume à la surface de l'œuf, s'incagine peu à peu dans l'intérieur du ritellus. L'embryon se développe à l'intérieur de cette invagination (embryon entoblastique); il est donc très efficacement protégé et le rôle des membranes embryonnaires est, de ce fait, très effacé ; elles se fol'ment cependant par le procédé ordinaire, mais elles suivent le mou-

(1) Gr.1Ber (V.). - Vergleïchen.7e Studien uber die Keimhüllen unul die Rückenbilsung der Insekten (Denkschr-Akad. wiss. Wien. 188s). 
rement de l'embryon et sont elles-mêmes entraînées à l'intérieur du vitellus (Fig. 133). Au moment de l'éclosion, les membranes se rompent au point où s'est fermé le repli amniotique, et c'est par cet orifice
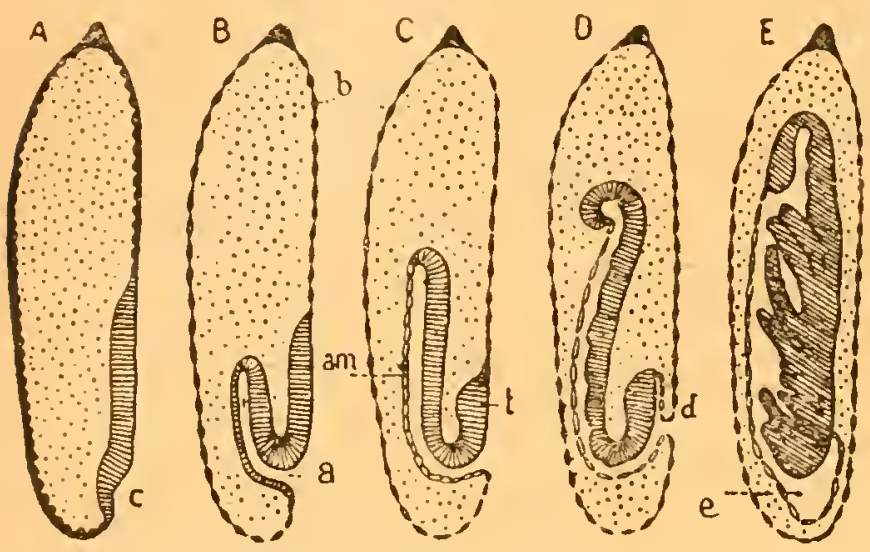

Fig. 139. - Cinq positions successives de l'em. bryon chez les Insectes entoblastiques (d'après BRANDT). que le corps de l'embryon se libère petit d petit de ses enveloppes.

$4_{1}^{\circ}$ Insectes ectoptychiques. - On réunit dans ce groupe tous les Insectesdontlesembryons accomplissent leurs transformations à la surface du vitellus ( $C_{0}$ léoptères, certains Orthoptères, Nésroptères, Diptères, etc.). Ici les membranes se forment par le procédé typique le plus régulier (embryors ectoblastiques) (Fig. 140).

Dans la grande majorité des cas, la cavité amniotique se ferme complètement; ce sont les Insectes holoptychiques de Graber (ex.: stcnobothrus; inais parfoisaussi, cependant, les membranes embryonnaires, n'étant pas suffisamment développées, la cavité amniotique reste ouverte sur la face ventrale; c'est le cas des Insectes hémiptnckiques de Graber (certáins Diptères: Musca: $\mathrm{Ce}^{-}$ cidomiga).

En résumé,depuis les Thysanoures, dépourvus d'enveloppes, jusqu'aux Insectes
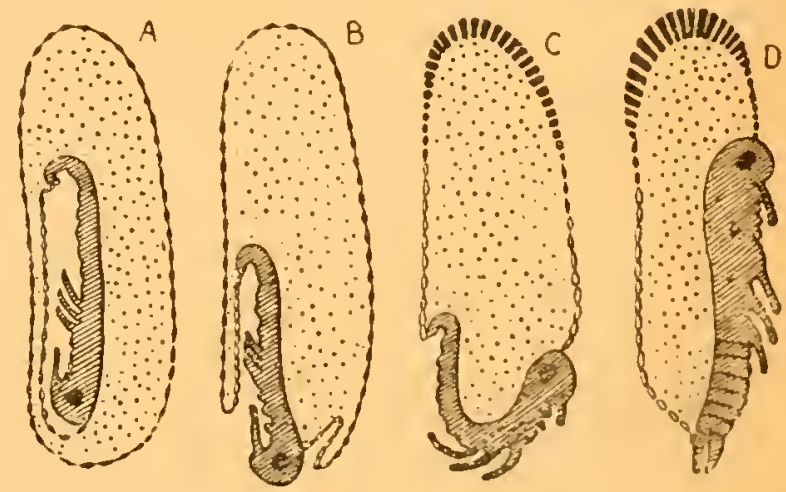

Fig. 140. - Les positions successives de l'cmbryon chez les Insectes ectoblastiques (d'après AYERS).

holoptychiques les plus parfaits sous ce rapport, nous voyons les membranes embryonnaires se perfectionner par une série de processus dont le but évident est de protéger l'embryon en voie de formation. Ce résultat, bien que très important, n'a cependant pas toute la valeur phylogénétique qu'on serait ten té de lui accorder, parce ru'il y a des variations tris nrrandes, et ce, dans un même 
groupe. Tout dépend, comme nous l'avons dit, de la richesse de l'œuf en réserves nutritives.

$3^{\circ}$ Origine des membranes embryonnaires. - Plusieurs hypothèses ont été émises; toutes contiennent probablement une part de vérité, mais celle qui nous paraîtrait tenir le plus grand compte des données de la phylogénie, serait celle qui combinerait la théorie Wheeler avec celles de Ryder et de Grassi.

On sait, en effet que, chez certains Myriapodes (Géophilus, par exemple), la zone germinative recouvre l'œuf presque tout entier ; à mesure que l'embryon s'accroît, il éprouve, de la part de la coque rigide de l'œuf, une résistance qui l'oblige à s'invaginer dans !'intérieur du vitellus.

Si nous passons maintenant aux Insectes, par suite de la diminution du nombre des métamères (Hémıptères), la bandelette épaissie qui doit donner naissance à l'embryon sera beaucoup moins étendue; il y aura donc une tendance graduelle à la dévagination; mais, en même temps, la partie limitante du blastoderme, restée mince, et se développant plus rapidement en surface, arrivera à former un repli qui, petit à petit, recouvrira l'embryon tout entier. Les rudiments de ce repli, qu'on observe chez. les Thysanoures, leur dévcloppement plus avancé, mais encnre incomplet chez les Insectes hémiptychiques, (Musca, Cecidomya), nous paraissent des étapes très claires de cette évolution. Par suite, comme le fait remarquer très justement Henneguy, la formation des membranes embryonnaires résulterait donc "d'une diminution de longueur de l'embryon en passant des Myriapodes aux Insectes ». On conçoit aussi trèミ nettement comment, à un mode de déreloppement primitivement endoblastique, a succédé le développement ectoblastique, ainsi que tous les états intermédaires qui ont dî, rationnellement, le précéder. 


\section{CHAPITRE II}

\section{FORMATION DES FEULLETS EMBRYONNAIRES}

La théorie féconde des trois feuillets blastodermiques (ectoderme, mésoderme et endoderme), introduite dans la science par von Baer, a eu une grande influence sur les progrès de l'embryogénie pendant trente années ; elle a conduit Hæckel à formuler sa fameuse théorie de la gastrula.

Mais, chez les Insectes, la présence d'un vitellus nutritif abondant amène une perturbation notable dans la marche des phénomènes; aussi la formation des trois feuillets y est-elle plus difficile à suivre que dans la plupart des autres groupes d'animaux.

Nous ne pouvons pas entrer dans le détail de toutes les discussions qui ont eu lieu à ce sujet, et nous allons nous borner ici, en résumant les conclusions concordantes d'Heymons et de Lécaillon, à présenter la question sous une forme aussi brève et aussi élémentaire que pussible.

Ectoderme. - Nous avons vu comment (p. 220) les deux premiers replis du blastoderme, en se soudant, arrivent à former la cavité amniotique. Aussitôt après cette soudure, la gouttière, formant le fond du repli, est reportée vers le dos par suite de l'extension des bords de la bandelette germinative; comme cette gouttière va sans cesse en s'approfondissant, il arrive que l'embryon se trouve bientôt isolé à l'intérieur de la cavité 
amniotique et recouvert par un nouveau tégument qui est l'ectoderme.

Mésoderme. - A part quelques légères variantes, la formation du mésoderme paraît se faire d'une façon très uniforme chez les Insectes. I] se produit, ainsi que l'ont indiqué Graber et Kowalewsky, par une invagination ectodermique en forme de gouttière à la surface de la bandelette primitive (p. 219); les bords de l'invagination, se rapprochant peu à peu, il en résulte un tube qui se sépare de l'ecto- derme, s'aplatit au-dessous de lui, et, finalement, s'étale pour former le mésoderme
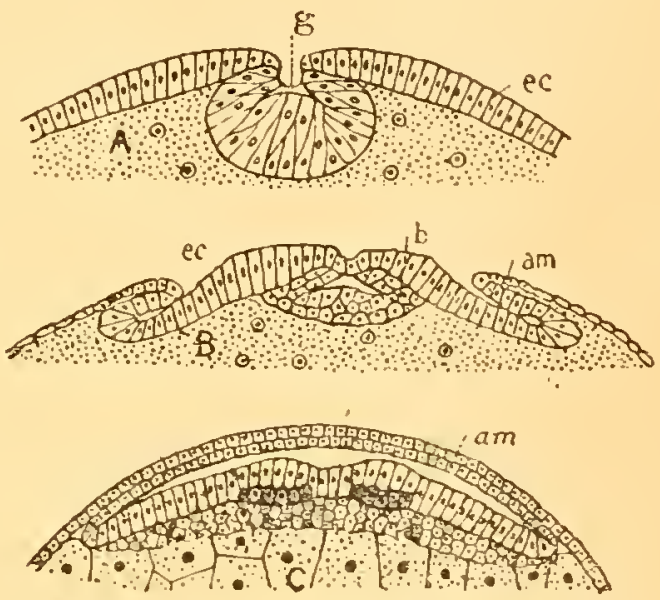

Fig. 141. - Trois star?es c.e la formation des feuillets embryonnaires chez l'Hyirophile; $e c$, ectoderme; $g$, lilastopore : am. replis amniotiques (d'après Heiner).

(Fig. 141). Heymons confirma cette manière de voir dans son travail sur les Orthoptères, en ce qui concerne la Forficule; Lécaillon arrive aux mêmes conclusions dans ses recherches sur l'embryogénie des Chry ysomélides.

Erdoderme. - L'ère des discussions n'est pas close, relativement au mode de formation de l'endoderme; voici, résumé en quelques mots, ce que l'on peut dire de général sur ce sujet.

Le véritable endoderme des Insectes est représenté par les grandes cellules vitellines qui se différencient, à l'intérieur de l'cuf, après là formation du blastoderme ; mais, au lieu de prendre part, comme on aurait pu s'y attendre, à l'édification de l'intestin 'moyen, ces cellules (D) sont, dans la majorité des cas, détournées de leur rôle naturel ; elles doivent assimiler l'abondante réserve 
nutritive de l'œuf, afin de la transmettre aux autres cellules de l'embryon (Fig. 142).

Mais alors, pendant ce temps, partent, du fond du pro-

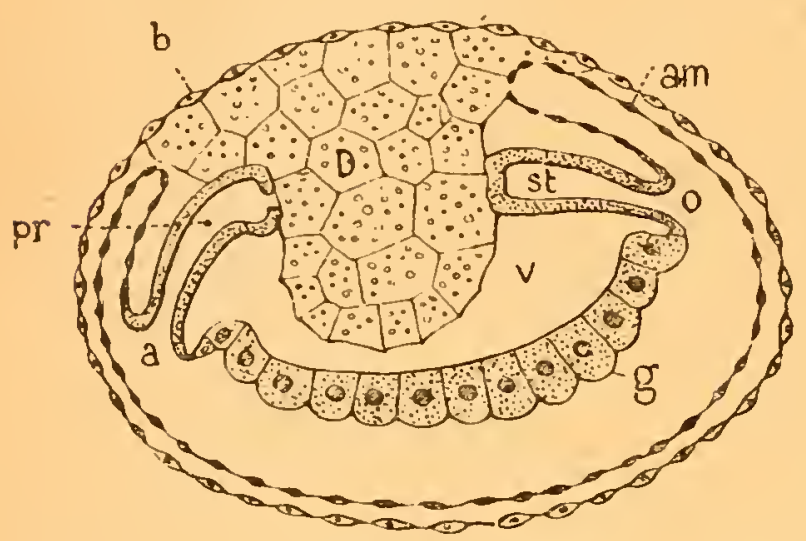
tocdeum $(p r)$ et du stomodeum $(s t)$, deux replis en forme de calottes, qui s'avancent l'un vers l'autre, et qui, s'insinuant entre le vitellus et le mésoderme, arrivent à former, par

Fig. 142. - Formation de l'endoderme chez leur réunion, un sac la Forficule; 0 , hlastoderme; am, amnios: $v$. clos à l'intérieur du-
sinus sanguin ventral ; $g$. ganglions nerveux (d’après F. Hennegu r).

quel le vitellus nutritif est renfermé tout entier; la cavité qui en résulte devient l'intestin moyen. Des communications s'établissent ensuite avec les extrémités de l'intestin antérieur et de l'intestin postérieur, de sorte que, vers le moment de l'éclosion, le tube digestif se trouve constitué dans toute son étendue.

Toutefois, le mode de formation que nous venons de décrire ne s'observe pas chez tous les Insectes; chez les Thysanoures, en effet, d'après Heymons, nous rentrons dans le cas normal, en ce sens que ce sont les grandes cellules vitellines qui donnent la paroi de l'intestin moyen. Il èn est de même, paraît-il, chez les Libellulides.

En résumé, c'est donc seulement chez les Thysanoures et les Libellulides, que l'épithélium de l'intestin moyen dérive normalement de l'endoderme; partout ailleurs, chez les Insectes, l'épithélium entier du tube digestif est d'origine ectodermique. 


\section{CHAPITRE III}

\section{DÉVELOPPEMENT POSTEMBRYONNAIRE}

TEсHNique. - Suivre les développements de ce chapitre sur les exemples indiqués dans le texte.

\section{$1^{0}$ - Les premières phases de la vie larvaire.}

Tous les changements que nous avions étudiés jusqu'ici se sont produits à l'intérieur de l'œuf et ont abouti à la formation d'un embryon. Or, à l'époque de l'éclosion, lorsque l'embryon des Insectes devient libre, il est rare que son développement soit complètement terminé; le petit être qui sort de l'œuf est, en général, un organisme incomplet, mais qui cependant doit vivre d'une vie indépendante et pourvoir lui-même aux besoins de son existence: c'est la larve. Pour acquérir la forme définitive de l'adulte, cette larve devra subir une série de transformations graduelles, qui constituent ce qu'on appelle les métamorphoses; au cours de ces transformations, on verra apparaitre un certain nombre d'organes nouveaux tandis que d'autres, devenus inutiles, disparaîtront.

Mais ce n'est pas tout; dans la grande majorité des Insectes, la larve, avant d'arriver à l'état adulte, subit encore plusieurs mues qui lui permettent de grandir; 
puis, elle traverse un état particulier, celui de nymphe ou chrysalide, pendant lequel elle conserve une immobilité plus ou moins complète.

Les Insectes qui présentent ainsi quatre étapes successives dans le cours de leur évolution : œuf (embryor), larve, nymphe et adulte (imago), sont dits : Insectes à métamorphoses complètes ou Holométaboliens (1); ceux qui, au contraire, ne subissent aucune modification, et qui possèdent déjà, au sortir de l'œuf, la forme qu'ils conserveront pendant toute leur vie, sont dits : Insectes sans métamorphoses ou Amétaboliens.

Mais, entre ces deux modes extrèmes d'évolution, il existe un certain nombre d'intermédiaires que nous nous efforcerons de caractériser.

Les anciens avaient remarqué, sans aucun doute, les transformations des Insectes, et ils connaissaient bien les métamorphoses des Papillons : " Erluca genus est qux, rupto cortice cui includitur, fit papilion ". (Pline. Lib. II, cap. 23) ; mais c'est à Linné que nous devons les termes employés pour désigner les différents stades de l'évolution des Insectes, depuis la sortie de l'œuf jusqu'à l'état adulte.

Cependant, toujours sous l'influence des idées de Swammerdam (p. 9) (2), Linné considérait que, sous le premier état, " la forme réelle de l'insecte est masquée, d'où le nom de larva (= masque); dans le deuxième état, l'insecte immobile est emprisonné dans ses téguments, crmme une momie dans son maillot; Linné appelle ce stade pupa (= poupée). - Mais le terme de pupe a été réservé depuis à certaines formes spéciales, et ce stade est

(1) Du gree : olos enticr et metalote cliangenent.

(2) 'Théorie de l'enloitement des germes. 
aujourd'hui désigné par le terme général de mymphe (1). Enfin, l'Insecte adulte était pour Linné l'imago (= imag'e), attendu qu'ayant dépouillé son masque, n'étant plus déguisé, il est devenu en quelque sorte le véritable représentant "l'image de son espèce ».

L'un des caractères les plus remarquables de la métamorphose chez les Insectes, c'est que le passage d'un stade à l'autre se fait, en quelque sorte, brusquement et non pas graduellement, par l'apparition de parties nouvelles comme cela a lieu, par exemple, chez les Crustacés.

Ces faits avaient vivement frappé les premiers observateurs; et, ils en étaient venus à admettre, naturellement, qu'à chaque étape de la métamorphose, l'Insecte se transformait entièrement en un animal nouveau.

Nous savions pourtant depuis longtemps qu'il n'en est rien, car Swammerdam (1738), étudiant l'évolution de la Piéride du chou, avait démontré que le Papillon était déjà tout formé sous la peau de la Chrysalide.

\section{2o - Métamorphoses des Insectes.}

Il y a, comme nous l'avons dit, des degrés dans les transformations que subissent les Insectes, ce qui justifie, dans une certaine mesure, les anciennes expressions de métamorphoses complètes et de métamorphoses incomplètes.

10 Ixsectes sans métamorphoses = Amétabolie. - Dans certains cas, l'œuf est assez riche en substances de réserves pour permettre à l'embryon d'accomplir tout son développement à son intérieur; le jeune Insecte sort alors de l'œuf avec la forme qu'il conservera tonte sa vie ; une série de mues lui permettront de grandir, et il sera adulte lorsque ses organes génitaux seront arrivés à maturité :

(1) Te nom de Chysalide s'emploie, de préférence, lorsqu'il s'agit de la nymphedes Papillons. 
c'est le cas, par exemple, des Thysanoures (Lépismc) et des Collem-

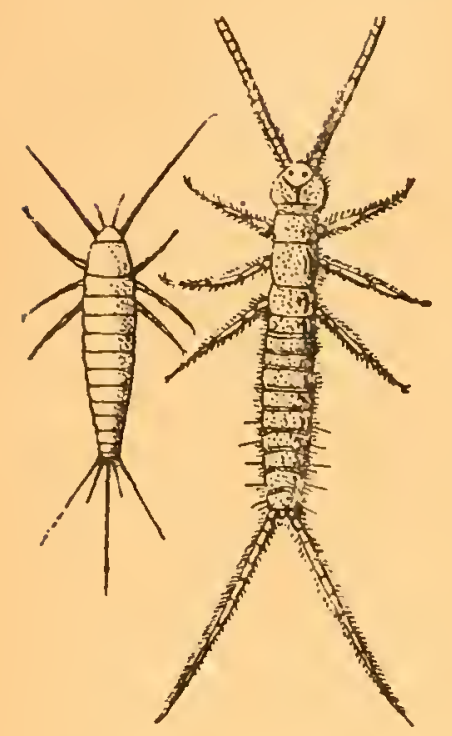

Fig. 143. - Insectes Thysanoures. A gauche un Lópisme: à droite, le Campodé. bules (Fig. 143).

Il faut encore ranger dans ce groupe un certain nombre d'Insectes adaptés à la vie parasitaire, et qui, de ce fait, sans aucun doute, présentent une ametabolie acquise: tels sont les Poux, la Punaise des lits, les femelles aptères et parthénogénétiques des Pucerons.

$2^{\circ}$ Métamorphose graduelle = Paurométabolie. - Ici encore, l'Insecte sort de l'œuf avec une forme très voisine de celle de l'adulte; toutefois il n'a pas d'ailes, et ses organes reproducteurs sont à l'état rudimentaire. Ces organes se développent au cours des mues successives que subit la larve; et, finalement, les ailes deviennent reconnaissables sous la peau. On donne génć. ralement le nom de nymplie à cette dernière forme transitoire de l'évolution; mais, comme le dit Henneguy, "cette pseudonymphe ne cesse de se mouvoir et de prendre de la nourriture; elle a exactement le même genre de vie que la larve et que l'adulte, et ne paraît être le siège d'aucun phénomène histologique."

On rencontre ce cas chez les Orthoptères et la plupart des Hémiptères (Fig. 144).

$3^{\circ}$ Métamorphose graduelle a NYMPHE IMMORILE = Hypométabolie. - Ce cas très remarquable d'évolution se rencontre, parmi les Hémiptères, dans la famille des Cicadidés. a Ici, la larve diffère de
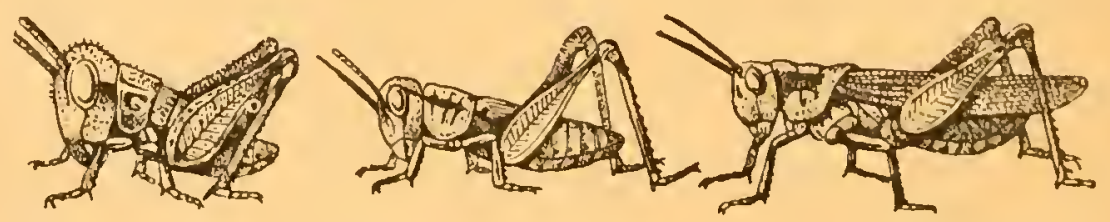

Fig. 144. - Mélamorphoses graduelles d'un Orthoptè re (Metanoplus fémur-rubrum) (d'après EmerToN).

l'adulte, non seulement par l'absence des ailes, mais encore par son genre d'existence. "Elle vit sous terre, dans les galeries qu'elle creuse à l'aide de ses membres fouisseurs et se nourrit de la sève des racines. Sa croissance est tellement lente qu'aux Etats-Unis, 
d'après l'intéressant rapport qu'en a donné Ch. Riley, en 1886 (1), la Cigale septemdéccnnaire et ses variétés mettent de treize à dix-sept années à se transformer en adultes, après avoir subi pour le moins vingt-cinq à trente mues; c'est l'exemnle le plus long d'évolution larvaire qui ait été observé jusqu’ici chez les Insectes (2).

A la larve succède une nymphe d'abord mobile puis ensuite immobile.

"Les mâles des Coccides passent également par un stade de nymphe immobile enfermée dans un petit cocon."

40 Métanorphose mcomplète = Hémimétabolic. - Chez certains Névroptères (Odonates, Fphémérides, Perlides), les larves sont aquatiques; leur organisation est donc très différente de celle des adultes qui sont aériens. A la suite de nombreuses mues, au cours desquelles elles se rapprochent graduellement de la forme adulte, sans cesser toutefois d'être

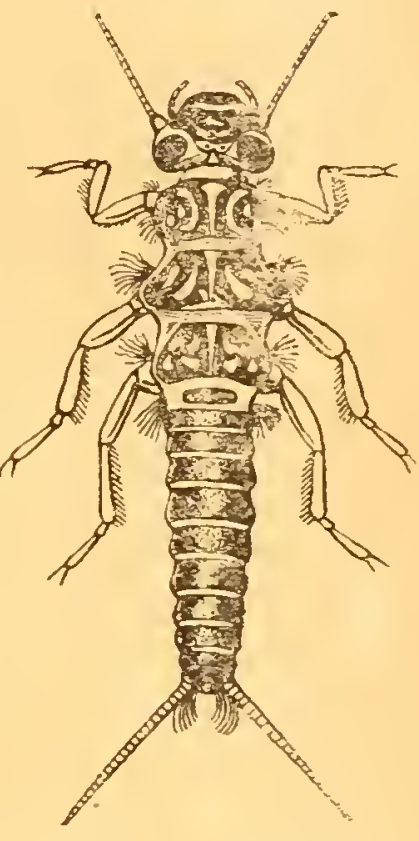

Fig. 145. - Larre de Névroptère (Perla bipunctata) (d'ap. Mrall). actives, ces larves finissent par acquérir des rudiments d'ailes: c'est pour elles la phase nymphale (Fig. 145).

Lorsqu'elles sont sur le point de se transformer, elles sortent de l'eau et s'attachent solidement par les pattes à quelque plante aquatique; leur peau, vite desséchée à l'air, se fend bientôt sur la ligne dorsale et l'Insecte parfait sort de son fourreau.

L'adaptation à un nouveau régime exige ici de profonds changements dans l'organisation, surtout dans l'appareil locomoteur : c'est une véritable "mítamnr l'ose fonctionnelle".

5c Métanorphose complète = Holométabolie.-- Considérons l'un des cas les plus faciles à observer, celui qui nous est offert par le Papillon blanc du chou ( Trerls lrassico) (Fig. 146).

La femelle de ce Papillon pond, sur les feuilles du chou, de petits œufs qui donnent naissance à une larse éruciforme, vulgairement désignée sous le nom de chenille. (.ette chenille grandit rapidement;

(1) RILEx (CH.). - The periodical Cicada (Report of the Commission of Agriculture. Washington, 1886, p. 25\%.)

(2) Ce fait paraît avoir été noté pour la première fois par le professeur Kalm, élève de Linné, en 1753 (Traerls in N. A. vol. II, p. 6). 
elle subit plusieurs mues, et, au bout de dix-huit à vingt jours, ce transforme en chrysalide (= nymphe) complètement immobile.

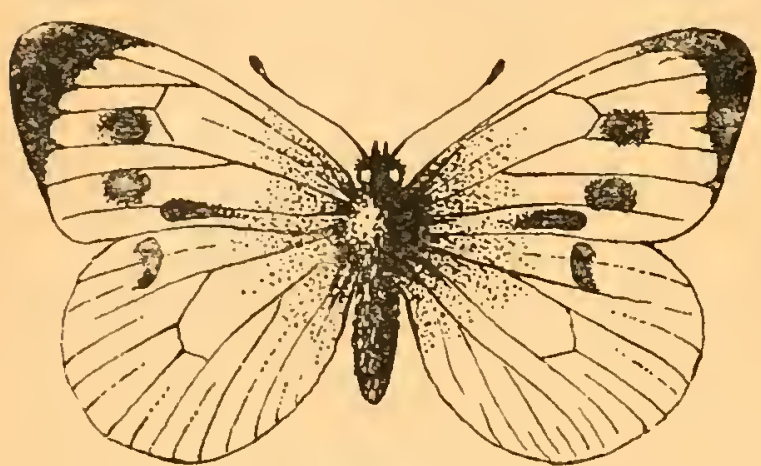

Fig. 146.

Ta Piéride du Chou (d'après wature). Après un temps variable. qui dépend de la température, le tégument de la chrysalide se fend sur le dos, livrant passage à un Papillon complet, ayant sa forme et sa taille définitives. Le Papillon n'est plus broyeur comme l'était sa larve; sa bouche est maintenant sur le type suceur.

On voit, par conséquent, que, pour arriver à sa forme définitive, le Papillon présente, l'œuf compris (embryon), quatre formes successives bien distinctes. L'ensemble de ces particularités, et surtout la présence de la chrysalide immobile, ne se nourrissant pas, c'est là ce qui caractérise la métamorphose dite complète (Lépidoptères, Coléoptères, Hyménoptères, Diptères, etc.).

Il convient aussi de dire que, presque toujours, la nymphe des Insectes holométaboliques est le siège de phénomènes d'histolyse ef d'histogénèse très accentués.

$6^{\circ}$ Hypermétamorphose. - Une place à part doit être réservée aux Insectes vésicants dans le chapitre des métamorphoses. Leur développement dépasse incontestablement, en complication, tout ce que nous avons décrit jusqu'à présent ; c'est pourquoi Fabre, qui l'a découvert en 1857, l'a désigné sous le nom d'hypermétamorphose.

L'hypermétamorphose est surtout caractérisée par l'existence de plusieurs formes larvaires très différentes, avant la transformation en nymphe. Les deux dernières formes de larves sont toujours séparées par un état de pseudonymphe qui, d'après Künckel d'Herculais, n'est autre chose qu'une phase d'attente, un enkystement, qu’il désigne sous le nom d'Hypnodie.

Quoi qu'il en soit, ces remarquables adaptations des larves chez les V'ésicants montrent nettemenl l'influence du mode de vie sur la mirche de l'évolution.

En réalité, la distinction des six types de développement que nous venons d'établir n'a aucune valeur 
a'solue; ce n'est là qu'un cadre, destiné à grouper provisoirement nos connaissances sur le dóveloppement postembryonnaire des Insectes. Entre ces différents types, existent de nombreuses formes de passages qui montrent que l'évolution d'un Insecte est progressive et continue; elle commence à la segmentation de l'œul et se poursuit avec des vitesses rariables, mais sans arrèt, jusqu'à l'uchèvement de l'adulte. La phase de nrmphe imnobile n'est ell-mêne' qu'un arrèt apparent dans l'ontogénie.

\section{$3^{\circ}$ - Difiérentes formes de larves.}

Les larves des Insectes - dont la vie est, en général, très longue -. ont subi, beaucoup plus que les adultes, les influences du milieu dans lequel elles ont vécu. Telles que nous les voyons aujourd'hui, ce sont des formes d'adaptation, souvent très éloignées du type ancestral qui a été, pour toutes, le point de départ commun.

A l'état parfait, les Insectes ne subissent plus aucuñ modificalion; la forme adulte nous représente donc, en définitive, pour chaque individu, le dernier degré de l'adaptation, autrement dit l'aboutissant de la phylogénie.

Il faudra dès lors, de toute nécessité, lorsqu'on voudra apprécier les relations de parenté entre les Insectes, considérer uniquement les formes larvaires à l'origine même de leur développement libre, c'est-à-dire avant que les agents extérieurs, la fixation, la vie aquatique ou parasitaire, n'aient modifié leur forme primitive. Sous ce rapport, en ce qui concerne les différents groupes d'Insectes, nous pouvons déjà faire d'intéressantes et utiles constatations.

Je reproduis ici, d'après Lubbock, les larves d'une Ephémère, d'un Méloë, d'un Dytique, d'un Stylops et d'un Campodé (Fig. 147), qui appartiennent à trois, sinon à quatre groupes différents. Il est facile de voir que toutes ces larves ont un facies commun, et qu'elles se rapprochent toutes, plus ou moins, de la forme adulte du Campodé. Si la ressemblance est frappante entre les larves, par contre, les différences qui existent entre les adultes sont considérables. L'aspect extérieur des formes adultes ne nous donne donc que des indica- 
tions vagues sur les relations de parenté ; l'étude comparée des larves, au contraire, nous amène immédiatement à concevoir une parenté très générale, non définie, il est vrai, non démontrée, mais extrêmement probable.

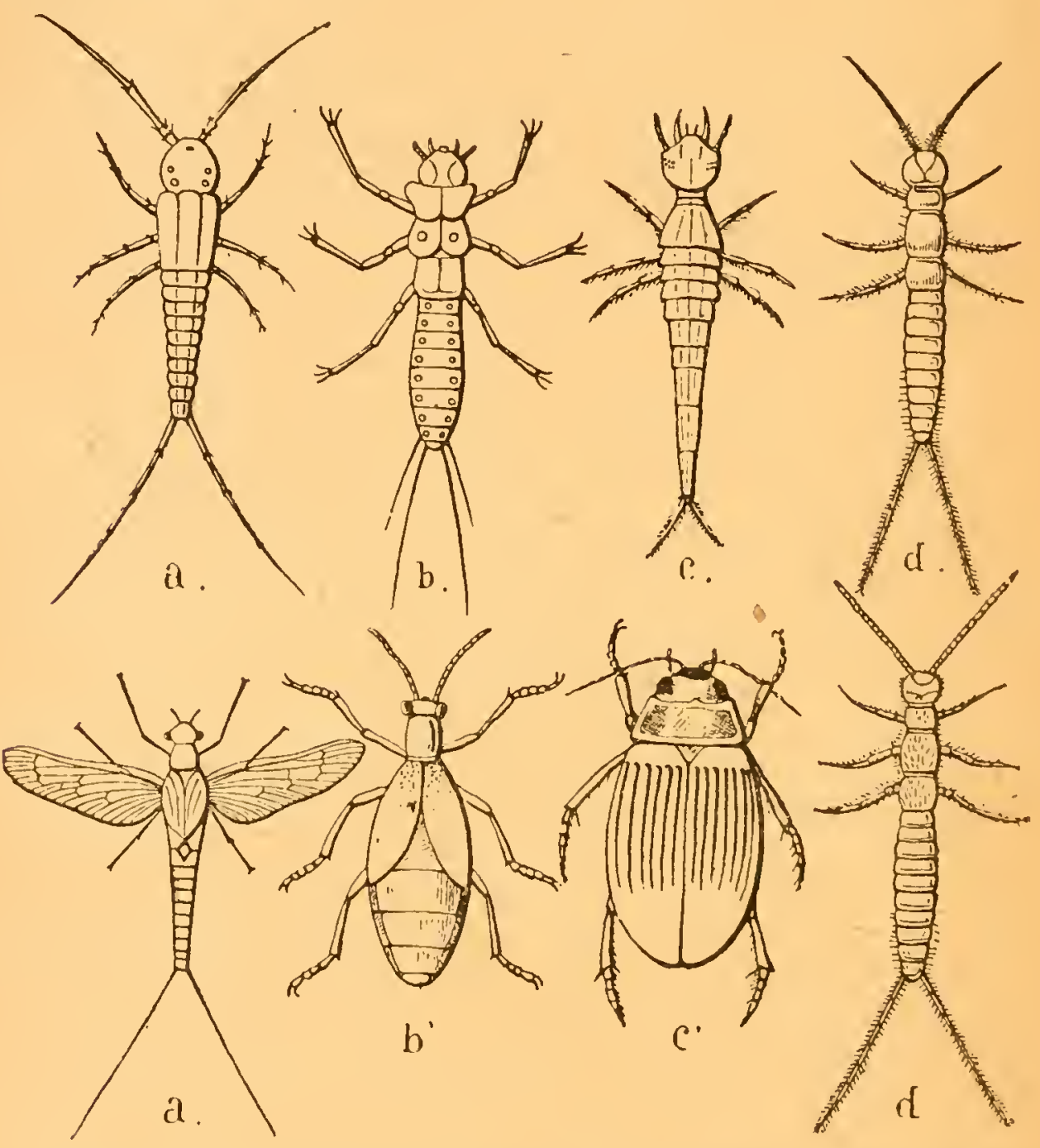

Fig. 147. - Chlö̈on : $a$, sa larve ; $a$, insecte parfait. - Melo" : $b$. sa larve $; b^{\prime}$ insecte parfait. - Dutique: $r$, sa larve; $c^{\prime}$, insectc parfait. Campodé : $l$, sa larve ; $d$ '. insezte parfait (d'avrès .J. LuBBoct).

Un autre groupement de larves pourrait donner lieu aux mêmes remarques; on verrait que, tout en s'éloignant des précédentes par un certain nombre de points, elles conservent cependant entre elles des caractères communs appréciables an premier coup d'œil.

Partant de ces principes, et conformément aux vues que MacLeay a appliquées, le premier, aux Coléoptères, on peut distinguer 
aujourd'hui huit types principaux de larves; ce sont les larves : campodéiformes, staphyliniformes, élatériformes, éruciformes, scarabéiformes, curculioniformes, sermiformes et naupliformes, auxquelles il conrient d'ajouter le cas très remarquable et jusqu ici unique du Prơsopistoma.

$1^{\circ}$ Larves campodéiformes. - Ce sont celles qui ressemblent le plus aux curieux Thysanoures habitant la vase humide et qui constituent le genre Campodea. Ces larves, en général pourvues de trois longues paires de pattes, se meuvent avec une grande agilité pour aller à la recherche de leur nourriture; elles portent des antennes longues, leur bouche est conformée sur le type broyeur et leur abdomen, souvent aplati, est terminé par des filaments plus ou moins longs.

On rencontre ces larves carnassières dans des

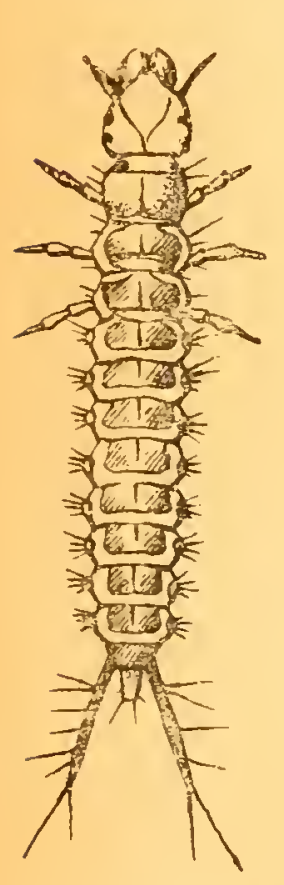

Fig. 149

Larve d'un Coléoptère. Notiophilus biguttatus

(d'après

SCHIODTE). groupes très différents (Thysanoures. Névroptères, Coléoptères, etc.) (Fig. 14\%).

¿ Larves staplyyliniformes. - Par leur aspect et leur conlormation générale, les larves de ce groupe se rappro-

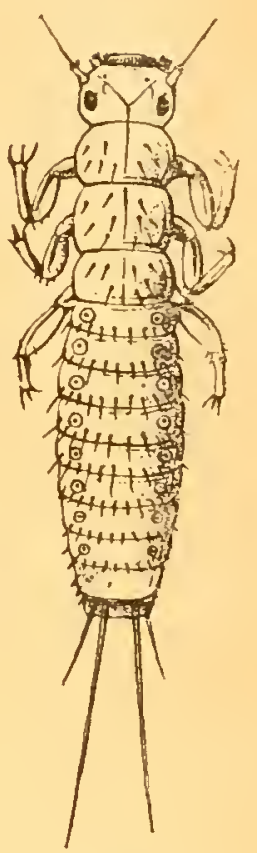

Fig. 148. Triongulin d’un Néloë (d'après

BEAVRECARD ). chent plus ou moins de celles des brachélytres (Staphylins). Leur tête porte des antennes courtes, mais leur bouche est armée de fortes mâchoires; les pattes, moins longues que dans le groupe précédent, leur permettent cependant de se mouvoir activement; le dernier segment du corps est quelquefois terminé par deux petits appendices droits ou recourbés (Fig. 149).

:o Larves élatériformes. - Ces larves possèdent trois paires de pattes thoraciques qui leur permettent encore de se déplacer avec une certaine activité; leurs antennes sont très courtes; l'abdomen ne poite jamais de fausses-pattes, et il n'est iamais non plus terminé par des appendices analogues ì reux qu'on observe dans les groupes précérients (F ig. 150).

$4^{\circ}$ Larves éruciformes. - Du mot latin erura, cherille; les larves de ce groupe ont le corps mou, sensiblement cylindrique; bien que leur régime soit généralement végétarien, 
les pièces de la bouche sont cependant conformées pour broyer les

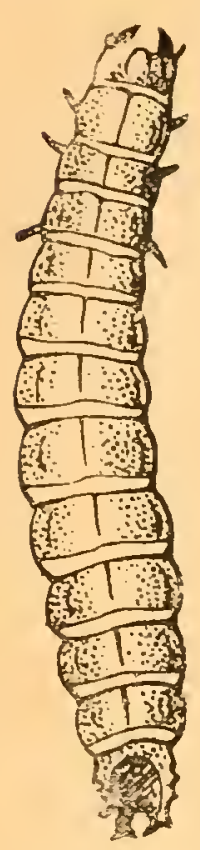

Fig. 150 . Larve d'un Coléoptère ( Elatéricle') (d'après SCHIONTE). aliments:; leurs antennes sont extrêmement courtes. Toutefois, leur caractère essentiel est la pré. sence d'appendices locomoteurs (iallsses-prttcs) en forme de mamelons articulés sur quelques-uns des segments de la partie postérieure de l'abdomen ( 151 ig.

Chez les larves éruciformes des Lépidoptères, auxquelles on réserve précisément le nom de chenilles, le nombre des fausses-pattes abdominales varie de 4 (Géométrides) à 10 (Sésies) (1), et il y a six ocelles de chaque côté de la tête. Tandis que,

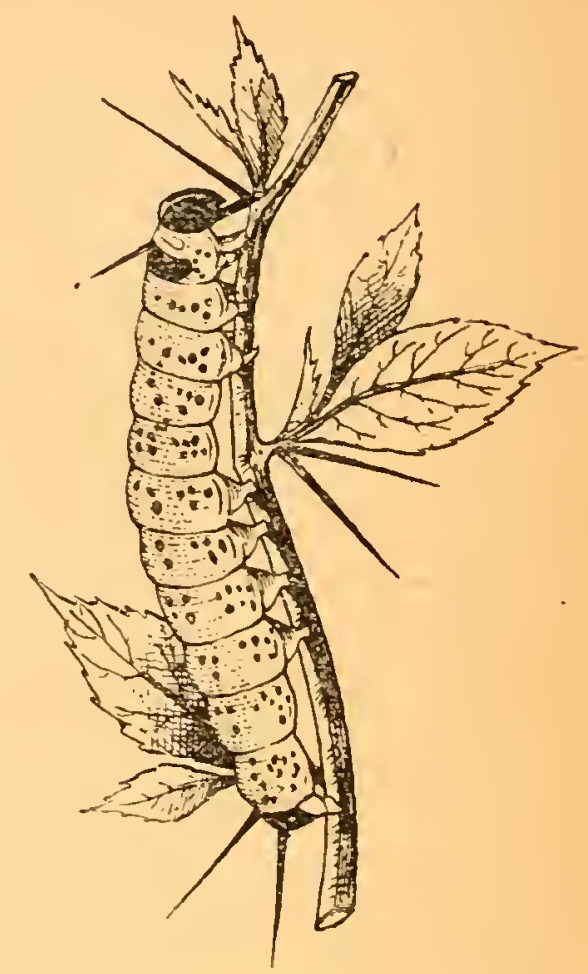

Fig. 151. - Larve (Chenille) d'un Lépidontère (d'après nature). dans les fausses-chenilles des Tenthrèdes (Hyménoptères), le nombre des pattes abdominales est toujours de 14 ou de 16 , et il n'y a qu'un seul ocelle de chaque côté de la tête.

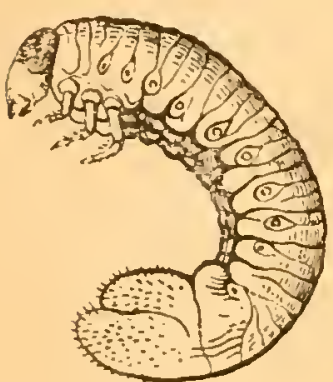

Fig. 152.

Larve du Hameton (151).

5. Larves searabéiformes. - Gomme type de ce groupe, on peut prendre la larve du Hanneton (Ver blane); le corps est mou, cylindrique; il existe trois paires de pattes thoraciques, mais si courtes qu'elles servent à peine aux déplacements de l'animal. Toutes ces larves sont phytophages et leur bouche est constituée pour broyer les aliments.

La plupart de ces larves ont le corps courbé en arc: il en résulte que, lorsqu'elles sont extraites du milieu où elles vivent, elles ne peuvent se tenir eñ équilibre sinon sur le côté du corps (ex.: Lamellicornes) (Fig. 152).

(1) Ce nombre n'est que très rarement supérieur ì dix. Paskard a cependant décrit une Chenille de Bombycide. ou le nombre des fausses pattes est de 14 (sept oalres) : c'est, il me semble, le seul exemple connu. 
$6^{\circ}$ Larves cureulioniformes. - Nous trouvons, dans ce groupe, les mêmes caractères généraux que dans les larves scarahéiformes; mais ici, les pattes sont complètement atrophiées. Ces lalves, qui vivent presque constamment en parasites, à l'intérieur des tissus des végétaux, ne peuvent se déplacer que par la contraction de leur corps (Ex.: Curculionides (1), Hyménoptères porte-aiguillon). La tête est toujours distincte (Fig. 153).

70 Larves vermiformes. - Les larves de ce groupe sont caractérisées par l'absence complète de pattes et d'organes visuels; le corps, ovoïde ou allongé, est très variable dans sa forme, mais la tête n'est plus distincte. L'appareil buccal, cela va sans dire, est également très peu visible; il est réduit à deux crochets chitineux qui servent à dilacérer les

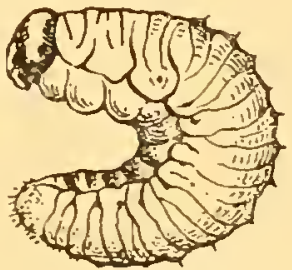

Fig. 153.

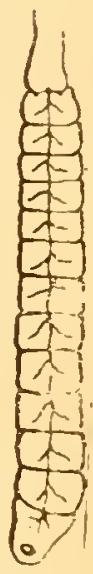

Fig. 154.

Larves d'un Curculionide (15?); d'une moucbe (154).

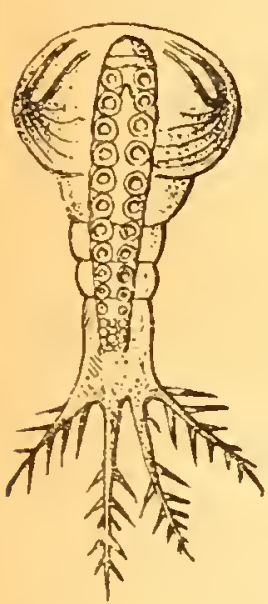

substances alimentaires (ex.: Mouches) (Fig. 15\%) (2)

Cher quelques Syrphides, on peut trouver, sur les segments abdominaux, de petits appareils de fixation comparables aux fausses-pattes des chenilles.

So Larves naupliformes. - Ce nom peut s'appliquer aux larves de certains petits Hyménoptères (Flatygaster) vivant en parasites dans les larves des Cécidomyes et décrites par Ganin en 1869 (3).

La partie antérieure du corps de ces larves est formée d'un large bouclier portant de courtes antennes (Fig. 155). L'abdomen, tout d'abord formé

Fig. 155. - de cinq segments, se termine par plusieurs filaments Larve naupliforme du

Platyzaster (d'ap. GANIN) ciliés. Vivant en parasites dans un milieu très riche en substances nutritives, ces larves ne tardent pas à perdre leurs appendices locomoteurs; leur corps devient ovale, en même temps que la métamérisation s'efface;

(1) Il y a cependant quelques larves de Curculionides (Pissodes, Hylobius) qui possèdent des pattes rudimentaires.

(2) On les dẻsigne encore sous le nom de larves helminthoïdes.

(3) Canin, leur trouvant une certaine ressemblance arec un petit crustace très commun cans les eaux donces, le Cyclops, les résınait sous le nom re larres cyclopéennes. Tous les naturalistes comprendront pourquoi nous préférons l'épithète de naupliforme. 
finalement, une dernière mue les amène à prendre l'aspect curculioniforme des Hyménoptères aculéates.

Nous retrouvons là la particularité essentielle de l'hypermétamorphose, car on peut admettre que la larve cyclopéenne du Platygaster est l'homologue du triongulin des Vésicants.

9० Larve du Prosopistoma. - Enfin, nous ne devons pas clore l'étude systématique des larves d'Insectes, sans au moins mentionner la très

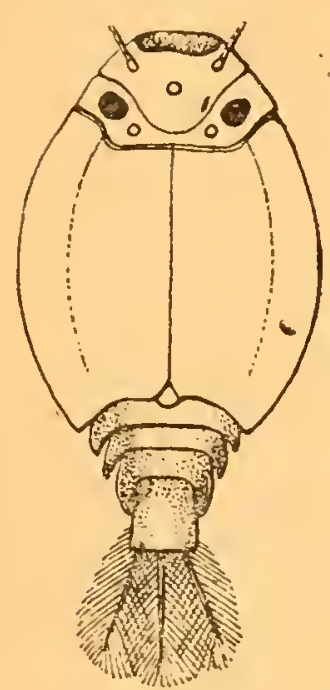
curieuse larve du Binorle à queue, découverte, en 1768 , par Geoffroy, dans une mare des environs de Paris.

Ce curieux animal, considéré pendant longtemps comme un Crustacé, est, en réalité, une larve de Névroptère de la famille des Ephémérides (Fig. 156).

Péoténie. - La néoténie est la persistance des caractères larvaires chez un adulte; ex. : femelles des Lampyrides, des Drilides, etc.

Tels sont les différents groupes de larves que l'on peut établir chez les Insectes. Laquelle, maintenant, parmi toutes ces formes, doit-on considérer Fig. 156.- Larve comme la plus ancienne? Quelle est celle qui, en

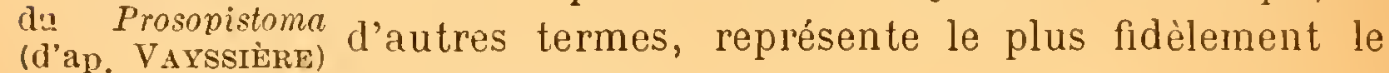
type ancestral?

Je sais bien que les formes larvaires éruciformes et helminthoïdes avaient d'abord induit en erreur un certain nombre de naturalistes, qui avaient cru voir, dans ces types d'adaptation, le rappel d'une lointaine parenté avec les Annélides. L'idée pouvait séduire, mais ce n'est qu'une apparence ; c'est bien, en réalité, la larve hexapode et campodéiforme qui représente le type normal et primitif, tandis que toutes les autres larves, plus ou moins érucoïdes, n'en sont que des modifications dues au parasitisme (1).

Donc, sans aucun doute, les Campodés de la famille des Thysanoures sont, dans la nature actuelle, les animaux qui ont conservé le plus fidèlement les caractères du type ancestral commun à tous les Hexapodes.

Ces généralités étant établies, nous pourons maintenant passer ì l'étude morphologique des larves:

(I) Je prends ce terme dans l'une de ses acceptations restreintes, car tous les animaux, sans exception, sont plus ou moins parasites. 


\section{DEUXIÈM PARTIE}

\section{Biologie générale des larves.}

\section{CHAPITRE IV}

\section{IMORPHOLOGIE EXTERNE DES LARVES}

Tecirique. - Pour tout ce qui concerne la morphologie extérieure et l'anatomie des larves, on peut prendre la Chenille $d u V e r$ is soie ou la larve du Hanneton ( Ver blan乞), ainsi que les exemples spéciaux indiqués dans le texte.

Les détails dans lesquels nous sommes entrés à propos des Insectes parfaits, vont nous permettre d'être plus bref en ce qui concerne la morphologie générale des larves; leur structure est d'ailleurs toujours beaucoup plus simple que celle des adultes.

10 Téguments et exosquelette. - La différenciation des trois régions du corps étant peu accentuée chez les larves, le squelette externe de ces dernières est toujours beaucoup plus simple que celui des adultes. Les sclérites des différentes parties du corps sont presque semblables, et seule souvent, la présence des pattes, permet de reconnaitre les trois sezments du thorax; cette possibilité, cela se conçoit, s'efface même chez les larves apodes.

Il convient cependant de noter ici une particularité curieuse qu'on peut observer dans quelques rares familles d'Insectes. Sur les 
larves des Lampyres, par exemple, chacune des plaques chitineus ss qui correspondent au mésothorax et au métathorax, recouvre devx métamères et cette particularité, moins accentuée toutefois, s'étend même à tous les segments abdominaux (Fig. 157). Cette dispo ition, comme le fait remarquer Henneguy, est intéressante à un double

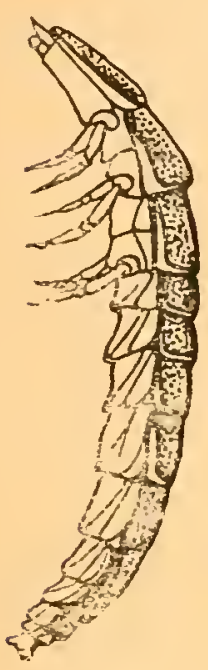

Fig. 157 .

T, arve

d'un Lampyre vue de cưté (d’après KOLBE). point de vue: "parce qu'elle permet d'établir lis parenté des Insectes aver les Myriapodes et parce qu'elle explique la situation intersegmentaire des stigmates."

On retrouve des traces d'une disposition semblable chez'd'autres larves de Coléoptères et chez les Nérroptères, tout au moins dans le genre Raphidia.

La couche de chitine qui revêt le corps des larves est toujours moins épaisse que chez l'adulte; cependant, les larves libres et actives sont, à cet égard, beaucoup mieux protégées que les larves apodes et immobiles.

La coloration des téguments est également très variable; les larves qui vivent à la lumière sont toujours très colorées (chenilles), tandis que celles qui vivent dans le sol, ou à l'intérieur des tissus des végétaux, sont blanches ou jaunâtres.

Les téguments des larves libres sont aussi fréquemment protégées pardes poils; ex.: Coléoptères, Dermestes, Anthrenus: Lépidoptères, Lasiocampa potatoria, Bomby: ru!r, etc., etc. Les poils de beaucoup de chenilles sont même quelquefois en rapport avec des appareils venimeux et possèdent drs propriétés urticantes; ce sont alors des organes de défense (ex. : Cnethocampa pityocampa, processionnaires du Pin.)

Enfin, dans l'épaisseur de la peau des larves, peuvent existrr des glandes cutanées, absolument semblables a celles des adultes el jouant le même rôle (ex. : Chrysomélides).

$2^{\circ}$ La tête et ses appendices. - La tôte est la région dn corps la mieux différenciée; cependant, ches les Diptères, li plupart des larves n'ont pas de tête distincte; le prenier segment du corps est une sorte de capsule mandibulaire on pseudocéphulur, renfermant seulement le pharynx et les muscles des crochets maslicateurs.

Par ailleurs la tête porte les mêmas appendices que shez. l'adulte.

ANTENREs. - Ies antennes sont bien développérs dans les lares à mútamorphoses graduelles, mais tonjours très courtes; chez les 
Ephémérides seuls, les antennes de la larve sont plus longues que celles de l'adulte.

Un peu en avant des antennes on observe, chez certains Diptères aquatiques du groupe des Bibionides (Fig. 15.3) (Simulium), des appendices longuement ciliés qui jouent un rôle important dans la nutrition. Par leurs mouvements continuels et rapides, les cils déterminent, dans l'eau, un courant qui amène vers la bouche les particules alimentaires dont la larve se nourrit. Des organes semblables s'observent, de même, chez les larves des Cousins.

Ocelles. - L'appareil visuel des larves est toujours très simple

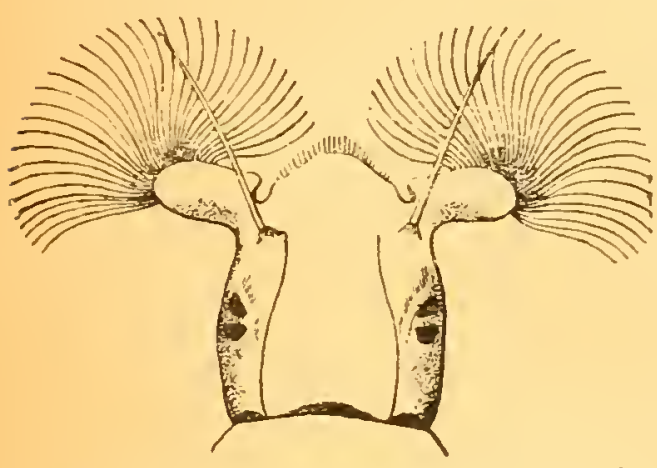

Fig. 158. - Houppes céphaliques de la larve des Simulies (d'après MIALL). chez les Insectes; dans la grande majorité des cas, il n'existe que des ocelles, en nombre variable et disposés synétriquement sur les côtés de la tête (Byrrhides) ; dans la seule famille des Névroptères on trouve des larves pourvues d'yeux composés (Lilellulides).

Par contre, la plupart des larves vivant à l'abri de la lumière dans la terre, dans le bois, dans les tissus des végétaux, sont aveugles ou simplement' pourvues d'organes risuels très rudimentaires ( Vers blancs; larves des Hyménoplères, des Diptères, etr.)

Pí̇ces buccales. - Presque partout chez les larves, la bouche est constituée sur le type broyeur et elle renferme, cela va sans dire, lsas mêmes pièces que chez l'adulte; il n'y a guère d'exceptions que pour les larves très dégradées de certains Diptères (Musca), où l'appareil masticateur n'est plus représenté que par deux crochets rudimentaires.

Toutefris, chez d'antres Insectes, les pièces buccales de la larve présentent de très curieuses adaptalions, elles pellvent devenir de véritables appareils de succion, de préhension. sans que pour cela le plan général du type broyeur soit effacé. On peut observer des modifications de ce genre, parmi les Coléoptères, chez les Dysticides ; parmi les Névroptères chez les Myrméléonides, les Hémérobides et kes Libellulides.

Ainsi par exemple, chez les Dytiscides, les longues mandibules en forme de crochet des lurves sont percées d'un trou à leur extre- 
mité libre et creusées intérieurement d'un canal qui communique avec l'intérieur de la bouche.

Ces larves peuvent ainsi sucer le sang de leurs victimes, lorsqu'elles les ont saisies avec leurs mandibules.

Chez les larves des Libellules, la partie supćrieure de la bouche (mandibules et mâchoires) ne présente rien de particulier; mais la lèvre inférieure s'allonge en un apparcil articulé, qui, au repos, vient s'appliquer sous la tête qu'il cache en grande partie; d'où le nom de masque donné par Réaumur à cet appareil. "Lorsqu'une proie passe à portée de la larve, celle-ci projette brusquement son masque en avant et s'empare de sa victime, à l'aide des crochets 'qui le terminent."

Dans le but de résumer ces différentes modifications, Braner a divisé les Insectes en trois groupes, suivant la manière dont l'appareil buccal est constitué successivement, chez la larve et chez. l'adulte:

10 Insectes ménorhynques. - Appareil buccal toujours constitué sur le type suceur, aussi hien chez la larve que chez l'adulte (Hémiptères).

$2^{\circ}$ Insectes ménognathes. - Appareil buccal broyeur chez la larve et chez l'adulte (Névroptères, Orthoptères, Coléoptères, etc.).

$3^{\circ}$ Insectes métagnathes. - Appareil broyeur chez la larve, mais suceur ou lécheur chez l'adulte (Diptères, Lépidoptères, Hyménoptères).

\section{Morphologie interne des Jarves.}

1. Tube digestif et glandes annexes. - Danz son ensemble, le tube digestif des larves est constitué ${ }_{\text {c }}$ comme celui des adultes, mais il est plus court et presque toujours rectiligne, sauf chez les Diptères, où il présente quelques circonvolutions. On y distingue les mêmes régions que chez l'adulte; mais, le fait le plus caractéristique à signaler est l'absence de communication entre l'intestin moyen et l'intestin postérieur chez un certain nombre d'Insectes (Abeilles, Guêpes, Fourmilion). Les aliments, toujours liquides, absorbés par ces larves, s'accumulent dans l'intestin moyen, et les parties non digérées sont rejetées plus tard par la voie buccale, quelques temps avant la nymphose.

TUBes dF MALigir. --- Toujours plus courts et moins nombreux 
que chez l'adulte. On en trouve quatre seulement ${ }^{\top}$ dans les larves des Abeilles, alors qu'ils sont en très grand nombre chez l'adulte.

Chez les Orthoptères (Bilattes, Grillons) le nombre des tubes de Malpighi augmente avec l'âge.

2 Appareil circulatoire et tissus adipeux. La disposition générale de l'appareil circulatoire est la même chez la larve et chez l'adulte.

Le tissu adipeux est toujours très développé cher les larves, c'est ̀̀ lui que la plupart d'entre ellẹ doivent leur coloration jaunâtra.

3- Appareil respiratoire. -- Le système tra-héen des larres f.st constitué, dans son ensembli, comme calui des adultes, mai:; il présente cependan! un certain nombre de variations dues als régime et au mode de vie.

La forme de ces expansions externes est extrêmement variable suivant les groupes d'Insectes que l'on considère (Fig. 159).

C'est toujours pour obéir à la même nécessité, augmentation de la surface respiratoire, que les tubes s'aliongent, se ramifient, s'aplatissent et se transforment en lames, ainsi qu'on l'observe chez les Gyrinides, les Phryganides, les Ephémérides, les Perlides, etc.

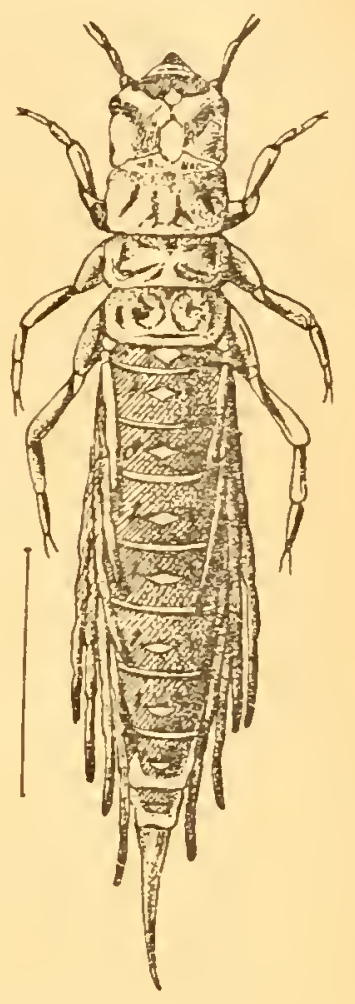

Fig. 159. - Larve du Sialis lutarius Niévroptère (d'après MIALL).

Tous ces appareils, si divers en apparence, ne sont donr, en

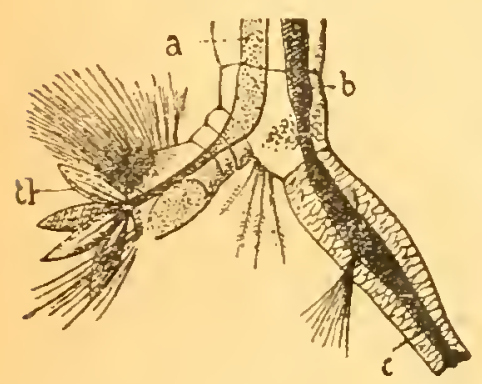

Fig. 100. Région postérieure du corps de la larve riu Cousin, Divt. a. tube igestil ; tl, branchies lamelleuses : $b, c$, tibe iracheen ablominal ( '’après RAScHKE). définitive, que des adaptations d'un seul et mème organe.

Schiner et Palmen ont défini, par ail. leurs, cinq types principaux de larves en se basant sur la distribution relative des ouvertures stigmatiques.

$1^{0}$ Les larves à système trachéen holop. neustique, qui se rencontrent surtout chez les Insectes aériens à métamorphoses gra. duelles (Orthoptères, Hémiptères), sont celles qui portent des ouvertıres stigmatiques ouvertes sur tous les segments du corps, à l'exception bien entendu de la tête et du prothorax.

$2^{\circ}$ Les larves à système piripneustique se rencontrent plus sourent 
parmi les Insectes à métamorphoses complètes. Ici, en effet, les stigmates situés sur le méso et sur le métathorax sont fermés; seuls

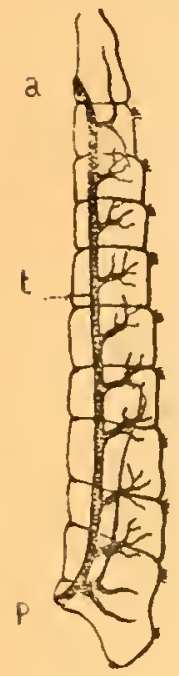

Fig. 161 .

-Larve de

la Mouche commune, distri -

bution des trachées (l'après

JANG). les stigmates abdominaux sont ouverts. Il existe bien une paire d'ouvertures stigmatiques sur le prothorax, mais ces ouyertures ne persistent pas chez l'adulte.

$3^{\circ}$ Les larves métapneustiques se rencontrent chez la plupart des Insectes aquatiques. Ces larves, dépourvues de branchies et devant respirer l'air en nature, ne possèdent de stigmates qu'à la partie postérieure de leur corps. Il existe alors fréquemment une disposition qui permet à la larve de rester suspendue la tête en bas à la surface de l'eau (Fig. 160) (Cousin, Stratiomys).

$4^{\circ}$ Les larves amphipneustiques; ce nom s'applique à quelques larves parasites, telles que celles des Estrides, des Mouches, qui ne possèdent que deux paires d'ouvertures stigmatiques, l'une placée à la partie antérieure du corps, l'autre à la partie postérieure (Fig. 161).

$5^{\prime}$ Les larves apneustiques. - Enfin, il existe un certain nombre de larves qui ne peuvent plus respirer l'air en nature; elles doivent emprunter l'oxygène dont elles ont besoin à l'air dissous dans l'eau. Ces larves, cela se conçoit, sont privées de stigmates, et leur système trachéen, complètement clos, ne s'ouvre jamais au dehors (Fig. 162). La respiration, dans ce eas, est purement cutanée(Chironomus, Simulium).

Branchies rectales des LibelluLIDES. - Chez les larves des Libellules, la disposition de l'appareil respiratoire est des plus curieuses qu'on puisse imarriner. On ne voit pas de Jranchies laté: rales externes lamelleuses ou buissonnantes, comme dans la plupart des autres larves aquatiques; ici, les branchies sont fixées à l'intérieur de la partie terminale du tube digestif (rectum); elles sont donc complè-

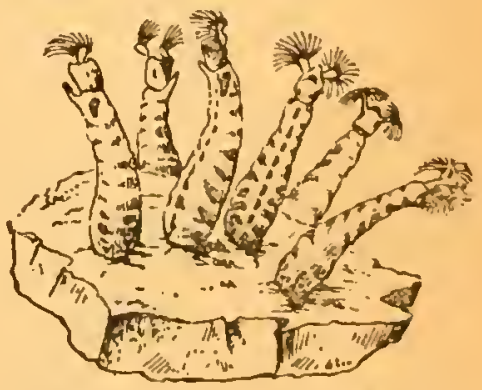

Fig. 102. - Larveq de Simulium Dilt, fixéeg sur une pierre (d'après MIALI. tement cachées. Ces branchies, en forme de lamelles, sont disposées sur six bourrelets longitudinaux et leur nombre peut dépasser 24.000. (Voir, pour plus de détails, le volume dẹ I'E. S. consacré aux Névroptères). 


\section{CHAPITRE V}

\section{FONGTIONS DE RELATIONS}

Techrigue. - Ver blanc ou Chenille du Ver à soie.

$1^{\circ}$ L'appareil musculaire de la larve est toujours plus simple que chez l'adulte et la disposition métamérique plus clairement conservée.

$2^{\circ}$ Système nerveux et organes des sens. - Plus nettement encore que chez l'adulte, le système nerveux des larves conserve le plan primitif des Arthropodes, il est dilaté. Il existe toujours une paire de ganglions nerveux par segment; mais, le plus souvent, pendant la nymphose, il se produit une concentration générale de la chaine ganglionnaire dans le sens longitudinal, de sorte que, sauf quelques rares exceptions, la chaîne nerveuse de l'adulte est toujours plus courte que celle de la larve.

Henneguy, en comparant l'appareil nerveux des larves à celui des adultes, a montré qu'on pouvait distinguer cinq combinaisons différentes (1).

$1^{\circ}$ Insectes isoncures. - Le système nerveux ne subit pas de contraciion longitudinale pendant la nymphose; il est le même chez la larve et chez l'adulte, sauf quelquefois un léger rapprochement des gan-

(1) Nous donnons ces groupements uniquement ì titre documentaire, car ils n'ont pas la moin're valeur an: woint "e vue strstématique." 
glions dans la région thoracique. Le long de la chaîne ventrale, les deux ganglions de chaque anneau se rapprochent l'un de l'autre et peuvent même se fusionner en une seule masse sur la ligne médiane du corps. Ce cas s'observe chez certains Coléoptères (Timarcha tenebricosa) et chez certains Diptères.

$2^{\circ}$ Insectes hétéroneures, - Le système nerveux reste dilaté chez la larve; mais il se concentre de plus en plus chez l'adulte (Abeilles Chrysomélides).

$3^{\circ}$ Insectes centroneures. - Le système nerveux est concentré de la même manière chez la larve et chez l'adulte, ex. : Diptéres.

‘́ $^{0}$ Insectes dicentroneures. - Le système nerveux est concentré dans les deux cas; mais plus chez la larve que chez l'adulte (larves éruciformes et curcuilioniformes). Ainsi, dans le Ver blanc, les dix ganglions de la chaîne ventrale sont réunis en une seule masse en arrière du cerveau.

$5^{\circ}$ Insectes incersoneures. - Le système nerveux est concentré chez la larve et dilaté chez l'adulte (Fourmilion).

Toutes ces modifications, on le devine aisément, sont en rapport avec le degré d'activité de la larve et le mode d'adaptation qu'elle a subi.

Cerveau. - Bien qu'il soit généralement plus simple, on retrouve cependant, dans le cerveau des larves, les nêmes parties fondamentales que chez l'adulte.

Les parties centrales du système nerveux se forment de très bonne heure et sont d'origine exclusivement ectodermique. On voit se produire, sur la face ventrale de l'embryon, à droite et gauche de la ligne médiane, deux épaississements indépendants (plaque nerveuse) qui s'étendent depuis la partie postérieure du corps jusque dans les lobes céphaliques, où ils présentent un renflement marqué. Ce renflement est le lobe procéphalique; c'est lui qui, par c'es modifications successives, donnera toute la partie du système nerveux renfermée dans la tête (Fig. 163).

D'après Viallanes, les cellules de la plaque nerrense 
se divisent en deux et on a ainsi, par une sorte de clivage, une couche externe de dermatoblastes et une couche interne de ganglioblastes (Wheeler). La couche dermatogène superficielle restera l'ectoderme de l'embryon; quant à la couche gangliogène, elle produira les cellules nerveuses proprement dites, qui s'organiseront pour former les ganglions.

\section{Développement des ganglions cérébroïdes.} - Au début, les deux lobes procéphaliques (lp) sont complètement indépendants; ils sont placés un peu en avant de chaque còté de la bouche. Bientôt, chacun des lobes procéphaliques se recourbe vers le dehors, et se divise en trois renflements, séparés par des étranglements (Fig. 164, B).

D'après leur position ces trois renflements

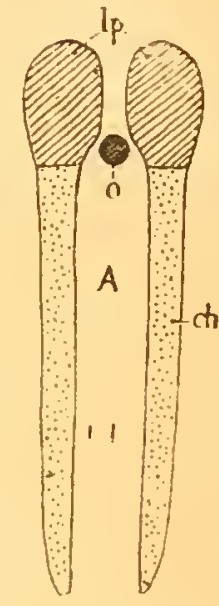

Fig . 163. -Lobes procéphaliques (li) et les deux moitiés de Ia chaîne ventrale,

o. bouche. ont reçu les noms de deuxième ou troisième lobe protocérébral; le troisième se continue avec la plaque nerveuse qui, à cette époque du développement,
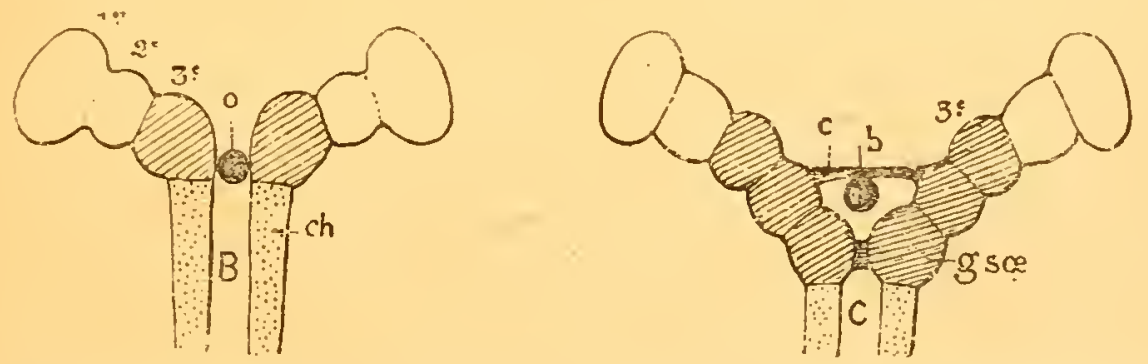

Fir. 164. - Evolution des trois lobes protocérébraux $1^{\mathrm{er}}, 2^{\mathrm{e}}$ et $3^{\mathrm{e}}$ lobes protocérébraux; $n, b$, bouche; ch, chaine ventrale; $a s a$, ganglions cérébroïles; c, commissure supra-buccale

ne présente encore aucune trace de segmentation (Fig. 164, B).

Un peu plus tard le troisième renflement se divise à son tour, il donne : le troisième lobe protocérébral pro- 
prement dit, le deutocérébron et le tritocérébron. (Fig. 164, C). Nous avons donc, en définitive, cinq paires de renflements nerveux successifs issus du lobe procéphalique.

Examinons le sort ultérieur de ces divers renflements (1).

Le $1^{\mathrm{er}}$ lobe protocérébral subit une délamination superficielle qui le divise en deux couches : la couche externe, plaque optique de Patten, donnera l'œil composé; la couche interne, neurollastique, donnera naissance aux fibres postrétiniennes.

Le 2 e lobe protocérébral servira à constituer le nerf optique et la masse médullaire qui s'y rattache.

Le 3 e lobe protocérébral, le deutocérébron et le tritocérébron qui le suivent, ne subiront presque aucune modification ; c'est leur ensemble qui constitue les trois paires de ganglions que l'on décrit sous le nom de ganglions cérébrö̈des ou sus-csophagiens.

Nerfs. - D'après Henneguy, les nerfs naissent des ganglions et ils se développent, " du centre à la périphérie, sous forme de bourgeons de fibrilles qui s'allongent et se dirigent vers les parties où ils doivent se terminer ». (Les Insectes, loc. cit. p. 369).

ORGANES DES SENS. - Les organes des sens sont très peu connus chez les larves; leur développement-principalement celui de l'appareil de la vision - n'a été étudié que dans quelques cas particuliers, par Grenacher et Patten pour les ocelles des Coléoptères (1887) ; et plus récemment, par Redikorgew (1900) pour ceux des Hyménoptères.

(1) L'ensemble des trois lobes protocérébraux constitue pour Viallanes la région qu'il désigne sons le nom de protocérébron. 


\section{CHAPITRE VI}

\section{FONCTIONS DE REPRODUCTION}

$1^{\circ}$ Organes génitaux. - Les ébauches génitales des larves n'atteignent leur développement complet que pendant la période nymphale; la seule remarque qu'on puisse faire c'est que, en général, chez les Insectes à métamorphoses complètes, l'évolution de la glande femelle est un peu en retard sur celle de la glande mâle.

On sait, depuis les travaux de Weismann (1863), que l'apparition des glandes sexuelles est très précoce; elles apparaissent, tout à fait au début de la période embryonnaire, sous forme de deux groupes de cellules placés au pôle postérieur de l'œuf, et qui sont, d'après Lécaillon, d'origine blastodermique.

Plus tard, au moment où se forme l'invagination qui donne naissance à l'intestin terminal (proctodeum), ces deux groupes de cellules sont séparés et refoulés latéralement. Par suite de ce refoulement, elles pénètrent entre les "lames mésodermiques de chaque segment abdominal ", ce qui explique la disposition métamérique qu'elles présenten: pendant un certain temps et qu'elles conservent même pendant toute la vie chez les Insectes inférieurs (Thysanoures).

Chez les Insectes plus élevés, les cavités cœlomiques des segments successifs se fusionnent; il en résulte que 
les cellules génitales se trouvent renfermées dans deux cordons, d'origine mésodermique, situés de chaque côté du corps, l'un àdroite, l'autre à gauche du tube intestinal. Mais, les cellules ne persistent dans ces tubes que du

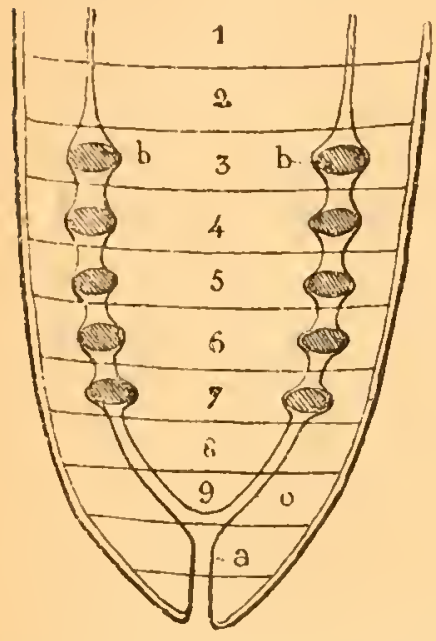

Fig. 165. - Origine métamérique des organes reproducteurs : $a$, canal ovarien: $b$, îlots de cellules génitiles: $n$, oviductes ; $1-9$ segments abdouinaux. troisième au septième segment; toute la région du tube située en avant du troisième segment s'aminciræ et, chez les femelles, deviendra le tube de Muller; en même temps, la partie située en arrière du septième s'organisera pour devenir l'oviducte (Fig. 165).

La marche des phénomènes qui aboutissent à la formation des glandes génitales est à peu près la même chez tous les Insectes, les seules variations notables qui peuvent se produire portent, semble-t-il, sur l'apparition plus ou moins précoce des initiales au pôle postérieur de l'œuf.

En même temps que les conduits génitaux apparaissent, comme nous venons de le voir, sous forme de deux cordons pleins, d'origine mésodermique et placés de chaque côté du tube digestif, deux invaginations ectodermiques, partant de l'extérieur, vont à la rencontre de ces conduits ; c'est là, suivant les cas, l'origine du cana! éjaculateur chez les mâles, ou du vagin chez la femelle. Sauf chez les Ephémérides, où elles restent séparées pendant toute la vie, ces deux invaginations se réunissent toujours sur la ligne médiane du corps en un conduit commun. 


\section{CHAPITRE VII}

\section{BIOLOGIE DES LARVE8}

Maintenant que nous connaissons les particularités les plus intéressantes de la structure des larves, étudions quelques-uns des points les plus importants de leur évolution biologique.

Éclosion. - Les conditions dans lesquelles se fait l'éclosion, ont été observées dans presque tous les groupes d'Insectes, mais le nombre des faits connus est encore trop petit pour qu'on puisse formuler les lois qui régissent cet acte important, s'il en existe.

On peut considérer cinq cas particuliers principaus.

10 Lorsqu'il s'agit d'œufs présentant un chorion épais, une partie de cette membrane présente, généralement, une zone de moindre résistance, différente du reste de l'enveloppe par sa structure. Une partie de l'œuf se détache alors sous forme d'une calotte, et la jeune. larve sort par l'orifice ainsi produit.

Ce cas s'observe chez beaucoup de Lépidoptères, chez un certain nombre d'Hémiptères (Pentatome) et, parmi les Orthoptères, chez quelques Phasmides (Phyllies).

$2^{\circ}$ Quelquefois, le chorion est uniforme dans toute son étendue, et il n'existe pas de zone de moindre résistance; dans ce cas, que l'on peut, par exemple, obser- 
ver chez le Ver à soie, la jeune larve s'ouvre un passage en rongeant, avec ses pièces buccales, la partie de l'enveloppe la plus voisine de sa tête.

$3^{\circ}$ Par extraordinaire, lorsque le chorion de l'œuf est peu résistant comme cela a lieu pour les œufs de certains Diptères (Mouche à viande), la jeune larve provoque la rupture de l'enveloppe par les mouvements de contraction de son corps à l'intérieur. En général, dans ce cas, la paroi se fend longitudinalement, ce qui semble indiquer qu'une ligne de déhiscence existe, mais qu'elle est beaucoup moins marquée que dans les deux premiers cas.

$4^{\circ}$ Chez les Orthoptères de la famille des Acridiens, ainsi que l'ont montré les recherches de Kunckel d'Herculais (1890), les larves sortent de l'œuf encore entourées de leur amnios, mais elles se débarrassent bientôt de cette enveloppe par un procédé bien curieux.

Elles possèdent, à cet effet, entre la tête et le thorax, une membrane molle que Kunckel désigne sous le nom d'ampoule cervicale; les jeunes Acridiens peuvent faire saillir, à volonté, cette membrane, du côté dorsal, en la remplissant de sang. Ils se servent tout d'abord de cetto membrane pour soulever le couvercle de l'oothèque dans laquelle ils sont renfermés. A peine la jeune larve est-elle parvenue à porter sa tête au dehor's, que l'ampoule cervicale entre, de nouveau, en jeu ; cette fois, le sac amniotique, considérablement distendu, se rompt, et le jeune Acridien. est désormais libre.

5- Enfin, certains Insectes possèdent un appareil spécial, un ruptor osi, selon l'heureuse expression de Riley, à l'aide duquel ils percent la paroi du chorion. Chez la Forficule, cet appareil consiste en une épine 
rigide placée entre les deux yeux. Cet appareil, dont le rôle unique est de favoriser l'éclosion, disparait chez les larves dès la première mue et il n'en reste, cela se conçoit, aucun vestige chez l'adulte.

Mues. - Toutes les larves, aussi bien celles des Insectes métaboliens que celles des amétaboliens, changent de peau un certain nombre de fois dans le cours de leur vie. L'enveloppe chitineuse, dont elles sont revêtues, et qui s'oppose, dans une certaine mesure, à l'accroissement du corps, tombe; il s'en forme
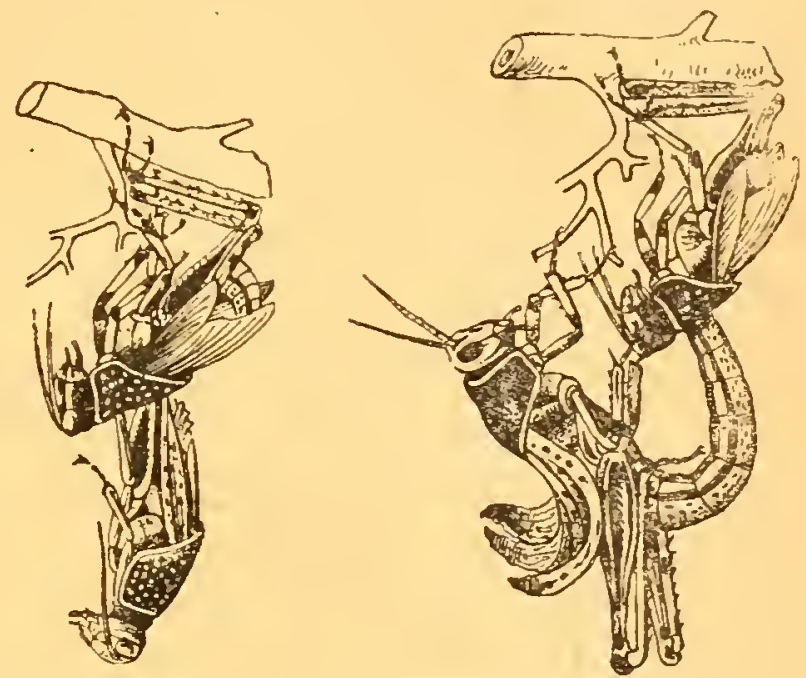

Fig. 166. - Deux phases de la mue chez Schistocera perenrina (ORTIIOPT.) (d'après KUNCEEL D'H ERCULAIS).

dessous une nouvelle, qui tombe de même, et ainsi de suite. C'est à ce phénomène qu'on a donné le nom de mue ou ecdysis. L'ancienne peau, abandonnée au hasard de la mue, et qui souvent conserve l'aspect de la larve, a reçu le nom d'exuvie (Fig. 166).

Mécanisme de la mue.-Les anciens entomologistes, imbus des idées de Swammerdam, pensaient que les différentes peaux de la larve étaient emboìtées les unes dans les autres. La vérité, c'est qu'une nouvelle couche tégumentaire se forme au dessous de l'ancienne par le travail des cellules hypodermiques; celles-ci devenant plus nombreuses, il en résulte que la nouvelle couche tégumentaire est obligée de se plisser sous l'enveloppe chitineuse rigide; un liquide spécial, sécrété par de grandes cellules hypodermiques, s'accumule entre l'ancien tégument et le nouveau. Le premier tégument, inextensible, finit par se rompre; alors, le second, devenu libre, tandis 
qu'il est encore mou et flexible, s'étend; et la jeune larve, bénéficiant alors de l'accroissement en surface de son enveloppe tégumentaire, -accroît, tout pendant que l'élasticité de la couche chitineuse le permet. Pantel a, en effet, constaté, sur la larve du Thrixion (Tachinaires), que, dans l'intervalle de deux mues successives, la taille de la larve peut augmenter du simple au double.

Chez certains Diptères ( $M$ uscidés), la larve ne sort pas de son enveloppe chitineuse au moment de la dernière mue ; elle se transforme. en nymphe dans son intérieur, c'est pourquoi Linné a donné, à la nymphe ainsi enveloppée, le nom de pupe (1).

Coloration DES LaRves. - La couleur des téguments change souvent au cours des métamorphoses; il en est de même dẹs dessins qui les caractérisaient. Ces modifications curieuses paraissent en rapport avec les phénomènes d'histolyse et d'histogénèse partiels qui s'accomplissent au moment des mues.

Nourriture des larves. - Le régime alimentaire des larves est très varié ; toutefois, les exigences'de l'adaption ont fait que chacune d'elles ne peut utiliser qu'une nourriture très spécialisée.

On peut sous ce rapport établir trois grandes subdivisions :

10 Les larves carnivores (Ex.: Carahes, Nécrophores, Libellulides etc.). qui se nourrissent de proies vivantes ou mortes mais toujours d'origine animale.

$2^{\circ}$ Les larves phytophages (Ex. : Chenilles), dont la nourriture est exclusivement s'égétale.

$3^{\circ}$ Les larves polyphages (Ex.: Blattes, Dermestes). Bien que manifestant souvent une préférence très marquée, les larves de ce groupe acceptent toutes les substances organiques, quelle qu'en soit l'origine.

Le régime des larves phytophages peut même être quelquefois strictement défini ; ainsi on trouve dans ce groupe des larves:

phyllophages (mangeuses de feuilles: Lépidoptères, Chrysomélides), rhizophages (mangeuses de ranines: Ver Blanc), xylophagres mangeuses de bois: Cécambycides, Buprestidcs, mycophages (mangeuses de champignons: Cryptophagilles), coprophages (excréments des herbivores : Bousiers), polliniphage (mangeuses de pollen : Anthonome).

L'adaptation de certaines espèces phy tophages est même tellement

1) Du latin pupa, poupés. 
étroite, que ces larves ne peuvent vivre qu'aux dépens d'une plante bien déterminée; il en est même qui, peut-être, changent de nourriture dans le cours de leur développement. Tel est probablement le cas de la Chenille du Bombyx rubi, si difficile à élever, et qui refuse obstinément les feuilles de Ronce qu'on lui offre au printemps.

\section{Influence des agents physiques.}

Nous avons vu (p. 197) que la température possède une influence très marquée sur la durée des mues et sur l'ensemble du développement. Il doit exister, pour chaque espèce, une température optimum, au-dessous et au-dessus de laquelle la marche normale des "phénomènes vitaux est plus ou moins troublée. Ainsi, par exemple, dans les conditions ordinaires, c'est-à-dire à une température moyenne de $22^{\circ}$ à $24^{\circ}$, le Ver à soie reste à l'état larvaire pendant trente-cinq jours ; tandis qu'à $45^{\circ}$ son évolution se fait en 14 jours. Inversement, à une température de $16^{\circ}$, il peut rester pendant cinquante jours avant de subir la nymphose.

On croit, en général, qu'une température très basse exerce une action nuisible sur la vie des larves; beaucoup de personnes croient même volontiers que les Insectes meurent pendant les hivers très rigoureux; c'est là une idée absolument fausse qu'il faut détruire (1). Un ancien naturaliste allemand, Justi (1753), a congelé des Vers à soie à tel point que leur corps, devenu fra gile comme du verre, se brisait sous le moindre choc; or, en réchauffant lentement ces larves, il les a vues " revivre, manger et filer leur cocon ".

(1) Pour que les Insectes (ou leur larves) meurent par le froid, il faudrait qu'ils fussent exposés, pendant quelques heures. à des températures inférieures à $-60^{\circ}$. 
Dimorphisme saisonnier. - Si les Insectes peuvent supporter, sans mourir, de très basses températures, l'expérience nous montre cependant qu'ils ne sont pas insensibles allx actions de la chaleur et du froid. Les variations de la température provoquent même, sur certains d'entre eux, principalement sur les Lépidoptères, des modifications tellement profondes, que les adultes d'une même espèce, qui naissent à des époques plus ou moins tardives dans l'année, sont souvent assez différents les uns des autres pour qu'on ait cru à l'existence de deux espèces distinctes. Ce phénomène. bien connu des lépidoptérologistes, constitue le dimorphismesaisonnier. L'exemple classique est celui qui nous est offert par Vanessa geographica.

Les individus de cette espèce, nés au printemps, et qui ont par conséquent passé l'hiver à l'état de chrysalides, possèdent une teinte générale fauve avec de nombreuses taches brunes : c'est Vanessa levana. Miais cette Vanesse pond ; la larve et la chrysalide de cette ponte estivale évoluent rapidement, et, en juillet, donnent naissance à une deuxième génération de papillons, entièrement d'un brun noir avec une bande blanche au milieu des ailes supérieures : c'est la variété prorsa. Quelques chrysalides tardives de cette variété prorsa peuvent même ne donner leur adulte qu'en septembre; on a alors une variété intermédiaire, que les lépidoptérologistes ont nommée prima. On ne peut guère attribuer à une autre cause qu'aux variations de la température, ces curieuses modificalions d'une espèce dans le court espace d'une année.

Jl va sans dire que les œufs pondus par les variétés prorsa et porima donnent, au printemps suivant, de nouvelles générations de levana et le cycle recommence.

Le premier, E. Berce entreprit l'étude expérimentale de ces curieuses variations chez Vanessa geographica; il constata qu'en maintenant à la chaleur les chenilles de la génération prorsa, il obtenait en quelque sorte à volonté la variété porima.

L'action du froid fut également expérimentée sur cette espère par Weismann ; il soumit, pendant quatre semaines, dans une glacière, à la température de $-1^{\circ}$, des chrysalides issues de la génération prorsa; les papillons qu'il obtint présentaient, pour la plupart, la coloration alaire et les maculations de les'ana.

Un grand Papilionide de l'Amérique du Nord (Papilio Ajax) revêt également trois formes saisonnières différentes; deux formes de printemps à teintes claires: $P$. telamonides et $P$. Walshii; plus une 
f srme d'été, $P$. Marcellus, à teinte foncée. Naturellement, ici encore, les œufs de Marcellus reproduisent la variété claire à évolution hibernale.

A l'exemple de Berce et de Weismann, de nombreux biologistes ont cherché à déterminer quelles sont les variations provoquées sur les adultes par la chaleur agissant continûment, soit sur les cufs, soit sur les larves, soit sur les chrysalides; les travaux qui ont eu le plus grand retentissement dans cette vole sont ceux du Dr Max Standfuss, que j'expose ici, d'après F. Henneguy et d'après l'excellent résumé qui en a été donné dans les Annales de la Société entomologique de France, 1900, p. 82, par 11. Deckert.

Standfuss montra, en 1895, que la chaleur, agissant sur les œufs, produit une accélération de l'évolution qui se fait sentir jusque dans le cours du développement de la larve. De même, des chenilles, élevées à une température moyenne de $25^{\circ}$, ont montré, non seulement une abréviation de la vie larvaire, mais ont fourni des adultes dont la taille s'est trouvée réduite.

Mais ce sont les expériences réalisées avec les chrysalides qui ont donné les résuitats les plus remarquables; le savant directeur du Musée entomologique de Zurich admet que l'action de la chaleur se traduit de quatre façons différentes sur les adultes; on peut obtenir :

$1^{\circ}$ Des variations saisonnières, analogues à celles qui existent normalement dans la nature, comme par exemple $V$. levana-prorsa et lesana-porima.

$2^{\circ}$ Des formes et des races locales, analogues à celles que l'on rencontre actuellement dans certaines localités bien définies et bien connues. Ainsi, par exemple, les exemplaires de Vanessa urticœ, obtenus de chrysalides reliroidies, sont identiques ou presque à ceux de $V$. urticce, var. polaris.

$3^{\circ}$ Des formes aberrantes, telles qu'on en voit quelquefois apparaitre çà et là dans la nature : c'est ainsi que Vanessa Io peut donner la variation $F i$ scheriet $V$. cardui la valiation elymi.

$4^{\circ}$ Des formes phylétiques, c'est-à-dire des variations inconnues actuellement dans la nature, mais qui ont peut-être existé dans le passé, ou qui pourront exister dans l'avenir, si les conditions nécessaires à leur production viennent à se trouver réalisées. Ainsi, certaines formes de Vanessa Io, et de $V$. Antiopa, obtenues par l'action du froid, rappellent à s'y méprendre le type spécifique de $V$.urtico. 
Inversement, des chrysalides chauffées de $V$. urticœ donnent des adultes qui tendent à se rapprocher de Vanessa Io. On peut donc en conclure que ces deux espèces ont probablement une origine commune.

Ce problème est troublant. Dans tous les cas, ces recherches montrent nettement qu'on ne saurait plus admettre une limite fixe, infranchissable, entre l'espèce et la variété ; et, qu'entre les espèces elles-mêmes, on entrevoit des transitions possibles.

ACTION DE LA LUMIÈRE. - Un certain nombre d'auteurs ont également cherché à déterminer quelle était l'action de la lum:èr sur le développement des larves. Pien que les résultats ne soient pas très concordants, on peut admettre que ce sont les radiations virilettes qui favorisent le plus la rapidité du développement.

En ce qui concerne les chrysalides, C. Venus (1888), ayant élevé à Dresde des chenilles de V anessa urticce ên les exposant aux rayons d'un soleil intense, obtint des chrysalides jaunes d'où sortirent des Papillons identiques à V anessa ischnusa de Corse et de Sardaigne.

En résumé, les actions combinées de la chaleur et de la lumière suffisent probablement à expliquer les différences de coloration que l'on observe sur une même espèce, dans la nature, suivant la saison et suirant les conditions de milieu où elle vit.

Dans le volume qui traitera des Lépidoptères, un bref exposé sera donné des expériences d'Oudemans (1898) et de Crampton (1899) sur le déterminisme des couleurs sexuelles che\% les Papillons. 


\section{CHAPITRE VIII}

\section{LA NYMPHE DES INSECTES}

Tecinique. - Se procurer, par élevage, un certain nombre de nymphes de la Pieride du chou; il suffit, pour cela, de nourrir des chenilles dans une boîte recouverte de toile métallique.

$1^{0}$ Les phénomènes précurseurs de la nymphose. Lorsqu'elle a atteint le terme de sa croissance, la larve des Insectes subit une dernière transformation, remarquable surtout chez les Holométaboliens : elle change de $i \varepsilon a u$, passe, en quelque sorte, par une période de vie ralentie et devient une nymphe; c'est là le phénomène de la nymphose.

Au moment où elle est sur le point de se transformer en nymphe, la larve cesse de manger ; elle rejette au dehors le peu d'aliments qui restait dans son tube digestif et se retire dans un endroit plus ou moins abrité.

zo Les différentes formes de nymphes. - Le stade de nymphe immobile ne se rencontre réellement que chez les Insectes à métamorphoses complètes; chez les Insectes qui ne subissent pas de métamorphoses, ou qui arrivent à l'état parfait par une série de transformations graduelles, la nymphe ne diffère de l'adulte que par le plus faible développement de ses ailes. Dans ce cas, comme le fait très justement remarquer Henneguy, 
il est impossible de dire " où commence et où finit la phase nymphale $n$ et le nom de nymphe s'applique donc, " conventionnellement ", à la phase du développement qui précède immédiatement l'état adulte.

Quoi qu'il en soit, en ce qui concerne les Insectes à

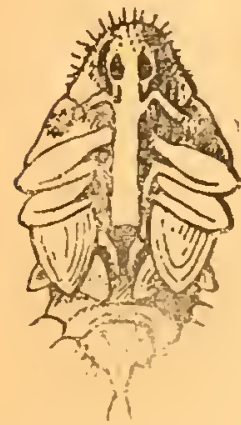

Fig. $16 \%$. Une nymphe de Curculionide Anthonome.Col (d'ap. nat.) métamorphoses complètes, on peut, dans la pratique, distinguer trois types principaux de nymphes:

10 Les Nymphes proprement dites, caractérisées par ce fait que tous les membres sont libres et déjà isolés; le corps tout entier est donc recouvert par une enveloppe très fine qui s'applique exactement sur chacun d'eux. Ce sont les pupes incomplètes de Linné (Coléoptères, Nérroptères, etc.) (Fig. 167).

$2^{\circ}$ Les Chrysalides; on réserve spécialement ce nom aux nymphes des Lépidoptères.

Ici, les membres, ainsi que les différentes parties du
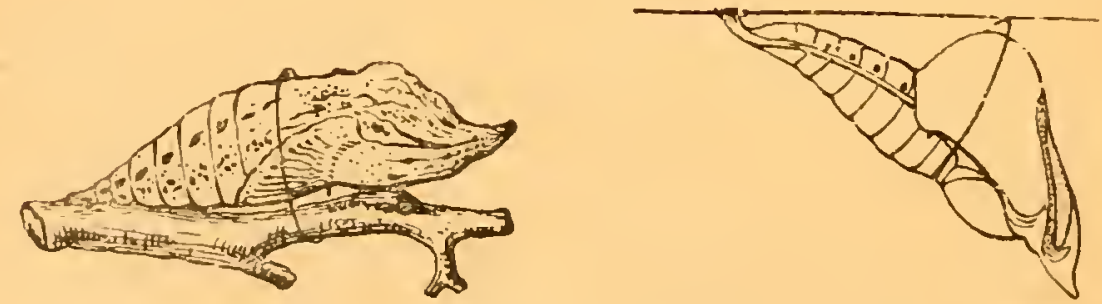

Fig. 168. - Deux chrysalides de Iépidortères, à gauche I'iérile

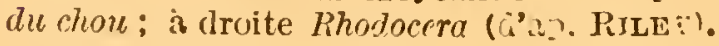

corps de l'adulte, sont encore visibles, mais il existe une enveloppe commune, qui recouvre le tout, et qui tient les membres appliqués contre le corps. Ce sont les pupes emmaillotées de Linné (Fig. 168).

$3^{\circ}$ Pupes. On donne ce nom aux nymphes qui restent 
enfermées sous la peau de la larve au moment de la dernière mue ; on ne peut, par conséquent, dans ce cas, distinguer aucune partie du corps de l'adulte : ce sont les pupes resserrées de Linné.

Il est à peine besoin de dire, qu'entre ces trois types bien définis et en quelque sorte classiques, il existe de nombreux intermédiaires.

3' Ornementation des nymphes. - Les nymphes des Insectes holométaboliens sont, en général, arrondies, ovoïdes (Diptères) ou légèrement coniques (Lépidoptères nociurnes) ; toutefois celles des Rhopalocères (Lépidoptères diurnes) sont presque toujours anguleuses et colorées de façon très variable.

L'enveloppe des nymphes est, le plus souvent, dépourvue d'appendices; lorsqu'il en existe on les retrouve toujours chez l'adulte.

4. Anatomie interne des nymphes. -- On ne saurait donner la description des organes internes de la nymphe, puisque la plupart de ces organes sont dans un état de continuelle transformation. C'est, en effet, pendant la période nymphale que s'accomplissent les phénomènes d'histolyse et d'histogénèse que nous décrirons plus loin (p. 263).

Malgré leur état de mort apparente, les nymphes sont simplement dans un śtat de vie ralentie; dans leur organisme, toutes les grandes fonctions de la vie végétative s'accomplissent comme par le passé, bien qu'avec une intensité beaucoup moindre; on savait notamment, depuis Réaumur, que des chrysalides, immergées dans l'huile, périssaient toujours par asphyxie.

Certains Insectes éprouvent une assez forte diminution de poids pendant la nymphose ; cette perte est surtout sensible, cela se conçoit, chez les chrysalides qui filent un cocon. Ainsi, par exemple Dandolo a constaté que, chez un Ver à soie d'assez grande taille' prêt à filer, la larve pesait $3 \mathrm{gr}$. 66 . Au bout de huit jours, la ch rỵ salide et son cocon, ensemble, ne pèsent plus que $2 \mathrm{gr}$. 18. La perte 
totale de poids, subie par l'animal dans ce court laps de temps, a donc été de $1 \mathrm{gr} .48$.

Bien que les nymphes des Insectes holométaboliens ne puissent, en général, se déplacer, elles ne sont pas cependant, pour cela, complètement immobiles; ainsi, par exemple, chez les chrysalides des Lépidoptères, les anneaux de l'abdomen conservent une certaine mobilité ; cela tient vraisemblablement à ce que, rendant les phénomènes d'histolyse, la destruction des muscles de cette région n'est jamais absolument complète. 


\section{CHAPITRE IX}

\section{HISTOLYSE ET HISTOGÉNÈSE}

Tecirique. - Il est nécessaire de durcir les tissus et de pratiquer des coupes minces dans les organes que l'on veut étudier. La technique demande un apprentissage près d'un naturaliste exercé. On en trouve quelquefois la description dans les mémoires spéciaux.

10 Phénomènes intimes de la nymphose. - Il n'y a guère plus de 50 ans que l'on connaît les phénomènes intimes de la métamorphose chez les Insectes. Sous l'influence des idées de Swammerdam et de Réaumur, les naturalistes avaient toujours cru que, "pendant la métamorphose, il y avait simplement développement d'organes préexistants chez la larve ".

Cependant, les organes qui jouent le rôle le plus important dans ces transformations, ceux que Weismann a désignés sous le nom de aisques imaginaux, avaient été aperçus dpuis longtemps, mais personne n'en avait compris la significalion.

Étant donnée la place qu'ils occupent, Lyonet, dès 1762, en était arrivé à soupçonner qu'ils pourraient bien "s̀tre les principes des ailes ". Léon Dufour, l'hahile entomologiste de Saint-Sever, les avait aussi aperçus chez les larves des Mouches, mais il les considérait comme " des ganglions d'une nature particulière ".

C'est Weismann, comme nous l'avons dit, qui comprit, le premier, Ix véritable signification de ces corps et détermina le rôle important joué par eux au cours des métamorphoses. "Il leur donna le nom de disques imaginaux, à cause de leur forme discoïle et reconnut que ce sont, en réalité, "de petites vésicules dans lesquelles on distingue une partie centrale plus épaisse, destinée à produire un appendice (patte, aile, etc.), et une partie périphérique "en rapport avec l'hypoderme environnant.

De plus, le profasseur Weissmann appela l'attention sur les cu- 
rieux phénomènes de dégénérescence qui se produisent à l'intẻ. rieur du corps de la nymphe. Il fit remarquer que les cellules des tissus larpaires se fragmentent et subissent une sorte de liquéfaction ; il donna le nom d'histolyse à l'ensemble de ces phénomènes.

C'est alors à ce moment qu'apparaissent, toujonrs d'après Weismann, au milieu des tissus liquéfiés de la larve, des globules granuleux, nés par formation libre; et 'c'est alors, aux dépens de ces matériaux, que se formeront "les tissus et les organes nouveaux, muscles, trachées, corps adipeux, etc..., etc."

A part la formation des cellules libres, les vues de Weismann ont été confirmées par tous les naturalistes qui se sont occupés de l'embryo-

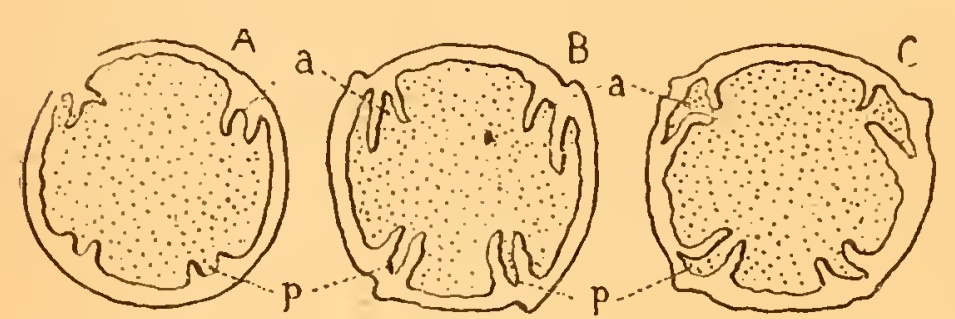

Fig. 169. - Diagrammes indiquant le mode de formation des appendices par l'intermédiaire des disques imaginaux: $a$, bourgeons donnant naissanre ?aux ailes ; $p$, bourgeons des pattes. 'Imité de GRABER). génie des Insectes, et l'on peut résumer ainsi les faits considérés aujourd'hui comme acquis.

10 Dans la cavité générale des larves, on trouve, dans chaque segment, audessous de l'hypoderme larvaire, quatre petits corps blancs, disposés par paires; ce snnt les disques imaginaux ou hisoblastes (Fig. 169, a) qui, plus tard, " détermineront la chutel de l'hypoderme en se substituant à lui. Toutefois, dans la tête, par suite de la fusion intime des métamères, on observe une réduction notable dans le nombre et dans les dimensions des disques. De même, dans la région abdominale, qui ne porte jamais d'appendices ${ }_{2}$ " les disques imaginaux apparaissent tardivement et ne s'invaginent pas à l'intérieur du corps".

20 Les disques imaginaux dérivent de l'hypoderme; ils se forment par une invagination ayant l'aspect d'un petit sac rattaché à la couche hypodermique par un court pédicule. Bientôt après, ces sacs s'accroissent, s'aplatissent, et s'étalent en membranes poul remplacer les tégumerits de la larve qui vont disparaître ; ̀̀ un moment donné, leur partie centrale s'épaissit et de cet épaississement naîtra un appendice : antenne, patte ou aile.

Les disques imaginaux se forment donc toujours allx points où doivent naître les appendices-; lorsqu'ils sont complètement développés ef réunis les uns aux autres, dans un même segment et dans 
les segments voisins, un nouveau tégument se trouve ainsi constituś sous le tégument primitif : celui-ci peut alors tomber et disparaîl'e.

Fn résumé, il y a deux phénomènes bien distincts à considérer pendant la nymphose :

La destruction des tissus de la larve (histolyse).

L.: réédification des tissus de l'adulte (histogérièse).

Cette destruction et cette réédification marchent de pair, de sorte qu'un organisme nouveau se constitue pendant que l'ancien disparait.

3o Disques imaginaux. - Les organes désignés solıs ce nom sont : tantôt de petits sacs pédonculés, tantôt des bourgeons saillants à la face. in térne de l'hypoderme, tantôt de simples épaississements de la paroi du corps, parfois même des cellules isolées çà et là, à la surface ou à l'intérieur des tissus (Fig. 170).

Nous ne les décrirons que sous leur forme la plus parfaite, c'està-dire sous celle d'un sac pyriforme, dont la cavité in térieure (ca: ité péripodale cp.) est limitée par une paroi (membrane péripodale $m p$.) notablement épaissie vers le fond du sac.

Au cours de leur développement, les disques imaginaux n'apparaissent pas à la même époque, suivant les différentes régions du corps de l'Insecte; très précoces dans la région thoracique, ils sont au contraire

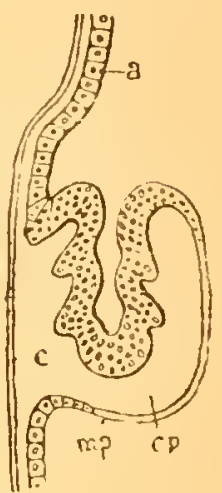

Fig. 170.Formation des disques imaginaux (d'après ANGLAS). beaucoup plus tardifs dans la région abdominale, et ce sont ces différences de rapidité quil sont causes des valiations que no!ıs a vons signalées plus haut (Fig. 171).

Évolution des disques imaginaux. -- Prenons le cas des ailes, qui a été suivi aver soin par Gonin chez les Lépidoptères. Au moment où se forme le bourgeon, qui garnit le fond de la cavité péripodale et qui deviendra l'appendice en question, on voit un tronc trachéen pénétrer dans son intérieur et s'y ramifier, au fur et à mesure que l'aile se développe.

Petit à petit, l'aile se dégage de la cavité péridonale; le sang qui pénètre à l'intérieur la distend et le réseau trachéen la consolide : il suffit de quelques heures pour que l'aile atteigne son développement complet; mais, comme elle est encore enfermée sous la peau de la nymphe, elle reste plissée, tandıs qu'une cuticule solide se 
différencie à la surface. Lorsque l'Insecte sortira de l'enveloppe nymphale, il suffira que l'air circule dans toutes les branches du réseau trachéen your produre l'étalement des membranes alaires.

Il est alors facile de comprendre l'utilité d'un remaniement de. l'hypoderme larraire au moment où l'Insecte adulte va sortir descin enveloppe nymphale.

Les Insectes, en effet, ne s'accroissent plus lorsqu'ıls sont à l'état adulte; il fant done qu'ils aient acquis tout leur développement pendant la vie nymphale et pendant le temps, très court, oì leurs téguments encore souples peuvent s'étendre. La surface totale du corps de l'Insecte est incomparablement plus grande que celle de

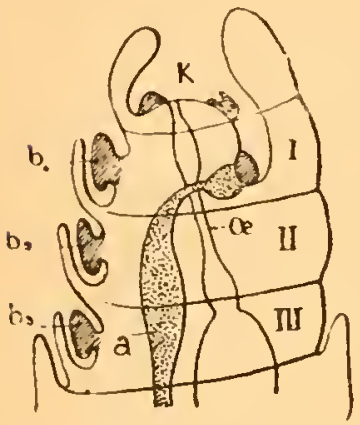

Fig. 171. - I.es disques imaginaux daurs la région céphalique de la Mouche : $k$, vésicule céphalique; $x$, osophage; $a$, syst. nerveux; I-III, anneaux thoraciques; $b_{1}, b_{2}, b_{3}$, disques imaginaux cles pattes

d’après VAN Rrss). la larve. Or, ce résultat ne peut être obtenu que si l'hypoderme larvaire est profondénent remanié.

La larve, sous sa forme de nymphe, a, ì sa disposition, une certaine quantité de maticre qu'elle ne peut augmenter, puisqu'elle ne se nourrit plus; l'hypoderme larvaire sera done remanié, à très pell de chose près, avec les mêmes éléments que ceux qui le constituent déjà'; mais ces éléments, nous le savons, ce sont de grosses cellules qui se divisent pour donner des cellules plus petites et par conséquent plus nombreuses ; la surface couverte par ces petites cellules est supérieure à la surface hypodermale de la larve, bien que la quantité primitive de matière ait à peine augmenté, problème souvent résolu de cette façon en biologie.

¿o Histogénèse des organes. - Tous les autres appendices de l'adulte prennent naissance, comme les ailes, par le jeu de disques imaginaux particuliers.

Toutefois, dans les larves pourvues de pattes, les membres locomoteur's de l'adulte se forment bien au niveau des pattes larvaires, mais non il leurs dépen., comme on le croyait d'après les expériences de Réaumur, la patte larvaire ne donne naissance qu'aux tarses; les 1 rois autres parties de la patle de l'adulte se forment par un épaississement hypodermique, véritable disque imaginal, bourgeonnant à la base de la patte de la larve el s’intercalant, pál consé- 
quent, entre l'extrémité de la patte larvaire et sa racine : c'est ce qu'on appelle le bourgeon fémoro-tibial.

Dans les antennes et les pièces buccales, nous trourons le même mode de développement, c'est toujours un bourgeon hypodermique qui fonctionne à la base de l'organe larvaire de façon à l'allonger.

10 Appareil Digestif. - Les transformations que subit l'appareil digestif sont d'autant plus grandes que le régime alimentaire de la larve diffère plus de celui de l'adulte ; en général, l’épithélium, de même que la tunique musculaire de l'intestin, sont frappés de dégénérescence et remplacés par des lissus de nouvelle formation.

Le nouvel épithélium est régénéré par de nombreuses petites cellules placées à la base des grandes cellules larvaires, et formant, dans chaque segment, une sorte d'anneau imaginal; l'épithélium larvaire est donc rejeté à l'intérieur du tube digestif par un phénomène d'exuviation, tout à fait comparable à celui qui produit les mues externes.

$2^{\circ}$ Apparell respiratoire. -.. Il suffit de considérer la positioi! des orifices stigmatiques, pour s'assurer que, souvent, l'appareil respiratoire des adultes diffère notablement de celui des larves : ce dernier devra donc subir des transformations profondes pendant la nymphose.

Voici, à titre dlexemple, les dispositions le plus fréquemment réalisées pour la pénétration de l'air à l'intérieur de l'organisme chez les Muscidés.

10 Chez les larves, l'air pénètre par deux ouvertures stigmatiques situées à la partie postérieure du corps.

$2^{\circ}$ Chez les nymphes, les orifices respiratoires n'existent que sur le thorax.

$3^{\circ}$ Chez l'adulte, on compte six paires de stigmates latéraux.

On conçoit que, pour passer de l'une à l'autre de ces dispnsitions, au cours des métamorphoses, il faudra des remaniements internes.

En général, les trachées de la larve disparaissent complètement pendant la nymphose ; cependant, d'après Kowalevsky, quelques grandes cellules de l'hypoderme persistent et sont le point de départ du nouveau réseau trachéen. Ces cellules sont donc de véritables trachéoblastes, mais leur évolution est insuffisamrnent connup.

$3^{\circ}$ Appareil circulatoire. - Ici, tous les auteurs sont d'accord; le vaisseau dorsal et ses dépendances ne subissent que de très faibles 
transformations pendant la nymphose. On conçoit d'ailleurs très bien que le cœur de la larve puisse suffire à la circulation del'adulte ; et, d'autre part, le fait que le vaisseau dorsal continue à fonctionner et à se contracter chez les nymphes, indique que cet organe n'est le siège d'aucun phénomène de dégénérescence histogénique.

Quant au sang, c'est le liquide incolore qui remplit la cavité générale du corps et dans lequel baignent tous les organes; les modifications chimiques qu'il subit ont été mal étudiées jusqu'ici. On trouve dans ce liquide de grosses cellules libres douées de mourements amiboïdes : ce sont des leucocytes ou amibocytes. Certains auteurs leur attribuent un rôle phagocytaire important dans l'histolyse.

$4^{\circ}$ Appareil nerveux. - Le système nerveux n'est modifié que dans ses dispositions topographiques (concentration). Ici encore, tous les auteurs sont d'accord, les ganglions cérébroïdes et ceux de la chaîne ventrale passent de la larvo à l'adulte sans subir l'histolyse.

Quant aux organes des sens, les modifications qu'ils subissent étant très mal connues, ce que l'on sait pour chaque groupe sera expliqué dans les volumes spéciaux.

$5^{\circ}$ Appareil musculaire. - Il était facile de prévoirque, parsuite du développement considérable des organes locomoteurs (patles et ailes), le système musculaire de la larve allait devenir insuffisant pour les besoins de l'adulte; cet appareil doit donc subir des remaniements très profonds pendant la nymphose. Presque tous les musclez larvaires disparaissent, en effet, par histolyse (= myolysè) ; las faisceaux dont ils sont formés commencent par se détacher de. la cuticule; ensuite ils se fragmentent, subissent une sorte de fonte et sont enfin, peu à peu, remplacés par des muscles de nouvelle formation

$6^{\circ}$ Appareil reproducteun. - Les organes reproducteurs, cela se conçoit, ne subissent en aucune façon l'histolyse pendant la nymphose; ils continuent à se développer, de manière que les éléments sexuels aient atteint leur maturité au moment où l'adulte devient libre.

$5^{\circ}$ La marche de I'histolyse. - Mais quel est le mécanisme de cette transformation? Ici. les avis des auteurs sont très partagés.

Opinion de Kowaleusky. - D'après Kowalevsky, c'est. sous l'action des globules du sang que se produirait l'histolyse musculaire ; mais, comme il a été démontré que d'autres cellules peurent 
également jouer un rôle phagocytaire, il en résulte que les leucocytes ne sont probablement pas les agents uniques de cette désagrégation. A la phase de reconstruction (histogénèse), ce seraient les cellules mésodermiques des disques imaginaux qui deviendraient les éléments des nouveaux muscles.

Opinion de Berlèse. - Pour Berlèse, les muscles larvaires, principalement ceux qui ne sont d'aucune utilité pour l'adulte, subissent une dissolution complète, une myolyse totale. Toutefcis, cette myolyse ne porte que sur la substance contractile; les noyaux, mis en liberté, persistent et continuent à vivre. Ces noyaux vont grandir et se multiplier; plus tard, ils se transformeront en myocytes allongés, qui seront le point de départ des nouveaux muscles de l'adulte. Rerlèse, comme on le voit, ne fait pas, comme Kowalevsky, intervenir lez disques imaginaux dans l'histogénèse des muscles.

Opinion de Pérez. - Charles Pérez a émis une opinion nouvẹlle en 1972. Pour lui, les muscles larvaires destinés à disparaître, présentent, à leur périphérie, de très petits noyaux qui sont de véritables histoblastes. A mesure que la fibre larvaire se détruit, ces histoblastes se développent, et ils aboutissent finalement à la formation des muscles de l'adulte; le myoplasme est donc en quelque sorte réemployé; dans tous les cas, la plasmolyse phagocy taire n'est que partielle et non pas totale comme le croyait Kowalevsky.

L'opinion de Pérez me parait très rationnolle. A mon aviz, il existe des histoblastes dans tous les tissus; quelquefni blastes peuvent être des simples noyaux isolés, c'est le cas pour les muscles; d'autres fois ce sont des cellules complètes, ainsi qu'on l'observe pour les trachées et pour l'épithélium de l'intestin ; enfin, dans les cas les plus connus, ce sont les groupes de cellules que les auteurs ont depuis longtemps résignés sous les noms de disques imaginaux.

\section{Considérations générales sur les Métamorphoses.}

$1^{0}$ Les carses d’ l'histolyse. - Les deux processus fondamentaux de l'histolyse et de l'histogénèse, s'exerçant simultanément et en sens inverse, résument èvidemment la marche de toutes les métamorphoses; 
malheureusement, sur l'origine même de ces phénomènes nous n'avons que des données très incomplètes, et il a été impossible jusqu'ici de formuler les lois qui les régissent.

Quelques auteurs ont cependant essayé de donner une explication acceptable de ces phénomènes ; le résumé de leurs opinions montrera suffisamment que la solution de ces importantes questions reste encore à trouver. Si les remarques suivantes, clairement résumées par Henneguy, permettent de serrer de plus près la question, elles ne la résolvent pas.

Incontestablement " c'est chez les Insectes holométaboliens, à larves apodes (Muscidés), que les phénomènes d'histolyse et d'histogénèse sont les plus marqués, tandis que chez les Insectes holométaboliens à larves hexapodes, il n'y a souvent qu'un remaniement, ure rénovation de certains tissus et formation d'organes nouveaux, en rapport avec des fonctions nouvelles. "

D’£utre part, " il est proṕable que l'on trouvera tous les stades de passage entre la simple évolution graduelle des tissus et des organes, chez les Insectes paurométaboliques, et la néoforınation d'organes chez les holométaboliques, quand on aura observé ce qui se passe chez les Insectes à métamorphoses graduelles et à hypermétamorphoses $"$. Ce sont les appareils digestif et locomoteur qui subissent, cela va sans dire, les transformations les plus profondes au moment de la métamorphose, puisque le passage à l'état adulte se traduit le plus sourent par un changement romplet dans le mode de locomotion (apparition des ailes) et dans le régime alimentaire (passage du type broyeur au type suceur, Lépidoptères). I'appareil respiratoire n'est lui-même 
fortement modifié que si l'Insecte passe de la vie aquatique à la vie aérienne ou inversement. Tous les autres appareils de la larve, qui peuvent servir aux besoins de l'adulte, persistent et échappent aux phénomènes de l'histolyse.

Voici maintenant un résumé des principales théorıes proposées pour appliquer les phénomènes de l'histolyse.

10 Théorie des blastèmes. - Cette théorie, dont les bases furent posées pour la première fois, en 1863, par Weismann, reprise et complétée par Viallanes en 1882, admet que, pendant la nymphose, les cellules larvaires subissent une désagrégation donnant naissance à des sortes de blastèmes aux dépens desquels se formeraient d'autres éléments cellulaires plus petits. Ce sont ces éléments nouveaux, dont l'importance a été plus tard démontrée par Pérez, qui servent à l'édification des nouveaux tissus et par suite des noureaux organes.

L'interprétation de Viallanes n'est pas soutenable aujourd'hui même en ce qui concerne l'histogénèse des muscles, où il avait cru trowver son principal appui.

20 Théorie de la phagocytose (1). - Édiliée par Kowalersky en 1887, cetto théorie renferme certainement une grande part de vérité ; mais c'est à tort qu'on a considéré la phagocytose comme la cause même de l'histolyse. On peut, en effet, lui faire une objection très grave; pourquoi, à un moment donné, les phagocytes s'attaquentils à certains organes tandis qu'ils en respectent d'autres, placés en apparence dans les mêmes conditions?

Il ne serait peut-être pas impossible de répondre à cette objec. tion, mais ce n'est pas ici le lieu d'une semblable discussion.

3. Théorie de la crise de maturité génitale. - Charles Pérez (2), qui a le premier formulé cette théorie en 1899, part de ce principe que "le fait primordial de l'histolyse est la prolifération subite et aciive des disques imaginaux, venant à la suite de la surnutrition

(1) Kowalevsix (A). - Zur embryonalen Entwickelung der Musciden, Biol. Centr. 1886. T. VI. 1. 49-54.

(¿) Pere7. (Ch.). - Bull. Soc. ent. 1899, p. 398. 
de la larve et de la pléthore des réserves dans le corps gras :. Ces phénomènes seraient, en d'autres termes, la manifestation de la lutte pour la vie qui éclate, à un moment donné, entre les différentes cellules de l'organisme: grosses cellules larraires d'une part, et petites cellules des histoblastes, d'autre part.

La prolifération rapide de ces petites cellules. rompant !'équilibre préexistant, provoquerait la désagrégation des tissus larvaires, c'està-dire la crise générale. Quelle serait alors la cause de cet avantage, entièrement au profit des cellules histoblastiques ? Pérez a étayé sa théorie d'une hypothèse ingénieuse : il admet, pendant la métamorphose, et sous l'influence d'une excitation due au développement des cellules génitales, la sécrétion, par les disques imaginaux, de produits particuliers qui seraient des stimulines pour les cellules histoblastiques et des toxines pour les éléments larvaires.

Cette théorie, très séduisante, a pour point de départ un fait positif certainement très remarquable, à savoir l'extraordinaire disproportion qui existe entre le volume des cellules larraires et celui des histoblastes; quant à la cause qui oblige ces éléments à évoluer en sens inverse, l'hypothèse des toxines, fut-elle même démontrée, reculerait la question mais ne la résoudrait pas.

$4^{\circ}$ Théorie de la lyocytose (1). - Tous les auteurs paraissent d'accord sur ce point : au moment de la métamorphose, les tissus qui doirent disparaître par histolyse, présentent toujours les signes d'une altération chimique plus ou moins profonde, de sorte que les phagacytes, lorsqu'ils interviennent, s'attaquent toujours à des tissus en roie de décomposition. Or, d'après Anglas, il existe de nombreux exemples où les choses se passent différemment et où les phagocytes n'interviennent pas. Dans ce cas, les tissus larvaires, d'après lui, se dissoudraient purement et simplement dans le liquide caritaire, sous l'action de diastases sécrétées par les leucotyces, et c'est ensuite, dans cette sorte de blastème général, que les éléments histoblastiques puiseraient les matériaux nécessair_s à lour évolution. Cette sorte de digestion, par les éléments figurés du sang au sein du liquide caritaire, a reçı d'Anglas le nom de lyocytose pour rappeler qu'elle se fait principalement par l'intermédiaire des leucocytes; malheureusement, jusqu'ici, il a été impossible de mettre en évidence l'e sistence des ferments actits.

(1) Anglas (J). - C. R. Soc. biol. nov. 1899. 
$5^{\circ}$ Théorie de l'asphyxie (1). - Voici une théorie qui a sérieusement retenu l'attention des biologistes; elle a été formulée par Bataillon en 1893, à la suite de ses recherches sur la nymphose du Ver à soie. Cet auteur pose, en principe, que la métamorphose est un ensemble de phénomènes asphyxiques; et, d'après lui, les phénomènes de dégénérences signalés précédemment ne seraient que la conséquence de l'asphyxie.

Sans aucun doute, sous l'enveloppe chitineuse étanche, il y a accumulation de $\mathrm{CO}^{2}$ dans le corps de la nymphe; mais ce gaz, ainsi que les autres produits nocifs, doit se distribuer uniformément dans le sang ainsi que dans la cavité générale ; on ne comprend pos alors pourquoi certains organes, tel que l'appareil digestif, disparaissent, brusquement histolysés, tandis que d'autres persistent et résistent à l'asphyxie.

En résumṕ, jusqu'à ce jour, aucune théorie n'a fourni une explication entièrement acceptable des phénomènes essentiels de la métamorphose; tout ce qu'on sait, c'est que les tissus et les organes qui subissent le plus rapidement l'histolyse, sont ceux qui ont cessé de fonctionner, ceux qui sont réduits à l'inaction, et qui sont devenus inutiles à l'Insecte par suite du changement radical qui s'est produit ou qui va se produire dans son mode de vie ! Mais alors, conclut MI. Henneguy, " on est conduit à se demander pourquoi la larve change de mode d'existence ", ce qui revient à poser le problème de l'origine des métamorphoses.

Nous ne pouvons pas aborder ici cette question, dont le seul exposé nous entraìnerait hors du cadre assigné à cet ouvrage ; il semble d'ailleurs que sa solution ne pourrait être donnée que par "une étude approfondie des documents paléontolugiques"; or, nous sllons voir que ces documents sont encore trop peu nombreux ou trop incomplets, pour qu'on puisse en faire état dans une question si difficile.

(1) B.taillox (E.). Bull. sc. France et Belgique. T. XXV. Paris, 1893, in-8º 



\title{
LIVRE TROISI ÈM E
}

\author{
PREMIERE PARTIE \\ ENTOMOLOGIE ÉCONOMIQUE \\ CHAPITRE I
}

\section{LES INSECTES DANS LES TEMPS GEOOLOGIUES \\ Paléoentomologie.}

Bien que très riches, les documents paléontologiques que nous possédons sur les Insectes, ne permettent pas de suivre l'évolution des principaux groupes à travers les époques géologiques. On sait seulement - et cette notion se dégage nettement des observations qui ont été faites dans tous les pays - que les Insectes, ne subissant aucune métamorphose, ont apparu sur la terre avant les métaboliens. La plupart, en effet, des formes anciennes qui ont été identifiées, peuvent être rapportées aux trois grandes classes d'amétaboliens : Névroptères, Orthoptères et Hémiptères.

\section{$1^{\circ}$ INSECTES DES TEMIPS PRIMAIRES}

Il faut rayer de la science l'empreinte silurienne des grés de Jurques (Calvadoi), que Ch. Brongriart arait décrite sous le nom de Palæo!lattina Dourıliei. Il a été démontré que cette empreinte, long- 
lemps considérée comme une aile d'Insecto (Rl.stiidés), n'étart aưre chose qu'une pointe génale de Trilobite.

ÉPOQUE DÉvONIENNE. - C'est dans les schistes dévoniens ou calbonifères du Canada, (Nouveau-Brunswiek), qu'ont été signalés, par Scudder, les premiers débris d'ailes que l'on puisse inconstestablement rapporter à des Insectes; mais, étant donné le mauvais état de conservation de ces débris, qui semblent appartenir à des groupes d'Éphémérides, et qui sont d'ailleurs en très petit nombre (sir śchantillons seulement), il est à peu près impossible de se faire une opinion, et de leur assigner une position définitive dans la classification.

Des faits observés, on peut cependant conclure que l'ordre d'cs Archiptères (Orthonévroptères) existait déjà pendant les temps déroniens.

ÉPOQUE GARBONIFERE. - Mais c'est surtout à l'époque carbonifère que les Inseetes deviennent nombreux et variés; cet accroissement des espèces est vraisemblab!ement dû à l'extension des continents et au développement des grandes forêts de Cryptogames qui donnèrent naissance à la houille.

Le; nombrax documents recueillis dans les schistes carbonifères de Commentry (. fllier), permettent de donner un aperçu suflisammert net de la faune entomologique à cette époque.

Tout d'abord. un fait remarquable est celui-ci : les Insectes de cette période étaient généralement de grande taille. quelques-uns d'entre eux n'avaient pas moins de $0 \mathrm{~m} .70$ d'envergure : les ancêtres des Thysanoures étaient représentés par le Dasyleptus Lucasi, voisin des Lépismes et des Machiles.

Dans le groupe des Orthoptères, on rencontre de nombreuses formes, que l'on peut rapprocher des Blattes, des Phasmes, des Criquets et des Locustides; tous ces Insectes présentent un caractère commun : les ailes de la seconde paire sont à peine plus larges que celles de la première et ne sont pas encore parcourues par ces nervures en éventail qui, chez les formes actuelles, permettent aux ailes - malgré leur étendue - de se replier entièrement sous les élytres.

Cette particularité avait paru ci importante à Scudder qu'il arait proposé de désigner tous ees Orthoptères paléozoïques sous le nom J'Orthoptéroüdes.

Parmi ces formes, oì cesont, semble-t-il, les Paleoblattidés (ancêtres des Blatles) qui dominent, nous nous bornerons à signaler ici la 
merveilleuse empreinte d'Ertólattina Gaudryi, récemment publiée par M. Agnus dans le Bulletin de la Société entomologique de Frunce, 1903, p. 292.

On såit aussi que, chez les Blattes de l'époque actuelle, les oufs sont renfermés dans une coque ovigère (p. 211) oì les jeunes restent abrités pendant un certain temps après l'éclosion; or, chose remarquable, il est probable que les Blattidés de l'époque houillère n'araient pas encore acquis ce caractère, car leur abdomen était terminé par une tarière (i), ce qui indique qu'elles devaient pondre leurs $œ u$ fs un à un, comme les Grillons actuels.

I.es Phasmidés étaienl représentés par les genres Prolophasma, I'itanophasma, elc.;l'une desplus belles espè-

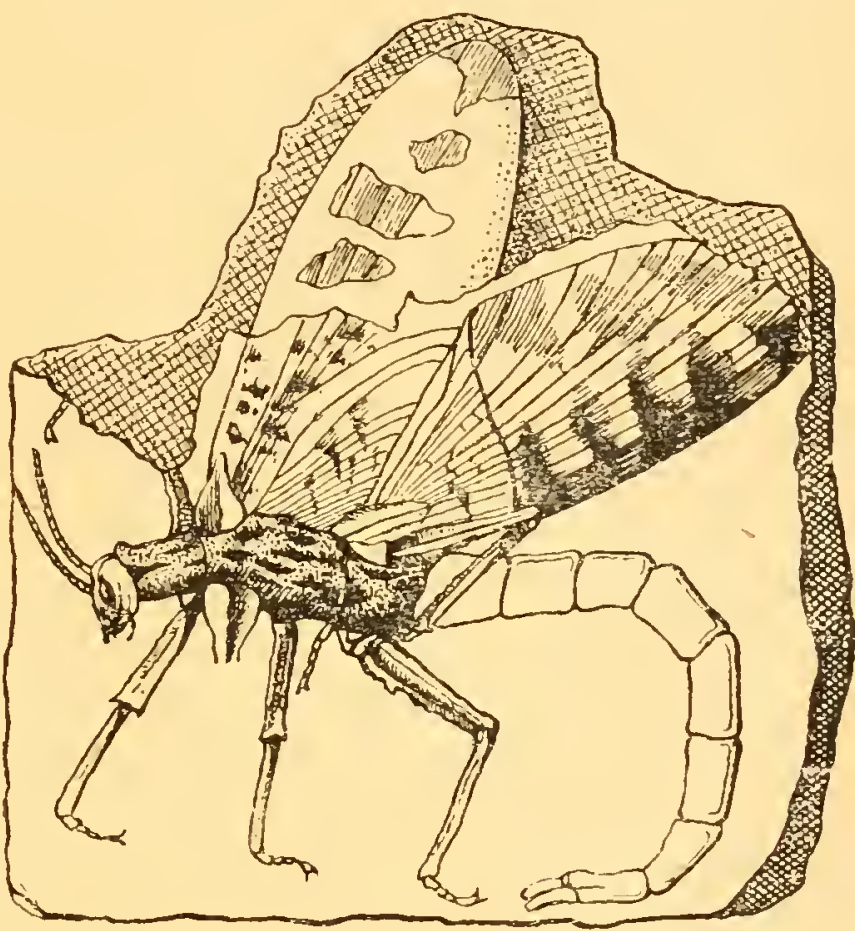

Fig. 17:2. - Jrotophasma I.umasi Orth. 1/4 graudeur naturelle. Terrain houiller de Commentry (d'après CH. BroNGNiART). ces, Protophasma Dumasi (Fig. 172), nous montre qu'à rette époque, ces Insectes étaient pourvus de deux parres d'ailes bien développées, tandis que les Phasmes actiels sont, ou bien complètement aptères, ou bien on les ailes antérieures réduites à l'état d'écailles : Titanophasma Fayoli, dont le corps seul est connu, ne devait pas mesurer moins de vingt-huit centimètres de long. Chez ces deux espèces, ainsi qu'on paut le voir, le corps rappelle les Orthoptères, tandis que les ailes sont semblables à celles des Névroptères.

Les Névroptères sont beaucoup plıs nombreux et plus diversifiés que les Orthoptères, ce qui tendrait à prouver qu'ils sont d'origine plus ancienn:; les ancêtres des Odonates (Protagrion), des Myrmé-

1) Brovgniart (Ch.). - Ann. géol. T. VI. 1889, p 788. 
léonides (Protascalaphus) et les grandes Éphémérides d!ı genre Meganeura, dont les atles seules ne mesurent pas moins de trente-trois centimètres, sont représentés par des formes aussi abondantes que variées.

Quant aux Hémiptères, ils comprennent un certain nombre de genres qui rappellent, jusqu'à un certain point, les Fulgores et. les Cicadelles; on y rapporte également le curieux Eugereon Bcerkengi:i, trouvé par Dohrn dans le permien de Bohême : cet Insecte est un Névroptère par la nervation de ses ailes, mais un véritable Hémiptère, par les pièces de sa bouche qui sont disposées pour piquer.

En somme. toutes les formes que nous venons d'énumérer possèdent in certain nombre de carartères communs : ce sont des Insectes n'ayant pas encore acquis le degré d'adaptation qui caractériso les formes d'aujourd'hui; ils se rapprochent des Orthoptères, des Névroptères et des Hémiptères, sans appartenir exclusivement ì l'un ou' l'autre de cos ordres. Ce sont, en réalité, des types synthétiques, d'où sont sortis plus tard, dans le cours des temps secondaires, tous les ordres actuellement vivants. A cause du mode de nervation de teurs ailes, ll paraît utile, ainsi que Scudder l'a proposé, de réunir tous ces types primilifs en un groupe phylétique sous le nom de Palxolictyoptera.

Il y a to:It lieu de croire que les Coléoptères, les Hyménoptùres el les Lépidoptères n'existaient pas encore comme groupes distincts pendant l'ère paléozoïque ; les débris douteux qu'on a trouvés ne permettent pas de se prononcer avec certitude sur ce sujet.

I! paraît aussi bien certain que la plupart des Insectes étaient dépourvus de métamorphoses complètes; leurs larves ne différaient sans doute de l'adulte que par l'absence d'ailes.

\section{INSECTES DES TEMPS SECONDAIRES}

Le; types actuels d'Insertes ne font leur apparition qu’à l'époque secondaire, et leur évolution paraît terminée à l'époque tertiaire ; ils se sont accrus et perfeclionnés en même temps que la flore terrestre. Aux ordres synthétiques de l'ère paléozoïque, qui, petil à petit, se différencient, succèdent des formes nouvolles. Dans le trias, ̀̀ côté des Librllules et des Hémiptères, déjà presque sen- 
blables à ceux qui vivent de nos jours, se trouvent les premièrcs traces certaines de Coléoptères (1).

Les Diptères et les Hyménoptères apparaissent dans le lias; quant aux Lépidoptères, on ne les rencontre que beaucoup plus tard, dans les dépôts d'eau douce du jurassique moyen.

Il est remarquable de constater que les débris de Coléoptères qu'on a recueillis dans le trias, (Curculionites prodomus, à Vaduze, (Allemagne), soient déjà des Rhynchophores; ce type d'Insfcte étant l'un de ceux que nous considérons comme les plus modi. fiés par l'adaptation.

Mais c'est surtout dans le lias de Schambelen, en Argovie, que les Coléoptères sont abondants; plus de 110 espèces ont été signalées par M. Heer ; ce sont surtout des Buprestides, des Byrrhides et dès Chrysomélides. Les eaux étaient déjà habitées par des Gyrinites et des Hyirophilites, ce qui fait supposer l'existence du type clasicérien (2). Enfin les Coléoptères carnassiers étaient déjà représentés par les Calosoma et les Carabites.

Quoi qu'il en soit, il convient aussi de remarquer que certaines familles ne sont pas encore représentées, tels sont, par exemple, les Cérambycides et les Coccinellides, ainsi que la plupart des Xylophages. L'existence des Diptères est douteuse; quant aux Hyménoptères, ils ne sont représentés, à Schambelen, que par quelques rares fragments d'ailes.

A l'époque jurassique, la faune entomologique des célèbres calcaires lithographiques de Solenhofen nous montre que l'ordre des Coléoptères se complète; on y trouve des Coccinellides et un grand nombre d'Hétéromères. Les Hémiptères et les Névroptères ont de nombreux représentants; les Diptères sont devenus communs, ce sont surtout des Tipulides. Oppenheim, en 1885, a fait connaître plusieurs Papillons du jurassique moyen et du jurassique supérieur. Enfin M. Geinitz a observé, dans les grès verts de Saxe, des morceaux de bois perforés qui lui paraissent attester la présence des Cérambycides à l'époque crétacée.

(1) Ch. Brongniart pense que les Coléoptères décrits par Bnckland et Prestwjch Eans les terrains houillers de l'Angleterre sont très problématiques ( $A$ nn. gecl. Y.r. 1020) ; tontefois Dathe (1855) a réellement découvert, dans le culm carbonifère rie Silésie, des tlytres, très bien conservès, qui paraissent, avoir appartenu à des Coléoptères roisins res Carabiques et à des Ténébrionicies (Aun. genl. 1888. p. 726).

(2) C. Hoclabert. - Phylogénie, p. 106. 
En résumé, l'évolution des Insectes, pendant la durée de l'ère mésozoïque, montre que les vrais Orthoptères ont remplacé les Orthoptéroïdes des temps primaires; les Hémiptères et les Névroptères vrais ont succédé aux formes ambiguës de l'époque houillère ; les Insectes sont de plus en plus nombreux. Cependant, tous les ordres ne sont pas également représentés, et c'est toujours le tyre des Insectes broyeurs qui domine, Coléoptères, Nésroptères, Orthoptères. Ceux qui vivent plus spécialement sur les fleurs, comme les Abeilles et les Papillons, n'ont fourni que de très rares débris, ce qui laisse à penser que le groupe des plantes à fleurs devait être encore peu développé ; petit à petit, les Insectes à métamorphoses incomplètes sont remplacées par des holométaboliens véritables, mais c'est dans le tertiaire surtout que les différents ordres acquerror. la diversité et la perfection qui caractérisent la faune actuelle.

\section{in INSECTES: IDES TEMPS |TEITIAHES}

L'ère mésozoïque vient de nous montrer un perfectionnement très notable de la faune entomologique; mais, c'est dans le cours des temps tertiaires (ìre néozö̈que) que va s'achever cette évolution et que vont apparaitre, en grand nombre, les Insectes à métamor-

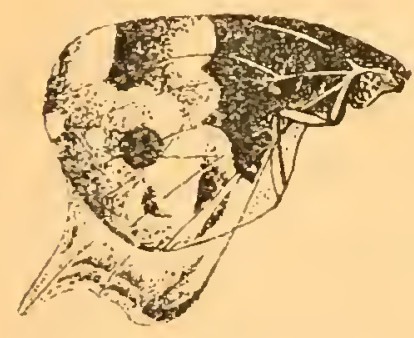

Fig. 173. - Neorinopsis scpulta, papillon fossile de l'époque tertiaire (d’ap. Poisduval),

phoses compiètes, qui jusqu'à présent avaient été pauvrement représentés. Aux groupes carnassiers et phytophages, qui vivaient pendant les temps secondaires, s'ajoutent des formes qui recherchent plus spécialement le suc des fleurs et les aliments liquides, les Papillons deviennent plus abondants (Fig. 173).

L'ordre des Coléoptères est toujours nombreux et prédominant; et, si on se rapporte aux documents fournis par les lignites oligocènes du Siebengebirge, on voit qu'ils représentent, à peu près, la moitié de la faune entomologique de cett? époque. Mais, dans la grande formation lacustre d'Giningen (miocène), dont la faune est la miêx connue de toutes, sur 5.081 échantillons d'Insectes recueillis par M. Herr, 2.456 appartiennent à l'ordre des Coléoptères; comme dans la nature, les Rhynchophores sont déjà en grande majorité (Fig. 174). 
Parmi les gisements fossilifères de l'époque néozoïque, l'un des plus intéressants est certainement l'ambre jaune des côtes de la mer Baltique.

L'ambre, que l'on désigne encore sous le nom de succin, est, con me on le sait, une résine fossile prodinite par les immenses forêts de Conifères qui couvraient, à l'époque oligocène, la Finlande et la Scandinavie. De nombreux Insectes, englués par cette résine, alors que, fluide, elle s'écoulait des arbres, s'y sont admirablement conservés. Nous représentons ici, à titre d'exemple, une très curieuse larve de Myrméléonide (Ascalaphus?) qui nous a été récemment communiquée par tert M. le $D^{r}$ R. Klebs, de Königsberg (Fig. 175).

En France, les gypses d'Aix, en Provence, ont

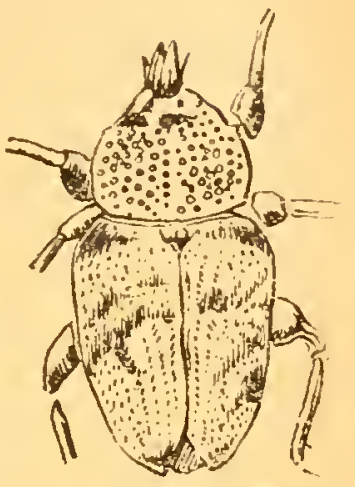

Fig. 174. - Coléoptère fossile de l'époque tertiaire Hipporhinus Heeri Oust.

(d'ap. Oustalet) fourni, en 1838, à M. le comte de Saporta, le premier exemplaire à

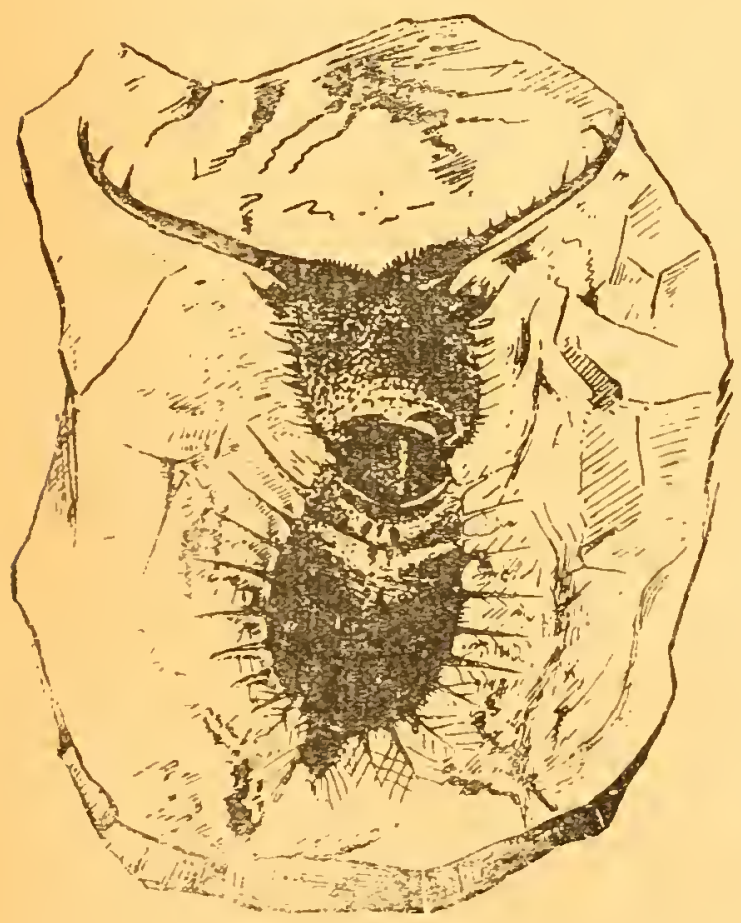

Fig. 175. - Larve de Myrmélèonide (Néropt.) découverte dans l'ambre de la Baltique, par il. le Prof. Klebs

(l'après une photographie de l'anteur). peu près complet d'un papilIon fossile (1) : le (Néorinopsis sepulta, Nymphalidé) (Fig. 173); à côté des Phalénides, on trouvait des Cossus, des Zygènes, des Sésies et des chenilles de Satyrides; mais, sauf Pontia, les Rhopalocères sont presque tous des genres éteints; de même, les travertins de Sézanne (Marne), dont la faune a été si habilement reconstituée par M. MunierChalmas, ont fourni de nombreux exemplaires d'Insectes. Les marnes de Florissant( Colorado-Amérique), ont foumi des'empreintes tellement bien conservées qu'on peut distinguer les descins formés par les écailles des ailes.

(1) Le Sphinx Surlleni, des schistes lithographiques de Solenhofen( lias), n'est, en effet, représenté que par un fragment de trompe enroujé. 
En résumé, les données fournies par la paléontologie, bien que très incomplètes, permettent d'établir, ainsi qu'il suit, la généalogie des hexapodes.

Les Insectes, au début, étaient tous des Palæodictyoptères, et de ce premier phylum sont sortis, tout d'abord, les Névroptères et les Orthoptères. Un rameau détaché des Orthoptères, vers la fin des temps paléozoiques, aurait donné naissance aux Coléoptères, tandis que les Hyménoptères et les Hémiptères seraient issus des Névroptères. Quant aux Lépidoptères, ils dériveraient des Phryganides (Nérroptères), qui les ont précédés dans le cours des temps secondaires. Les Diptères seraient ensuite descendus des Hémiptères, par modification des pièces de la bouche et par atrophie des ailes postérieures.

Telles sont, dans leurs grandes lignes, les conclusions que l'on peut formuler aujourd'hui sur l'évolution des Insectes dans le cours des temps géologiques. Le tableau suivant, complétera, pour les détails, un certain nombre de points, sur lesquels il nous a été impossible de nous étendre ici. 
LES INSECTES DANS LES TEMPS GÉLOGIQUES 283

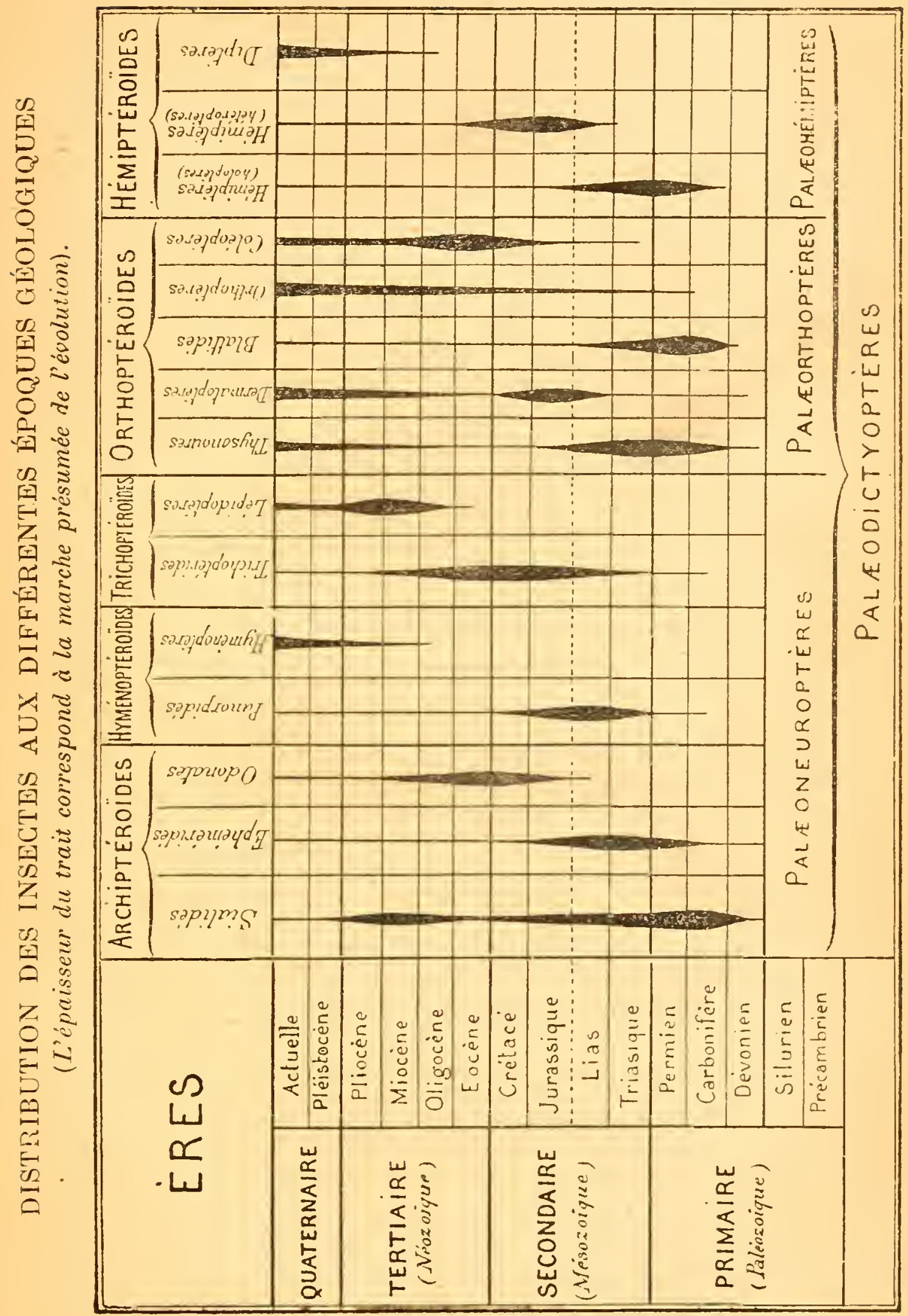




\section{CHAPITRE II}

\section{MOYENS DE DÉFENSE CHEZ LES INSECTES}

De tous temps, les couleurs brillantes des Insectes, les formes si variées et parfois si bizarres qu'ils présentent; ont excité un vif sentiment d'admiration chez les naturalistes; toutefois, les anciens entomologistes n'accordaient qu'un simple intérêt de curiosité à ces phénomènes, et il a fallu bien des siècles avant que les hommes osassent se demander quelle était la raison d'être de cette diversité infinie.

La réponse à cette question, qui nous parait si natu. relle aujourd'hui, commence à sortir lentement de son obscurité.

Il y a quelque cinquante ans, à la suite de notre grand Lamarck, le célèbre naturaliste anglais Ch. Darwin a nettement posé les conditions du problème, en montrant que les causes primordiales, qui ont autrefois déterminé ces merveilleuses variations, étaient les mêmes que celles qui agissent de nos jours : en première ligne, la lutte pour l'existence. Qu'il le veuille ou non, aucun animal n'échappe à cette obligation; les mieux armés survivent et se reproduisent, transmettant leurs qualités à leurs descendants; quant aux plus faibles, ils disparaissent : c'est la loi de sélection naturelle.

Nous n'avons pas à étudier ici suivant quelles moda- 
lités multiples s'exerce la sélection naturelle chez les Insectes; nous nous bornerons à enregistrer des faits, et nous verrons plus tard s'il est possible d'en donner une explication rationnelle.

Les Insectes, cela n'est nullement douteux, ont à se défendre contre de nombreux ennemis : les oiseaux, les parasites, et une foule d'autres animaux insectivores. Or, ils auront d'autant plus de chances d'être épargnés, qu'ils seront mieux protégés mécaniquement ou plus difficiles à découstir. Beaucoup d'entre eux, en effet, unt acquis des ressemblances très frappantes avec les objets les plus divers. Les uns ressemblent à des feuilles vertes (Phyllies) (Fig. 176), ou sèches;

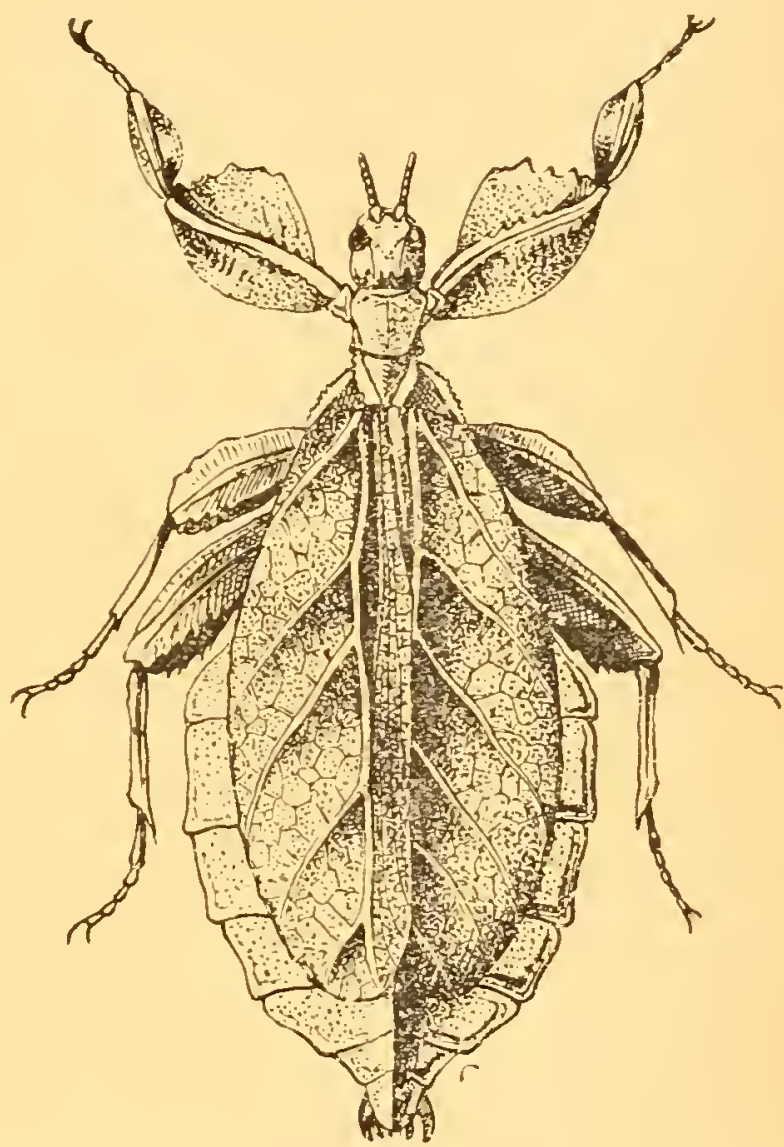

Fig. 176. - La Phyllie feuille sèrhe (rothopt.), exemple de mimétisme (d'après DraḱpiL).

d'autres à des branches mortes, à des fragments de lichen, à des fleurs, à des excréments d'oiseaux et même à d'autres insectes vivants.

Dans certains cas, l'imitation se borne à la couleur (homochromie), mais elle peut parfois s'étendre à la forme et mème aux attitudes : c'est alors le mimétisme proprement dit. La ressemblance est quelquefois si 
parfaite que les animaux - comme nous-mêmes peuvent y être trompés; les remarquables expériences de Poulton et Sanders ne laissent aucun doute à ce sujet (1).

Toutes ces variations, qui paraissaient inexplicables il y a trente ans, sont donc, en réalité, des attributs de défense naturels et, comme le dit excellemment M. Cuénot, "l'étude de ces moyens de défense constitue l'un des chapitres les plus intéressants de la biologie".

Il est hors de doute que toute variation, tendant à dissimuler l'Insecte, contribuera à assurer sa conservation et cela, d'une façon d'autant plus efficace, que la ressemblance sera plus parfaite.

Les procédés de défense les plus répandus chez les Insectes peuvent être rapportés à trois catégories :

10 Si l'Insecte n'a été pourvu d'aucune arme défensive, il a alors tout intérêt à éviter l'attaque et à passer le plus possibleinaperçu; il y arrive de trois manières: soit en menant une vie cachée (hypogéisme) ; soit en se confondant avec le milieu qui l'entoure (homochromie) ; soit enfin en imitant d'autres insectes bien défendus "de façon à profiter de la confusion et à être laissé de côté \# : c'est Jà le mimélisme, au sens le plus restreint de ce mot.

20 Lorsque l'Insecte est pourvu de moyens de dél'ense mécaniques (carapace, épines, poils risides, vol rapide) ou physiologiques (liquides caustiques ou odorants, lumière, etc.), qui lui permettent de moins redouter les attaques de ses ennemis, alors il ne craint pas d'ètre vu et il vit à la surface du sol ou sur les plantes: c'est le cas de la plupart des Coléoptères (Coccinellides),

(1) Poultoni et Sanders (C. B.). - Rep. of Mceting. brit. Assoc. Bristol. 1898 p. 900-909. 
des Hémiptères, d'un grand nombre d'Hyménoptères, etc.

30 Enfin quelquefois, l'Insecte, imparfaitement protégé, trouve, inconsciemment bien entendu, dans l'association avec un autre insecte ou un animal quelconque, la sécurité qui lui manque, en même temps qu'une table assurée (parisi isme).

Nous nous occuperons seulement ici, aree quelques détails, de l'homochromie et du mimétisme proprement dit.

Homochromie.- Tont le monde sait que, chez un grand nombre d'animaux, la couleur dominante du corps se rapproche beancoup de celle du milieu où ils virent; c'est là un moyen de défense, purement passif, acquis par adaption au cours de longs siècles, et qui souvent peut atteindre un très haut degré de perfection.

L'homochromie mimétique, cela se conçoit facilement, aura pour l'Insecte son maximum d'utilité, non seulement lorsqu'elle s'harmonisera avec la teinte générale environnante, mais surtout lorsqu'elle s'étendra, ainsi que nous l'avons indiqué plus haut, à ia copie exacte des particularités de la surface et de la coloration (nervure des feuilles, écorce des arbres, brindilles de bois). Ce phénomène est trop général chez les animaux, et particulièrement chez les Insectes, pour qu'on ne lui reconnaisse pas une haute signification, au point de vue de la conservation des espèces.

Cependant l'homochromie n'est pas uniquement une propriété défensive; elle peut être aussi quelquefois offensive; certains Insectes, en effet, s'ils sont dissimulés par une coloration suffisamment parfaite, peuvent en profiter pour approcher plus facilement leurs proies sans en être aperçus.

Mimétisme.. - Mimétisme veut dire faculté d'imiter; mais, avec le sens précis qu'on lui réserve aujourd'hui, cette expression s'applique, plus spécialement, aux homologies de forme et d'attitude.

Le mimétisme, comme son nom l'indique, ne comprend pas seulement la couleur, mais encore tout ce qui a trait à la forme extérieure des objets; l'Insecte ne se contente plus de s'harmoniser, comme coloration, avec le milieu ambiant, mais il imite, il mime 
(d’où le nom de mimétisme), une autre espèce très bien armée au point de vue de la défense; le résultat de cette adaptation est facile à saisir ; l'espèce mimante, quoique inoffensive et comestible, sera généralement délaissée par ses ennemis, qui la confondront avec l'espèce mimée " dont le goût est souvent rebutant et la cuirasse indigeste ".

De même que l'homochromie, le mimétisme peut être offensil; c'est le cas de certains Insectes qui vivent en parasites, comme par exemple les Volucelles, qui profitent de leur déguisement pour déposer leurs œufs dans les nids des Guêpes et des Bourdons.

Nous allons maintenant étudier brièvement, dans les différents ordies d'Insectes, soit à l'état de larve, soit sous la forme adulte, les procédés de défense mimétique les plus généralement employés (1).

\section{INSECTES A METAMORPHOSES GRADUELLES}

10 Névroptères. - Ici, les procédés de défense sont très variés ; les Névroptères les plus parfaits de la famille des Odonates ont un vol rapide et très puissant, ce qui leur permet ie cap-

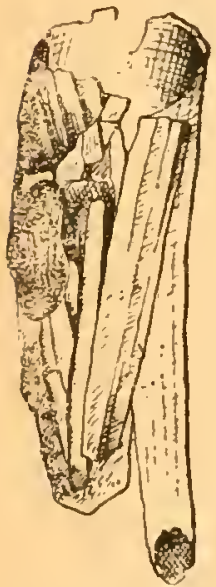

Fig. 177. F) surreau ie l'hrygani le (imité de SI I T.ILA). turer leur's proies et en même temps d'échapper allx poursuites de leurs ennemis. Tout le monde a vul ces grandes Libellules, que l'on désigne vilgairement sous le nom de Demoiselles, planer au-dessus des eaux et faire la chasse à tous les Insectes plus faibles qu'elles.

Dans cet ordre, beauroup do larves sont aquatiques (ex. : Phryganes) et leurs téguments sont mous; elles se construisent alurs, à l'aide de petites coquilles ou de débris végétaux, des fourreaux protecteurs à l'intérieur' desquels elles trouvent un abri (Fig. 17\%). D'antres, cəlles des Fourmilions, par exemple, s'enfoncent dans le sahle, dont elles possèdent la couleur: d'antres enfin, comme les Termites, trouvent dans l'association, une sécurité quə ne possèdent jamais les individus isolés.

$2^{\circ}$ Orthoprères. - La plupart des Orthoptères ètant phytophages vivent sur les arbres, ou à terre parmi les herbes, aussi l'homo chromie est-elle très répandue; tout le monde sait que la grande Sauterelle verte (Locusta viridissima) est

(1) Tin ce qui concerne chaque ordre d'Insectes, ce sujet sera traité avee beacouß plus de détails dảns les volumes spéciaux de l’E.s. 
très difficile à apercevoir, lorsqu'elle est immobile sur le feuillage.

La plupart des Criquers possèdent la couleur grise des rocailles ou de la terre nue; ceux des prairies ont toujours la couleur de l'herbe qui les nourrit.

Les Phasmes, animaux complètement dépourvus d'arme défensive, se laissent tomber à terre lorsqu'on les inquiète, et ils présentent alors, à s'y méprendre, l'aspect d'un morceau de bois sec. Mais ?e cas le plus remarquable est celui des Phyllies, dont les élytres élargies, d'un beau vert, reproduisent exactement l'aspect d'une feuille vivante avec ses dents et ses nervures (Fig. 176).

Chez les Mantes, l'homochromie revêt un caractère offensif ; lorsque la Mante prie-Dieu est à l'affût, immobile sur un buisson, las autres Insectes s'approchent d'elle sans défiance, ce qui lui permet de les saisir brusquement avec ses pattes antérieures armées d'épines.

$3^{\circ} \mathrm{HÉ}$ ulPtères. - I I i, les moyens de défense les plus répandus sont d'ordre physiologique; la plupart des Hémiptères sécrètent des liquides d'odeur désagréable qui suffisent, vraisemblablement, à écarter leur's ennemis ; l'émission de ces liquides se fait certainement sous l'influence de la volonté, car les Punaises des bois, que tout le monde connait, ne répandent leur mauvaise odeur que lorsqu'on les inquiète.

L'homochromie, à peu près inutile dans ces conditions, est, en effet, peu répandue; quelques espèces sont même ornées de couleurs voyantes qui tranchent fortement avec celles du milieu environnant.

Un cas très remarquable de mimétisme protecteur est celui du Réduse masciué. A l'état de larve et de nymphe, cet Insecte se recouvre de poussière, de flocons de laine ou de toiles d'araignées, au point de devenir méconnaissable. ll faut d'ailleurs éviter sa piqûre avecsoin, car elle est douloureuse et fréquemment suivie d'accidents infectieux.

\section{INSERTES A METTMORPHOSES COMPLETES}

10 Coléoptéres. - Chez les Coléoptères, ce sont les moyens de défense mécaniques et physiologiques qui sont les plus répandus. Chez presque tous, la cuirasse de chitine est très dure et les protège efficacement. Ceux dont les téguments sont peu résistants comme les Vélö̈, les Jélíphores, etc., émettent, lorsqu'ils sont inquiétés, des liquides odorants ou caustiques qui rebutent leurs

LES INSECTES. $-2^{\mathrm{e}}$ édit. 
ennemis. Les deux modes de protection se complètent d'ailleurs tellement, dans cet ordre, qu'au seul aspect d'un Insecte, on pourrait presque dire quels sont ses moyens de défense habituels.

L'homochromie, qui n'est guère utile qu'aux espèces phytophages, se rencontre, en effet, chez un grand nombre de Curculionides, dont les élytres copient la couleur des écorces. Mais le cas le plus remarquable est celui d'un grand Péricalide de Java, le Mormolyce phyllodes, qui vit sous les trones d'arbres renversés, et dont les élytres ressemblent à des feuilles mortes.

2ग HYMÉnoptères. - Le mimétisme est peu répandu parmi les Hyménoptères, qui sont, pour la plupart, admirablement armés avec leur aiguillon et leur appareil venimeux; les espèces qui ne possèdent pas d'aiguillon mordent avec fureur et peuvent lancer un fluide caustique jusqu'à 50 centimètres de distance (Fourmis); tous ceux qui ont essayé de visiter l'intérieur d'une fourmilière ont pu vérifier ce fait.

En revanche, les larves nues de certains Hyménoptères (Abeilles; Grıêpes, etc., etc)., assez mal protégées par leurs nids, sont la proie de nombreux ennemis.

Le parasitisme est fréquent, et l'un des cas les plus curieux est celui des Psythyres, dont la livrée imite si parfaitement celle des Bourdons, qu'ils peuvent aller et venir au milieu de ceux-ci, vivre sans rien faire aux dépens des provisions communes, et même confier leur descendance aux soins des nourrices de la colonic sans jamais être inquiétés.

3" LÉrid ortères.- - lci, les moyens de défense sont tellement variés qu'il est impossible d'en donner une idée, mêne incomplète. Sous las trois états de chenille, chrysalide et adulte, le mimétisme et l'homochromie se combinent de cent façons différentes.

La plupart du temps, la couleur des chenilles se confond exactement avec celle de la plante nourricière ; d'autres fois, les chenilles portent de longs poils rigides (Chelcnides), on sécrètent des liquides âcres, à odeur désagréable (7.ygizna). Les chrysalides des papillons diurnes sont, en général, suffisamment protégées par leur carapace durcie; celles des papillons de nuit, on bien s'abritent dans la terre (Phalène ìu (iroseiller), ou bien s'enveloppent d'un cocon de soie dont la couleur se confond avec celle du milieu environnant.

Chez. les adultes, ce sont évidemment les phénomènes de coloration protectrice qui sont les plus remarquables; au repos, $90 \mathrm{c} / 0$ des 
Pap'llons sont tellement bien dissimulés sur le support qu'ils ont choisi. qu'il est extrêmement difficile de les apercevoir. L'exemple le plus parfait que l'on connaisse est celui des grands Nymphalidéz malais appartenant au genre Kallima, dont les ailes, colorées en dessous comme des feuilles, à diverśs degrés de dessiccation, sont en outre munies d'une forte nervure médiane. Lorsqu'un de ces heaux Lépidoptères est au repos,sur le côté d'une petite branche d'arbre, il est impossible de le distinguer des feuilles voisines, si l'on n'est pas prévenu.

Les Papillons à couleurs voyantes ne possèdent, ainsi qu'on devait s'y atiendre, que des moyens de défense chimiques: c'est un liquide âcre ou à odeur désagréable qu'ils émettent à rolonté, tels sont. par exemple, les Héliconides et les Danaïdes des régions tropicales; il parait que ces magnifiques Insectes n'essayent jamais de se cacher, néanmoins, ils ne sont jamais attaqués par les oiseaux insectivores.

Enfin, quelques Lépidoptères sont protégés par leur ressemblance avec des espèces bien défendues et bien armées, tel est, par exemple; la Sésie apiforme, qui mime si parfaitement le gros Frelon qu'on hésite toujours avant de la saisir avec les mains.

4o Diptères. - Chez les Diptères, le mịmétisme, quel qu'il soit, revêt surtout un caractère offensif ; la plupart des espèces mimantes sont, en effet, des parasites, ce qui leur permet d'approcher de leur hôte sans attirer son attention et sans l'effrryer.

Limites du mimétismo. - Il ne faudrait pas croire, à la vérité, que les phénomènes si remarquables du mimétisme et de l'homochromie, puissent suffire à expliquer toutes les particularités de la biologie des Insectes. Si leur rôle est utile et incontestable, il convient cependant de faire quelques réserves; l'hypothèse darwinienne de la sélection naturelle, si simple et si convaincante en apparence, n'a peut-être pas joué, dans ces phénomènes, le rôle dominant qu'on lui prête : il y a tant de hasards dans la vie des espèces qu'il est difficile de comprendre comment un avantage si mince peut conférer pour toujours, à une espèce donnée, une invulnérabilité presque absolue. 11. Charles Oberthür, l'éminent lépidop téologiste renna:s a égalemen faił, à ce sujet quelques remarques intéressantes: "Rarement, dit-ii, les ennemis naturels des espèces sont trompés par la ressembiance el. its savent très bien décourrir les êtres nécessaires j leur alimentation." 


\section{C.HAPITRE III}

\section{DISTRIBUTION CEOGRAPHIQUE DES INSECTES}

On a constaté, pour les Insectes, le mème phénomène que pour tous les autres animaux; les diverses régions de la terre présentent une faune qui les caractérise en quelque sorte aussi bien que les accidents géographiques.

Or, ce fait n'est pas dû au hasard; il en faut chercher la raison dans les modifications géologiques qui se sont accomplies à la surface du globe, plus encore que dans les conditions de température, de régime et de locomotion, que l'on considérait autréfois comme prépondélantes.

En ce qui concerne les Insectes remontant à l'époque primaire, et dont l'évolution était déjà, en grande partie, achevée à l'origine des temps secondaires, il faudrait, pour se rendre compte do leur distribution géographique, reconstituer les continents de l'époque paléozoïque.

Ainsi, par exemple, les Coléoptères, les Hémiptères hétéroptères, les Orthoptères eux-mèmes, volent mal el ne se servent pas volontiers de leurs ailes comme moven de locomotion ; on peut, sous un certain point de vue, les considérer connme des animaux terrestres. En revanche, la plupart des Névroptères, les lépidoptères, los Hyménoptères, los Diptères, sont lrunchement aćriens counme. les Oisuaux, et deviaient, ce semble, présenter le mème 
mode de distribution; il n'en est rien cependant et roici pourquoi.

La plupart de ces Insectes bons roiliers sont des animaux à métamorphoses; ils sont astreints à vivre, au moins dans la première phase de leur existence. c'est-à-dire sous la forme de larre, sur une plante déterminée. La propagation d'une espèce ne peut done se faire qu'en présence de la plante nourricière, ou, tout au plus, en présnnee " de plantes de la même famille qui peuvent la remplacer ». L'évolution de la faune entomologique est done le plus sourent, sous la dépendance immédiate de la flore, ce qui diminur considérablement au point de rue des migrations, l'a rantage que possèdent les Insectes aériens sur les Insectes terrestres.

Il est bien certain que les Lépidoptères, par exemple, dont les chenilles sont presque exclusircment régétariennes, emigrent moins facilement que les Libellules, yui se nourrissent d'Insectes, et dont les larres virent dans les eaux douees. Partout, en effet, où existe le moindre cours d'eau, les Libellules pourront fonder de nourelles colonies.

\section{T. IXSETES MALVAIS VOILIERS}

La distribution géograpbique des Insectes qui volent mal ou volent très peu, se fait d'arrè̀s les mêmes lois que celles des animaux terrestres. Sous ce rapporl, ce sont les Coléoptères qui sont les mieux connus, et tout ce qui va suivre s'appliquera presque exclusivement à cel ordre d'Insertes.

Les mœurs et le mode de nutrition sont pourtant très variés dans ce groupe ; à côté des Carabiques, qui sont surtout des Insectes marcheurs, nous trouvons les Dytiscides et les Hydrophilides qui vivent dans l'eall, tandis que certains Longicornes, comme les Leptura, "volent də fleur en flur et sont prezque aussi aériens que les Pa- 


\section{DISTRIBUTION GÉOGRAPHIQUE DES INSECTES}

pillons. "Dans certaines circonstances, les I.eptura peuvent donc ĉtre aussi transportés par le vent; il en résulte que, sous le rapport géonémique, les Carabiques et les Hydrocanthares, dont les larves sont d'ailleurs carnassières, pourront bien mieux servir à caractériser une région que les Lamellicornes, les Longicornes, et, d'une manière générale, que tous les Coléoptères dont les larves sont phytophages.

Il est vrai cependant que ces larves peuvent être transportées par les bois flottés ; mais, ainsi que le fait très judicieusement remarquer

REGION ARCTIQUE (P.UURE EN COLEOPTĖRS)

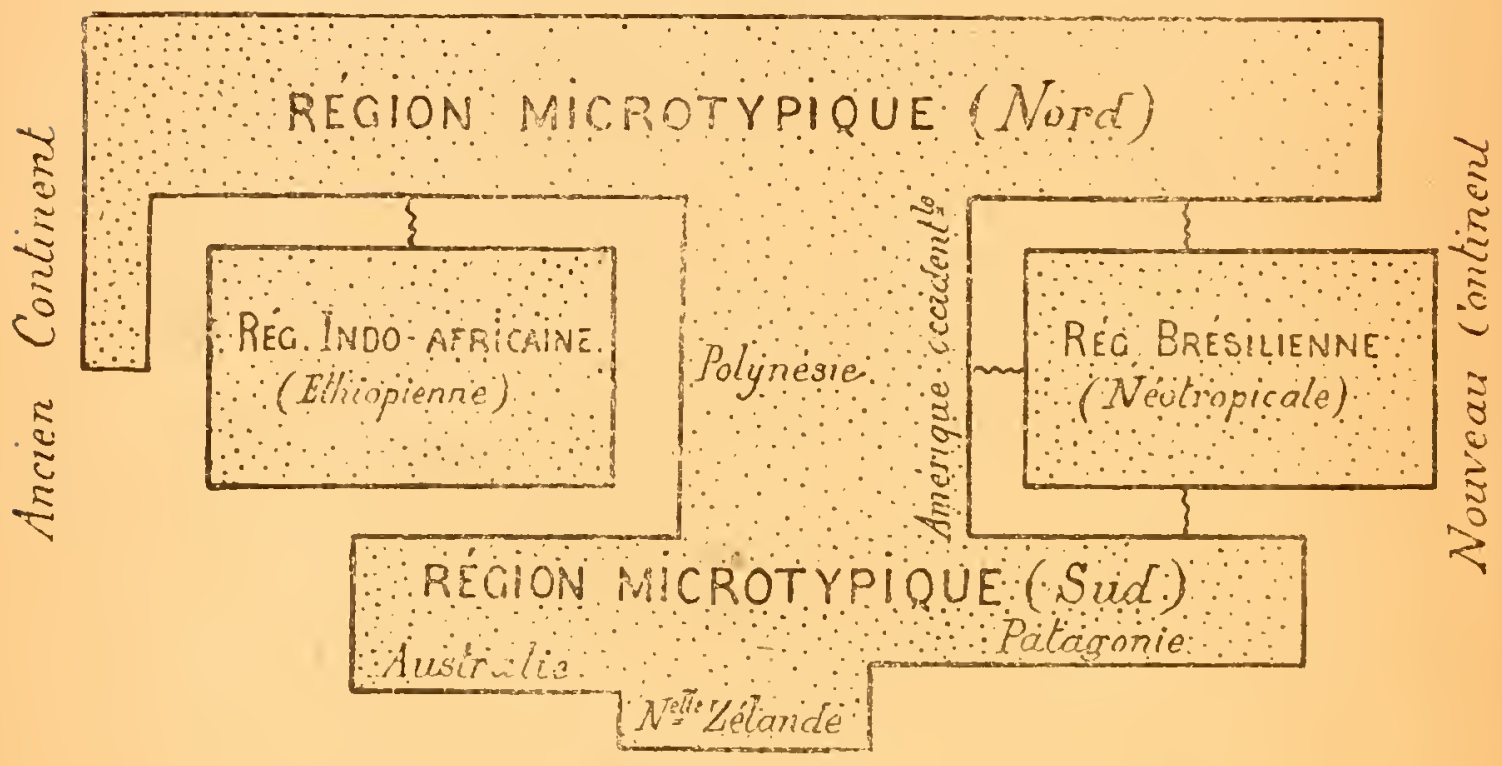

REGION ANTARCTIRUE (PAUVRE EN COLÉOPTĖRES)

Fig. 178. - Schéma des régions entomologiques terrestres (d’après E. L. Trouess.urT).

M. le Dr Trouessart, " nous ignorons encore jusqu'à quel point les larves, sorties des culs pondus par ces immigrants, ont pu s'accommod'r des essences for stières que leur fournissen! la nour elle patrie."

Quoi qu'il en soit, le naturaliste anglais $\Lambda$. Murray admet, pour les Coléoptères, trois grands centres primitifs de dispersion, qui correspondent sensiblement aux trois régions suivantes à la surface du globe :

$1^{\circ}$ Région indo-africaine ou éthiopienne.

$2^{\circ}$ Région brésilienne ou néotropicale.

$3^{\circ}$ Région microtypique, s'étendant très loin vers les deux pôles, et ainsi nommée à cause de la petite taille de ses représentants, comparés à ceux des deux autres régions. Le tableau qui précède 
(Fig. 178) permet de donner une notion assez précise des idées de Murray.

En examinant ce tableau, on est immédiatement frappé par l'analogie qui existe entre la faune de la zone tempérée australe et celle de la zone tempérée boréale. Ce fait, en opposition absolue arec les résultats fournis par l'étude des Vertébrés, nous améne encore à cette conclusion déjà formulée : c'est que l'évolution du type Coléoptère était, vraisemblablement, déjà achevée tout à fait au début de l'époque mésozoïque.

Il est certain que, pendant l'ère primaire, les zones climatériques étaient très étendues et peu différenciées ; les Insectes, trouvant partout les mêmes conditions d'existence, devaient avoir une répartition géographique très vaste et à peu près uniforme. Mais, lor'squ'au cours des temps secondaires, les mers jurassiques envahirent les régions équatoriales, elles isolèrent, dans les différentes régions du globe, des faunes localisées ayant cependant une origine et un fonds d'espèces communs.

Ces considérations, beaucoup mieux que la supposition d'une migration lente et progressive le long de la chaîne des Andes, permettent de comprendre pourquoi les genres Carabus, Asida, Helops, Opatrum, peuvent s'observer dans l'Amérique australe, alors que leurs représentants sont beaucoup moins nombreux dans l'Amérique du Nord.

Parmi les Coléoptères, la grande division des Carabidés peut être considérée comme caractéristique de la région paléarctique. Cette région possède, en effet, à elle seule, plus de $300 / 0$ des espèces connues, et c'est là qu'on rencontre exclusivement les types de grande taille, tels que Procerus et Procrustes.

Le genre Damaster est spécial à l'archipel du Japon; les genres Anthia et Graphupterus sont, en quelque sorte, particuliers à la région éthiopienne; Agra caractérise la région brésilienne: mais, déjà plus au sud, dans les Andes du Chili, se trouve le curieux genre Antarctia, qui représente, dans ces régions, les Amara de la faune paléarctique.

\section{INSECTES BONS VOILIERS}

Ainsi qu'on devait s'y attendre, la distribution géographique de ces Insectes présente une certaine analogie arec celle des Oiseaux. 
10 LÉPIDOPTÉRES. - Les renseignements suivants, empruntés à l'excellent ourrage de M. le Dr T rouessart, donneront une première indication sur les principaux centres de dispersion des Lépidoptères à la surface du globe.

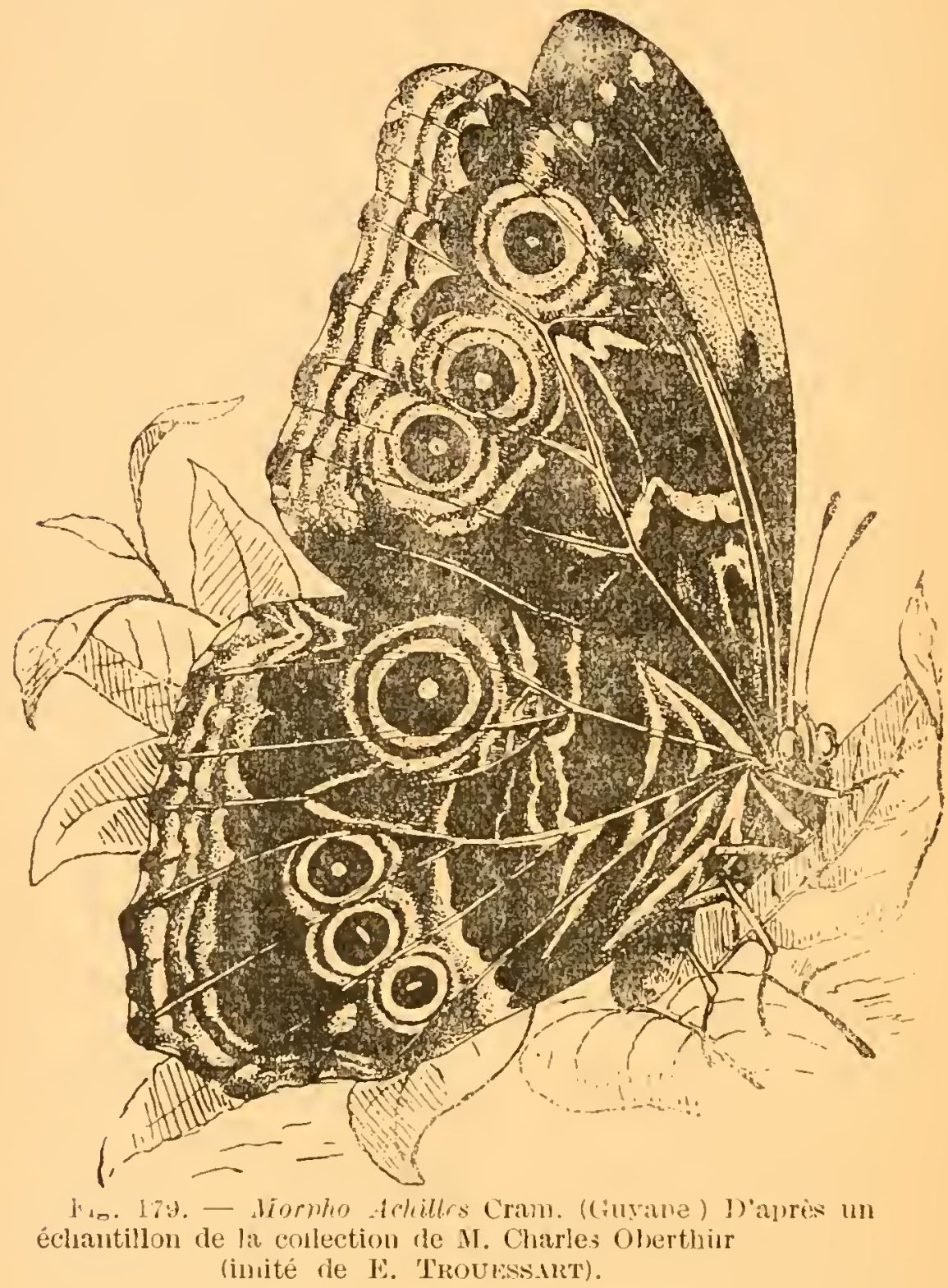

a. - La Région européenne est caractérisée par les genres Argynis, Melitoea, Lycœna, etc.

b. - La Région indienne paraît être la patrie des grands Ornithoptères et des Parnassiens.

c. - La Régrion américaine renferme, aver les genres P'apilio, des Héliconides et des Nymphalides de grande taille (Fìn. 179). 
Bien entendu, ces diverses régions empiètent souvent les unes sur les autres, et il est absolument impossible d'étahlir entre elles une limite précise.

2'Névroptéres. - Les Odonates, dont le rol est très puissant, se rencontrent dans presque toutes les parties du monde; toutefois, les renres Libellula et Fschna paraissent manquer dans les îles de la Polynésie et à la Nouvelle-Zélande, où ils sont représentés par les Cordulines. En revanche, les A arionides sont absolument cosmopolites.

3. Orthoptére๔. - N'après M. I. Bolivar, le savant professeur de l'Université de Madrid, si on laisse de côté les espèces émigrantes (Pachytilus, Acritium), qui sont seules capables d'un vol soutenu, la Jistribution géographique des Orthoptères aurait les plus grandes analogies avec celle des Coléoptìres. Il y a, en effet, dans ce groupe, des genres à allure lente, tels que les Mantes, les Phasmes, qui ne se déplacent pas plus que les Insectes terrestres les plus franchement :édentaires.

L'étude des antres familles nous permettrait de faire des constatations identiques, mais ce sujet sera traité arec tous les développəments qu'il comporte, dansles volumes spéciaux de cette collectior. 


\section{CHAPITRE IV}

\section{ROIE ÉCONOMIQUE DES INSECTES}

De tous temps, les animaux ont été associés aux divers épisodes de la vie de l'homme et on les voit fréquemment apparaitre dans les légendes qui constituent la primitive histoire des nations les plus anciennes.

\section{Io LES INSECTES ET LES RELIGIONS ANTIQUES}

De tous les Insecles, celui qui a joué le plus grand rôle dans l'antiquité est le Scarabée sacré (Ateuchus sacer), que les Égyp-

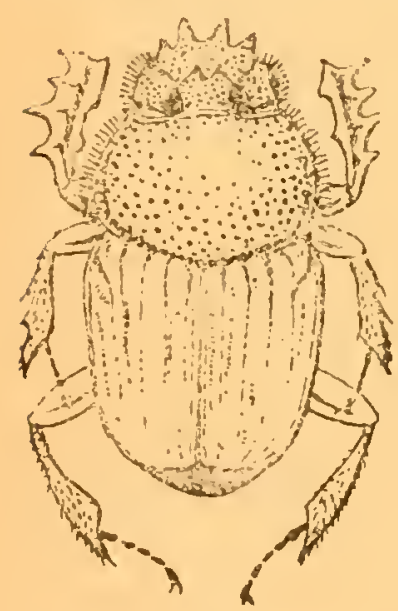

Fig. 180. - Je Scarabèe sacrẻ (.Leuchus stacer), Coléontère de la famille des Lamell!cornes (d'ap. uat.) tiens vénéraient, et qu'ils ont représenté sous les aspects les plus variéc (Fig. 180).

Ce cros Coléoptère, de conleur noire, que l'on rancontre çà et là dane le Midi de la France, appartient ì la famille des Lamellicornes; son corps arrondi et convexe mesure I rois centimètres de longueur environ; la tête élargie porte généralement deux tubercules et présente, en avant, six dents plus ou moins développées.

On rencontre toujours le Scarabée dans les endroits chauds et sablonneux; il recherclic les excréments des herbivores pour confectionner les curieuses boules dont il fait provision pour les temps de disette, et qu'an prix des plus grands efrorts, il roule avec ses pattes de derrière jusqu'au terrier qu'il s'est prépare (1) (F'is. 181).

(1) D'oin les noms de Roule-boule et de Pilulaires, yu'on donne encore aux Coléoptères qui. comme le Searabèe. composent leurs boules avec des matières stercoraires 
Tant de particularités bizarres devaient attirer l'attention. "Messagers du printemps, dit Latreille (1), annonçant par leur reproduction le renouvellement de la nature; remarquables par le singulier instinct qui leur apprend ̀̀ réunir les excréments en manière de corps sphériques qui doivent renfermer les germes de leur race, occupés sans cesse, comme le Sisyphe de la mythologie, à faire rouler ces corps, ces animaux parurent offrir aux prêtres égyptiens l'emblème des tra-

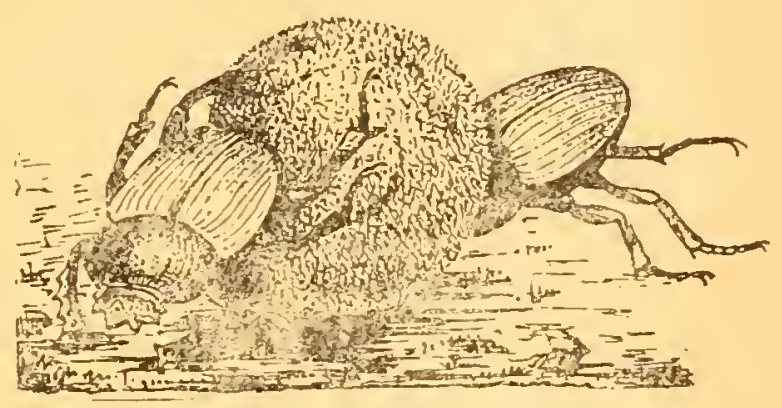

Fig. 181. - Deux Scarabées roulant leur houle. vaux d'Osiris et du Soleil."

Comme ces Insectes ne diffèrent presque pas entre eux, et que, mâle et femelle prennent une part égale aux travaux nécessaires à la conservation de la race, les Égyptiens, les croyant tous du sexe masculin, imaginaient, pour le Scarabée, la génération spontanée.

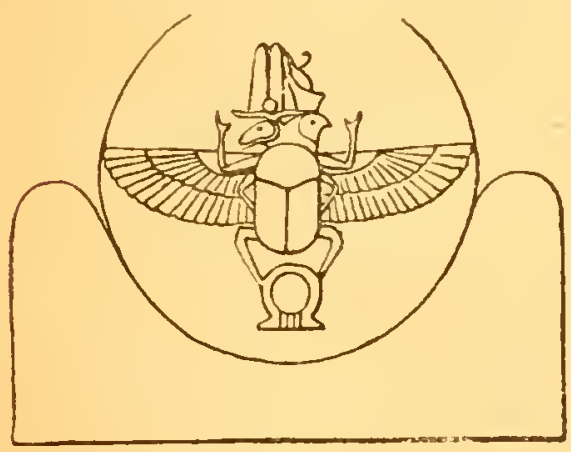

Fig. 182. - Motif ornant la frise du grand temple d'Edfou (d'après H. Boussac. Le Faturaliste, p. 150.) "Il n'est ni engendré ni porté dans lo corps d'une femelle, dit Hor-Apollon; lorsqu'il veut procréer, il prend de la fiente de bosuf et lui donne la forme du monde; il la roule avec ses pattes de derrière, du levant au couchant, et se retourne ensuite vers le levant. Il dépose ensuite sa boule dans la terre oì elle reste pendant 28 jours, temps pendant lequel la race des Scarabées prend la vie. Le 29 e jour il ouvre sa boule et la jette dans l'eau, les animaux en sortent, ce sont. les Scarabées. "

Les Égyptiens disaient encore que le Scarabée a trente doigts, représentant les 30 jours du mois ; ils entendaient par là les articles des tarses; mais ici, l'observation n'est pas exacte, attendu que les Ateuchus (Fig. 180) n'ont jamais de tarses aux pattes antérieures.

Les Égyptiens vénéraient, en réalité, quatre espèces distinctes de

(1) Latreille (P.A.).-2.T. V.p. 251. 
Coléoptères coprophages, l'Ateuchus sacré, l'A. égyptien, d'un vert doré magnifique ; un Géntrupe (Scarabée bicorne) et un Copris, ces deux derniers, il est vrai, présentent des tarses de cinq articles à tous les tarses.

Les anciens attribuaient également au Scaraliée pilulaire un grand

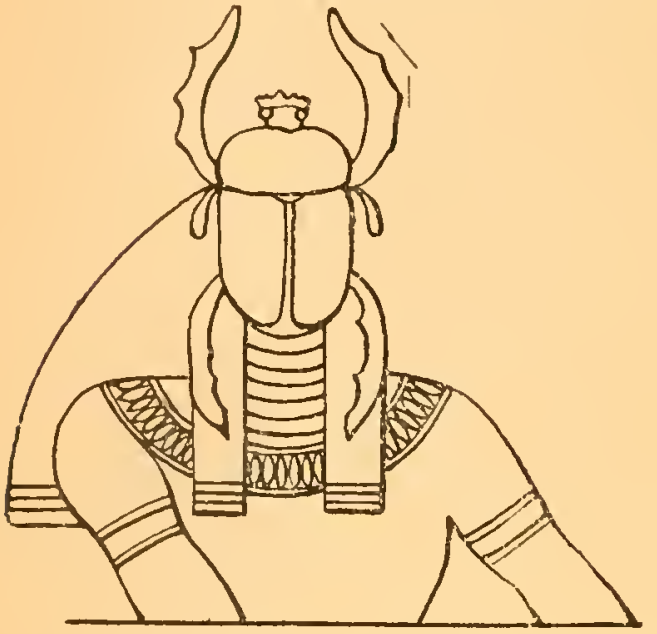

Fig. 183. - La tête du dieu Khépra (d'après H. Boussac). nombre de propriétés médicinales. Pline recommande notamment, contre les maux d'oreilles, de placer sur le corps, soit comme amulette, soit à l'état d'onguent, l'un de ces Insectes. De nos jours, parait-il, ce remède est encore en usage; et, dans la Haute-Égypte, les mères suspendent, au cou de leurs enfants, un Scarabée vivant, enveloppé dans un petit sac de toile (1).

Les légendes concernant les Insectes sont tellement nombreuses qu'il nous est impossible de les rapporter toutes ici ; d'ailleurs, aucun autre Insecte n'a jamais joué, dans lia vie politique et religieuse d'une nation, un rôle aussi important que le Scarabée sacré dans l'ancienne Égypte (Fig. 182 et 183).

\section{I1 ${ }^{\circ}$ INSECTES OBJETS DE LUXE ET DE PARURE}

De tout temps, les Insectes ont fourni à la bijouterie des modèles nombreux et variés ; très souvent même on est parvenu à les imiter avec une grande perfection.

Mais la mode, capricieuse, ne s'est pas toujours contentée de ces imitations plus ou moins heureuses. Il était du meilleur ton, il y a une soixantaine d'années, d'employer les Insectes aux reflets métalliques pour rehausser l'éclat des coiffures de bal; cette mode ne s'est pas généralisée à cause des dépenses considérables q'1'elle occasionnait. On employait alors, en effet, quelquefois, ces magnifiques papillons bleus de la Guyane: Morpho Rcthenor ou M. Cypris,

(1) H. Bouss 1 c. - Ie Scarabée sacré. - Le Nituraliste, 1905, 1). 136-149 
dont un seul échantillon ne valait pas moins de 250 francs. Les commerçants naturalistes de cette époque étaient assez hahiles pour donner à ces coûteux hochets un peu de solidité, en étendant sur les ailes un vernis transparent à la gomme laque. "Dans cet état, dit le Dr Boisduval, s'il ne lui arrivait, aucun accident, il pouvait servir pour deux ou trois soirées."

D'autres Papillons du Brésil, plus communs, et par conséquent moins coûteux, étaient également recherchés, les Chlorippe Laurentia et cyanea, notamment, eurent leurs heures de grande faveur; enfin un Lycænide indigène très élégant, le Polyommate de la Verge d'Or (Polyommatus Virgaurece), n'était pas non plus dédaigné.

En même temps que les Papillons, les Coléoptères eurent aussi très souvent les honneurs de la mode. Une sorte de petit Hanneton aux élytres bleus, Hoplia ccrulea, commun dans le centre de la France, se vend quelquefois par millier's; mais les brillantes espèces exotiques, tolles que Chrysochroa divittata, Julodis equisignata d'Asie, sont toujours plus recherchées malgré

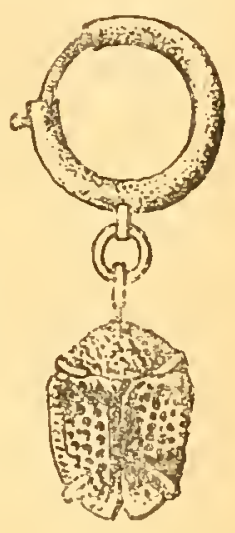

Fig. 184. Casside sertie dans un cadre métallique et formant pendeloque. leur prix élevé.

Une belle espèce de Curculionide américain, Prœpocies resalus, ainsi qu'une Casside, (Desmánota variolosa), grâce à leurs téguments très solides, sont fréquemment montés en broches, en boutons et en épingles de cravates (Fig. 184). Les Indiens de la région du RioNapo confectionnent encore d'élégantes pendeloques avec les grands Buprestes américains on même seulement aver. leurs élytres.

\section{INSECTES UTILES A L'ALIMENTATION}

Au point de vue de l'alimentation, cela est hors de doute, i'homme dit civilisé ne tire aucun parti des Insectes. Sous ce rapport, les anciens étaient plus pratiques que nous; il en est de même encore, actueilement d'un certain nombre de peuplades, que nous considérons comme sauvages, et chez lesquelles les linsecips, ou leurs larves, sont fort en honnolir au point de vue. culinaire.

F.t de fait, pourquoi un Inserte serait-il inférieur à un Crabe? lls 
appartiennent, tous les deux an groupe des $\Lambda$ rthropodes et, incontestablement, le régime ordinairement végétarien de l'Insecte est toujours beaucoup plus "raffiné " que celıi du Crabe.

Il n'y a donc là qu'une question de préjugé, qui se dissipera sans doute un jour. Il y a plus de cinquante ans, un savant médecin Irançais, le Dr Virej, membre de l'Institut, après avnir étudié scientifiquement la question, arrirait dejà nettement à rette conclusion : "L'homme peut manger des Insectes; rien dans son orgarusation anatomique ni dans ses fonctions ne s'y oppose. "

Dans presque tous les groupes d'Insectes se trouvent des espèces qui peuvent être utilisées au point de vue alimentaire.

10 Manne. - Les légendes bibliques rapportent que, dans leur fuite à travers le désert, les Israélites vivaient d'une sorte de manne "tombée du ciel ". Or, cette substance n'était autre chose qu'un. exsudation sucrée, produite par le Tamarix mannifera, à la suite de la piquîre d'un hémiptère (Chermes manniparus). Cette substance, fluide pendant le jour, à cause de la chaleur du soleil, tombait en gouttelettes sur le sable. Flle se solidifiait pendant la nuit, ce qui explique pourquoi " elle apparaissait le matin comme la rosée, sous forme de grains menus, semblables à de la gelée blanche. "Chaque Israélite avait le droit d'en recueillir un gomor (environ 2 litres), car " elle disparaissait aussitôt que le sol était échauffé par les rayons du soleil. "(Moïse, Exode, XVI).

Le mêmo Tamarix, le même Kermès, existent toujours en Arabie ; la sécrétion produite par la piqûre de l'Insecte se produit toujours comme au temps de Moïse, mais les habitants, mieux pourvus d'aliments, n'en font plus aucun usage aujourd'hui.

20 Orthoptères - De toute antiquité, les Orthoptères ont joué un rôle important dans l'alimentation des peuples de l'Orient. Moise permettait aux Hébreux de manger quatre espèces de Sauterelles : Arbé, Solam, Chagal et Slargol (Lévitique, I, chap. 21). Voici les caractères qu'il leur assignait : "Slargol a une bosse et une queue; Chagal, une bosse et pas de queue ; Solam, une queue, pas de bosse ; Arbé, ni queue ni bosse ". On reconnaît là, très nettement, quelquesuns des plus grands Pamphagides syriens; quant à la queue, c'est purement et simplement l'oviscapte des femelles.

Les anciens Grecs, si raffinés en toutes choses, ne dédaignèrent pas d'utiliser les Orthoptères. Aristophane nous apprend que les Béotiens apportaient, au marché d'Athènes, des Sauterelles qui figu- 
raient ensuite avec honneur sur les tables; un auteur grec a même composé un traité de l'acridophagie. D'après la lecture de cet ourrage, II. Daguin croit pouvoir affirmer que " les Arabes, les Lybiens, les Mauritaniens, les Éthiopiens, les Abyssins, etc., qui, de nos jours, sont encore de grands amateurs de Sauterelles ", peuvent être considérés comme les descendants directs des acridophages de l'antiquité.

Voici, d'après Diodore de Sicile, le procédéqui était usité pour la préparation des Sauterelles chez les Éthiopiens : "Comme leur" pays est riche en sel, ils en saupoudrent les Sauterelles, tant pour les rendre plus savoureuses, que pour les conserver plus longtemps, jusqu'au retour de la saison qui en ramène d'autres. "

Actuellement, en Palestine, on frit les Sauterelles dans l'huile de sésame ; dans l'Arabie Pétrée, on en fait une farine après les avoir desséchées au soleil; en Abyssinie, on les grille légèrement. Les Arabes d'Algérie les préparent comme nous le faisons pour les crevettes, en les faisant simplement bouillir dans l'eau salée; ils recueillent et préparent les Criquets, non seu'ement pour leur usá," personnel, mais pour en faire le commerce. Au siècle dernier, ¿̀ Bagdad (Perse), on apportait les Sauterelles par sacs, sur le marché, comme des céréales.

Beaucoup de voyageurs se sont trouvés à l'occasion d'éprouver le goût des Sauterelles; les avis sont partagés, cela se conçoit; mais si quelques-uns les apprécient sans enthousiasme, aucun du moins n'en parle aveć répugnance.

L'acridophagie se pratique même en France; je ne sais plus oì l'un des plus savants professeurs du Muséum de Paris, M. le Dr Trouessart, raconte que l'une de ses plus grandes surprises, étant enfant, fut de voir, à Poitiers, "des gamins faisant l'école buissonnière " cloquer des Sauterelles après leur avoir arraché les grandes pattes de derrière.

$3^{0}$ Hémiptères. - Les Hémiptères fournissent peu d'éléments à l'art culinaire ; toutefois, concurremment avec les Sauterelles, les Grecs mangeaient aussi les Cigales (Cicada plebeja). Aussi, le compilateur Elien s'indigne-t-il " contre ces Orientaux qui, quoique pourvus de toutes sortes de nourritures exquises, broyaient sous leurs mâchoires ces célestes bestioles consacrées aux Muses ».

Actuellement encore, au Laos, le long des rives du Mékong, les femmes capturent des Cigales à la glu, soit pour leur nourriture, 
soit pour les vendre au marché. Enfin, au Mexique, on recueille les $œ u f s$ de certains Hémiptères aquatiques (Corixa et Notonecta); ces œufs séchés, et assez semblables à des grains de semoule, servent alors à confectionner des gâteaux et des potages.

40 Diptères. - Au dire de Livingtsone, les nègres qui habitent les rivages septentrionaux du lac Nyassa, recueillent les moucherons qui voltigent par myriade pendant la nuit, dans la belle saison. Ils confectionnent, avec ces Insectes bouillis, de grosses galettes dont ils sont très friands et dont le goût. paraît-il, rappelle celui du caviar.

$5^{\circ}$ Hyménoptères. - "A l'état parfait, dit M. Daguin, les Hyménoptères sont peu musclés et fort coriaces; aussi sont-ils délaissés nar les gastronomes" qui plutôt s'adressent aux larves. C'est ainsi qu'à l'île de Timor, les indigènes mangent, comme un mets délicieux, les larves d'Abeilles ; il en est de même, paraît-il, aux îles Bahama et à la Guyane pour les chrysalides et les larves des Guêpes sauvages.

Au Mexique, d'après M. Ernest André, le savant historien des Hyménoptères, les Indiens recherchent les nids de la Fourmi à miel (1) et ils sucent l'abdomen de cet Insecte avec délices.

fo Névroptères. - Plusieurs voyageurs, notamment de Humboldt, dans ses Relations historiques (2), rapportent que les galibis de la Guyane recueillent, au printemps et à l'automne, de grosses Fourmis rolantes, dont l'abdomen est une sorte de sac, renfermant avec les œufs "la liqueur nourricière des larves ". Après avoir fait nne ample provision de ces animaux, ils les cuisent dans un vase en y ajoutant de la farine ou de la graisse; ils obtiennent ainsi un mets qu'ils désignent sous le nom de Kamaka, et qui est, paraìt-il, très agréable.

La même coutume se retrouve au Brésil, où les Fourmis sont même l'objet d'un commerce très florissant ; ạprès les avoir torréfiées comme des grains de café, les femmes indigènes les colportent dans les rues en criant " va iça!" ce qui veut dire, pour manger! On verid aussi des préparations de qualités différentes, mais les plus recherchées sont les grosses femelles, dont l'adbomen est gonflé par les œufs.

D'après l'opinion de Latreille, et il ne saurait y avoir' de doute à ce sujet, les espèces qui font l'objet des deux relations précédentes

(1) Myrmecocystus melliger.

(2) La Nature, année 1888, t. I. 1. 221. 
ne sont pas de vraies Fourmis, mais bien des Termites, Insectes sociaux de la famille des Névroptères. D'ailleurs, aux Indes, dans l'Amérique du Sud et en Afrique, où ils sont abondants, les vrais 'Termites constituent un gibier courant. A Java, on les vend sur les marchés d'un grand nombre de villes.

70 Coléoptères. - Certaines larves de Coléoptères ont joui, à juste titre, d'une célébrité considérable. Qui ne connaît le fameux Cossus des Romains?

Les Cossus, au dire de Pline, sont de gros vers blancs qui vivent dans l'intérieur des chênes, dont ils dévorent le bois. Les gourmets considèrent ces larves comme une nourriture de luxe, dont le goût est exquis, surtout lorsqu'elles ont été "engraissées avec de la farine ». Il ne saurait être question ici du Cossus ligniperda, gros Lépidoptère nocturne, dont la larve vit aussi dans l'intérieur des arbres; Pline ajoute, en effet, que le Cossus avait des cornes (antennes) et qu'il faisait entendre un petit bruit strident: "Omnes tamen in cerastem figurantur sonumque edunt parsuli stridoris (1)."

Ainsi donc, le Cossus des Romains n'était autre que la larve du Grand-Capricorne (Cerambyx heros) (Fig. 185).

De même, les larves des gros Prioniens (Acanthophore serraticorne), Coléoptères appartenant à la même

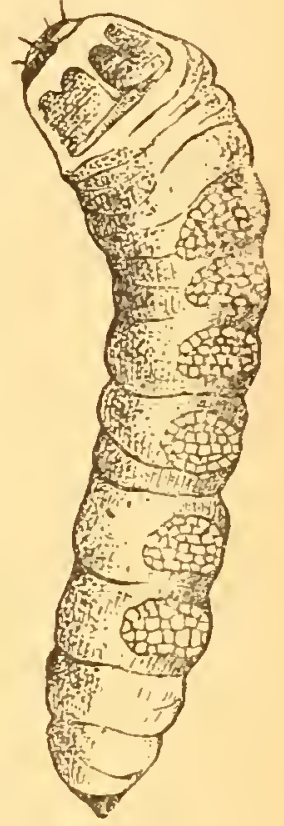

Fig. 185. Larve du GrandCapricorne (Ce rambyx heros).

Col. famille que le Capricorne, constituent, paraît-i], encore de nos jours, un mets très recherché au Brésil et à la Guyane. Auguste Saint-Hilaire, qui a eu la curiosité de se renseiginer par lui-même sur le goût de ces larves, assure que leur saveur "rappelle celle de la crème la plus délicate ". Mile Sybille de Mérian, dans son célèbre Voyage à Surinam, indique que les indigènes de la Guyane mangent avec délices les larves du Cerambyx cervicornis L., du Passalus irterruptus L. et le "Ver palmiste " (Curculio palmarum L.)

Mêmes faits en Australie, constatés par Lumholtz, de 1880 à 1884. Les noirs recueillent des larves de longicornes et de lépidoptères dans

(1) Tous (ces vers) se transforment en un Ceraste (Capricnrne) et font entendre une petite stridulation. 
le tronc des arbres tombés; il les cuisent, de la façon la plus simple, en les jetant dans la braise où elles deviennent croquantes en un clin d'œil ; ce mets, dit l'auteur, est supérieur à l'omelette de nos pays.

Enfin, il n'est pas jusqu'au vulgaire Hanneton qui n'ait eu son jour de célébrité culinaire. En 1897, M. de Fonvielle, vice-président de la Société d'insectologie de Paris, indiqua que "le meilleur moyen de se venger de ce ravageur de nos cultures "était de le manger; au point de vue gastronomique, le Hanneton - préparé à la sauce rrevette - constituerait, paraît-il, un plat irréprochable.

go Lépidontères. - Les larves des Lépidoptères, malgré les poils dont elles sont souvent recouvertes, sont utilisées pour la nourriture en certains pays. Au Natal, notamment, les indigènes sont très friands d'une grosse chenille velue qui vit en familles nombreuses sur une espèce de Mimosa; ils font rôtir la chenille pour griller les poils et la mangent ensuite avec tous les signes de la plus grande satisfaction.

Le sustillo des Péruviens est également une chenille très recherchée.

Les Chinois eux-mêmes ne rejettent pas toujours les chrysalides du Ver à soie dont ils ont dévidé le cocon; il les pralinent dans. du sucre, pour assurer mieux leur conservation, et les servent ensuite sur leurs tables comme des dragées.

Enfin Boisduval raconte que, dans les villes de Colombie, où la civilisation est aussi avancée qu'en Europe, on vend souvent, notamment sur les marchés de Bogota, par petites mesures, comme des noisettes ou des châtaignes, les chrysalides d'une grande Hespérie, dont la chenille vit sur les Acarias.

On ferait un volume entier sur l'utilisation des Insectes dans l'alimentation, mais il nous est impossible de nous étendre davantage sur cette intéressante question. Avec M. E. Daguin, auquel nous avons emprunté la plupart des détails qui précèdent (1), nous terminerons par cette pensée profonde de Rrillat-Savarin : "Celui qui invente un plat nouveau fait plus pour le bonheur de ses semblables que tous les philosophes, les littérateurs, les savants et les politiques réunis!."

\section{INSECTES UTILISES LN MEDECINE}

Depuis lez temps les plus reculés, la médecine utilise les Insectes, tantôt pour les substances actives qu'ils renferment (Cantharides),

(1) Daguin (H). - Le Nrturaliste, 1900, $n^{09} 285-290$. 
tantôt pour les produits qu'ils fabriquent (A beilles, Vers à soie, Cochenille, etc.). Trcis familles, celles des Coléoptères, des Hyménoptères et des Hémiptères, fournissent des espèces utilisables au point de vue médicinal.

10 Coléoptères. - Les Méloïdes seuls, appartenant à la grande sub. division des Hétéromères, sont employés à cause de leurs propriétés vésicantes. La plupart de ces Insectes contiennent, en effet, dans leur test, et surtout dans leurs organes génitaux, une substance active, âcre, extrêmement toxique, la cantharidine, ayant la propriété de déterminer une vésication rapide lorsqu'elle est mise en contact avec la peau.

L'espèce la plus commune en France est la Cantharide, à vésicatoires (Lytta resicatoria), bel Insecte cle quinze à vingt-cinq m:llimètres de long, aux élytres mous, d'un vert doré très brillant (Fig. 186).

Les Cantharides se rencontrent en juin et juillet sur les Fresnes, les Lilas et les Troènes, dont elles

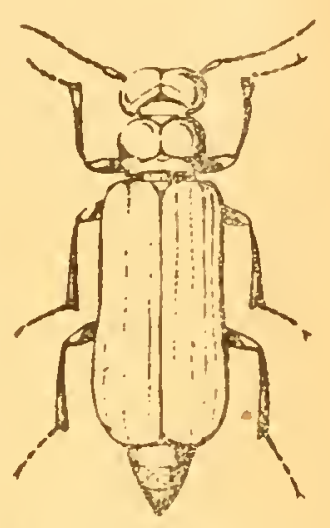

Fig. 186. - Cantharide officinale (Lutta resicatoria) Col. (d'apr. nat.) rongent les femilles avec avidité ; elles décèlent leur présence par une odeur vive et très désagréable.

La pharmacie utilise surtout la poudre de cantharides, obtenue en pulvérisant les Insectes préalablement desséchés ; cette poudre entre ensuite dans un certain nombre de préparations, telles que les teintures alcooliques et éthérées de Cantharides, l'huile de Can-

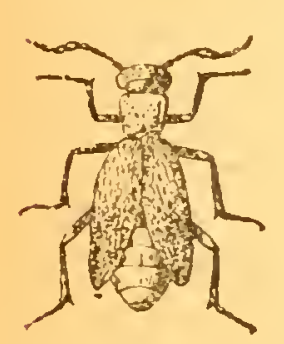

Fig. 18: Le Melo prin. tanir. $r$ insecte résicant Col.

(d'ap. nat.) tharides, les pommades épispastiques, l'emplâtre iésicatoire, les mouches de Milan, etc., etc.

On récolte peu les Cantharides en France, hien qu'elles y soient communes et que le commerce les cote actuellement, suivant la provenance, entre $5 n \mathrm{fr}$. et $100 \mathrm{fr}$. le lilog. Celles que l'on trouve en pharmacie viennent généralement d'Espagne, d'I talie, de Chinc ou de Russie.

Les autres Méloïdes, appartenant aux genres Cerocoma, Mylabris, Meloë, Epicauta, possèdentles mêmes propriétés que la Cantharide et sont employés aux mêmes usages dans divers pays (Fig. 187).

Il est inutile, je pense, de rapporter ici, avec détails, toutes les légendes qui avaient cours au siècle dernier, relativement aux propriétés thérapeutiques des Insectes; tout au plus peut-on indiquer 
à titre de simple curiosité historique, que les punaises guérissaient, de la fièvre intermittente; les mouches, écrasées et appliquées sur la tête, avaient la propriété de faire repousser les cheveux; la poudre de perce-oreille, mélangée à l'urine de lièvre, était souveraine contre la surdité, etc., etc.

Nos connaissances actuelles ne nous permettent pas de croire davantage aux propriétés antirabiques de la Cétoine dorée, préconisée contre la rage au milieu du siècle dernier, et qui, au dire d'un savant médecin (!), aurait donné d'excellents résultats dans la Russie méridionale.

Comme tant d'autres, ces remèdes ont fait leur temps ; nos pères en ont profité, pendant qu'ils possédaient la propriété de guérir!

2r Hyménoptères (Miel et Cire). - Les Abeilles fournissent, à la máttière médicale, leur miel et la cire.

L'importance du miel a considérablement diminué depuis la découverte du sucre, cependant, on l'utilise encore pour un certain nombre de préparations officinales (mellites), telles que le liiel rosat, le Sirop de longue vie, etc.

Il servait autrefois, et sert encore dans certains pays (Bretagne) pour la fabrication de l'hydromel.

La cire est un produit de sécrétion naturul fourni par les Abeilles: on se contente de la purifier pour les usages médicinaux et elle entre exclusivement dans les préparations pour l'usage externe : emplâtres, cérats, onguents, et quelques pommades.

Ses usages industriels sont beaucoup plus importants.

$3^{\circ}$ Hémipteres. - Les diverses substances désignées sous le nom de laques sont produites par la piqûre de certaines Cochenilles, vivant sur les plantes, à la manière des Pucerons (voir p. 195).

Enfin, parmi les Insectes eux-mêmes, utilisés quelquefois, on peut citer la Cochenille (Coccus cacti) qui vit sur le Cactus Nopal ; le Kiermès du Chêne vert (Chermes ilicis), employé autrefois pour la préparation d'un électuaire (Confection Alicrmes), et qui ne sert plus, de nos jours, qu'à colorer la fameuse liqueur d'Alkermes, fabriquée suriout à Florence par les moines du couvent de Sainte-Marie-Nouvelle.

\section{Vo INSECTES UTILES 1 L'INDUSTRIL}

De tous les produits fournis par les Insectes, le plus important, sans contredit, est la soie fabriquée par un Lépidoptère noclurne 
d'origine asiatique, le Bombyx du Mûrier. La préparation et le tissage de la soie étaient pratiqués, par les Chinois, plus de 5.000 ans avant notre ère ; aujourd'hui cette industrie est répandue dans tous les pays, mais cependant la France tient toujours le premier rang; Lyon est le centre de production le plus important du monde entier.

Ver a sole. - Sous l'influence de l'élevage, le Ver à soie s'est profondément modifié ; il a notamment perdu la faculté de voler ; il se contente de remuer les ailes. La chenille, très poilue au moment de l'éclosion, comme celle de tous les Bombycidés, perd ses poils et devient blanche (Fig. 188).

Lorsque l'accouplement a eu lieu, on fait pondre la femelle sur de petits carrés d'étoffe, et les oufs, ainsi fixés, sont livrés au commerce sous le nom de graine de Ver à soie; il faut environ 1.200 de ces œufs pour donner le poids de 1 gramme.

En France et en Italie, le Ver à soie ne donne généralement qu'une

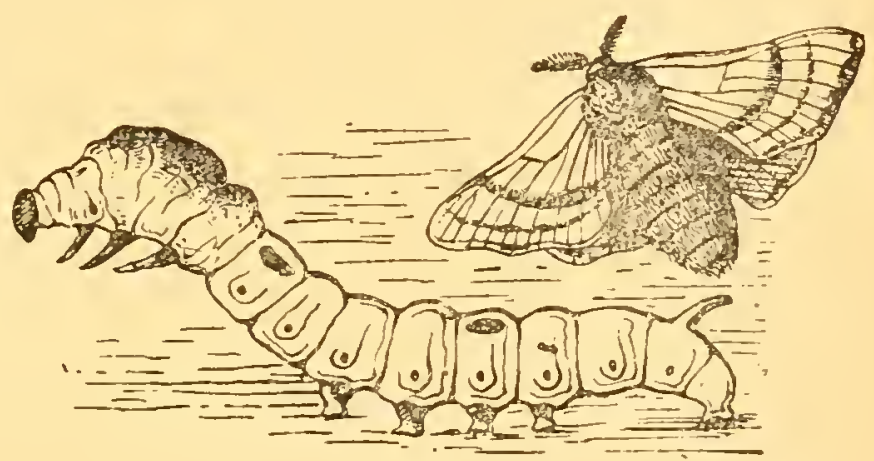

Fig. 18.. - Le Ver a sole (ぶ ricaria mori) et sa chenille.

seule génération par an, on dit que la race est univoltine; mais, dans son pays d'origine, en Chine et au Japon, il peut donner deux et même trois générations, on a alors les races bicultines et polycoltine: (1).

Dans les races françaises univoltines, on doit retarder l'éclosion des œufs jusqu’à l'épaque oì le Mûrier développe ses feuilles; à cet effet, on prolonge la durée d'hibernation à l'aide d'appareils réfrigérants où les cuîs sont maintenus à la température de - 20. Inversement, pour obtenir l'éclosion à l'époque la plus favorable, on emploie des chambres d'incubation où la température est mainte. nue aux ensirons de 19?.

La durée normale de l'évolution du Ver à soie, depuis l'éclosion

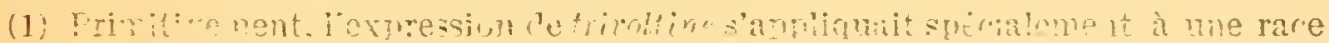

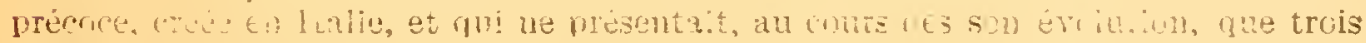

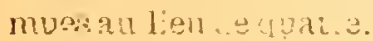


jusqu'à la montọe (1), est de 32 à 38 jours, mais on est arrivé à l'abaisser à 20 en se servant d'étuver.

Line once de graine $(30$ grammes) donne en iron 30.000 chenilles, quin'absorbent pas moins de 350 kilogr. de feuilles de Mûrif r pa: jour.

La scic. - Pour dévider les cocons, on les jette dans l'eau chauff́́e à $70^{\circ}$ ou $80^{\circ}$; l'eau dissout la substance collante qui agglutine les fils ensemble (voir p. 134); ensuite, après avoir réuni ces fils par cinq ou six, on les enroule sur un petit dévidoir.

Chaque cocon, pesant o gr. 1 environ, est formé d'un seul fil de soie dont la longueur peut atteindre 1.000 mètres; mais, au dévidage, on n'obtient guère, en réalití, comme soie marchande, qu'un fil de 280 à 300 mètres.

Lo jaune d'or était la couleur primitive des cocons du Ver à soie ; les couleurs blanches ou verdâtres, si répandues aujourd'hui, représenteraient des phénomènes d'albinisme, analogues à ceux qui ont atteint la chenille, el seraient le résultat de la domestication. Il e t possible qu'il $y$ ait eu, au début, deux espèces très roisines, l'une à soie jaune, l'autre à soie blanche, confondues aujourd'hui en une

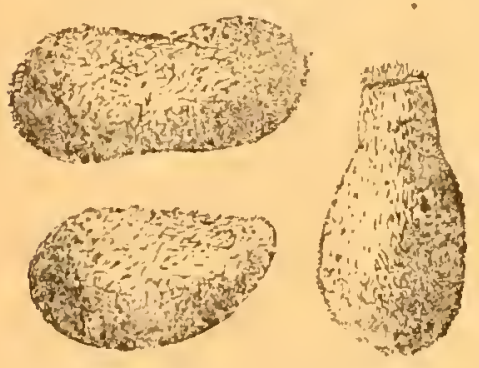

Fig. 1:9. - Diverass fornes de Cocons suivant les races. seule àlasuite de nombreux et très anciens croisements.

Les cocons isolés se vendaient, avinul la guerra, selon la beauté, de 3 francs à $3 \mathrm{fr} .50$ le kilog.

LES LÉPIDOPTÉRES SERRIGIGÉYIS.-Cln connaît, en France, quatre races de V'er's à soie qui se distinguent les unes des autres par la forme des cocons et par la couleur de la soie.

a. La race du Var, donnant un cocoll jaune pâle, renflé à ses deux extrémités (Fig. 189).

b. La race des Pyrénées, donnant un cocon jaune, pointu à l'une de ses extrémités.

c. La race des Alpes.

a. La race des Cévennes, dont les cocons sont jaunes ou blanes.

En plus du Bombyx du Mûrier, on connait encore une quinzane ('espèces, dont lej chenilles peuvent fournir de la soie utilisable, voici les principales:

(1) Lipoque ou la dhenille monte il l'extrénite des branches de bruyere pour filer son rocon. 
Saturnia Cynthia (Bombyx de l'Ailante) originaire du Japon; la chenille vit sur le Vernis du Japon et s'est très bien acclimatée en France. Elle donne une soie grossière que l'on a essayé d'utiliser pour la fabrication des cordages.

Bombyx Yama-mai (Chine).

Saturnia Mylitta (Ver à soie du Chêne) donne la soie qui sert à

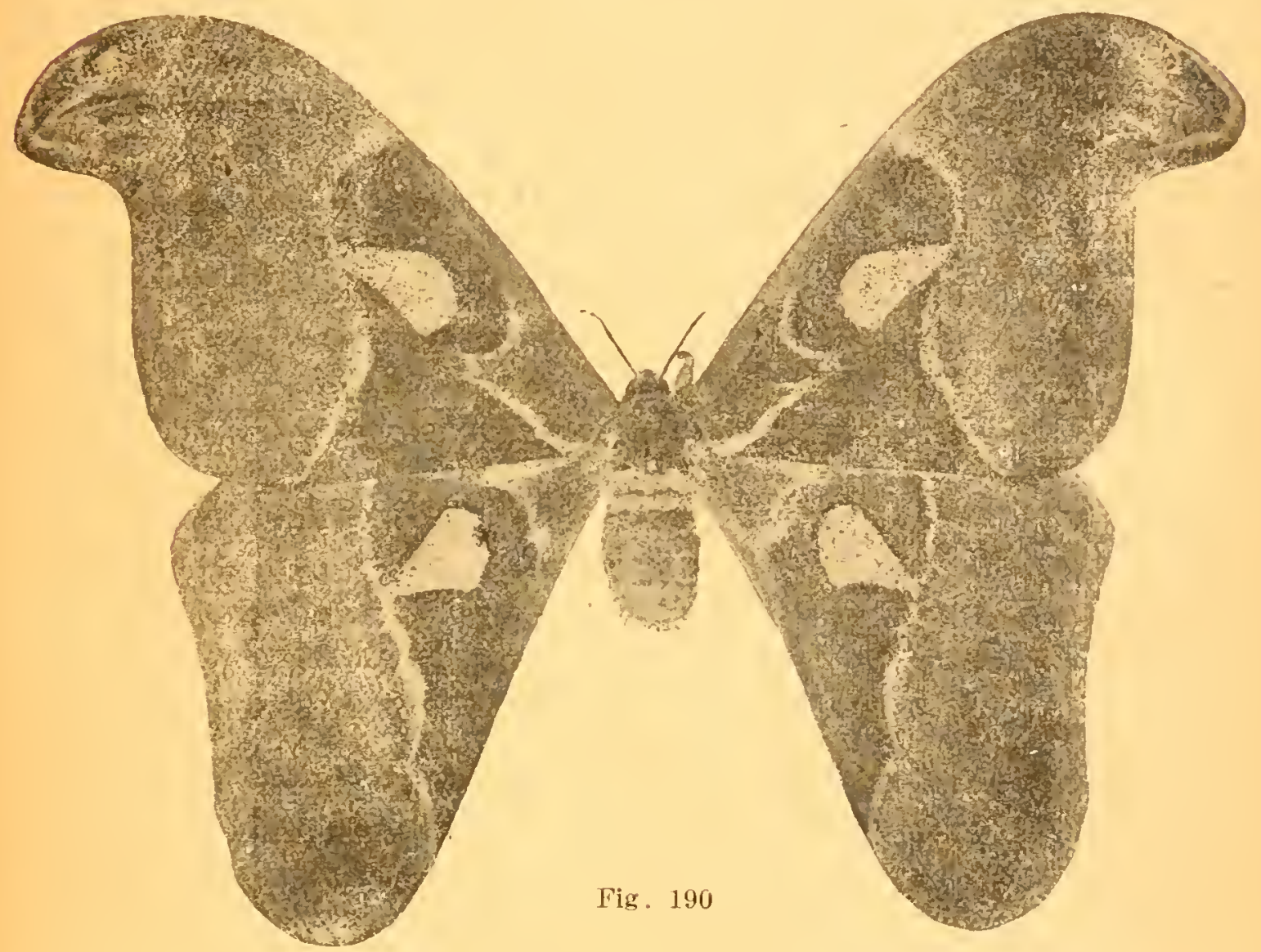

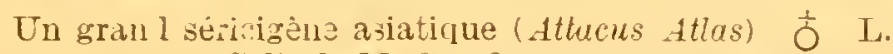
(Coll. de M. Ch. OBerthlr).

fabriquer les étoffes dites tussohr; le Papillon lui-même est appelé tussah par les Indiens; sa chenille donne un cocon presque aussi gros qu'un œuf de pigeon, mais le tussah ne se cultive pas en captivité comme le Bombyx du mûrier. On récolte ses cocons à l'état sauvage, sur les arbres oi l'on a placé préalablemen i les jeunes chenilles. Sa soie, d'excellente qualité, est l'objet d'un commerce considérable dans l'Inde et au Bengale.

Satirnia Cucropia. - Vit aux États-Unis sur le Prunier, le Poirier, etc. Il est l'objet d'une culture rationnelle à la Nouvelle-Orléans. 
Bombyx Rhadama, donne une excellente soie exploitée à Madagascar.

Suturnia purr, vit en France, sur divers arbres; sa chenille donne une soie grossière mais très résistante.

Attacus fillas (Fig. 190 ) ; c'est l'un des plus grands Bombycidés de l'Asie orientale; donne une soie de très bonne qualité.

Saturnix Pernyi (Chine). Vit sur le Chêne et se laisse facilemen: domestiquer; c'est avec la soie qu'il produit que l'on fabrique le ponqé de Chine.

11 existe encore, de par le monde, al! moins 200 espèces produisant une soie non utilisable; chose curieuse, toutes appartiennent à la famille des Bombycidés.

En dehors des Lépidoptères séricigènes, brièrement énumérés. ci-dessus, on peut encore citer quelques Hyménoptères et quelques Hémiptères, utilisés directement ou indirectement par l'industrie.

Hyménoptères. - Le Cynips gallæ tincturia, vit sur le Chêne à galle dans l'Asie mineure; par sa piqûre, il produit ces espèces de boules connues dans le commerce sous le nom de Noix de galle.

Le Cynips ficus carico, avec lequel on pratique la caprification des figues (1).

Hémiptrires. - Quelques espèces de Cochenilles (Coccus) vivent aussi sur les plantes à la manière des Pucerons.

La Cochenille du Nopal (Coccus cacti). Cultivée au Mexique depuis la plus haute antiquité sur les Opuntia, fournit la belle substance rouge connue sous le nom de carmin.

La Cochenille à cire (Coccus sinensis). Vit en Chine, sur les rameaux de certains Sumacs, de certains Frênes, qui, à la suite de sa piqûre, produisent une excrétion comparable à la cire des Abeilles.

La gomme-laque est produite, dans l'Inde et en Indo-Chine, par la picuire du Coccus Lacca sur plusieurs Figuiers et sur les Crotons. Enfin, d'après Boisduval, les habitants des côtes de la Ciuinée príparent une sorte de savon en broyant un petit Harpalide (IIypolichus saponarius, très commun dans ces contrées ar ec de la cendre de Baobab.

Nous ne rous étendrons pas davantage sur ce sujet; les quelque: exemples qui précèdent suffisent pour montrer les services que les Insectes peuvent rendre aux arts el a l'industrie.

(1) Opúration qui consiste à faire piquer les figues par ce petit Hyménoptère pour hâter leur maturité. 


\section{CHAPITRE V}

\section{LES INSECTES ET L'AGRICULTURE}

\section{I' LE HOLE DE L'ENTOMOLOGIE ÉCONOMIQUE}

Dans le munde entier, l'attention des pouvoirs commence à ètre sérieusement attirée sur le rôle des Insectes nuisibles, dans leur's rapports avec l'Agriculture, et l'Entomologie économique constitue aujourd'hui l'une des branches les plus importantes de la zoologie et. de la brulogie appliquées.

Nous ne pouvons, cela va sans dire, qu'effleurer cette raste question; aussi, nous bornerons-nous i donner ici un résumé des effurts qui sont faits dans les divers pays nour rendre la lutte agricole aussi rationnelle et auss fficace que possible. Nous suivrons pour cela, la très intéressante Notice que M. le Prof. Gurtel pabiia, il y a quelques dix ans, dans les Archives de Zoologie expérimentale (1).

Les Stations entomologiques. - Il est bien certain que l'importation des plántes exotiques, avec leurs parasites; la multiplicité et l'énorme rapidité des movens de transport, rendent, de jour en jour, plus urgente la lutte contre les Insectes nuisibles.

(1) Gubet (F.). - Sur la créution l'une Station entomologinue à la Fuculte des sciences de Rennes. (Arch. de Zool, expérim. et génèr. Paris, Schieicher, 1907 vol. VI, ') XCIII-CI).

LES INSECTES, - $2^{\mathrm{e}}$ édit. 
Les Etats-Unis d'Amérique et le Canada ont été irs premiers à comprendre les résultats que pouvait donsor la lutte scientifiquement engagée, aussi sont-ils arris à créer une organisation d'Entomologie appliquée !?:il n'a d'égale en aucun pays.

La division d'Entomologie du Département de l'A frrlculture, dirigée par M. L. O. Howaro, et dont le siegu est à Washington, comprend un nombreux personne? de savants dont les travaux sont consignés dans des Recueils spéciaux, répandus avec la plus grande libéralité, non seulement dans les Etats-Unis, mais enecre dans tout le monde entier.

Certains Etats, comme ceux de Massachusets, Ne:YYork, Illinois et Missouri, ont leurs entomologis: is officiels, et sont pourvus de laboratoires admirablement. aménagés; les autres possèdent, au moins, une " $A$ şricultural expériment Station ".

Les progrès réalisés dans ces dernières années sous l'influence de cette organisation ont été considérables, surtout en ce qui concerne l'application des insecticiles aux grandes cultures.

En Europe, l'Italie a été la première à suivre l'cxemp:" donné par les Etats-Unis. La Station royale d'Entom logie agricole de.Florence, dont la direction fut suces:sivement confiée à des sarants comme TARGIoriTozetri et BerLese, a été fondée en 1875. D'autres dét? même genre existent actuellement à Portici, à Milain, à Turin. Le budget de la suule Station de Florence s'b?vait, en 1908, à 16.000 lires, sàns compter le traitemont dia personnel.

En Suède el en Norvège, chacun des deux royaumes possède un entomologiste d'Etat, dont le ròle est de 
frurnir des expertises sur toutes les questions relatives ¿nx ravages des Insectes et d'indiquer aux agriculteurs los muyens à employer pour lutter avec succès contre Is s ennemis de leurs cultures.

L'Allemagne possède, depuis quelques années, une Station entomologique d'État à Berlin-Dahlem et le Vinistre de l'Agriculture se fait rendre compte, régulir rement chaque année, des dégàts occasionnés par les rectes nuisibles. Les renseignements sont recueillis ver les Ecoles forestières de Tharandt et d'Eberswalde; at Halle, le professeur Holruxg possède un laboratoire (:entomologie expérimentale.

L'entomologie appliquée n'a pas encore, semble-t-il, rf çu d'organisation officielle en Autriche; mais, comme ri Allemagne, l'enseignement de l'entomologie forestire y élait, arant la guerre, très florissant.

En Hongrie, il existe, depuis 1881, à Budapest, une Station entomologique d'Etat parfaitement organisée; ccite station est dirigée par le Prof. Jablonowski ; un 1414, son budget dépassait 10.000 florins.

La belgique possède, depuis 1891, un service officiel d'entomologie appliquée, dirigé par M. le Prof. Strierin. L'enseignement pratique est, en outre, donné à l'Inslitut agricole de Gembloux, qui fournit gratuitement aux particuliers, aux Sociétés et aux journaux, tous les renseignements concernant les Insectes nuisibles.

I'Angleterre possède également un service ofliciel d'entomologie appliquée, placé sous la direction de M. CEcil Wasburton. Ce service, installé à Cambridge, possède maintenant descollections et un laboratoire de recherches.

En 1894, il a été créé, à l'Université d'Amsterdam, un laboratoire mixte de phỵtopathologie et d'entomo- 
* logie appliquées, dont le M. Ritzema-Bos estle Directeur. La Hollande possède, en outre, une Station agroncmique importante à Java.

La Russie possède un service complet d'entomologie appliquée, qui fonctionne depuis une vingtaine d'annéc:s ( Prof. J. Portschinski) au Ministère de l'Agriculture is Saint-Pétersbourg. Ce bureau central de renseignements possèdait autrefois un budget annuel d'environ 40.000 francs. Il ćtait en rapport continuel avec les agriculteur:s et envoyait des entomologistés exercés aux endroits où ce produisent des dégìts, soit pour y étudier des Insectcs peu connus, soit, pour y diriger les travaux de destruction.

Il y avait, en outre, plusieurs stations en province.

Celie de Kiev, dirigée par M. le Prof. Walibemar Posi'in Low.

Celle de Kichinev (Bessarabie), M. le Prof. I. KrasSILTCFIK.

Celle de Simféropol (Crimée), Prol: S. Mokschetsk y (1).

Comme on le voit, presque tous les grands Etats européens ont imité, de leur mieux, l'exemple des EtatsUnis; quant à la France, qui, en sa qualité de pays essentiellement agricole, aurait dô se tenir à la tête do ce mouvement, elle n'est maiheureusement pas dotée comme elle devrait l'être pour cela.

Actuellement; notre pays possède seulement huit laboratoires d'entomologie appliquée ayant une existence officielle ou semi-officielle, ce sont:

10. A Paris, la Station entomologique dépendan:

(1) 11 est probable que la gnerre a apportè de grands changenients dans 1 : organisations entomologiques de la Russie: mas il nous a éte impossibla d'avoir ancun renseignement ì ce sujet. 
du Ministère de l'Agriculture et installée à l'Institut agronomique (Direct. M. le Prof. Paul Marcial).

2o. A Montpellier, le laboratoire d'entomologie de l'Ecole nationale d'Agriculture. Orgunisé autrefois par Valéry-Mayet, il est dirigé aujourd'hui par M. le Prof. Pration; M. le Dr Branc, préparateur:

30. A Rouen, le laboratoire régional d'entomologie, entretenu par la ville et par le département de la SeineInférieure. Directeur M. Paul Noel (1).

$4_{1}{ }^{\circ}$. A Rennes, la Station entomologique de Bretagne, annexée au laboratoire de Zoologie de la Faculté des Sciences, fut fondée par l'auteur de eet ouvrage en 1904 et dirigee administrativement par M. le Prof. F. Guitel (2). Elle a aujourd'hui pour préparateur M. I. Pourllande, irigénieur agronone.

50. A Bordeaux, la Station entomologiquo dépend du laboratoire de Zoologie de la Faculté dés Sciences. Olficiellement organisée, en 1913, par la municipalité, the est actueliement dirigée par M. le $\mathrm{D}^{\mathrm{r}}$ J. Fertiud. Son organe de publicité est le Bulletin de la Société d'Etude et de Vulgarisation de la Zoologie agricole, fondé en 1902.

60. A Blois, la Station entomologique, dirigée par M. Vezin, s'est vccupée principalement, ces dernières ariaées, de l'étude des Pucerons et de la Cochylis.

70. La Station entoirologique de Beaune (Côte-d'(Or), dirigée par M. Paillor, a abordé l'étude très intéresante des bactéries entomophytes et des Insectes parasites.

(1) M. Paul Noec est mort pendant la guerre et nous ne connaissons pas le nom de sori sucresseur.

(2) M, le Prof. GCitel vient lni-même ce s'éteinćre prématurément après une rruelle maladie : c'est une grande perte pour la science et pour l'entomologie. 
80. Nous sarons, par ailleurs, que plusieurs autres centres universitaires lrançais, Strasbourg notamment, sous la direction. de M. le Prot. E. Torsent, assisté de M. Paul Schekilin, se proposent d'organiser très énergiquement la lutte contre les Insectes nuisibles; mais nous manquons de renseignements à ce sujet.

Il est à peine besoin de dire que tous ces ćtablissements rendent de très grands services, mais ils sont oncore en trop petit nombre, dans un grand pays comme le nôtre, dont la première occupation est l'Agriculture.

\section{II ${ }^{\circ}$ LES GRANDS PROHLEMES ENTOMOLOGIQUES}

Pour donner maintenant une idée des questions économiques
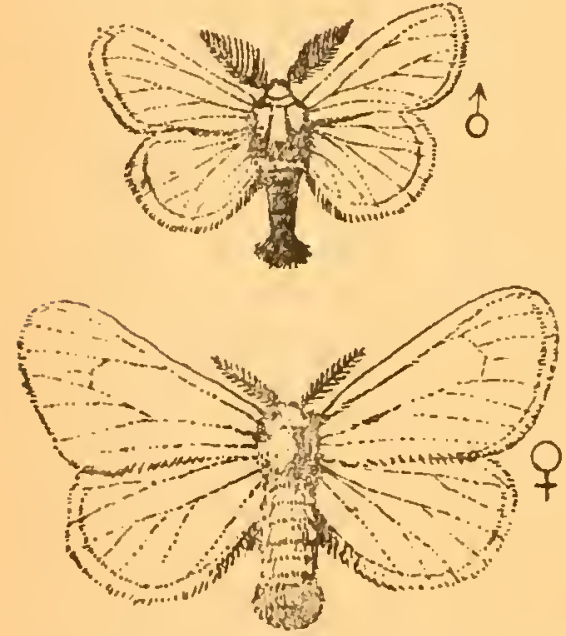

Fig. 191. - Le Liparis chrysorrhea, Téyidopt. mâle et femelle. que les établissements d'entomologie appliquée sont appelés à résoudre, nous dirons quelques mots de la vaste expérience entreprise en 1905-1906, par le gouvernement des États-Unis, pour combattre le Liparis chrysorrhea, qui menaçait d'une destruction complète les forêts de la Pensylvanie. (rig. 191).

La chenille du Liparis chrysorrhen. lépidoptère voisin des Bombyciclés, s'attaque au feuillage d'un grand nombre d'arbres, et c'est spécialement pour combattre ses ravages qu'a été édictée la loi du 15 mars 1796 sur l'échenillage. En France, heureusement, cette espèce a des parasites très nombreux qui l'empêchent de se propager an deliz d'une certaine limite. Or, vers 1890 , le Liparis chrysnrrhea fut importé accidentellement anx États-Unis; les parasites américains attaquèrent aussitôt ses chenilles, mais trop faibles ou trop peu nombrenx, l'extension dı Liparis prit bientôt les proportions d'un vrai désastre; le fléau allait s'aceroissant d'année en année, et il fallut renoncer à l'espoir de voir les parasites américains suffire pour 
l'enrayer. Le problème qui se posa immédiatement fut donc celui-ri : il fallait au plus tôt, introduire en Amérique les parasites européens du Lipais chrysorrhea.

M. L. O. Howard, chez du Bureau de l'Entomologie au Département de l'Agriculture à Washington, spécialement versé dans l'étude du parasitisme chez les Insectes, entreprit, tout exprès, le voyage d'Europe, dans le but de se mettre en relations avec les entomologistes européens. Prenant alors pour point de départ l'intéressante découverte de MI. le Professeur Jablonowsky, de Budapest, qui a établi que les nids d'hiver du Liparis chrysorrhea renfermaient de très nombreux parasites, M. Howard prit ses dispositions pour assurer l'expédition, en Amérique, d'environ 100.000 nids du Liparis, provenant de différentes parties de l'Europe centrale.

La Station entomologique de Rennes, mise au courant des démarches de M. L. Howard par II. René Oberthür, eut l'honneur de contribuer à la réalisation de cette vaste expérience par un enroi de 15.000 nids, dont la plupart furent récoltés dans l'Indre, anx environs de c'luis et de La Châtre par le Prof. G. Houlbert.

Pour l'élevage des chenilles et de leurs parasites, une maison de la ville cle Saugus, à quelques kilomètres de Boston, a été partiellement aménagée en laboratoire. D'autre part, trois grands arbres, infestés par le chrysorrhea, ont été emprisonnés dans une vaste toile métallique formant une immense cage. On put ainsi recueillir les parasites qui sortaient des chenilles et les étudier.

La remarquable expérience, organisée par les Américains, îut conduite avec une science consommée de la biclogie des Insectes. Elle a déjà donné des résultats très encourageants et sa réussite aboutira très probablement, pour la région des F́tats-Unis, à un état d'équilibre analogue àcelui qui s'estétabli naturellement en Europe, entre le parasite et les parasités.

D'ailleurs, il y a un précédent: une expérience analogue a été réalisée avec succès, il y a près de 30 ans, par le savant entomologiste C. V. Riley, prédécesseur de M. Howard (voir p. 31).

L'Icerya Purchasi, cochenille originaire d'Australie, avait été introduite en Californie, ver's 186s. Elle s'y acclimata et se propagea même avec une si prodigieuse rapidité, qu'elle menaçait, à un moment donné, de ruiner complètement la culture des orangers.

Un biologiste américain, M. Kicbele, fut envoyé en Australie. arec mission de rechercher les parasites naturels de l'Icerya. ll rapporta, 
à son retour, une petite Coccinelle (Nosius cardinalis) qui s'acclimata parfaitement, et qui, au bout de deux années, avait presque entièment débarrassé la région des Icerya. Un entomologiste allemand, iI. Schaufuss, a cru devoir contester ces résultats, en 1910, dans le no 1 du Deutschen Entomologischen National Muséum; mais il a certainement confondu les expériences de M. Kœbele avec celles enireprises, à l'aide du Chilocorus similis, sur le San Jose Scale.

Des expériences analogues ont été récemment reproduites en Portugal et en Italie avec le même succès, toujours contre l'Icerya des orangers.

11 nous semble inutile d'insister ; on voit quels immenses services peusent rendre aujourd'hui, à l'Agriculture, les Stations entomoloriques scientifiquement installées; on peut, en effet, estimer à 500 !nillions de francs, les pertes annuelles occasionnées par les Insectes nuisibles (1).

'Tous les Insectes, heureusement, ne sont pas nuisibles au même leggré ; il en est même d'utiles, mais il n'existe, cela va de soi, aucun c rractère extérieur qui permette de distinguer un Insecte utile d'un Insecte nuisible; les seules remarques qu'on puisse faire à ce sujet sont les suivantes: d'une manière générale, toutes les espèces carnassières sont utiles; inversement, la plupart des espèces phytophages sont nusibles.

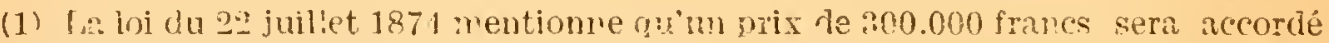
Jour l'btat, a l'insesteur d'an moyen economicue qui bourrat. dans la renéralité .ses terrains, cètruire ie Phylloxera on empécher ses ravages. 


\section{CHAPITRE VI}

\section{LE PARASITISME CHEZ LES INSECTES}

L'énumération qui précède nous a amené à considérer les insectes dans leurs rapports avec l'homme et avec. les végétaux ; sous couleur d'un simple problème d'entomologie économique, c'est en réalité la grande question du Parasitisme qui se pose. C'est encore là un des côtés du rôle biologique c'es Insectes qu'il n'est plus permis d'ignorer aujourd'hui.

Il serait tout à fait impossible d'étudier ici, avec toute l'étendue qu'elle comporterait, la question du parasitisme chez les Insectes : nous allons simplement dresser un plan, dont les chapitres successifs pourront être déve.. loppés dans les volumes spéciaux de cette collection.

10 Insectes parasites de l'Homme et des Animaux. Deux ordres d'Insectes, principalement, les Hémiptères et les Diptères, renlerment des espèces parasites de l'homme et des animaux (1).

A. Hémiptères. - Nous citerons seulement, dans ce groupe, la punaise des lits et les innombrables espèces de Puux.

I.a plupart de ces animaux sont des Insectes nocturnes, qui se guident exclusivement par l'odorat. Leur piquire serait peu douloureuse, si l'Insecte ne déposait, dans la plaie, une salive irritante rraisemblablement destinée à empêcher le sang de se coaguler.

(1) Mentionnons seulement pour mémoire les rares Coléoptères Platypsillus castoris, vivant en parasites sur les Castors du Phône, du Canada, etc.; Amllunpinus J dskyi petit staphylinide, virant à la façon des Acariens, sur la peau des petits rongeurs au Pérou et à la Nourelle-Grenare. 
D'autre part, ces parasites, étant en contact continuel avec le corps et les vêtements, doivent contribuer, dans une large mesure, à la dissémination des maladies microbiennes (tuberculose).

a) Punaise des lits (Acanthia lectularia L.). - C'est un insecte aplati, de forme ovale, long de 4 à 5 milli-

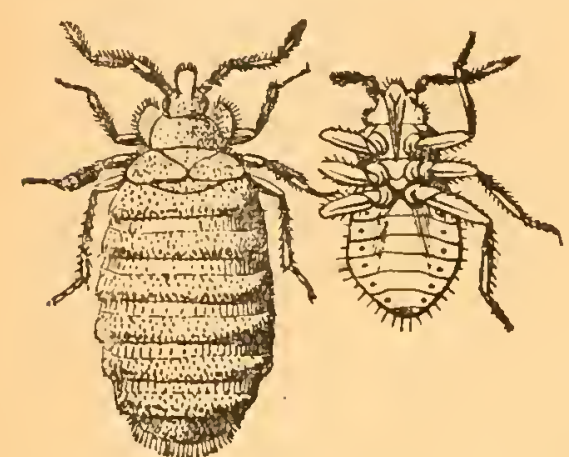

Fig. 192. - La punaise des lits (Acanthia lectularia) et sa larve (d'après How ARD et MARLATT)

mètres; il se tient dans les fentes des boiseries, sous les papiers de tentures, dans les jointures des meubles et peut supporter un long jeùne, ce qui explique comment il se conserve quelquefois pendant plusieurs années dans les locaux inhabités (Fig. 192).

b) Poux. - Vivent en parasites sur les Mammifères, sur les Oiseaux et suuvent sur l'Homme. Leur développement se fait sans métamorphoses; les femelles pondent des ouís allongés, fixés vers la base des poils ou sur les vêtements à l'aide d'une substance agglutinante et désignés sous le nom de lentes. On sait aujourd'hui qu: le Pediculus vestimentiest l'agent de propagation le plus actil du typhus exanthératique. Cette terrible maladie, qui suit oujours les grandes guerres et les famines, a causé, de 1914 à 1917, des épidémies effrayantes et sxtrêmement meurtrières, en Serbie, en Roumanie ut dans les camps de prisonniers en Allemagne.

B. Diptères. -- Un grand nombre de Diptères possèdent, comme les Hémiptères, un appareil vulnérant formé par les pièces de la bouche modifiées. Il est nécessaire de distinguer plusicurs catégories, car tous ne sont pas parasites au mème degré. 
Chez les uns, le parasitisme est obligatoire, comme par exemple les diverses espèces des genres Hypoderma, Dermatobya et le Sarcopsylla penetrans (Puce pénétrante) qui, à l'état de larve, se tiennent sous la peau ; les Estrides, qui vivent dans le tube digestif des herbivores.

Chez d'autres, le parasitisme est facultatif, en ce sens que leurs larves, qui vivent normalement à l'intérieur des matières organiques en décomposition, peuvent vivre accidentellement dans les tissus des animaux vivants : c'est le cas de certaines mouches: Compsomyia macellaria, Sarcophaga carnaria, ruficornis et magnifica.

Enfin, d'autres peuvent servir d'agents de transmission à diverses maladies parasitaires, exemple :

Les Pllces qui, par leur piqùres, jouent un rôle capital dans la dissémination de la peste et de la furonculose.

Le Stomoxys calcitrans qui, dans nos pays, peut être considéré comme l'agent de dissémination de la Bactéridie charbonneuse.

La Mouche Tsé-isé (Afrique tropicale et occidentale) inncule, par sa piquûre, le Trypanosome de Gambie, agent de la maladie du sommeil. Les nombreuses espèces de Taons agissent évidemment dans le mème sens.

La Mouche dumestique, qui pose sur nos aliments ses pattes souiliées de germes infectieux est, à n'en pas douter, un agent redoutable de transmission de la tuberculose, du choléra, de la fièvre typhö̈de, de la dysenterie bacillaire et do la diarrhée verte des enfants.

Enfin, les Moustiques, toujours dangereux, non seulement à cause des piquires douloureuses qu'ils peuvent occasionner, mais parce qu'ils sont les agents de transmission d'un certain nombre d'affections redoutables.

Les Anophèles inoculent l'hématozoaire du palu- 
disme, agent des fièvres dites paludéennes (Laverax).

Les Stégomies transmettent la fièvre jaune.

Les vulgaires Cousins sont des agents de transmission de la Filariose (Filaria Bancrofii) à la Guyane et dans les Antilles.

C. Coléoptères. -- Enfin, parmi les ('oléoptères, un n'a signalé jusqu'ici que la larve d'un Nécrobia, trouvéé à Rennes, en 1910, dans un kyste de la sclérotique. Cette larve fut décrite et représentée p:r M. C. Housв $\mathrm{k}$ T dans les Archives de Parasitologie.

$2^{\circ}$ Insectes parasites des végétaux.--Tous les Insectes phytophages sont, à proprement parler, parasites des plantes sur lesquelles ils vivent; mais, le nombre de ces Insectes est si grand, qu'il nous est impossible de traiter cette question dans son entier. Nous nous bornerons spécialement à étudier ici le cas, d'ailleur's très fréquent, où les tissus de la plante réagissent en produisant, une déformation que l'on désigne vulgairement sous

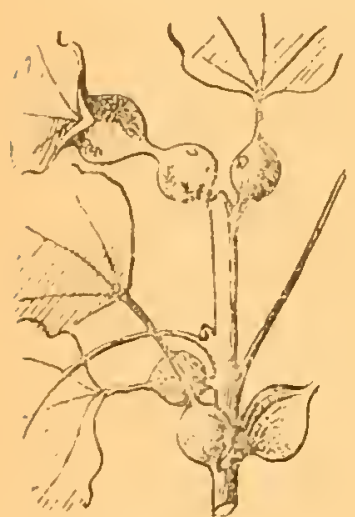

Fig. 193. - Exemvle ile gralles ( Entomocécilies) pro iuites par les Insertes (d'alrèz DARB)CX et H(O) (1) AR). le nom de galle, et, scientifiquement, sous celui de Cécidie.

Cécidologie. - La Cécidologie est aujourd'hui l'un des chapitres les plus intéressants de la pathologie végétale.

L'origine des Cécidies (gralles) est variable; elles peuvent être produites soit par un parasite animal, soit par un parasite végétal, d’où la distinction en :

Zoocécidies, déformations dues à la présence d'un parasite anımal (très souvent un Insecte).

Phytocćcidies, déformations dues à un parasite végélal (Champignon ou Bactérie).

Nous ne nous occuperons, bien entendu, ici que des zoucícilies et móme spécialement parmi celles-ci des lintomocérilies (grilles produites par les Insectes) (rig. 193). 
Entomocécidies. - Dans tout le règne animal, la classe des Insectes est sans contredit celle qui fournit le plus grand nombre d'agents producteurs de galles (1); presque tous les ordres renferment des représentants cécidogènes, mais ce sont les Diptères, les Hémiptères et les Hyménoptères qui en fournissent le plus. Quant aux Névroptères, dont toutes les espèces sont carnivores. il semble qu'ils n'ont pas jusqu'ici de représentant gallicole bier, connu.

Suivant l'Insecte qui les produira, nous aurons donc à distinguer diverses catégories d'entomocécidies.

$1{ }^{\circ}$ Coléoptérocécidies. - Bien qu'il soit l'un des plus riches de la classe des Insectes, l'ordre des Coléoptères ne renferme guère qu'une cinquan-

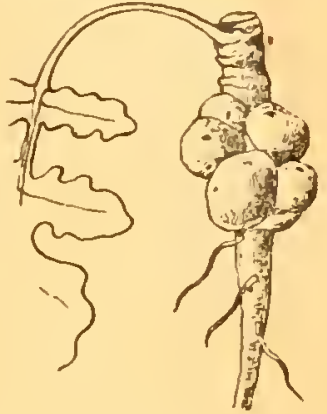

Fig. 194. - Galle produite à la base de la tige du Chou nar le Ceutorhynchus sulvicollis Col.

(d'ap. J. KIEFFER). taine d'espèces cécidogènes. Les familles qui en fournissent le plus sont les Cérambycides, les Buprestides et les Curculionides.

D'après 11. l'abbé Kieffer (Feuille des jennes Vaturalistes, 1892, p. 53), les déformations produites, par ces Insectes, sur les plantes.

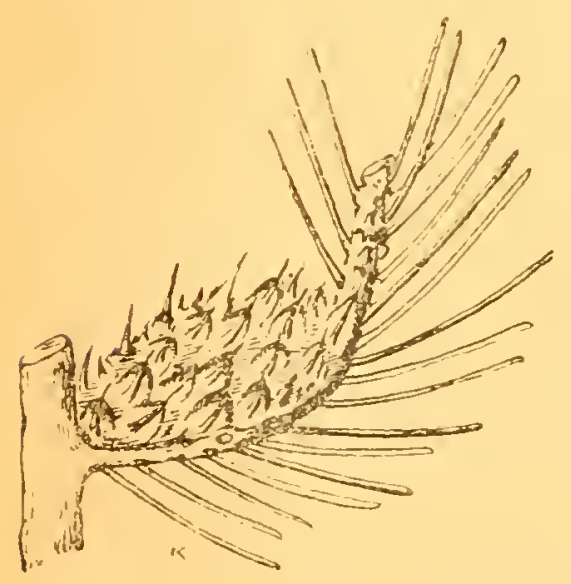

Fig. 195. - Cécidie produite par l'Adelges abietis Hèmipt.

(d'après J. KIEFFER). sa présentent généralement sous la forme d'un renflement contenant une ou plusieurs cavités hermétiquement closes (Fig. 194).

$2^{\circ}$ Lépidopierocécidies. - Les cécidies dues aux Lépidoptìres ressemblent beaucoup à celles produites par les Coléoptères; comme ces dernières. elles produisent le gonflement d'un organe quelconque de la plante nourricière. L'une des mieux connues estcelle de Conchylis hilarana, qui détermine un renflement fusiforme à la base des tiges del'Artemisia crmpestris.

$3^{\circ}$ Hémiflérncéridies. - Les galles produites par les Hémiptères ne se présentent guère que sous la forme d'une boursouflure ou d'un enroulement marginal des feuilles; l'enroulement se distinguera facilement de celui produit par les Hyménoptères ou les Diptères,

(1) Voir Cutulogue Darboux et Houard.

LES IXsECTES. - 2e éait. 
parce qu'il est beaucoup plus lâche, et qu'on trouve, presque toujours à l'intérieur, les dépouilles de l'Insecte qui les a habitées (Fig. 195).

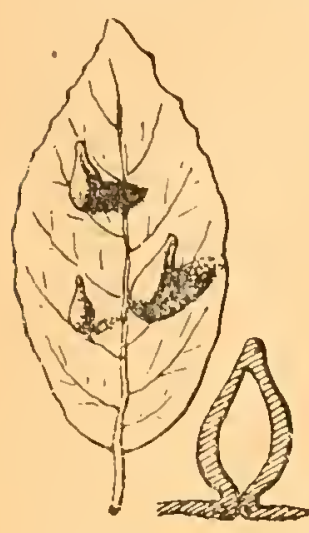

Fig. 196 . Galle conique produite sur les feuilles du Hêtre par le Mikiola fagi Dip.

(d'ap. DarDoux et HOUARD).

Deux ou trois seulement doivent leur origine à des hétéroptères; toutes les autres sont produites par les homoptères; les nodosités galloïdes, occasionnées par le Phylloxera sur les racines de la vigne, sont malheureusement trop connues.

$4^{\circ}$ Diptérocécidies. - Les galles produites par les Insectes de ce groupe sont excessivement nombreuses;; la plupart sont dues à la famille des Cécydomyes (Mikiola faรi) (Fig.,196). Parmi celle dues aux Muscidés, la plus commune est celle rle l'l/rophora cardui, sur la Circium arvense, décrite il y a plus de 200 ans par Godart pour la première fois.

$5^{\circ}$ Hyménoptérocéridies. - Ce sont les Insectes de cet ordre qui déterminent les céridies les plus remarquables (Fig. 197). La plupart sont produites par les Cynipides, un certain nombre par des Tenthrédines; enfin, quelques-unes seulement par des Ptéromalides des genres Euruthoma et Idosoma.

Ce sont les Cynipides qui déterminent ces déformations volumineuses, connues depuis la plus haute antiquité, très communes sur les Chênes et sur les Rosiers sauvages (bédégards).

La galle des Cynipides la plus anciennement connue est la Noix de Galle ou galle d'Alep, produite, sur le Quercus lusitanica (Grèce et Asie mineure), par le Diplolepis gallæ tinctoriæ.

60 Orthoptérucécidics. - D'après M. l'abbé J. Kieffer (Synopsis des zoocécidies d'Europe, p. 234), on ne peut guère citer, dans cet ordre, que le genre Thrips qui soit susceptible de produire, sur les plantes, des réactions cécidiformes.

$3^{0}$ Les parasites des Insectes. - Si les

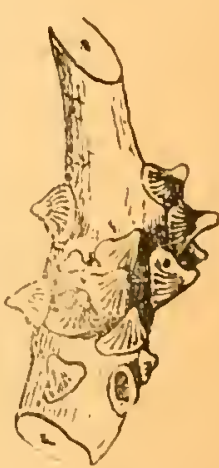

Fig. 197.Galles produites sur le Chêne par l'. Indricus Sieboldi Hymén. (d'après D.ARBOIX et Hor.tin).

Insectes parasites sont nombreux, ils peuvent, à leur tour, etre parasités par un certain nombre d'organismes inférieurs, animaux ou végétaux. Un Insecte 
donné, peut mâme être parasité par un autre Insecte.

10 Animaux parasites des Insectes. - Un certain nombre de Protozoaires, appartenant principalement à la classe des Grégarines, vivent dans le tube digestif de différents Insectes. On peut citer les espèces suivantes: Stylorhynchus longicollis chez les Blaps, et Gregarina blattarum chez les Blattes: Gr. polymorpha, dans la larve du Tenebrio molitor et Actinocephalus stellilormis chez les Carabes.

Dans l'embranchement des Némathelminthes, certains Gordills ne sont pas rares dans la cavité viscérale des Acridiens; les embryons de Gigantorhynchus gigas et moniliformis subissent les premières phases de leur évolution dans les larves du Hanneton, de la Cétoine dorée, etc.

$2^{\circ}$ Insecies entomophages. - Sans nous arrêter ici aux Stylopides (Strepsipteres), dont les frmelles aptères vivent toujours en parasites sur l'abdomen des Guêpes, des Abeilles et des Bourdons, nous appellerons surtout l'attention sur les Insectes dits entomophages, dont les larves se développent dans le corps des autres Insectes, et dont l'importance est si grande au point de rue économique.

Les entomophages appartiennent, presque tous, à l'ordre des Hyménoptères; les uns, comme par exemple les Cerceris, les Sphex, alimentent

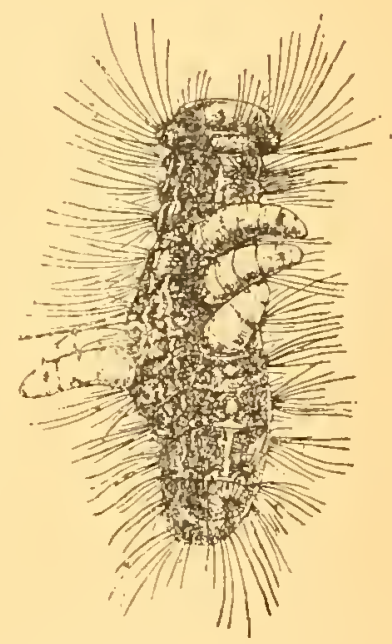

Fig. 198. - Larve d'un Hyménoptère parasilc (Glyplapanteles), sortant c'une Chenille pour se chrysalider (grand. nat.). leurs larves arec des proies vivantes, préalablement anesthésiées d'un coup d'aiguillon. D'autres (Ichneu- 
monides), déposent leurs œuls sous la peau de leurs victimes: c'est ainsi que la Chenille du Papillon blane du Chou peut ètre envahie par une armée de petites larves appartenant à un Braconide, le Microgaster glomerator (Fig. 198). Tous ces Hyménoptères, entomophages à lå façon des Ichneumonides et des Braconides, sont de puissants auxiliaires de l'Agriculture (1), parce qu'ils détruisent un nombre incalculable de chenilles d'Hyponomeutes qui, sans eux, auraient tôt fait de ronger le feuillage de nos haies et de nos arbres fruitiers.

$3^{\circ}$ Microphites parasites des Insectes. - Une famille entière d'oomycètes, les Entomophthorées vivent en parasites dans le corps de divers Insectes et les tuent rapidement. C'est ainsi que, chaque année, à l'automne, on peut voir, sur les vitres de nos maisons, les Mouches envahies par le mycélium blanc et floconneux de l'Empusa muscr. D'autres fois, ce sont les chenilles de la Piéride du Chou, qui sont envahies par' l'Entomophthora radicans.

Pendant quelques années, on a caressé l'espoir de détruire les larves du Hanneton (Ver's blancs) en les infestant d'une Mucorinée parasite, le Botrytis tenella; les résultats n'ont pas répondu à l'attente des expérimentateurs.

Des Bactéries peuvent aussi envahir le corps des Insectes, exemple la pébrine du Ver à snie, la flacherie, etr.

(1) Marchal (P). - Ctilisalion des Inereits aucitinires mfomophanes dans la lutte contre les Insertes nuisibles ì l'Agricullure. (Amm. de l'Institut agronom. Paris, J.B. Baillière, 1907. T. VI.) 


\section{DEUXIENAE PARTIE \\ Entomologie appliquée.}

\section{CHAPITRE VII \\ CLASSIFICATION DES INSECTES}

I० Principe de la Classification. -- En tenant compte des caractères fournis par les ailes, par les pièces de la bouche et par la nature des métamorphoses, on peut classer les Insectes en neuf ordres, ainsi que l'indique le tableau suivant:

Io Insectes primitifs, dépourvus d'ailes (Aptérygogènes) ; pas de métamorphoses (Amétahola). 10 TiHYSAnoures

IIo Insectes pourvus d'alles (Ptérygogènes).

Métamorphoses incomplètes (Hémimétabola).

Pièces buccales conformées pour broyer.

1 Quatre ailes souvent inégales et possédant de nombreuses nervures.

20 Archiptéres

Quatre ailes inégales, les supérieures cornées et transformées en élytres.

Pièces buccales conformées pour piquer.

30 ORTHOPTÉRES

40 Hémiptères

Métamorphoses complètes (Holométabola)

Pièces buccales conformées pour broyer.

( Quatre ailes, sensiblement égales et riche-

ment veinées.

io Nétroptères

1 Quatre ailes inégales : les supérieures transformées en élytres.

6 o Coléoptères

Pièces bucciles conformées pour brover et lécher.

7O HYMÉNOPTĖRES

Pièces buccales conformées pour piquer ou aspirer les liquides.

I Quatre ailes couvertes d'écailles.

So l.éPIDOPTĖRES

\{ Deux ailes seulement.

9" Diptéres 
Tel qu'il est présenté ici, ce tableau suppose une fixité de caractères qui n'existe point dans la nature. Le lecteur qui essayerait de s'en servir pour déterminer l'état civil d'un Insecte inconnu, pourrait évidemment réussil dans un grand nombre de cas très simples et pour ainsi dire classiques, mais aussi parfois, il risquerait fort de s'égarer.

Classer des Insectes et les déterminer sont deux choses très différentes; aussi pour remédier, dans la mesure du possible, à l'insuffisance analytique du cadre précédent, nous donnons, ci-après, une table dichotomique de détermination, s'appliquant à tous les Insectes et tenant compte de toutes ies modifications qui peuvent se présenter dans les différents groupes.

IIo Tableaux analytiques de détermination permettant de ranger rapidement un Insecte donné dans l'crdre auquel il apppartient.

Io Insectes aptères (ou pourvu seulement de rudiments d’ailes).

Pièces de la bouche cachées dans une cavité de la tête, de telle manière que leur extrémité seıle est visible.

Physanoures

1. Pièces de la bouche proéminentes, visibles et

- disposées pour mordre. • . . . .

Pièces de la bouche visibles, disposées pour piquer ou sucer.

Tête prolongée en un long bec à l'extrémité

2.

duquel se trouvent les pièces de la bouche

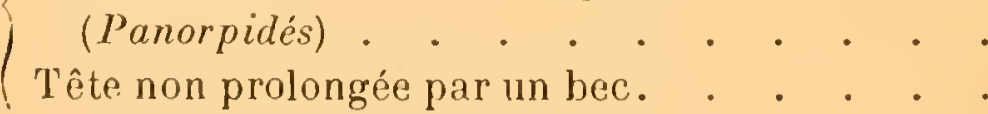

Archiptéres 3 
(Insectes ayant toujours moins de 4 millimètres

de long; ayant une forme rappelant celle des Poux (Mallophages). . . . . . Archiptéres

3. Insectes ayant plus de 4 millimètres de longueur (exception pour quelques Fourmis); la forme n'est jamais celle d'un Pou. . .

(Abdomen muni à sa partie postérieure d'ap-

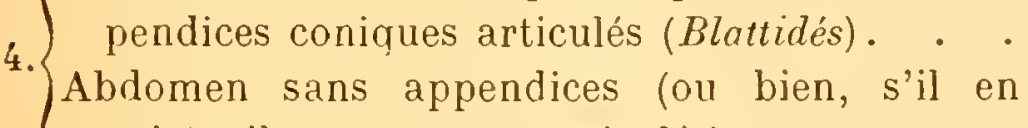

Orthoptères

existe, ils ne sont pas articulés). . . .

Pattes postérieures très longues conformées

5. pour sauter (Locustidés, Acrididés, Gryllidés) Orthoptères

Toutes les pattes conformées pour marcher.

Abdomen réuni au thorax par une très large base . . . . . . . . . . . .

6. Abdomen fortement rétréci à sa base et relié au thorax par une sorte de pédoncule (Fourmis aptères $)$. . . . . . . . .

HYMÉNOPTÉR'S

Corps allongé, très étroit; antennes de 12 à 25

7. $\left\{\begin{array}{c}\text { articles (Phasmidés) . • } \\ \text { Corps ovale, antennes courtes de } 11 \text { articles } \\ \text { aumaximum . . . . . . . . }\end{array}\right.$

Orthoptéres

Coléoptères

Petits insectes parasites, toujours fixés aux végétaux (tiges ou feuilles), présentant l'aspect d'une écaille ou d'un bouclier; le corps est souvent recouvert d'un enduit cireux ou cotonneux (Coccidés) . . . . . . . Hémiptères

8. Corps recouvert de longs poils, formant une fourrure complète; bouche formant un tube enroulé sous la tête. . . . . . . . LÉPIDOptères

Corps nu ou recouvert seulement par des poils ou des soies isolés. . . . . . . .

(Tarses de 5 articles ; prothorax intimement soudé 9. $\left\{\begin{array}{l}\text { au mésothorax et au métathorax. . } \\ \text { Tarses de 1-2-3 articles; prothorax bien isolé } \\ \text { (Physopodes) . . . . . . . . . . }\end{array}\right.$

Diftères

HÉMIPTÉRES 
II Insectes ailés (les élytres des Coléoptères et des Perceoreilles sont comptés comme des ailes dans ce tablea1).

1. $\{$ Insectes avec deux ailes. . . . . . . . 2

Insectes avec quatre ailes. . . . . . . 6

2. Ailes cornées, coriaces ou parcheminées. . . 3

Ailes entièrement membraneuses. . . . . 5

Mâchoires visibles; pièces de la bouche dis-

3. posées pour broyer. . . . . . .

Mâchoires non visibles; pièces de la bouche disposées pour piquer ou sucer. . . . Hémiptères

Ailes cornées, sans nervures visibles (1). Pattes postérieures conformées pour la marche. ColÉoptères

4. Ailes parcheminées, portant de nombreuses nervures; pattes postérieures conformées pour sauter . . . . . . . . . .

OrTIOPTÉRES

$\int$ Adbomen pourvu de longues soies caudales

5. (Ephémérides) • • • • • • • • .

(Les deux paires d'ailes différentes dans leur

structure; les supérieures cornées ou parche-

6. minées, les inférieures membraneuses. .

Les deux paires d'ailes semblables, toutes les deux membraneuses . . . . . . . .

Archiptères

Diptéres

Ailes antérieures coriaces à la base, membra-

7. neuses à leur extrémité. (Hétéroptères). ·

HÉMIPTĖRES toute leur étendue.

Ailes antérieures cornées, sans nervures appa8. $\left\{\begin{array}{l}\text { rentes, formant deux étuis (élytres) pour } \\ \text { protéger les ailes membraneuses qui sont en } \\ \text { dessous . . . . . . . . . . } \\ \text { Ailes antérieures coriaces ou parcheminées } \\ \text { avec un riche réseau de veines. . . . }\end{array}\right.$

(1) Il ne faut yas confondre avec les nervures, les stries ou leg granulations régulières qui ornevt les élytres des Coléoptères. 
Abdomen terminé par deux crochets cornés 9. en forme de pinces (Forficulidés). . . . Orthoptères Abdomen non terminé par deux crochets cor. nés • . . . . . . . . . . . Coléoptères

Ailes postérieures pliées en forme d'éventail;

10.) pièces de la bouche disposées pour broyer. . Orthoptéres Ailes postérieures non pliées; pièces de la

l bouche disposées pour piquer (Homoptères). Hémiptères

Dernier article des tarses vésiculeux, dépourvu

11. de griffes (Physopodes). . . . . . Hémiptères

Dernier article des tarses non vésiculeux et pourvu de griffes. . . . . . . . 12

Ailes recouvertes d'écailles, entièrement ou.

12. tout au moins le long des nervures. . . . LÉPIDoptéres Ailes nues, transparentes, simplement garnies de poils • • • • • • . • . • . 13

Ailes nettement veinées, avec un certain nombre de veines et de nervures transversales. ARchiptEREs

13. Ailes avec des nervures ramifiées, mais pas de nervures transversales, ou tout à fait sans nervures . . . . . . . . . . 14

14. $\{$ Tarses de 2 ou 3 articles (Corrodentia, PlécopTarses de 4 ou 5 articles.
(Abdomen orné de soies caudales à son extrê15. mité (Epliémérides) • • • • • • Archiptères Abdomen sans filaments ni soies à son extrémité • • • • • • • • • • • 16

Prothorax corné ; mandibules bien développées, 16. palpes courts • • • • • • • • • Hyménoptíres Prothorax membraneux; mandibules invisibles, palpes longs ('Trichoptères). . . . . Archiptères 


\section{LES PRINCIPAUX ORDRES D'INSECTES}

Io INSECTES SANS MÉTAORPHOSE (Amétabola).

1e Ordre: Thysanoures.

(Type : Lépisme).

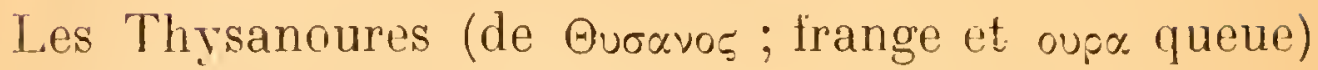
sont des Insectes de petite taille, complètement privés d'ailes et ne subissant aucune métamorphose. Leur, corps, velu ou couvert, d'écailles, porte, à son extrémité postérieure, des appendices ciliés de forme et de dimensions variables.

lls vivent dans les endroits obscurs et humides; les uns courent avec rapidité (ex.: Lépismes),

Fig. 199. - Achorutes murorum, très co mmmun a u pied des murs. les autres peuvent sauter à la façon des Puces et des Altises (ex.: Podurelles). La constitution des pièces buccales (mâchoires et mandibules) permet de les rapprocher des Orthoptères et. notamnent des Blattes (Fig. 199).

On a divisé les Thysanoures en trois sous-familles : Cumpodéidés, Lépismidés et Poduridés, d'après la forme du corps, la dimension des antennes et la constitution des appendices abdominaux.

Comme exemple de Thysanoure, nous pouvons citer le Lépisme du sucre, petit animal allongé, un peu déprimé, brillant comme de l'argent, qui s'enfuit avec agilité lorsqu'on remue les vieux papiers conservés dans les placards humides.

\section{Ordre: Archiptères.}

(Ortuonévroptéres. Pseunonéroptires).

(Type: Éphémère).

On range suus ce nom un certain nombre d'Insectes qui faisaient autrefois partic de l'ordre des Néoroptères, 
et qui ne s'en distinguent, en réalité, que par la plus grande simplieité des métamorphoses (Fig. 200).

Leur appareil buccal est conformé sur le type broyeur; leurs ailes, au nombre de quatre, grandissent progressivement et sont parcourues par des nervures nombreuses mais peu différenciées, où dominent les branches transversales.

Les représentants les mieux connus de cet ordre, en France, sont les Ephémères et les Libellules, dont les larves mènent une vie aquatique. Les

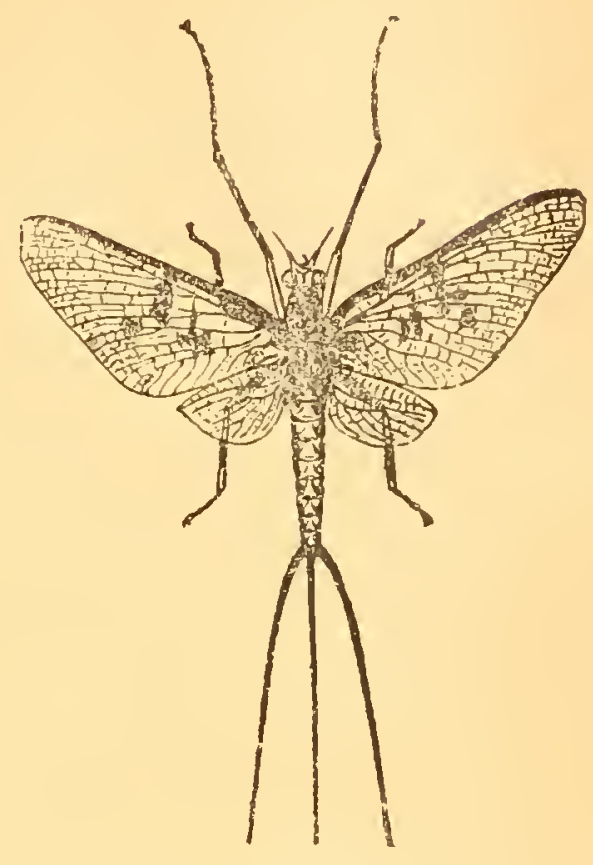

Fig. 200.- Ephémère commune: abondante au printemps au bord des eaux. (d'ap. nat.).

Terrnites, si remarquables par leurs instincts sociaux, vivent dans les régions chaudes du globe.

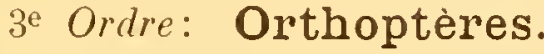

\section{(Type: La Grande Sauterelle verte).}

On peut considérer les Orthoptères comme un rameau dérivé des Archiptères : la bouche est également conformée sur le type broyeur et les métamorphoses sont incomplètes. Lorsque les larves sortent de l'œuf, leur forme rappelle, de très près, celle de l'adulte, sauf qu'elles sont privées d'ailes et d'organes génitaux ; les transformations qu'elles subissent se bornent donc à l'accroissement du corps et des ailes; les organes sexuels se développent peu à peu et la larve, finalement, arrive à l'état 
d'Insecte parfait (Fig. 201). Mais jamais, à aucun moment de leur existence, ces larves ne présentent la phase de pupe ou de nymphe immobile qu'on observe chez les Insectes à métamorphoses complètes.

Les ail's inférieures des Orthoptères présentent à peu

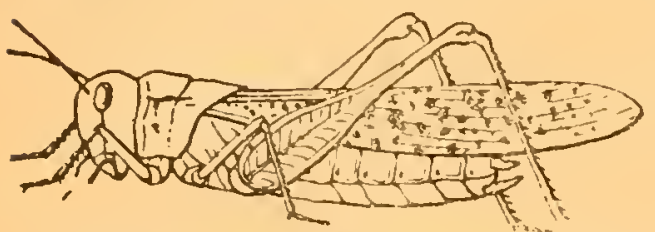

Fig. 201. - Le Criquet cendró le plus grand des Acridiens français.

(8-10 centim.) près la même nervation que celles des Archiptères ; mais, de plus, elles peurent se plier, dans le sens de la longueur, à la façon d'un éventail (Fig. 201) ; les ailes supérieures sont transformées en élytres.

On trouve les Orthoptères dans toutes les parties du monde; cependant leur nombre diminue à mesure qu'on s'éloigne de l'équateur dans la direction des pôles.

Les principaux représentants de cet ordre en Europe sont : les Forficules, les Blattes, les Mantes, les Phasmes, les Acridiens, les Locustes et les Grillons.

IIo Insectes A MétAMORPhoses Ingomplètes (Hémimétabola).

\section{4: Ordre: Hémiptères.}

(Type: Punaises des bois).

Les Hémiptères (emilus demi et pteron aile) ont reçu ce nom à cause de la conformation de leur's ailes supérieures qui, dans les espèces les plus parfaites, sont cornées à leur base et membraneuses à leur extrémití: ce sont donc des demi-élytres, d'où le nom d'Hémiptères.

La bouche des Hémiptères est conformée pour piquer ; elle comprend, en eflet, un rostre pointu, assez dur pour 
pénétrer dans le curps des animaux ou dans les tissus des végétaux.

Les métamorphoses sont incomplètes et la jeune larve diffère peu de l'adulte, sauf, comme toujours, par l'absence d'ailes et d'organes génitaux ; toutefois, les ailes n'apparaissent qu'à l'avant-dernièrè mue. La parthénogénèse se rencontre normalement chez les Aphidés et chez les Coccidés.

Comme beaucoup d'Hémiptères ont leurs quatre ailes semblables, on a été obligé de diviser cet ordre en deux sections :

10 Hétéroptères, qui possèdent des demi-élytres bien caractérisés $\left(P_{l l-}\right.$ - naise des Bois (Rhaphinaises) (Fig. 202).

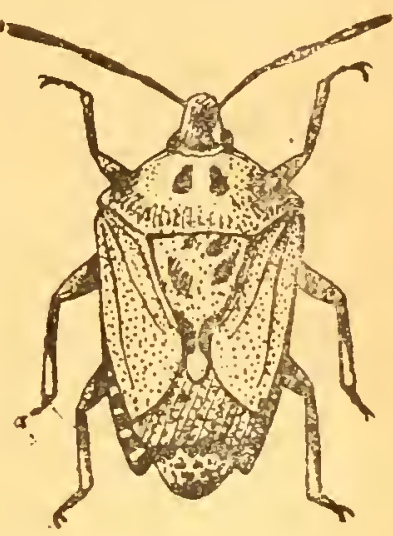

Fig 202. - La Pugaster nebulosa)

(d'après nat.).

$2^{0}$ Les Homopteres, dont les quatre ailes sont semblablés (Cigales).

Les Hémiptères sont répandus dans toutes les parties du monde; beaucoup de formes comptent parmi les plus dangereux parasites des végétaux (Phylloxera, Cochenilles, etc.).

IIIo Insectes a MétamorPhoses complètes (Holométabola).

$5^{e}$ Ordre: Coléoptères.

(Type: Hanneton).

Les Coléoptères se reconnaitront toujours facilement aux caractères suivants:

10 Les ailes de la première paire sont dures, elles constituent des sortes d'étuis cornés appelés élytres (1),

(1) D'où le nom de Coléoptères : koleos étui et pteron aile. 
immobiles pendant le vol et qui servent seulement à recouvrir et à protéger les ailes inférieures membra-

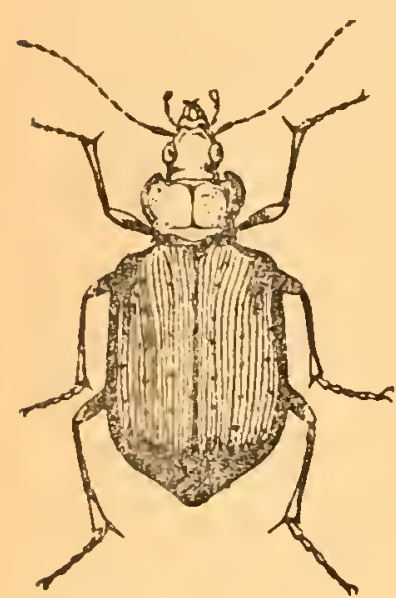

Fig. 203. - Le Calo. some sycophante (d'après nat.). neuses (Fig. 203). Mais, comme les ailes membraneuses, sont généralement très longues, elles doivent se replier en travers pour s'abriter sous les élytres.

$2^{\circ}$ La bouche est conformée pour broyer les aliments, car la plupart des Coléoptères se nourrissent de substances solides.

$3^{\circ}$ Enfin, tous subissent des métamorphoses complètes.

Cet ordre paraît être le plus nombreux de tous, parmi les Insectes, car il ne renferme pas moins de 100.000 espèces.

\section{6e Ordre: Névroptères.}

(Type: Fourmilion).

Les Névroptères dérivent évidemment des Archiptères, dont ils présentent tous les caractères; mais ici, les métamorphoses sont complètes.

Quelques Névroptères sunt adaptés à la vie tẹrrestre, comme par exemple les Fourmilions, les Ascaluphes (Fig. 204), etc.; mais d'autres ont

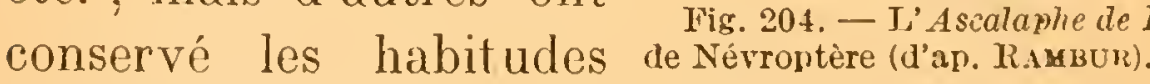

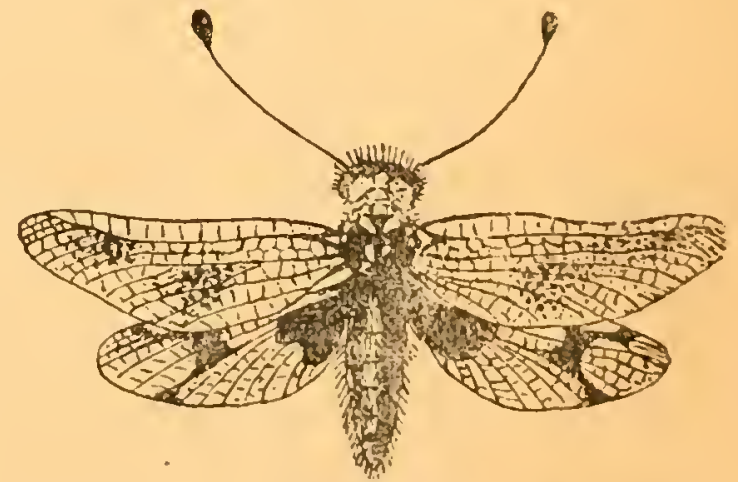

Fig. 204. - J'A scalaphe de Hongrie, type ancestrales, tels sont les Phryganes, dont les larves sont. aquatiques et vivent à l'intérieur de fourreaux qu'elles 
se fabriquent avec des grains de sable, des brindilles de bois ou de petites coquilles.

Les ailes des Phryganes sont couvertes de poils ou d'écailles ; leur bouche, profondément modifiée, tend à devenir une sorte de trumpe très courte, formée par les deux mòchoires accolées.

Tous ces caractères permettent de considérer les Phryganides comme les ancètres des Lépidoptères.

7e Ordre: Hyménoptères.

(Type: Abeille, Bourdons).

Les Hyménuptères normaux possèdent tous quatre ailes transparentes parcourues par des nervures peu nombreuses; de plus, les ailes inférieures, toujours beaucoup plus petites que les ailes supérieures, sont rattachées à ces dernières par une rangée de poils crochus. Cette disposition, qui oblige les deux ailes à se mouvoir en même temps, est certainement très avantageuse pour le vol (Fig. 205).

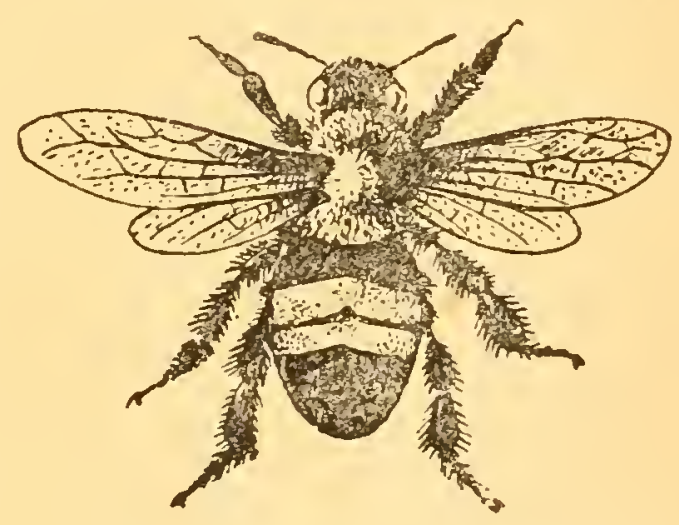

Fig. 205. - Un Bourdon, type d'Hyménoptère.

La bouche des Hyménoptères est constituée sur le type lécheur, c'est-à-dire que toute sa partie supérieure (lèvre et mandibules) ressemble au type broyeur, tandis que la partie inférieure (màchoires et lèvre inférieure) forme une sorte de languette servant à l'Insecte à recueillir les aliments liqquides, dont il fait sa nourriture, comme à l'aide d'un pinceau. 
Les Hyménoptères subissent tous des métamorphoses complètes, et plusieurs espèces, commo par exemple, les Abeilles, les Guêpes, les Fourmis, vivent en sociétés admirablement organisées.

\section{Se ordre: Lépidoptères.}

\section{('Type: Vanesse).}

L'ordre des Lépidoptères comprend tous les Insectes que, dans le langage courant, on désigne sous le nom de Papillons. Leur caractère principal consiste en ce que

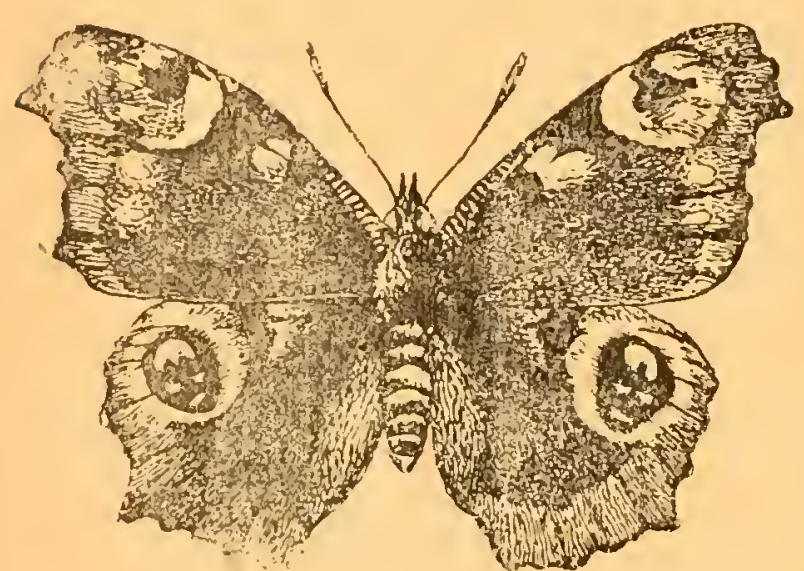

Fig. 206. - Le Paon de jour (Vanosso Io), type de Lénidoptère (d'ap. nat.). leurs quatre ailes sont. lecouvertes d'une multitude de petites écailles diversement colorées, se recouvrant comme les tuiles d'un toit. Aussitôt qu'on touche un Papillon, ces écailles se détachent sous forme d'une fine poussière qui se colle aux doigts (Fig. 206).

La bouche est disposée pour la succion ; elle est constituée par une trompe très long'ue, qui s'enroule en spirale lorsque l'Insecte est au repos, et qui se déroule quand il veut aller puiser sa nourriture au lond de la corolle des fleurs.

Les métamorphoses sont toujours complètes, et la nymphe immobile est désignée sous le nom de Chrysalide, la larve elle-même porte le nom de Chenille.

On peut distinguer deux sous-ordres parmi les Papillons : 
10 Les Papillons diurnes (Rhopalocères), dont les ailes sont relevées au repos.

$2^{\circ}$ Les Papillons nocturnes (Hétérocères), dont les ailes sont horizontales au repos.

Il existe quelques Lépidoptères utiles (Ver à soie), mais la plupart sont très nuisibles, à cause de la voracité de leurs chenilles, qui se nourrissent fréquemment de feuilles.

\section{Ordre: Diptères.}

\section{(Type: Mouche).}

Ces Insectes sont très faciles à distinguer de tous les autres puisque, comme leur nom l'indique, leur corps ne porte jamais que deux ailes. Ce sont les deux ailes de la paire antérieure qui ont persisté ; toutefois, celles de la partie postérieure n'ont pas complètement disparu; elles se retrouvent, sur les côtés du corps, sous forme de petites massues appelées balanciers, recouvertes par une sorte de petite écaille nommée cuilleron. Malgré la modification profonde qu'ils ont subie, les balanciers jouent certainement encore un rôle important dans la locomotion,

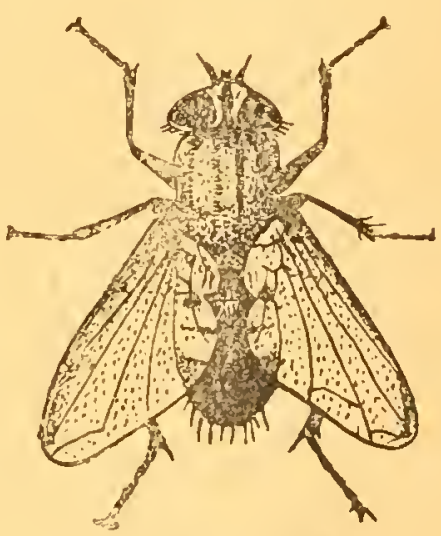

Fig. 207: - La Mouche commune, type de Diptère (d’ap. nat.. grossie 3 fois). car si on enlève, à une Mouche, ses balanciers, elle devient incapable de diriger son vol (Fig. 207).

La bouche est conformée pour aspirer les liquides.

Les métamorphoses sont complètes, et les larves, quoique très actives, sont presque toujours dépourvues de pattes ; la chrysalide est ovoïde et ne laisse voir, à 
la surface de ses téguments, aucun des organes de l'Insecte parfait.

Beaucoup de Diptères vivent en parasites sur les animaux ou sur les végétaux; quelque-uns, par leur piqûre (Moustiques, Mouches), peurent inoculer les maladies les plus dangereuses (paludisme, maladie $d u$ sommeil, fièrre jaune, etc.).

Dans les volumes suivants de l'Encyclopédie scientifique, et notamment dans ceux qui traiteront des Coléoptères, on trouvera des tíbleaux analytiques illustrés, qui permettront de déterminer tous les genres européens et mème un très grand nombre d'espèces françaises. 


\section{INDEX BIBLIOGRAPHIQUE}

Amaxs (P). Essai sur le vol des Insectes (Res. des Sc. nat. Montpellier $3^{\text {e }}$ série, t. II, 1883 et $t$. III, 5 pl., 188', in- $\left.8^{\circ}\right)$.

Anglas (J). 1. Sur l'his tolyse et l'his togénèse des muscles des Hyménoptères pendant la métamorphose. (Bull. de la Soc. entomol. de France, no 18, 1899 p. 348, Paris, in-8º).

- 2. Observations sur les métamorphoses internes de la Guêpe et de l'Abeille. Thèse (Bull. sc. France et Belgique. T. XXXIV, Paris, 1900, in-8 ${ }^{\circ}$.

- 3. Les Phénomènes des Métamorphoses internes (Scientia.

Série biologique. Paris, Gauthier-Villars, 1902, in-120.)

Audourx (J. V.). Recherches anatomiques sur le thorax des animaux articulés et celui des Insectes hexapodes en particulier ( $A n n$. des Sc. nat. Zooi.t. I. 1824, Paris, in-8 ${ }^{\circ}$, nombr. planches).

\section{B}

Balbiaxi (E. G.). La Parthénogénèse (Journal de micrographie, $2^{e}$ année, Paris, 1878 , in $-8^{\circ}$

Ballowitz (E.). Untersuchungen uber die Struktur der Spermatoz.oen, etc. Die Spermatozoen der Insekten. I. Coléopteren (Zeitschr. f. wissensch. Zool., t. I, Leipzig 1890, mit. 4 Taf.)

Bataillon (E.). La métamorphose du Ver à soie et le déterminisme évolutif. (Bull. scient. France et Belgique, t. XXV, Paris, 1893, in-8. Beauregard (H). Les Insertes vésicants, Paris, Alcan, 1890, in-80.

Berlese (A.). Gili Insetti. Organizzazione, embryologia, abitudine e rapporti coll'uomo, 2 vol. Milano, 1906-1909, in-4º.

Branc (L.). Étude sur la sécrétion de la soie et la structure du brin et de la bave dans le Bomby.r mori. Lyon, 1889. $4 \mathrm{pl}$.

Blanchard (E.). 1. Histoire des Insectes, 2 vol. Paris 1845, in-8 a avec $20 \mathrm{pl}$.

- 2. Recherches anatomiques et zoologiques sur le système nerveux des Insectes. (Ann. des Sc. nat. zool., t. 'T, 1847, Paris. 8 pl.) 
- 3. Les Insectes, métamorphoses, mœurs et instincts, 1 vol. Paris, 1866, gr. in $-8^{\circ}$ avec 200 fig. et 40 pl. $\left(2^{e}\right.$ édit 1876).

BoвRетzкy (N.). Ueber die Bildung des Blastoderms und der Keimblitter bei Insecten. (Zeitschr.fur wissensch.Zool.. t. XXXI, Leipig $1877,1 \mathrm{pl}$.)

Bonnet (Ch.). Trnité d'Insectologic ou observations sur les Pucerons Pari-, 1745,2 vol. in-12', \& pl.

Bordas (L.). 1. Les glandes défensives ou glandes anales des Coléoptères. (Anr. de la Faculté des Sc. de Marseille, t. IX, fasc. 5, Marseille $189\left(1,2 \mathrm{pl}\right.$. in $\left.-8^{\circ}\right)$.

- 2. Recherches sur les organes reproducteurs mâles des Coléoptères. (Ann. des Sc. nat. zool., $10^{\mathrm{e}}$ série, t. XI. Paris, 1900. 10 pl. in-8\%.)

'Boussac (H.). Le Scarabée sacré. (Le Naturaliste). 'E. Deyrolle, Paris 1905, in-40 p. 136-149.

Brauer (F.). Betrachtung uber die Verwandlung der Insekten in

Sinne der Descendenz-Thenrie. (lerhandlung de k. k. zorl-hol. Gesell. Wien. Bd. XIX, 1869).

Bremi (A. E.). Merveilles de la nature. Les Insectes, les Myriapodes et les Arachnides par Künckel-d'Herculais, Paris, J. B. Baillière, 2 vol. in-4 avec $36 \mathrm{pl}$.

Brongniart (Ch.). 1. Note sur quelques Insectes fossiles du terrain houiller qui présentent au prothorax des appendices aliformes. (Bull. de la Soc. Philomathique de

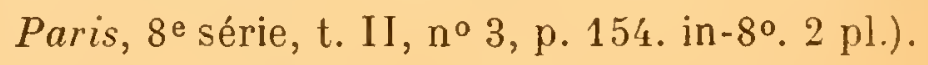

- 2. Recherches pour servir à l'histoire des Insectes fossiles des temps primaires, précédées d'une étude sur la nervation des ailes des Insectes. Saint-Étienne, 1893 , atlas de $37 \mathrm{pl}$. in-folio.

\section{C}

CAND rize (E.). Les moyens d'attaque et de défense chez les Insectes. (Bull. Acad. roy. de Belgique, t. XXXVIII, 1884, Biluxelles, in $-8^{\circ}$ ).

Guabunen (J.). Essaj sur le vol des Insectes. (Mémorrss du Muséum d'Ilist. not. Paris, 1820-1822, vol. VI, VII et VIIl, in-4º). 
Chatro (J.). Morphologie comparée des pièces maxillaires, mandibulaires et labiales chez les Insectes broyeurs. 1 vol. Paris, 1884.

Снододкоwsкy (N.A.). 1. Ueber den Bau der Testikel bei Schmetterlingen. (Zool. Anzeiger, t. III, 1880).

- 2. Ueber die Hoden der Lepidopteren (Zool. Anzeig. Leipzig, t. VII, 1884, in-80).

Ciraccio (G. V.). Dell'anatomia minute de quei muscoli che negl' Insetti muovono le ali. (Rend. Accad. Ss. Bologna, 1882 et Mem. Accad. Bologna, $4^{\text {e }}$ sépie, t. VIII).

Co.rstock (J. H.). 1. A manual for the Study of Insects. I thaca, 1895, 1 vol. in $-8^{\circ}$.

2. The Wings of Insects. An exposition of the uniform Terminology, etc. New-York, 1918. 1 vol. gr. in $-8^{\circ}$ 430 pp. $10 \mathrm{pl}$. et $427 \mathrm{fig}$. dans le texte.

Cuénot (L.). 1. Moyens de défense dans la série animale (Encyei. scient. des Aides mèm. 1 vol., Paris, 1892, in-120).

- 2. Études sur le sang, son rôle et sa formation dans la série animale. Part 2. Invertébrés. Note préliminaire. (Arch. de zool. expériment., $2^{\mathrm{e}}$ série 1888 et

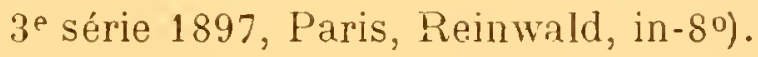

Da gurx (E.). Les Insectes comestibles dans l'antiquitéet de nos jour" ; (Le Naturaliste, Paris, E. Deyrolle, nos 285-290, 1900, in-4º).

Darboux et Houard (C.). Catalogue systématique des Zoocécidies de l'Europe et du bassin méditerranéen. (Bull. scient. de la France et de la Belgique $6^{\text {e }}$ série, t. XXIV, Paris, 1901, gr. in-80, 540 pages. 850 fig.) -- 2e édit. Hermann, Paris, 1907.

Dewitz (H.). Beiträge zur Kenntniss der postembryonal. Gliedmassenbildung bei den Insecten. (Zeitschr. f. wissens Zool. Bd. XXX, suppl. Leipzig, 1878, in-8\%).

Dufour (L.). Recherches ana tomiques sur les Carabiques et sur plusieurs autres Coléoptères. (Ann. Sc. nat. zool., Paris, t. II, III, IV, V, VI, VII, 1824-26 et nombreux autres Mémoires jusqu'en 1862). 
Fabre (J.H.). 1. Mémoire sur l'Hypermétamorphose et les mœurs des Méloïdes (Ann. des Sc. natur. zool., $4^{\mathrm{e}}$ série, t. VII, Paris, 1857 , in $\left.-8^{\circ}\right)$.

- 2. 2e Mémoire. (Ann. des. Sc. nat. zool., $4^{\mathrm{e}}$ série, t. IX. Paris, 1858 , in $\left.-8^{\circ}\right)$.

Faivre (E.). Recherches expérimentales sur la distinction de la sensibilité et de l'excitabilité dans les différentes parties du système nerveux d'un Insecte, le Dutiscus marginalis. (Ann. des. Scienc. nat. Zool. $5^{\mathrm{e}}$ série. T. I. 1864, in-8º.

Fauvel (Albert). Faune gallo-rhénane ou species des Insectes qui habitent la France, la Bclgique, etc. Caen, Le Blanc-Hardel, 1868, in- $8^{\circ}$.

Forel (A.). Expériences et remarques critiques sur les sensations des Insectes. (Recueil zorl. Suisse, t. IV. Genève, 1887. Appendice. t. IV, in $\left.-8^{\circ}\right)$.

\section{G}

Gadeaude Kerville (H.). Les Insectes phosphorescents, Rouen, 1er fasc. 55 p. 4 pl. color. 1881 , 2e fasc. 135 pp. 1887 ; in-80.

G.izagnaire (I.). Des glandes chez les Insectes. (C. R. Acad. des Sciences, Paris, t. CII, 1886, in- $4^{\circ}$.

Grard (A.). Convergence et pœcilogonie chez les Insectes (Bull. Soc. entom. de France, t. XLIII, 1894.

Girard (M.). 1. Les Insectes. Traité élémentaire d'entomologie, 3 vol., Paris 1873-1885, gr. in- $8^{\circ}$.

- 2. Les métamorphoses des Insectes. Paris, Hachette, $5^{\mathrm{e}}$ édit. 1879 , in-12.

Graber (V.). Die Insekten. I Theil. Der Organismus der Insekten. II e Theil. Vergleichende Lebens-und Entwicklungsgeschichte der Insekten. München 1877, 2 vol. in-12 ${ }^{\circ}, 404$ fig.

Grassi (B.). Les ancêtres des Myriapodes et des Insectes. Anatomie comparée des Thysanoures et considérations générales sur l'organisation des Insectes. (Archis. italicn. Biol. vol. XI. Turin, 1889.

Guiter (F.). Sur la création d'une Station entomologique à la Faculté des Sciences de Rennes. (Archives de sonl. cxpérimcutale et générale, vul. VI. (p. XCIII, Paris, Schleicher, in-8\%). 
H

HaAse (E.). Die Abdominalanhange der Insekten mit Berücksichtjgung der Myriapoden. (Morphol. Jahbr. Bd. XV, Leipzig, 1889, in- $8^{\circ}$.

HaUSER (G.). Recherches physiologiques et histologiques sur l'organe de l'odorat chez les Insectes. (Archiv. de zool. experim., t. VIII, Paris, 1880, in- $\left.8^{\circ}\right)$.

Her (Osw.). Die Insektenfauna der Tertiärgebilde von Eningen und Radaboj. Leipzig. 3 Theilde 1847-53, in-40.

Henneguy (L. P.). 1. Les modes de reproduction chez les Insectes. (Bull. de la Soc. philomatique, ge série, t. I, Paris, 1899 , in-80).

2. Les Insectes. Morphologie, Reproduction, Embryogénie. Paris. Masson, 1904, arec 622 fig. et 4 pl. en couleur hors texte, gr. in- $5^{\circ}$.

Heynoxs (R.). Entwickelungsgeschichtliche Untersuchungen an I.epismr saccharina. (Zeitschr. f. wissens, zoologie, t. XLI I, Leiprig, in- $\left.8^{\circ}\right)$.

H JULBERT (C.). 1. Rapports naturels et phylogénie des principales familles de Coléoptères. (Bull. des Sciences nat. de la Faculté des Sc. de Paris. A. Fourneau, 1894. 4 Année).

- 2. Le système tarsal; étude d'entomologie systématique. (.Miscellanea entomolonica. Narbonne, 1895. T. II!. in- $\left.8^{\circ}\right)$.

- 3. La Loi de la Taille et l'évolution des Coléoptères. (Congrès internat. de Zoologie. II onaco, 1913. g. in-8, 46 pp. 14 fig. dans le texte).

Howard (L. O.). A Study in Insect parasiitsm. (Divis. of Entomol. 7ecínical, série no 5 , Washington, 1897, in-8º.)

\section{.)}

JCQuelix-Duvar (G.). Genera des Coléoptères d'Europe comprenant leur classif. en familles naturelles, 4 vol. Paris. A Deyrolle, 1857$1862, \mathrm{pl}$. gre in $-8^{\circ}$.

Janet (Ch.). 1. Essai sur la constitution morphologique de la tête de l'Insecte. G. Carré et Naud, Paris, 1899. in-80 avec $7 \mathrm{pl}$. 
- 2. Anatomie du corselet de la Myrmica rubra Reine (Mém de la Soc. zool. de France. Paris. T. XI. 1898, p. VI.)

\section{$\mathbf{K}$}

Kieffer(J.-J.). Synopsis des Zoocécidies d'Europe, (Ann. de la Soc. entom. de France, Paris, 1901, in- $\left.8^{\circ}\right)$.

Kirby (W.) et Spence (W.). Introduction to entomology, 1815-1826.

Kolbe (H.-J.). Einführung in die Kenntniss der Insel:ten, Berlin,

F. Dümmler, 1895. 324 fig. in- $8^{\circ}$.

Kowalevsky (A.). Beiträge zur Kenntniss der nachembryonalen Entwickelung der Musciden. (Zevtschr. \%. wissensch. zool, t. XLV, Leipzig, 1877, in-8\%).

KünckeL-D'Herculais. (J.). Observations sur l'hypermétamorphose ou hyprodie chez les Cantharidiens. La phase dite de pseudochrysalide considérée comme phénomène d'enkystement. (Ann. de la Soc. entom. de France, Paris, t. LXIII, 1894).

I.

Lacaze-Duthiers (H.). Recherches sur l'armure génitale femelle des Insectes. (Ann. des Sc. nat. zool. t. XII, 1849 ; t, XIV, 1850 ; t. XVIII, 1852 ; t. XIX, 1853, Paris, in- $\left.\delta^{\circ}\right)$.

Lacordare (Th.). Introduction ì l'Entomologie, Paris, Roret, 183'1838, 2 vol. in $-8^{\circ}$ avec 2 's planches.

La ueEre (A.). La raison d'être des métamorphoses chez les Insectes. A (Ann. de la Soc. entomol. de Belgique, t. XLIII, 1899, Bruxells, in $-8^{\circ}$.

LAxg (A.). Traité d'Anatomie comparće et de zoologie, Paris, 1891. 1 vol. in- $8^{\circ}$ av. fig. (Trad. (r. Curtel).

Latreille 1. Précis des caractères génériques des Insertes disposts dans un ordre naturel, Brives, an V (1796), in- $8^{\circ}$, $208 \mathrm{pp}$.

- 2. Des Insectes peints ou sculptés sur les monuments antiques de l'Ḱgypte. (Mémolies du. Muséun d'Misl. naturelle, Paris 1819, 21 pp. 1 pl. in- $-_{1}^{\circ}$.)

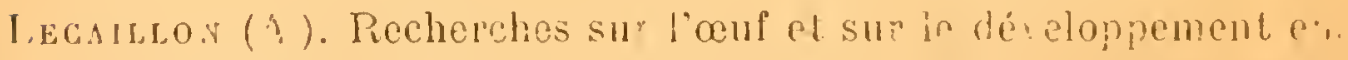
bryonnatre de quelques Chrysomólides. 1. Disiré, Patí: 189. 4 pl. in $-8^{\circ}$. 
LEYDIG (F.) Besträge zur Anatomie und Histnlogie der Incekten. (Zool. Anzeig. Leipzio, 1887).

LeE (J.). La dynamique des phénomènes de la rie. Paris, Alcan, 1908, in-8 (Trad. H. DAUDIN et SCH.モFFER).

Luввоск (J). De l'origine et des métamorphoses des Insectes (Trad. j. (irolous. Paris, C. Reinwald, 1880. in-12, 7 pı.

\section{M}

Marey (E.I.). Mémoire sur le vol des Insectes et des Oiseaux. (Aun des Sc. nat. Zool., 5e série, t. XII, 1869 et t. XV, 1872, 42 fig. Paris, in $\left.-8^{\circ}\right)$.

Manchal (Paur). 1. Utilisation des Insectes auxiliaires entomophages dans la lutte contre les Insectes nuisibles à l'Agriculture. (Annales de l'Institut agronomique, 2e série, t. VI, Paris, 1907, in-80).

- 2. L'Entomologie appliquée en Europe. (Bull de la Soc. nat. d'acclimatation de France. Paris, 1896, in-8\%).

3. Les Sciences biologiques appliquées à l'agriculture et la lutte contre les ennemis des plantes aux États-Unis, Paris (Lhomme), 1916, $1 \mathrm{vol}$. in-80.

Marer (Paul). Ueber Ontogenie und Phylogenie der Insekten, Iena, 1876. (Zeitschrift fur Naturissenschaft. X. Band. in-8º, p. 121221 avec 4 planches).

Mitil. (L.) and Dexir (A.). The structure and Life-history of the cockroach, (Periplaneta orientalis). (An introduction to the stud"i of Insects. London, 1887, in-80).

II vgativi (P.). Ricerche sul canale digerente dei Lamellicorni fitofagi. (Larve e Insetti perfetti). (Mitteil, zool. Station zu Neapel t. IX, Berlin, 1889-1891, 7 pl).

MénIY (P.). Les parasites articulés che: l'hommesetles animaux utiles. Paris, Masson, $1895,1 \mathrm{rol}$. in-80 et atlas XXI pl.

\section{X}

Nawport (F.). On the L'se of the Antenne of insects. (Trans entom. Soc. London, t. II, 1840. in-80).

LES IXSECTES. - 2e édit. 
Овевтий (Ch.). 1. Observations sur les lois qui régissent les variations, chez les Insectes Lépidoptères. (Feuile des Jnes. Nat., $3^{\text {e }}$ série, no 277. Paris, 1893, in-8\%).

- 2. Du Mimétisme chez les Insectes. (Feuill. des. Jnes. Nat. $n^{\text {or }} 304,308,309,1896 . n^{\circ} 313,1897$, Paris, in $\left.-8^{\circ}\right)$.

Osbory (H.). The duty of economic entomology. (Bull. no 7, New Série, Washington. 1898, in-8 ${ }^{\circ}$, p. 6-13).

OUSTA LET (E.). Recherches sur,les Insectes fossiles des terrains tertia.res de la France, Paris, Masson, 187/4. 1 vol. in-8 ${ }^{\circ} .1^{\text {re }}$ part., 17/i p., $6 \mathrm{pl}, 2^{\mathrm{e}}$ parı., $381 \mathrm{p} ., 6 \mathrm{pl}$.

\section{I'}

PACKARD (A.S.). A Text-book of Entomolog!, including the anatomy, physiology, embryology and metamorphoses of Insects. NewYork. The Macmillan Company, 1903, in- $8^{\circ}$.

Perez (Ch.). Sur la métamorphose des Insectes. (Bull. de la Soc. entomol. de France, No 20, p. 398, Paris 1899, in-8).

Plateau (N). 1. Sur la force musculaire des Insectes. (Bull. de l'Acad. :ou. de Belgique, 2e série, t. XX, Bruxelle; 1865 et t. XXII, 1866).

- 2. Recherches expérimentales sur la vision cher. les Insectes.

(Bull. Acar. Belg. 1885-1889 Bruxelles in-'(\%).

?eyerimitofr (P. de). La larve des Insectes Metabola et les idées de Fr. Brauer. (Feuill. des Jnes. Nat., nos 398,399 , Paris, in-8º.

Peytouratu (S. A.). Contribution à l'étude de la Morphologie del'Armure gémitale des Insectes. J. Durand Bordeaux, 1895, $22 \mathrm{pl.}$

\section{$\mathbf{R}$}

Redtenbagher (J.). Vergleichende Studien über das Flugelge ider der Insekten. (Ann. des. kl. naturhist Hofmuseums zu. Wien. 1886. mit 12 Taf.)

RuLE (C. V.). 1. The periodical Cicada.( Report of the Commission of A sriculture, Washington, 1886, in-8\%).

- 2. Sur l'importation artificielle des parasites et ennemis naturels des Insectes nuisibles aux régitaux, (C. R. des séanres dil Congries international te zoologie. Pris, 1889). 
$\mathbf{S}$

Schrteder (Ch.). Was schützt den Falter ? (Illustr. Wochenschir. f. fintomol. I. Jahrg. Neudamm 1896, in-8 ${ }^{\circ}$ ).

ScUdDER (S. H.). Palæodictyoptera, or the affinity and classification o! palro: oic Herapoda. Boston, 1885.

Silfvenius (A. J.). Beiträge zur metamorphose der Trichopteren. ( icta Socictatis pro Fauna et Flora fennica, t. XXVII. Helsingfors. 190\%, in-8. mit \& Tafeln).

Siltala (A. I.). Ueber die postembryonale Entwiclilung der Trichopteren Larren. (Zocl. Jahrbüchern. Supplementbanil. IX. G. Pischer. Jena, 1907, in-80, 1; pl.).

STANDFUs (M.). On the causes of variation and coloration in the imago stage of Butterflies, with suggestion on the etablissement. of new species. (The Entomologisi, rnl. XXVIII, London 1896, in $\left.-8^{\circ}\right)$.

Straus-Durckimen (H. F.). Considérations générales sur l'anatomie comparéc des animaux articulés, auxquelles on a joint l'anatomie descriptive du Hanneton vulgaire. Paris, 1828, avec atlas de 19 planches.

\section{$\mathbf{T}$}

Tower (W. J.). The developpement of colors and color pattern of Coleoptera, with observations upon the developpement of color in other orders of Insects (Publications of the University of Chicago, 1903, t. X, gr. in-80).

Trimex (R.). Protective ressemblance in Insects (The Entomologist. t. XVIII, London, 1885, in-8 $\%$ \%

Trouessart (Dr E.). La Géographie zoologique. (Biblinth. scient. contemp. Paris, J.-P. Baillière, 1890, in-12).

\section{V}

VANEY (C.). Contribution à l'étude des larves et des métamorphoses des Diptères. Thèse. (Ann. dc l'Unio. de 1.yon, t. I. 1902, in-80).

VAYSSIÈre (A.). Recherches sur l'organisation des larves des Éphémérides, (Ann. des Sr. nat. zool., 6e série, t. XIII, Paris, 1882, in $\left.-8^{\circ}\right)$. 
VIALLANes (H.). Études histrologiques et organologiques sur les centres nerveux et les organes des sens des animaux articulés. Nombreux Mémoires. (Ann. des sc. nat. arıl., $6^{\mathrm{e}}$ el, $7^{\mathrm{e}}$ série, Parı, 188 -1991 , in $\left.-8^{\circ}\right)$.

\section{W}

Warrer (N.). Spontane Fortpflanzung bei Insektenlarven. (Denkschrift. de Kais Kasan'schen Unicers, 1862).

Weisuann (A.). Studien zur Descendenztheorie. I. Ueber den Saison-Dimorphismus der Schmetterlinge. - II. Die Entstehung der Zeichnung bei den Schmetterlings-Paupen. Leipzig, 1876, in $-8^{\circ}$ ).

Westwood (J. O.). An Introduction to the motern classification o. Inscots. 2 vol. Longmann and C. London, 1838-4n,.in-8n).

WheEler (W. M.). The primisive number of Malpighian vessels in Insect.s. (Psyche. Cambr. 1. II, 189?, in-8\%).

Woodwort $($ C. W.). The wings veins of Insects (University of California pubiications. Technicnl Bulletins, vol. I, 1906, Sacramento, gr. in-3").

Il ne fallait pas songer à donner ici une bibliographie complète de la littérature entomologique; nous nous sommes borné à l'indication des ouvrages généraux les plus importants, réservant pour les volumes qui suivront les travaux plus spécialisés.

Le lecteur qui voudra appronfondir une question, trouvera d'ailleurs, dans la plupart des auteurs cités, une bibliographie étendue ; mais, nous signalons tout particulièrement sousce rapport les ouvrages de Lang, Kolbe, Packard, HexNEGUY et BERLESE. 


\section{TABLE ALPHABÉTIQUE}

DES AUTEURS ET DES MATIÉRE

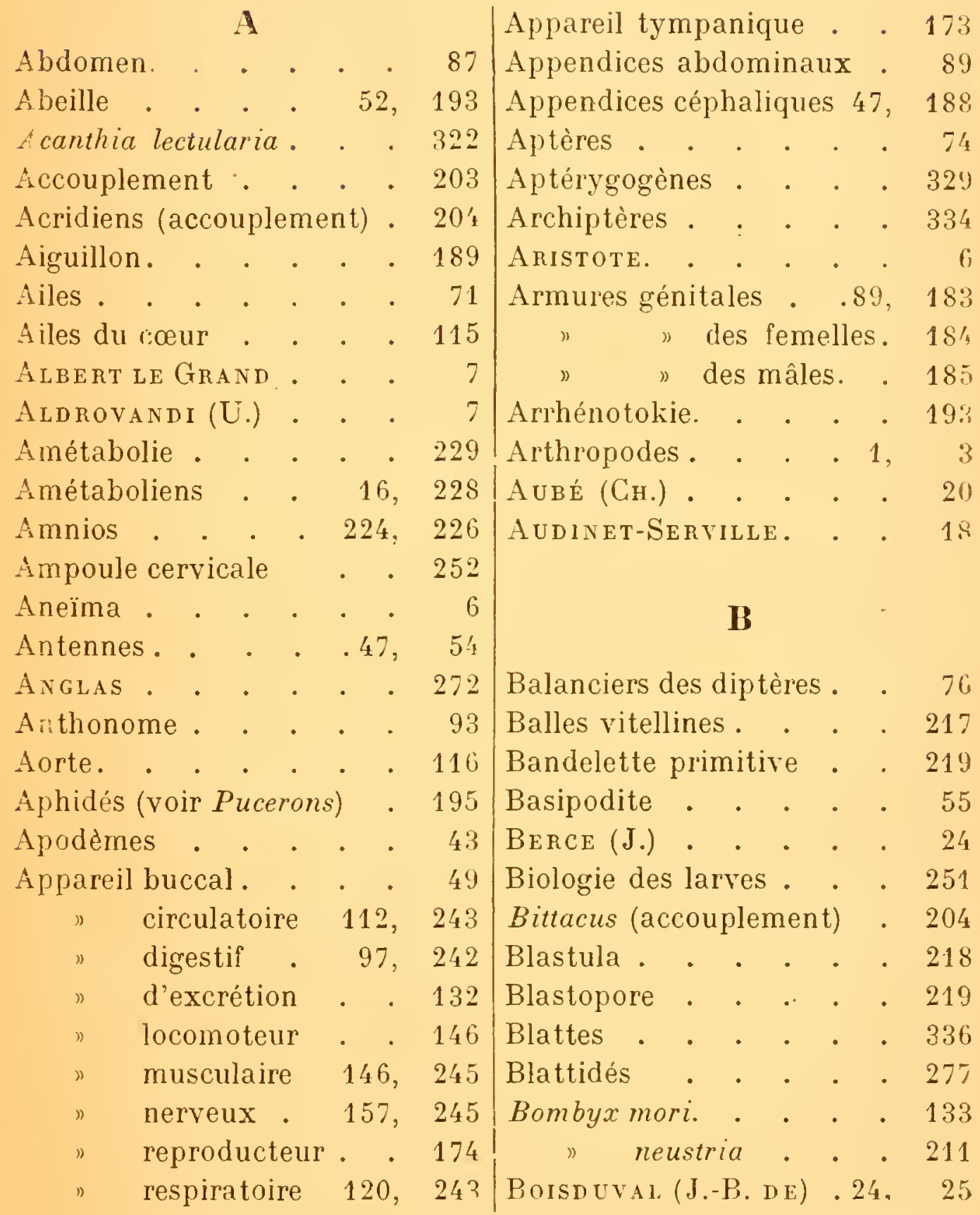


Bonvet (Сh.) . . . $11 /$ Chenille . . . . 231

Bordas (L.) . . 177, 185 Chevrolat (A) . . . 27

Bouche des insectes. . . 49 Chironomus. . . . 187

Bourdonnement. . . 172 Chitine . . . 39, 41

Braconides . . . 134 Chorion . . . 214, 215

Brachycère; . . . . 49 Chrysalide . . 228, 261

Branchies permanentes . 129 Chrysomélides . . 47, 2:10

" rectales . . . 24 4́ Cicada septendecim. . 231

" trachéennes. . 129 Circulation . . . 117

Brisout de Baryevile (l..) 29 Cire . . . . . . 308

Broyeurs (Insectes). . . 50 Classification des Insectes. 329

Brullé (G.). . . . 22 Claprillie (J) . . 13

Buprestides . . . . 25't Cloeon dimidiatum . . 85

Burneister $(\mathrm{H})$. 16, 23 Clou scolopal . . . 170

Byrrhides . . . . 241 Clypæus . . . . . ${ }_{4} 6$

G Coccinellides . . . 280

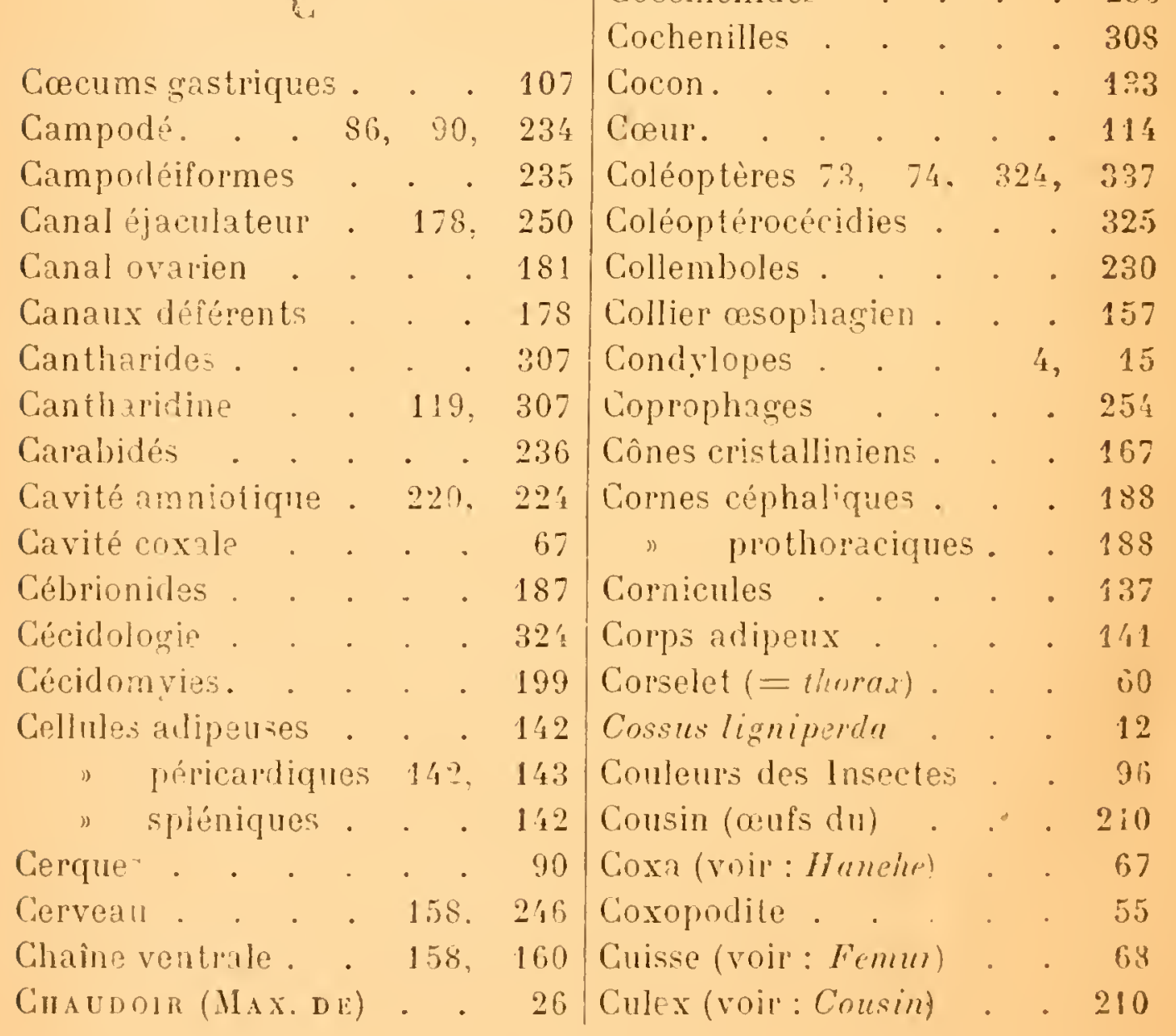


Curculioniformes • . . $235 /$ Elytres . . . . . 72

Curculionites prodomus . 279 Elytroptères. . . . 80-81

Cuticule primaire . . 39, 40 Emboîtement des germes . 9

Cuticule secondaire . . 40 Embryon . . . 219

Cuvier (G). . . . . 1'1

\section{?}

Dasileptus Lucasi . . . 276

Decticus rerrucivorus . . 91

Dégénérescence (voir : Histolyse). . . . . 264

DEJEAN (A.). . . . . 19

Dermaptères . . . . 13

Dermatoblastes. . . . 24?

Deutocérébron . $58,159,248$

Développement postembryonnaire

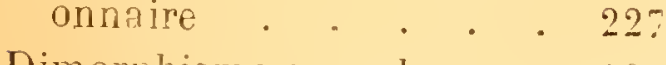

Dimorplisme sexuel . = $180^{\circ}$

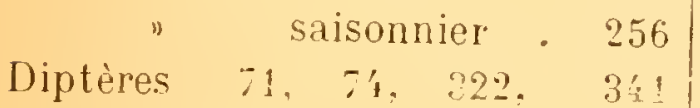

Diptèrocécidies . • . . 326

Disques imaginaux . 12, 265

Distribution géograplique 292

Dufour (L.). • . . . 17

Dupoxchel (A.-J.) . . 18

Durée de la vie . . . 20 ?

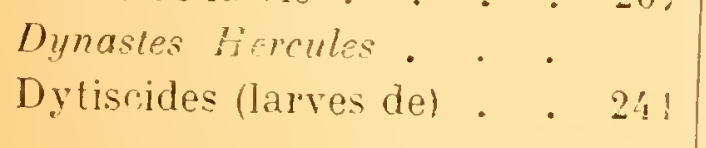

$$
\text { E }
$$

Ecaille:

Ecdysis (vojr: inues) 95

Eclosion dies larves.

Ectobaitima Gaudryi

Ectoderme

Ectoptychiques.

Ecusson.

Epistome

" ectoblastique.

entoblastique

Eneïma . . . . . 6

Encyrtus (ponte de l') . . 209

Endoderme . . . . 225

Endopodite . . . . 56

Entoma . . . . 6

Entomocécidir . . . 325

Entomologie . . 2

Entonnoir . . . 104

Entothorax . . . 43

Entoptychiques . . . 221

Ephéméridés . . . 243

Ephippigers . . . . 173

Epicrâne, . . . 46, 47

Epimères. . . . . 43

Epimérites . . . . 184

Epipharynx . . . . 57

Episternes . . . 43

Episternites . . . . 184

Epoque carbonifere. 46

Epoque dévonienne _. $\quad 276$

Eruciformes . . 235

Exopodite . . 56

Exuvie (voir: inlue) . . 253

Elatérilormes

\section{6}

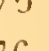


Fémur (voir : Cuisse) .

Fermeture des stigmates . 128

Fibres musculaires . . 154

Fibrilles musculaires . . 155

Fibroïne . . . . 135

Flagellum (voir antennes).

Force musculaire des Insectes

Fossettes olfactives. - -165

Fourmis . 137

Fourmilion (larve) . . . 242

Frein . . . . . 77

Front. . . . . 46

Fingore porte-lanterne . . $\quad$ i44

Funicule (voir : antennes).

\section{G}

Gaines ovariques . 180, 181

Galea. . . . . . 55

Galles (voir: Cécidies) . . 324

Ganglioblastes . . . 247

Ganglions abdominaux. . 160 " cérébroïdes 157,248 " sous-œsophagiens

. . 157, 248

$\operatorname{Gastrula} \cdot \cdot \cdot \cdot \cdot \cdot 11$

Geminger (M.) • . . 28

Génération spontanée . . 8

Geoffroy (E. L.) · . 12

Gésier . . . . 101

Gesner (C.) . . . . . 7

Girard (M) • . . . . 28

Glandes anales . . . . 110

défensives. . 138

dermiques . . 140

venimeuses . . 137

" cirières
Glandes coxales

» cutanées . • . 96

138

) salivaires . . 106

" sébifiques $139,182,211$

" séricigènes . . 132

Globules polaires . • 216

Globules sanguins . . . 118

Glossina morsitans . . . 322

Gedart (J.). • . . .

Goureau (Cil.) . . . 22

Goût (sens du) . . . . 16'

Grès . . . . . 135

Grillons . . . . . 336

Guenée (A). • • • . 26

Guitel (Prof. F.) • • . 317

Gyrinides (larve des) • . 129

\section{H}

Hanches (voir : Pattes) . 67

Hanneton . . . . 236

Herold (Ed, voN) . . 28

Helminthoïdes . . . . 237

Hémiélytres. . . . 336

Hémimétabolie . . . 231

Hémiptères 72, 74, 321, 336

Hémiptérocécidies . • 325

Hémiptychiques • • . 222

HenNeguY (Fro!. 1.) 221，248

Hétérorères . . . . 341

Hétćromères. . . . 307

Hétéromorplivar. . . 55

Hétéroparthénogénèse 193,195

Hétéroptères . . . 337

Hexapodes . . . 4, 14

Histoblastes . . . . 12

Histogénèse . . 264, 26 ;

Histoire de l'Entomo-

logie. . . . . 5 
Histolyse . . . 264, 269 Holométabolie . . . . 231

Holométaboliens . . . 228

Holoptychiques. . . . 222

Homochromie . . 285, 287 Homoptères. . . . . 337

Howard (L. O.). 31, 314, 319 Hydrophile . . . . 121

Hydrous caraboïdes . . . 122

Hyménoptères . 58, 7/4, 339 "entomophages . 327

" phytophages . 254. " parasites . . 209

Hyménoptères porte-aiguillon 237 (thorax des) . . 66

Hyménoptérocécidies . . 326

Hypermétamorphose . . 232

Hypnodie . . . . . 232

Hypoderme . . . . . 39

Hypogéisme . . . . 286

Hypométabolie . . . 230

Hyponomeutes . . . 328

Hypopharynx . . . . 56

Icerya Turchasi. . $\quad 319,320$

Ichneumonides . . . 213

Imago . . . . 228, 229

Insecte (Type) . . 2, 3, 34

Insertion des muscles . . 155

Insectes broyeurs . . . 50

Insectes comestibles . . 302 " entomophages. . 32;

" lécheurs . . . 52

" piqueurs . . . 53

" suceurs. . . . 53

Insufflation des chenilles . 10

Intestin antérieur . • . 99
Intestin moyen . . $\quad 99,101$

" postérieur. 99, 102

Isoparthénogénèse . . . 193

\section{J}

Jabot . . . . . 100

JacQuelin du Vat. (C.) . . 2'́

Jambe (voir : Tibia) • • 69

Janet (Сн.) . . $151 \quad 156$

Japyx • . . 86,96

Joues. . . . . . 46

JUSSiEU (A-.L.) . . . . 13

\section{$\mathbf{K}$}

Lallima . . . . . 291

KALT (E.) . . . . . 167

Kamaka . . . . 304

Kítby (W. F.) . . .14. 16

L.

Labium (lèvre infér re) . 51, 56

Labre (lèvre supre) . . $\quad 59$

Lacordatre (Тh.) . . . 23

Lacune péricardique . . 117

Lamellicornes . . 48, 236

Lampyre. . . 144, 240

Lampyrides (org. lumineux) 189

Langue (hypopharyna) . . 56

Languette . . . 52, 56

Larves . . . . . 227

" amphipneustiques . 244

" apneustiques . . 244

" campodéiformes . . 235

" curculioniformes . . 237

" cyclopéennes. . . 238

" élatériformes . . . 235 
Larves éruciformes .

$235 \mid$ LyOXET (P.)

" helminthoïdes.

237

"holopneustiques . . 243

" métapneustiques. . 244

, naupliformes . . 237

„ péripneustiques . . 243

" scarabæiformes . . 236

"staphyliniformes. . 235

"vermiformes . . . 237

Latreille (P.) • • . . 13

LeAch (G.-L.) • . . . 16

LE CONTE (J.) • • . 27

Lécheurs (Insectes). • • 52

Lépidoptères. . 72, 74, 340 " séricigènes - 310

Lépidoptérocécidies . • • 325

Lépismes • • . . . 86

Leucocytes . .118, 119, 272

Leeuvenhogk (A. De) • • 10

Lèvre inférieure. . . 51, 56

Lèvre supérieure . . . 59

Libellules (accouplement) . 205

Lichtenstein • • • 28

Ligule • • • • . 56

LINNÉ (CH.). . . . . 11

Liparis chrysorrhea. 318, 3 $\gg$ dispar . . . . 199

Lister (M.) . . . . 10

Lobes cérébraux . . . 248

Lobes optiques . . . . 248

" procéphaliques . . 246

Lobe protocérébral . 247, ¿48

Locusta viriaissima. . . 288

Locustidés . . . . 62

Longicornes . . . . 48

Lucanides . . . . 187

Lucane Cerf-volant. . 51, 187

Isucioles . . . . . 189

Lyocytose . . . . 27:2

Machilis .

M

Mâchoires . . . 51, 55

Malacodermes . . . 185

Malpighi (M.) • . 8

Mandibules . . $51, \quad 56$

Manne des Hébrevx . . 302

Mantes . . . . . 2\$1

MARChal (Prof. P.). . . 317

Marseul (A. de) . . . 29

Maturité génitale . . 271

Maxillaires (palpes). . . 51

Mayer (Paul) . . . 34

VIAYet (Prof. Valéry) • . 317

Vécanisme de la respiration 130

Meigen (Сh.) . . . . 18

Mcloë proscarabæus. . . 307

Méloïdes . . . . 307

Melolontha oulgaris. . 124

Membranes embryonnaires 219

Membrane ectotrachéale - 124

"endotrachéale . 125

Menton . . . 52, 56

Mériax (Sybille Df) • . 10

Mésenteron . . 99, 101

Mésoderme . . . 225

Mésonotum . . . . 61

Mésotergite . . . 63

Mésosternum . . . 61

Mésothorax . $60,61,63$

Métabola . . . . . 16

Métamères . . . . 41

Métamérie . . . . 41

" de la títe. . 57

Métamorphoses . . 226, 230

Métanotum . . . . 61

Métasternum . . . 61 
Métathorax . - $60,61,65 \mid$ Nervures indépendantes 78, 83

Microgaster glomerator. . 327 Nervure marginale . . . 81

Micropyle . . . . 216 Nervures principales 78, 81

Miel

308 Nervures tranverses. $79,8 \%$

Mrlitère (P) . . . 28

Mimétisme . . 285, 287

Neuromères . . . . 158

Mormolyce phyllodes . 290

Neuroptères . . . 80

Morpho Rethenor

300

Névroptères . 72, 74, 79, 339

Morpho Cypris .

Mouches .

300

:

Mouche ('Tsé-tsé)

Mucoïdine

Mues (=ecdysis)

323

13 ś

253

Mulsant (E.) . . . 25

Muqueuse intestinale 103, 104

Noel (Dir. P.)

317

Nopal (cactus) . . 308, 312

Notonectes . . 69, 210

Muscles . . . . . 146

Muscles aliformes . . . i15

" de la tête..
" du thorax .
" de l'abdomen .
" jaunes de Leydig.
" moteurs des ailes.

148

149

153

154

150

" des pattes.

Muscles (structure des)

152

153

Mycophages

254

Myriapodes .

Myrméléon

281

Notum . . . . . 42

Novius cardinali: . . 320

Nymphalidés • . . 296

Nymphe . . . 228, 261

Nymphose . . . . 26.3

$$
0
$$

Oberthur (Charles) 24, 291

Oberthur (René) . . 27, 319

Ocelles . . . 166, 242

Occiput . . . . 46

Odonates . . 63, 231

Odorat (sens de l') . . . 165

Eil . . . . 54, 166

Enocytes . . 142, 143

Esophage . . . . 100

Estrides . . . . 323

Euf controlecithe . . . 215

CEufs . 208, 209, 210, 214

Vaissance des premières nervures.

78

Naupliformes

Necrobia (paras. de l'œii)

Némocères

Néoptères . . . 80 ,

Neorinopsis sepulta. . .

Néoténie . . . . . 238

Neris . . . 160 ,

Nervation des ailes.
23 J Oligonéphridés . . . 109

324 Olinier (A.) . . . . 13

49 Ommatidie . . . . 167

81 Ongles . . . . . 70

280 Onychophores . . . 44

238 Oothèque . . . . 211

248 Origine des ailes . . 8'

78 Organes auditifs . 169, 171 
Orranes chordotonaux. . $170 \mid$ Parthénogénèse normale . 193

$"$ copulateurs 181,184

"tympaniques ${ }^{\circ} .171$

$"$ des sens . 163, 248

" du goût . . 164

"de l'odorat. . . 165

" de l'ouie . . . 169

"du toncher. . . 163

" de la vision . . 166

Organes génitaux . 174, 249

" " des mâles . 176

" "des femelles. 179

"lumineux . . . 144

Oiganes pulsatiles . . 117

" splérniques . . 145

Ornéodes . . . . . 73

Orthoptères . 73, 74, 335

Orthoptérooécidies . . 326

Orthonévroptères

Oryctes nasicornis

Osmétérium

Osseleis.

Ostiole

Ovaires

Oviductes

Oviscapte

Ovules

I3

Pudogénèse.

Pilxoblattina Dourillei.

Piléoentomologie

P.lpes labiaux

") naxillaires

Papilio Ajax

Paraglosses.

Paraptères

Pardsitisme cher les Insectes 821

Parthénogrénèse.
$19 ?, \quad 199$

334 185

139

85

114

180

$180, \quad 181$

18?, 183

180, 181

275

275

51

51 Pièce basilaire

257 "prébasilaire

56 Piquenrs (Insectes).

7 'i

Plantule.

Plenres.

Pline (IAncien) accidentlle. 195

larvale. 198 expérimentale 201

75

67

230

14

38

48

32.2

89

178

114

167

$\left.4_{1}\right\}^{\prime}$

126

21

$-31$

273

272

62

99

287

144

288

285

255

195

$25 / 4$

324

242

46

46

53

70

41

7 
Poche copulatrice

Podurides

Poils .
" tactiles

" sensitifs

Polynéphridés

Ponte des œufs

Postépistome

Postscutellum

Præscutum

Préformation

Presse

Proctodæun (Intestin post ${ }^{\mathrm{r}}$ )

Progénèse

Pronotum . 61,

Prosternum . . . 61,

Prosopistoma (larve du)

Protagrion

Protentomon (théorie du).

Prothorax . . 60,61,

Protophasma Dumasi

Protocérébron

Proventricule . . . 101

Pseudonévroptères . . . 334

Pseudonymphe . . . 233

Pteronarcys regalis . . . 129

Ptérophores.

Ptérygogènes

Puce.

Pucerons(parthénogenèst des) 195

Pucerons (migration des)

192

Puceron lanigère

197

Punaise des lits.

322

Pupe.

229 ,

Pygidium

Pyrophorus noctilucus.

\section{$\mathbf{R}$}

Races de vers à soie.

bivoltine.

94

65

207

47

43

9

200

59

Races polyvoltine.

199

93

43

136

62

233

35

62

77

59

(1)

4 San

Sang des Insectes
Sarcolemme"

Savigny (Lelorgne de) . . 16

Savigny (théorie de). . . 49

Scape (voir:Antennes). . 48

Scarabéiforme . . . . 237

Scarabée sacré . . . 298

Scha um (R.) . . . . . 23

Schïрte (J. (.). . . . 27

SchœNHER . . . . $\quad 16$

Sclérites . . . . . 42

89 Scolophophore . . . . 70

44 Scolytides (accouplement) . 206

Scutellum . . . . . 43

Scutum . . . . . . 43

Segment médiaire - . $\quad 66$

LES INSECTES, $-2^{\mathrm{e}}$ édit. 
Segment prémandibulaire . Segments abdominaux . Sogmentation de l'œuf Səns "du goût

" de l'odorat

$$
\text { " l'ouie }
$$

"du toucher

" de la vision

Séreuse

Séricine

Serville (Aüdinet)

Simulies

Sinus ventral

Soie (structure) . . 132

SPENCE (W.).

Spermatogénèse. :

Spermatophores

Spermatosyzygie

Spermatozoïdes ${ }^{7}$ (Spermatozoaires)

Stations entomologiques

Stemmates

Sternites

Sternorhabdites .

Sternum .

Stigmates

Stomodæum .

Stomoxys calcitrans.

Straus-Durckheim (H.) .

Strepsiptères

Stridulation .

Styles

Stylops (dimorphisme des)

Suceurs (Insectes) .

Sustillo

Synapsis.

Système musculaire

"nerveux
59

163

166

220

134

18

$241, \quad 244$

11:

134

41

$1 \%$

179

178

179

235

313

166

43

185

43

126

323

327

90,91

201

53 306

179

146

157
Système nerveux viscéral . 161

Sivamuerdam (J.) . . .

\section{$\mathbf{T}$}

Tachinaires .

Taons . . . . 32";

Tarière . . . 180, 183

Tarse . . . . . . 69

Tégulæ . . . . . . 74

Téguments . . . . . 38

" (ornementation des) 92

Téléphores . . . . 96

Tempes . . . . . 45

Temps primaires . . 275

" secondaires . . 278

"tertiaires. . . 280

Tenthrèdes . . . . . 236

Tentorium . . . . 47

Térébrants . . . . 327

Tergites . . . . . 43

Tergorhabdites . . . 185

Tergum . . . . . . 43

Termitas . . . 305, 335

Testicules . . . 176, 177

Tête . . . . . 45

Tétraptères . . . . . 72

Théorie de l'asphyxie . . 273

" des blastèmes . . 271

" de Fritz-Müller. 85

"de Gegenbaur . 85

" de la lyocytose . 272

" de la phagocytose. 271

"de la vision. . . 169

"du vol. . . 150

Thorax . . . . . . 60

\(appendices du) . 67

Thysanoures . . .74, 331

Tibia (voir : Jambe) . 69

Timarcha tenebricosa . . 119 


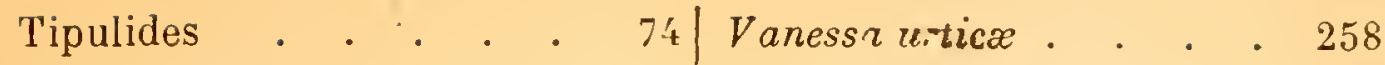

Tissu adipeux . . . . 141 " Io . . . . 258

Titanophasma Fayoli. . 277 " Antiopa . . 257

Trachées . . . . . 123

Ventricule chylifique $\quad 101$

" fasciculeuses . . 123 Ventriculites . . . 114

" tubuleuses . . 123 Ver à soie . . 133, 134

" vésiculeuses . . 124 Ver luisant . . . . 189

Trachéates . . . . 3

3 Vermiformes . . . 237

Triongulin . . . . 235 Vertex . . . . 46

Tritocérébron . . 159, 24i Vésicants (Insectes) . 30, 238

Trochanter . . . . 68 Vésicules abdominales . . 98

Trompe . . . . . 104 Vésicule céphalique. . . 253

Trypanosome . . . 322 . séminale . 178, 205

Tubes de Malpighi. 108, 242 Vision (sens de la) . . 166

Tube digestif . . 97, 242 Vitellus . . . . . 215

Tychoparthénogénèse . . 198 Viviparité . . . . 196

\section{$\mathbf{U}$}

Uranidine . . . . 119

Uromères . . . . $\quad 87$

Urosomes . . . . 87

Urosternites . . . 87

Urotergites . . . . 87

Utérus (voir : Vagin) : 181

\section{V}

Vagin . . . 181, 251)

Vaisseau dorsal . . . 113

Valuisnieri (A.) . . . 10

Valvule cardiaque . . 104

- cesophagienne 102, 104

Vanessa geographica . . 256

"levana. . . 257

- porima . . 257

" prorsa. . . . 257

Xylophages . . . . 254

\section{$\mathbf{X}$}

Yeux . . . 47, 54

Yeux acones . . . 167

Yeux à facettes . . . 167

Yeux composés. . . 167

Yeux eucones . . . 168

: pseudacones . . 168

Zetterstedt (J. W.) . . 19

Zone germanitive • . 219

Zoocécidies . . . . 324

Zoonite. . . . . 41 



\section{TABLE SYSTÉMATIQUE DES MATIERES}

Préface. . . . . . . . . . . . . XI

Tableau synoptique des Arthropodes . . . . XII

I. Généralités sur l'Entomologie . . . . . : . 1

II. Définition du type Insecte . . . . . . 2

\section{INTRODUCTION. - Histoire de l'Entomologie.}

I. L'Entomologie dans l'antiquité et au Moyen-Age. 5 II. L'Entomologie dans les temps modernes . . . 7 III. L'Entomologie dans les temps actuels. . . . 16

\section{LIVRE PREMIER}

\section{PREMIÈRE PARTIE. - Morphologie externe.}

\section{CHAPITRE PREMIER}

Caractères généraux des Insegtes. . . . . 33

Théorie du Protentomon . . . . . . . . . 35

\section{CHAPITRE II}

Tégunents des Insegtes * . . . . . . 38

Cuticule; origine de la membrane cuticulaire. . 39

Propriétés de la Chitine . . . . . . . . 41

Constitution des segments (métamérie) . . . 42

Les trois régions du corps. . . . . . . . 44 


\section{CHAPITRE III}

La Tête et ses appendices . . . . . . . . 45

Pièces buccales; théorie de Savigny . . . . . 49

Signification des pièces céphaliques . . . . . 54

Origine métamérique de la tête . . . . . . 58

\section{CHAPITRE IV}

Le Thorax et Ses appendiges . . . . . . . . 60

Les trois parties du Thorax . . . . . . . . 60

Thorax des Hyménoptères . . . . . . . . . 66

Appendice du thorax : pattes et ailes. . . . . 67

Classification des Insectes d'après les ailes . . . 74

\section{CHAPITRE V}

Nervation des alles • . . . . . . . . . . 78

Naissance des premières nervures . . . . . 78

Évolution et classification des nervures . . . . 81

Origine des ailes . . . . . . . . . . . 84

\section{CHAPITRE VI}

L'Ábdomen et ses appendices . . . . . . . . 87

Constitution de l'abdomen . . . . . . 87

Membres abdominaux des Thysanoures . . . 90

Styles et Cerques. . . . . . . . . . . . 91

\section{CHAPITRE VII}

Ornementation et armature des téguments • $\quad 92$

Poils et leurs principales adaptations . . . . 93

Écailles . . . . . . . . . . . . 95

Glandes cutanées et glandes cirières . . . . . 96 


\section{DEUXIÈME PARTIE. - Fonctions de nutrition.}

\section{CHAPITRE VIII}

L'APpareil digestif et SES ANNexes . . . . 9 97

Anatomie du tube digestif . . . . . . . . . 103

Organes annexes du tube digestif . . . . . 105

Glandes salivaires . . . . . . . . . . 106

Cocums gastriques. . . . . . . . . . 10 \%

Tubes de Malpighi. . . . . . . . . . 108

Physiologie de la digestion . . . . . . . 110

\section{CHAPITRE IX}

Appareil circulatolre et circulation. . . 112

Historique de la circulation . . . . . . . . 112

Appareil circulatoire . . . . . . . . . 112

Mécanisme de la circulation . . . . . . 117

Organes pulsatiles des Hémiptères . . . . . 117

Le sang des Insectes . . . . . . . . 118

CHAPITRE $\mathrm{X}$

Historique de la Respiration . . . . . . . 120

I:Appareil respiratoire et ses annexes . . . 120

I. Insectes aériens : trachées. . . . . . . . 123

Origine des trachées . . . . . . . 125

Stigmates, leur structure . . . . . . . 126

Appareil d'occlusion des stigmates . . . 128

II. Insectes aquatiques : branchies . . . . . . 128

Branchies permanentes . . . . . 129

Mécanisme de la respiration . . . . . . 130

\section{CHAPITRE XI}

Appareil de sécrétion et d'épuration . . . . . 132

Épuration de l'organisme . . . . . . . . 132

Soie et glandes séricigènes . . . . . . . . . 132 
Constitution de la soie. . . . . . . . . . 134

Glandes cirières . . . . . . . . . . 136

Glandes venimeuses . . . . . . . . . 137

Glandes défensives . . . . . . . . . . . 138

Glandes à cément (glandes sétifiques) . . . . . 139

Glandes dermiques . . . . . . . . 170

\section{CHAPITRE XII}

Réserves nutritives . . . . . . . . . . 141

Corps adipeux et ses dérivés . . . . . . . 141

Cellules adipeuses . . . . . . . . . . . 142

Cellules péricardiques . . . . . . . . . . 143

Enocytes . . . . . . . . . . 143

Organes lumineux . . . . . . . . 144

Organes spléniques . . . . . . . . . . . 145

\section{TROISIEME PARTIE. - Fonctions de relations.}

\section{CHAPITRE XIII}

Appareil musculaire . . . . . . . . . . . 146

I. Dispositions générales des muscles . . . . 146

A. Muscles de la tête. . . . . . . . . 148

B. Muscles du thorax. . . . . . . . . 149

Théorie du rol. . . . . . . . . . 150

C. Muscles de l'abdomen . . . . . . . 153

II. Structure des muscles. . . . . . . . . 153

III. Insertion des muscles sur les téguments . . . 155

IV. Force musculaire des Insectes . . . . . 156

\section{CHAPITRE XIV}

APPareil Nerveux ET ORganes des SENS . . . 157

Système nerveux central . . . . . . . 157

Cerveau . . . . . . . . . . . . . 158

Chaîne ventrale . . . . . . . . . . 160

Système nerveux viscéral . . . . . . . . 161 


\section{CHAPITRE XV}

ORGANES DES SENS . . . . . . . . . . 163

Sens du toucher. . . . . . . . . 163

Sens du goût. . . . . . . . . . 164

Sens de l'odorat. . . . . . . . . . . 165

Sens de la rision . . . . . . . . . . 166

Théorie de la Vision . . . . . . . . . 169

Sens de l'ouïe . . . . . . . . . . . 169

Organes chordotonaux . . . . . . . . 170

Bruits produits par les Insectes . . . . . . 171

A. Bourdonnement . . . . . . . . 172

B. Stridulation . . . . . . . . . 172

C. Appareil tympanique des Cigales . . . 173

\section{QUATRIÈME PARTIE}

Fonctions de reproduction.

\section{CHAPITRE XVI}

APPAREIL REPRODUCTEUR . . . . . . . . 174

Généralités sur l'appareil reproducteur . . . . 174

Évolutiun de l'appareil reproducteur . . . . . 175

Appareil reproducteur des mâles . . . . . 176

A. Testicules . . . . . . . . . . . 177

B. Canaux déférents . . . . . . . 178

C. Canal éjaculateur . . . . . . . . . 178

D. Glandes accessoires . . . . . . . . 178

Appareil reproducteur des femelles . . . . 179

A. Ovaires et gaines ovariques . . . . . 180

B. Oviductes et canal ovarien . . . . . 181

C. Poche copulatrice et réservoir séminal. . 181

Fécondation . . . . . . . . . . 182 


\section{CHAPITRE XVII}

Armures Génitales . . . . . . . . . . . . 183

10 Armure génitale des femelles . . . . . . 184

$2^{\circ}$ Armure génitale des mâles . . . . . . 185

\section{CHAPITRE XVIII}

Caractères Sexuels Secondatres • . . . . 186

Tariations dans la taille, les appendices, etc. . . 187

\section{CHAPITRE XIX}

Reproduction asexuée : La Parthénogénèse . . . 190

Généralités sur la Parthénogénèse . . . . . . 190

Parthénogénèse normale (Isoparthénogénèse) • . 193

Parthénogénèse cyclique (Hétéroparthénogénèse) . 195

Parthénogénèse accidentelle (Tychoparihénogénèse). 198

Parthénogénèse larvale (Pædogénèse) . . . . 199

Parthénogénèse expérimentale . . . . . . 201

\section{CHAPITRE XX}

Divers actes de la reproduction sexuée . • . 203

Divers modes d'accouplement des Insectes . . . 203

Ponte des œufs - leur nombre - mode de ponte. 207

\section{CHAPITRE XXI}

L'Ceuf et ses enveloppes. . . . . . . . . 212

Structure de l'œul . . . . . . . . . . . 214

Segmentation. - Formation du blastoderme - . $21 \%$ 


\section{LIVRE DEUXIEME \\ PREMIÈRE PARTIE. - Embryogénie.}

\section{CHAPITRE I}

Développentent de L'EMBryon * . . . . . . 219

Formation des membranes embryonnaires. . . 219

Modifications dans la formation des membranes embryonnaires . . . . . . . . . . . 221

Origine des membranes embryonnaires . . . 223

\section{CHAPITRE II}

FORMATION DES FEULLETS EMBRYONNAIRES . • . . 224

\section{CHAPITRE III}

UÉveloppenent POSTEMBRYONAARE 。 • . . . 227

Les premières phases de la vie larvaire. . . . 227

Métamorphoses des Insectes . . . . . . 229

Différentes formes de larves . . . . . . . 233

\section{DEUXIÈME PARTIE}

Biologie générale des larves.

\section{CHAPITRE IV}

Morphologie externe des larves . . . . . . $23 !$

Morphologie interne des larves . . . . . . 242

Branchies rectales des Libellulides . . . . . . 244 


\section{CHAPITRE V}

FONCTIONS DE RElations. . . . . . . . . 245

Appareil musculaire des larves . . . . . . . 245

Système nerveux et organes des sens . . . . 245

Développement du cerveau . . . . . . 247

\section{CHAPITRE VI}

Fonctions de reproduction . . . . . . . 249

Organes génitaux et cellules reproductrices. . . 249

\section{CHAPITRE VII}

Biologie des larves . . . . . . . . 251

Modes d'éclosion des larves . . . . . . 251

Mues ; mécanisme de la mue . . . . . . . 253

Influence des agents physiques sur l'évolution . . 255

\section{CHAPITRE VIII}

La Nymphe des Insectes. . . . . . . . . 259

Phénomènes précurseurs de la nymphose . . . $\quad 259$

Différentes formes de nymphes . . . . . . 259

Ornementation des nymphes . . . . . . . 261

Anatomie internẹ des nymphes . . . . . . 261

\section{CHAPITRE IX}

Histolyse et Histogénèse . . . . . . . 263

Phénomènes intimes de la nymphose . . . . . 263

Disques imaginaux (Histoblastes). . . . . . 265

Histogénèse des organes . . . . . . . 266

La marche de l'histolyse . . . . . . . . 268

Considérations générales sur les métamorphoses . 269 


\section{LIVRE TROISIEME}

\section{PREMIÈRE PARTIE. - Entomologie économique.}

\section{CHAPITRE I}

Paléontomologie . . . . . . . . . . . 275

Les Insectes dans les temps géologiques . . . 275

Insectes des temps primaires . . . . . . . 275

Insectes des temps secondaires . . . . . . 278

Insectes des temps tertiaires. . . . . . 280

Distribution des Insectes aux différentes époques

géologiques .. . . . . . . . . . . . . 283

CHAPITRE II

Moyens de défense chez les Insectes. . . . 284

Homochromie . . . . . . . . . . 287

Mimétisme . . . . . . . . . . . 287

Insectes à métamorphoses graduelles . . . . . 288

Insectes à métamorphoses complètes . . . . . 289

\section{CHAPITRE III}

Distribution géographique des Insegtes . • . 292

Insectes mauvais voiliers . . . . . . . . 293

Les grandes régions entomologiques . . . . 294

Insectes bons voiliers . . . . . . . , 295

\section{CHAPITRE IV}

Rôle économique des Insegtes . . . . . . 298

Les Insectes et les religions antiques . . . . 298

Insectes objets de luxe et de parure . . . . . 300

Insectes utiles à l'alimentation . . . . . . . 301

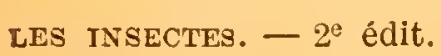


La manne des Iébreux. . . . . . . . . 302 Insectes utilisés en médecine : . . . . . 306

Les Coléoptères vésicants . . . . . . . . 307 Insectes utiles à l'industrie . . . . . . . . 308

La soie. Les Lépidojtères séricigènes . . . . . 310

\section{CHAPITRE V}

Les Insectes et L'Agriculture • • • • • • 313

Le rôle de l'Entomologie économique . . . . . 313

Les Stations entomologiques . . . . . . 313

Les grands problèmes entomologiques . . . 318

\section{GHAPITRE VI}

Le Parasitishe chez les Insectes . • . • $\$ 21$ Insectes parasites de l'homme et des animaux . . 321 Insectes parasites des végétaux. - Cécidologie. . 324 Les parasites des Insectes . • • • • . . 326

\section{DEUXIÈME PARTIE. - Entomologie appliquée.}

\section{CHAPITRE VII}

Clasification des Insectes • . . . . . . . 329

Principes de la classification . . . . . . 329

Tableaux analytiques de détermination, permettant de ranger rapidement un Insecte donné dạs l'ordre auquel il appartient . . . . . . 336

Caractères généraux des principaux ordres d'Insectes • . . . . . . . . . . . 334 Iridex bibliographigue • . . . . . . . . 343

TABLE ALPHABÉtiQUE DES AUteURS ET DES MATIL̀RES. 353 Table systématigue des matières. . . . . 365 


\section{ENCYCLOPÉDIE SCIENTIFIQUE}

Publiée sous la direction du Dr TOULouse

Nous avons entrepris la publication, sous la direction générale de son fondateur, le $D^{r}$ Tculouse, Directeur à l'École des Hautes-Études, d'une Excyclopénie sciextifinue de langue française dont on mesurera l'importance à ce fait qu'elle est divisée en 40 sections ou Bibliothèques el qu'elle comprendra environ 1000 volumes. Elle se propose de rivaliser avec les plus grandes encyclopédies étrangères et même de les dépasser, tout à la fois par le caractère nettement scientifique et la clarté de ses exposés, par l'ordre logique de ses divisions et par son unité, enfin par ses vastes dimensions et sa forme pratique.

\section{PLAN GÉNERAL DE I'ENCYCLOPÉDIE}

Mode de publication. - L'Encyccopédie se composera de monographies scientifiques, classées méthodiquement et formant dans leur enchaînement un exposé de toute la science. ('rganisée sur un plan systématique, cette Encyclopédie, tout en éritant les inconvénients des Traités, - massifs, d'un prix ğlobal élevé. difficiles à consulter, - et les inconvénients des Dictionnaires, où les articles scindés irrationnellement, simples chapitres alphabétiques, sont toujours nécessairement incomplets, - réunira les avantages des uns et des autres.

Du Traité, l'Encyclopédie gardera la supériorité que possède 
un ensemble complet, bien divisé et fournissant sur chaque science tous les enseignements et tous les renseignements qu'on en réclame. Du Dictionnaire, l'Encyclopédie gardera les facilités de recherches par le moyen d'une table générale, l'Index de l'Encyclopédie, qui paraîtra dès la publication d'un certain nombre de volumes et sera réimprimé périodiquement. L'Index renverra le lecteur aux différents volumes et aux pages où se trouvent traités les divers points d'une question.

Les éditions successives de chaque volume permettront de suivre toujours de près les progrès de la science. Et c'est par là que s'affirme la supériorité de ce mode de publication surtout autre. Alors que, sous sa masse compacte, un traité, un dictionnaire ne peut ètre réédité et renouvelé que dans sa totalité et qqu'à d'assez longs intervalles, inconvénients graves qu'atténuent mal des suppléments et des appendices, l'Encyclopédie scientifique, au contraire, pourra toujours rajeunir les parties qui ne seraient plus au courant des derniers travaux importants. Il est évident, par exemple, que si des livres d'algèbre ou d'acoustique physique peuvent garder leur valeur pendant de nombreuses années, les ouvrages exposant les sciences en formation, comme la chimie physique, la psychologie ou les technologies industrielles, doivent nécessairement être remaniés à des intervalles plus courts.

La lecteur appréciera la souplesse de publication de cette Ency. clopédie, toujours vivante, qui s'élargira au fur et à mesure des besoins dans le large cadre tracé dès le début, mais qui constituera toujour's, dans son ensemble, un traité complet de la Science, dans chacune de ses sections un traité complet d'une science, et dans chacun de ses livres une monographie complète. Il pourra ainsi n'acheter que telle ou telle section de l'Encyclopédie, sûr de n'avoir pas des parties dépare:llées d'un tout.

L'Encyclopédie demandera plusieurs années pour être achevée car pour avoir des expositions bien faites, elle a pris ses collaborateurs plutôt parmi les savants que parmi les professionnels de la rédaction scientifique que l'on retrouve généralement dans les œuvres similaires. Or les savants écrivent peu et lentement; et il est prélérable de laisser temporairement sans attribution certains ouvrages plutôt que de les confier à des auteurs insuffisants. Mais cette lenteur et ces vides ne présenteront pas d'inconvénients, puisque chaque livre est une curre indépendante 
et que tous les volumes publiés sont à tout moment réunis par l'Index de l'Encyclopédie. On peut donc considérer l'Encýclopédie comme une librairie, où les livres soigneusement choisis, au lien de représenter le hasard d'une production individuelle, obéiraient à un plan arrêté d'avance, de manière qu'il n'y ait ni lacune dans les parties ingrates, ni double emploi dans les parties très cultivées.

Caractère scientifique des ouvrages. - Actuellement, les lirres de science se divisent en deux classes bien distinctes : les livres destinés aux savants spécialisés, le plus souvent incompréhensibles pour tous les autres, faute de rappelèr au début des chapitres les connaissances nécessaires, et surtout faute de définir les nombroux termes techniques incessamment forgés, ces derniers rendant un mémoire d'une science particulière inintelligible à un savant qui en a abandonné l'étude durant quelques années; et ensuite les livres écrits pour le grand public, qui sont sans profit pour des savants et même pour des personnes d'une certaine culture intellectuelle.

L'Encyclopédie scientifique a l'ambition de s'adresser au public le plus large. Le savant spécialisé est assuré de rencontrer dans les volumes de sa partie une mise au point très exacte de l'état actuel des questions; car chaque Bibliothèque, par ses techniques et ses monographies, est d'abord faite avec le plus grand soin pour servir d'instrument d'études et de recherches à ceux qui cultivent la science particulière qu'elle représente, et sa devise pourrait être : Par les savants, pour les-savants. Quelques-uns de ces livres seront même, par leur caractère didactique, destinés à devenir des ouvrages classiques et à servir aux études de l'enseignement secondaire ou supérieur. Mais, d'autre part, le lecteur' non spécialisé est certain de trouver, toutes les fois que cela sera nécessaire, au seuil de la section, - dans un ou plusieurs volumes de généralités, - et au seuil du volume, - dans un chapitre particulier, - des données qui formeront une véritable introduction le mettant à même de poursuivre avec profit sa lecture. Un vocabulaire teclınique, placé, quand il y aura lieu, à la fin du volume, lui permettra de connaître toujours le sens des mots spéciaux. 


\section{II}

\section{ORGA NISATION SCIENTIFIQUE}

Par son organisation scientifique, l'Encyclopédic paraît devoir offrir aux lecteurs les meilleures garanties de compétence. Elle est divisée en sections ou Bibliothèques, à la tête desquelles sont placés des savants professionnels spécialisés dans chaque ordre de sciences et en pleine force de production, qui, d'accord avec le Directeur général, établissent les divisions des matières, choisissent les collaborateurs et acceptent les manuscrits. Le même esprit se manifestera partout: éclectisme et respect de toutes les opinions logiques, subordination des théories aux données de l'expérience, soumission à une discipline rationnelle stricte ainsi qu'aux règles d'une exposition méthodique et claire. De la sorte, le lecteur, qui aura été intéressé par les ouvrages d'une section dont il sera l'abonné régulier, sera amené à consulter avec confiance les livres des autres sections dont il aura besoin, puisqu'il sera assuré de trouver partout la même pensée et les mêmes garanties. Actuellement, en effet, il est, hors de sa spécialité, sans moyen pratique de juger de la compétence réelle des auteurs.

Pour mieux apprécier les tendances variées du travail scientifique adapté à des fins spéciales, l'Encyclopédie a sollicité, pour la direction de chaque Ribliothèque, le concours d'un savant placé dans le centre même des études du ressort. Elle a pu ainsi réunir des représentants des principaux colps savants, Établissements d'enseignement et de recherches de langue française :

Institut.

Académie de Médecine.

Collège de France.

Huséum d'Histoire naturelle. Ecole des Hautes-Etudes.

Sorbonne et Ecole normale.

Facultés des Sciences.

Facultés des Leitres.

Facultés de Médecine.

Institut Pasteur.

Ecole des Ponts et Chaussées.

Ecoles des Wines.
Ecole Polytechnique.

Conservatoire des Arts et Métier's. Ecole d'Anthropologie.

Institut National agronomique.

Ecole vétérinaire d' Aljort.

Ecole supéricure d'Electricité.

Ecole de Chimie industrielle de Lyon.

Ecole des Beaux-Arts.

Ecole des isciences politiques.

Obscriatnire de Paris.

IIôpitaur de Paris. 


\section{III}

\section{BUT DE L'ENCYCLOPEDIE}

Au xvin siècle, "l'Encyclopédie " a marqué un magnifique mouvement de la pensée vers la critique rationnelle. A cette époque, une telle manifestation devait avoir un caractère philosophique. Aujourd'hui, l'heure est venue de renouveler ce grand effort de critique, mais dans une direction strictement scientifique ; c'est là le but de la nouvelle Encyclopédie.

Ainsi la science pourra lutter avec la littérature pour la direction des esprits cultivés, qui, au sortir des écoles, ne demandent gruère de conseils qu'aux cenvres d'imagination et à des encyclcpédies où la science a une place restreinte, tout à fait hors de proportion avec son importance. Le moment est favorable à cette tentative; car les nouvelles générations sont plus instruites dans l'ordre scientifique que les précédentes. D'autre part, la science est devenue, par sa complexité et par les corrélations de ses parties, une matière qu'il n'est plus possible d'exposer sans la collaboration de tous les spécialistes, unis là comme le sont les producteurs dans tous les départements de l’activité économique contemporaine.

I un autre point de rue, l'Encyclopédıe, embrassant toutes les manifestations scientifiques, serrira comme tout inventaire à mettre au jour les lacunes, les champs encore en friche ou abandonnés, - ce qui expliquera la lenteur avec laquelle certaines sections se développeront, - et suscitera peut-être les travaux nécessaires. Si ce résultat est atteint, elle sera fière d'y aroir contribué.

Elle apporte en outre une classification des sciences et, par ses divisions, une tentative de mesure, une limitation de chaque domaine. Dans son ensemble, elle cherchera à refléter exactement le prodigieux effort sciéntifique du commencement de ce siècle et un moment de sa pensée, en sorte que dans l'avenir elle reste le document principal où l'on puisse retrouver et consulter le témoignage de cette époque intellectuelle.

On peut voir aisément que l'Encyclopédie ainsi conçue, ainsi réalisée, aura sa place dans toutes les bibliothèques publiques, universitaires et scolaires, dans les laboratoires, entre les mains 
des savants, des industriels et de tous les hommes instruits qui veulent se tenir au courant des progrès, dans la partie qu'ils cultivent eux-mêmes ou dans tout le domaine scientifique. Elle fera jurisprudenc:- ce qui lui dicte le devoir d'impartialité qu'elle aura à remplir.

Il n'est plus possible de vivre dans la société moderne en ignorant les diverses formes de cette activité intellectuelle qui révolutionne les conditions de la vie; et l'interdépendance de la science ne permet plus aux savants de rester cantonnés, spécialisés dans un étroit domaine. Il leur faut, - et cela leur est souvent difficile, - se mettre au courant des recherches voisines. A tous l'Encyclopédie offre un instrument unique dont la portée scientifique et sociale ne peut échapper à personne.

\section{IV}

\section{CLASSIFICATION DES IATIÈRES SCIENTIFIQUES}

La division de l'Encyclopédie en Bibliothèques a rendu nécessaire l'adoption d'une classification des sciences, où se manifeste nécessairement un certain arbitraire, étant donné que les sciences se distinguent beaucoup moins par les différences de leurs objets que par les divergences des aperçus et des habitudes de notre esprit. Il se produit en pratique des interpénétrations réciproques entre leurs domaines, en sorte que, si l'on donnait à chacun l'étendue à laquelle il peut se croire en droit de prétendre, il envahirait tous les territoires voisins; une limitation assez stricte est nécessitée par le fait même de la juxtaposition de plusieurs sciences.

Le plan choisi, sans viser à constituer une synthèse philosophique des sciences, qui ne pourrait être que subjective, a tendu pourtant à échapper dans la mesure du possible aux habitudes traditionnelles d'esprit, particulièrement à la routine didactique, et à s'inspirer de principes rationnels.

Il y a deux grandes divisions dans le plan général de l'Encyclopédic: d'un côté les sciences pures, et, de l'autre, toutes les technologies qui correspondent à ces sciences dans la sphère des applications. A part et au début, une Bibliothèque d'introduc- 
tion générale est consacrée à la philosophie des sciences (histoire des idées directrices, logique et méthodologiel.

Les sciences pures et appliquées présentent en outre une divi. sion générale en sciences du monde inorganique et en sciences biologiques. Dans ces deux grandes catégories, l'ordre est celui de particularité croissante, qui marche parallèlement à une rigueur décroissante. Dans les sciences biologiques pures enfin, un groupe de sciences s'est trouvé mis à part, en tant qu'elles s'occupent moins de dégager des lois générales et abstraites que de fournir des monographies d'êtres concrets, depuis la paléontologie jusqu'à l'anthropologie et l'ethnographie.

Étant donnés les principes rationnels qui ont dirigé cette classification, il n'y a pas lieu de s'étonner de voir apparaître des groupements relativement nouveaux, une biologie générale, - une physiologie et une pathologie végétales, distinctes aussi bien de la botanique que de l'agriculture, - une chimie physique, etc.

En revanche, des groupements hétorogènes se disloquent pour que leurs parties puissent prendre place dans les disciplines auxquelles elles doivent revenir. La géographie, par exemple, retourne à la géologie, et il y a des géographies botanique, zoologique, anthropologique, économique, qui sont étudiées dans la botanique, la zoologie, l'anthropologie, les sciences économiques.

Les sciences médicales, immense juxtaposition de tendances tıès diverses, unies par une tradition utilitaire, se désagrègent en des sciences ou des techniques précises; la pathologie, science de lois, se distingue de la thérapeutique ou de l'hygiène, qui ne sont que les applications des données générales fournies par les sciences pures, et à ce titre mises à leur place rationnelle.

Enfin, il a paru bon de renoncer à l'anthropocentrisme qui exigeait une physiologie humaine, une anatomie humaine, une embryologie humaine, une psychologie humaine. L'homme est intégré dans la série animale dont il est un aboutissant. Et ainsi, son organisation, ses fonctions, son développement s'éclairent de toute l'évolution antérieure et préparent l'étude des formes plus complexes des groupements organiques qui sont offerts par l'étude des sociétés.

On peut voir que, malgré la prédominance de la préoccupation pratique dans ce classement des Bibliothèques de l'Encyclopédie scientifique, le souci de situer rationnellement les sciences dans leurs rapports réciproques n’a pas été négligé. Enfin il est à peine 
besoin d'ajouter que cet ordre n'implique nullement une hiérarchie, ni dans l'importance ni dans les difficultés des diverses sciences. Certaines, qui sont placées dans la technologie, sont d'une complexité extrême, et leurs recherches peuvent figurer-parmi les plus ardues.

Mode de publication. - Les volumes, illustrés pour la plupart, seront publiés dans le format in-18 jésus et cartonnés. De dimensions commodes, ils auront 350 pages environ, ce qui représente une matière suffisante pour une monographie ayant un objet défini et important, établie du reste selon l'économie du projet qui saura éviter l'émiettement des sujets d'exposition. 


\title{
TABLE DES BIBLIOTHÈQUES
}

\author{
Directeur : D- TouloUSE, Directeur de Laboratoire à l'Êcole \\ des Hautes-F̂tudes. \\ Secrétatre géséral : H. Piéron,
}

Directeurs des Bibliothèques:

1. Philosophie des Sciences. A. Rex, professeur d'Histoire de la Philosophie dans ses rapports arec la Science à la Sorbonne.

I. SCIENCES PURES

\section{A. Sciences mathématiques :}

2. Mathematiques . . . J. DrAch, chargé de cours à la Facultẻ des Sciences de l'Université de Paris.

3. Mécanique .

J. Drach, chargé de cours à la Faculté des Sciences de l'Université de Paris.

\section{B, Sciences inorganiques :}
4. Physique . . . . A. LEDdc, professeur adjoint de physique à la Sorbonne.
5. Chimie physique . . J. Perris, professeur de chimie physique à la Sorbonne.

6. Chimie . . . . A. Pictet, professeur à la Faculté des Sciences de l’Université de Genève.

7. Astronomie et Physique J. Mascart, professeur à l'Université, directeur céleste . . . . . de l'Observatoire de Lyon.

8. Météorologie . . . J. Mascart, professeur à l'Université, directeur de l'Observatoire de Lyon.

9. Minéralogie et Pétrogra- A. LACroIx, secrétaire perpétuel de l'Académie phie . . . . . des Sciences, professeur au Muséum d'Histoire naturelle.

10. Géologie

1I. Boule, professeur au Muséum d'Histoire naturelle, directeur de l'Institut de Paléon. tologie humaine.

11. Océanographie physique. J. RIchıRn, directeur du Musée Océanographique de Monaco. 


\section{Sciences biologiques normatives :}

12 Biologie générale. . . M. Caullery, professeur de zoologie à la Sorbonue.

13. Physique biologique . A. Inibert, professeur à la Faculté de Médecino de l'Université de Montpellier.

14. Chimie biologique - . G. Bertrand, professeur de chimie biologique à la Sorbonne, professeur a l'Institut Pasteur.

15. Physiologie et Patholo- L. MANGIN, de l'Institut, directeur du Muséum gic végétales d'Histoire naturelle.

16. Physiologie . . . . J.-P. LANGLors, professeur agrégé à la Faculté de Médecine de Paris, directeur de la Rerue générale des Sciences.

17. Psychologie . . . E. Toulouse, directeur de Laboratoire à l'Ecole des Hautes-Etudes, médecin en chef de l'asile de Villejuif.

18. Sociologie. . . . G. RrChalid, professeur à la Faculté des Lettres de l'Université de Bordeaux.

19. Microbiologie et Parasitologie.

A. Calmetre, professeur à la Faculté de Mẻdecine de l'Université, directeur de l'Institut Pasteur de Lille, et F. BESANcon, professeur à la Faculté de Médecine de l'Université de Paris, médecin des Hôpitaux.

\section{Sciences biologiques descriptives :}

21. Paléontologie.

M. Boule, yrofesseur au Muséum d'Histoire naturelle, directeur de l'Institut de Paléontologie humaine.

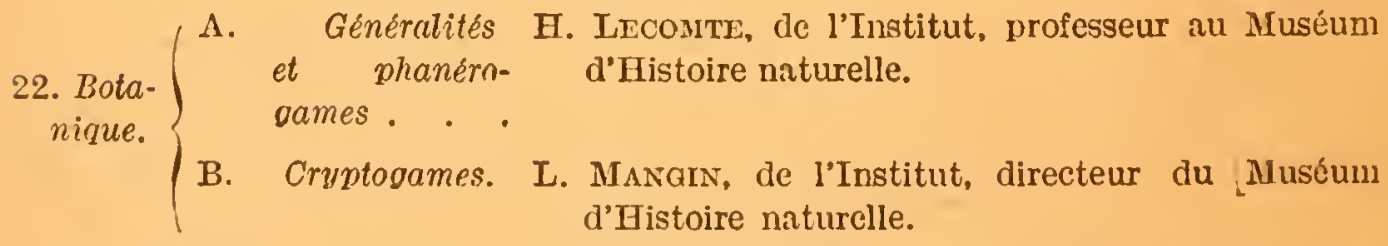

23. Zonlogie . . . . G. LorseL, dirccteur de T,aboratoire ì l'Ecole des Hautes-Litudes. 
24. Anatomie et Embryologie

25. Anthropologie et Ethnographie . . . .

26. Economie politique . .
G. Lorset, directeur de Laboratoire à l'Ecole des Hautes-Etudes.

G. PaPillault, directeur-adjoint du Laboratoire d'Anthropologie à l'Ecole des Hautes-Etudes, professeur à l'Ecole d'Anthropologie.

G. Renard, Professeur d'Histoire du Travail au Collège de France.

II. SCIENCES APPLIQUÉES

\section{A. Ściences mathématiques :}

27. Mathématiques appli- M. D’Ocagne, professeur à l'Ecole Polytechnique quées . . . . . et à l'Ecole des Ponts et Chaussées.

28. Mécanique appliquée et M. D’OcAgne, professeur à l'Êcole Polytechnique génie. . . . . et à l'Êcole des Ponts ei Chaussées,

\section{B. Sciences inorganiques :}

29. Industries physiques . .

H. Chaumat, professeur au Conservatoire des Arts et Métiers, sous-directeur de l'Ecole supérieure d'Electricité de Paris.

30. Photographie - . . A. Serewetz, soug-directeur de l'Ecole de Chimie industrielle de Lyon.

31. Industries chimiques . . J. DERôME, inspecteur général de l'Instruction publique, inspecteur cles Etablissements classès.

32. Géologie et minéralogie L. CAIEUX, professeur au Collège de France et à appliquses . . . l'Institut national agronomique.

33. Construction . . . N..

\section{Sciences biologinnes :}

34. Industries biologiques

35. Botanique appliquee et agriculture.

A. Phanisegames,

B. Cryptogames,

36. Zoologie appliqué

G. Bertrand, professeur de chimie biologique à la Sorbonne, professeur à l'Institut Pasteur.

H. LeCoMTe, de l'Institut, professeur au Muséum d'Histoire naturelle.

L. Manain, de l'Institut, directeur du Muséum d'Histoire naturelle,

J. Pellegrix, assistant au Muséum d'Histoire natırelle. 
37. Thérapeutique oénérale et pharmacologie. . .

38. Hygiène et médecine publiques . . . .

39. Psychologie appliquée

40. Sociologie appliguée.
G. Pouchet, membre de l'Académie de Iédecine, professeur à la Faculte de Médecine de l'Université de Paris.

A. Calmetre, professeur à la Faculté de Médecine de l'Université, directeur de l'Institut Pasteur de Lille.

E. Toulouse, directeur de Laboratoire à l'Ecole des Hautes-Etudes, médecin en chef de l'asile de Villejuif.

M. Albert Maire, bibliothécaire à la Sorbonne, est chargé de l'Index cee l'Encyclopédie scientiftque.

Paris-Lille. Imp. A. Taffin-Lefort. - 53-8-19. 



SMITHSONIAN INSTITUTION LIBRARIES

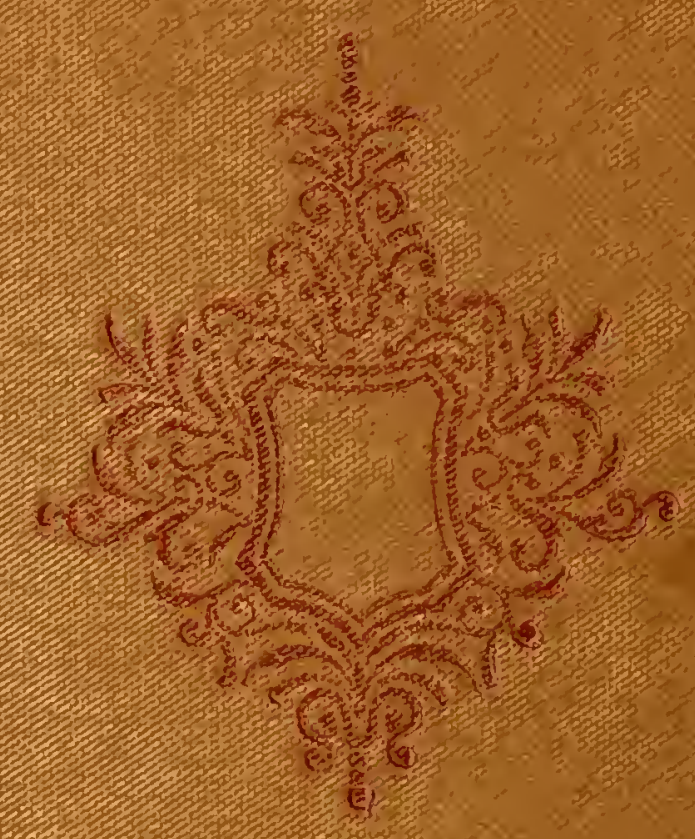

